\title{
Organic Thin Film \\ Transistor Integration \\ by
}

Flora M. Li

\author{
A thesis \\ presented to the University of Waterloo \\ in fulfillment of the \\ thesis requirement for the degree of \\ Doctor of Philosophy \\ in
}

Electrical and Computer Engineering

Waterloo, Ontario, Canada, 2008

CFlora Man-Wah Li 2008 
I hereby declare that I am the sole author of this thesis. This is a true copy of the thesis, including any required final revisions, as accepted by my examiners.

I understand that my thesis may be made electronically available to the public. 


\begin{abstract}
This thesis examines strategies to exploit existing materials and techniques to advance organic thin film transistor (OTFT) technology in device performance, device manufacture, and device integration. To enhance device performance, optimization of plasma enhanced chemical vapor deposited (PECVD) gate dielectric thin film and investigation of interface engineering methodologies are explored. To advance device manufacture, OTFT fabrication strategies are developed to enable organic circuit integration. Progress in device integration is achieved through demonstration of OTFT integration into functional circuits for applications such as active-matrix displays and radio frequency identification (RFID) tags.

OTFT integration schemes featuring a tailored OTFT-compatible photolithography process and a hybrid photolithography-inkjet printing process are developed. They enable the fabrication of fully-patterned and fully-encapsulated OTFTs and circuits. Research on improving device performance of bottom-gate bottom-contact poly $\left(3,3^{\prime \prime \prime}\right.$-dialkyl-quarterthiophene) (PQT-12) OTFTs on PECVD silicon nitride $\left(\mathrm{SiN}_{\mathrm{x}}\right)$ gate dielectric leads to the following key conclusions: (a) increasing silicon content in $\mathrm{SiN}_{\mathrm{x}}$ gate dielectric leads to enhancement in field-effect mobility and on/off current ratio; (b) surface treatment of $\mathrm{SiN}_{\mathrm{x}}$ gate dielectric with a combination of $\mathrm{O}_{2}$ plasma and octyltrichlorosilane (OTS) selfassembled monolayer (SAM) delivers the best OTFT performance; (c) an optimal $\mathrm{O}_{2}$ plasma treatment duration exists for attaining highest field-effect mobility and is linked to a "turnaround" effect; and (d) surface treatment of the gold ( $\mathrm{Au}$ ) source/drain contacts by 1octanethiol SAM limits mobility and should be omitted. There is a strong correlation between the electrical characteristics and the interfacial characteristics of OTFTs. In particular, the device mobility is influenced by the interplay of various interfacial mechanisms, including surface energy, surface roughness, and chemical composition. Finally, the collective knowledge from these investigations facilitates the integration of OTFTs into organic circuits, which is expected to contribute to the development of new generation of all-organic displays for communication devices and other pertinent applications. A major outcome of this work is that it provides an economical means for organic transistor and circuit integration, by enabling use of the well-established PECVD infrastructure, yet not compromising the performance of electronics.
\end{abstract}




\section{Acknowledgements}

I feel truly blessed, honored, and thankful to be approaching the end of my Ph.D. studies. Blessed...to be granted the opportunity to pursue my doctoral degree. Honored...to have interacted with brilliant and talented individuals who have enlightened, inspired, and helped me through my studies. Thankful...to be surrounded by loving and kind souls who have offered constant support and encouragement.

My utmost appreciation goes to my supervisor, Professor Arokia Nathan, who has been a wonderful mentor, an inspiration, and a true visionary. His ingenious advices guided me to many extraordinary opportunities, invaluable experiences, and academic achievements. I am absolutely grateful for his guidance, support, friendship, encouragement, trust and countless stimulating discussions.

My deepest gratitude goes to my family. It is my greatest fortune/blessing to have extraordinarily amazing parents, David and Adda Li, who have offered unconditional love, support, understanding, and nourishment. I am truly thankful for the freedom and luxury to explore my academic endeavors. Special thanks go to my grandmother, my brother Ben, and my sister Christina for bringing me joy and laughter when most needed.

I am indebted to Professor Beng Ong and Dr. Yiliang Wu from Xerox Research Centre of Canada (XRCC), who have offered invaluable insights and constant support. Much of the work in this thesis could not have been possible without their help, their expertise, and their magical organic materials.

I like to express my sincere appreciation to my fellow researchers and colleagues at the Giga-to-Nano Research Laboratory for their contributions to this work, for sharing their insights and expertise, for their assistance, and their guidance. These talented individuals include Dr. Jackson Lai, Dr. Kai Wang, Dr. Mohammad R. Esmaeili-Rad, Dr. Reza Chaji, Maryam Moradi, Dr. Yuri Vyangrenko, Prof. Peyman Servati, Hyun-Jung Lee, Prof. Andrei Sazonov, Dr. Isaac Chan, Dr. Denis Striakhilev, Dr. Sarswati Koul, Richard Barber, Robert Mullins, and Eliza Ho. It has been my pleasure and my honor to work with you.

Many thanks go to all my dear friends in Toronto and my extended family in GoodLife, for your persistent support and encouragement. I sincerely thank Uncle Norman and Auntie Anna for their superb generosity and kindness. Special acknowledgement goes to my 
superstar coach, Graham Patterson, for going the extra mile to keep me on track, positive, enthuse, and laser focus in thesis writing. Your daily motivation and words of wisdom will never be forgotten. I lovingly extend my gratitude to James for his adoring support and care during final stages of this work.

I acknowledge the financial support from Natural Sciences and Engineering Research Council (NSERC) of Canada, Ontario Centres of Excellence (OCE), and University of Waterloo.

Lastly, I want to thank everyone who have educated, inspired, and enlightened me along the way, to make my academic journey at University of Waterloo a truly remarkable and colorful experience.

"Do not go where the path may lead, go instead where there is no path and leave a trail." $\sim$ Ralph Waldo Emerson 


\section{To My Parents,}

My achievements are your achievements. 


\section{Table of Contents}

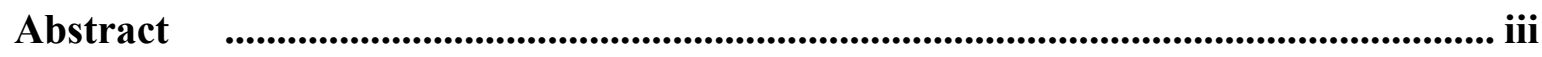

Acknowledgements..........................................................................................................................iv

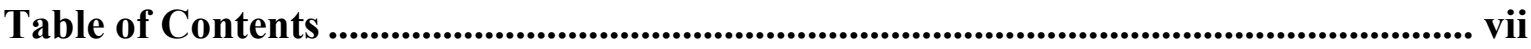

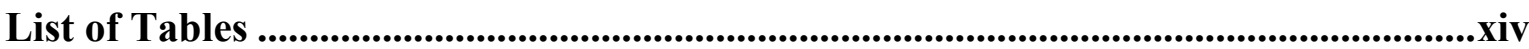

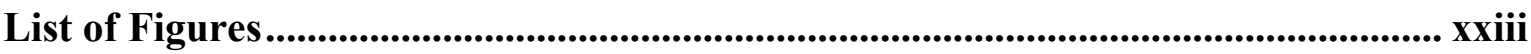

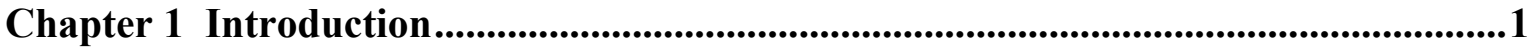

$1.1 \quad$ Organic Electronics: History and Market Opportunities .................................................... 3

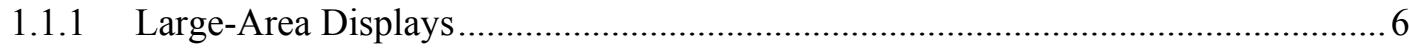

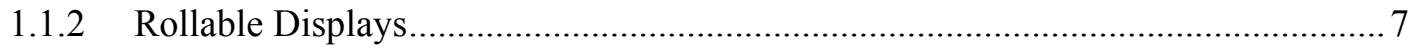

1.1.3 Radio Frequency Identification (RFID) Tag ................................................

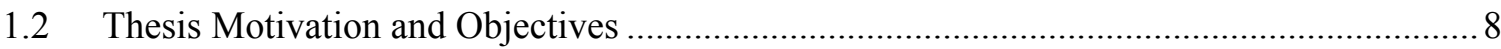

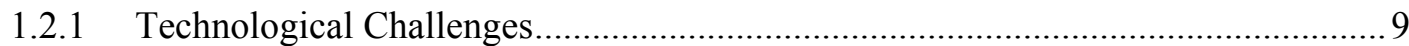

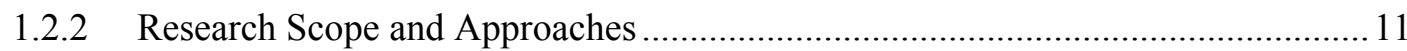

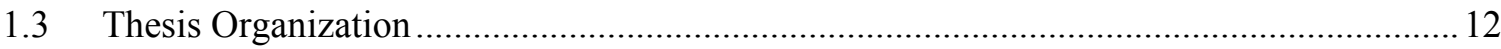

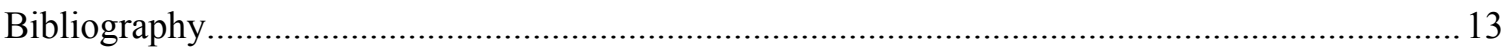

Chapter 2 Organic Thin Film Transistor (OTFT) Overview ....................................16

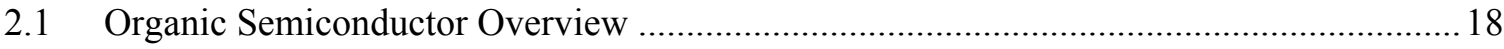

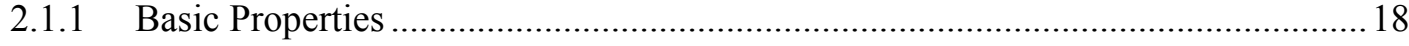

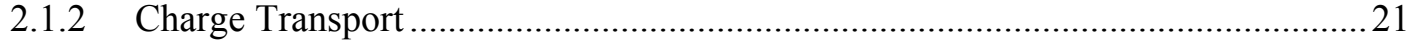

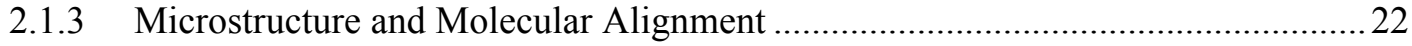

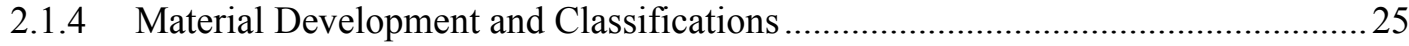

2.1.5 Sensitivity to Environmental Influences............................................................... 30 


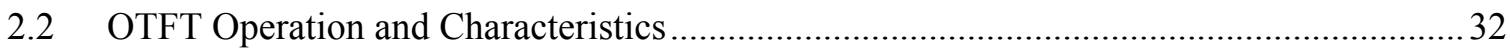

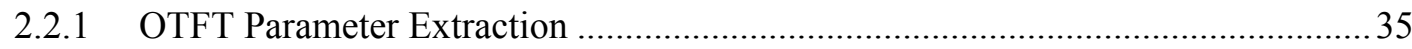

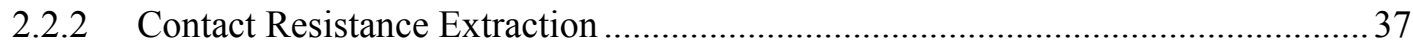

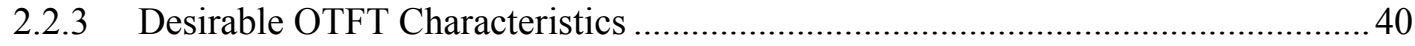

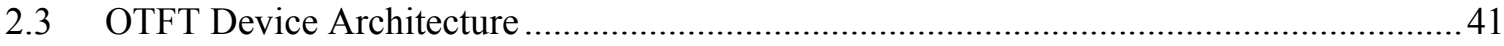

2.3.1 Top-Contact and Bottom-Contact OTFTs .............................................................. 41

2.3.2 Top-Gate, Bottom-Gate, and Dual-Gate OTFTs ...................................................... 42

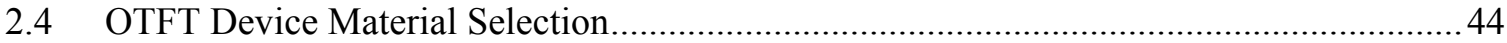

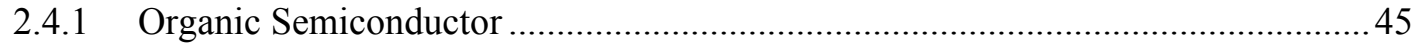

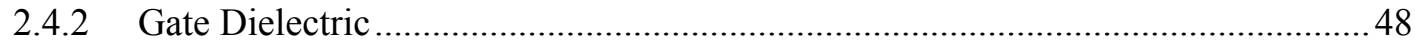

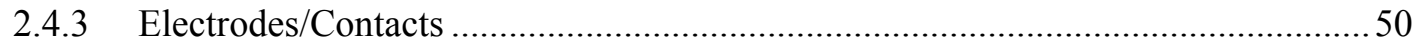

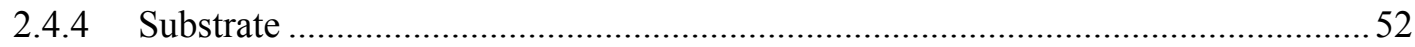

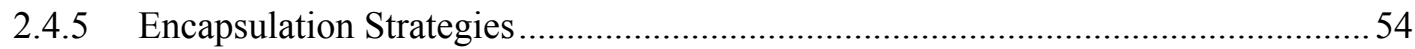

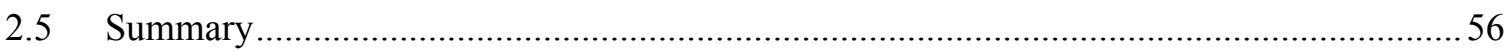

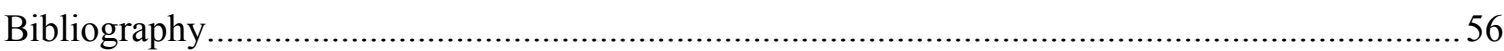

Chapter 3 OTFT Integration Strategies ............................................................62

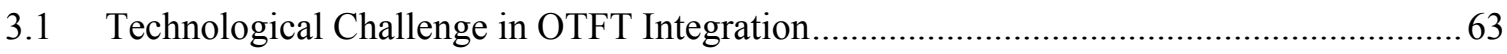

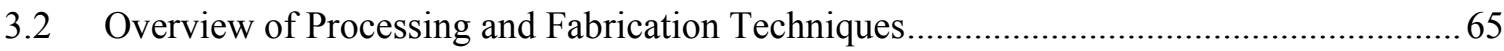

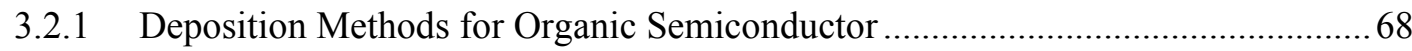

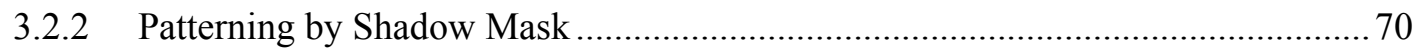

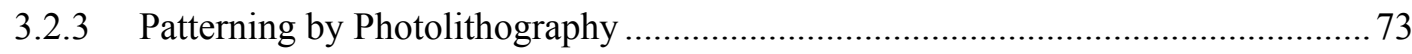

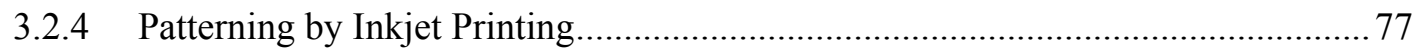

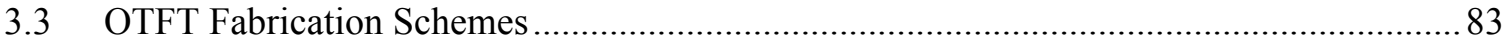

3.3.1 Basic 1-Mask Processing Scheme for Bottom-Gate OTFT ................................... 84

3.3.2 Photolithography Scheme for Fully-Patterned and Fully-Encapsulated Bottom-

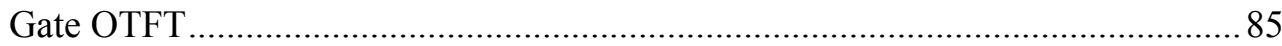

3.3.3 Hybrid Photolithography-Inkjet Printing Scheme for Fully-Patterned Bottom-Gate

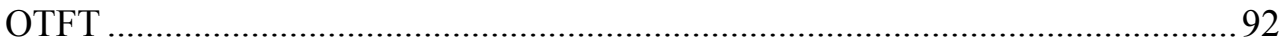

3.3.4 Photolithography Scheme for Top-Gate and Dual-Gate OTFTs ........................... 96

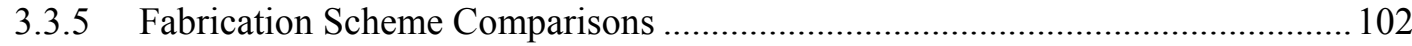

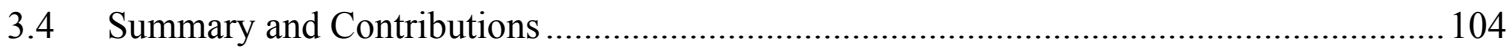

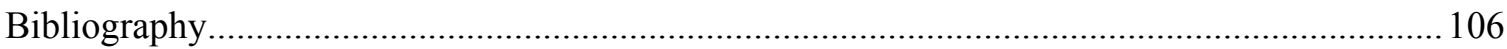




\section{Chapter 4 Gate Dielectric by Plasma Enhanced Chemical Vapor Deposition

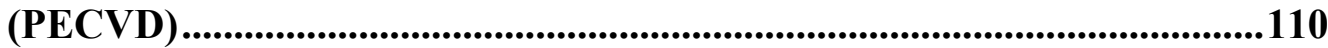

4.1 Experimental Details and Characterization Methods ………………………………........112

4.1.1 Deposition Conditions of PECVD Silicon Nitride $\left(\mathrm{SiN}_{\mathrm{x}}\right)$................................ 112

4.1.2 Thin Film Characterization Methods.................................................................... 113

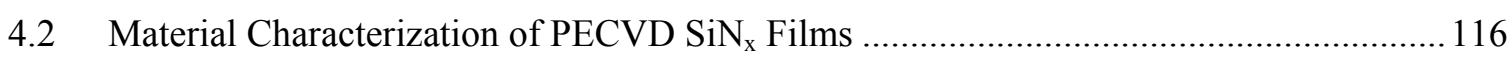

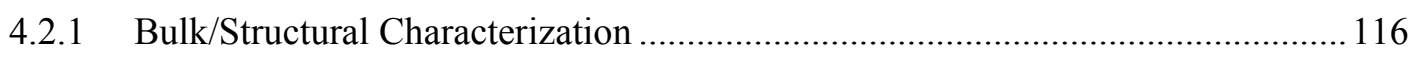

4.2.2 Surface Characterization................................................................................. 123

4.2.3 Electrical Characterization.............................................................................. 130

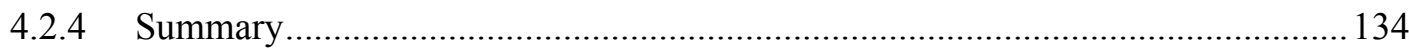

4.3 Electrical Characterization of OTFTs with PECVD Gate Dielectric …………………...... 134

4.3.1 $300^{\circ} \mathrm{C} \mathrm{SiN}_{\mathrm{x}}$ Gate Dielectrics....................................................................... 136

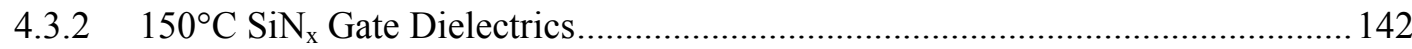

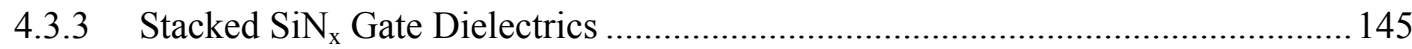

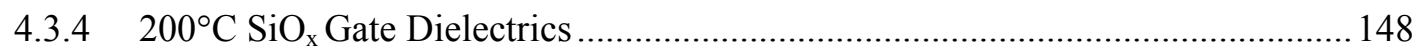

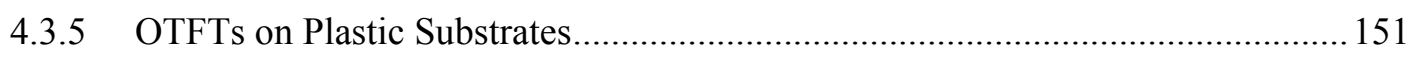

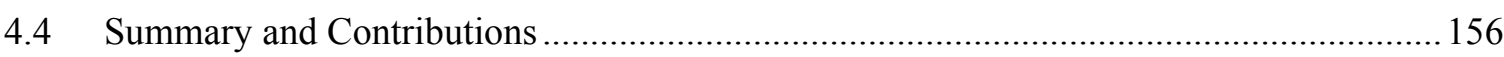

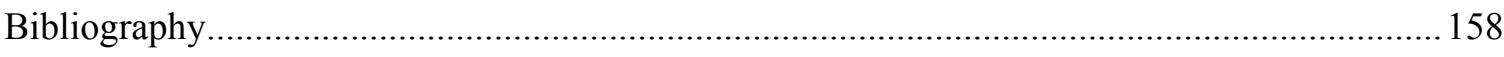

Chapter 5 Dielectric Interface Engineering........................................................................162

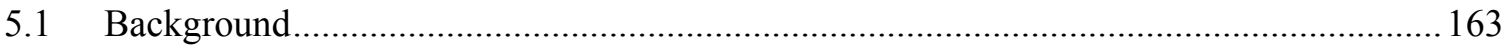

5.1.1 Self Assembled Monolayer (SAM) ………………………………………...... 163

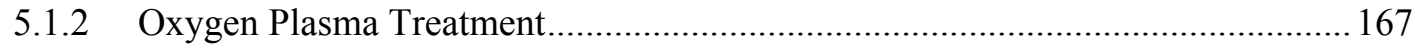

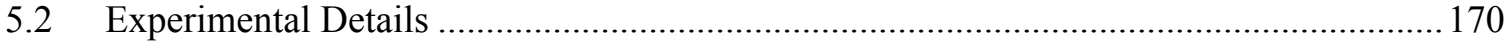

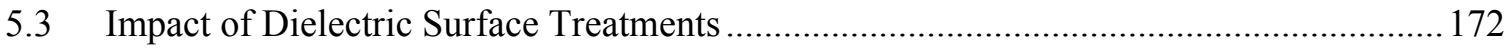

5.3.1 Electrical Characterization.......................................................................... 172

5.3.2 Interface Characterization........................................................................... 176

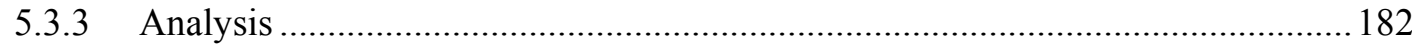

5.4 Impact of Oxygen Plasma Exposure Conditions …………………………………...... 184

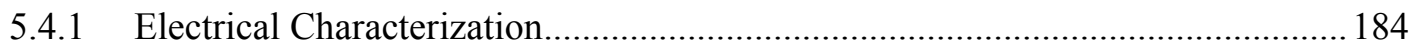

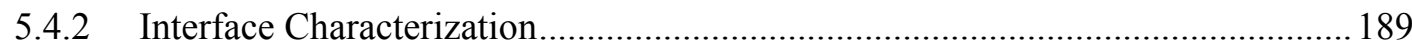

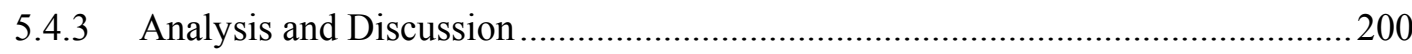




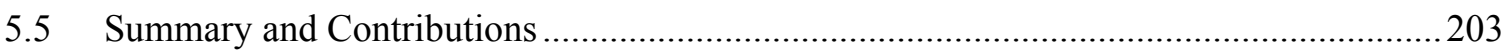

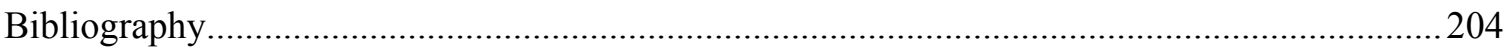

Chapter 6 Contact Interface Engineering...........................................................207

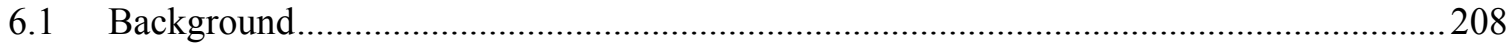

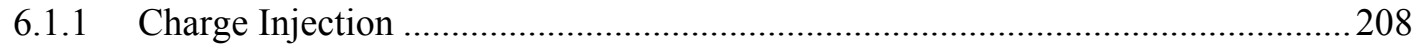

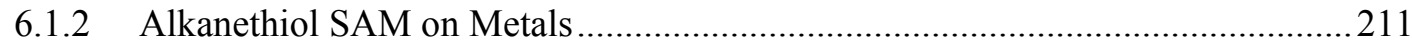

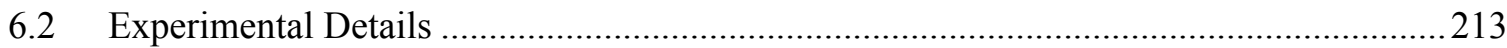

6.3 Impact of Contact Surface Treatment by Thiol SAM...................................................2214

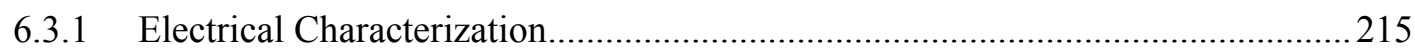

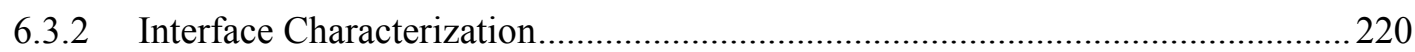

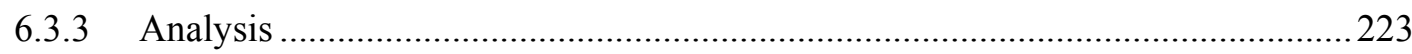

6.4 Impact of Execution Sequence of Surface Treatment................................................226

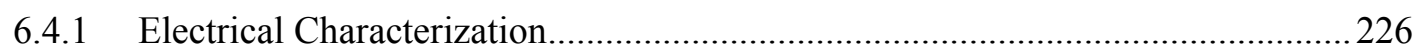

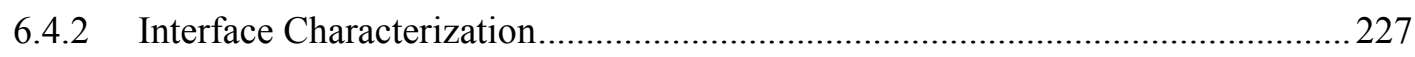

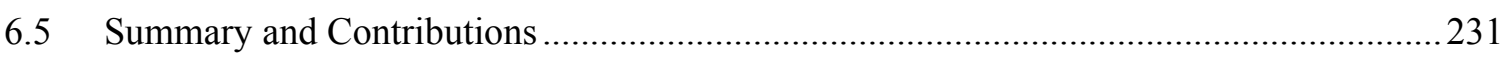

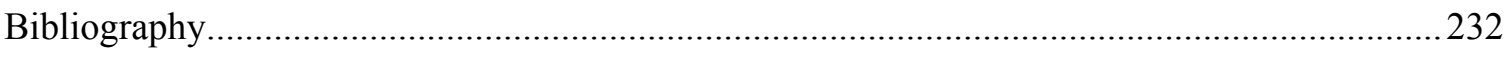

Chapter 7 OTFT Circuits and Systems.............................................................235

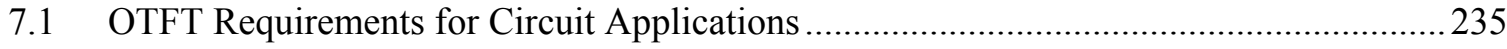

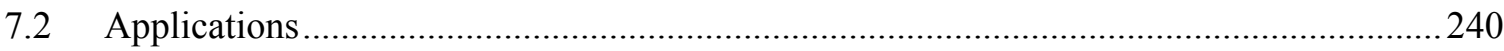

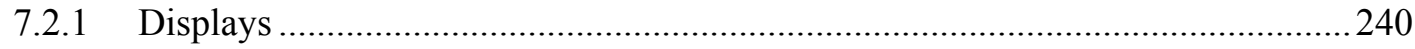

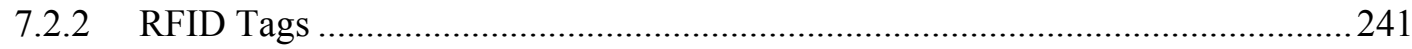

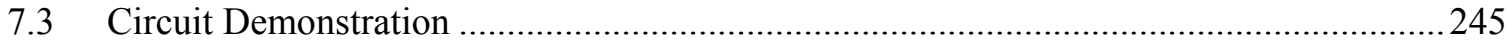

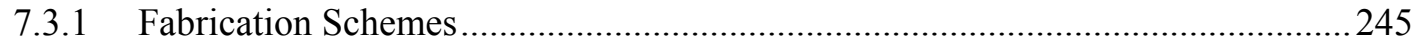

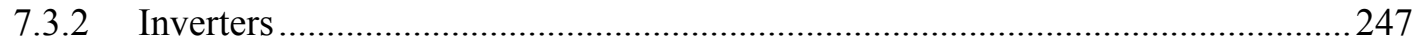

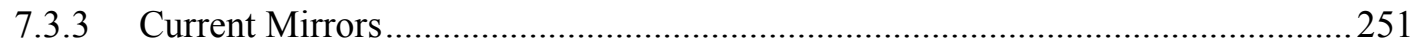

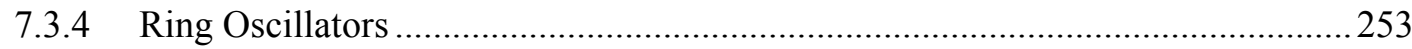

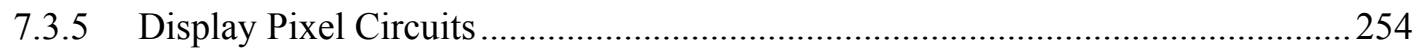

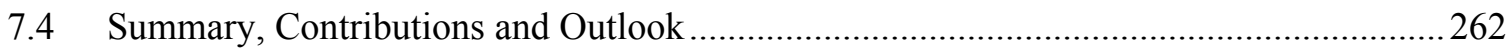

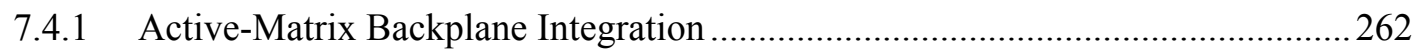

7.4.2 Back-End Process Integration: Bonding \& Packaging ......................................2. 263

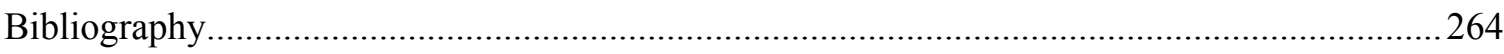


Chapter 8 Conclusions..........................................................................................................268

Chapter 9 Research Contributions....................................................................273

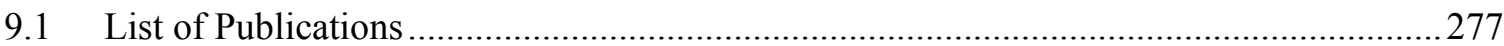

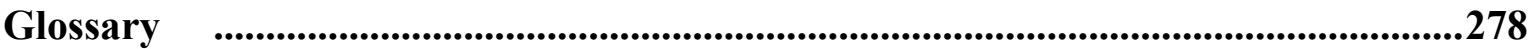




\section{List of Tables}

Table 1.1. Comparison of OTFTs and amorphous silicon (a-Si) TFTs......................................6

Table 2.1. Properties, representative chemical structure and typical mobility of the three main classes of organic semiconductors. The mobility of silicon is included for comparison

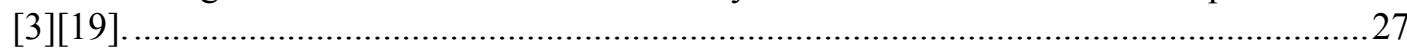

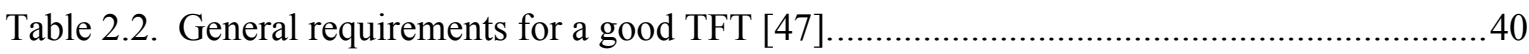

Table 2.3. Device material consideration for the OTFT fabrication in this thesis...........................45

Table 2.4. Work function $\left(\Phi_{\mathrm{M}}\right)$ of selected metals [63]............................................................51

Table 2.5. Properties of flexible substrate material candidates [68] ............................................53

Table 3.1. Deposition and patterning techniques employed for OTFT fabrication in this research.

Table 3.2. Key advantages of the inkjet printing process for the fabrication of organic electronics [29][31].

Table 3.3. Experimental summary of the impact of selected printing parameters on the quality of inkjet printed PQT-12 organic semiconductor thin film.

Table 3.4. Summary of strengths and weaknesses of the various OTFT integration approaches developed in this research. The approaches are grouped by the technique used for depositing/patterning organic layer: photolithography and inkjet printing.

Table 3.5. Comparing device performance of fully-patterned OTFTs fabricated by various approaches. Best values of field-effect mobility and on/off current ratio measured are shown.

Table 3.6. Comparison of the strengths and drawbacks of the fabrication/patterning techniques considered in this thesis.

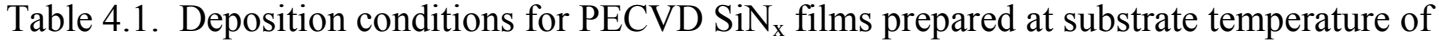
$300^{\circ} \mathrm{C}$ and $150^{\circ} \mathrm{C}$. $\mathrm{SiN}_{\mathrm{x}}$ films were deposited in a parallel-plate PECVD reactor operating at an excitation frequency of $13.56 \mathrm{MHz}$.

Table 4.2. Unique surface properties of silicon-rich $\operatorname{SiN}_{\mathrm{x}}$ (when compared to nitrogen-rich $\mathrm{SiN}_{\mathrm{x}}$ ) to account for the improved mobility in PQT-12 OTFTs. The qualities quoted are measured on $\mathrm{O}_{2}$ plasma and OTS SAM treated Si-rich $\mathrm{SiN}_{\mathrm{x}}$ surfaces.

Table 4.3. Impact of silicon-rich $\mathrm{SiN}_{\mathrm{x}}$ gate dielectric on OTFT and nc-Si TFT............................141

Table 4.4. Description of stacked $\mathrm{SiN}_{\mathrm{x}}$ gate dielectric samples 
Table 4.5. Material characterization data for stacked $\mathrm{SiN}_{\mathrm{x}}$ films, including refractive index, $[\mathrm{N}] /[\mathrm{Si}]$ ratio, and dielectric constant. Data for Si-rich $\mathrm{SiN}_{\mathrm{x}}$ and $\mathrm{N}$-rich $\mathrm{SiN}_{\mathrm{x}}$ are included for comparison.

Table 4.6. Various plastic substrates and PECVD gate dielectric employed for PQT-12 OTFT fabrication in this study. The corresponding effective field effect mobility $\left(\mu_{\mathrm{FE}}\right)$, on/off current ratio $\left(I_{\mathrm{ON}} / I_{\mathrm{OFF}}\right)$ and threshold voltage $\left(V_{\mathrm{T}}\right)$, extracted in the saturation region, are shown.

Table 4.7. Surface roughness of $150^{\circ} \mathrm{C} \mathrm{PECVD} \mathrm{SiN}_{\mathrm{x}}$ on $\mathrm{Si}$ wafer and plastic substrate. 152

Table 4.8. Summary of key observations on comparative study of OTFT with different PECVD gate dielectrics.

Table 5.1. The impact of surface conditions on the molecular orientation of organic semiconductor (e.g., pentacene and P3HT).

Table 5.2. Dielectric-semiconductor interface engineering experiments. 171

Table 5.3. Dielectric surface treatment experiment: sample descriptions.

Table 5.4. Changes in threshold voltage $V_{\mathrm{T}}$, switch-on voltage $V_{\mathrm{SO}}$, and inverse subthreshold slope $S$ of OTFTs with different dielectric surface treatments. Data are extracted from PQT-12 OTFTs on $300^{\circ} \mathrm{C} \mathrm{SiN}_{\mathrm{x}}(\mathrm{x}=1.08)$ gate dielectric, with $W / L=500 \mu \mathrm{m} / 10 \mu \mathrm{m}$, in the saturation region $\left(V_{\mathrm{DS}}=-40 \mathrm{~V}\right)$ (see Figure 5.5).

Table 5.5. Comparison of effective field-effect mobility, contact angle and surface roughness for samples with different dielectric surface treatment. Data shown is obtained by averaging results from a number of samples.

Table 5.6. Various $\mathrm{O}_{2}$ plasma recipes used in this experiment, with chamber pressure at 150 mTorr, and $\mathrm{O}_{2}$ gas flow rate at $30 \mathrm{sccm}$. RIE and ICP is abbreviation for reactive ion etching and inductively coupled plasma, respectively. DC bias directly controls the RIE power.

Table 5.7. OTFT device parameters as a function of $\mathrm{O}_{2}$ plasma exposure duration. Data are extracted from PQT-12 OTFTs on $150^{\circ} \mathrm{C} \mathrm{SiN}(\mathrm{x}=1.28)$ gate dielectric, with $W / L=500$ $\mu \mathrm{m} / 10 \mu \mathrm{m}$, in the saturation region $\left(V_{\mathrm{DS}}=-60 \mathrm{~V}\right)$ (refer to Figure 5.12).

Table 5.8. Summary of the impact of $\mathrm{O}_{2}$ plasma exposure duration and RIE power on surface properties of $\mathrm{SiN}_{\mathrm{x}}$ and on OTFT device mobility. $\mathrm{t}^{*}$ denotes the "turnaround point", defined as the exposure duration that generated maximum effective mobility.

Table 5.9. Summary of key observations from dielectric interface engineering experiments.......204

Table 6.1. Source contact resistance $R_{\mathrm{S}}$ of bottom-contact P3HT and F8T2 OTFTs made with different metal contacts $\left(T=300 \mathrm{~K}, V_{\mathrm{GS}}=-20 \mathrm{~V}\right)$. $\mathrm{IP}_{\mathrm{S}}$ is the ionization potential of the semiconductor, $\Phi_{M}$ is the measured work function of the metal, and $\varphi_{\mathrm{B}}$ is the estimated Schottky barrier height at the metal/organic interface (adapted from [4]).

Table 6.2. Contact-semiconductor interface engineering experiments.

Table 6.3. Percentage change in mobility and contact resistance for PQT-12 OTFT on $150^{\circ} \mathrm{C} \mathrm{SiN}$ $(x=1.60)$, based on data in Figure 6.5 and Figure 6.6, respectively. \% Change is calculated as "(Thiol - NoThiol)/NoThiol".

Table 6.4. Experiment on execution sequence of OTS and thiol surface treatments 
Table 6.5. Summary of key observations from contact interface engineering experiments..........232

Table 7.1. A concise comparison of OTFT characteristics demanded by various applications. The most critical parameter(s) in each application is identified by the symbol $\diamond \ldots \ldots \ldots \ldots \ldots \ldots \ldots . .236$

Table 7.2. Calculation of maximum intrinsic switching frequency for OTFTs...........................237

Table 7.3. Reported display prototypes using OTFTs [12]...................................................240

Table 7.4. Frequency bands and applications of RFID systems [20] ......................................241

Table 9.1. Summary of research contributions of this Ph.D. thesis...........................................274 


\section{List of Figures}

Figure 1.1. A broad range products and technologies inspired by organic electronics [2]..............2

Figure 1.2. Examples of commercial opportunities for OTFTs................................................2

Figure 1.3. Pictorial illustration on cost versus performance comparison between silicon

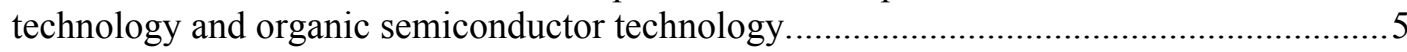

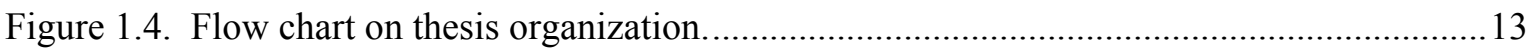

Figure 2.1. Cross section of a basic OTFT structure, in bottom-gate and top-contact configuration.

Figure 2.2. Chemical structure of three conjugated polymers: (a) polyacetylene, (b) polythiophene

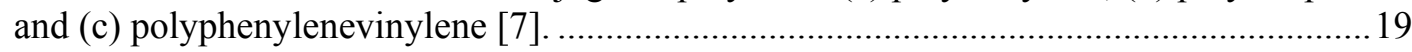

Figure 2.3. (a) Conjugated and (b) non-conjugated structure of an organic molecule [8]..............19

Figure 2.4. Representative energy band diagram of an organic semiconductor............................20

Figure 2.5. (a) Chemical structure of PQT-12 organic semiconductor. (b) GIXRD and (c) transmission electron diffraction pattern of a PQT-12 film. (d) Schematic illustration of lamellar $\pi-\pi$ stacking in a PQT-12 film (adapted from [15]).

Figure 2.6. Two different orientations of ordered P3HT domains with respect to the dielectric substrate surface (adapted from [12]).

Figure 2.7. Schematics of ideal alignment of organic semiconductor building blocks with strong $\pi$ -

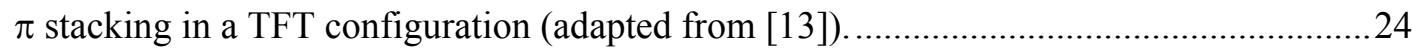

Figure 2.8. Progress in the performance of organic semiconductors (adapted from [3][18]).........26

Figure 2.9. (a) Two types of arrangements for P3HT. (b) Head-to-tail regioregular P3HT [19]....29

Figure 2.10. Chemical structure of (a) PQT and (b) PBTTT [15][31] .......................................29

Figure 2.11. Simplified illustration of the operation of a TFT with p-type semiconductor [7].......33

Figure 2.12. Typical electrical characteristics of a p-type OTFT. (a) Transfer curve $\left(I_{\mathrm{D}}-V_{\mathrm{GS}}\right)$ in the saturation regime $\left(V_{\mathrm{DS}}=-40 \mathrm{~V}\right)$, and $(\mathrm{b})$ output curves $\left(I_{\mathrm{D}}-V_{\mathrm{DS}}\right)$ at different gate voltages. The device shown here is PQT-12 OTFT on PECVD SiN $\mathrm{S}_{\mathrm{x}}$ gate dielectric. Inset of (a) shows molecular structure of PQT-12, which serves as semiconductor in the device. ........35

Figure 2.13. Extraction of OTFT device from electrical characteristics: (a) $g_{\mathrm{m}}-V_{\mathrm{GS}}$ plot for extracting $\mu_{\mathrm{FE}}$ and $V_{\mathrm{T}}$, and (b) $I_{\mathrm{D}^{-}} V_{\mathrm{GS}}$ plot for deducing $I_{\mathrm{ON}} / I_{\mathrm{OFF}}$ and subthreshold slope....36 
Figure 2.14. Contact resistance extraction. (a) Exemplary plot of $R_{\mathrm{m}} W$ vs. $L$ for different values of $V_{\mathrm{GS}}$ for an OTFT at a given $V_{\mathrm{DS}}$, to extract $A$ and B. (b) Exemplary plot of $B$ vs. $A$ at a given $V_{\mathrm{DS}}$, to extract $R_{\mathrm{DS}} W$ and $\Delta L$.

Figure 2.15. Cross section of two bottom-gate OTFT configurations: (a) top-contact and (b) bottom-contact.

Figure 2.16. Cross-section of (a) top-gate, (b) dual-gate, and (c) bottom-gate OTFTs. Devices shown are in bottom-contact configuration.

Figure 2.17. Interaction between the three key device layers in an OTFT. 44

Figure 2.18. Energy band diagram at the contact-semiconductor interface, illustrating the concept of work function $\left(\Phi_{\mathrm{M}}\right)$, ionization potential (IP) and injection barrier $\left(\varphi_{\mathrm{B}}\right)$ at the contact. Representative values for $\mathrm{p}$-type polymer semiconductor and contact material are shown.51

Figure 3.1. Cross sectional diagram of (a) top-contact OTFT and (b) silicon n-type MOSFET (NMOS).

Figure 3.2. Fabrication sequence of bottom-gate OTFT by the shadow mask patterning approaches, for (a) bottom-contact and (b) top-contact configuration.

Figure 3.3. Illustration of individually addressable gates on the same substrate, which is a basic requirement to enable circuit implementation.

Figure 3.4. Basic photolithography process: the exposure and etching process that allows one to transfer a pattern to the film/wafer.

Figure 3.5. Cross section illustration of OTFTs with (a) single capping layer, and (b) stacked

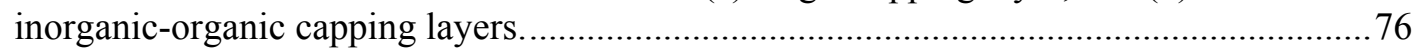

Figure 3.6. Comparison on the process complexity of photolithography and inkjet printing. ........78

Figure 3.7. Surface-energy-assisted inkjet printing to improve image resolution. (a) Schematic view of inkjet deposition of PEDOT solution along the hydrophobic PI strip. (b) Control of ink spreading by a "dewetting pattern" of hydrophobic lines that confines the organic ink to specific hydrophilic regions on the surfaces. (c) Topographic AFM image of the transistor channel defined by PI, and the PEDOT source and drain electrodes. The width of the strip (channel length) is $5 \mu \mathrm{m}$ (adapted from [6]).

Figure 3.8. Fabrication sequence of bottom-gate bottom-contact OTFT on highly-doped Si substrate with a 1-mask patterning step to define the source/drain contacts (by photolithography or shadow mask).

Figure 3.9. Fabrication sequence of fully-patterned fully encapsulated bottom-gate bottom-contact OTFT by a 4-mask photolithography process.

Figure 3.10. Two photolithographically-defined bottom-gate OTFT structures with (a) photoresist passivation and (b) parylene-photoresist passivation.

Figure 3.11. Cross section illustration and photograph of a fully-patterned OTFT fabricated using 4-mask photolithography scheme with parylene-photoresist passivation.

Figure 3.12. (a) Transfer and (b) output characteristics of a fully-patterned bottom-gate bottomcontact PQT-12 OTFT with $\mathrm{SiN}_{\mathrm{x}}$ gate dielectric on glass substrate. Polymer semiconductor layer was directly patterned using photolithographically, with direct deposition of photoresist on PQT-12 layer. 
Figure 3.13. (a) Transfer and (b) output characteristics of a fully-patterned bottom-gate bottomcontact PQT-12 OTFT with $\mathrm{SiN}_{\mathrm{x}}$ gate dielectric on glass substrate. Polymer semiconductor layer was photolithographically patterned with parylene passivation layer.

Figure 3.14. Hybrid photolithography-inkjet printing fabrication scheme for fully-patterned bottom-gate bottom-contact OTFT.

Figure 3.15. Cross section illustration and photograph of a fully-patterned OTFT fabricated using hybrid photolithography-inkjet printing scheme.

Figure 3.16. (a) Transfer and (b) output characteristics of a fully-patterned bottom-gate bottomcontact PQT-12 OTFT with $\mathrm{SiN}_{\mathrm{x}}$ gate dielectric on glass substrate. Organic semiconductor layer was deposited by inkjet printing.

Figure 3.17. Photograph of OTFTs with inkjet printed organic semiconductor showing (a) a thin uniform layer and (b) a thicker non-uniform layer.

Figure 3.18. Illustration of inkjet printing direction: (a) horizontal and (b) vertical printing..........95

Figure 3.19. Fabrication sequence of dual-gate OTFT by a 5-mask photolithography process. Topgate OTFT can be fabricated by excluding steps (1), (8) and (9).

Figure 3.20. Top-gate P3HT OTFT on glass substrate, fabricated by photolithography. (a) Transfer characteristics $\left(I_{\mathrm{D}}-V_{\mathrm{GS}}\right)$. (b) Output characteristics $\left(I_{\mathrm{D}}-V_{\mathrm{DS}}\right)$.

Figure 3.21. Dual-gate P3HT OTFT on Si wafer. Bottom-gate dielectric is $\mathrm{SiO}_{2}$, and top-gate dielectric is PECVD SiN $\mathrm{x}$. (a) Transfer characteristics $\left(I_{\mathrm{D}^{-}} V_{T G}\right)$ of the dual-gate OTFT as a function of top-gate voltage. (b) Output characteristics $\left(I_{\mathrm{D}}-V_{\mathrm{DS}}\right)$ of the dual-gate OTFT at various top-gate voltages.

Figure 4.1. Contact angle $(\theta)$ of surfaces with different wettability: (a) water droplet on a hydrophobic surface showing large contact angle, and (b) water droplet on a hydrophilic surface showing small contact angle.

Figure 4.2. Fourier transform infrared (FTIR) spectroscopy of the $300^{\circ} \mathrm{C}$ PECVD SiN films...117

Figure 4.3. Fourier transform infrared (FTIR) spectroscopy of the $150^{\circ} \mathrm{C}$ PECVD $\mathrm{SiN}_{\mathrm{x}}$ films....117

Figure 4.4. Normalized N-H and $\mathrm{Si}-\mathrm{H}$ bond concentration as a function of $\mathrm{NH}_{3} / \mathrm{SiH}_{4}$ gas flow ratio for $\mathrm{SiN}_{\mathrm{x}}$ films deposited at $150^{\circ} \mathrm{C}$. Data is normalized to $[\mathrm{N}-\mathrm{H}]$ value for the sample deposited with $\mathrm{NH}_{3} / \mathrm{SiH}_{4}$ gas flow ratio of 20 .

Figure 4.5. N-H and $\mathrm{Si}-\mathrm{H}$ bond concentration as a function of $\mathrm{NH}_{3} / \mathrm{SiH}_{4}$ gas flow ratio for $\mathrm{SiN}_{\mathrm{x}}$ films deposited at substrate temperatures of $150^{\circ} \mathrm{C}$ and $300^{\circ} \mathrm{C}$

Figure 4.6. Wavenumber of the Si-N stretching band as a function of $\mathrm{NH}_{3} / \mathrm{SiH}_{4}$ gas flow ratio for PECVD SiN ${ }_{x}$ films. Inset plots the changes in $\mathrm{Si}-\mathrm{N}$ wavenumber versus $[\mathrm{N}] /[\mathrm{Si}]$ ratio of the films.

Figure 4.7. Refractive index at wavelength of $637 \mathrm{~nm}$ as a function of $\mathrm{NH}_{3} / \mathrm{SiH}_{4}$ gas flow ratio for $300^{\circ} \mathrm{C}$ and $150^{\circ} \mathrm{C} \mathrm{SiN}_{\mathrm{x}}$ samples.

Figure 4.8. Nitrogen to silicon ratio $([\mathrm{N}] /[\mathrm{Si}])$ of the $300^{\circ} \mathrm{C} \mathrm{SiN}_{\mathrm{x}}$ and $150^{\circ} \mathrm{C} \mathrm{SiN}_{\mathrm{x}}$ films as a function of gas flow ratio. 
Figure 4.9. Water contact angle of $\operatorname{SiN}_{x}$ surface before $(O)$ and after $(\boldsymbol{\square})$ surface treatment with oxygen plasma and OTS SAM, for four different $\mathrm{SiN}_{\mathrm{x}}$ films (denoted by $\mathrm{NH}_{3} / \mathrm{SiH}_{4}$ gas flow ratios).

Figure 4.10. Surface roughness of $150^{\circ} \mathrm{C}$ and $300^{\circ} \mathrm{C} \mathrm{SiN}_{\mathrm{x}}$ films as a function of $\mathrm{NH}_{3} / \mathrm{SiH}_{4}$ gas flow ratios, after surface treatment with $\mathrm{O}_{2}$ plasma and OTS SAM.

Figure 4.11. Chemical composition of three different $300^{\circ} \mathrm{C} \mathrm{SiN}$ films $(x=1.50,1.34,1.08)$, plotted as atomic distribution at the film surface as measured by XPS. Three surface conditions were considered for each of the $300^{\circ} \mathrm{C} \mathrm{SiN}$ films: (a) as-deposited (bare) surface, (b) $\mathrm{O}_{2}$ plasma treated surface, and (c) $\mathrm{O}_{2}$ plasma and OTS SAM treated surface 128

Figure 4.12. XPS measurement comparing chemical composition of silicon-rich $300^{\circ} \mathrm{C}$ and $150^{\circ} \mathrm{C}$ $\mathrm{SiN}_{\mathrm{x}}$ films deposited at gas flow ratio of $\mathrm{NH}_{3} / \mathrm{SiH}_{4}=5$. Two surface conditions are considered: (a) as-deposited (bare) and (b) $\mathrm{O}_{2}$ plasma treated surface.

Figure 4.13. Leakage current density as a function of electric field for $300^{\circ} \mathrm{C} \mathrm{SiN}_{\mathrm{x}}$ dielectrics deposited at various $\mathrm{NH}_{3} / \mathrm{SiH}_{4}$ gas flow ratio. The film thickness is approximately $300 \mathrm{~nm}$. Measurements were performed using MIS capacitor structure. 130

Figure 4.14. Leakage current density as a function of electric field for $150^{\circ} \mathrm{C} \mathrm{SiN}_{\mathrm{x}}$ dielectrics deposited at various $\mathrm{NH}_{3} / \mathrm{SiH}_{4}$ gas flow ratio. The film thickness is approximately $300 \mathrm{~nm}$. Measurements were performed using MIS capacitor structure.

Figure 4.15. Dielectric constant $\left(\varepsilon_{\mathrm{r}}\right)$ for $\mathrm{SiN}_{\mathrm{x}}$ films as a function of $\mathrm{NH}_{3} / \mathrm{SiH}_{4}$ gas flow ratio...... 132

Figure 4.16. $C-V$ characteristics of various $300^{\circ} \mathrm{C} \mathrm{SiN}$ dielectrics at $1 \mathrm{kHz}$, in both forward (negative to positive) and reverse (positive to negative) sweep directions. Flatband voltage $\left(V_{\mathrm{FB}}\right)$ is extracted from forward curves and is plotted as a function of $\mathrm{NH}_{3} / \mathrm{SiH}_{4}$ gas flow ratio.

Figure 4.17. $C-V$ characteristics of various $150^{\circ} \mathrm{C} \mathrm{SiN}$ dielectrics at $1 \mathrm{kHz}$, in both forward (negative to positive) and reverse (positive to negative) sweep directions. Flatband voltage $\left(V_{\mathrm{FB}}\right)$ is extracted from forward curves and is plotted as a function of $\mathrm{NH}_{3} / \mathrm{SiH}_{4}$ gas flow ratio.

Figure 4.18. Chemical structure of poly(3,3"'-dialkylquarterthiophene) (PQT). In the case of PQT12, alkyl side chain is $\mathrm{R}=\mathrm{n}-\mathrm{C}_{12} \mathrm{H}_{25}$. (Adapted from [28].) ....

Figure 4.19. Schematic cross-section of the OTFT structure considered in this study. 135

Figure 4.20. Electrical characteristics of PQT-12 OTFT on $300^{\circ} \mathrm{C} \operatorname{SiN}_{\mathrm{x}}(x=1.08)$ gate dielectric with $W / L=1000 \mu \mathrm{m} / 20 \mu \mathrm{m}$ : (a) Transfer characteristics $\left(\log \left|I_{\mathrm{D}}\right|-V_{\mathrm{GS}}\right)$ and (b) output characteristics $\left(I_{\mathrm{D}}-V_{\mathrm{DS}}\right)$. Inset of (a) shows a well-behaved linear plot of $\sqrt{I_{D}}-V_{G S}$ in the saturation regime.

Figure 4.21. Comparison of PQT-12 OTFT characteristics on $300^{\circ} \mathrm{C} \mathrm{SiN}$ gate dielectric of varying film composition. (a) Transfer characteristics, (b) effective field-effect mobility, (c) on/off current ratio and threshold voltage as a function of the $[\mathrm{N}] /[\mathrm{Si}]$ ratio of $\mathrm{SiN}_{\mathrm{x}}$ film.

Figure 4.22. Electrical characteristics of PQT-12 OTFT on $150^{\circ} \mathrm{CPECVD} \operatorname{SiN}_{\mathrm{x}}(x=1.60)$ gate dielectric with $W / L=1000 \mu \mathrm{m} / 20 \mu \mathrm{m}$ : (a) transfer characteristics $\left(\log \left|I_{\mathrm{D}}\right|-V_{\mathrm{GS}}\right)$ and (b) 
output characteristics $\left(I_{\mathrm{D}}-V_{\mathrm{DS}}\right)$. Inset of (a) shows a well-behaved linear plot of $\sqrt{I_{D}}-V_{G S}$ in the saturation regime.

Figure 4.23. (a) Effective field-effect mobility and (b) on/off current ratio of PQT-12 OTFT (W/L $=1000 \mu \mathrm{m} / 20 \mu \mathrm{m})$ plotted as a function of $\mathrm{NH}_{3} / \mathrm{SiH}_{4}$ gas flow ratio for $150^{\circ} \mathrm{C}$ and $300^{\circ} \mathrm{C}$ $\mathrm{SiN}_{\mathrm{x}}$ gate dielectric. Data corresponds to measurements in saturation $\left(V_{\mathrm{DS}}=-40 \mathrm{~V}\right)$ region.

Figure 4.24. Schematic cross-section of the OTFT structure with stacked $\mathrm{SiN}_{\mathrm{x}}$ gate dielectric. .. 145

Figure 4.25. Fourier transform infrared (FTIR) spectroscopy of the stacked $\mathrm{SiN}_{\mathrm{x}}$ films, deposited

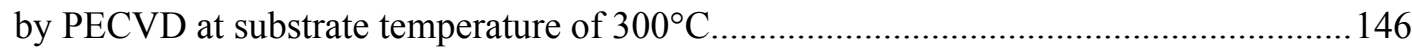

Figure 4.26. Effective field-effect mobility of PQT-12 OTFT with stacked $\mathrm{SiN}_{\mathrm{x}}$ gate dielectrics. Mobility of OTFT with Si-rich $\operatorname{SiN}_{\mathrm{x}}(x=1.08)$ and $\mathrm{N}$-rich $\operatorname{SiN}_{\mathrm{x}}(x=1.50)$ is also shown for comparison.

Figure 4.27. Electrical characteristics of PQT-12 OTFT on PECVD $\mathrm{SiO}_{\mathrm{x}}$ gate dielectric with $W / L$ $=1000 \mu \mathrm{m} / 20 \mu \mathrm{m}$ : (a) transfer characteristics $\left(\log \left|I_{\mathrm{D}}\right|-V_{\mathrm{GS}}\right)$ and (b) output characteristics $\left(I_{\mathrm{D}}-V_{\mathrm{DS}}\right)$. Inset of (a) shows a linear plot of $\sqrt{I_{D}}-V_{G S}$ in saturation regime.

Figure 4.28. Schematic cross-section of the bottom-gate top-contact OTFT on plastic substrate. 151

Figure 4.29. (a) Transfer and (b) output characteristics of PQT-12 OTFT on PET substrate with $\operatorname{PECVD~SiN}_{\mathrm{x}}$ gate dielectric $(W / L=1000 \mu \mathrm{m} / 90 \mu \mathrm{m})$.

Figure 4.30. (a) Transfer and (b) output characteristics of PQT-12 OTFT on Kapton substrate with PECVD SiN ${ }_{\mathrm{x}}$ gate dielectric $(W / L=1000 \mu \mathrm{m} / 90 \mu \mathrm{m})$.

Figure 4.31. (a) Transfer and (b) output characteristics of PQT-12 OTFT on Kapton substrate with PECVD $\mathrm{SiO}_{\mathrm{x}}$ gate dielectric $(W / L=1000 \mu \mathrm{m} / 90 \mu \mathrm{m})$.

Figure 4.32. Flowchart illustrating the relationship between $\mathrm{SiN}_{\mathrm{x}}$ film composition, interface properties, and OTFT performance.

Figure 5.1. (a) Simplified illustration of three main parts of a SAM system, and (b) the formation of alkylsiloxane SAM by adsorption of alkyltrichlorosilanes from solution on a $\mathrm{Si}_{-} \mathrm{SiO}_{2}$ substrate (adapted from [9]). 164

Figure 5.2. Schematics of different P3HT chain orientations, (a) edge-on and (b) face-on, according to interfacial characteristics (adapted from [12]). 166

Figure 5.3. (a) Diagram of a RIE system. (b) Potential distribution as a function of distance between electrodes. Wafer is placed on a smaller electrode, which is biased negatively relative to the plasma. Ions accelerate from plasma to wafer due to a DC bias electric field.

Figure 5.4. Schematic cross-section of the OTFT structure considered in this dielectric-surface treatment study.

Figure 5.5. (a) Output and (b,c) transfer characteristics of PQT-12 OTFTs on $300^{\circ} \mathrm{C}$ PECVD $\mathrm{SiN}_{\mathrm{x}}$ $(x=1.08)$ with different dielectric surface treatments. The transfer characteristics are shown on semi-logarithmic scale in (b) and as the square root of the drain current in (c). 
Figure 5.6. (a) Effective mobility and (b) on/off current ratio of PQT-12 OTFT with $\mathrm{SiN}_{\mathrm{x}}$ gate dielectrics under various dielectric surface treatment conditions. Measurements were collected in saturation region. Each data point corresponds to an average value from three

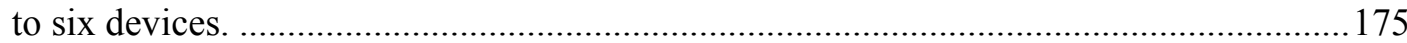

Figure 5.7. Factor of improvement in effective mobility, relative to untreated devices, for PQT-12 OTFT after various types of dielectric surface treatments. Measurements were collected in saturation region.

Figure 5.8. Photographs from contact angle measurement of $\operatorname{SiN}_{\mathrm{x}}$ surface after various dielectric surface treatments.

Figure 5.9. (a) Water contact angle measurement and (b) mean surface roughness of $\mathrm{SiN}_{\mathrm{x}}$ surfaces after various dielectric surface treatments.

Figure 5.10. AFM images showing surface topography of $\mathrm{SiN}_{\mathrm{x}}$ surface after various dielectric surface treatments. The mean surface roughness $\left(R_{a}\right)$ is indicated.

Figure 5.11. Atomic composition from XPS measurements of $\mathrm{SiN}_{\mathrm{x}}$ surfaces with different surface treatments.

Figure 5.12. (a) Output characteristics and (b,c) transfer of PQT-12 OTFTs on $150^{\circ} \mathrm{C} \mathrm{PECVD}$ $\operatorname{SiN}_{\mathrm{x}}(x=1.28)$ gate dielectric treated with different $\mathrm{O}_{2}$ plasma exposure duration. The transfer characteristics are shown on semi-logarithmic scale in (b) and as the square root of the drain current in (c). All samples were treated with $\mathrm{O}_{2}$ plasma, OTS SAM and 1Octanethiol SAM.

Figure 5.13. (a) Effective mobility and (b) on/off current ratio as a function of $\mathrm{O}_{2}$ plasma exposure duration of PQT-12 OTFT with $150^{\circ} \mathrm{C} \mathrm{SiN}_{\mathrm{x}}(x=1.28)$ gate dielectric. Data was collected in the saturation region. All samples were treated with $\mathrm{O}_{2}$ plasma, OTS, and 1-octanethiol. Here, each data point corresponds to an average value from three to six devices.

Figure 5.14. Effective mobility as a function of $\mathrm{O}_{2}$ plasma exposure power of PQT-12 OTFT with $\mathrm{SiN}_{\mathrm{x}}$ gate dielectric biased in saturation region. All samples were treated with $\mathrm{O}_{2}$ plasma and OTS SAM.

Figure 5.15. Water contact angle of $150^{\circ} \mathrm{C} \operatorname{SiN}_{x}(x=1.28)$ surface treated with (a) $\mathrm{O}_{2}$ plasma only and (b) $\mathrm{O}_{2}$ plasma/OTS SAM, as a function of $\mathrm{O}_{2}$ plasma exposure duration.

Figure 5.16. Surface roughness as a function of $\mathrm{O}_{2}$ plasma exposure duration for $\mathrm{SiN}_{\mathrm{x}}$ surface treated with $\mathrm{O}_{2}$ plasma and OTS SAM. Measurements performed using tapping-mode AFM. These samples are treated with $\mathrm{O}_{2}$ plasma Recipe (I) in Table 5.6.

Figure 5.17. Surface roughness as a function of $\mathrm{O}_{2}$ plasma exposure power for $\mathrm{SiN}_{\mathrm{x}}$ surface treated with $\mathrm{O}_{2}$ plasma and OTS SAM. Measurements performed using tapping-mode AFM.....192

Figure 5.18. (a) XPS spectrum in survey scan mode of an untreated $\mathrm{SiN}_{\mathrm{x}}$ surface and $\mathrm{O}_{2}$ plasma treated $\mathrm{SiN}_{\mathrm{x}}$ surfaces after $80 \mathrm{sec}$ and $600 \mathrm{sec}$ exposure. (b) Chemical composition (represented by atomic percentage) of the $\mathrm{SiN}_{\mathrm{x}}$ top surface extracted from (a). $\mathrm{O}_{2}$ plasma exposure was done using Recipe (I) in Table 5.6.

Figure 5.19. Changes in chemical composition (represented by atomic percentage) of $\mathrm{O}_{2}$ plasma treated $\mathrm{SiN}_{\mathrm{x}}$ surface at various exposure powers.

Figure 5.20. (a) Si $2 \mathrm{p}$, (b) N 1s, and (c) $\mathrm{O} 1 \mathrm{~s}$ spectra of $150^{\circ} \mathrm{C} \mathrm{SiN}_{\mathrm{x}}(x=1.28)$ upon successive ion sputtering in XPS depth-profiling measurement. (d) Atomic percentage as a function of 
sputtering time, calculated based on XPS spectra in (a)-(c). Three $\mathrm{SiN}_{\mathrm{x}}$ samples were considered: as-deposited (untreated) $\mathrm{SiN}_{\mathrm{x}}$, and $80 \mathrm{sec}$ and $600 \mathrm{sec} \mathrm{O}_{2}$ plasma treated $\mathrm{SiN}_{\mathrm{x}}$.

Figure 6.1. Energy level diagrams for metal and p-type semiconductor contact interface (assuming negligible interface states). (a) Small work function metal leads to interface barrier and poor contact. (b) Large work function metal leads to reduced interface barrier and improved contact.

Figure 6.2. Pictorial illustration of alkanethiol SAM on Au surface. (Adapted from [10].).........212

Figure 6.3. Schematic cross-section of the OTFT structure considered in this contact surface treatment study.

Figure 6.4. (a) Effective mobility and (b) on/off current ratio of PQT-12 OTFT with $150^{\circ} \mathrm{C} \mathrm{SiN} \mathrm{N}_{\mathrm{x}}(x$ $=1.60$ ) gate dielectrics in the absence ("no thiol") or presence ("with thiol") of 1octanethiol SAM modification of Au contact surfaces. Four scenarios of gate dielectric surface treatments were considered. Measurements collected in saturation region.

Figure 6.5. Effective mobility of PQT-12 OTFT with $300^{\circ} \mathrm{C} \operatorname{SiN}_{\mathrm{x}}(x=1.08)$ gate dielectrics in the absence ("no thiol") or presence ("with thiol") of 1-octanethiol SAM modification of $\mathrm{Au}$ contact surfaces. Four scenarios of gate dielectric surface treatments were considered. Measurements were collected in saturation region of OTFT operation.

Figure 6.6. Contact resistance of PQT-12 OTFT on $150^{\circ} \mathrm{C} \operatorname{SiN}_{\mathrm{x}}(x=1.60)$ gate dielectrics in the absence ("no thiol") or presence ("with thiol") of 1-octanethiol SAM modification of $\mathrm{Au}$ contact surfaces. Four scenarios of gate dielectric surface treatments were considered. Measurements were collected in linear region of OTFT operation.

Figure 6.7. Saturation mobility versus contact resistance of PQT-12 OTFT on $150^{\circ} \mathrm{C} \mathrm{SiN}_{\mathrm{x}}(x=$ 1.60) gate dielectrics.

Figure 6.8. Water contact angle of $150^{\circ} \mathrm{C} \mathrm{SiN}{ }_{x}(x=1.28)$ surface after various types of dielectric surface treatment, and in the absence or presence of 1-octanethiol exposure.

Figure 6.9. Mean surface roughness measured by AFM for $\mathrm{SiN}_{\mathrm{x}}$ surfaces after $\mathrm{O}_{2}$ plasma/OTS SAM surface treatment, in the absence or presence of 1-octanethiol SAM treatment......222

Figure 6.10. Atomic percentage detected by XPS on $\mathrm{SiN}_{\mathrm{x}}$ surface after $\mathrm{O}_{2}$ plasma/OTS SAM surface treatment, in the absence or presence of 1-octanethiol treatment. (Experimental error $= \pm 5 \%$ ).

Figure 6.11. Comparison of (a) effective mobility and (b) on/off current ratio of PQT-12 OTFTs subjected to different execution sequence of OTS and Thiol SAM treatment.

Figure 6.12. Water contact angle for Thiol/OTS and OTS/Thiol treated $\mathrm{SiN}_{\mathrm{x}}$ surfaces. 229

Figure 6.13. Surface roughness for Thiol/OTS and OTS/Thiol treated $\mathrm{SiN}_{\mathrm{x}}$ surfaces. 229

Figure 6.14. Atomic percentage detected by XPS on the surface of $\mathrm{SiN}_{\mathrm{x}}$ for different execution sequences of OTS and 1-Octanethiol SAM treatments.

Figure 7.1. Cross sectional diagram of a vertical OTFT structure. .238

Figure 7.2. (a) Typical setup of a RFID system. (b) Key modules/components of a RFID tag $[20][21]$. 
Figure 7.3. A simple diode-based half-wave rectification stage for RFID tag applications [22]. .243

Figure 7.4. Circuit schematic of a 1-bit OTFT based RFID tag. Power is coupled into the tag circuit using inductive coupling between the reader and the tag. $\mathrm{L}_{t}$ and $\mathrm{C}_{t}$ form a resonant tank that powers the tag circuitry directly, without a separate rectification stage. The lower figure shows a scaled-down schematic of the logic circuitry [22] ..................................24

Figure 7.5. Development strategy for OTFT-based RFID tag circuitries for this thesis.

Figure 7.6. Photograph of a variety of OTFT circuits on a 3" glass wafer, fabricated using the integration schemes developed in this thesis. In this photo, circuits were fabricated using photolithography approach with parylene passivation (scheme \#2).

Figure 7.7. Schematic diagram and top-view photograph of OTFT inverter circuits with (a) saturation load and (b) depletion load. (c) Cross-section diagram of an inverter with saturated load. The photographs in (a) and (b) correspond to top-view layout of actual PQT-12 OTFT circuits $(W / L=200 \mu \mathrm{m} / 25 \mu \mathrm{m}$ for both T1 and T2) fabricated by photolithography approach with parylene buffer/passivation layer (scheme \#2).

Figure 7.8. Voltage-transfer characteristics of a p-type inverter circuit with saturation load, fabricated using photolithographically-defined PQT-12 OTFT with photoresist as passivation (scheme \#1).

Figure 7.9. Voltage-transfer characteristics of a p-type inverter circuit with depletion load, fabricated using photolithographically-defined PQT-12 OTFT with photoresist as passivation (scheme \#1).

Figure 7.10. Voltage-transfer characteristics of a p-type inverter circuit with saturation load, fabricated using the photolithography approach with parylene passivation (scheme \#2). 250

Figure 7.11. Voltage-transfer characteristics of a p-type inverter circuit with saturation load, fabricated with inkjet printed PQT-12 layer (scheme \#3). .251

Figure 7.12. Current Mirror: (a) circuit schematic, and (b) micrograph showing top view of an actual circuit fabricated using hybrid photolithograph-inkjet method (scheme \#3). Photo shows $W / L=200 / 25$ for both T1 and T2

Figure 7.13. Transfer characteristic of current mirror circuits built using PQT-12 OTFTs on $\mathrm{SiN}_{\mathrm{x}}$ gate dielectric with $(W / L)_{2} /(W / L)_{1}=1$. Circuit was fabricated using the photolithography approach with photoresist passivation (scheme \#1).

Figure 7.14. Transfer characteristic of current mirror circuits built using PQT-12 OTFTs on $\mathrm{SiN}_{\mathrm{x}}$ gate dielectric with $(W / L)_{2} /(W / L)_{1}=2$. Circuit was fabricated using the photolithography

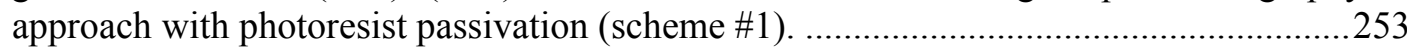

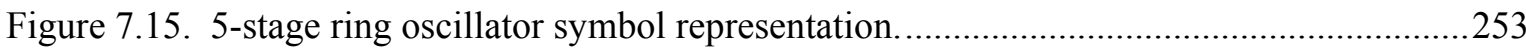

Figure 7.16. (a) 5-stage ring oscillator with saturation load. (b) 5-stage ring oscillator with depletion load. Circuit schematic and micrograph of the top-view of the fabricated structure (using scheme \#2) are also shown $(W / L=200 \mu \mathrm{m} / 25 \mu \mathrm{m}$ for the transistors

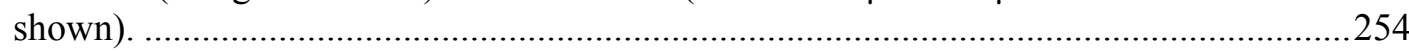

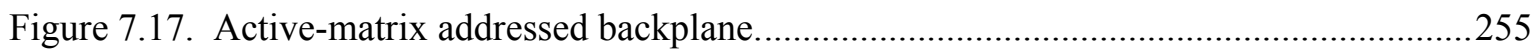

Figure 7.18. Conventional 2-TFT AMOLED pixel circuit: (a) schematic diagram, (b) micrograph of the top-view of the fabricated structure (using scheme \#2) .........................................256 
Figure 7.19. Current-voltage characteristic of a conventional 2-T pixel circuit for AMOLED, built using PQT-12 OTFTs on $\mathrm{SiN}_{\mathrm{x}}$ gate dielectric. Circuit was fabricated using the photolithography approach with parylene passivation (scheme \#2).

Figure 7.20. Current-voltage characteristic of a conventional 2-T pixel circuit for AMOLED, built using PQT-12 OTFTs on $\mathrm{SiN}_{\mathrm{x}}$ gate dielectric. Circuit was fabricated using the photolithography approach with photoresist passivation (scheme \#1).

Figure 7.21. Compensating 2-TFT AMOLED pixel circuit: (a) schematic diagram, (b) micrograph of the top-view of the fabricated structure (using scheme \#2). A diode-connected TFT (TLD) and a capacitor $\left(\mathrm{C}_{\mathrm{LD}}\right)$ are used to emulate the OLED, Also, since the TLD is under stress, its threshold voltage increases, which resembles the OLED voltage shift.

Figure 7.22. Input-output transfer characteristic of a compensating 2-T pixel circuit for AMOLED, built using PQT-12 OTFTs on $\mathrm{SiN}_{\mathrm{x}}$ gate dielectric. Circuit was fabricated using the photolithography approach with parylene passivation (scheme \#2).

Figure 7.23. Photograph showing a section of a $4 \times 4$ array of AMOLED PQT-12 OTFT pixel circuits, based on compensating 2-TFT circuit design (using fabrication scheme \#2)......260

Figure 7.24. 4-TFT current mirror AMOLED pixel circuit: (a) schematic diagram, (b) photomicrograph of the top-view of the fabricated structure (using scheme \#2). 261

Figure 7.25. Architecture of a vertically integrated AMOLED display, featuring pixels with dualgate OTFTs. 263

Figure 7.26. Photograph of packaged and wire-bonded OTFT circuits in a dual inline package (DIP). 



\section{Chapter 1}

\section{Introduction}

Organic semiconductor technology has attracted considerable research interest as it holds great promises for large area, low-end, lightweight and flexible electronics applications [1]. Intrigued by their processability advantages, compatibility with large area flexible substrates and unique material properties with versatile functionalities (e.g., electrical, optical, sensing and magnetic capabilities), organic electronic materials can bring exciting and revolutionizing opportunities for broad-impact applications requiring large-area coverage, mechanical flexibility, low-temperature processing, and, especially, low cost. Thus, organic electronics appeal to a broad range of devices and products including transistors, diodes, sensors, solar cells, and lighting. Figure 1.1 depicts a number of application domains that can benefit from the versatility of organic electronics technology [2]. Since their proof of concept in the 1980s, the impressive development in organic semiconductor materials have led to performance properties that are competitive with amorphous silicon, increasing their aptitude for practical commercial applications [3].

Transistors based on organic semiconductors as the active layer to control current flow are referred to as organic thin film transistors (OTFTs). Transistor is a fundamental building block for all modern electronics. A number of commercial opportunities has been identified for OTFTs, including flexible displays, active-matrix flat panel displays based on liquid crystal pixels (LCDs) or organic light-emitting diodes (OLEDs), electronic paper (e-paper), low-end data storage such as smart cards, electronic identification and tracking devices (e.g., radio-frequency identification (RFID) tags), low-cost disposable electronic products, and sensor arrays; more applications continue to evolve as the technology matures [4]. Figure 1.2 illustrates a few commercial opportunities envisioned for OTFTs. 


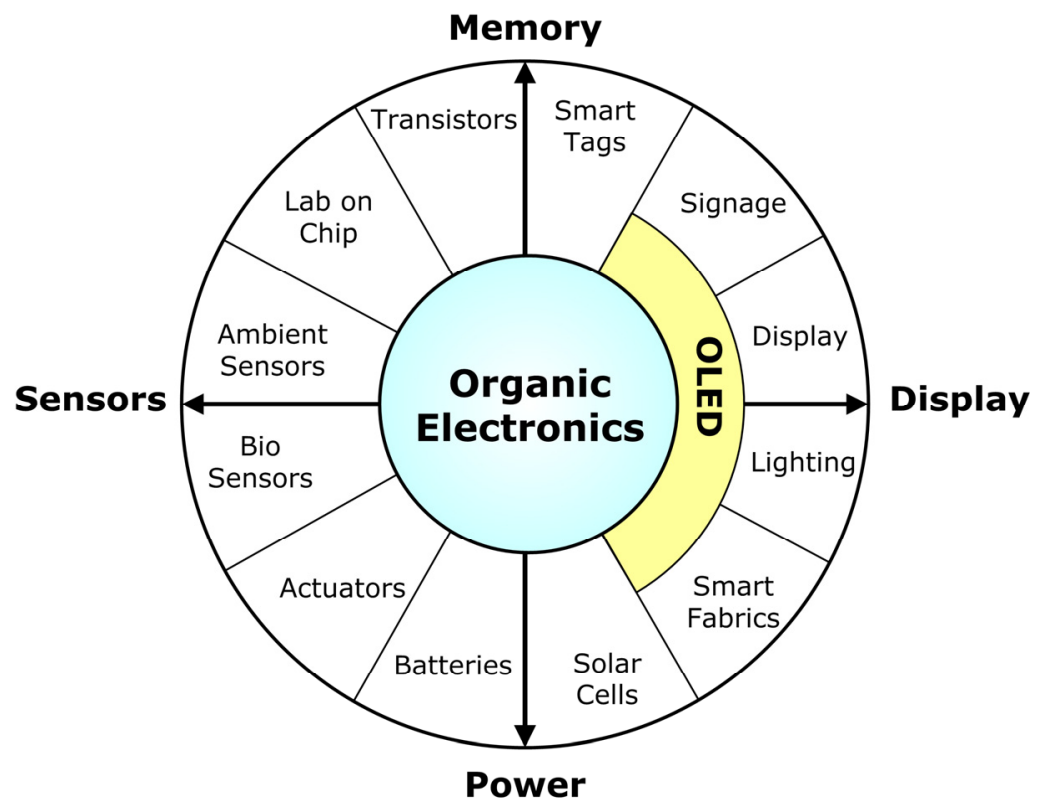

Figure 1.1. A broad range products and technologies inspired by organic electronics [2].
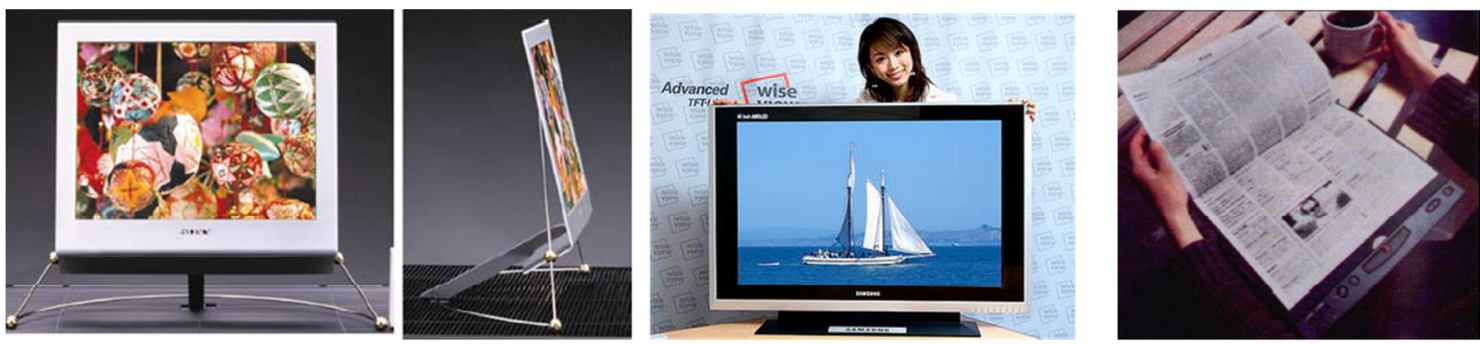

Large Area Displays (Sony, Samsung)

E-Papers (Plastic Logic)
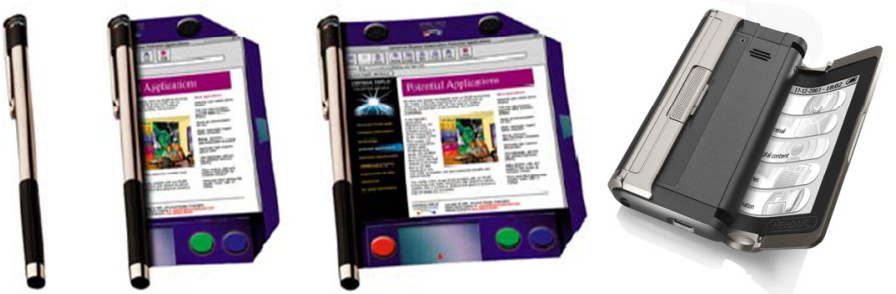

Rollable \& Portable Displays (UDC, Polymer Vision)

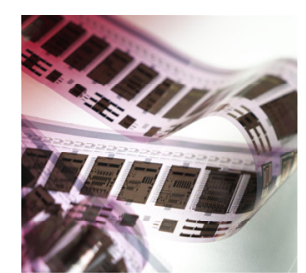

RFID Tags

(PolyIC)

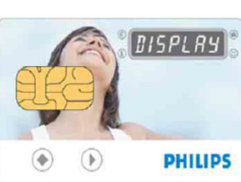

Smart Cards (Philips)

Figure 1.2. Examples of commercial opportunities for OTFTs.

The unique features which give organic electronics a technological edge are its simpler fabrication methods and compatibility for flexible electronics. Fabrication of organic electronics 
can be done using simpler equipment such as an evaporator, a spin-coater and a printer, and can be carried out in a regular laboratory environment. For example, solution-processible organic thin films can be deposited by spin coating, enabling fast and inexpensive coverage over large areas. Printing techniques (e.g., inkjet printing) can be used to deposit soluble organic inks, presenting an efficient solution for fabrication of organic devices. In addition, low temperature processing and mechanical flexibility of organic materials make them highly favorable for implementation of flexible electronics on robust substrates or on non-conventional form factors. In general, organic electronic devices are not expected to compete with silicon devices in the production of high-end products, especially because organic semiconductors have lower electrical performance (e.g., speed) than silicon. Instead, organic electronics technology is intended to complement conventional silicon technology, and is expected to thrive in a different market domain targeting lower resolution, lower end, cost-effective and mass-produced items, such as identification tags, smart cards, and pixel drivers for display and sensor technology. A more detailed overview of the market opportunities for organic electronics is presented in Section 1.1. The motivation and objectives underlying this thesis research framework are presented in Section 1.2. The organization of this thesis is outlined in Section 1.3.

\subsection{Organic Electronics: History and Market Opportunities}

Historically, organic materials (or plastics) were viewed as insulators, where applications are commonly seen in inactive packaging, coating, containers, mouldings, etc. Research on the electrical behavior of organic materials commenced in the 1960s [5]. Photoconductive organic materials were discovered in the 1970s and were used in xerographic sensors. The announcement of conductive polymers in the late 1970s [6], and of conjugated semiconductors and photoemission polymers in the 1980s [7], gave a new impulse to the activity in the field of organic electronics. Polyacetylene was one of the first polymers reported to be capable of conducting electricity [8], and it was discovered that oxidative doping with iodine causes the conductivity to increase by 12 orders of magnitude [9]. This discovery and development of highly-conductive organic polymers was credited to Alan J. Heeger, Alan G. MacDiarmid, and Hideki Shirakawa, who were jointly awarded the Nobel Prize in Chemistry in 2000 for the 1977 discovery and development of oxidized, iodine-doped polyacetylene. 
Today, the continual evolution of organic semiconductor material with improved stability, processability, functionality and performance has led to fabrication of high-performance organic electronic devices in laboratory environments [10][11][12][13][14]. The remarkable advancement in organic semiconductor materials has recently prompted transition of the technology from academic research environment towards industrial research and development (R\&D) and commercialization. The shift towards industrial $R \& D$ is evident from the establishment of several government-sponsored research initiatives [15][16], the founding of various organic electronics driven associations and companies [17][18][19], and the development of IEEE standards for testing of organic electronics devices [20]. One example is the European Commission (EU) funded project called NAIMO (Nanoscale Integrated processing of self-organising Multi-functional Organic Materials); the project ambition is to create technological environment to develop a new sustainable industry of organic electronics to improve the quality of life of European citizens [21]. The increased cooperative efforts between academic, industry, and government are vital to the development of a strong materials and manufacturing infrastructure, in order to drive organic electronics towards early stages of commercialization [22][23][24][25][26].

The outlook for low-cost production of organic electronics is a key driver for market opportunities in this area. To achieve these cost targets, low-cost materials, cost-effective processes, and high-volume manufacturing infrastructure are required. The development of highvolume roll-to-roll manufacturing platforms, for fabrication of organic circuits on continuous, flexible, low-cost substrates, has been reported; these platforms are based on the integration of lithography, vacuum deposition, and printing technologies. It was forecasted that an organic semiconductor fabrication facility can be built for US\$40 million [3]. The estimated costs are substantially lower than the costs of existing silicon semiconductor fabrication facilities, which currently exceeds US\$3 billion. The high cost of silicon-based foundries can be attributed to the sophisticated wafer processing and handling equipment, high-resolution lithography tools, wafer testing equipment, clean-room environment, and costly chemical distribution and disposal facilities. In contrast, the cost reduction forecasted for an organic electronic manufacturing facility is expected to derive from lower cost materials, less sophisticated equipment, simpler manufacturing technologies, less stringent demands on clean-room settings, and reduced waste output. However, the potential savings in manufacturing cost of organic electronics comes with the tradeoff of lower performance. 
Figure 1.3 provides a conceptual view of the cost-and-performance sectors served by silicon technology and organic semiconductor technology. It must be noted that organic semiconductor devices do not offer the same electrical performance as silicon devices. While silicon technology is aimed for high-end, high performance and high processing power electronic products, organic semiconductor technology appeals to lower-end, cost-effective disposable electronics products.

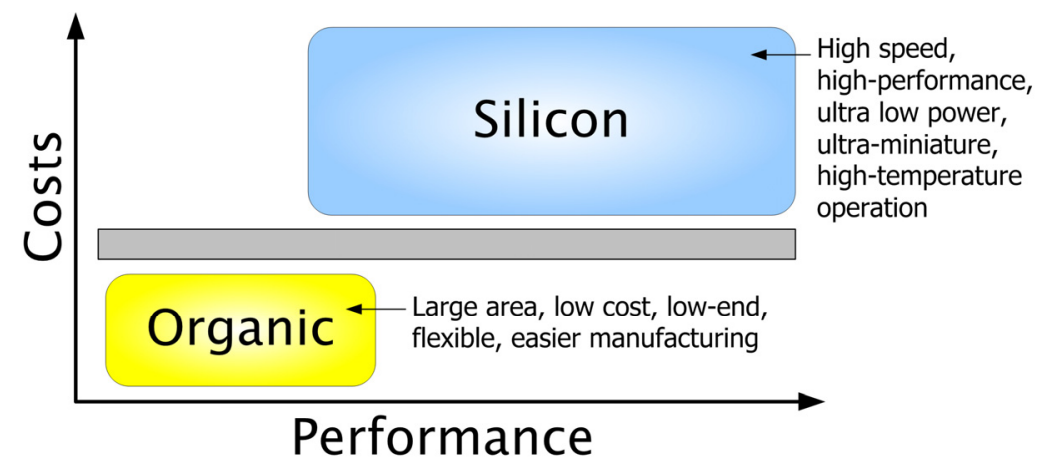

Figure 1.3. Pictorial illustration on cost versus performance comparison between silicon technology and organic semiconductor technology.

One of the most frequently discussed opportunities for organic electronics is their integration and use for the driver backplane of flexible displays. Specifically, printed organic semiconductor materials are strong candidates for novel electrically active display media. RFID tags are another avid application for OTFTs. An overview of these OTFT-based applications and their current market status are presented next. Note that at present, amorphous silicon (a-Si) TFTs and polycrystalline silicon (poly-Si) TFTs are the key backplane technology used in flat panel display products. Therefore, OTFTs are not intended to displace a-Si TFTs in large-area high resolution flat panel displays; instead, OTFTs will have a bigger impact on lower-cost flexible displays and electronic paper applications. The key features of OTFT and a-Si TFT are compared in Table 1.1. 
Table 1.1. Comparison of OTFTs and amorphous silicon (a-Si) TFTs.

\begin{tabular}{|c|c|c|}
\hline & OTFT & a-Si TFT \\
\hline Material & $\begin{array}{l}\text { Organic semiconductor as active layer; } \\
\text { P-type, n-type, ambipolar }\end{array}$ & $\begin{array}{l}\text { a-Si as active layer; } \\
\mathrm{N} \text {-type }\end{array}$ \\
\hline Processing & $\begin{array}{l}\text { Spin-coat, print, evaporation; } \\
\text { Low temperature (e.g., room } \\
\text { temperature) }\end{array}$ & $\begin{array}{l}\text { Plasma enhanced chemical vapor } \\
\text { deposition (PECVD); } \\
T<350^{\circ} \mathrm{C}\end{array}$ \\
\hline \multirow{3}{*}{$\begin{array}{l}\text { Mobility } \\
\text { Substrate \& } \\
\text { Form Factor } \\
\text { Mechanical } \\
\text { Flexibility }\end{array}$} & Can be comparable to a-Si & $\sim 1 \mathrm{~cm}^{2} / \mathrm{V}-\mathrm{s}$ \\
\hline & $\begin{array}{l}\text { Variety of substrates \& form factor; } \\
\text { Mechanically flexible }\end{array}$ & $\begin{array}{l}\text { Glass (most common), } \\
\text { Plastic (in development) }\end{array}$ \\
\hline & More bendable & More fragile and brittle \\
\hline $\begin{array}{l}\text { Electrical } \\
\text { Stability }\end{array}$ & $\begin{array}{l}\text { More rapid degradation, but } \\
\text { degradation stabilizes } \\
\text { (may be favorable for devices that turn } \\
\text { on for a longer time) }\end{array}$ & $\begin{array}{l}\text { Slower bias-induced degradation, } \\
\text { but degradation does not stabilize }\end{array}$ \\
\hline Pros & Potentially no cleanroom, lower cost & More mature and stable \\
\hline Cons & $\begin{array}{l}\text { Process challenge; device performance, } \\
\text { stability, and lifetime }\end{array}$ & $\begin{array}{l}\text { Mechanical flexibility (stress), } \\
\text { Higher processing temperature }\end{array}$ \\
\hline $\begin{array}{l}\text { Key } \\
\text { Applications }\end{array}$ & $\begin{array}{l}\text { Numerous: displays, RFID tags, sensors, } \\
\text { disposable electronics }\end{array}$ & $\begin{array}{l}\text { Circuits for large-area displays and } \\
\text { sensors array backplane }\end{array}$ \\
\hline Outlook & $\begin{array}{l}\text { New opportunities: smaller/flexible } \\
\text { displays, disposable electronics, smart } \\
\text { textiles }\end{array}$ & $\begin{array}{l}\text { Continue to excel in AMLCD, } \\
\text { AMOLED, active-matrix sensor } \\
\text { technologies }\end{array}$ \\
\hline
\end{tabular}

\subsubsection{Large-Area Displays}

The application of OTFTs for large area displays has been demonstrated by a number of companies and research institutions. For example, Plastic Logic Ltd. demonstrated the integration of an OTFT-driven backplane to a Gyricon display in 2003 [27]. The active-matrix display backplane was inkjet printed and drove a 3000-pixel display that was fabricated on glass. The active-matrix backplanes consisted of arrays of OTFTs that switched each pixel within the display. In early 2007, Plastic Logic secured \$100 million venture capital funding to build the world's first factory to produce plastic electronic devices [18]. Such investment exemplifies the promising outlook for the potential commercialization and growth of the organic electronics industry.

A number of corporations have also invested in R\&D for OTFT-driven large area displays; examples include Sony, Samsung, Kodak, LG Philips, Motorola, 3M, and Hewlett-Packard. In 2007, Sony demonstrated a 2.5 inch active-matrix organic light emitting diode (AMOLED) display driven by OTFTs [28]; LG Philips' LCD Division presented a high resolution active-matrix liquid 
crystal display (AMLCD) with OTFT-driven backplane based on solution processing fabrication methods [29]; and Samsung Electronics reported active-matrix display using printed OTFTs [30].

\subsubsection{Rollable Displays}

The mechanical flexibility of organic materials makes them particularly attractive for rollable or flexible displays. Polymer Vision, a spin off from Royal Philips Electronics, is a pioneer in demonstrating the capability of rollable displays. Their displays are produced by combining ultrathin flexible OTFT-driven active-matrix backplane technology and flexible electronic ink (EInk) display technology. In January 2008, Polymer Vision introduced their first rollable display product, called Readius ${ }^{\circledR}$. The product is a pocket-sized device which combines a 5" rollable display with high speed connectivity. Thus, the product merges the reading-friendly strengths of electronic-readers with the high mobility features of mobile phones, along with instant access to personalized news and information [31]. Demand for larger mobile displays is accelerating as telecom players push mobile content and mobile advertisements to compensate for shrinking growth in voice revenues. The solution is to unroll the display when needed and simply store away when not in use. Therefore, rollable display enabled devices are expected to be an emerging commodity for new generations of portable communication devices, thus presenting exciting commercial opportunities for OTFT-driven display backplane technology.

\subsubsection{Radio Frequency Identification (RFID) Tag}

One of the frequently promoted applications for organic electronics is the radio frequency identification (RFID) tag. RFID tag is a wireless form of automated identification technology that allows for non-contact reading of data, which makes it effective for manufacturing, inventory and transport environments where bar code labels are inadequate. By passing items equipped with RFID tags through a doorway similar to theft detectors at a store, a computer system would instantly identify and record the items. Silicon versions of RFID tags are currently in use. The cheapest silicon RFID chips cost about 50 cents (US\$) each, which is rather costly to implement in most situations. The industry consensus is that the price of silicon RFID tags will drop to 10 cents, but it is not yet realized [32]. In contrast, organic semiconductor RFID tags can be a cost-effective alternative because they can be printed directly onto retails product along with the antenna. 
Organic-based RFID tags are anticipated to be as inexpensive as one cent each, thus justifying their use even on low-priced items like groceries. It is envisioned that in an RFID-enabled grocery store, customers can simply pass their shopping cart through a RFID sensor which instantly calculates the total purchase price, without the need of cashier. Other advantages of organic-based RFID tags over silicon-based tags include mechanical flexibility (e.g., bendable) and direct fabrication onto large area substrates using simple printable methods. Contrarily, in the case of silicon RFID tags, silicon chips are first fabricated by standard high-end CMOS process and are subsequently attached/bonded to plastic substrate to form the RFID tag. Thus, the processing of organic RFID tags is simpler than silicon-based tags.

The attractiveness of printed organic semiconductor materials and manufacturing platforms has provoked the involvement of several companies (e.g., 3M, Siemens), start-ups (e.g., OrganicID, ORFID Corp.) and research institutions to develop technology for organic-based RFID tags [25][26]. The latest breakthrough in organic RFID was the demonstration of a 64-bit inductivelycoupled passive plastic RFID tag on plastic substrate, operating at $13.56 \mathrm{MHz}$ and with a read distance of over $10 \mathrm{~cm}$; these specifications are approaching item-level tagging requirement. This achievement paves the way for low-cost high-volume production of RFID tags, with the potential to use as barcode replacement [19][33].

\subsection{Thesis Motivation and Objectives}

With continual advances in the material properties and processing technologies, this thesis on OTFT integration is motivated by the need to advance organic electronics from fundamental research to practical applications and to develop the needed technology kernels for realization of low-cost organic memory and logic circuits, as well as displays and imagers on mechanically flexible plastic substrates. The research emphasis/scope is not on material development, but on device engineering and process integration. The key objectives of this thesis are to advance OTFT technology in the area of device performance and device manufacture, and to demonstrate feasibility of OTFT integration into functional organic circuits. The present technological challenges in device performance and manufacturing are discussed in Section 1.2.1. The research approaches undertaken to address these technological challenges and to fulfill the thesis objectives are outlined in Section 1.2.2. 


\subsubsection{Technological Challenges}

Organic electronics have reached early stages of commercial viability. Personal electronic devices incorporating small displays based on OLEDs are now available. However, many challenges still remain and are currently hindering the wide adoption of OTFT in electronic devices. The shortcomings of OTFTs include limited charge carrier mobilities, poor device stability and lifetime, high contact resistance, and limited availability of robust/mature patterning techniques that are compatible with organic thin films. These technical challenges can be grouped into two categories: device performance and device manufacture.

\subsubsection{Device Performance}

One of the limitations of organic semiconductor materials, compared to silicon technology, is its intrinsically lower mobility. Most organic semiconductor thin films are composed of a mixture of polycrystalline and amorphous phases. The hopping process between molecules in the disordered regions often limits charge-carrier mobilities in organic semiconductor films [34]. To improve charge transport and device mobility, the disorder phase must be suppressed. The two most common tactics considered are:

(i) Tuning the molecular structure of the organic semiconductor during material preparation. Examples include modifying molecular parameters (e.g., regioregularity, molecular weight, side-chain length, doping level) during material synthesis, and altering processing conditions during material deposition (e.g., thermal annealing, solvent selection, film thickness, deposition methods and parameters) [10][11][12].

(ii) Exploiting interfacial phenomena to improve molecular ordering of the semiconductor layer during device processing. Examples of interfacial phenomena include semiconductor alignment using self-assembled monolayers (SAMs), surface-mediated molecular ordering, surface dipoles, physical alignment, and photoalignment [35]. Surface control using SAMs is a well-known technique for such interface modifications and can provide microscopically good interface regulations.

This thesis expands on the latter tactic, where the device interfaces are engineered to enhance device performance. An OTFT has two critical interfaces: the interface between the gate dielectric and organic semiconductor, and the interface between source/drain contacts and organic semiconductor. These two interfaces dictate charge transport and charge injection in OTFTs, 
respectively, and have an overriding influence on the device characteristics. In this thesis, interface modification techniques for these two device interfaces are investigated, with an attempt to enhance device performance.

One of the research objectives in this thesis is to enable the integration of a plasma enhanced chemical vapor deposited (PECVD) gate dielectric with a solution-processible organic semiconductor for OTFT fabrication. PECVD silicon nitride $\left(\mathrm{SiN}_{\mathrm{x}}\right)$ was studied as the gate dielectric. Past experiments reported limited OTFT performance with PECVD SiN $\mathrm{x}$ gate dielectric, and attributed the limited performance to surface roughness and unfriendly (or non-organicfriendly) interfaces of $\mathrm{SiN}_{\mathrm{x}}$. However, there is an interest to explore PECVD $\mathrm{SiN}_{\mathrm{x}}$ as a gate dielectric candidate for OTFTs owing to its good dielectric properties and its suitability for large area flexible electronics. We believe the critical factors to enable integration of $\mathrm{SiN}_{\mathrm{x}}$ in organic semiconductor applications is the quest for a suitable $\mathrm{SiN}_{\mathrm{x}}$ composition and an agreeable interface modification process. Strategies to address these factors and to enable use of $\mathrm{SiN}_{\mathrm{x}}$ with organic materials while delivering acceptable device performance are presented in this thesis.

\subsubsection{Device Manufacture}

Since a major driving force behind OTFT technology is the manufacture of low-end, low cost and disposable electronic devices, this demands a fabrication process that allows high volume production at low cost. Moreover, the fabrication technique should be able to produce standalone devices, device arrays, and integrated circuits (ICs) of acceptable operating speed, functionality, reliability, and lifetime. However, an integrated process that can meet the above requirements for production of high yield, stable OTFTs is currently non-existent.

Conventional photolithography processes for manufacturing of silicon-based microelectronics are not completely amenable to organic electronics. Although photolithography has the advantage of producing high resolution, complex device structures with excellent precision, the process must be tweaked to ensure compatibility with organic materials. Advanced printing techniques (e.g., inkjet printing, nanoimprinting) that take advantage of the solution-processability of organic materials are highly favorable for achieving the goals of low-cost and high-volume production. Inkjet printing technology is particularly attractive because it offers the advantages of fast, direct imaging and single-step print processing, precise deposition of the organic ink only where it is needed (thus reducing waste), compatibility with flexible substrates, large area processing and high material usage efficiency. However, because the requirements of printing electronic functions are 
very different from those of printing visual images, the adaptation of inkjet systems for processing organic electronic devices will require extensive optimization of printing parameters and processing conditions; in addition, technological concerns such as layer continuity and multilayer registration must be resolved. Inkjet printed organic devices with good performance have been demonstrated; however, low device yield is an issue. This thesis addresses challenges in OTFT manufacture by exploring a hybrid manufacturing approach that combines photolithography process with a novel inkjet printing technique, thus delivering an integration strategy with workable manufacturing yields while lowering costs compared to conventional processes.

\subsubsection{Research Scope and Approaches}

As the material properties and processing technology for organic electronics continue to advance and mature, the next phase of development is directed at integrating OTFTs into circuitries to implement practical electronic devices. In lieu of pursuing organic semiconductor material development (where the strength in this area resides in chemical physicists), this thesis strives to advance OTFT research from an engineering and integration perspective. By utilizing and assimilating existing materials, techniques and resources, this research explores a number of approaches to deliver higher performance devices and demonstrate feasibility of organic circuits for practical applications. The key focus areas of this thesis include:

$\phi$ Development of OTFT fabrication strategies to enable circuit integration (Chapter 3);

$\$$ Optimization of PECVD gate dielectric composition and structure to improve OTFT performance (Chapter 4);

$\phi$ Investigation of interface engineering methodologies to enhance the dielectricsemiconductor interface and the contact-semiconductor interface (Chapter 5 and 6);

$\phi$ Finally, the scientific and technical knowledge acquired from these investigations are applied to demonstrate integration of OTFTs into functional circuits for active-matrix display and RFID applications (Chapter 7).

Research activities were primarily conducted at the Giga-to-Nano $(\mathrm{G} 2 \mathrm{~N})$ research facility at the University of Waterloo. Device fabrication and characterization were conducted in-house. Research collaboration with Xerox Research Centre of Canada (XRCC) has granted access to Xerox's high quality, high performance, stable organic semiconductor material; this was a key ingredient for the novel research outcomes attained in this thesis research. Xerox's solution-processible poly $\left(3,3^{\prime \prime \prime}-\right.$ 
dialkylquarterthiophene) (PQT-12) polymer semiconductor form the basis for majority of the OTFT experimental work [10].

The initial phase of the research involved the development of deposition/patterning techniques for the fabrication of OTFTs. These were performed in conjunction with the design, fabrication, optimization, and systematic characterization of OTFTs based on different material systems. Key technical challenges related to the performance and manufacturing of OTFTs were addressed. The final phases of the research engaged in design, realization and evaluation of OTFT circuits.

It is envisioned that the outcomes from this research on OTFT integration will contribute to the development and advancement of OTFT ICs for a new generation of all-organic displays, communication devices, and other pertinent applications.

\subsection{Thesis Organization}

This thesis is divided into nine chapters. We begin with an introduction to organic electronics and market opportunities for OTFT technologies in Chapter 1, along with the motivation and objectives of this thesis. Chapter 2 examines the OTFT technology in greater depth, with a review of fundamental properties of organic semiconductors and discussion of OTFT operation, device architectures, and material selection. Chapter 3 presents integration strategies developed through the course of this research to enable fabrication of OTFT circuits.

With the aspiration to improve OTFT device performance, optimization of PECVD gate dielectrics is explored in Chapter 4. Interface engineering strategies to improve charge transport by dielectric-semiconductor interface treatment methods and to enhance charge injection by contactsemiconductor interface modification techniques are investigated in Chapter 5 and Chapter 6, respectively. The objectives for these investigations are to enhance OTFT characteristics via functionalizing the gate dielectric material and the device interfaces, and to develop better understanding on materials and device/interface physics for OTFTs.

The scientific and technical knowledge gained from these investigations are assimilated to demonstrate integration of OTFTs into functional circuits in Chapter 7. Finally, the major conclusions and the key research contributions of this thesis are summarized in Chapter 8 and Chapter 9, respectively. The structural design of this thesis is summarized in Figure 1.4, which illustrates the flow of the various research topics towards fulfilling the thesis objectives in advancing device manufacture, device performance and OTFT circuit integration. 


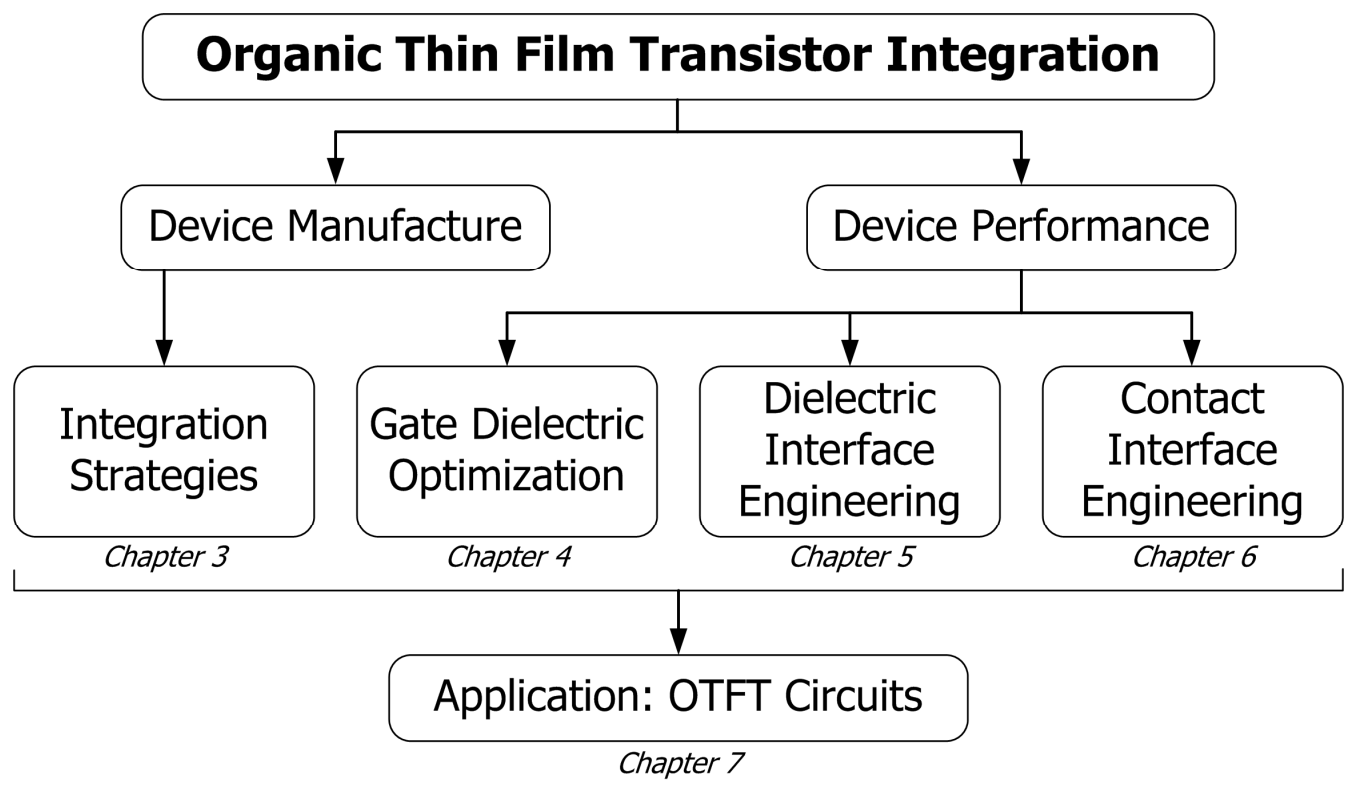

Figure 1.4. Flow chart on thesis organization. 


\section{Bibliography}

[1] C. Reese, M. Roberts, M.M. Ling, and Z. Bao, "Organic thin film transistors," Materials Today, 7(9), 20 (2004).

[2] Orgatronics. Website: http://www.orgatronics.com/.

[3] M. Chason, P. W. Brzis, J. Zhang, K. Kalyanasundaram, and D. R. Gamota, "Printed Organic Semiconducting Devices," Proc. Of IEEE 93 (7), 1348 (2006).

[4] A. Afzali, C.D. Dimitrakopoulos and T.L. Breen, "High-Performance, Solution-Processed Organic Thin Film Transistors from a Novel Pentacene Precursor," J. Am. Chem. Soc., 124, 8812 (2002).

[5] J.M. Shaw and P.F. Seidler, "Organic electronics: Introduction," IBM J. Res. \& Dev. 45(1), 3 (2001).

[6] "The 2000 Nobel Prize in Chemistry," (2000). www.nobel.se/chemistry/laureates/2000/press.html.

[7] G. Horowitz, "Organic Field-Effect Transistors,” Adv. Mater., 10(5), 365 (1998).

[8] T. Ito, H. Shirakawa, and S. Ikeda, "Simultaneous polymerization and formation of polyacetylene film on the surface of concentrated soluble Ziegler-type catalyst solution," J. Polym. Sci.: Chem. Ed. 12(1), 11 (1974).

[9] C.K. Chiang, C. R. Fincher, Y.W. Park, A.J, Heeger, H. Shirakawa, E.J. Louis, S. C. Gau, and A.G. MacDiarmid, "Electrical Conductivity in Doped Polyacetylene," Phys. Rev. Lett. 39, 1098 (1977).

[10] B.S. Ong, Y. Wu, and P. Liu, "Design of High-Performance Regioregular Polythiophenes for Organic Thin-Film Transistors," Proc. IEEE 93(8), 1412 (2005).

[11] I. McCulloch, C. Bailey, M. Giles, M. Heeney, M. Shkunov, D. Sparrowe, M. Suzuki, and R. Wagner, "Stable Semiconducting Thiophene Polymers and Their Field Effect Transistor Properties," 2005 Mater. Res. Soc. Spring Meeting, Symposium I: Organic Thin-Film Electronics, I7.10 (2005).

[12] I. McCulloch, M. Heeney, C. Bailey, K. Genevicius, I. MacDonald, M. Shkunov, D. Sparrowe, S. Tierney, R. Wagner, W. Zhang, M.L. Chabinyc, R.J. Kline, D. McGehee, and M.F. Toney, "Liquid-crystalline semiconducting polymers with high charge-carrier mobility," Nature Materials 5, 328 (2006).

[13] Y. Lin, D. Gundlach, S. Nelson, and T. Jackson, "Pentacene-based organic thin-film transistors," IEEE Trans. Electron Devices 44(8), 1325 (1997).

[14] H. Sirringhaus, T. Kawase, R. H. Friend, T. Shimoda, M. Inbasekaran, W. Wu, and E. P. Woo, "High-resolution inkjet printing of all-polymer transistor circuits," Science 290, 2123 (2000).

[15] "Project brief: Printed organic ASICs: A disruptive technology," National Institute of Standards and Technology (NIST) (2000).

http://jazz.nist.gov/atpcf/prjbriefs/prjbrief.cfm?ProjectNumber=00-00-4209 
[16] "Project brief: Printed organic transistors on plastic for electronic displays and circuits," National Institute of Standards and Technology (NIST) (2000).

http://jazz.nist.gov/atpcf/prjbriefs/prjbrief.cfm?ProjectNumber=00-00-4968

[17] Organic Electronics Association. Website: http://www.oe-a.org

[18] “UK in plastic electronics drive," BBC News, 3 January 2007. http://news.bbc.co.uk/2/hi/business/6227575.stm

[19] PolyApply: The Application of Polymer Electronics to Ambient Intelligence. Website: http://www. polyapply.org.

[20] "IEEE Standard for Test Methods for the Characterization of Organic Transistors and Materials," IEEE Standard P1620 ${ }^{\mathrm{TM}}$ (2004). http://grouper.ieee.org/groups/1620/

[21] NAIMO Project. Website: http://www.naimo-project.org.

[22] T. Jackson, Y. Lin, D. Gundlach, and H. Klauk, "Organic thin-film transistors for organic light-emitting flat-panel display backplanes,” IEEE J. Sel. Topics Quantum Electron. 4(1), 100 (1998).

[23] H. Huitema, G. Gelinck, J. van der Putten, K. Kuijk, C. Hart, E. Cantatore, P. Herwig, A. van Breemen, and D. de Leeuw, "Plastic transistors in active-matrix displays," Nature 414, 599 (2001).

[24] B. Crone, A. Dodabalapur, Y. Lin, R. W. Filas, Z. Bao, and A. La-Duca, "Large-scale complementary integrated circuits based on organic transistors," Nature 403, 521 (2000).

[25] R. Rotzoll, S. Mohapatra, V. Olariu, R. Wenz, M. Grigas, O. Shchekin, K. Dimmler, and A. Dodabalapur, "13.56 MHz Organic Transistor Based Rectifier Circuits for RFID Tags," Mater. Res. Soc. Symp. Proc. 871E, I11.6.1 (2005).

[26] P.F. Baude, D.A. Ender, M.A. Haase, T.W. Kelley, D.V. Muyres, and S.D. Theiss, "Pentacene-based radio-frequency identification circuitry," Appl. Phys. Letts. 82, 3964 (2003).

[27] S. Burns, C. Kuhn, J. D. MacKenzie, K. Jacobs, N. Stone, D.Wilson, P. Devine, K. Chalmers, N. Murton, P. Cain, J. Mills, R.H. Friend, and H. Sirringhaus, "Active-matrix displays made with ink-jet-printed polymer TFT," presented at the Soc. Information Display Int. Symp., Exhibition, and Seminar, Baltimore, MD (2003).

[28] Organic semiconductor analyst direct, Vol. 5, Issue 39, October 2007.

[29] H. Kang, "High Resolution OTFT-AMLCD using Solution Process," presented at Printed Electronics USA 2007, San Francisco, CA, USA (2007).

[30] S. Lee, "Active Matrix Display Using TFT Array", presented at Printed Electronics USA 2007, San Francisco, CA, USA (2007).

[31] "Polymer Vision pioneers world's first production of rollable displays," Press Release 1012-2007, Polymer Vision (2007).

[32] P. Heremans, S. Verlaak and T. McLean In: P. Gamota, P. Brazis, K. Kalyanasundaram and J. Zhang, Editors, Printed Organic and Molecular Electronics, Kluwer Academic Publishers, Boston, Dordrecht, New York, London (2004). 
[33] “Organic RFID Breakthrough,” Technology News Daily, 02-11-2008. http://www.technologynewsdaily.com/node/9175

[34] N. Hiroshiba, R. Kumashiro, N. Komatsu, Y. Suto, H. Ishii, S. Takaishi, M. Yamashita, K. Tsukagoshi, and K. Tanigaki, "Surface Modifications using Thiol Self-Assembled Monolayers on Au Electrodes in Organic Field Effect Transistors," Mater. Res. Soc. Symp. Proc. 965, 0965-S08-03 (2007).

[35] Y.D. Park, J.A. Lim, H.S. Lee, and K. Cho, "Interface engineering in organic transistors," Materials Today 10, 46-54 (2007). 


\section{Chapter 2}

\section{Organic Thin Film Transistor (OTFT) Overview}

By definition, organic materials describe a large class of chemical compounds whose molecules contain carbon. The dividing line between organic and inorganic is somewhat controversial and historically arbitrary, but generally speaking, organic compounds have carbon-hydrogen bonds, and inorganic compounds do not [1]. Until about 40 years ago, all carbon based organic compounds and polymers ${ }^{1}$ were regarded as insulators. Organic polymer materials (or plastics) were utilized as inactive packaging and insulating materials. This narrow perspective rapidly changed as a new class of polymer known as conductive polymer was discovered in the 1960s-1970s [2][3][4]. Today, there is tremendous research efforts focused on using conductive polymers for electronic fabrication. Depending on the resistivity levels, conductive polymers can behave as semiconductors (referred to as "organic semiconductors"), or they can be highly doped to behave as conductors or metals.

Organic semiconductor is loosely defined as any organic material that has semiconductor properties. Both short chain (oligomers) and long chain (polymers) organic semiconductors are known. There are two major classes of organic semiconductors, with considerable overlap: organic charge-transfer complexes, and various "linear backbone" polymers derived from polyacetylene. Charge transfer (CT) complexes are obtained by pairing an electron donor molecule with an

\footnotetext{
${ }^{1}$ Polymer is a long, repeating chain of atoms, formed through the linkage of many molecules called monomers. In this report, the discussion of polymers is limited to organic polymers (despite inorganic polymers also exist).
} 
electron acceptor molecule, and are characterized by electronic transition(s) to an excited state. One example of CT complex is a TTF-TCNQ crystal, where tetrathiafulvalene (TTF) serves as a donor and tetracyanoquinodimethane (TCNQ) serves as an acceptor. TTF-TCNQ crystal was the first organic conductor to show almost metallic conductivity (in 1972). Organic semiconductors based on linear-backbone polymers are obtained from doped conjugated polymers. In this thesis, discussion of "organic semiconductor" pertains to this class of polymer-based organic semiconductors.

Transistors based on organic semiconductors as the active layer to control current flow are commonly referred to as organic thin film transistors (OTFTs), or sometimes organic field effect transistors (OFETs). The simplest OTFT configuration is shown in Figure 2.1. Generally speaking, a TFT is composed of three main parts: a thin semiconductor layer, a dielectric (or insulator), and three electrodes (gate, source, and drain). The source (S) and drain (D) electrodes directly contact the semiconductor, whereas the gate $(G)$ electrode is separated from the semiconductor by a dielectric layer. The gate turns the device on and off with an applied voltage, and thus controls the current flow $\left(I_{\mathrm{DS}}\right)$ in the semiconductor between the source and drain electrodes.

\begin{tabular}{|c|c|} 
Source & Drain \\
\hline Organic Semiconductor \\
\hline Gate Dielectric \\
\hline Gate \\
\hline Substrate \\
\hline
\end{tabular}

Figure 2.1. Cross section of a basic OTFT structure, in bottom-gate and top-contact configuration.

This chapter presents an overview on OTFT technology. The organic semiconductor, which is the heart or foundation of the OTFT, is introduced in Section 2.1. The fundamental properties of organic semiconductors and recent progresses in material development are reviewed. The basic operation and characteristics of the OTFT are discussed in Section 2.2, along with the parameter extraction techniques used for characterizing OTFT performance. The device architectures and material systems, specific to this thesis research, are examined in Section 2.3 and Section 2.4, respectively. Please note that the terms "organic" and "polymer" are used interchangeably in this thesis. Generally speaking, "organic" covers a wider scope of materials, where "polymer" is a subset of organic. The research reported in this thesis focus primarily on polymer semiconductors. 


\subsection{Organic Semiconductor Overview}

The operation and performance of OTFTs are largely dictated by the characteristics of the active organic semiconductor layer. In particular, mobility of the device is related to the efficiency of charge transport through the semiconductor channel. This section begins with a review on the unique conjugated chemical structure that gives rise to electrical conduction in organic semiconductor materials. Typical charge transport mechanisms in organic materials are presented. These models pertain primarily to non-crystalline (or disordered) organic semiconductors (e.g., polymers); the transport mechanism in well-organized organic molecular crystals is different, and is outside the scope of this thesis. The recent progresses in material development and classifications of organic semiconductors are considered.

\subsubsection{Basic Properties}

Polyacetylene was one of the first polymers reported to be capable of conducting electricity [5], and it was discovered that oxidative doping with iodine causes the conductivity to increase by 12 orders of magnitude [6]. The "doped" form of polyacetylene had a conductivity of $10^{5}$ Siemens per meter $(\mathrm{S} / \mathrm{m})$. As a comparison, an insulator such as teflon has conductivity of $10^{-16} \mathrm{~S} / \mathrm{m}$, and a metal such as silver and copper has conductivity of $10^{8} \mathrm{~S} / \mathrm{m}$ [4]. However, polyacetylene reacted rapidly and irreversibly with oxygen, was insoluble in organic solvents, and was difficult to process. Progress was made with the discovery of polythiophene (PT) and polyphenylenevinylene (PPV), which exhibited better characteristics than polyacetylene [7]. Figure 2.2 shows the chemical structure of these three conductive polymers.

All electrically conductive polymers share two principal properties. The first is the presence of "conjugated double bonds" along the backbone of the polymer. In conjugation, the polymer consists of alternate single and double bonds between the carbon atoms. This alternating structure can be observed in polyacetylene, displayed in Figure 2.2(a). The second property is that the polymer must be "doped", implying that electrons are removed (through oxidation) or introduced (through reduction). These extra holes or electrons can move along the molecule to contribute to electrical conductivity [4]. 
<smiles>CC=CC=CC=CC=CC</smiles>

(a) Trans-polyacetylene

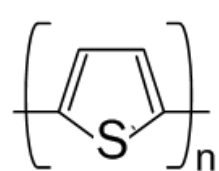

(b) Polythiophene

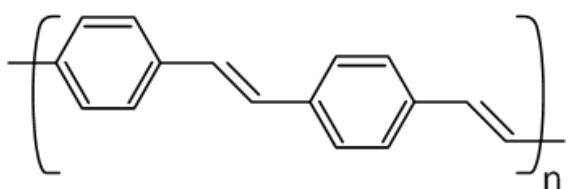

(c) Polyphenylenevinylene

Figure 2.2. Chemical structure of three conjugated polymers: (a) polyacetylene, (b) polythiophene and (c) polyphenylenevinylene [7].

In the conjugated double bond structure, the single bond is a sigma $(\sigma)$ bond, and the double bond consists of a $\sigma$-bond and a pi $(\pi)$ bond [8]. $\sigma$-bonds, the strongest type of covalent bonds, require that both atoms give an electron from the $s$ orbital. Thus, the electrons that form the $\sigma$-bond are attached to the two nuclei and are localized. $\pi$-bonds are direct sharing of electrons between two atoms' $p$ orbitals. $\pi$-bonds are weaker than $\sigma$-bonds because their orbitals are further away from the positively charged nucleus. Normally, the electrons that form a $\pi$-bond are localized. However, in conductive polymers, $\pi$-orbitals of the neighboring double bonds overlap due to the conjugated structure, as illustrated Figure 2.3(a). This overlapping results in weakly localized (or "delocalized") $\pi$-electrons that can move from one bond to another or move along the entire molecule. Therefore, delocalization, accomplished by the continuous overlapping $\pi$-orbitals of the conjugated backbone, makes the conduction of charge carriers along the polymer chain (i.e., intramolecular transport) possible [8].

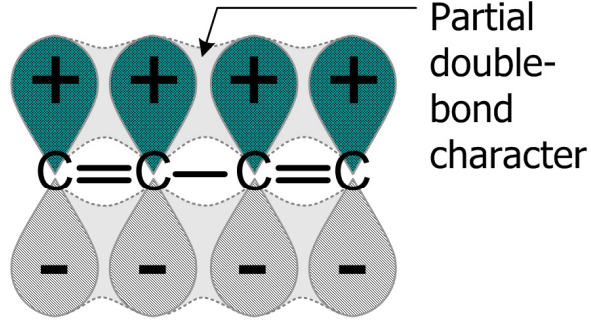

(a) Conjugated molecule

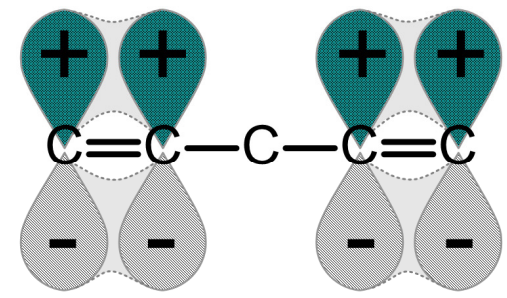

(b) Non-conjugated molecule

Figure 2.3. (a) Conjugated and (b) non-conjugated structure of an organic molecule [8].

The system of alternating double and single bonds in the conjugated backbone gives rise to a separation of bonding and anti-bonding states, resulting in the formation of a forbidden energy gap and a spatially delocalized band-like electronic structure, as illustrated in Figure 2.4. The highest occupied molecular orbital (HOMO) consists of bonding states of the $\pi$-orbitals with filled 
electrons, and is analogous to the valence band in silicon. The lowest unoccupied molecular orbital (LUMO) consists of empty higher energy anti-bonding $\left(\pi^{*}\right)$ orbitals, and is analogous to the conduction band. The energy difference between the HOMO and LUMO defines the band-gap energy $\left(E_{\mathrm{G}}\right) . E_{\mathrm{G}}$ depends on the chemical structure of the repeating unit, and generally decreases with the number of repeat units in the chain [4]. $E_{\mathrm{G}}$ of conjugated polymers is typically in the energy range of 1-4 eV. This band-like structure, along with low electronic mobility, is responsible for the semiconducting properties observed in conjugated polymers. Due to the disordered nature of organic materials, conduction mainly takes place via phonon-assisted hopping and polaronassisted tunneling between localized states; this is contrast to crystalline semiconductors (e.g., silicon) where conduction occurs in energy bands through delocalized states.

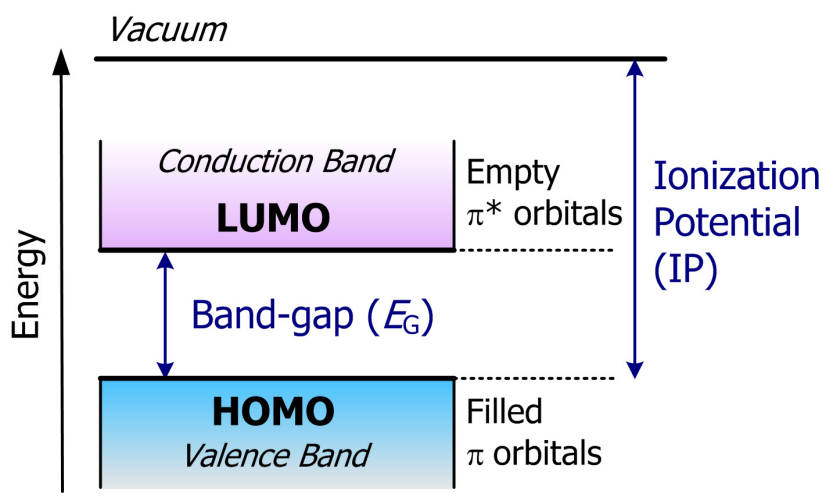

Figure 2.4. Representative energy band diagram of an organic semiconductor.

Most conductive polymers in their neutral state are wide band-gap semiconductors and exhibit very low conductivities [7]. To increase electrical conductivity, doping is required. The conductivity of a conjugated polymer can be modified by chemical doping or electrochemical doping, where oxidation and reduction reactions are used to achieve p-type (electron removal) and n-type (electron addition) doping, respectively [4]. These doping processes generate mobile charge carriers which move in an electric field, giving rise to electrical conductivity. In most cases, conductive polymers are doped by oxidative reactions; thus, p-type conductive polymer materials are more common, with holes as the majority transport carriers.

Highly conjugated organic materials can work as semiconductors because of their strong $\pi$ orbital overlap. When an electron is added or a hole is injected, the resultant charge becomes delocalized across the conjugated system. This injected charge acts as a carrier for current conducting through the molecule and thru the organic semiconductor thin film. Transistors based 
on organic semiconductors as the active layer to control current flow are commonly referred to as organic thin film transistors (OTFTs).

\subsubsection{Charge Transport}

In conductive polymers, the charge transport is relatively easy along a conjugated molecular chain (i.e., intramolecular transport) because of the strong $\pi$-orbital overlap. However, due to the amorphous structure of polymer materials and the weak intermolecular interaction, charge transport between molecules (i.e., intermolecular transport) is more difficult. Polymer semiconductor often contains polycrystalline phases intermixed in disorder phases. These disorder segments tend to hinder charge transport. Typically, the charge transport between molecules is described as a thermally activated tunneling of charge carriers, commonly referred to as "hopping" [9][10]. Hopping of carriers from chain to chain occurs between localized states; this rather inefficient conduction mechanism is responsible for the limited carrier mobility in amorphous organic semiconductors.

A main difference between the delocalized transport in crystalline inorganic semiconductor and the localized "hopping" transport in amorphous organic semiconductor is that, in the former, the transport is limited by phonon scattering, whereas in the latter, it is phonon assisted [9]. Accordingly, the charge mobility decreases with temperature in conventional crystalline semiconductors, and the reverse relationship applies for organic materials. The temperature dependence of the mobility $(\mu)$ of such "hopping" transport generally follows the form:

$$
\mu=\mu_{0} \exp \left[-\left(T_{0} / T\right)^{1 / \alpha}\right],
$$

where $T$ is temperature, $T_{0}$ and $\mu_{0}$ are material parameters, and $\alpha$ is an integer ranging from 1 to 4 [9]. The boundary between localized and delocalized transport processes is often taken at mobility near $0.1-1 \mathrm{~cm}^{2} / \mathrm{V}$-s. The mobility in highly ordered molecular crystals is close to this limit, thus there is controversy as to whether the conductivity in these materials should be described by localized or delocalized transport.

In addition to temperature dependence, the mobility of organic semiconductors becomes field dependent at high electric field (e.g., $>10^{5} \mathrm{~V} / \mathrm{cm}$ ) [9]. This phenomenon occurs through a PooleFrenkel mechanism, in which the applied field modifies the potential near the localized states in such a way as to increase the tunnel transfer rate of carriers between sites. The general fielddependence of the mobility is described by: 


$$
\mu(E)=\mu(0) \exp \left[\frac{q}{k T} \beta \sqrt{E}\right],
$$

where $\mu(0)$ is the mobility at zero field $(E=0), \beta=\left(\varepsilon / \pi \varepsilon \varepsilon_{0}\right)^{1 / 2}$ is the Poole-Frenkel factor, and $E$ is the magnitude of the electric field.

Gate voltage dependent mobility is often observed in organic semiconductors. This dependence stems from the fact that as the gate voltage increases, injected charge-carriers tend to fill the traps, so trapping becomes less efficient and charge transport improves. This behavior is described by the multiple trapping and release (MTR) model, which assumes delocalized transport is limited by a distribution of traps near the band edge [11]. The model also predicts thermally activated mobility. Interestingly, as the quality of OTFT devices improves, gate bias dependence and thermally activated mobility tend to be less encountered, which suggests these are defect-induced effects [11].

The charge transport and mobility in organic semiconductors are also limited by macroscopic factors such as poor contacts between different crystalline domains in the material or disorder in the material [4]. Therefore, molecular ordering and alignment of the conjugated segments in a thin film plays a critical role in attaining efficient charge transport between molecules, as discussed next.

\subsubsection{Microstructure and Molecular Alignment}

Most organic semiconductor thin films are composed of a mixture of polycrystalline and amorphous (disorder) phases. Charge transport via hopping of carriers between molecules in the disordered regions often limits charge carrier mobility. Thus, it is important to suppress the disorder phases and grain boundaries to achieve efficient charge transport. By ensuring the polymer chains or the organic molecules lie/stack close together in an orderly fashion, more efficient carrier transport can be achieved [4]. However, organic semiconductors tend to have poor self-organizing properties, due to their weak London or Van der Waals intermolecular bonds. Therefore, clever molecular design and proper interface preparation are needed to achieve a well-stacked and wellordered intermolecular structure in the polymer film.

The microstructure of an organic thin film can be characterized using glacing incidence $\mathrm{x}$-ray diffraction (GIXRD) and transmission electron diffraction measurements. An example is shown in Figure 2.5 for poly(3,3"'-dialkyl-quarterthiophene) (PQT-12) organic semiconductor thin film, 
where GIXRD and transmission electron diffraction were used to analyze molecular parameters and the $\pi-\pi$ stacking structure. Transmission electron diffraction analysis in Figure 2.5(c) indicates a $\pi$ - $\pi$ stacking distance of $3.7 \AA$ (along the face-to-face direction). The XRD pattern in Figure 2.5(b) renders information on interchain ordering, where the diffraction peaks correspond to interlayer distance of $\sim 17.3 \AA$ (i.e., intermolecular spacing along the lamellar $\pi-\pi$ stacking direction) [14]. ${ }^{2}$ These results signify the formation of lamellar $\pi$ - $\pi$ stacking structural orders in the thin film as well as a preferential orientation of the lamellar (100) axis normal to the substrate. Accordingly, it can be concluded that PQT-12 possesses an excellent ability to organize into highly ordered lamellar $\pi-\pi$ stacking structures (Figure 2.5(d)) whose orientation to the substrate could be manipulated through proper alignment layers and techniques [14][15].
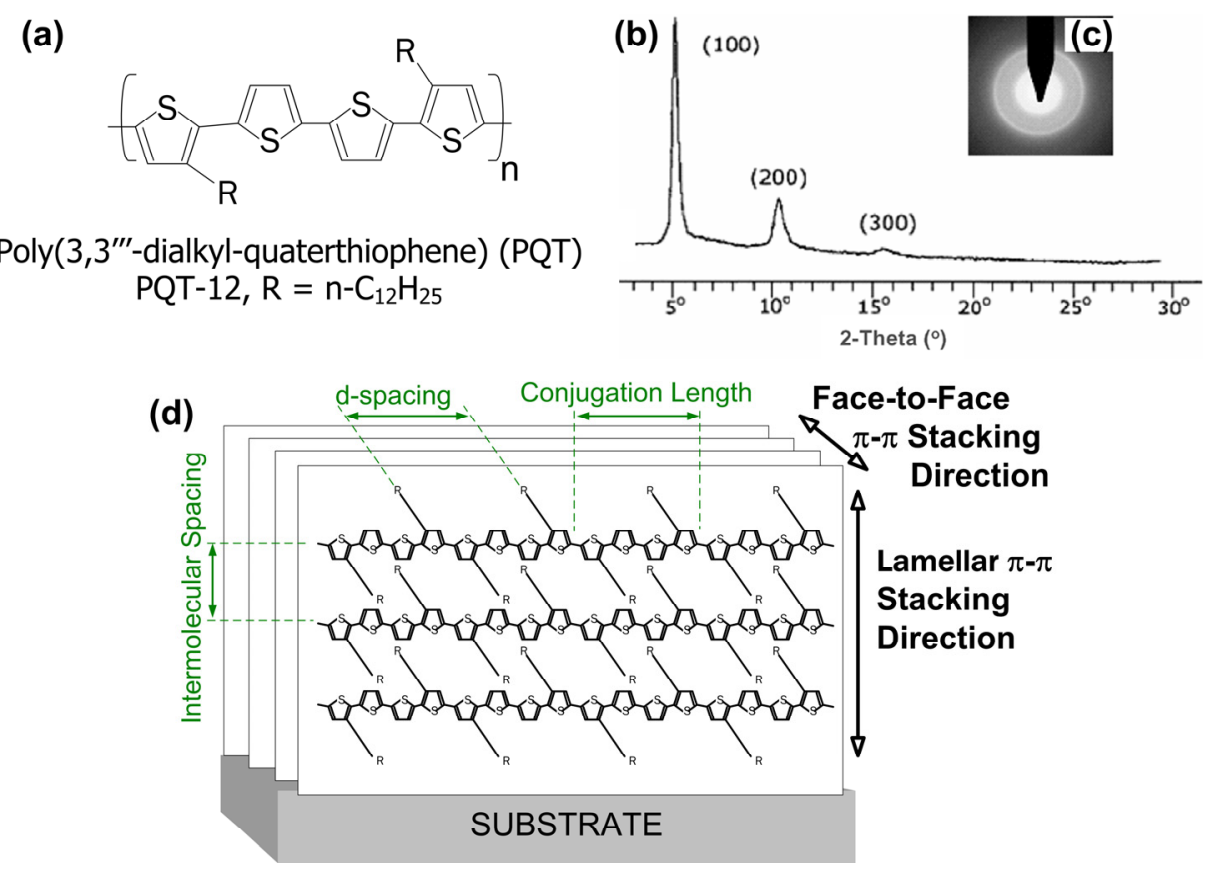

Figure 2.5. (a) Chemical structure of PQT-12 organic semiconductor. (b) GIXRD and (c) transmission electron diffraction pattern of a PQT-12 film. (d) Schematic illustration of lamellar $\pi-\pi$ stacking in a PQT-12 film (adapted from [15]).

In addition to stacking order, the orientation and alignment of the polymer chains in the device structure have a strong influence on the performance of polymer OTFTs. It was reported that

\footnotetext{
${ }^{2}$ According to Bragg's law $2 d \sin (\theta)=n \lambda$, for the diffraction peak at $2 \theta=5.1^{\circ}$, the interlayer distance $(d)$ of $\sim 17.3 \AA$ can be calculated. Assuming $n=1$ (measurement done in air) and $\lambda=1.54 \AA$ (wavelength of the $\mathrm{X}$-ray used in XRD measurement).
} 
poly(3-hexylthiophene) (P3HT) may adopt two different orientations depending on the processing conditions and dielectric surface properties (Figure 2.6): (a) with thiophene rings oriented edge-on (i.e., perpendicular to) relative to dielectric surface, or (b) with the chains oriented face-on (i.e., parallel to) the surface [12]. The mobility differed by more than a factor of 100, and higher device mobility was achieved for P3HT OTFTs with the edge-on arrangement.

(a)

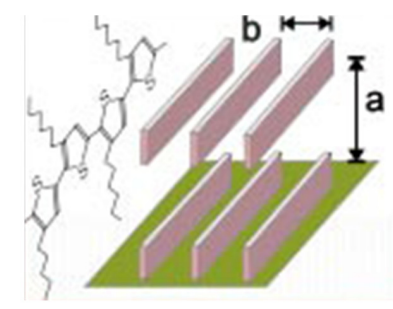

Edge-on Orientation (b)

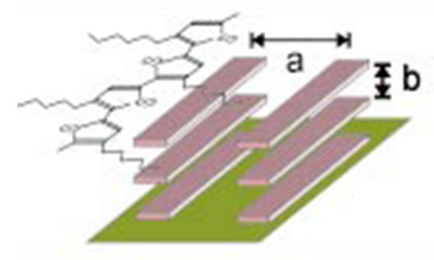

Face-on Orientation

Figure 2.6. Two different orientations of ordered P3HT domains with respect to the dielectric substrate surface (adapted from [12]).

The alignment of the polymer chains relative to the electrodes of an OTFT is also important. The ideal alignment of the organic molecules is such that charge transport is parallel to the substrate with in-plane electrodes, where the strong $\pi-\pi$ stacked building blocks are uniaxially aligned in a direction parallel to the current flow in the channel region, as illustrated in [13].

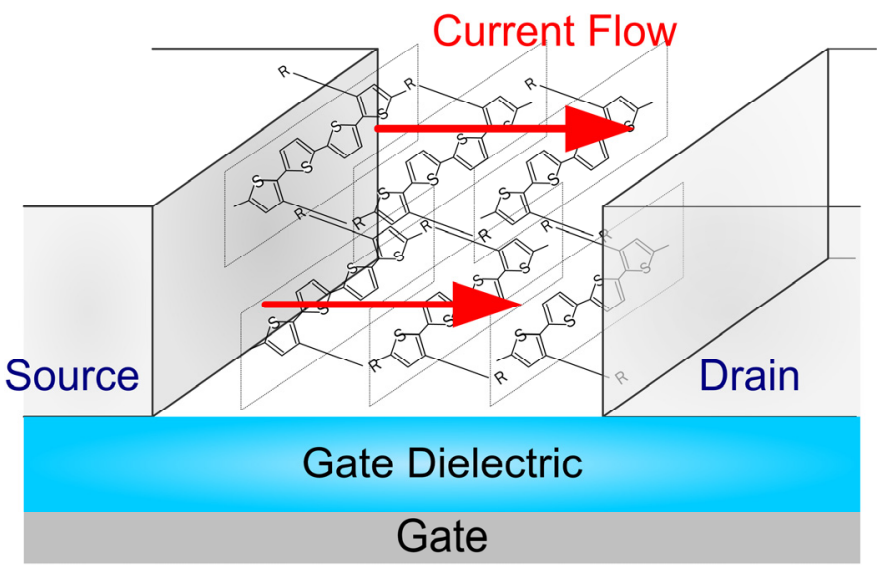

Figure 2.7. Schematics of ideal alignment of organic semiconductor building blocks with strong $\pi-\pi$ stacking in a TFT configuration (adapted from [13]). 
In many cases, the molecular structure and morphology of the organic semiconductor are largely determined by the underlying surface properties. In the case of bottom-gate OTFTs, careful preparation and functionalization of the dielectric surface prior to semiconductor deposition is essential to facilitate device enhancements. The impact of dielectric surface conditions on electrical characteristics of OTFTs is studied in Chapter 4 and Chapter 5.

\subsubsection{Material Development and Classifications}

In the last two decades, the use of organic semiconductors in thin film transistors (TFTs) has gained considerable interest due to their potential application in low-cost integrated circuits (ICs). Most of the initial efforts were focused on increasing the mobility and on/off ratio of the OTFTs by improving existing materials, synthesizing new materials, experimenting with innovative chemistry and processing methods, and by improving self-assembly and ordering of materials [3]. Figure 2.8 summarizes the mobility improvements achieved in various organic semiconductors. Since the research on OTFTs commenced in the 1980s, dramatic progresses in device performance were witnessed in the last two decades. In particular, the mobility of polythiophene has improved by five orders of magnitude [3]. OTFTs using pentacene as the organic semiconductor material have achieved mobilities comparable to that of amorphous silicon $\left(\mu \sim 1 \mathrm{~cm}^{2} / \mathrm{V}-\mathrm{s}\right)$. Amorphous silicon (a-Si:H) is commonly used for fabrication of thin film transistors (TFTs) to drive pixels in flatpanel active-matrix liquid crystal displays (AMLCDs) and active-matrix organic light-emitting diode displays (AMOLEDs) [16][17]; some of these applications coincide with the applications envisioned for OTFTs. As the mobility of organic semiconductors improves and approaches that of a-Si:H, OTFTs can offer a promising alternative to a-Si:H TFT technology, especially for applications involving flexible plastic substrates (e.g., flexible displays).

Organic semiconductor materials can generally be categorized into three main classes: smallmolecules, oligomers, and polymers [3]. Small-molecules are characterized by smaller molecular mass, shorter molecular chains, and fewer carbon atoms than polymers. On the other hand, polymers possess longer chains and larger molecular mass, contain more carbon atoms, and have good solubility. The properties of oligomers are intermediate between that of small-molecules and polymers. In terms of processability, small-molecule and oligomer compounds usually exhibit limited solubility in organic solvents; as a result, they are normally deposited by vacuum evaporation. In contrast, polymer semiconductors have higher solubility, which makes them 
particularly well-suited for simpler and lower cost solution-based processing methods such as spincoating and inkjet printing. Table 2.1 lists the key properties and a representative chemical structure for each type of organic semiconductors. Additional information on small molecule and polymer organic semiconductors, as well as n-type organic materials, is presented next.

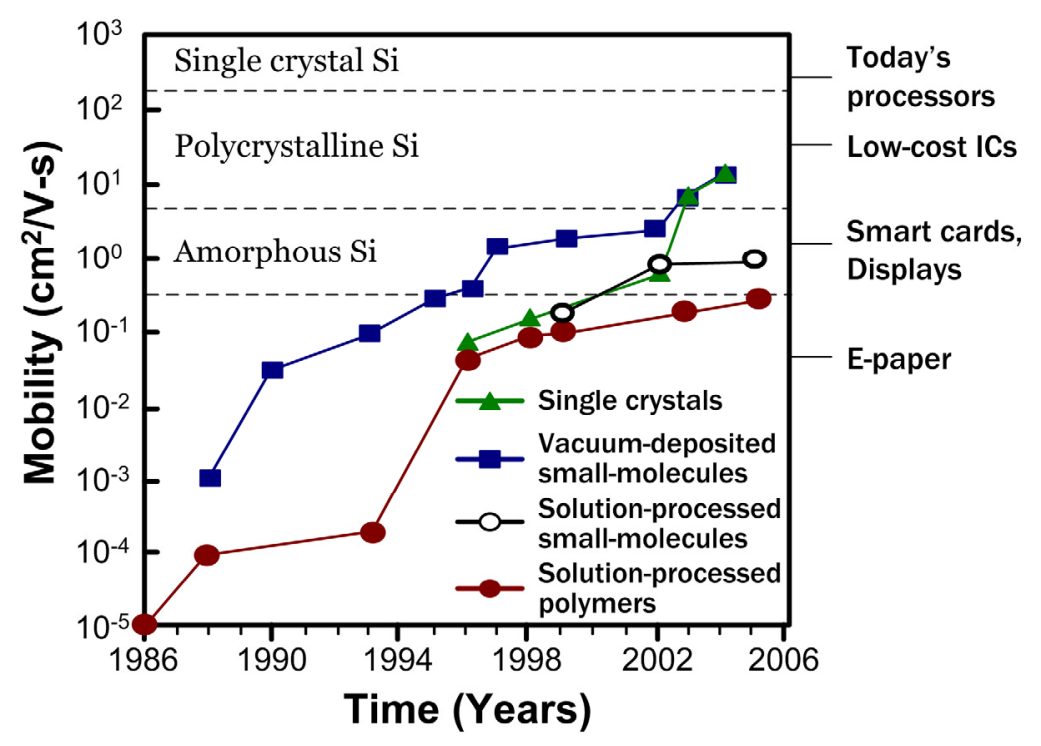

Figure 2.8. Progress in the performance of organic semiconductors (adapted from [3][18]).

\subsubsection{Small Molecules}

Evaporated small-molecule organic semiconductors typically exhibit higher mobility and better semiconducting characteristics than polymer semiconductors, owing to higher molecular ordering and ability to form well-ordered film. The most frequently studied p-type small-molecule organic semiconductor is pentacene because of its chemical and thermal stability as well as superior mobility compared to other organic materials. The field-effect mobility of vacuum deposited pentacene-based thin film transistors are typically reported in the range of $0.5-1.5 \mathrm{~cm}^{2} / \mathrm{V}-\mathrm{s}$, and as high as $5 \mathrm{~cm}^{2} / \mathrm{V}$-s, which is comparable to devices using amorphous silicon as the semiconductor material [20][21]. The reported on/off current ratio of such devices was $10^{5}-10^{8}$. The high performance of pentacene is attributed to its ability to form single-crystal-like or polycrystalline films upon vacuum deposition onto gently heated (about $80^{\circ} \mathrm{C}$ ) substrates.

The insolubility of pentacene may present as a shortcoming in terms of processability. Researchers at IBM have developed solution-processible pentacene based on precursor route, with 
initial mobility $\left(0.02 \mathrm{~cm}^{2} / \mathrm{V}\right.$-s $)$ significantly lower than vacuum-deposited pentacene, due to a lower degree of molecular ordering [22]. More recently, Kawasaki et al. reported an OTFT that was made using an inkjet-printed pentacene channel layer having a mobility of $0.15 \mathrm{~cm}^{2} / \mathrm{V}$-s (one of the highest values reported for inkjet printed OTFTs) and a current on-to-off ratio of $10^{5}$ [23]. The mobility strongly depends on the film morphology, which can be controlled by the processing conditions, such as solution concentration, substrate temperature, solvent composition, and environmental conditions during film drying.

These results showed that deposition of good quality pentacene film requires vacuum evaporation. This approach is undesirable if low-cost organic devices are to be fabricated in the long run. To build cost-effective organic electronics on a mass scale, solution-processing techniques that operate at low temperature and normal room conditions are preferred. This can reduce the cost and complexity of the manufacturing infrastructure, and enhance compatibility with a wider variety of flexible substrates. Polymer-based OTFTs, fabricated by spin-on, drop-casting, or printing techniques, can satisfy these specifications.

Table 2.1. Properties, representative chemical structure and typical mobility of the three main classes of organic semiconductors. The mobility of silicon is included for comparison [3][19].

\begin{tabular}{|c|c|c|c|}
\hline Classes & Properties & Representative Chemical Structure & $\begin{array}{l}\text { Typical Mobility } \\
\left(\mathrm{cm}^{2} / \mathrm{V}-\mathrm{s}\right)\end{array}$ \\
\hline $\begin{array}{l}\text { Small- } \\
\text { molecules }\end{array}$ & $\begin{array}{l}\text { - Low molecular mass } \\
\text { ( 20-40 carbon atoms) } \\
\text { - Size: } 1 \mathrm{~nm} \\
\text { - Polycrystalline } \\
\text { - Vapor-deposited }\end{array}$ & & $\begin{array}{l}\sim 1 \\
\left(>5 \mathrm{~cm}^{2} / \mathrm{V}-\mathrm{s} \text { was }\right. \\
\text { reported })\end{array}$ \\
\hline Oligomers & $\begin{array}{l}\text { - Short molecular chains (made } \\
\text { of only a few monomers or } \\
\text { structural units) }\end{array}$ & thio & $<1$ \\
\hline Polymers & $\begin{array}{l}\text { - Long molecular chains (made } \\
\text { of repeating units of the } \\
\text { monomer) } \\
\text { - High molecular mass ( }>50 \\
\text { carbon atoms) } \\
\text { - Size: } \sim 10-1000 \mathrm{~nm} \\
\text { - Semi-crystalline, or amorphous } \\
\text { - Solution processed (high } \\
\text { solubility) }\end{array}$ & $\begin{array}{l}(3- \\
\text { HT })\end{array}$ & $\leq 0.1$ \\
\hline Silicon & - Inorganic semiconductor & $\begin{array}{c}\text { Crystalline silicon } \\
\text { Polycrystalline silicon } \\
\text { Amorphous silicon (a-Si:H) }\end{array}$ & $\begin{array}{c}300-1000 \\
50-100 \\
\sim 1\end{array}$ \\
\hline
\end{tabular}




\subsubsection{Polymers}

Polymer semiconductors exhibit structural stability, tunable electrical properties, and solubility, which are customizable by designing and shaping the polymer chain structures. The conjugation length and rotational freedom determine the semiconductor polymer functionality and environmental sensitivity, while the alkyl side chains determine polymer solubility [24]. The mobility of polymer semiconductors stands roughly one order of magnitude below that of vacuumdeposited small-molecules, and is attributed to the poorer molecular ordering of solution-processed polymer materials [25]. Devices fabricated using solution processible regioregular poly(3hexylthiophene) (P3HT) resulted in field-effect mobility typically on the order of $10^{-5}$ to $10^{-2}$ $\mathrm{cm}^{2} / \mathrm{Vs}$, depending on the processing conditions (e.g., in air or oxygen-free environments, annealing), dielectric material, and dielectric-semiconductor interface properties. The highest mobility reported was $0.1 \mathrm{~cm}^{2} / \mathrm{V}$-s from a soluble regioregular poly(3-hexylthiophene) (P3HT), with greater than $98.5 \%$ head-to-tail (HT) linkages [26][27]. The chemical structure of a head-totail regioregular P3HT is displayed in Figure 2.9(b) [26]. Head-to-tail (HT) is one of the two possible arrangements for the 3-alkyl substituents (represented by " $R$ " in Figure 2.9(b)) in the P3HT structure; ${ }^{3}$ the other arrangement is known as head-to-head $(\mathrm{HH})$, as depicted in Figure 2.9(a). If P3HT consists of both HH and HT moieties, it is "regiorandom"; if only one type of arrangement is present (either HH or HT), it is "regioregular". High regioregularity is critical for higher molecular ordering, and thus high mobility, in polymer OTFTs. In contrast, regiorandom P3HT films usually yield lower mobility due to a higher degree of disorder in the regiorandom structure [26][28]. Alternative techniques to improve the mobility of P3HT OTFT have also been reported, including optimizing the choice of solvent, thermal annealing, optimizing the chain length, and using chemical treatment of the substrate surface prior to deposition of the polymer semiconductor film [12][28][29]. Poly(9,9'-dioctyl-fluorene-co-bithiophene) (F8T2) is another relatively air-stable polymer, and displayed mobility of $0.01-0.02 \mathrm{~cm}^{2} / \mathrm{Vs}$ after special interface preparation and high-temperature annealing [30].

\footnotetext{
${ }^{3}$ An alkyl is a functional group of an organic chemical that contains only carbon and hydrogen atoms, which are arranged in a chain. Its general formula $\mathrm{C}_{\mathrm{n}} \mathrm{H}_{2 \mathrm{n}+1}$, and is usually found to be attached to other hydrocarbons.
} 
(a)

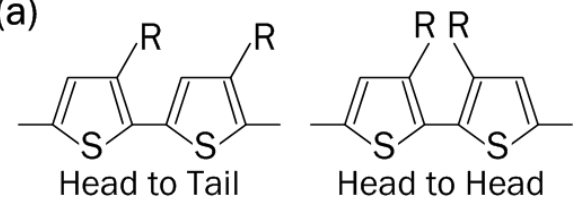

(b)

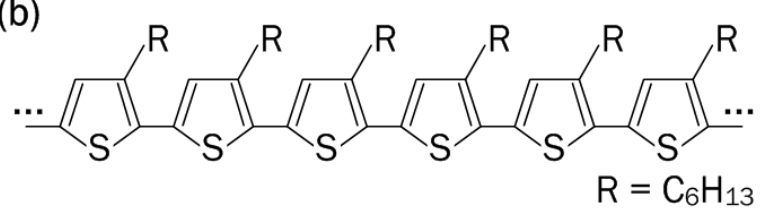

Figure 2.9. (a) Two types of arrangements for P3HT. (b) Head-to-tail regioregular P3HT [19].

A notable advance in the conjugated regioregular polythiophene family is the development of poly(3,3"'-dialkyl-quaterthiophene)s (PQT) by Ong et al. at Xerox Research Centre of Canada, with considerably enhanced oxygen resistance, solution processability, and self-assembly [15]. The typical device mobility is $0.07-0.12 \mathrm{~cm}^{2} / \mathrm{V}$-s with a current on/off ratio of $10^{6}$ under ambient conditions. Most of the research work presented this thesis are conducted using PQT-12 polymer semiconductor. Through dielectric and interface optimization, mobility of $0.2 \mathrm{~cm}^{2} / \mathrm{V}$-s and on/off ratio of $10^{8}$ are obtained for PQT-12 OTFT on silicon nitride $\left(\mathrm{SiN}_{\mathrm{x}}\right)$ gate dielectric (See Chapter 5). A more recent advancement in solution-processible semiconductor is a new liquid crystalline poly(2,5-bis(3-alkylthiophen-2-yl)thieno[3,2-b]thiophene) (PBTTT) polymer, developed by Merck Chemicals. PBTTT transistor showed good mobilties and stability in the range of $0.2-0.6 \mathrm{~cm}^{2} / \mathrm{V}-\mathrm{s}$ [31]. The chemical structure for PQT and PBTTT is presented in Figure 2.10.

(a)

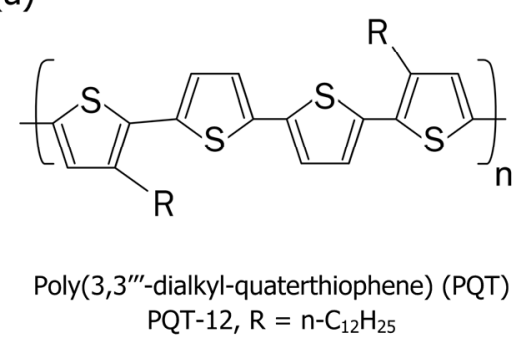

(b)

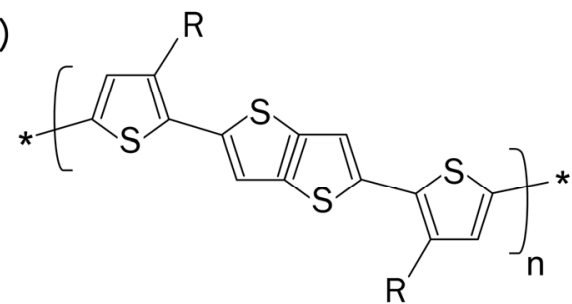

Poly(2,5-bis(3-alkylthiophen-2-yl)thieno[3,2-b]thiophene) (PBTTT). R = $\mathrm{C}_{10} \mathrm{H}_{21}, \mathrm{C}_{12} \mathrm{H}_{25}, \mathrm{C}_{14} \mathrm{H}_{29}$

Figure 2.10. Chemical structure of (a) PQT and (b) PBTTT [15][31].

\subsubsection{N-Type Semiconductors}

To make p-n junction diodes, bipolar transistors, and complementary circuits, n-type organic semiconductors are required. The majority of organic semiconductors exhibit p-type characteristics, which means that they primarily transport holes $\left(\mathrm{h}^{+}\right)$rather than electrons, and that holes are more easily injected than electrons. Comparatively, there are considerably fewer reports on n-type organic semiconductors [32][33][34]. In general, most n-type organic semiconductors 
have relatively lower field-effect mobilities, poorer stability, and limited solubility compared to ptype polymers. Examples of n-type material include fullerene $\left(\mathrm{C}_{60}\right)$ and $\mathrm{N}, \mathrm{N}$ '-dialkyl-3,4,9-10perylene tetracarboxylic diimide derivates, which currently have the highest electron mobility of up to $0.5 \mathrm{~cm}^{2} / \mathrm{V}$-s [11][35]. A major problem with most n-type organic semiconductors is their high instability in ambient conditions, especially oxygen and moisture [21][25]. Limited number of ntype compounds with improved air stability was identified (e.g., hexadecafluoro-phthalocyanine $\left(\mathrm{F}_{16} \mathrm{CuPc}\right)$, diperflurorohexylquarter-thiophene (DFH-4T)); these materials typically have lower mobilities. N-type compounds also tend to have lower solubility than p-type polymers; phenylC61-butyric acid methyl ester (PCBM) is one of the few soluble n-type polymers [36].

More extensive research efforts are required to improve the properties of n-type organic semiconductors. The recent discovery of ambipolar functionality in some organic materials, where a single semiconductor layer exhibits both n-type and p-type transport capabilities, appears promising for the implementation of complementary logic OTFT circuits and is expected to increase the versatility of OTFTs [17][37]. Another route to achieve ambipolar conduction is to interpenetrate network of two compounds, one p-type and one n-type. Meijer et al. used a PPV:PCBM blend to fabricate ambipolar OTFTs and CMOS-like inverters [38].

From a manufacturing perspective, to truly realize the advantages of organic materials in device applications, liquid phase processing techniques by spin-coating, casting, or printing are strongly desired. Soluble polymer semiconductors combined with large-area printing techniques are particularly favorable from the manufacturing cost standpoint. This thesis research focuses on solution-processible polymer semiconductors. More details on the selection of device materials used for this thesis research are given in Section 2.4.

\subsubsection{Sensitivity to Environmental Influences}

The high sensitivity of most organic semiconductors to ambient conditions or environmental influences, such as oxygen, humidity, light, and temperature, poses a huge limitation on the performance and stability of OTFTs [16][39][40][41]. For example, atmospheric oxygen and moisture, as well as photo-induced oxidation, have a doping effect on the organic material, causing an increase in the conductivity, which subsequently degrades the on/off current ratio of OTFTs [28]. Moreover, the current-voltage (I-V) characteristics of OTFTs tend to shift with bias-stress, thermal-stress, and exposure to atmosphere [16]. This results in changes in important device 
parameters such as conductivity, mobility, threshold voltage, on/off current ratio, and subthreshold characteristics.

The effect of air exposure on the conductivity of P3HT was studied by Allport et al. [42], in which the $I-V$ characteristics of a $\mathrm{P} 3 \mathrm{HT} /$ aluminium diode were measured after fabrication (in vacuum) and after one week of exposure to air in a clean environment. Increases in both the forward and reverse currents were observed after exposure to air, and were attributed to an increase in doping concentration and in conductivity. Many polymer semiconductors easily absorb oxygen and water molecules in air, and often become increasingly p-type doped. As a result of (photoinduced) oxidative doping, the free carrier density increases and the conductivity of the material increases [42].

The uncontrolled increase in conductivity can severely deteriorate the performance of OTFTs, possibly resulting in higher off-current and lower on/off current ratio. Abdou et al. [43] studied the interaction of oxygen with conjugated polymers and observed that oxygen forms a reversible charge transfer complex (CTC) with the polymer. It was suggested that CTC is largely responsible for the generation of charge carriers in semiconducting $\pi$-conjugated polymers when exposed to air, which modulated the electronic properties of the polymer. Analysis of P3HT OTFTs under increasing pressures of oxygen revealed that the formation of CTC resulted in an increase in carrier concentration, increase in conductivity, and decrease in charge carrier mobility. With prolonged or higher intensity exposure of oxygen, the proper transistor behavior was lost [43]. The degree of carrier generation by oxidative doping upon air exposure is expected to increase as the ionization potential (IP) of the polymer is lowered. The ionization potential, defined as the energy difference between the HOMO levels and vacuum, is dependent on the effective $\pi$-conjugation lengths of the polymer. An extensive $\pi$-conjugation along the polymer chain leads to a lower IP for polythiophenes, and thus a greater tendency to be oxidatively doped. On the other hand, conjugated polymers with shorter effective $\pi$-conjugation lengths (such as small-molecule materials) possess higher IPs, and thus greater resistance against oxidative doping [15]. Therefore, organic semiconductors possessing low IPs and having an amorphous nature are predisposed to oxygen diffusion into the bulk, and are particularly susceptible to oxidative doping.

To reduce the degradation induced by atmospheric factors, fabrication and processing of these air-sensitive organic semiconductors can be done in inert environment (e.g., vacuum, dry nitrogen). P3HT OTFTs fabricated in inert environment tend to exhibit improved performance than those fabricated in air [16]. Moreover, most of the high performance solution-processed OTFTs reported 
in the literature involved fabrication and measurement in inert environment, to reduce unwanted doping effects. Other reported techniques to address atmosphere-induced degradation involve treating the polymer film with a reducing agent (e.g., ammonia) to de-dope the polymer film, which have resulted in an increase in the on/off ratio [29]. Annealing presents another technique to recover the polymer from degradation.

A more effective solution to address this material limitation is to develop air-stable organic materials; an excellent example is the polythiophene derivative (PQT) developed by Ong et al. [15]. PQT-12 OTFTs fabricated and measured in air demonstrated excellent performance compared to other commercially available P3HT OTFTs fabricated in air. Merck Chemicals Ltd. has also reported thiophene polymer semiconductor with improved air stability. However, long term degradation of OTFT is inevitable; thus, passivation/encapsulation layers and other strategies must be considered to extend the device lifetime. More discussion on encapsulation techniques is presented in Section 2.4.5.

\subsection{OTFT Operation and Characteristics}

The TFT structure is well adapted to low conductivity materials, and is currently used in amorphous silicon and polycrystalline silicon transistors [25]. There are a number of functional and structural differences between TFTs and the conventional metal-oxide-semiconductor field-effect transistor (MOSFET) used in crystalline silicon ICs. First, there is no depletion region in TFT to isolate the device from the substrate. Low off-currents $\left(I_{\mathrm{OFF}}\right)$ in TFTs are only guaranteed by the low conductivity of the semiconductor. ${ }^{4}$ A second crucial difference is that the TFT operates in the accumulation regime while MOSFET operates in the inversion regime. This implies that the conduction channel in TFT is formed by accumulating majority carriers at the gate dielectric and semiconductor interface, as illustrated in Figure 2.11(a). In this figure, a TFT with a p-type semiconductor is turned "on" by applying a negative bias to the gate electrode. This sets up an electric field in the dielectric layer that induces positive charge carriers (i.e., holes) to accumulate in the semiconductor at the dielectric-semiconductor interface. This accumulation layer forms a conducting channel in the semiconductor, allowing charges carriers to be driven from the source to

\footnotetext{
${ }^{4}$ Contrary to the standard silicon MOSFETs, OTFTs do not have blocking p-n junction near the contacts to prevent the current from flowing between source and drain when the channel is not formed yet. As a result, the off-current $\left(\mathrm{I}_{\mathrm{OFF}}\right)$ is relatively high in OTFTs compared to MOSFETs [26].
} 
the drain by applying a bias to the drain electrode. Here, the source serves as the reference (grounded) electrode. When TFT is in the "off" state (Figure 2.11 (b)), the gate voltage is set so that there is no conducting channel in the semiconductor layer. This implies that, ideally, no current should flow between the source and drain electrode, even with an applied $V_{\text {DS }}$ bias [7]. Figure 2.12 shows typical electrical characteristics of a p-type OTFT; the transition from "off" state to "on" state of the OTFT can be detected by the abrupt increase in drain current in the transfer $\left(I_{\mathrm{D}}-V_{\mathrm{GS}}\right)$ characteristics near $V_{\mathrm{GS}}=0 \mathrm{~V}$ in Figure 2.12(a).

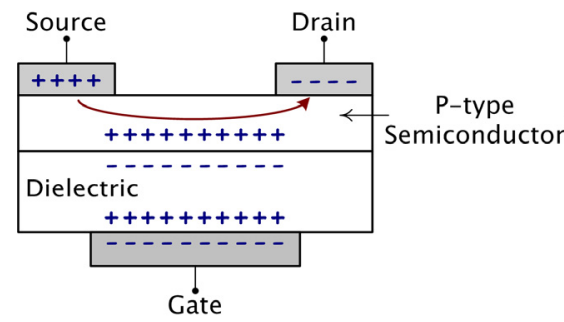

(a) "ON" State $\left(V_{G}<0\right)$

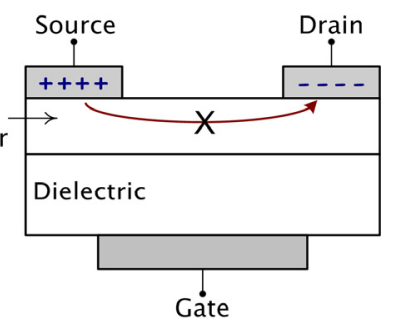

(b) "OFF" State

Figure 2.11. Simplified illustration of the operation of a TFT with p-type semiconductor [7].

TFT's gate-dielectric-semiconductor structure operates like a capacitor. Assuming the ideal case, application of a gate voltage induces an equal charge of opposite polarity in the semiconductor near the dielectric-semiconductor interface, as shown in Figure 2.11(a). This charge forms a conducting channel if it is of the same type (polarity) as the majority charge carrier being injected into the semiconductor from the contacts. Conductance of the channel is proportional to the gate voltage.

At low drain voltages, the current flowing between drain and source through the channel, identified as $I_{\mathrm{DS}}$, follows Ohm's law; $I_{\mathrm{DS}}$ is proportional to drain and gate voltages. This operation mode is referred to as linear regime. The linear current can be described by:

$$
I_{D S, \text { lin }}=\mu_{F E} C_{i} \frac{W}{L}\left(V_{G S}-V_{T}-\frac{V_{D S}}{2}\right) V_{D S} .
$$

As drain voltage increases and approaches gate voltage, $V_{\mathrm{DG}}$ drops to zero and pinch-off of the channel occurs. At this point, the channel current $\left(I_{\mathrm{DS}}\right)$ becomes independent of the drain bias. This regime is called the saturation operation. Thus, for $V_{\mathrm{DS}}$ more negative than $V_{\mathrm{GS}}, I_{\mathrm{DS}}$ tends to saturate owing to the pinch-off of the accumulation layer. The saturation current is approximated by:

$$
I_{D S, s a t}=\frac{1}{2} \mu_{F E} C_{i} \frac{W}{L}\left(V_{G S}-V_{T}\right)^{2} .
$$


(a)

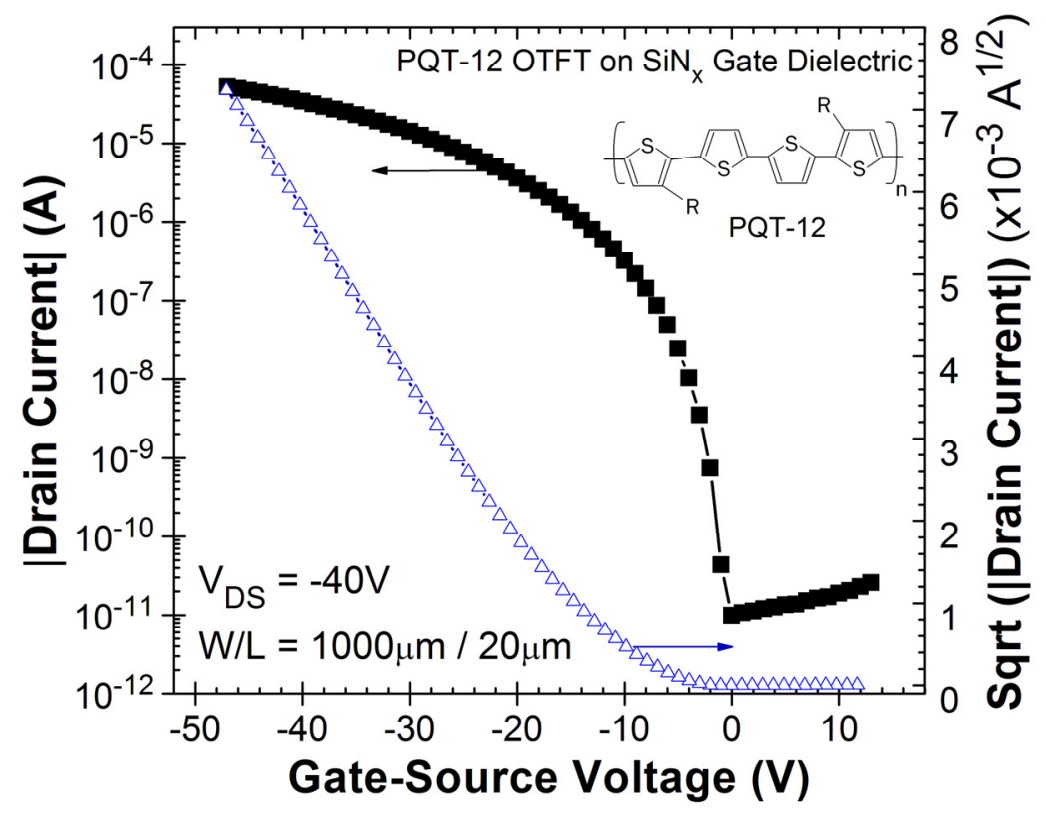

(b)

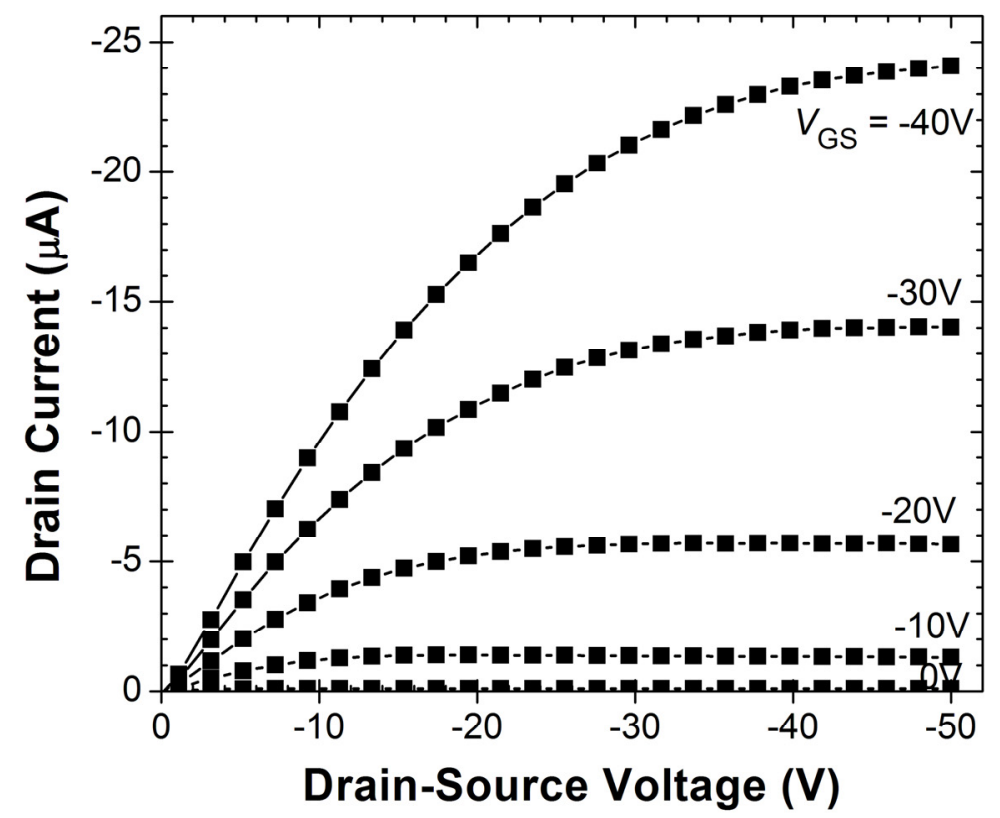

Figure 2.12. Typical electrical characteristics of a p-type OTFT. (a) Transfer curve ( $\left.I_{D}-V_{G S}\right)$ in the saturation regime $\left(V_{D S}=-40 \mathrm{~V}\right)$, and $(b)$ output curves $\left(I_{D}-V_{D S}\right)$ at different gate voltages. The device shown here is PQT-12 OTFT on PECVD SiN $\mathrm{N}_{\mathrm{x}}$ gate dielectric. Inset of (a) shows molecular structure of PQT-12, which serves as semiconductor in the device. 
The transition from linear to saturation regime is observable in the output $\left(I_{\mathrm{D}}-V_{\mathrm{DS}}\right)$ characteristics in Figure 2.12(b). In these equations, $\mu_{F E}$ is the field-effect mobility, $C_{\mathrm{i}}$ is the capacitance per unit area of the dielectric layer, $L$ is the channel length, $W$ is the channel width, $V_{\mathrm{T}}$ is the threshold voltage, $V_{\mathrm{GS}}$ is the gate-source bias, and $V_{\mathrm{DS}}$ is the drain-source bias. $V_{\mathrm{T}}$ is usually defined as the gate voltage at which the channel conductance (at low $V_{\mathrm{DS}}$ ) is equal to that of the whole semiconductor layer.

The operation and performance of OTFTs are generally governed by two key mechanisms: (1) charge transport in the organic semiconductor layer, and (2) charge injection and extraction at the source/drain contacts. Field-effect mobility $\left(\mu_{\mathrm{FE}}\right)$ is often used to characterize the efficiency of charge transport in a device, and contact resistance $\left(R_{\mathrm{C}}\right)$ provides a measure of charge injection efficiency in an OTFT. The extraction of $\mu_{\mathrm{FE}}$ and $R_{\mathrm{C}}$, as well as other relevant device parameters, is discussed in the next section.

Note that $\mu_{\mathrm{FE}}$ is commonly used as a figure a merit for reporting OTFT performance by the research community. However, the accurate extraction and modeling of $\mu_{\mathrm{FE}}$ remain controversial, primarily due to incomplete understanding of the OTFT physics, artifacts in the extraction procedure (e.g., contact resistance, threshold voltage shifts, stress, geometry dependence), and the use of over-simplified models. As a result, the extracted value of $\mu_{\mathrm{FE}}$ may not give a precise representation of OTFT performance. These concerns are not as critical in this thesis because $\mu_{\mathrm{FE}}$ is used to systematically compare device performance within various sets of experiments. Nonetheless, the absolute value of the extracted $\mu_{\mathrm{FE}}$ should be analyzed with caution when it is assessed against data published in the literature.

\subsubsection{OTFT Parameter Extraction}

Current-voltage equations in Eqns. (2.3) and (2.4) provide a simple approach to approximate the OTFT characteristics, and are used in this thesis to provide a basis to characterize and compare a variety of OTFT devices. The key device parameters of interest include: effective field-effect mobility $\left(\mu_{\mathrm{FE}}\right.$, measured in $\mathrm{cm}^{2} / \mathrm{V}$-s $)$, on/off current ratio $\left(I_{\mathrm{ON}} / I_{\mathrm{OFF}}\right)$, threshold voltage $\left(V_{\mathrm{T}}\right.$, in volt), and contact resistance $\left(R_{\mathrm{C}}\right)$. In particular, $\mu_{\mathrm{FE}}$ describes how rapidly the charge carriers can move through the material, and is often used as a figure of merit for comparing the performance of various organic semiconductor materials. High $\mu_{\mathrm{FE}}$, as well as high $I_{\mathrm{ON}} / I_{\mathrm{OFF}}$, are desirable qualities for OTFTs. For analyzing the subthreshold behavior, switch-on voltage $\left(V_{\mathrm{SO}}\right)$ and inverse 
subthreshold slope $(S$, in $\mathrm{V} / \mathrm{dec})$ are studied. $V_{\text {SO }}$ is defined as the gate voltage where the current starts to increase in the semi-logarithmic $I_{\mathrm{D}}-V_{\mathrm{GS}}$ plot (Figure 2.12(a)).

In this thesis, $\mu_{\mathrm{FE}}$ and $V_{\mathrm{T}}$ are extracted from transconductance $\left(g_{\mathrm{m}}\right)$ measurements. In the linear regime, $g_{\mathrm{m}, \text { lin }}$ is expressed as:

$$
g_{m, l i n}=\frac{\partial I_{D, l i n}}{\partial V_{G S}}=\mu_{F E} C_{i} \frac{W}{L} V_{D S} .
$$

Accordingly, the effective mobility in the linear regime, $\mu_{\mathrm{FE}, \text { lin }}$, can be extracted as follows:

$$
\mu_{F E, l i n}=\frac{g_{m, l i n}}{C_{i} V_{D S}} \frac{L}{W}
$$

In the saturation regime, $g_{\mathrm{m}, \text { sat }}$ is expressed as:

$$
g_{m, s a t}=\frac{\partial I_{D, s a t}}{\partial V_{G S}}=\mu_{F E} C_{i} \frac{W}{L}\left(V_{G S}-V_{T}\right) .
$$

By plotting $g_{\mathrm{m}, \mathrm{sat}}$ versus $V_{\mathrm{GS}}$ and performing linear curve fitting, $\mu_{\mathrm{FE}}$ and $V_{\mathrm{T}}$ can be extracted from the slope and intercept, respectively, of the linearly fitted curve (see Figure $2.13(\mathrm{a})$ ). $I_{\mathrm{ON}} / I_{\mathrm{OFF}}, V_{\mathrm{SO}}$ and subthreshold slope are easily deducible from $I_{\mathrm{D}}-V_{\mathrm{GS}}$ curve (see Figure 2.13(b)). Calculation of contact resistance is discussed in Section 2.2.2.

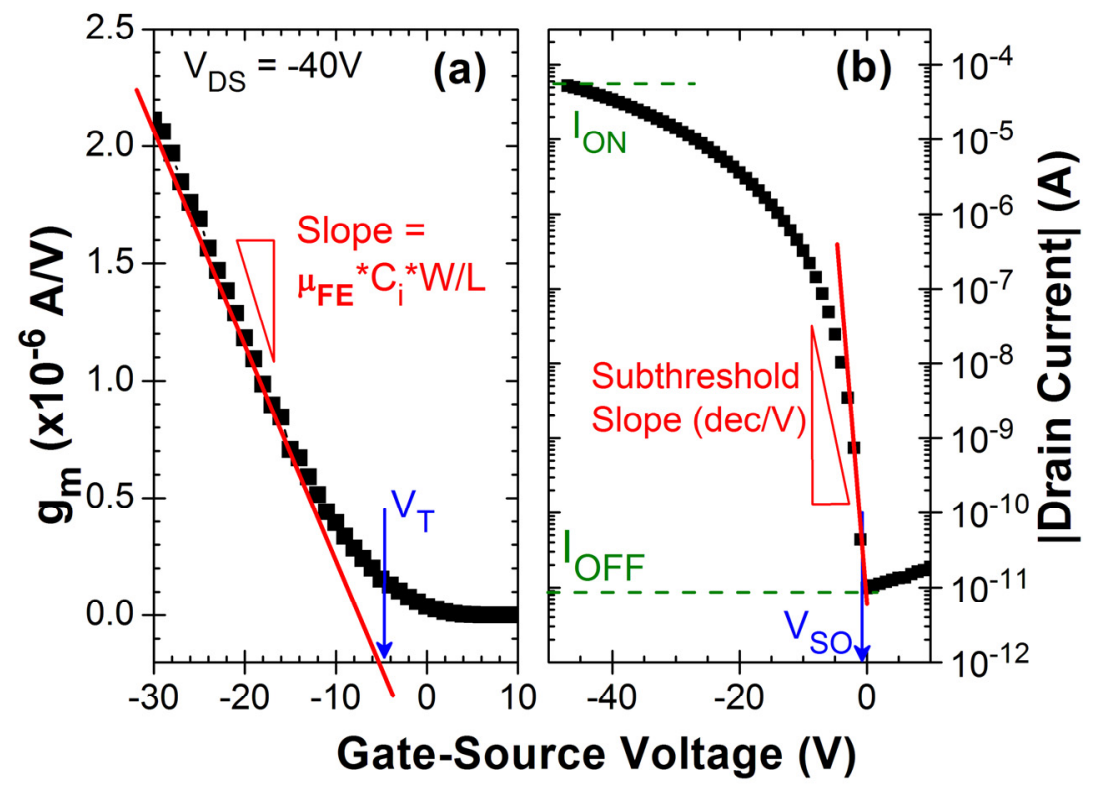

Figure 2.13. Extraction of OTFT device from electrical characteristics: (a) gm- $V_{G s}$ plot for extracting $\mu_{\mathrm{FE}}$ and $V_{\mathrm{T}}$, and (b) $I_{\mathrm{D}}-V_{\mathrm{GS}}$ plot for deducing $I_{\mathrm{ON}} / I_{\mathrm{OFF}}$ and subthreshold slope. 
In most cases, the current-voltage equations in Eqns. (2.3) and (2.4) can satisfactorily describe the actual OTFT curves; however, their derivation is based on several approximations that are not always fulfilled by organic semiconductors. These assumptions include [11]:

- Gradual channel approximation: the electric field along the channel is much lower than that across it, thus the voltages vary gradually along the channel from the drain to the source. This assumption is valid when the distance between the drain and source is much larger than the thickness of the dielectric.

- The mobility, $\mu_{\mathrm{FE}}$, is constant.

Furthermore, this over-simplified model, derived based on concepts of silicon MOSFETs, overlooks many unique properties of OTFTs (e.g., disordered nature of amorphous organic semiconductor materials, contact resistance, voltage-dependent mobility, absence of an inversion layer). Thus, the extracted device parameters are subjective to error. A more comprehensive model for TFTs based on disordered/amorphous semiconductors is outlined in [44]; this model can offer better insight on device behavior and provide more accurate extraction of device parameters. Improved models of OTFT based on different types of organic semiconductors continue to evolve through a growing understanding and stronger knowledge-base on OTFT physics.

\subsubsection{Contact Resistance Extraction}

Contact resistance $\left(R_{\mathrm{C}}\right)$ provides a means to evaluate the efficiency of charge injection. Devices with ohmic contacts and efficient charge injection are characterized by low contact resistance values. To investigate the role of contacts on device performance, a gated transmission line model is used for extraction of contact resistance and channel resistance. The extraction procedure used in this thesis is adapted from the comprehensive TFT model for disordered semiconductors in references [44] and [45]. Output characteristics of TFTs with different channel lengths are used for extraction. The measured resistance of a TFT with unit width, denoted by $R_{\mathrm{m}} W$, can be written as [44]:

$$
R_{m} W=\frac{V_{D S}}{I_{D S, L I N}} W=R_{D S} W+A L, \text { where } A=f\left(R_{c h}\right) .
$$

Here, $R_{\mathrm{m}}=V_{\mathrm{DS}} / I_{\mathrm{DS}, \text { lin }}$ is the measured resistance between drain and source electrodes, $R_{\mathrm{DS}} W$ is the contact resistance for a unit width TFT, and $A$ is parameter related to channel resistance $\left(R_{\mathrm{ch}}\right)$. The 
total measured resistance, $R_{\mathrm{m}} W$ is calculated at a small $V_{\mathrm{DS}}$ and plotted as a function of $L$ for each $V_{\mathrm{GS}}$, as depicted in Figure 2.14(a). To facilitate evaluation of linear component and nonlinear component of contact resistance, Eqn. (2.8) is rewritten as:

$$
R_{m} W=B+A L
$$

where

$$
B=R_{D S} W+A \Delta L, \text { and } A=f\left(R_{c h}\right) .
$$

According to Eqn. (2.9), linear fitting of a $R_{\mathrm{m}} W$ vs. $L$ plot gives $B$ and $A$ as coordinate intercept point and the slope, respectively. Sets of $(A, B)$ values are obtained by plotting Eqn. (2.9) at various $\mathrm{V}_{\mathrm{GS}}$. Subsequently, plotting $B$ as a function of $A$ gives a line with $R_{\mathrm{DS}} W$ and $\Delta L$ as the coordinate intercept point and slope, respectively. This is illustrated in Figure 2.14(b). From these data, we extract two key parameters:

- $\quad R_{\mathrm{DS}} W[\mathrm{ohm}-\mathrm{cm}]$ gives the constant part of the contact resistance, i.e., linear component;

- $\Delta L[\mu \mathrm{m}]$ represents the voltage-dependent part of contact resistance, i.e., non-linear component.

This extraction approach is applied in Chapter 6 to evaluate the effectiveness of contact interface treatments of OTFTs.

Of particular importance is the relative influence of the contact resistance $R_{\mathrm{C}}$ at the organic semiconductor-metal interface and the channel resistance $R_{\mathrm{ch}}$ formed at the organic semiconductordielectric interface. Depending on the charge carrier mobility in the semiconductor and the quality of contact interface, contact resistances may dominate device operation and limit device performance. For devices with large contact resistance, where there is a significant portion of the total $V_{\mathrm{DS}}$ dropped across the contacts, the extracted field-effect mobility tends to be underestimated [46]. In addition, contact resistances may mask intrinsic bulk effects within the organic semiconducting layer, leading to misleading device parameter extraction. Therefore, it is important to minimize the contact effects in the operation of OTFTs. This necessitates a better understanding of the charge injection mechanisms, as well as a more comprehensive device model to account for contact resistance effects and correct extraction of mobility values. Overall, increased knowledge in charge transport and charge injection mechanisms is beneficial to assist in delivering enhanced device performance and in designing OTFT circuits for practical applications. 
(a)

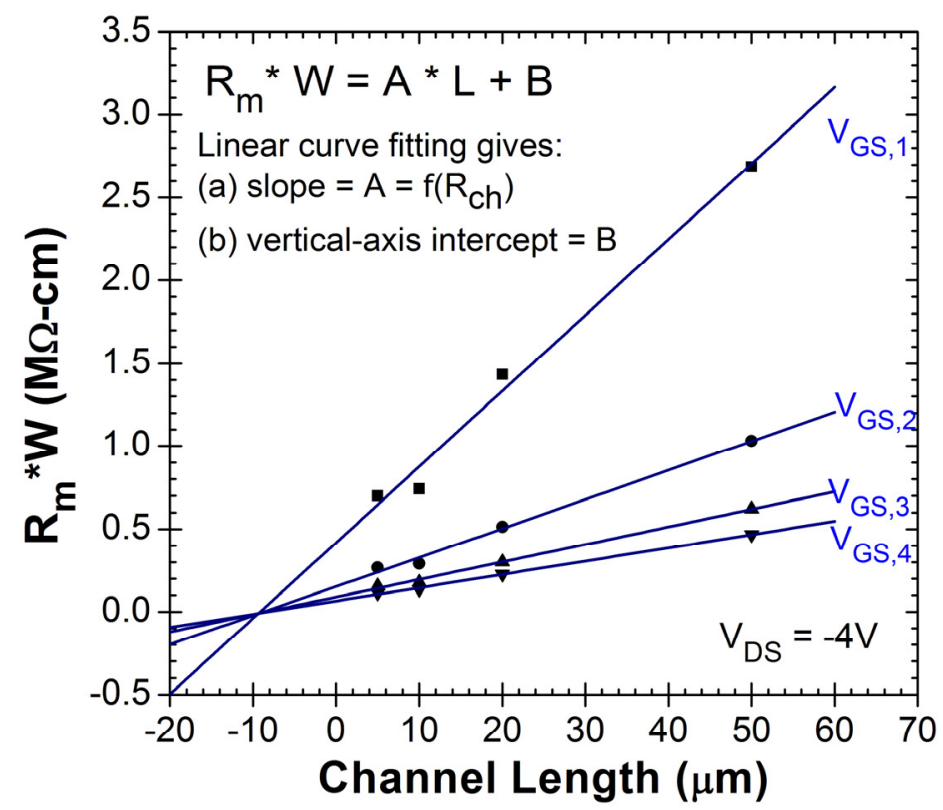

(b)

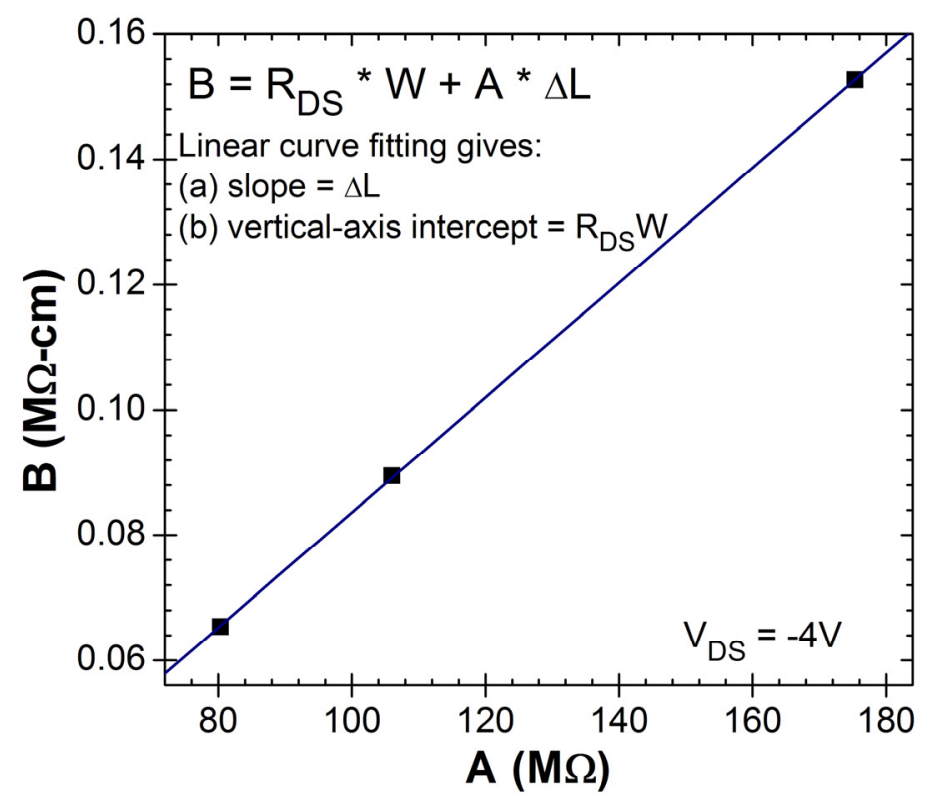

Figure 2.14. Contact resistance extraction. (a) Exemplary plot of $R_{\mathrm{m}} W$ vs. $L$ for different values of $V_{G S}$ for an OTFT at a given $V_{D S}$, to extract $A$ and B. (b) Exemplary plot of $B$ vs. $A$ at a given $V_{D S}$, to extract $R_{\mathrm{DS} W}$ and $\Delta L$. 


\subsubsection{Desirable OTFT Characteristics}

The preferable characteristics of a "good" OTFT typically include high field-effect mobility, high on/off drain current ratio, low leakage current, minimal threshold voltage shift, sharp subthreshold slope, and low contact resistance. These general performance requirements are summarized in Table 2.2. In most cases, the performance of an OTFT is dictated by two key mechanisms:

1) Charge transport (related to $\mu_{\mathrm{FE}}$ ) in the semiconductor layer, which is dictated by the quality of the semiconductor layer and the dielectric-semiconductor interface;

2) Charge injection at the contacts (related to $R_{\mathrm{C}}$ ), which is dictated by the energetic matching and compatibility between contact and semiconductor materials and the corresponding interface.

In this thesis, attempts to enhance charge transport (or channel quality) are done through optimizing the dielectric-semiconductor interface, as addressed in Chapter 4 and Chapter 5. Charge injection is examined in Chapter 6 by investigating contact-semiconductor interface treatment strategies.

Table 2.2. General requirements for a good TFT [47].

\begin{tabular}{|c|c|}
\hline Requirement & Descriptions \\
\hline $\begin{array}{l}\text { High field-effect } \\
\text { mobility }\left(\mu_{F E}\right)\end{array}$ & $\begin{array}{l}\text { - Determined by quality, crystallinity, ordering, and microstructure of the } \\
\text { semiconductor layer. } \\
\text { - Requires a low defect density in the semiconductor layer and at interfaces. } \\
\text { - Mobility influences switching speed in transistors. }\end{array}$ \\
\hline $\begin{array}{l}\text { High on/off drain } \\
\text { current ratio } \\
\left(I_{\mathrm{ON}} / \mathrm{I}_{\mathrm{OFF}}\right)\end{array}$ & $\begin{array}{l}\text { - Depends on the quality of the semiconductor and gate dielectric layers } \\
\text { - Requires minimum leakage during "off-state" and maximum drain current } \\
\text { during conduction. }\end{array}$ \\
\hline $\begin{array}{l}\text { Low threshold } \\
\text { voltage }\left(V_{T}\right) \text { and } \\
\text { minimal } \\
\text { threshold voltage } \\
\text { shift }\left(\Delta V_{T}\right) \text {. }\end{array}$ & $\begin{array}{l}\text { - Low } V_{T} \text { signifies lower operation voltages. } V_{T} \text { depends on the bulk and } \\
\text { interface states of the dielectric film. } \\
\text { - Minimal } \Delta V_{T} \text { is critical for stability reasons. } \Delta V_{T} \text { is dependent on interfacial } \\
\text { stresses, and on the creation of metastable defects in the semiconductor } \\
\text { layer under a gate bias. }\end{array}$ \\
\hline $\begin{array}{l}\text { Sharp } \\
\text { subthreshold } \\
\text { slope (S) }\end{array}$ & $\begin{array}{l}\text { - Sharp S (i.e., small value in units of V/dec) for faster switching of the TFT. } \\
\text { - Requires a low density of deep gap states localized at and/or near the } \\
\text { semiconductor/dielectric interface }\end{array}$ \\
\hline $\begin{array}{l}\text { Low leakage } \\
\text { current (lleak) }\end{array}$ & $\begin{array}{l}\text { - When a TFT is off, leak needs to be small for higher retention of the stored } \\
\text { charge, especially in display applications. leak is determined by the quality of } \\
\text { gate dielectric, the thermal generation current, the interface state density } \\
\text { (ideally low), and interfacial stress. }\end{array}$ \\
\hline $\begin{array}{l}\text { Low contact } \\
\text { resistance }(R c)\end{array}$ & $\begin{array}{l}\text { - Depends on the charge injection efficiency at the semiconductor-contact } \\
\text { interface. High Rc limits current drive and switching speed. }\end{array}$ \\
\hline
\end{tabular}




\subsection{OTFT Device Architecture}

A number of OTFT structures can be obtained by varying the relative placement of the gate and source/drain electrodes with respect to the semiconductor layer. The resulting devices are described as top-contact, bottom-contact, top-gate, bottom-gate, or dual-gate structures. Each design possesses distinctive strengths and weaknesses in terms of operating mode and ease of fabrication. Thus, each structure may find unique usage in specific applications/configurations.

\subsubsection{Top-Contact and Bottom-Contact OTFTs}

Depending on the arrangement of the source and drain contacts relative to the semiconductor layer, two configurations are possible as depicted in Figure 1.3: top-contact (or staggered) OTFT and bottom-contact (or co-planar) OTFT. For top-contact OTFTs, the source and drain electrodes are placed on top of the semiconductor layer. For bottom-contact OTFTs, the organic semiconductor is deposited onto the gate dielectric and the prefabricated source and drain electrodes. The top-contact structure can have a performance advantage over bottom-contact devices in terms of lower contact resistance. On the other hand, the bottom-contact structure enjoys simpler and more robust processing schemes over top-contact structures. These differences are explained next.

Top-contact and bottom-contact OTFTs often show different electrical performance. Considering bottom-gate devices, the application of gate voltage induces a conductive channel at the interface between the organic semiconductor and the dielectric. For a bottom-contact OTFT, the carrier exchange between the channel and the source/drain contacts is limited to narrow lines along the contact edges (i.e., at the edge of the contacts along the width of the channel), where the contact and the induced-channel intersect (Figure 1.3(b)). Research showed that this small contact area tends to generate large contact resistance in bottom-contact OTFTs [48]. In contrast, top-contact OTFTs usually have relatively larger areas for carrier injection at the contact-semiconductor interface, resulting in smaller contact resistance (Figure 1.3(a)). Due to more pronounced contact effects in bottom-contact designs, field-effect mobility extracted from the electrical characteristics of top-contact OTFTs often exceed those calculated for bottom-contact devices, even in the case of identical materials, device dimensions, and process conditions [20]. This is due to artifacts in the empirical device models used for OTFT parameter extraction, where mobility often exhibits contact resistance dependence. 
From a processing perspective, the bottom-contact design is preferable over top-contact structure. This is because the deposition of the organic semiconductor constitutes the last fabrication step in bottom-contact OTFTs (assuming no encapsulation), and is not limited or affected by other processing steps. Thus, there is greater flexibility in selecting a patterning/deposition method for the various device layers. If an inorganic dielectric is used, the source/drain contacts can be patterned by photolithography technique to deliver higher resolution features. On the other hand, the top-contact design is susceptible to physical damage of the organic layer from subsequent processing of source/drain contacts, or from metal-semiconductor reactions (which may influence the contact resistance). With the top-contact architecture, contacts are often deposited through shadow masks, with substantial loss of resolution. Moreover, the metal-organic interaction during metal deposition may deteriorate device performance. For this thesis research, fabrication processes are geared towards bottom-contact structures to ensure minimum processinduced disruption to the organic semiconductor layer.

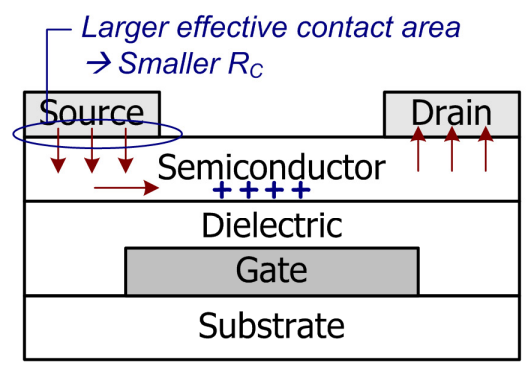

(a) Top-Contact OTFT

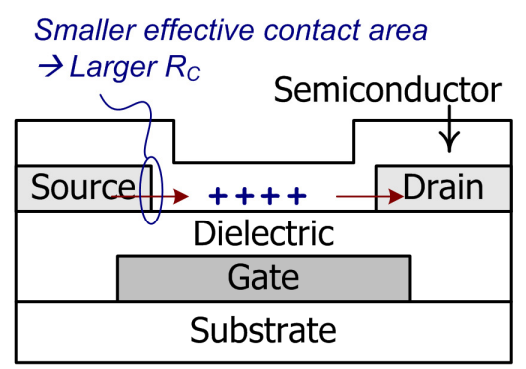

(b) Bottom-Contact OTFT

Figure 2.15. Cross section of two bottom-gate OTFT configurations: (a) top-contact and (b) bottom-contact.

\subsubsection{Top-Gate, Bottom-Gate, and Dual-Gate OTFTs}

Based on the arrangement of the gate electrode(s) relative to the semiconductor layer, OTFTs can be classified as bottom-gate, top-gate, or dual-gate, as illustrated in Figure 2.16. A feature of topgate OTFT is that the gate dielectric can provide encapsulation and protection of the organic semiconductor layer. Encapsulation is essential because most organic materials are chemically sensitive to environmental influence. However, the top-gate design poses some process integration challenges. First, the gate dielectric and gate electrode have to be deposited and structured on top of the organic semiconductor, and this process must preserve the organic material. Secondly, 
vertical interconnects and vias between the conductive layers have to be built through the organic semiconductor; this necessitates developing a compatible patterning/etching process for the organic layer. In this thesis research, we demonstrated the first fully-encapsulated top-gate P3HT OTFT using silicon nitride $\left(\mathrm{SiN}_{\mathrm{x}}\right)$ as the passivating gate dielectric, featuring a tailored etch process for patterning of the organic layer [49]; the results are presented in Section 3.3.

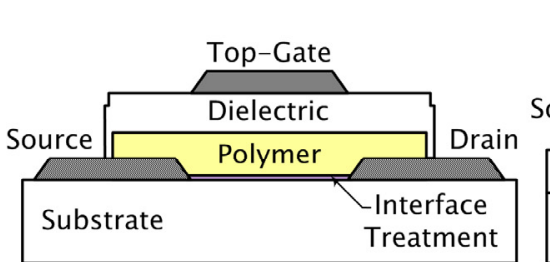

(a) Top-gate OTFT

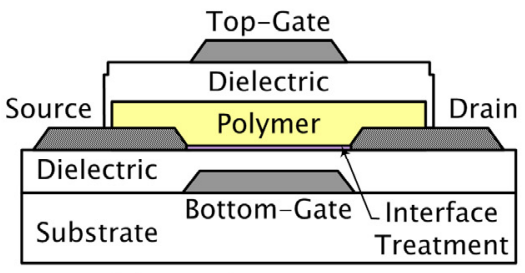

(b) Dual-gate OTFT

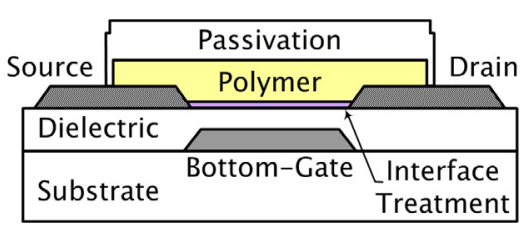

(c) Bottom-gate OTFT

Figure 2.16. Cross-section of (a) top-gate, (b) dual-gate, and (c) bottom-gate OTFTs. Devices shown are in bottom-contact configuration.

Comparatively, the fabrication of bottom-gate OTFT is much simpler. Since the deposition of the organic semiconductor layer occurs after preparation of the gate and gate dielectric, there is a greater flexibility on the choice of materials and fabrication techniques. Most of the published research on OTFTs adopted the bottom-gate design, and have demonstrated encouraging results. A relevant concern of this structure is the need for passivation of the organic layer. Passivation and encapsulation plays an important role in prolonging the lifetime and stability of OTFTs, especially because most functional organic materials are very sensitive to environmental influences (including air, oxygen, moisture, water) and light, often leading to significant degradation or failure of devices [39][40][41].

In dual-gate OTFTs, the voltage bias on the bottom-gate has a distinct influence on the threshold voltage, subthreshold slope, on-current, and leakage current of the top-gate TFT. Similarly, there is a dependence of the bottom-gate TFT characteristics on the top-gate bias. This dual-gate arrangement offers the ability to control selected TFT parameters, making it attractive for circuit applications that demand high threshold voltage control and good reliability. The dual-gate OTFT also lends itself as a highly functional test structure for characterization of density of states (DOS) at the interfaces of the active organic and dielectric layers, to evaluate the interface integrity and provide insight into the underlying transport mechanisms [50]. Another promising application of dual-gate OTFT is its use as an on-pixel circuit element for vertically integrated backplane electronics in active matrix displays and imagers. The use of dual-gate OTFT can potentially 
improve the aperture ratio of the device and permit shielding of parasitic effects in the vertically integrated backplane electronics. (This concept is elaborated in Section 7.4.1). Dual-gate OTFTs can be implemented by extending the fabrication procedure of top-gate OTFTs. Since processing must be done on the organic semiconductor layer, there is added processing complexity that requires careful consideration when designing a fabrication procedure for dual-gate OTFTs. In this thesis research, we demonstrated one of the first dual-gate polythiophene OTFTs fabricated by photolithography methods [51]. The fabrication schemes that originated from this research to implement top-gate, bottom-gate and dual-gate OTFTs are presented in Section 3.3.

\subsection{OTFT Device Material Selection}

As illustrated in the cross-sectional structure of OTFTs in Figure 2.1, the functional device layers are the semiconductor, the gate dielectric, and the electrodes. Since these layers are actively involved in the operation of OTFTs, the choice of materials and the compatibility between materials influence device performance. Figure 2.17 depicts the key interaction mechanisms between various device layers. There are two additional material layers that require consideration for OTFT fabrication: the substrate and the encapsulation. The substrate presents a platform for material deposition; in the case of organic electronics, there is an application-driven aspiration towards flexible substrates. Encapsulation provides protection/passivation for the functional device layers, which is particularly important for organic electronics due to sensitivity of organic materials to environmental influences.

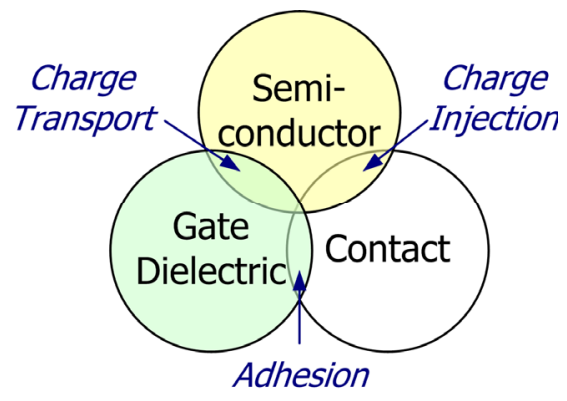

Figure 2.17. Interaction between the three key device layers in an OTFT.

Table 2.3 summarizes the selection of device materials used for OTFT research in this thesis. The research focused on solution-processible p-type polymer semiconductors, inorganic gate 
dielectrics and metallic electrodes. The various device layers are discussed in greater depth in this section. Well-established electronic materials were chosen to allow this research to focus on device integration and circuit development (instead of developing new materials). Solution-processible polymer semiconductor was selected specifically for this research because solution-based fabrication methods (e.g., inkjet printing, microcontact printing, and nanoimprinting) are projected to be the main workhorse for manufacturing of organic electronics in the near future. Solution processing of OTFTs can eliminate the need for expensive vacuum chambers and lengthy pumpdown cycles, and can potentially reduce the cost and complexity of the fabrication processes [28].

Table 2.3. Device material consideration for the OTFT fabrication in this thesis.

\begin{tabular}{|ll|}
\hline OTFT Device Layers & Material Choices \\
\hline \multirow{3}{*}{ Semiconductor } & Regioregular poly(3-hexylthiophene) (P3HT); Aldrich \\
& Poly(3,3'"-dialkyl-quaterthiophene) (PQT-12); Xerox \\
& Poly(2,5-bis(3-alkylthiophen-2-yl)thieno[3,2-b]thiophene) (PBTTT); Merck \\
\hline \multirow{3}{*}{ Dielectric } & Thermal silicon dioxide $\left(\mathrm{SiO}_{2}\right)$ \\
& PECVD silicon nitride $\left(\mathrm{SiN}_{\mathrm{x}}\right)$ \\
& PECVD silicon oxide $\left(\mathrm{SiO}_{\mathrm{x}}\right)$ \\
\hline \multirow{2}{*}{ Electrodes } & Source/Drain contacts: $\mathrm{Au}, \mathrm{Cr}$ \\
& Gate electrode: $\mathrm{Mo}, \mathrm{Al}$ \\
\hline \multirow{5}{*}{ Substrate } & Silicon wafer \\
& Glass wafer \\
& Plastic substrate: Kapton, polyethylene naphthalate (PEN), Poly(ethylene \\
& terephthalate) (PET) \\
Encapsulation & Parylene \\
& Photoresist \\
& PECVD silicon nitride $\left(\mathrm{SiN}_{\mathrm{x}}\right)$ \\
\hline
\end{tabular}

\subsubsection{Organic Semiconductor}

Organic semiconductor is the core foundation of an OTFT. The semiconductor governs the charge transport in an OTFT. High quality and high performance semiconductor is a pre-requisite for high performance transistor. Motivated by the quest for low-cost manufacturing methods, this research focused on solution-processible organic polymer semiconductors. Initial experiments used regioregular poly(3-hexylthiophene) (RR-P3HT, $\left.\left(\mathrm{C}_{10} \mathrm{H}_{18} \mathrm{~S}\right)_{\mathrm{n}}\right)$, from Aldrich-Sigma Company, as the organic semiconductor layer. P3HT was chosen because it was commercially available and it had one of the highest reported mobilities among polymer semiconductor materials at that time [25][28][26]. However, our implementation of functional, high performance P3HT OTFTs was hindered by a number of challenges. First, many commercial organic semiconductors are very 
sensitive to air and moisture; thus, the lifetime and stability of the resulting OTFTs are a concern (especially as our processing and measurements were done in ambient, and nitrogen ambient for organic processing was unavailable). Secondly, the purity of the commercial-grade organic materials is questionable (despite claims of $99.9 \%$ purity). Impurities are manifested as defects in the organic semiconductor films, which can cause undesirable leakages and traps that obstruct proper device operation. Thirdly, the quality of commercial organic semiconductors tends to vary from batch to batch; this can have a negative impact on uniformity of TFT characteristics and device yield. As a result, preliminary trials of P3HT OTFTs displayed high gate leakage currents, primarily due to impurities in the commercial-grade P3HT. Issues with impurities and instabilities of commercially available organic semiconductors are a well-known problem among researchers; however, these issues were not documented in the literature. Publications reporting high performance P3HT OTFTs typically employed ultra-high purity P3HT materials that were synthesized in-house or were subjected to additional purification steps (typically by thermal sublimation) to eliminate/reduce possible impurities and contaminations in the product; moreover, processing and testing were typically done in inert (nitrogen) environment. Unfortunately, neither purification equipment nor nitrogen-purged chambers were accessible for this research; thus, limited device performance was measured for P3HT OTFTs.

A more effective and direct approach to attain OTFTs with improved performance and lifetime necessitates the development of stable polymer materials and an encapsulation strategy. Xerox Research Centre of Canada (XRCC) has successfully developed a new family of polythiophene materials, called poly(3,3'"-dialkylquarterthiophene) (PQT-12), with excellent stability and high mobility [15]. The core of this thesis work was conducted using PQT-12 polymer semiconductor [52]. Please refer to Figure 2.10(a) for the chemical structure of PQT-12.

More recently, Merck Chemicals introduced a new class of semiconducting liquid crystalline poly(2,5-bis(3-alkylthiophen-2-yl)thieno[3,2-b]thiophene) (PBTTT) polymers, featuring a fused ring structure (see Figure 2.10(b)). PBTTT OTFT demonstrated one of the highest mobilities and decent stability amongst solution-processed polymer semiconductors [31]. We recently demonstrated that high mobility can be achieved with PBTTT on PECVD $\mathrm{SiN}_{\mathrm{x}}$ gate dielectric with minimal surface treatment [53]; this is an important breakthrough as it signifies the potential to simplify OTFT fabrication process steps by clever selection of appropriate semiconductordielectric material pairs. 
A number of factors can affect the quality and properties of the organic semiconductor layer, including solvent selection, deposition conditions, and surface properties. Different solvents produce films with varying degrees of molecular ordering, uniformity, and continuity [26]. A variety of solvents has been reported for P3HT, including chloroform, toluene, $p$-xylene, hexane, chlorobenzene, 1,1,2,2,-tetrachloroethane, and teterachloroethylene. Researchers observed variations in the mobility of P3HT OTFT by two orders of magnitude depending on the choice of solvent [28]. OTFT device performance is also influenced by the deposition rate (e.g., spin coating speed of the polymer semiconductor layer) and temperature (substrate temperature), which affect the morphology of the semiconductor. Therefore, the choice of solvent, concentration of the polymer solution, and the deposition parameters must be optimized to yield good quality films. In the case of PQT polymer semiconductor, experiments have shown that 1,2-dichlorobenzene solvent gave optimal device performance. Additional enhancements can be achieved by surface or interface treatment [12][26][27][29] and post-deposition treatments such as annealing [54]; these processes can improve molecular ordering and contribute to improved device performance. Interface treatment techniques for OTFTs are examined in Chapter 5 and Chapter 6.

The main incentive of using polymer semiconductors is their compatibility with simple solutionbased processing techniques. Spin-on methods are the simplest approach for depositing polymers uniformly across the entire substrate. The solvent is then dried off by evaporation after deposition. This technique can generate uniform films ( $\sim 100 \mathrm{~nm}$ thick), as required in most OLED and OTFT devices. Although solution-based deposition methods can offer numerous advantages such as highspeed deposition over large substrate areas, concerns related to solvent interaction must be considered. The solvents used for one polymer layer may interact with previously applied polymer layers, thereby limiting the complexity and performance of the structures that can be achieved. Thus, the polymer solutions must be cautiously prepared and optimized to avoid chemical interactions in multi-layer organic devices. Another critical drawback of simple spin-on deposition methods is the inability to locally pattern the electronic device. Patterning of the organic layer is needed for OLED color displays, to define the red (R), green $(G)$ and blue (B) polymer OLED subpixels. The organic semiconductor layer of the OTFTs requires proper patterning for device isolation and circuit realization; these issues are discussed in Chapter 3. 


\subsubsection{Gate Dielectric}

The gate dielectric is one of the most critical materials for organic transistor performance. It plays an important function in establishing field-effect operation in TFTs. A general specification for the gate dielectric of TFTs is summarized as follows: the dielectric material has to withstand electric fields of about $2 \mathrm{MV} / \mathrm{cm}$ without breakdown, must have good insulating properties and low rate of charge trapping at lower electric fields, and it should form a high quality interface with the semiconductor layer [55]. In addition, the dielectric films should possess low trapping density at the surface, low surface roughness, low impurity concentration, and compatibility with organic semiconductors. Dielectric materials can be classified as inorganic or organic. Examples of inorganic gate dielectrics include silicon dioxide $\left(\mathrm{SiO}_{2}\right)$, silicon nitride $\left(\mathrm{SiN}_{\mathrm{x}}\right)$, titanium oxide $\left(\mathrm{TiO}_{2}, k=41\right)$ and aluminum oxide $\left(\mathrm{Al}_{2} \mathrm{O}_{3}, k=8.4\right)$ [56]. The OTFT research documented in this thesis focuses primarily on inorganic gate dielectrics; more specifically, plasma enhanced chemical vapor deposited (PECVD) silicon nitride $\left(\mathrm{SiN}_{\mathrm{x}}\right)$ and silicon oxide $\left(\mathrm{SiO}_{\mathrm{x}}\right)$ thin films. The key benefits of using inorganic dielectrics include their maturity, good dielectric strength and integrity, and availability of high-quality films. The mature manufacturing processes and deposition technologies are capable of depositing a pinhole-free gate dielectric layer with a thickness of a few hundred angstroms [24]. The well-known dielectric characteristics of these materials significantly reduce process variability. PECVD gate dielectrics are investigated in depth in Chapter 4.

A high-k dielectric (e.g., $\mathrm{Al}_{2} \mathrm{O}_{3}, \mathrm{TiO}_{2}$ ) is another candidate for gate dielectric in OTFTs because its high-k value can increase the intrinsic gate capacitance $\left(C_{\mathrm{i}}=k \varepsilon_{0} A / d\right)$ of a transistor, which in turn increases the current output (since $I_{\mathrm{D}} \propto \mu C_{\mathrm{i}}$ ). Thus, high-k dielectrics can partially compensate for the relatively low mobility $(\mu)$ of the organic semiconductors and, can enable device operation at lower drive voltages. However, a key shortcoming of high-k materials is their higher degree of disorder and surface roughness, which can create traps, reduce mobility, and lead to localization of carriers at the dielectric-semiconductor interface [56].

Although OTFTs with high quality inorganic gate dielectrics on rigid substrates have demonstrated noticeably improved performance over the past two decades, the insoluble and rigid nature of most inorganic dielectric materials prompted researchers to investigate solutionprocessible organic dielectrics in order to fulfill the inherent merits of OTFTs for low-cost processing and mechanical flexible. Examples of organic gate dielectrics for OTFTs include polyvinylphenol (PVP), polyvinylalcohol (PVA), polymethyl-methacrylate (PMMA), and polyimide [57][58][59][60][61]. Comparatively, organic dielectric materials are less matured; on- 
going research is needed to enhance material quality, dielectric strength, electrical integrity and other properties. However, organic dielectrics with good solubility are ideal for low-cost solutionbased processing methods (e.g., spin-coating, dip-coating, printing). Moreover, the low processing temperature of organic dielectrics is compatible with plastics substrates, making it suitable for flexible electronics applications.

Among the various polymer gate insulators, PVA appears promising because it has many merits such as a surface alignment effect by rubbing process, high dielectric constant, and compatibility with solution-based processes [57]. However, PVA tends to easily absorb moisture due to the presence of hydroxyl group (-OH) in the material. A possible remedy is to use cross-linked PVA as the gate dielectric, which exhibits higher endurance against moisture than uncross-linked PVA; moreover, the cross-linking process provides an opportunity for pattern definition by UV exposure. Insertion of a PMMA buffer layer on a cross-linked PVA gate insulator can provide an extra barrier against moisture penetration [57]. In addition, PMMA buffer layers on cross-linked PVA gate insulators have shown to improve the ordering of organic semiconductor layer owing to the excellent hydrophobicity of PMMA.

A more recent advancement is the development of very thin $(2.3-5.5 \mathrm{~nm})$ molecule-derived self-assembled organic dielectric multi-layers that enable operation of OTFTs at very low voltages (sub-1V) and without serious leakage currents [62]. Thin, nanostructurally ordered, pinhole-free, high-capacitance and low leakage organic dielectrics can be formed by solution phase deposition methods. This chemically-tailored siloxane-crosslinked polymeric nanodielectric represents a new and promising approach to high-performance, low-power dissipation OTFTs, while providing excellent dielectric properties an efficient low-cost solution-phase processing characteristics.

Although there have been successful demonstrations of OTFTs with organic gate dielectrics, most of these devices exhibit lower on/off ratio and higher leakage current compared to OTFTs with inorganic gate dielectrics. The surface roughness of the solution-processed organic dielectric is a key concern here, as the roughened dielectric-semiconductor interface can generate a higher density of interface traps, cause interface scattering, and degrade the mobility of bottom-gate OTFTs [61]. It is also important to ensure the solution-processible organic dielectric do not chemically disrupt the organic semiconductor. This requires careful consideration for solvent selection and deposition conditions.

In addition to the bulk properties of the dielectric material, the interface properties between the dielectric and organic semiconductor have a profound effect on device performance. The molecular 
ordering of the organic semiconductor film, and hence field-effect mobility of the device, is usually very sensitive to dielectric surface properties (e.g., surface roughness and surface energy). Interface modification methods, such as alkylsilane self-assembled monolayers (SAMs) treatment of the dielectric surface prior to semiconductor deposition, are often used to induce ordering of the polymer film (i.e., $\pi-\pi$ stacking between conjugated polymer molecules to form the path way for charge carrier hopping from molecule to molecule). These interface treatments can improve device mobility by as much as an order of magnitude. Optimization of the gate dielectric and the interface properties form a critical part of this thesis research; the results are presented in Chapter 4 and Chapter 5.

\subsubsection{Electrodes/Contacts}

The gate electrode and source/drain electrodes (also known as contacts) for OTFTs can be implemented using metals or organic conductors. Typically, the material specification for the gate electrode is relatively relaxed. Considerations for selecting gate material include high conductivity, compatibility with device layers, and low surface roughness in the case of bottom-gate structures. For example, molybdenum (Mo) is selected over aluminum (Al) for bottom-gate a-Si TFTs owing to molybdenum's smoother surface, which critically affects the quality of subsequent (or overlying) device layers.

In contrast, the choice of material for source/drain contacts is more stringent; conscientious energetic matching between contact and semiconductor is important to establish efficient charge injection in OTFTs. Ohmic contact is desirable, which requires the work function of the contact metal to match with the HOMO of the organic semiconductor in the case of p-type OTFT, and with the LUMO in the case of n-type OTFT. This implies high work function metal for p-type OTFT and low work function metal for n-type OTFT. The work function of various metals is listed Table 2.4. Gold $(\mathrm{Au})$ and platinum $(\mathrm{Pt})$ are favorable choices for their high work function, which has good electrical compatibility with the p-type organic semiconductors. Moreover, the environmentally stable nature of $\mathrm{Au}$ and $\mathrm{Pt}$ (in contrast to $\mathrm{Ca}$ and $\mathrm{Al}$ ) ensures low contact resistance at the contact-semiconductor interfaces by shunning from formation of surface oxide and eliminating chemical interaction with most organic materials. 
Table 2.4. Work function $\left(\Phi_{M}\right)$ of selected metals [63].

\begin{tabular}{|ccccccccccccc|}
\hline & $\mathrm{Ag}$ & $\mathrm{Al}$ & $\mathrm{Au}$ & $\mathrm{Cu}$ & $\mathrm{Cr}$ & $\mathrm{Mo}$ & $\mathrm{Ni}$ & $\mathrm{Pd}$ & $\mathrm{Pt}$ & $\mathrm{Ti}$ & $\mathrm{W}$ & $\mathrm{ITO}$ \\
\hline$\Phi_{\mathrm{M}}(\mathrm{eV})$ & 4.26 & 4.28 & 5.1 & 4.65 & 4.5 & 4.6 & 5.15 & 5.12 & 5.65 & 4.33 & 4.55 & $4.8-5.2$ \\
\hline
\end{tabular}

The interface between the source/drain contact and organic semiconductor requires low contact resistance, which is a function of both parasitic resistance and the energy barrier at the contactsemiconductor interface [24]. Low energy barriers necessitate matching the electrode work function $\left(\Phi_{\mathrm{M}}\right)$ with semiconductor ionization potential $\left(\mathrm{IP}_{\mathrm{S}}\right)$. Figure 2.18 shows the energy scheme at the contact-semiconductor interface. Material should be selected so that the barrier of hole injection $\left(\varphi_{\mathrm{B}}=\mathrm{IP}_{\mathrm{S}}-\Phi_{\mathrm{M}}\right)$ is minimized (for p-type OTFT), to allow for high charge injection efficiency at the contacts. In addition to energetic matching at the interface, the contact material must possess high conductivity for optimal OTFT performance [30]. Most metal-organic semiconductor junctions form a Schottky contact, indicating the presence of an injection barrier. Contact resistance is discussed in Chapter 6, along with techniques to improve the contactsemiconductor interface.

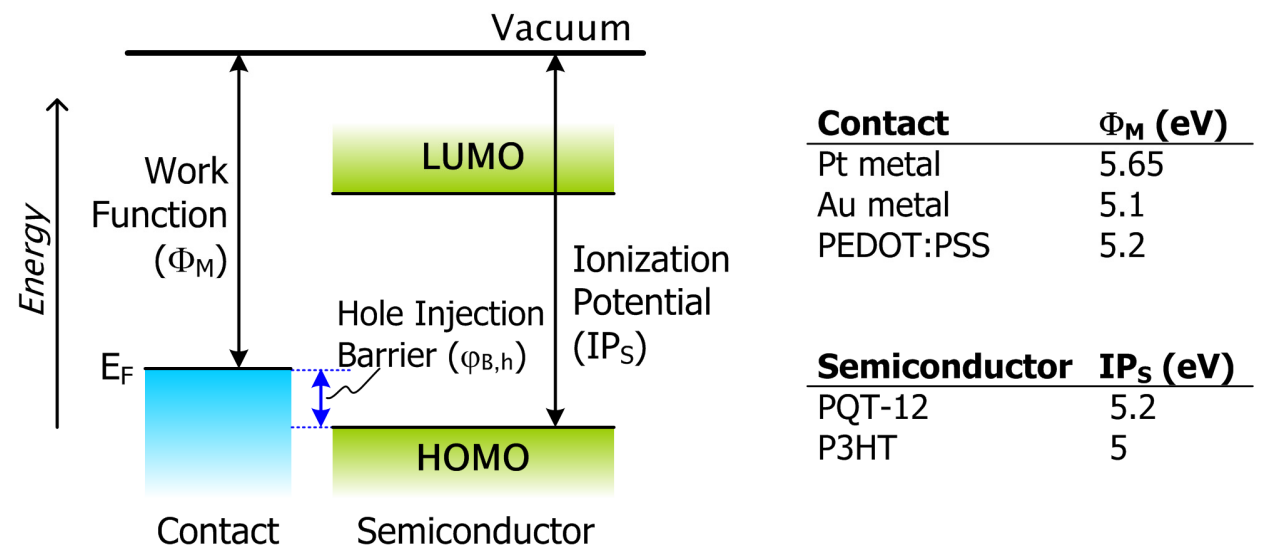

Figure 2.18. Energy band diagram at the contact-semiconductor interface, illustrating the concept of work function $\left(\Phi_{\mathrm{M}}\right)$, ionization potential (IP) and injection barrier $\left(\varphi_{B}\right)$ at the contact. Representative values for $p$-type polymer semiconductor and contact material are shown.

Metals are typically deposited by evaporation or sputtering; and thus require vacuum processing. To fulfill the inherent virtues of OTFTs such as low-cost processing and compatibility of flexible substrates, solution-processible organic conductors are favorable. Conductive polymers such as poly(3,4-ethylene dioxythiophene) doped with polystyrene sulfonic acid (PEDOT:PSS) and polyaniline (PANI) have been used as electrodes in OTFTs . Polymer conductors can be deposited 
by spin-coating, inkjet printing, screen-printing or soft-lithography methods (e.g., microcontact printing) [30][58][59][64]. More recently, solution-processible conductors based on $\mathrm{Au} / \mathrm{Ag} / \mathrm{Cu}$ nanoparticles, carbon nanotubes and nanowires are emerging as promising candidates for electrode material [65][66][67].

\subsubsection{Substrate}

Candidates for the substrate material for OTFTs include rigid substrates such as silicon wafers and glass substrates, and flexible substrates such as polyimide (or Kapton), poly(ethylene terephthalate) (PET), poly(ethylene naphthalate) (PEN), aluminum or stainless steel foil, paper and fabric. The choice of substrate affects the OTFT performance. Rigid substrates are advantageous for high planarity, optimal surface smoothness, ease of processing, and capability of high temperature annealing of organic semiconductors [24]. Planarity and surface smoothness are particularly critical to OTFT performance because they affect quality of the overlying thin film device layers. Large substrate surface roughness is expected to cause a greater density of trap states in the gate dielectric and at the dielectric-semiconductor interface for bottom-gate OTFTs, which can negatively impact the OTFT characteristics. For instance, Klauk et al. observed that pentacene OTFTs with PVP gate dielectric on PEN substrate have a slightly higher subthreshold swing and threshold voltage than OTFTs on glass substrate. These differences are attributed to the larger surface roughness of the PEN substrate compared to glass (15 $\AA$ for PEN versus $8 \AA$ for glass) [60].

Since organic electronics are envisioned to be flexible and lightweight, the ability to fabricate organic electronics on flexible substrates (e.g., plastic or foil) is of considerable interest for handheld devices and military applications. The low material cost, opportunity for using high-speed roll-to-roll printing processes for reduced manufacturing costs, and the flexible form factor are all marketable features of organic electronics. Criteria for selecting flexible substrate used in OTFTs include thermal, chemical and mechanical stability, surface roughness, and cost of the films. A number of flexible substrate candidates are listed in Table 2.5, along with their maximum process temperature and key material characteristics.

However, existing plastic substrates exhibit a number of shortcomings related to permeability, dimensional distortion, and temperature tolerance. Most plastics are permeable to water and oxygen, which may lead to rapid deterioration in organic device performance. Thus, it is vital to perform proper passivation of the plastic substrate prior to device fabrication. Also, inexpensive 
plastic materials typically cannot withstand high processing temperatures. Researchers are investigating various processing techniques to maintain the substrate temperature below $100^{\circ} \mathrm{C}-$ $150^{\circ} \mathrm{C}$. New plastic substrate materials that can withstand higher temperatures (e.g., up to $300^{\circ} \mathrm{C}-$ $350^{\circ} \mathrm{C}$ ) are also under development; however, these temperature-tolerant plastic substrates will likely involve higher costs [69]. Dimensional distortion (e.g., shrinking) of plastic substrates due to thermal effects can affect stability of organic electronics.

To improve the dimensional stability of flexible plastic substrates for device applications, plastic substrates can be subjected to thermal treatment prior to device fabrication. For example, a PEN substrate can be preshrunk in a vacuum oven at $200^{\circ} \mathrm{C}$ and at a rate of $3^{\circ} \mathrm{C}$ per minute, followed by cooling to room temperature at about $1{ }^{\circ} \mathrm{C}$ per minute. Klauk et al. [60] observed that OTFTs on untreated PEN exhibited dimensional distortions greater than $0.5 \%$, but OTFTs on pre-treated PEN substrate had dimensional distortion of less than $0.02 \%$. These observations suggested that thermal treatment of the plastic substrate prior to device processing can help to secure sufficient dimensional stability in the resulting organic device.

Using fabric or textile as a device substrate for organic electronics has recently gained increased visibility. Wearable electronics, such as clothing with sensors that monitor critical body health parameters, are beginning to be promoted by companies such as Infineon and Philips [70].

Table 2.5. Properties of flexible substrate material candidates [68].

\begin{tabular}{|c|c|c|}
\hline $\begin{array}{c}\text { Flexible Substrate } \\
\text { Material }\end{array}$ & $\begin{array}{l}\text { Maximum Process } \\
\text { Temperature }\left({ }^{\circ} \mathrm{C}\right)\end{array}$ & Characteristics \\
\hline Steel Foil & 900 & $\begin{array}{l}\square \text { Moderate chemical resistance, moderate CTE } \\
\bigotimes \text { Opaque, poor surface finish }\end{array}$ \\
\hline $\begin{array}{l}\text { Polyimide } \\
\text { (Kapton } ®)\end{array}$ & 275 & $\begin{array}{l}\square \text { Good chemical resistance, highest processing } \\
\text { temperature for plastic substrates } \\
\text { Orange color, higher cost, high moisture } \\
\text { absorption }\end{array}$ \\
\hline $\begin{array}{l}\text { Polyetheretherket } \\
\text { one (PEEK) }\end{array}$ & 250 & $\begin{array}{l}\text { Good chemical resistance, low moisture } \\
\text { absorption } \\
\bigotimes \quad \text { Amber color, expensive }\end{array}$ \\
\hline $\begin{array}{l}\text { Polyethersulphone } \\
\text { (PES) }\end{array}$ & 230 & $\begin{array}{l}\square \text { Clear, good dimensional stability, inexpensive, } \\
\text { moderate moisture absorption } \\
\bigotimes \text { Poor solvent resistance, expensive }\end{array}$ \\
\hline $\begin{array}{l}\text { Polyetherimide } \\
\text { (PEI) }\end{array}$ & 200 & $\begin{array}{ll}\square & \text { Strong } \\
\bigotimes & \text { Hazy-colored, expensive }\end{array}$ \\
\hline $\begin{array}{l}\text { Polyethylenenapth } \\
\text { alate (PEN) }\end{array}$ & 150 & $\begin{array}{l}\nabla \text { Clear, good chemical resistance, inexpensive, } \\
\text { moderate CTE, moderate moisture absorption }\end{array}$ \\
\hline Polyester (PET) & 120 & $\begin{array}{l}\square \text { Clear, good chemical resistance, inexpensive, } \\
\text { moderate CTE, moderate moisture absorption }\end{array}$ \\
\hline
\end{tabular}




\subsubsection{Encapsulation Strategies}

Because many organic semiconductors and conductive polymers are easily influenced by water, oxygen and other environmental elements present in ambient conditions, the lifetime and reliability of organic electronics are a critical concern. This demands an optimum strategy to protect organic electronics from environmental damage. Encapsulation presents a viable solution to protect organic electronic devices from the environment which helps to maintain device performance and prolong device lifetime. The proper encapsulation method for OTFTs should meet these basic criteria [71]: low-temperature deposition $\left(<80^{\circ} \mathrm{C}\right)$ to enable direct deposition on temperature-sensitive organic devices, intrinsically defect-free films to provide a reliable barrier against moisture and oxygen, compatibility with large-area substrate manufacturing systems (e.g., for displays), minimal effect on device performance, low cost, and good durability to provide long-term protection from moisture, oxygen, alkali ion or other contaminants.

Various encapsulation strategies have been investigated for organic devices, including metal/glass lid encapsulation for OLEDs, photoresist-based encapsulation, PVA-based encapsulation, and multilayer coating. For example, the current OLED technology uses monolithic encapsulation with metal or glass lids to protect the OLED devices. However, this approach is regarded only as a short-term expedient. Improved materials are needed as sealants and as dessicants to minimize/eliminate water and oxygen penetration into the device [69]. Photoresist materials have been investigated as an encapsulation material for polymer devices. Lu et al. [72] encapsulated their P3HT OTFTs with a thin layer $(1 \mu \mathrm{m})$ of the photoresist (AZ1312 SFD) using spin casting technique. The mobility of the encapsulated OTFT remained quite stable over a 60-day period compared to an unencapsulated device, implying enhanced electrical stability of the device in the long term. However, a decrease in mobility by $\sim 60 \%$ was observed after the encapsulation step, suggesting undesirable interaction between the photoresist and the organic semiconductor film [72]. Therefore, a more reliable and robust solution is required.

A similar resist-based encapsulation was demonstrated by Qui et al. [73], in which a $200 \mu \mathrm{m}$ thick UV curable resin was coated by a doctor blade method on the top of a pentacene OTFT for encapsulation. For an unencapsulated OTFT, the field-effect mobility decreased by $30 \%$ and the on/off current ratio decreased to $20 \%$ of the original value after storing the OTFT in the atmosphere for 500 hours [73]. In contrast, encapsulated OTFTs showed reasonable electrical 
stability. Degradation of the organic material was not reported after the coating process, implying that this UV curable resin may offer a feasible encapsulation solution for OTFTs.

Encapsulation of OTFTs using polyvinyl alcohol (PVA) has been studied [74]. Although PVA was able to passivate the OTFT from environmental effects, some groups have reported degradation in OTFT electrical characteristics after the PVA coating (formed by a wet process). The decrease in mobility is proposed to be due to the migration of PVA solution into the organic semiconductor layer, and/or due to the sheer stress by the viscosity of PVA when it was coated and dried.

To eliminate interaction between the solution-based chemicals, encapsulation based on multilayer coatings has been introduced. Lee et al. reported an encapsulation method by means of an adhesive multilayer formed by conventional lamination process. The adhesive multilayer consisted of polyacrylate based adhesive (15 $\mu \mathrm{m}$ thick) and Al (185 $\mu \mathrm{m}$ thick). For OTFT encapsulation, the $\mathrm{Al}$ film is adhered onto the semiconductor layer in a dry nitrogen atmosphere using a proper adhesive [74]. The passivated OTFTs showed no degradation after the encapsulation procedure and demonstrated reasonable stability in air overtime. This adhesive multilayer coating technique provides an excellent barrier against moisture penetration with a very low water vapor transmission rate, and is suitable for use as flexible and light-weight encapsulation for flexible OTFT devices [74]. Multilayer coatings based on alternating layers of inorganic and polymer materials are also under development [69]. These coatings are especially useful to compensate for the high porosity of plastic films, and are used as barrier layers to withstand moisture and oxygen penetration.

Inorganic gas barriers such as tin oxide $\left(\mathrm{SnO}_{2}\right)$, aluminum oxide $\left(\mathrm{AlO}_{\mathrm{x}}\right)$, and silicon oxide $\left(\mathrm{SiO}_{\mathrm{x}}\right)$ have been considered as encapsulation for OTFTs. Kim et al. reported long term stability of pentacene OTFTs encapsulated with transparent $\mathrm{SnO}_{2}$ thin film. Although the field effect mobility degraded from $0.62 \mathrm{~cm}^{2} / \mathrm{V}$-s to $0.5 \mathrm{~cm}^{2} / \mathrm{V}$-s with encapsulation, enhanced long-term device stability and lifetime was observed [75]. A caution when using these inorganic barrier layers is a potential for process-induced damage to the organic semiconductor layer; the damage may arise from exposure to energetic ions, $\mathrm{x}$-rays, electron beams, and high temperatures during deposition of the inorganic barrier layers.

Inorganic material such as $\mathrm{SiN}_{\mathrm{x}}$ is commonly used as passivation layers in silicon ICs. We have demonstrated P3HT OTFTs with $\mathrm{SiN}_{\mathrm{x}}$ encapsulation [49][50]. However, a reduced mobility is observed after depositing $\operatorname{SiN}_{\mathrm{x}}$ on top of the P3HT layer by PECVD. The reduction is believed to 
stem from plasma-induced material degradation, and can be circumvented by incorporation of a protective layer (e.g., an organic dielectric such as parylene) to eliminate direct plasma exposure of the organic semiconductor layer during PECVD process. Parylene is ideal for this purpose as it is routinely used as a conformal coating for many commercial and industrial applications [76]. Successful demonstration of OTFT devices and circuits with parylene passivation are reported in Chapter 3 and Chapter 7, respectively.

\subsection{Summary}

This chapter presented a background overview of OTFT technology. The fundamental properties of organic semiconductors and the basic operation of OTFT were reviewed. A procedure for the extraction of OTFT device parameters was explained to provide a basis for evaluation of OTFT characteristics. The device architecture and material selection considered for OTFT fabrication in this research were also specified. The discussion presented in this chapter lays a foundation for subsequent discussions and investigations reported in this thesis. 


\section{Bibliography}

[1] "Definition of organic compound," (2004). www.wordiq.com/definition/Organic_compound.

[2] E. Cantatore, G.H. Gelinck and D.M. de Leeuw, "Polymer Electronics: from Discrete Transistors to Integrated Circuits and Active Matrix Displays," IEEE Proceedings of the Bipolar/BiCMOS Circuits and Technology Meeting, 167 (2002).

[3] J.M. Shaw and P.F. Seidler, "Organic electronics: Introduction," IBM J. Res. \& Dev. 45(1), 3 (2001).

[4] "The 2000 Nobel Prize in Chemistry," (2000). www.nobel.se/chemistry/laureates/2000/press.html.

[5] T. Ito, H. Shirakawa, and S. Ikeda, "Simultaneous polymerization and formation of polyacetylene film on the surface of concentrated soluble Ziegler-type catalyst solution," J. Polym. Sci.: Chem. Ed. 12(1), 11 (1974).

[6] C.K. Chiang, C. R. Fincher, Y.W. Park, A.J, Heeger, H. Shirakawa, E.J. Louis, S. C. Gau, and A.G. MacDiarmid, "Electrical Conductivity in Doped Polyacetylene," Phys. Rev. Lett. 39, 1098 (1977).

[7] S.J. Higgins, W. Eccelston, N. Sedgi and M. Raja, "Plastic electronics," Education in Chemistry (2003). http://www.rsc.org/lap/educatio/eic/2003/higgins_may03.htm

[8] C.G. Cameron, "Enhanced Rates of Electron Transport in Conjugated-Redox Polymer Hybrids," Ph.D. thesis, Department of Chemistry, Memorial University of Newfoundland, Canada (2000). http://mmrc.caltech.edu/colin/science/past/PhD/html-thesis/node8.html

[9] C.D. Dimitrakopoulos and P.R.L. Malenfant, "Organic Field-Effect Transistors for Large Area Electronics," Adv. Mater. 14(2), 99 (2002).

[10] M.C.J.M. Vissenberg and M. Matters, "Theory of the field-effect mobility in amorphous organic transistors,” Phys. Rev. B 57, 12964 (1998).

[11] G. Horowtiz, "Organic Transistors," Organic Electronics: Materials, Manufacturing and Applications, H. Klauk (ed.). Wiley-VCH (2006).

[12] H. Sirringhaus, P.J. Brown, R.H. Friend, M.M. Nielsen, K. Bechgaard, B.M.W. LangeveldVoss, A.J.H. Spiering, R.A.J. Janssen, E.W. Meijer, P.T. Herwig, and D.M. de Leeuw, "Two-Dimensional Charge Transport in Self-Organized, High-Mobility Conjugated Polymers," Nature 401, 685 (1999).

[13] Y.D. Park, J.A. Lim, H.S. Lee, and K. Cho, "Interface engineering in organic transistors," Materials Today 10, 46-54 (2007).

[14] B.S. Ong, Y. Wu, P. Liu, and S. Gardner, "Structurally ordered polythiophene nanoparticles for high-performance OTFTs," Adv. Mater. 17, 1141 (2005).

[15] B.S. Ong, Y. Wu, P. Liu, and S. Gardner, "High-Performance Semiconducting Polythiophenes for Organic Thin-Film Transistors," J. Am. Chem. Soc. 126(11), 3378 (2004). 
[16] J. Ficker, A. Ullmann, W. Fix, H. Rost, and W. Clemens, "Stability of polythiophene-based transistors and circuits," J. App. Phys. 94(4), 2638 (2003).

[17] J. H. Schon, S. Berg, C. Kloc, and B. Batlogg, "Ambipolar Pentacene Field-Effect Transistors and Inverters," Science 287, 1022 (2000).

[18] T. Kelley, "High Performance Pentacene Transistors," Organic Electronics: Materials, Manufacturing and Applications, H. Klauk (ed.). Wiley-VCH (2006).

[19] E. Cantatore, "Organic Materials: A New Chance for Electronics?" Proceedings of the SAFE/IEEE Workshop, 27, November 2000.

[20] T.N. Jackson, Y-Y. Lin, D.J. Gundlach and H. Klauk, "Organic Thin-Film Transistors for Organic Light-Emitting Flat-Panel Display Backplanes,” IEEE J. Sel. Top. Quantum Electronics 4(1), 100 (1998).

[21] Z. Bao, A. Dadabalapur, H.E. Katz, R.V. Raju and J.A. Rogers, "Organic Semiconductors for Plastic Electronics," IEEE-TAB New Technology Directions Committee (NTDC), 2001 IEEE Workshop on New and Emerging Technologies (2001).

[22] A. Afzali, C.D, Dimitrakopoulos, and T.L. Breen, "High-performance, solution-processed organic thin film transistors from a novel pentacene precursor," J. Amer. Chem. Soc. Comm. 124, 8812 (2002).

[23] M. Kawasaki, M. Ando, S. Imazeki, Y. Sekiguchi, S. Hirota, H. Sasaki, S. Uemura, and T. Kamata, "Printable organic TFT technologies for FPD applications," Proc. SPIE 5940, 59400Q1-10 (2005).

[24] M. Chason, P. W. Brzis, J. Zhang, K. Kalyanasundaram, and D. R. Gamota, "Printed Organic Semiconducting Devices," Proc. Of IEEE. 93(7), 1348 (2006).

[25] G. Horowitz, “Organic Field-Effect Transistors,” Adv. Mater., 10(5), 365 (1998).

[26] Z. Bao, A. Dodabalapur, and A. J. Lovinger, "Soluble and Processable Regioregular Poly(3-hexylthiophene) for Thin Film Field-Effect Transistor Applications with High Mobility," Appl. Phys. Lett., 69, 4108 (1996).

[27] Z. Bao, Y. Feng, A. Dodabalapur, V.R. Raju and A.J. Lovinger, "High-Performance Plastic Transistors Fabricated by Printing Techniques," Chem. Mater. 9, 1299 (1997).

[28] C.D. Dimitrakopoulos and D.J. Mascaro, "Organic thin-film transistors: A review of recent advances,” IBM J. Res. \& Dev. 45(1), 111 (2001).

[29] H. Sirringhaus, N. Tessler, and R.H. Friend, "Integrated Optoelectronic Devices Based On Conjugated Polymers," Science 280, 1741 (1998).

[30] H. Sirringhaus, T. Kawase, R.H. Friend, T. Shimoda, M. Inbasekaran, W. Wu, and E.P. Woo, "High-Resolution Inkjet Printing of All-Polymer Transistor Circuits," Science 290, 2123 (2000).

[31] I. McCulloch, M. Heeney, C. Bailey, K. Genevicius, I. MacDonald, M. Shkunov, D. Sparrowe, S. Tierney, R. Wagner, W. Zhang, M.L. Chabinyc, R.J. Kline, D. McGehee, and M.F. Toney, "Liquid-crystalline semiconducting polymers with high charge-carrier mobility," Nature Materials 5, 328-333 (2006).

[32] Z. Bao, A. J. Lovinger, and J. Brown, "New Air-Stable n-Channel Organic Thin Film Transistors," J. Amer. Chem. Soc. 120, 207 (1998). 
[33] H.E. Katz, J.J. Johnson, A.J. Lovinger, and W. Li, "Naphthalenetetracarboxylic DiimideBased n-Channel Transistor Semiconductors: Structural Variation and Thiol-Enhanced Gold Contacts," J. Amer. Chem. Soc. 122, 7787 (2000).

[34] A. Babel and S.A. Jenekhe, "n-Channel Field-Effect Transistors from Blends of Conjugated Polymers," J. Phys. Chem. B 106(24), 6129 (2002).

[35] T.D. Anthopoulos, B. Singh, N. Marjanovic, N.S. Sariciftci, A.M. Ramil, H. Sitter, M. Colle, and D.M. de Leeuw, "High performance n-channel organic field-effect transistors and ring oscillators based on $\mathrm{C}_{60}$ fullerene films," Appl. Phys. Lett. 89, 213504 (2006).

[36] S.P. Tiwari, E.B. Namdas, V. Ramgopal Rao, D. Fichou, and S.G. Mhaisalkar, S.G., "Solution-Processed n-Type Organic Field-Effect Transistors With High on /off Current Ratios Based on Fullerene Derivatives," IEEE Electron Dev. Lett. 28, 880 (2007).

[37] T.D. Anthopoulos, S. Setayesh, E. Smits, M. Colle, E. Cantatore, B, de Boer, P.W.M. Blom, and D.M. de Leeuw,, "Air-stable complementary-like circuits based on organic ambipolar transistors, Advanced Materials," Adv. Mater. 18, 1900 (2006).

[38] E. J. Meijer, D. M. de Leeuw, S. Setayesh, E. van Veenendaal, B. -H. Huisman, P. W. M. Blom, J. C. Hummelen, U. Scherf \& T. M. Klapwijk, "Solution-processed ambipolar organic field-effect transistors and inverters," Nature Mat. 2, 678 (2003).

[39] J.I. Han, Y.H. Kim, S.K. Park, D.G. Moon, and W.K. Kim, "Stability of Organic Thin Film Transistors," Mat. Res. Soc. Symp. Proc. 814, I4.3.1 (2004).

[40] M.L. Chabinyc, F. Endicott, B.D. Vogt, D.M. DeLongchamp, E.K. Lin, Y. Wu, P. Liu, and B.S. Ong, "Effects of humidity on unencapsulated poly(thiophene) thin-film transistors," Appl. Phys. Lett. 88, 113514 (2006).

[41] M.L. Chabinyc, R.A. Street, and J.E. Northrup, "Effects of molecular oxygen and ozone on polythiophenes-based thin-film transistors," Appl. Phys. Lett. 90, 123508 (2007).

[42] P.P. Allport, P.S.L. Booth, G. Casse, W. Eccleston, S. Higgins, J. Marsland, and N. Sdghi, "Ionizing Radiation Detection Using Semiconducting Conjugated Polymers," (2003). http://hepwww.rl.ac.uk/pprp/Experimental_reports/ExpRep_2002/polymer.pdf

[43] M.S.A. Abdou, F.P. Orfino, Y. Son, and Steven Holdcroft, "Interaction of Oxygen with Conjugated Polymers: Charge Transfer Complex Formation with Poly(3alkylthiophenes)," J. Am. Chem. Soc. 119, 4518 (1997).

[44] P. Servati, D. Striakhilev, and A. Nathan, "Above-Threshold Parameter Extraction and Modeling for Amorphous Silicon Thin-Film Transistors," IEEE Trans. Electron Devices 50, 2227 (2003).

[45] P. Servati, "Amorphous Silicon TFTs for Mechanically Flexible Electronics," Ph.D. Thesis, University of Waterloo (2004).

[46] B.H. Hamadani, D.J. Gundlach, I. McCulloch, and M. Heeney, "Undoped polythiophene field-effect transistors with mobility of $1 \mathrm{~cm} 2 \mathrm{~V}-1 \mathrm{~s}-1$," Appl. Phys. Lett. 91, 243512 (2007).

[47] A. Nathan, "Large area thin film electronics", University of Waterloo, Waterloo, Ontario (2001). 
[48] M. Halik, H. Klauk, U. Zschieschang, G. Schmid, W. Radlik, S. Ponomarenko, S. Kirchmeyer, and W. Weber "High-mobility organic thin-film transistors based on $\alpha, \alpha^{\prime}$ didecyloligothiophenes,” J. Appl. Phys. 93, 2977 (2003).

[49] F.M. Li, S. Koul, Y. Vygranenko, A. Sazonov, P. Servati and A. Nathan. "Fabrication of RR-P3HT-based TFTs using low-temperature PECVD silicon nitride," Mater. Res. Soc. Symp. Proc. 871E, I9.3.1 (2005).

[50] P. Servati, K.S. Karim, and A. Nathan, "Static Characteristics of a-Si:H Dual-Gate TFTs," IEEE Trans. Electron Devices 50, 926 (2003).

[51] F.M. Li, S. Koul, Y. Vygranenko, P. Servati and A. Nathan, "Dual-Gate $\mathrm{SiO}_{2} / \mathrm{P} 3 \mathrm{HT} / \mathrm{SiN}_{\mathrm{x}}$ OTFT.” Mater. Res. Soc. Symp. Proc. 871E, I9.4.1 (2005).

[52] F.M. Li, Y. Wu, B.S. Ong, A. Nathan, "Organic Thin-Film Transistor Integration using Silicon Nitride Gate Dielectric,” Appl. Phys. Lett. 90,133514 (2007).

[53] F.M. Li, P. Dhagat, G.E. Jabbour, H.M. Haverinen, I. McCulloch, M. Heeney, and A. Nathan, "Polymer TFT without Surface Pre-Treatment on Silicon Nitride Gate Dielectric," Appl. Phys. Lett. 92 (2008). [Accepted for publication, in-press]

[54] M.J. Joung, C.A. Kim, S.Y. Kang, K.H. Baek, G.H. Kim, S.D. Ahn, I.K. You, J.H. Ahn, and K.S. Suh, "The application of soluble and regioregular poly(3-hexylthiophene) for organic thin-film transistors," Synthetic Metals 149, 73 (2005).

[55] A. Sazonov, D. Striakhilev, A. Nathan, and L.D. Bogomolova, "Dielectric performance of low temperature silicon nitride films in a-Si:H TFTs," J. Non-Cryst. Solids 299-302, 1360 (2002).

[56] G. Wang, D. Moses, A.J. Heeger, H.M. Zhang, M. Narasimhan, and R.E. Demaray, "Poly(3-hexylthiophene) field-effect transistors with high dielectric constant gate insulator," J. Appl. Phys. 59, 316 (2004).

[57] S.H. Jin, J.S. Yu, J.W. Kim, C.A. Lee, B.G. Park, J.D. Lee, and J.H. Lee, "PMMA BufferLayer Effects on Electrical Performance of Pentacene OTFTs with a Cross-linked PVA Gate Insulator on a Flexible Substrate,” SID 03 DIGEST, pp. 1088 (2003).

[58] F. Garnier, R. Hajlaoui, A. Yassar and P. Srivastava, "All-polymer field-effect transistors realized by printing techniques,” Science 265, 1684 (1994).

[59] M. Halik, H. Klauk, U. Zschieschang, G. Schmid, W. Radlik and W. Weber, "Polymer Gate Dielectrics and Conducting-Polymer Contacts for High-Performance Organic ThinFilm Transistors," Adv. Mater. 14, 1717 (2002).

[60] H. Klauk, M. Halik, U. Zschieschang, F. Eder, G. Schmid, and C. Dehm, "Pentacene organic transistors and ring oscillators on glass and on flexible polymeric substrates," Appl. Phys. Lett. 82, 4175 (2003).

[61] G.W. Kang, K.M. Park, J.H. Song, C.H. Lee, and D.H. Hwang, "The electrical characteristics of pentacene-based organic field-effect transistors with polymer gate insulators," Current Appl. Phys. 5, 297 (2005).

[62] A. Facchetti, M.H. Yoon, and T.J. Marks, "Gate Dielectrics for Organic Field-Effect Transistors: New Opportunities for Organic Electronics,” Adv. Mater. 17, 1705 (2005). 
[63] (a) http://environmentalchemistry.com/yogi/periodic/;

(b) http://www.chemsoc.org/exemplarchem/entries/2001/williamson/development.html

[64] T. Kawase, H. Sirringhaus, R.H. Friend et al., "Inkjet printing of polymer thin film transistors," Thin Solid Films 438, 279 (2003).

[65] Y. Wu, Y. Li, B.S. Ong, P. Liu, S. Gardner, and B. Chiang, "High-Performance Organic Thin-Film Transistors with Solution-Printed Gold Contacts," Adv. Mater. 17, 184 (2005).

[66] S. Gamerith, A. Klug, H. Scheiber, U. Scherf, E. Moderegger, and E.J.W. List, "Direct inkjet printing of $\mathrm{Ag}-\mathrm{Cu}$ nanoparticle and Ag-precursor based electrodes for OFET applications," Adv. Func. Mater. 17, 3111 (2007).

[67] Y.Y. Zhang, Y. Shi, F. Chen, S.G. Mhaisalkar, L.J. Li, B.S. Ong, and Y. Wu, "Poly(3,3didodecylquarterthiophene) field effect transistors with single-walled carbon nanotube based source and drain electrodes," Appl. Phys. Lett. 91, 223512 (2007).

[68] W. Wagner, H. Gleskova, J. Sturn and Z. Suo, "Novel Processing Technology for Macroelectronics," Chapter 5 from R. A. Street (Ed.), Technology and Applications of Amorphous Silicon, pp.222-251, Germany (2000).

[69] J.N. Bardsley, "International OLED Technology Roadmap," IEEE J. Sel. Top. Quantum Electronics 10(1), 3, (2004).

[70] D. Marculescu, R. Marculescu, S. Park, and S. Jayaraman, "Ready to wear," IEEE Spectr. 40, 28 (2003).

[71] "Flat Panel Displays," Symmorphix Inc., (2004). www.symmorphix.com/index.asp?pgid=16

[72] X. Lu, Z. Xie, M.S.A. Abdou, M.J. Deen, S. Holdcroft, "Studies of polymer-based field effect transistors," IEEE Canadian Conference on Electrical and Computer Engineering 2, 814 (1993).

[73] Y. Qiu, Y. Hu, G. Dong, L. Wang, J. Xie, and Y. Ma, " $\mathrm{H}_{2} \mathrm{O}$ effect on the stability of organic thin-film field-effect transistors," Appl. Phys. Lett. 83, 1644 (2003).

[74] J.H. Lee, G.H. Kim, S.H. Kim, S. C. Lim, Y.S. Yang, J. Oh, J.H. Youk, J. Jang, and T. Zyung "The novel encapsulation method for organic thin-film transistors," Current Appl. Phys. 5, 348 (2005).

[75] W.J. Kim, W.H. Koo, S.J. Jo, C.S. Kim, H.K. Baik, J. Lee and S. Im, "Passivation effects on the stability of pentacene thin-film transistors with $\mathrm{SnO}_{2}$ prepared by ion-beam-assisted deposition,” J. Vac. Sci. Technol. B 23(6), 2357 (2005).

[76] (a) http://www.conformal-coating.com/parylene_coating.htm.

(b) http://www.scscookson.com/parylene_knowledge/specifications.cfm 


\section{Chapter 3}

\section{OTFT Integration Strategies}

As described in the previous chapter, the availability of high performance organic semiconductor material systems is, undeniably, a principal ingredient for successful realization/implementation of organic electronics. Of similar importance is the establishment of robust and compatible fabrication infrastructure that can capitalize on these material systems for practical applications. Without a dependable fabrication process and integration strategy, researchers cannot reliably reproduce OTFT devices/circuits for proper characterization studies; as a result, advancement, maturation and deployment of OTFT technology would be hindered.

Development of robust processing schemes for manufacturing of OTFT circuits is a challenge due to the sensitivity of organic electronic materials to standard microelectronic fabrication methods and material compatibility limitations/issues. The device layout, architecture, and material selection have a strong bearing on the choice of processing techniques and the overall construct of the fabrication procedure. This chapter explores various aspects of OTFT fabrication. Development of OTFT fabrication strategies constitutes a major part of this thesis work; the technological challenges involved are addressed in Section 3.1. Deposition methods and patterning techniques for organic semiconductor materials are described in Section 3.2. The OTFT integration strategies transpired from this thesis research are presented in Section 3.3. To take advantage of the solutionprocessability of organic materials, this research focuses primarily on OTFTs based on soluble organic semiconductors. For a list of device materials used, please refer to Table 2.3. 


\subsection{Technological Challenge in OTFT Integration}

Although the OTFT device structures (as presented in the Chapter 2) appear relatively straight forward, implementation and fabrication of these devices can be difficult. One of the major challenges when developing an OTFT fabrication process is to address sensitivity of the functional organic layers. More specifically, the intricacy lies in formulating compatible methods to pattern the organic semiconductor layer in order to enable fully integratable OTFT circuits and systems. In addition, compatibility between various device material layers, to ensure minimum chemical interaction and process-induced material degradation, must not be overlooked.

Most of the early demonstrations of OTFT devices used a continuous/unpatterned layer of organic semiconductor [1][2]; this device configuration is predisposed to higher leakages and parasitic than silicon MOSFET devices. Figure 3.1 illustrates the structural difference between an OTFT and a silicon n-channel MOSFET (NMOS). MOSFET has lower leakages and lower offcurrents than OTFT owing to the presence of a depletion layer. In the case of an n-channel MOSFET (NMOS), a sufficiently high positive $V_{\mathrm{G}}$ results in the formation of an inversion layer at the dielectric-semiconductor interface. This inversion layer provides a conducting channel between the source and the drain, which turns the device on. One of the main advantages of the MOSFET structure is that the depletion region between the p-type substrate and the n-type channel provides isolation from other devices fabricated on the same substrate; this effectively suppresses parasitic leakage and cross-talk between individual transistors. Moreover, the $\mathrm{n}+$ regions below the source/drain contacts form reverse-biased $\mathrm{p}$-n junctions with the $\mathrm{p}$-type substrate. These reversebiased junctions are responsible for impeding leakage and generating very low off-currents.

In contrast, an OTFT operates by accumulation, and there is no reverse-biased junction to isolate the source and the drain when the device is turned off. Consequently, a leakage current always flows between source and drain through the semiconductor bulk, and thus, OTFTs are liable to higher off-currents and parasitic leakages than MOSFETs. Moreover, since many existing OTFT fabrication processes do not incorporate local isolation between adjacent OTFT devices, lateral electrical cross-talk is an issue for OTFTs fabricated on the same substrate. 


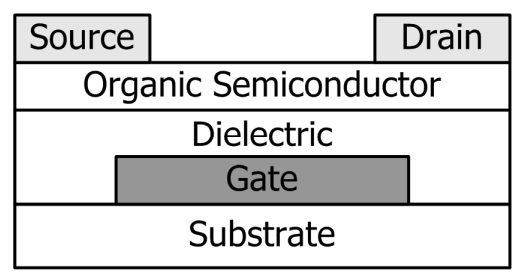

(a) Top Contact OTFT

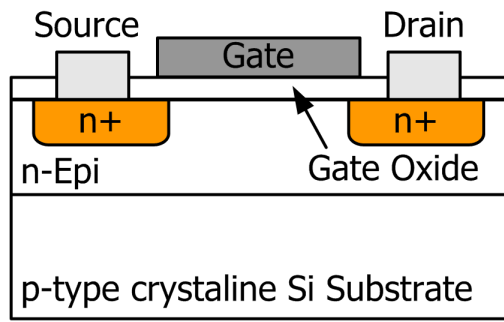

(b) Silicon NMOS

Figure 3.1. Cross sectional diagram of (a) top-contact OTFT and (b) silicon n-type MOSFET (NMOS).

To improve the reliability of OTFTs, minimize parasitic leakage and reduce cross-talk, it is crucial to develop appropriate isolation schemes and patterning techniques for the organic semiconductor. The semiconductor should be confined to the active channel region to isolate each transistor from neighboring devices. There are three key benefits to patterning the semiconductor: reduction of cross-talk, improvement of the on/off ratio, and removal of material from the optical path (as in the case of a backlit display, the backplane should be as clear as possible for maximum brightness) [3]. By removing semiconductor from the non-active transistor area, non-gated current carrying pathways (which contribute to a constant leakage current) can be removed; this decreases the off-current while making no significant changes to the on-current. Our experiments also showed that isolating the device via patterning of the organic semiconductor layer led to a reduction in gate leakage current. This suggests a possible contribution of gate leakage from the interaction between organic semiconductor (impurities or solvents) and gate dielectric; more research is needed to clarify this speculation. In addition, a means to integrate reverse-biased $p-n$ junctions in OTFT would be ideal, to permit further reduction in leakages and parasitic.

Accordingly, patterning of the organic semiconductor layer is important from a device performance perspective as well as an integrated circuit (IC) fabrication perspective. Unfortunately, developing compatible methods to achieve patterned organic films has been a major bottleneck. Most organic films, particularly soluble polymers, are very sensitive to solvents and chemical attacks, so they are typically not amenable to conventional photoresist-based photolithographic patterning approaches (e.g., photoresist coating, developing, film etching, and photoresist removal). In addition, the structure of organic materials is generally optimal at deposition and most posttreatments tend to degrade the material properties [3]. Exposure to liquid solvents, perhaps during a wet etching/patterning process, can degrade the charge transport properties of organic thin films. 
Therefore, most types of wet processing are typically unsuitable for patterning organic semiconductor materials.

The most desirable method for patterning the organic layer involves direct printing of the active materials, in which deposition and patterning occurs simultaneously in one single step, eliminating any etching steps. For example, in inkjet printing, the functional organic material is printed only in the desired area, thus reducing material cost/ waste and eliminating the need for subsequent patterning. Printing tools are the subject of extensive research and development, and factors such as resolution, printing speed, etc. need to be refined before large-scale manufacturing can occur [4][5]. An all-printed fabrication process, hence the term "printed electronics", is envisioned for the organic electronics industry in the long term.

Shadow masks also permit generation of patterned organic layers; however, this approach is limited to vacuum-deposited organic materials and is not applicable to solution-processed polymers. Other emerging patterning processes for organic semiconductor include laser ablation, selective deposition by substrate treatment, mold printing/patterning, microcontact printing, and soft lithography techniques [4][6][7][8][9][10].

For the current OTFT research work, we developed an organic-compatible photolithography fabrication approach for patterning the organic semiconductor layer, which involved investigating a number of passivation/masking materials and strategically tailoring the patterning steps to ensure minimum process-induced damage to the organic layer. A more versatile/pliable approach based on a hybrid photolithography-inkjet printing fabrication scheme was also conceived/formulated to enable robust and direct patterning of the organic semiconductor layer. Before presenting the details of these OTFT integration strategies in Section 3.3, Section 3.2 provides an overview of various patterning techniques.

\subsection{Overview of Processing and Fabrication Techniques}

To produce electronic devices, existence of a foolproof/impeccable fabrication process is as critically important as avaliability of high-performance materials. With regards to the OTFT fabrication schemes developed in this thesis (see Section 3.3), the device processing steps can be grouped into four categories: deposition, patterning, etching/removal, and interface/surface modification. 
- Deposition refers to depositing/coating a thin film of material onto the substrate. For this research, relatively standard thin-film deposition techniques are used [11][12], including plasma enhanced chemical vapor deposition (PECVD) for gate dielectric layer, sputtering and thermal evaporation for electrode layer, spin-coating or inkjet printing for solutionprocessed organic semiconductor layer, and chemical vapor deposition for polymer passivation layer.

- Patterning refers to shaping or altering the existing shape of the deposited materials, or directly depositing materials into a selected region to form a film with patterned structures. Three patterning approaches are considered: shadow mask, photolithography, and inkjet printing.

- Etching/Removal refers to removing material in selective regions from the wafer. Once an image is transferred from a mask to a wafer, one has to remove or etch material from selected regions to form the final device. Specially designed wet or dry etching processes allow selective removal of materials once the resist has been patterned. For the photolithography fabrication schemes outlined in Section 3.3, inorganic device layers are patterned by wet-etching. Organic layers are dry-etched in oxygen plasma to avoid chemical/solvent interactions between wet-etchants and organic device layers. Metal source/drain contacts are patterned by lift-off processes.

- Interface/surface modification refers to functionalizing the device interfaces for performance enhancements. For OTFTs, and other transistor technologies alike, the interfaces between device layers play a significant role in establishing functionality and performance. Interface modification has been a vital step for OTFT fabrication; it improves interfacial interaction and controls the quality/ordering/structure of subsequently organic deposited layers. Modifications are typically done on the dielectric surface and contact surface prior to deposition of the organic semiconductor layer, in the case of bottom-gate bottom-contact devices. Chapter 5 and 6 are dedicated to interface treatment and analysis.

Table 3.1 summarizes the deposition and patterning techniques used to process the various device layers for the OTFT fabrication schemes developed in this thesis research. Particulars of the processing sequence are laid out in Section 3.3. 
Table 3.1. Deposition and patterning techniques employed for OTFT fabrication in this research.

\begin{tabular}{|c|c|c|}
\hline Device Layer & Deposition Techniques & Patterning Techniques \\
\hline Gate Metal (Mo, Al) & Sputtering & $\begin{array}{l}\text { Photolithography, wet etching } \\
\text { with PAN55 }\end{array}$ \\
\hline $\begin{array}{l}\text { Gate Dielectric } \\
\text { (silicon nitride }\left(\mathrm{SiN}_{\mathrm{x}}\right) \text {, } \\
\left.\text { silicon oxide }\left(\mathrm{SiO}_{\mathrm{x}}\right)\right)\end{array}$ & $\begin{array}{l}\text { Plasma enhanced chemical } \\
\text { vapor deposition (PECVD) }\end{array}$ & $\begin{array}{l}\text { Photolithography, wet etching } \\
\text { with BHF6 }\end{array}$ \\
\hline $\begin{array}{l}\text { Source/Drain Contacts } \\
(\mathrm{Au}, \mathrm{Cr})\end{array}$ & Thermal evaporation & $\begin{array}{l}\text { 1) Photolithography and lift-off } \\
\text { 2) Shadow mask }\end{array}$ \\
\hline $\begin{array}{l}\text { Organic semiconductor } \\
\text { (PQT-12, P3HT, PBTTT) }\end{array}$ & $\begin{array}{l}\text { 1) Spin-coating } \\
\text { 2) Inkjet printing }\end{array}$ & $\begin{array}{l}\text { 1) Photolithography, dry } \\
\text { etching with } \mathrm{O}_{2} \text { plasma } \\
\text { 2) Inkjet Printing }\end{array}$ \\
\hline $\begin{array}{l}\text { Passivation or capping } \\
\text { layers }\left(\mathrm{SiN}_{\mathrm{x}} \text {, parylene) }\right.\end{array}$ & $\begin{array}{l}\text { 1) SiNx: PECVD } \\
\text { 2) Parylene: vapor deposition }\end{array}$ & Photolithography, dry etching \\
\hline
\end{tabular}

One of the key challenges in OTFT fabrication lies in patterning the device layers. Due to sensitivity of most organic thin films to chemicals/solvents used in standard photolithographic processes, careful/special considerations must be given when developing a compatible fabrication process for organic electronic devices. During the early stages of OTFT research, where material development was the main driving force, most researchers adapted a very simple bottom-gate OTFT device configuration ${ }^{7}$ with a single-patterning step done on source/drain contacts and a continuous organic semiconductor layer (see Figure 3.2). This procedure delivers a very simple and convenient platform to prototype devices and evalute different organic material systems, and circumvents complications associated with device patterning. For preliminary material characterization, an unpatterned semiconductor layer in single/discrete transistor configuration would suffice since the key interests are device mobility and interface properties. However, this approach is inadequate for creating highly integrated devices and robust circuits. Functional circuitries require individually-addressable gate electrodes, via holes through dielectric layer to make electrical interconnection between different metal layers, and patterned semiconductor layer to define transistor's active region. Therefore, patterning of the organic semiconductor active layer is crucial to fulfill various performance, functionality, and integration objectives:

\footnotetext{
${ }^{5} \mathrm{PAN}$ is a mixture of phosphoric acid $\left(\mathrm{H}_{3} \mathrm{PO}_{4}\right)$, acetic acid $\left(\mathrm{H}_{4} \mathrm{C}_{2} \mathrm{O}_{2}\right)$ and nitric acid $\left(\mathrm{HNO}_{3}\right)$, for etching metallization (e.g., $\mathrm{Al}$ )

${ }^{6} \mathrm{BHF}$ is hydrofluoric (HF) acid buffered with $\mathrm{NH}_{4} \mathrm{~F}$, for etching silicon oxide or silicon nitride

${ }^{7}$ The device configuration is a bottom-gate bottom-contact OTFT using a highly doped silicon (Si) substrate as a bottom gate, a continuous layer of gate dielectric, defining source/drain electrodes through a shadow mask or by lithographic means, and depositing a continuous organic semiconductor film by spin-coating or vacuum evaporation.
} 
- Patterning the active layer reduces cross-talk between adjacent devices, reduces parasitic resistance, and limits leakage current (off-current). As a result, the devices have better performance characteristics (e.g., smaller $I_{\mathrm{OFF}}$ and higher $I_{\mathrm{ON}} / I_{\mathrm{OFF}}$ ratio for OTFTs).

- Patterning is more important when OTFTs share a common gate, in which case the leakage through the gate dielectric may become significant.

- For circuit implementation, patterning the semiconductor layer is even more critical because transistors must be isolated from one another and have individually addressable gates to achieve circuit functionality.

- From an integration standpoint, a patterned polymer layer is required in applications such as color or backlit polymer/organic light emitting diode displays, where the OTFT pixel circuit should only consume a small footprint to get high-fill factor AMOLED system with OTFT backplane.

Three patterning techniques are considered in this thesis. The first is the shadow-mask technique, which provides a simple method to form patterns of the source and drain contacts for OTFTs. The second approach is the photolithography technique, which enables precise device definition and higher integration complexities. Lastly, inkjet printing enables direct deposition of patterned layers, which is envisioned to be an important work-horse for future generations of plastic or printed electronics. Each of these techniques are studied in greater detail in this section. The focus of this section is primarily on deposition (Section 3.2.1) and patterning (Section 3.2.2 to Section 3.2.4) techniques for organic semiconductor materials. On the other hand, the technologies used for processing metals and silicon based inorganic semiconductor/dielectric materials are relatively standard; please consult the appropriate references for details [11][12].

\subsubsection{Deposition Methods for Organic Semiconductor}

The two most common deposition methods for organic materials are vacuum evaporation and solution processing techniques (e.g., spin-coating, printing). These two methods are briefly discussed in this section. 


\subsubsection{Vacuum Evaporation}

Small-molecules and oligomer organic semiconductors are typically deposited by vacuum evaporation, which consists of heating the material under reduced pressure. The process is conducted in a very high or ultra-high vacuum chamber. The organic material is put into a metal boat, which is heated by Joule effects or with an electron gun, and the substrate is placed a few centimeters above the boat [1]. This technique is usually not applicable for polymers, because polymers tend to decompose by cracking at high temperatures. The main advantages of vacuum evaporation are the easy control of the thickness and purity of the deposited film, and the ability to realize highly ordered films by monitoring the deposition rate and the substrate temperature [1]. Patterned films can be realized by evaporation thru a shadow mask. The primary drawback is the need for sophisticated vacuum-based instrumentation, which is in contrast to the simplicity of spincoating or other solution-processing techniques.

\subsubsection{Solution-Processed Deposition}

Various techniques are available for processing soluble polymer semiconductor films from the liquid phase, which include spin-coating, casting, printing, and soluble precursor conversion. One of the most simple and effective ways to realize a nice polymer film is by spin-coating. When the technique is well handled, it allows the production of very homogeneous films with precise control of their thickness over relatively large areas. Control parameters of a spin-coating process include spin-coating speed, ramping rate, choice of solvent of the polymer solution, drying temperature, drying time, etc; these parameters need to be optimized to achieve film of desired thickness and uniformity. Spin-coating is the principal technique employed to deposit organic semiconductor in this thesis research. The spin-coating environment affects performance of air-sensitive organic materials. For instance, for extremely air-sensitive n-type organic materials, such as PCBM or $\mathrm{C}_{60}$ $[1][14][15]$, device processing and measurements must be done in inert environment (e.g., nitrogen) in order to attain functioning OTFTs with good mobility. Fortunately, PQT-12 polymer semiconductor is relatively stable in air; thus, spin-coating of PQT-12 can be performed in ambient conditions for the experiments conducted in this thesis.

Spin-coating requires polymers with good solubility. Solubility of conjugated polymers can be tuned by grafting solubilizing groups to the polymer backbone. An alternative approach is to use a soluble precursor polymer, which can undergo subsequent chemical reactions to convert the material to the desired conjugate oligomer or polymer. The precursor method has been applied for 
the preparation of pentacene [16] and polythienylenevinylene (PTV) films [13]. This precursor route technique typically produces polymer films with lower mobilities than evaporated films, due to a higher degree of molecular disorder in the film [13]. In general, solution-based processing techniques are more pertinent to polymers than small-molecules. Polymers have higher viscosity, which is essential to obtain uniform and sufficiently thick films. However, the mobility of polymer semiconductors is inferior to that of small-molecule semiconductors due to the more random orientation of molecular units or the relatively short conjugation length in polymer back bones [17].

A limitation of the spin-coating is the lack of patterning capability. Spin-coating forms a continuous film across the substrate. This shortcoming can be circumvented by direct printing techniques, which is discussed in Section 3.2.4. Alternative solution-based processing/patterning methods, including microcontact printing, screen printing, and nanoimprinting, also demonstrate excellent potential for organic electronics fabrication.

\subsubsection{Patterning by Shadow Mask}

Patterning by depositing vaporized materials through shadow masks is one of the earliest and simplest routes for making OLEDs and OTFTs. In this process, metals or low molecular weight organic molecules are evaporated from a source in a physical vapor deposition system and travel through openings in masks placed near the surface of the substrate. The deposition typically occurs under high vacuum $\left(10^{-8}-10^{-6}\right.$ Torr $)$ such that the mean free path of the evaporated species exceeds the distance between the source and substrate [18]. When this condition is satisfied, the evaporated material travels in a directional manner through the gaps in the mask and onto the substrate [12]. This technique is additive at the substrate level, which enables sequential deposition of multiple layers of different materials.

The shadow mask technique is attractive for OTFT fabrication because it can guarantee a simple, robust and low cost source/drain contact definition process without damage to underlying organic materials if present. A shadow mask can also provide a constructive tool to deposit patterned lowmolecular weight organic films, to define the active semiconductor of the OTFT. Since this thesis focuses on solution-processible polymer semiconductors, shadow mask is used only to define source/drain contacts.

Figure 3.2 displays the fabrication sequence of bottom-gate OTFT patterned by the shadowmask approach in this research work. Preliminary samples are fabricated on thermally oxidized 
silicon wafers, where the $\mathrm{SiO}_{2}$ serves as the gate dielectric and the highly doped $\mathrm{Si}$ substrate serves as the gate electrode. Other gate dielectrics tested include PECVD silicon nitride and PECVD silicon oxide. The dielectric surface is pre-treated with OTS SAM, to promote proper alignment of the polymer semiconductor molecules and to improve the quality of the dielectric-semiconductor interface. Following the deposition of the polymer semiconductor layer, the source/drain contact metal (typically gold) is deposited through a (stainless steel) shadow mask to define the transistor geometries for top-contact structures (see Figure 3.2(a)). A similar process can be applied to fabricate bottom-contact OTFTs (see Figure 3.2(b), but with the source/drain electrodes deposited directly on top of the dielectric surface and followed by spin-coating of the polymer semiconductor layer as the last step. Discrete OTFTs with different $W$ and $L$ have been fabricated using this shadow mask approach.

(1)

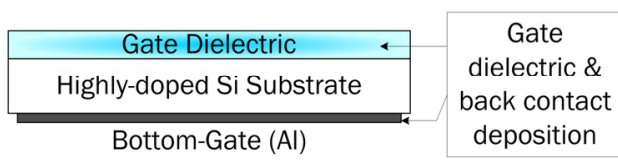

(2)

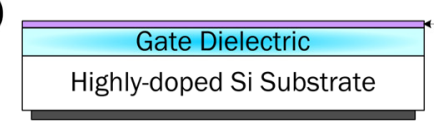

(3)

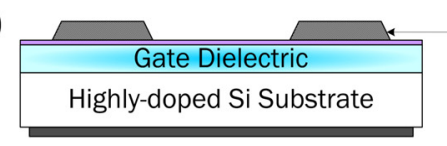

(4)

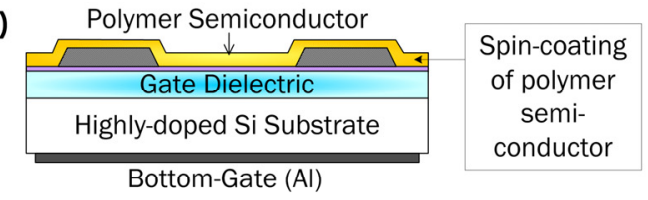

(a) Bottom-Contact OTFT
(1)

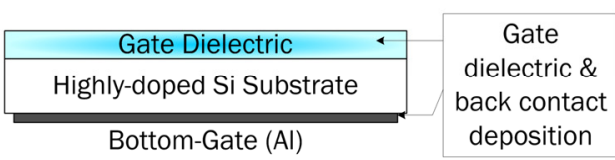

(2)

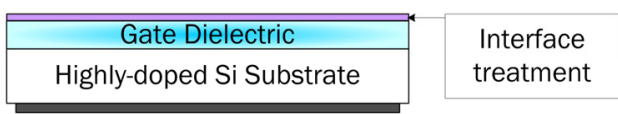

(3)

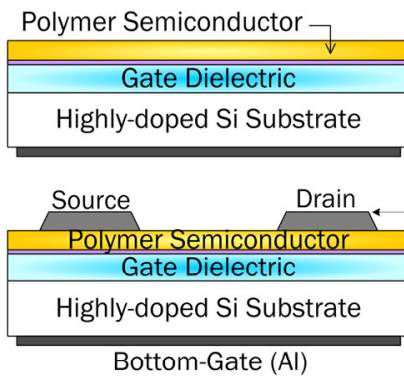

Spin-coating of polymer semiconductor S/D metal deposition via shadow mask

Figure 3.2. Fabrication sequence of bottom-gate OTFT by the shadow mask patterning approaches, for (a) bottom-contact and (b) top-contact configuration.

As demonstrated above, shadow mask fabrication process is quite simple. It provides a convenient approach to produce discrete OTFTs for prototyping and device characterization purposes. However, the above-described scheme has one major setback: it is not well-suited for fabrication of circuits with high complexity due to its inability to perform gate patterning. For the approach outlined in Figure 3.2, all transistors on the substrate share a common gate. To achieve proper circuit functionality, individually-addressable gates are required to drive/bias individual 
transistors in a circuit. Moreover, an unpatterned gate electrode results in considerable overlap/parasitic capacitances, which limit the circuit's speed performance. Figure 3.3 illustrates a more practical device configuration, with individual gates for each transistor. To extend the capability of the shadow mask process for circuit fabrication, several adaptations are necessary, including:

- Developing a method for patterning the gate electrodes, in order to realize circuits;

- Developing a method for patterning the polymer semiconductor layer, in order to reduce parasitic leakages; and

- Establishing a method to allow accurate alignment (e.g., fiducial marks) of the various masks/layers.

Multilevel registration with shadow masks has been reported by the use of physical mounting brackets, mask translating fixture and alignment systems [19]. Multiple organic light-emitting layers and electrodes can be patterned sequentially by successive shadow masks, and several companies have demonstrated organic devices, such as full-color OLED displays (15 inch active matrix OLED by Kodak-Sanyo, 13 inch active matrix OLED by Sony, Pictiva OLED display by Osram, etc) using this shadow mask approach [20][21]. However, the processing demands are more stringent for fabrication of OTFT circuitries, thus parameters such as multi-layer registration, alignment, and feature resolution still pose significant challenges for the shadow mask approach.

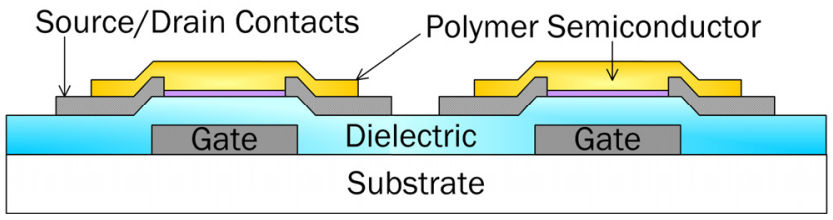

Figure 3.3. Illustration of individually addressable gates on the same substrate, which is a basic requirement to enable circuit implementation.

Another shortcoming of the shadow mask approach is its limited resolution compared to other patterning techniques. Conventional metal shadow masks, fabricated by photochemical machining or laser beam machining, have inherent limits on the process resolution and aspect ratio. The resolution of such masks is typically in the range of $\sim 50 \mu \mathrm{m}$, and must be in the same order as the mask thickness [22]. Practical resolution limits are set by (i) sizes of openings that can be generated in masks that retain sufficient rigidity (i.e., thickness) to be mechanical stable, (ii) mask-tosubstrate separation distances that can be reproducibly achieved without unwanted physical 
contact, and (iii) levels of directionality in the material flux. New technology to fabricate higher resolution shadow masks (since smaller $L$ is preferred for OTFTs) and/or adoption of alternate mask materials are needed. One research group reported a new shadow mask fabrication procedure, combining micro-electro-discharge machining (micro-EDM) and electrochemical etching (ECE), to produce high-aspect-ratio and high-resolution stainless steel shadow mask [23]. OTFT with $W=$ $150 \mu \mathrm{m}$ and $L=3.6 \mu \mathrm{m}$ was achievable with this new shadow mask. Novel use of a set of polymeric shadow masks to fabricate pentacene-based organic circuits was also demonstrated [24].

Although alternative patterning technologies (e.g., high-resolution rubber stamping, microcontact printing, soft lithography) can offer resolution better than $10 \mu \mathrm{m}$, each of these methods has fundamental drawbacks, including more complicated process steps, limited manufacturability for large area applications, and brittleness of the mask [23]. Therefore, by developing higher resolution shadow masks with precise multi-layer alignment capability, shadow masks may be adaptable for OTFT circuit fabrication and can potentially compete with other patterning technologies.

\subsubsection{Patterning by Photolithography}

Even though the industry forecasts printing technologies/processes to be a key manufacturing standard for organic electronics, there are a number of applications in organic electronics that can benefit from a robust photolithography process at this time. Photolithography is a well-established method commonly used in the semiconductor industry today. It enables circuit integration, through patterning and etching the multiple device layers, and creating vias and interconnects with modest alignment tolerances. Photolithography facilitates device fabrication that incorporates more than one function, for instance, integrating capacitors, transistors and diodes in the same device flow. Ability to implement higher resolution devices, as well as complex OTFT structures and integrated circuits, are other advantages of photolithography compared to shadow masking process. The reported development of a large area, high resolution lithography system with high volume, roll-toroll production capability exemplifies the potential and practicality of lithography-based patterning approach for organic electronics [5]. Our primary motivation for using photolithography is to provide a more immediate fabrication solution for organic electronics, as well as to bridge the technological transfer and development to an all-printed process for the near future. The basic

photolithography process is reviewed in Section 3.2.3.1. The challenges/concerns related to 
adaptation of photolithography process for organic electronics are considered in Section 3.2.3.2. The photolithography approaches developed in this research for OTFT fabrication are presented in Section 3.3.

\subsubsection{Photolithography Basics}

Photolithography is a process used in microfabrication to selectively remove parts of a thin film (or the bulk of a substrate). It uses light to transfer a geometric pattern from a photomask to a lightsensitive chemical (photoresist) on the substrate. A series of chemical treatments then engraves the exposure pattern into the material underneath the photoresist. The basic photolithography process steps are outlined in Figure 3.4. It typically begins with coating the substrate with photoresist, whose properties can be modified by exposure to ultraviolet (UV) light. UV light is passed through a photomask that manipulates the phase and/or amplitude to achieve patterend exposure on photoresist layer. The photomask can be in direct contact with (contact mode) or in proximity to (proximity mode) the photosensitive material. Alternatively, imaging optics can magnify or demagnify the mask image and project it onto the substrate (projection mode). UV exposure changes the chemical structure/solubility of the photoresist in selected region. Subsequent development of photoresist (in a developer solution) remove/dissolve the more soluble regions, leaving behind a patterned layer of photoresist. This patterned photoresist acts as an etch mask for the underlying film, where areas uncovered by photoresist are removed by wet- or dry-etching processes. Finally, photoresist is stripped and the image from photomask is replicated on the film/substrate.

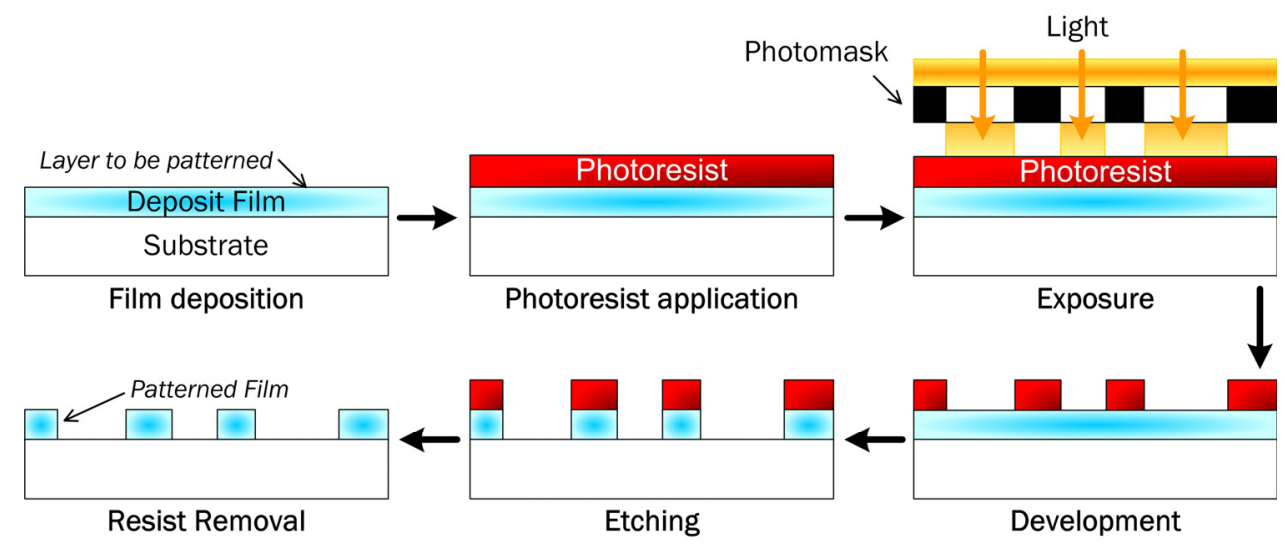

Figure 3.4. Basic photolithography process: the exposure and etching process that allows one to transfer a pattern to the film/wafer. 


\subsubsection{Photolithography Considerations for OTFTs}

Photolithography is the industry's workhorse for manufacturing of inorganic microelectronics and optoelectronics. The high speed, parallel patterning capability and high resolution features of (projection mode) photolithography also make it attractive for applications for organic devices. However, straightforward implementations can be difficult due to (i) the incompatibility of photoresists, solvents, developers, and UV light exposure with many organic active materials, (ii) challenges in resolution and registration caused by rough, and often dimensionally unstable, plastic substrates, and (iii) cumbersome implementations needed for large-area patterning [18]. Despite these challenges, photolithography still offers many attractive advanages for organic circuit integration, provided that we can meticulously and strategically design the processing sequence to avoid process-induced degradation of device properties. One possible consideration is to execute majority of the photolithographic steps prior to deposition of the active organic layers; however, this approach would limit device structures to bottom-gate bottom-contact configuration, as the one shown in Figure 2.16(c).

Alternatively, a more versatile strategy is to incorporate a passivating/capping layer (e.g., $\mathrm{SiN}_{\mathrm{x}}$, $\mathrm{AlO}_{\mathrm{x}}$, parylene, PVA) on top of active organic semiconductor to provide protection against chemical attacks during photlithography processing and from deposition of subsequent device layers. This concept is illustrated in Figure 3.5. Benefits of this approach are several-folds: enabling post-deposition patterning of the organic layer to realize a greater variety of OTFT architectures, incorporating passivation for the organic active layer, and more importantly, generating fully-patterned device for implementation of integrated circuits. Inorganic capping layers such as $\mathrm{SiO}_{2}, \mathrm{SiN}_{\mathrm{x}}, \mathrm{AlO}_{\mathrm{x}}$ have been used. Since these films have low permeability against solvents, oxygen, and moisture, they serve as excellent barriers to protect the underlying organic semiconductors from solvent processing. However, deposition of these inorganic barrier films may induce damage to the organic layer (e.g., thermal heating during evaporation, plasma exposure during PECVD, or physical damage from energetic ions during sputtering) [1][25][26][27]. These concerns can be eliminated with the use of organic-based capping layers (e.g., parylene, PVA) with better processing compatibility with the underlying organic semiconductor layer. Parylene is a vapor deposited polymer, deposited at room temperature. It is widely used as a tough and conformal coating, and is one of materials used in our process [28]. Another organic capping material is water soluble polyvinylalcohol (PVA). A concern with organic-based passivation films is their higher permeability to oxygen, moisture and solvents, when compared to inorganic 
candidates. A more comprehensive solution is delivered by a stacked inorganic-organic capping layer structure, where an inorganic barrier is deposited on top of the organic buffer. The inorganic barrier can effectively prevent diffusion of chemical/solvent into the organic semiconductor layer, while the organic buffer can minimize process-induced damage to the active organic layer.

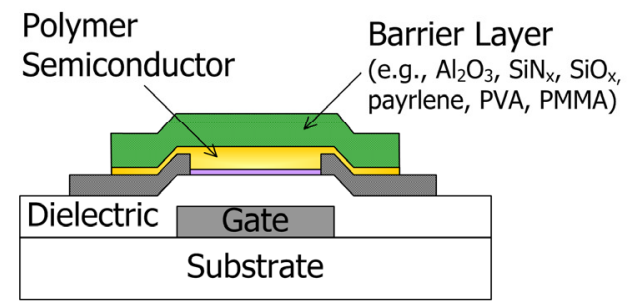

(a) Single Capping Layer

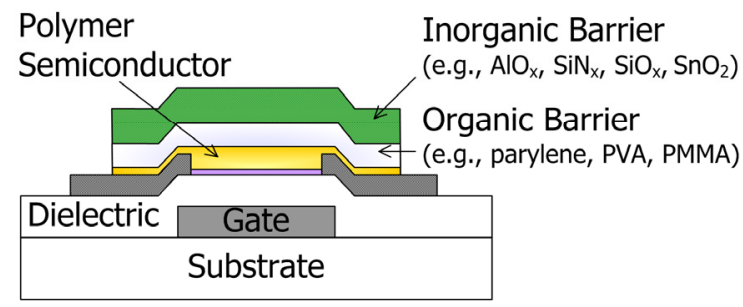

(b) Stacked Capping Layer

Figure 3.5. Cross section illustration of OTFTs with (a) single capping layer, and (b) stacked inorganic-organic capping layers.

Photolithography patterning can be executed on the capping layers to define the active channel region. The capping layers then serve as an etch mask to etch the uncovered regions of the underlying organic semiconductor layer. Oxygen plasma generated by a reactive ion etcher (RIE) is used to etch organic-based films in this research; dry etching is preferable over wet etching because it provides improved control and anisotropy.

This photolithography approach with capping layer has been successful applied for the fabrication of OTFTs and circuits (please refer to results in Chapter 7) [25][26][27]. However, reduced device performance is typically observed. Jia et al. studied the source of degradation for their P3HT OTFT with parylene $/ \mathrm{Al}_{2} \mathrm{O}_{3}$ capping layers [5]. For their process, the degradation occurred primarily during the atomic layer deposition (ALD) of $\mathrm{Al}_{2} \mathrm{O}_{3}$ and etching of capping layer. There was $30 \%$ degradation in mobility, a nearly two times reduction in drive current, and an increase in threshold voltage after the $\mathrm{ALD} \mathrm{Al}_{2} \mathrm{O}_{3}$ deposition. In the capping layer etching, a near $50 \%$ degradation in mobility was observed. The patterned devices displayed mobility of 0.02 $\mathrm{cm}^{2} / \mathrm{V}$-s [5]. For PQT-12 OTFT on $\mathrm{SiN}_{\mathrm{x}}$, devices patterned with parylene had mobility of 0.04 $0.12 \mathrm{~cm}^{2} / \mathrm{V}$-s, which is very comparable to unpatterned devices $0.05-0.14 \mathrm{~cm}^{2} / \mathrm{V}$-s a reduction. This shows the reliability of our process. Nonetheless, careful handling and process optimization is required to minimize any process-induced degradation using this approach.

Despite the maturity and effectiveness of the photolithography process for circuit fabrication, it is not the optimal solution for organic electronics in the long run, due to its relatively high costs 
and process complexity. Many research groups are assertively exploring innovative fabrication techniques (e.g., inkjet printing, nanoimprinting, microcontact printing) that can capitalize on the solution-processability offered by organic materials [7][8][9].

\subsubsection{Patterning by Inkjet Printing}

Inkjet printing is a precision micro-fluid dispensing technology for creating prints and images via non-contact printing onto a substrate. The inkjet printing system works by producing a fine jet of ink, called "ink-jet", through a printing nozzle of up to $50 \mu \mathrm{m}$ in diameter, and positioning these ink droplets as required on the substrate [29]. Inkjet printing (IJP), conventionally a popular consumer desktop publishing technology, is now an emerging and revolutionizing technique for fabrication of organic electronics and optical devices (e.g., OLEDs, OTFTs, organic solar cells). The rising field of "printed electronics" is receiving a great deal of attention. Plastic Logic, a Cambridge-based UK company whose core technology is in printed plastic electronics manufacturing, acquired large sums of investment in early 2007 to build the first factory to manufacture plastic electronics on a commercial scale [30]. This announcement further exemplifies the promising future of inkjet printing for electronic fabrication.

Inkjet printing, along with other solution-based processing methods (e.g., spin-coating, imprinting, stamping), can reduce fabrication costs and lead to large area, cost-effective reel-to-reel production of organic electronics. The advantages of inkjet printing technology over the photolithography process for silicon ICs include low capital investment, large area capability, elimination of high-temperature and vacuum deposition processes, compatibility with flexible substrates, ease of customization, quick cycle and turnaround times, robustness, and an environmentally friendly production process [31]. Because custom images can be generated using a software program, inkjet printing presents a low-cost and convenient method to create prototype organic devices. Figure 3.6 illustrates the simplicity of the inkjet printing fabrication process over the photolithography process, where printing combines deposition and patterning all in one step; thus, reducing processing steps and material wastages [13]. Table 3.2 summarizes the key advantages of using inkjet printing for OTFT fabrication. 


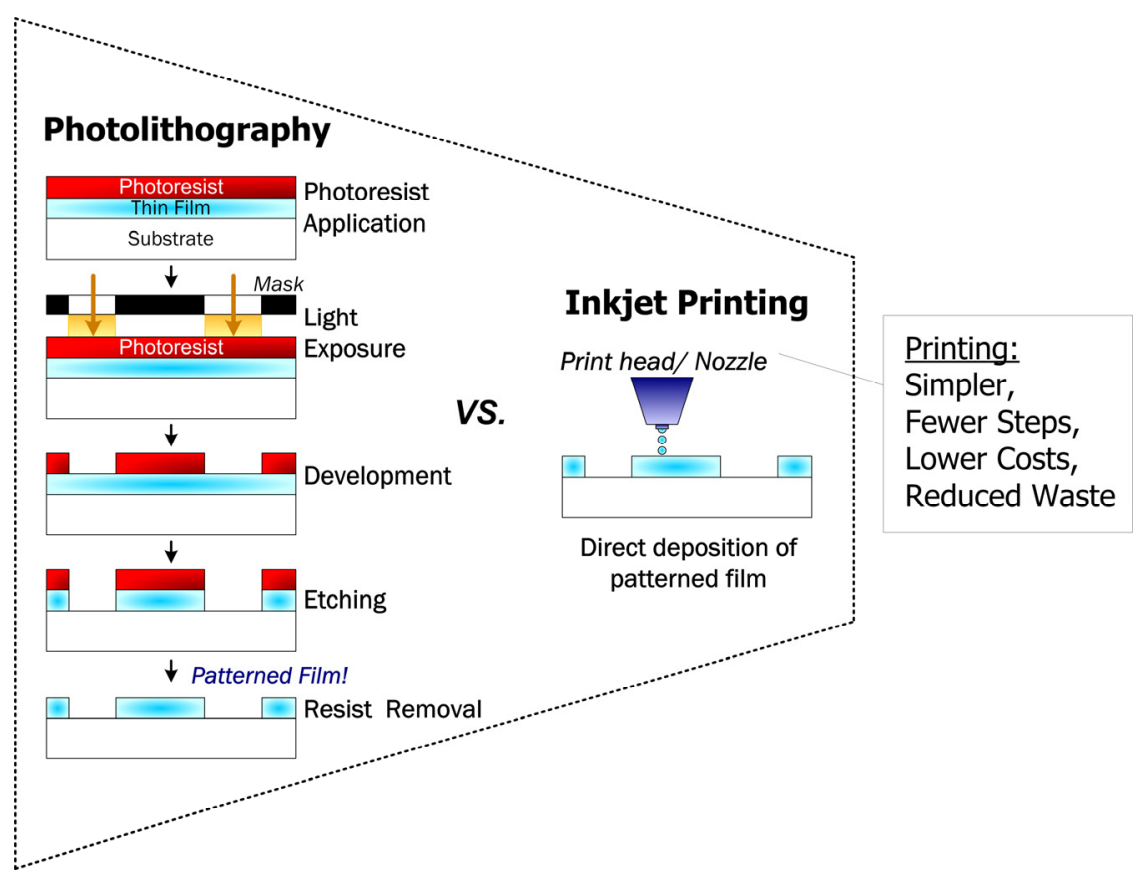

Figure 3.6. Comparison on the process complexity of photolithography and inkjet printing.

Table 3.2. Key advantages of the inkjet printing process for the fabrication of organic electronics [29][31].

\begin{tabular}{|c|c|}
\hline $\begin{array}{l}\text { Feature of } \\
\text { Inkjet Printing }\end{array}$ & Description \\
\hline $\begin{array}{l}\text { Additive } \\
\text { patterning } \\
\text { technique }\end{array}$ & $\begin{array}{l}\text { Allows simultaneous patterning and deposition of material, simplifying } \\
\text { the patterning process } \\
\text { Reduces consumption of material and energy } \\
\text { Minimal ink/chemical wastage } \\
\text { Circumvent use of chemicals such as photoresist or developer, which } \\
\text { can adversely deteriorate functional organic layers } \\
\text { Different kinds of material can be deposited by simply changing } \\
\text { solutions in an inkjet head }\end{array}$ \\
\hline $\begin{array}{l}\text { Non-contact } \\
\text { patterning } \\
\text { method }\end{array}$ & $\begin{array}{l}\text { No direct contact between substrate and equipment } \\
\text { Free from mechanical damages or contaminations to a device that } \\
\text { could arise from a direct contact of a device surface with a tool } \\
\text { Compatibility with a variety of substrates, irregular shapes or surfaces } \\
\text { Provides accurate alignment with patterns already on a substrate to } \\
\text { create multilayered devices }\end{array}$ \\
\hline $\begin{array}{l}\text { Computer- } \\
\text { controlled } \\
\text { process }\end{array}$ & $\begin{array}{ll}\text { Excellent flexibility and adaptability } \\
\text { Designs can be generated and modified quickly on the computer } \\
\text { Ideal for small volumes or complex designs } \\
\text { On-site design and printing functionality will enable new applications } \\
\text { such as personalized ICs or electronic tickets }\end{array}$ \\
\hline $\begin{array}{l}\text { Sufficiently } \\
\text { high print speed }\end{array}$ & $\begin{array}{l}\text { Ideal for high-throughput production } \\
\text { Higher print speed is achievable by increasing the jetting frequency and } \\
\text { the number of nozzles on a print head }\end{array}$ \\
\hline
\end{tabular}


Despite the many advantages, there are some challenges and limitations associated with inkjet printing organic electronics material. Because the requirements of printing electronic functions are very different from those of printing visual images, the adaptation of inkjet systems for processing organic devices will require careful considerations of many factors, including print-head performance (e.g., nozzle geometry, ink delivery, reliability, drive electronics); solvent compatibility with polymer material and its functionality (e.g., solubility, viscosity); print-head and liquid compatibility; polymer liquid-substrate interactions (e.g., surface tension vs. substrate energy); polymer solidification (e.g., adhesion, mechanical integrity, functionality); and placement accuracy [32]. More specifically, when using inkjet printing for OTFT fabrication, technological concerns pertain to film continuity, multi-layer registration/alignment, device resolution, and ink formulation. Some of these issues are explained below.

- Continuous and pinhole-free films: A feature of inkjet printing (and other non-contact printing techniques) is the utilization of discrete dots to form surface images, and the image resolution is measured by the number of dots per inch (dpi) [33]. However, this attribute can be a concern for electronic device fabrication, because the discrete dots must have electrical continuity for functional devices. Also, it must be possible to print pinhole-free layers to avoid shorting of devices. These requirements impose a challenge when designing printing systems for device fabrication.

- Printing multi-layer devices: Inkjet printing should have the capability to print multiple layers such that the various layers form discrete unmixed layers, or they mix and react to form a single material depending on the specific process goals [31].

- Higher image resolution: Typical image resolution for inkjet printing is $\sim 25 \mu \mathrm{m}$ [34]. Higher device resolution is desirable for fabricating OTFTs, because smaller channel lengths can result in higher output currents and faster switching speeds (refer to Eqns. (2.3) and (2.4)). The resolution of most direct printing techniques is often limited by difficulties in controlling the flow and spreading of the liquid inks on surfaces. One approach to overcome these resolution limitations is by "surface-energy-assisted printing", where the functional ink is deposited onto a substrate containing a predefined surface-energy pattern that is able to steer the deposited ink droplets into place. OTFTs with channel length of $500 \mathrm{~nm}$ have been demonstrated with this surface-energy-assisted inkjet printing method [35]. More discussion on the dewetting technique is provided in this section. 
- Ink formulation and printable materials: Solution-processible organic electronic material must be formulated into printable inks, with the right viscosity, surface tension, concentration, and solvent. Compatibility of the organic ink with the print-head, nozzle size (to avoid clogging), and other specifications of the printer system is also important. Ink formulation and printing parameters strongly influence the performance of printed device. Strong knowledge in material processing conditions and material properties is vital for developing a set of suitable, printable, and electrically functional materials systems for OTFT fabrication [33].

Researchers are actively addressing these technological challenges in order to develop a versatile inkjet printing technology that can gain further momentum in the field of organic or printed electronics.

\subsubsection{Inkjet Printing of OTFTs}

The relevance of inkjet printing to organic electronics is evident by vast reports in the literature. The earliest demonstrated applications is inkjet printed OLEDs using organic electroluminescent materials [31][36][37]. Investigations were also directed to inkjet printing of conducting lines as electrodes for OLEDs and OTFTs [38][39][40][35]. One of the key objectives here is to improve printing resolution for channel length reduction. A significant breakthrough was made with the introduction of a selective dewetting technique [6][35], which enabled patterning of submicron channels. There is also ongoing research focused on improving homogeneity, morphology and molecular ordering of the inkjet printed semiconductor film for OTFT fabrication [40][41][42]. In all applications, the printed media (i.e., inks) must be carefully adapted to the printing process, the print heads and the underlying structures.

Inkjet printing of OTFTs has been demonstrated by various research groups [4][6][29][39][43]. For example, Kawase et al. reported inkjet printing of a top-gate OTFT structure, using solutionprocessible polymer materials [6]. The source/drain electrodes were inkjet printed in air from an aqueous dispersion of water-soluble poly(3,4-ethylenedioxythiophene) doped with polystyrene sulfonic acid (PEDOT:PSS) on glass substrate. The active semiconductor layer was fluorenebithiophene copolymer $(\mathrm{F} 8 \mathrm{~T} 2)^{8}$ spin-coated from xylene solution. The gate insulator,

\footnotetext{
${ }^{8}$ F8T2, the abbreviation for poly(9,9-dioctylfluorene-co-bithiophene), is a nematic liquid crystalline conjugated polymer semiconductor. It can be preferentially oriented by rubbed polyimide layers.
} 
poly(vinylphenol) (PVP), was spin-coated from isopropanol solution. A gate electrode was printed on the gate dielectric to cover the channel. Different types of solvent were used to deposit those four layers to avoid dissolving or damaging the underlying layers or structures [6]. The inkjetprinted F8T2 OTFT has channel length $(L)$ of $50 \mu \mathrm{m}$ and channel width $(W)$ of $2 \mathrm{~mm}$ and demonstrated a relatively high on-off current ratio of $>10^{5}$ [6]. However, the inkjet-printed TFT has relatively low drain current $\left(I_{\mathrm{D}}\right)$, in the range of $0.4 \mu \mathrm{A}$ with $V_{\mathrm{DS}}$ and $V_{\mathrm{GS}}$ biased at $-40 \mathrm{~V}[6]$.

The channel length of $50 \mu \mathrm{m}$ may be too large to obtain sufficient drain current at a lower voltage. It is desirable to reduce $L$ to submicrometer dimensions to obtain sufficient output current at a lower voltage, since $I_{\mathrm{D}} \propto W / L$, and to allow faster switching speeds. However, device yield of the inkjet printing process is compromised by a decreasing channel length due to the fluctuation in flight direction of the inkjetted droplets [6]. In addition, inhomogeneous wettability of a substrate surface leads to irregularities in printed patterns and short circuit formation between source and drain electrodes. To suppress the uncontrolled spreading of the ink droplets, a novel surfaceenergy-assisted inkjet printing technique was demonstrated; this approach involves pre-patterning the substrate with a "dewetting pattern" of hydrophobic lines, which confines the organic ink to specific (hydrophilic) regions on the surface [34][41].

\subsubsection{Improved Resolution by Surface-Energy Assisted Inkjet Printing}

Sirringhaus and co-workers incorporated this "dewetting" prepatterning approach in their inkjetprinted OTFT [4]. The prepattern was prepared with polyimide (PI) by photolithography and by etching with $\mathrm{O}_{2}$ plasma. The surface of the patterned PI strip is hydrophobic and the surface of the etched regions is hydrophilic. The geometry of the PI strip defines the channel of the polymer TFT. When a droplet of a PEDOT aqueous dispersion (for source and drain electrodes) is inkjetted on to the etched region along the PI strip (see Figure 3.7(a)), the droplet spreads up to the edge of the PI strip and is confined in the hydrophilic etched region. The atomic force microscopy (AFM) image in Figure 3.7(b) shows that the dried PEDOT is successfully deposited up to the edge of the PI strip (or channel edge) and the spreading of droplet is effectively controlled. The success of this approach relies heavily on the wettability contrast between the two regions. Transistors with channel length as small as $2 \mu \mathrm{m}$ have been reported with this technique; the resolution is notably better than the standard inkjet process (with resolution around $25 \mu \mathrm{m}$ ). The transfer characteristics of an OTFT with $L=5 \mu \mathrm{m}$ and $W=3 \mathrm{~mm}$ exhibited an on-off switching ratio $>10^{5}$. The drain current was also higher than $10 \mu \mathrm{A}$ (with $V_{\mathrm{DS}}$ and $V_{\mathrm{GS}}$ biased at $-40 \mathrm{~V}$ ). The mobility $\left(\mu_{\mathrm{FE}}\right)$ was 
$\sim 0.02 \mathrm{~cm}^{2} / \mathrm{V}$-s, which is $2-3$ times larger than the mobility of non-prepatterned inkjet-printed polymer TFT [6]. More recently, devices with $500 \mathrm{~nm}$ channel length was demonstrated with this surface-energy-assisted inkjet printing method with stringent control over the flow of liquid ink droplets and optimization of the wetting/dewetting mechanisms [35].

One shortcoming of this prepatterning inkjet method that might limit if from full-scale manufacturing deployment is that it requires a photolithography or e-beam lithography step to create the high resolution dewetting pre-patterns. Photolithography might not be the most costeffective option for low-cost production of surface energy patterns in the long term. Other nonlithographic approaches for modifying the surface chemistry to get wettability contrast are under investigation; examples include soft lithographic stamping, embossing, and laser patterning.
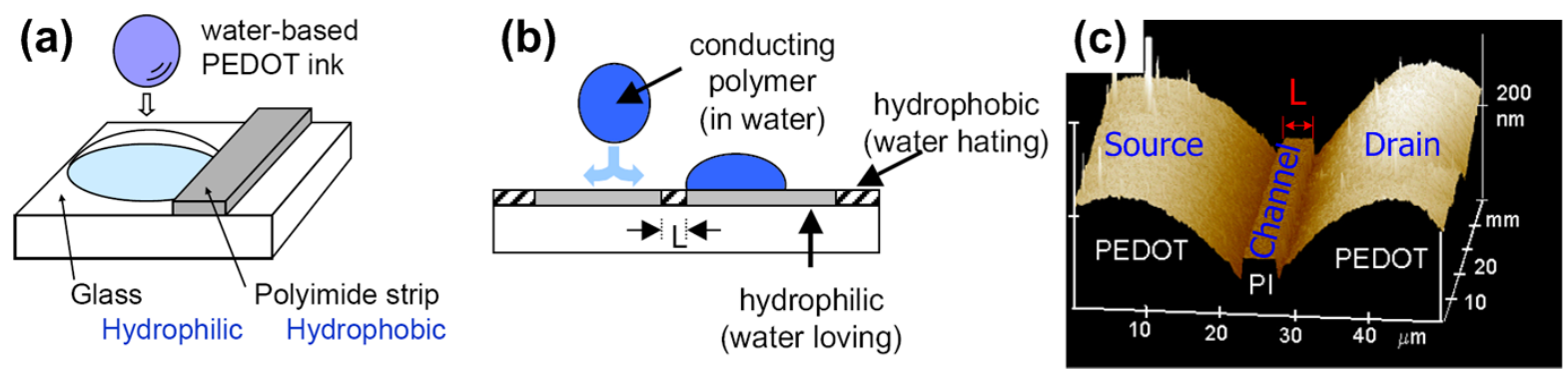

Figure 3.7. Surface-energy-assisted inkjet printing to improve image resolution. (a) Schematic view of inkjet deposition of PEDOT solution along the hydrophobic PI strip. (b) Control of ink spreading by a "dewetting pattern" of hydrophobic lines that confines the organic ink to specific hydrophilic regions on the surfaces. (c) Topographic AFM image of the transistor channel defined by PI, and the PEDOT source and drain electrodes. The width of the strip (channel length) is $5 \mu \mathrm{m}$ (adapted from [6]).

\subsubsection{Printing Peripheral Circuit: Vias and Interconnects}

Additional criteria to make inkjet printing fully adaptable for integrated circuit fabrication include the ability to form inter-layer electrical connections (i.e., implementation of vias and interconnects), and the capability to print other circuit elements including resistors, capacitors and pad electrodes. An inkjet printing process for creating via-holes based on the local dissolution of materials by inkjet deposition of a suitable solvent was demonstrated [4][6]. For example, to fabricate a via-hole through a polymer insulating layer of PVP, a droplet of isopropanol is first inkjetted at the appropriate location. The isopropanol locally dissolves the PVP, and excavates a hole that can be subsequently filled with a conducting polymer (e.g., PEDOT:PSS) to form the viahole interconnect. Resistors can also be inkjet printed by using a low conductivity polymer material 
such as PEDOT diluted with PSS. The resistance value can be adjusted over a wide range by varying the concentration of PEDOT in PSS, the length of the resistor structure, or the density of drop depositions [4]. The realization of inkjet-printed polymer TFTs, via-holes and resistors have permitted the fabrication of simple inverter circuits, which are the basic building blocks of a logic circuit. The first inkjet-printed inverters were reported by Sirringhaus et al. [4]. They fabricated an enhancement-load inverter using two p-type inkjet-printed F8T2 polymer transistors, where the drain and gate of the load transistor are connected together through a via-hole interconnect. An inkjet-printed resistance-load inverter was also demonstrated, where a printed resistor was used as the load element. The inkjet-printed polymer transistors used in these inverters are fabricated using the prepattern-dewetting approach, to attain channel lengths of $5 \mu \mathrm{m}$. Both circuit configurations demonstrated clean inverter action. These inkjet-printed inverters can be switched at frequencies up to a few hundred $\mathrm{Hz}$ [4]. Further improvements are expected with improving mobility of the polymer semiconductor and conductivity of the PEDOT electrodes, as well as decreasing the channel length and the source/drain-to-gate overlap capacitance. The performance of these inkjet-

printed all-polymer TFT circuits is believed to be adequate for applications such as active-matrix displays or identification tags [4].

\subsection{OTFT Fabrication Schemes}

The OTFT integration strategies originated from this thesis research are presented in this section. The key patterning techniques used are photolithography and inkjet printing. The processing sequence, device layout and photomask designs are varied to produce a variety of OTFT architectures.

The mask sets used for OTFT fabrication were designed to facilitate systematic characterization of OTFT's electrical behavior. These masks consist of OTFT designs with different geometries, with $W=20-6000 \mu \mathrm{m}$ and $L=5-400 \mu \mathrm{m}$ in discrete transistor or transistor array formats, are fabricated. Testing of these devices enables accurate extraction of device parameters (e.g., effective mobility, threshold voltage, contact resistance, off current), which are valuable for studying device physics and for device modeling. The masks also include simple OTFT circuits (e.g., inverters, current mirrors, source followers, ring oscillators), display pixel circuits, imaging pixel circuits, and phototransistors. Functional OTFT circuits were fabricated, which demonstrate the feasibility 
of OTFT integration using the strategies developed in this thesis. Preliminary results from circuit characterization are presented in Chapter 7.

Device fabrication was primarily conducted using PQT-12 OTFTs; please refer to Table 2.3 for a list of device materials used for this research. The 1-mask bottom-gate OTFT process was also used to fabrication solution-processed PBTTT OTFTs [44]. It also provides a convenient platform to demonstrate and evaluate solution-processed nanocomposite TFTs based on spin-coated singlewall carbon nanotubes (SWCNT), silicon nanowires (SiNW), zinc oxide ( $\mathrm{ZnO}$ ) nanowires, and zinc oxide tetrapods; this work was done in collaboration with University of Cambridge. Our hybrid photolithography-inkjet printing approach led to one of the first demonstrations of inkjet printing carbon nanotube TFTs and PQT-12/SiNW nanocomposite TFTs [45][46]. These results demonstrate the versatility of our integration strategies. The fabrication schemes and mask designs developed in this thesis can be easily extended for fabrication of TFTs based on other novel organic or nanocomposite thin-film material systems, thus providing a convenient platform for initial prototyping new devices.

\subsubsection{Basic 1-Mask Processing Scheme for Bottom-Gate OTFT}

The fabrication sequence of a bottom-gate bottom-contacts OTFT on highly doped Si substrate as gate, employing single patterning step (i.e., 1-mask process), is depicted in Figure 3.8. The source/drain contacts can be defined by shadow mask or by photolithography. Although this common-gate configuration may not deliver devices with the best overall performance (e.g., overlap capacitance will limit speed) and may not be highly compatible for circuit integration (e.g., lack of individually addressable gates), this design provides the simplest method to evaluate material systems and interfaces. Furthermore, it has the least number of processing variables and the lowest possibility of process-induced damage, thus one can focus strictly on evaluation and/or characterization studies of new material systems. This configuration and fabrication scheme serve as the basic platform for the dielectric and interface investigations reported in Chapter 4 to Chapter 6.

Initial stages of this research used a shadow mask to define the source/drain contacts. However, the image resolutions produced by evaporation through the shadow mask were poor (smallest channel that can be reliably reproduced is $90 \mu \mathrm{m}$ ). As discussed in Section 3.2.2, manufacturing high-resolution shadow masks is an industrial-wide challenge. Furthermore, the edges of the 
resulting source/drain contacts exhibited uneven (zigzag) ridges; these ridges inherited from fabrication of the actual shadow mask. Due to shadowing effect during deposition, the geometry of the deposited contacts often deviates slightly from the dimensions of the objects on the shadow mask. In contrast, photolithography provides a much cleaner, sharper, and well-controlled source/drain contact definition than the shadow-mask approach. Consequently, photolithography was the technique-of-choice for this 1-mask OTFT fabrication scheme. Bottom-gate OTFTs with photolithographically-defined bottom-contacts form the basis for the experimental work reported in Chapter 4 to Chapter 6 . One exception was the fabrication of OTFTs on plastic substrates (in Section 4.3.5), where the shadow mask was used to minimize potential damage to the thin film on flexible substrate (e.g., cracking) from handling or photolithography processing steps.

(1)

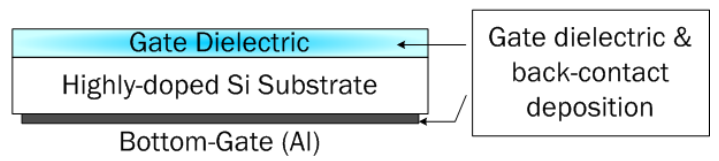

(2)

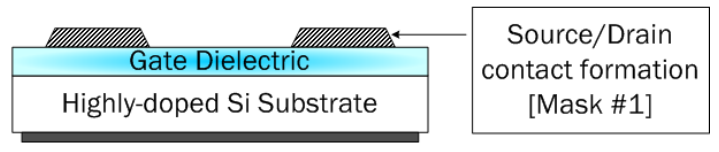

(3)

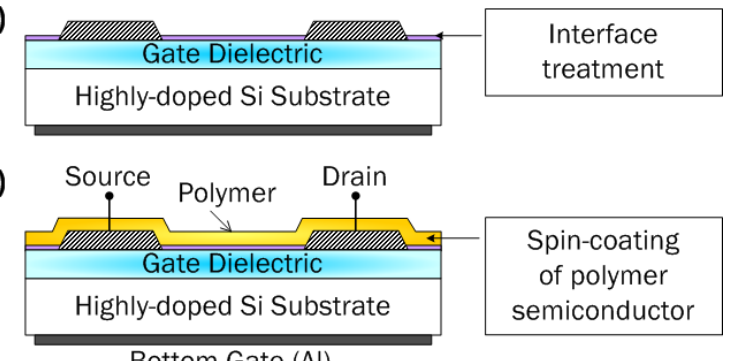

Figure 3.8. Fabrication sequence of bottom-gate bottom-contact OTFT on highly-doped Si substrate with a 1-mask patterning step to define the source/drain contacts (by photolithography or shadow mask).

\subsubsection{Photolithography Scheme for Fully-Patterned and Fully-Encapsulated}

\section{Bottom-Gate OTFT}

Figure 3.9 shows the processing scheme for a photolithographically-defined fully-patterned and fully-encapsulated bottom-gate bottom-contact OTFT, developed in this research. First, the 
bottom-gate metal is deposited and patterned (mask \#1), followed by deposition of the bottom-gate dielectric across the wafer. A second metal layer is deposited on the dielectric surface, and is subsequently patterned by photolithography to define the source/drain contacts (mask \#2). Prior to deposition of the organic semiconductor, interface treatment is performed on the dielectric and/or contacts to improve molecular ordering and quality of the organic semiconductor film, for enhanced device performance. (The role of interface treatment is analyzed in-depth in Chapter 5 and Chapter 6.)

(1)

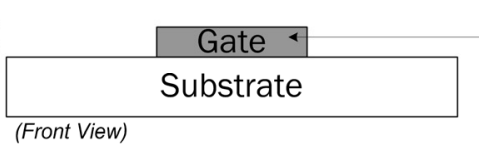

(2)

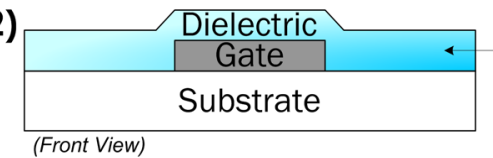

(3)

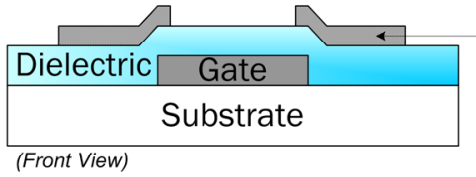

(4)

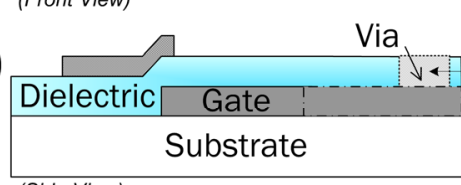

Gate dielectric deposition

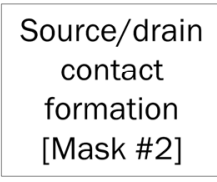

Via formation for gate contact [Mask \#3]
(5)

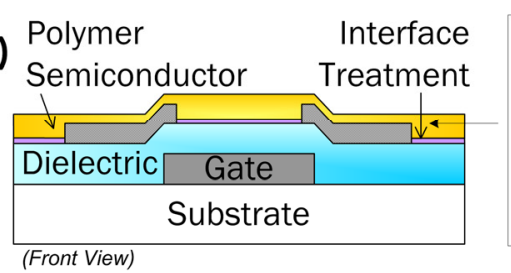

(6)

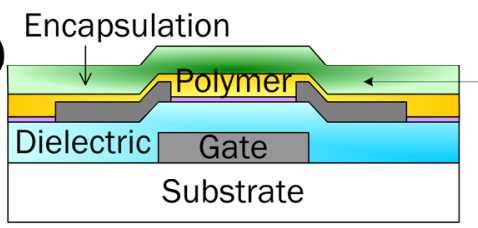

(7)

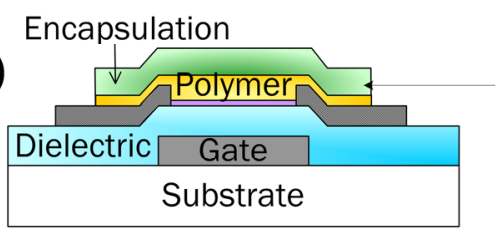

Subtractive patterning by oxygen plasma etching

Interface treatment \& spin-coating of polymer semiconductor

Encapsulationmasking layer deposition

Patterning and etching of polymer semiconductor [Mask \#4]

Figure 3.9. Fabrication sequence of fully-patterned fully encapsulated bottom-gate bottomcontact OTFT by a 4-mask photolithography process.

The most critical step in this process is patterning of the organic semiconductor layer. Two approaches were investigated:

1. Direct patterning, where the standard photolithography process was performed directly on the organic semiconductor layer. Photoresist was processed directly on the semiconductor, and was preserved on the final device to serve as passivation. Figure 3.10(a) illustrates a cross-section of the directly-patterned structure with photoresist passivation.

2. Indirect patterning, where a buffer layer was used using to isolate the organic semiconductor from photoresist. Parylene was used as buffer material in our process. 
Photoresist was processed on the buffer layer, and was later preserved on the device after processing to provide an additional level of passivation. Figure 3.10(b) shows a cross-section of the final structure with parylene-photoresist passivation.

Figure 3.11 displays a cross-sectional diagram and actual microphotograph of a fully-patterned parylene-passivated PQT-12 OTFT fabricated using the scheme presented in Figure 3.9; the microphotograph shows that well-defined device layers are produced using photolithography approach. OTFT circuits were successfully fabricated using this scheme; more details are presented in Chapter 7. Details of the direct and indirect patterning approaches are explained below.

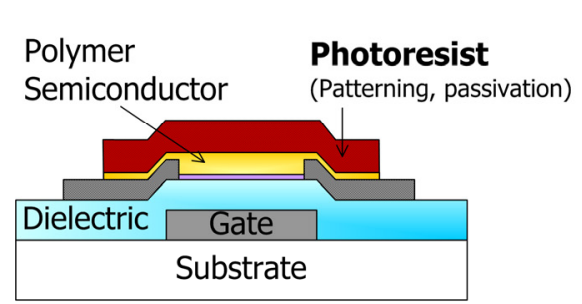

(a) Photoresist Passivation

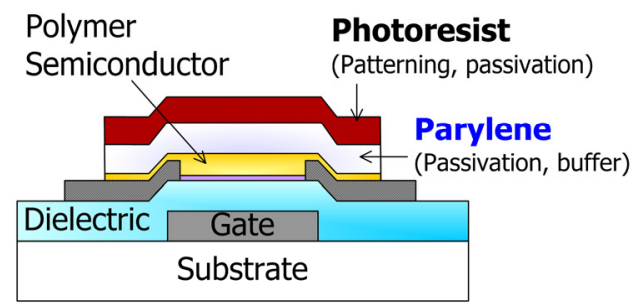

(b) Parylene-Photoresist Passivation

Figure 3.10. Two photolithographically-defined bottom-gate OTFT structures with (a) photoresist passivation and (b) parylene-photoresist passivation.

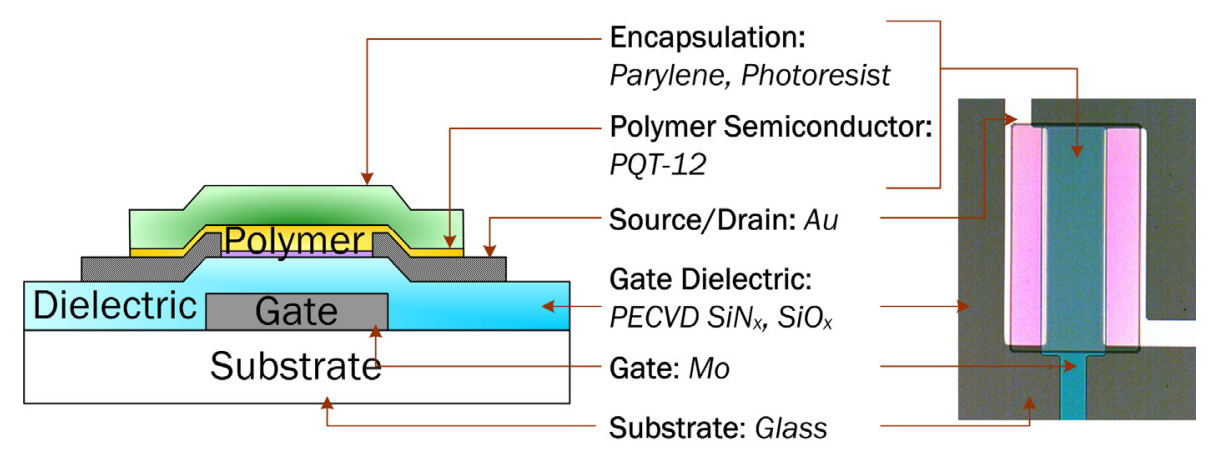

Figure 3.11. Cross section illustration and photograph of a fully-patterned OTFT fabricated using 4-mask photolithography scheme with parylene-photoresist passivation.

\subsubsection{Directly Patterned OTFTs}

With the direct patterning approach, spin-coating a uniform photoresist layer on the polymer semiconductor surface (PQT-12 in this experiment) across the entire 3" wafer can be a challenge. This can be due to inhomogeneity of the PQT-12 thin film surface and adhesion issues of photoresist on PQT-12. Imperfect photoresist coverage degrades the device yield across a single 3" 
wafer. To gain better control over the patterning process and to improve adhesion/uniformity of photoresist on PQT surface, it is recommended to fine-tune the processing parameters of photoresist (e.g., spin rate, baking temperatures), and to optimize surface energy of photoresist (e.g., by modifying the photoresist formulation or pre-treating the semiconductor surface).

Following spin-coating, the photoresist layer was patterned by the standard photolithography sequence: soft-baking, UV exposure, developing, and hard-baking. The organic semiconductor was carefully etched using oxygen plasma in reactive ion etching (RIE) mode, where the photoresist pattern served as a masking layer.

Functional OTFTs and circuits were produced using this direct patterning approach. Electrical characteristics of a photoresist-passivated directly-patterned OTFT are displayed in Figure 4.20. However, the resulting PQT-12 OTFTs displayed rather low mobility, in the range of 0.003-0.008 $\mathrm{cm}^{2} / \mathrm{V}$-s; this is an $\sim 10 \mathrm{x}$ reduction compared to the indirect patterning approach with a parylene buffer layer, discussed next. These results indicate that direct patterning using photoresist may induce unfavorable changes to the organic semiconductor layer. Damages may arise from chemical interactions with photoresist, developer solution, or oxygen plasma during etching. Nevertheless, the demonstration of functional OTFTs is a breakthrough, since it is generally reported that standard photolithography process are destructive to OTFTs. Therefore, with careful control and planning, concerns related to photolithography can be overcome to bring forth a compatible process for organic electronics fabrication.

\subsubsection{Indirectly Patterned OTFTs}

Indirect patterning presents a more reliable approach, where the organic semiconductor is encapsulated with a buffer layer prior to photolithographic patterning. Parylene-C film (200-300 $\mathrm{nm}$ thick), deposited using room temperature chemical vapor deposition (CVD) (Cookson CVD deposition system), was used as the buffer/passivation layer in our process. Parylene is an attractive masking/capping/dielectric/passivation material owing to its ability to form conformal pinhole-free coating, excellent dielectric strength $\left(2.2 \mathrm{MV} / \mathrm{cm}, \varepsilon_{\mathrm{r}}=2.95-3.15\right)$, temperature-independent electrical properties, high molecular weight $(\sim 500,000)$, high melting temperatures $\left(290^{\circ} \mathrm{C}\right)$, superior barrier properties (i.e., gas and moisture permeability) compared to other polymeric materials, good resistance to chemical attack at room temperatures, low solubility in organic solvents up to $175^{\circ} \mathrm{C}$, and good adhesion to a wide variety of substrates (where surfaces can be 
treated with silane to improve adhesion) [28]. More information on the material properties and processing procedure of Parylene is available in reference [28].

Patterning of the organic semiconductor with a parylene buffer layer is relatively straightforward. Spin-coating of photoresist on parylene results in a relatively uniform film across the wafer. Regular exposure and development steps were carried out to pattern the photoresist. Oxygen plasma reactive ion etching (RIE) is used to transfer the pattern onto parylene and the underlying organic semiconductor layer; photoresist served as an etch mask for parylene and semiconductor layers. Again, careful timing of the etching in oxygen plasma is extremely critical to avoid over-etching and to suppress/avoid any possible process-induced degradation of the organic semiconductor layer.

A processing concern in this approach is the possible penetration of water or solvent under the parylene layer (thru film edges) during photoresist developing and rinsing, which can affect the quality/properties of the underlying organic semiconductor layer. Possible remedies include the use of a thicker parylene layer, improving adhesion of the parylene on the organic semiconductor via interface treatment, and cautious device processing/handling. Despite these minor imperfections, parylene-passivated PQT-12 OTFTs on glass substrates displayed mobility of $0.04-0.12 \mathrm{~cm}^{2} / \mathrm{V}-\mathrm{s}$; this is comparable to unpatterned PQT-12 OTFTs fabricated with the same $\mathrm{SiN}_{\mathrm{x}}$ gate dielectric on $\mathrm{Si}$ wafer using the scheme reported in Section 3.3.1. The ability to maintain comparable device mobility suggests successful implementation of this parylene-passivated approach for photolithographically-defined fully-patterned and fully-encapsulated bottom-gate bottom-contact OTFT. Figure 3.13 illustrates the electrical characteristics of a parylene-passivated OTFT.

As an extension of this fabrication scheme, further process improvements can be achieved by incorporating an inorganic passivation layer (e.g., $\mathrm{SiN}_{\mathrm{x}}, \mathrm{SiO}_{\mathrm{x}}, \mathrm{AlO}_{\mathrm{x}}$ ) on top of parylene, to provide an additional level of protection against moisture, oxygen or solvent penetration. This multilayer passivation approach, as illustrated in Figure 3.5(b), is expected to minimize environmental sensitivity of the organic material, and improve the stability and lifetime of OTFTs. 
(a)

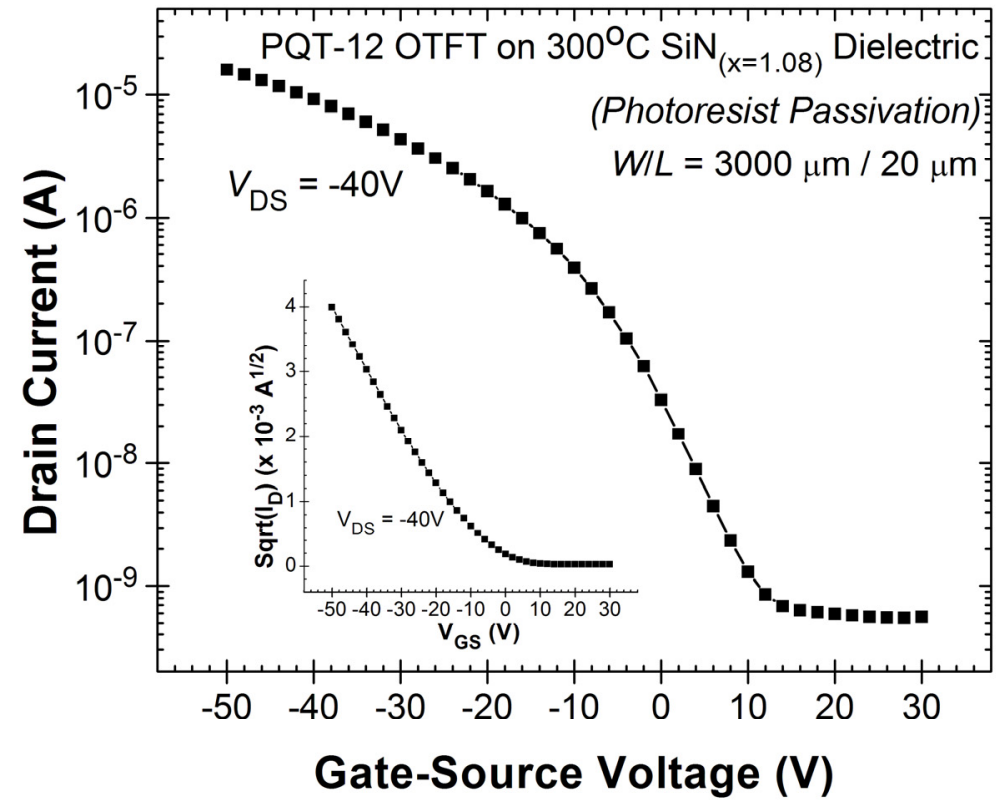

(b)

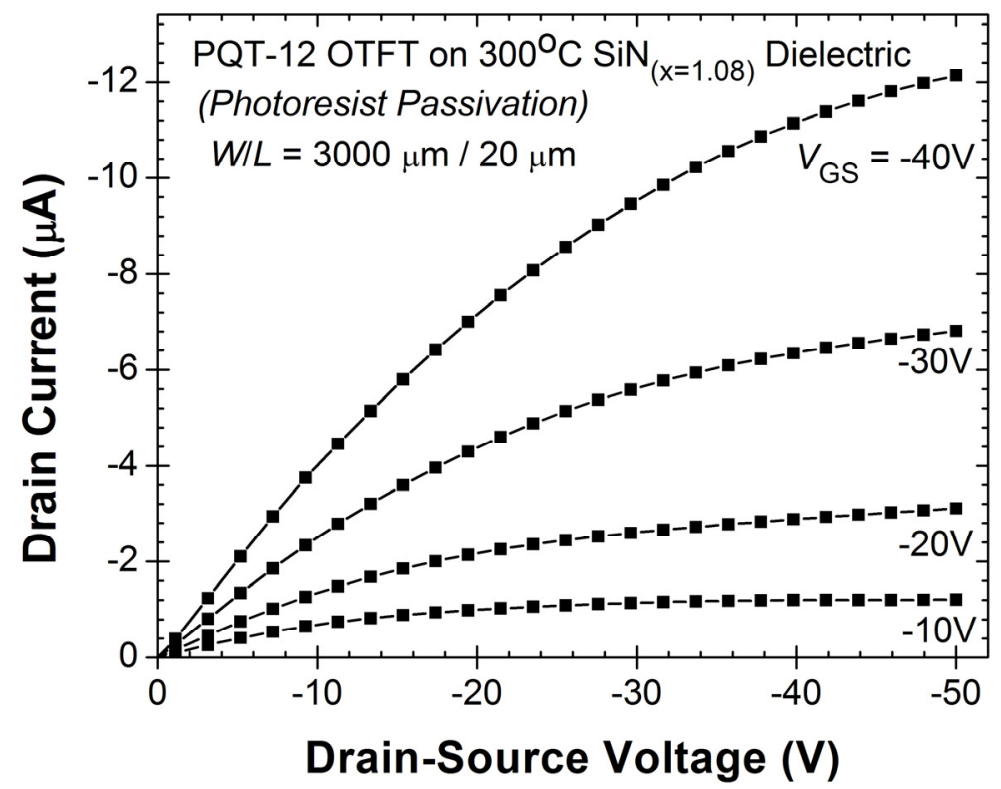

Figure 3.12. (a) Transfer and (b) output characteristics of a fully-patterned bottom-gate bottom-contact PQT-12 OTFT with $\mathrm{SiN}_{\mathrm{x}}$ gate dielectric on glass substrate. Polymer semiconductor layer was directly patterned using photolithographically, with direct deposition of photoresist on PQT-12 layer. 
(a)

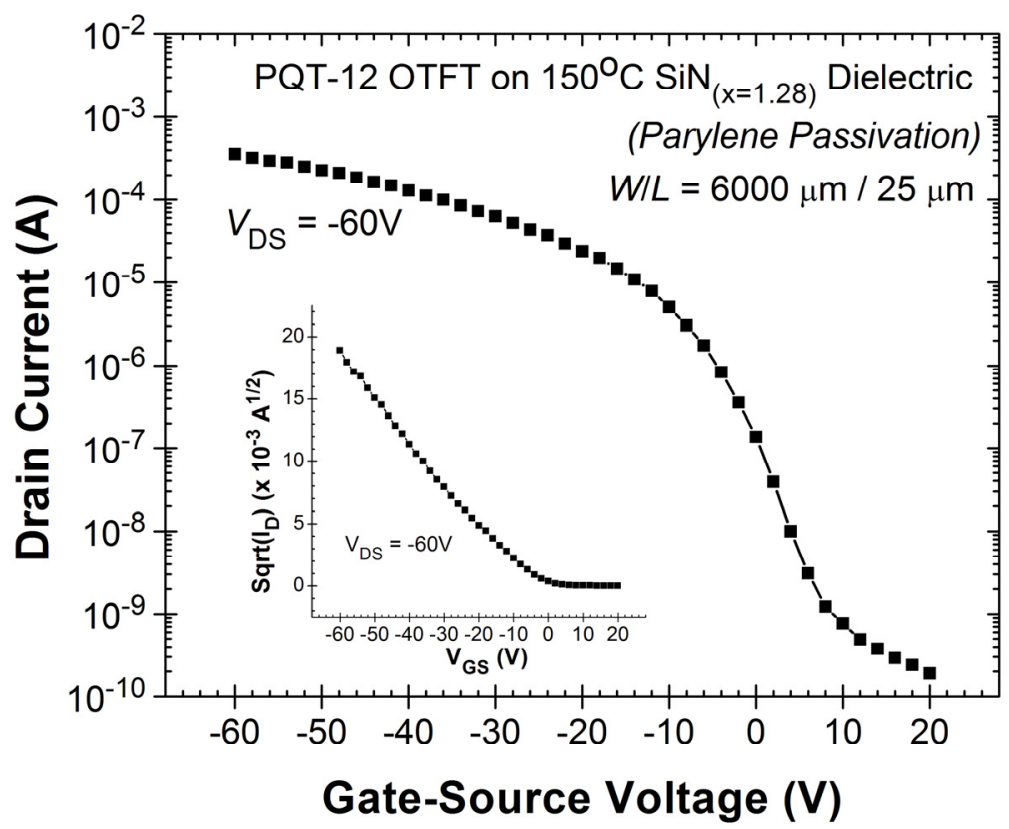

(b)

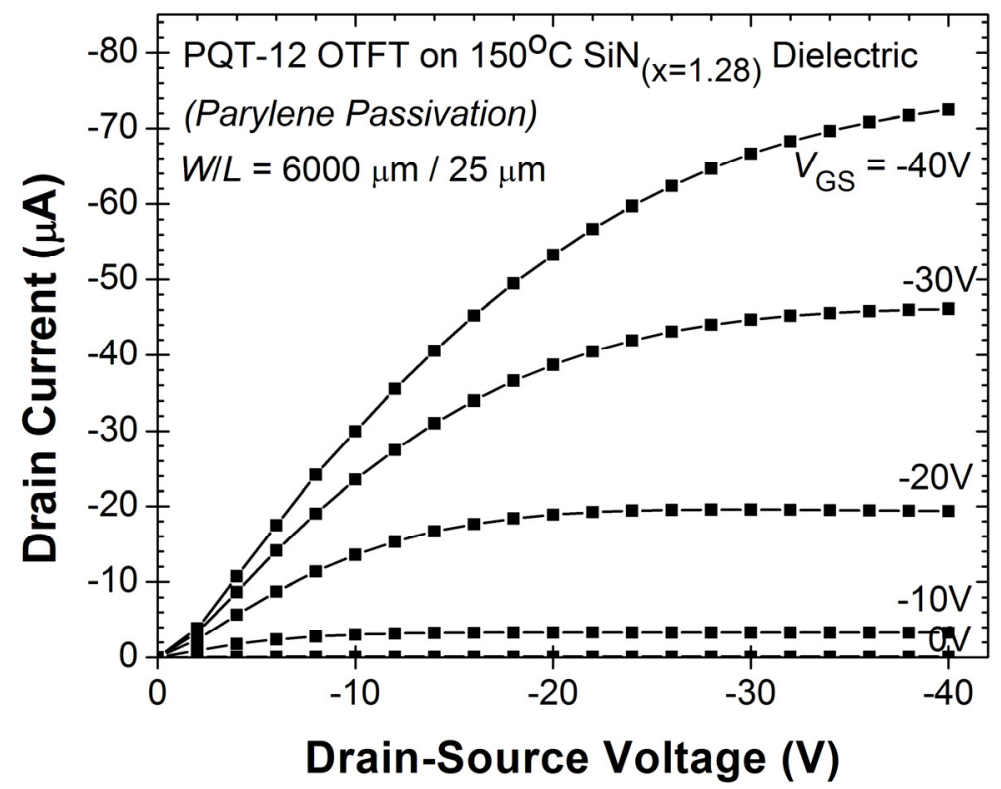

Figure 3.13. (a) Transfer and (b) output characteristics of a fully-patterned bottom-gate bottom-contact PQT-12 OTFT with $\mathrm{SiN}_{\mathrm{x}}$ gate dielectric on glass substrate. Polymer semiconductor layer was photolithographically patterned with parylene passivation layer. 


\subsubsection{Hybrid Photolithography-Inkjet Printing Scheme for Fully- Patterned Bottom-Gate OTFT}

We developed a hybrid photolithography-inkjet printing fabrication scheme to enable nondisruptive patterning of the organic semiconductor layer via printing, while providing the necessary feature resolution, patterned gate structures, and via-interconnect structures by photolithography means. Since an all-printed process is envisioned for organic electronics fabrication in the future, this hybrid process can help bridge the transfer to an all-printed fabrication process while providing an immediate solution for OTFT circuit integration. The fabrication sequence for our hybrid photolithography-inkjet printing approach to produce fully patterned bottom-gate OTFTs is depicted in Figure 3.14. Figure 3.15 displays a cross-section diagram and actual microphotograph of a fully-patterned PQT-12 OTFT fabricated using this hybrid inkjet printing scheme; the microphotography shows the inkjet printed polymer semiconductor has higher non-uniformity and inhomogeneity compared to the photolithographically-defined polymer layer (in Figure 3.11). PQT-12 OTFT circuits (including inverters, ring oscillators, pixel circuits) were successfully fabricated using this approach; results are documented in Chapter 7.

(1)

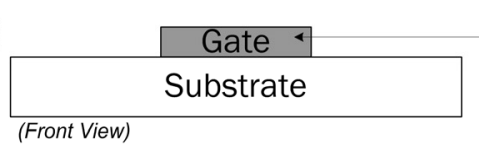

(2)

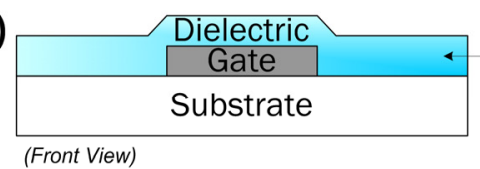

(3)

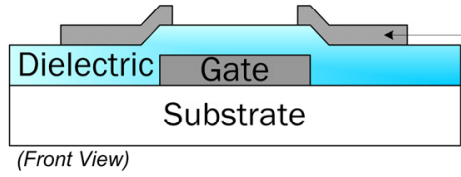

Gate formation [Mask \#1]

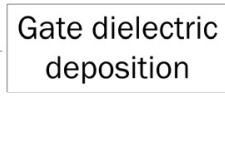

Source/drain contact formation [Mask \#2]
(4)

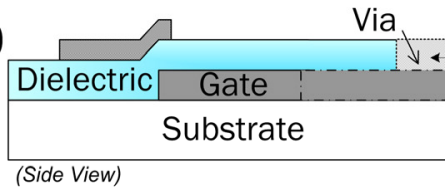

(5)

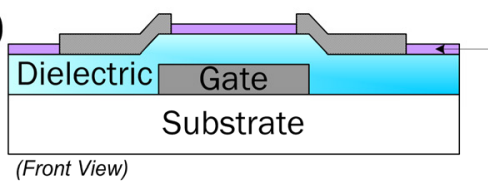

(6)

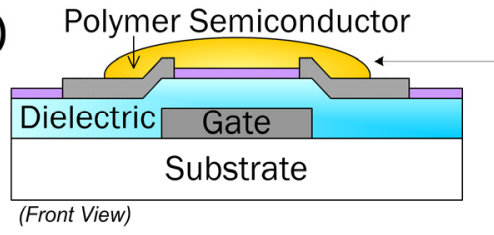

Via formation for gate contact [Mask \#3]

Interface treatment Inkjet Printing of polymer semiconductor

Figure 3.14. Hybrid photolithography-inkjet printing fabrication scheme for fully-patterned bottom-gate bottom-contact OTFT. 


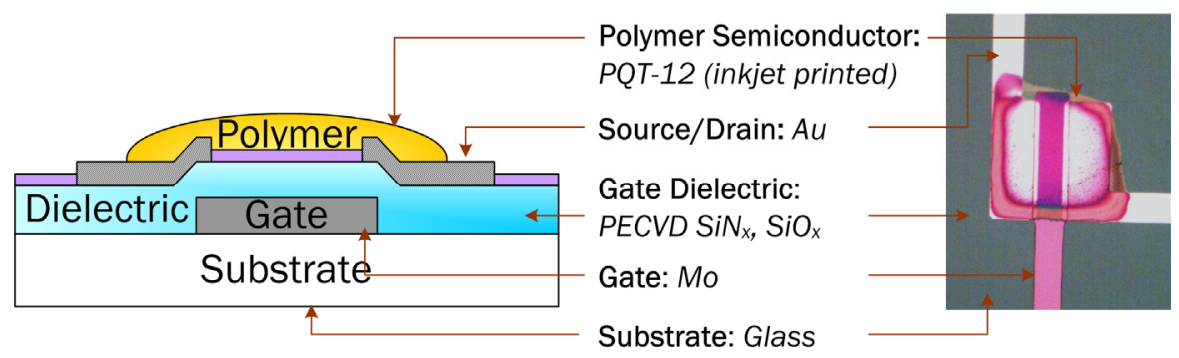

Figure 3.15. Cross section illustration and photograph of a fully-patterned OTFT fabricated using hybrid photolithography-inkjet printing scheme.

The gate electrode, source/drain contacts, and via/interconnect structure are defined by photolithography techniques. Organic semiconductor (PQT-12 in dichlorobenzene solvent) is inkjet printed onto the pre-patterned substrates using a Dimatix Materials Printer to define the transistor's active region [47]. Inkjet printed PQT-12 OTFTs on $\mathrm{SiN}_{\mathrm{x}}$ gate dielectric displayed effective mobility in the range of $0.005-0.03 \mathrm{~cm}^{2} / \mathrm{V}$-s. Typical electrical characteristics are shown in Figure 3.16. The effective mobility of OTFTs with inkjet printed PQT-12 is lower (by 5 to 10 times) than OTFTs with spin-coated PQT-12; this is attributed to non-uniformity and possibly discontinuity of the inkjet printed organic layer, as shown in Figure 3.17. Device variation across a wafer was also more pronounced with the inkjet printing approach. These observations indicate that better control over the inkjet printing process is important to achieve better device performance.

Experiments showed that the resolution, quality and performance (e.g., mobility) of the inkjet printed device are sensitive to ink formulation and a variety of printing parameters, including printing direction (see Figure 3.18), drop spacing, substrate and ink cartridge temperature, frequency and amplitude (or voltage) of inkjet driving waveform, etc. For instance, the choice of solvent and ink concentration affects the evaporation rate of the ink droplet, which in term influences configuration of the printed film (i.e., dot-like or ring-like). A low evaporation rate yields small dot-like film and a fast evaporation rate results in the wide ring-like film [48]. Ink formulation also determines the spreading of the ink droplet on the substrate, which affects film uniformity and image resolution. In addition, printing parameters or settings must be carefully controlled to deliver a continuous and uniform inkjet-printed organic layer needed for high performance OTFTs. Table 3.3 summarizes experimental observations on the impact of selected printing parameters on the quality of inkjet printed PQT-12 organic semiconductor thin film.

Researchers at Palo Alto Research Center (PARC) reported jet printed polythiophene OTFTs with mobility $\left(0.1 \mathrm{~cm}^{2} / \mathrm{V}\right.$-s $)$ comparable to spin-coated devices [43]. Thus, with technological 
optimization and precise control of the printing parameters, high performance inkjet printed OTFTs can be delivered. Moreover, the ease of patterning compared to other approaches make inkjet printing particularly promising and versatile for fabrication of OTFTs for low cost electronics.

(a)

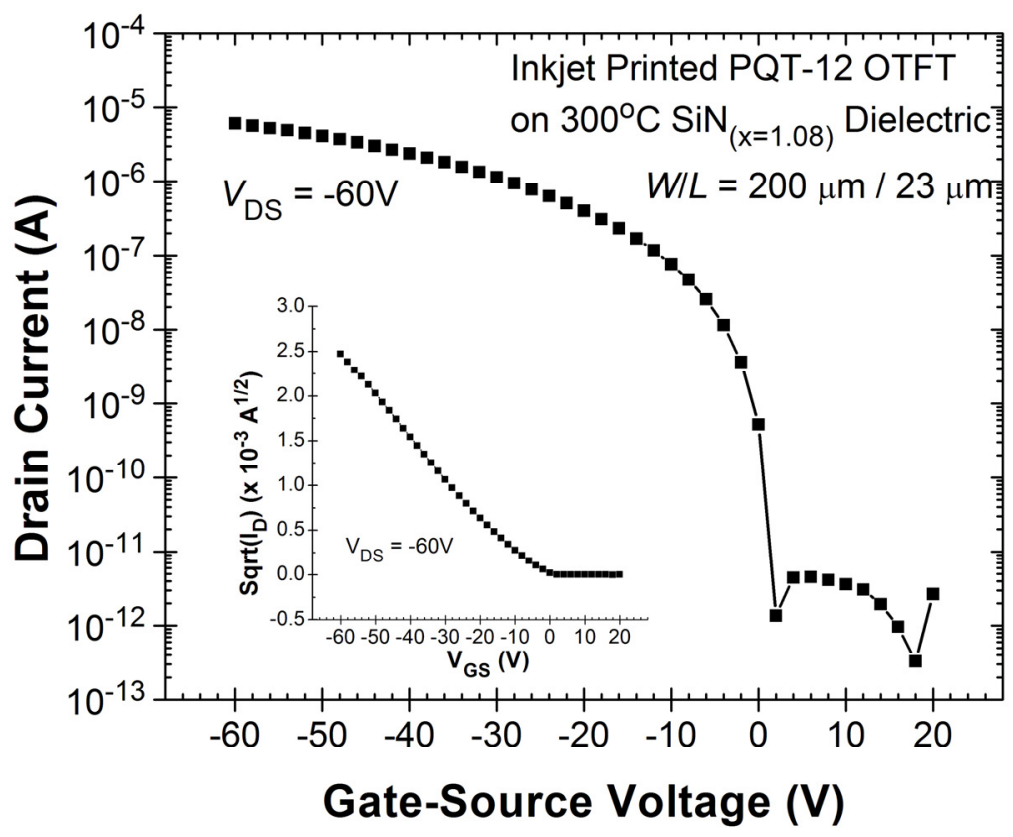

(b)

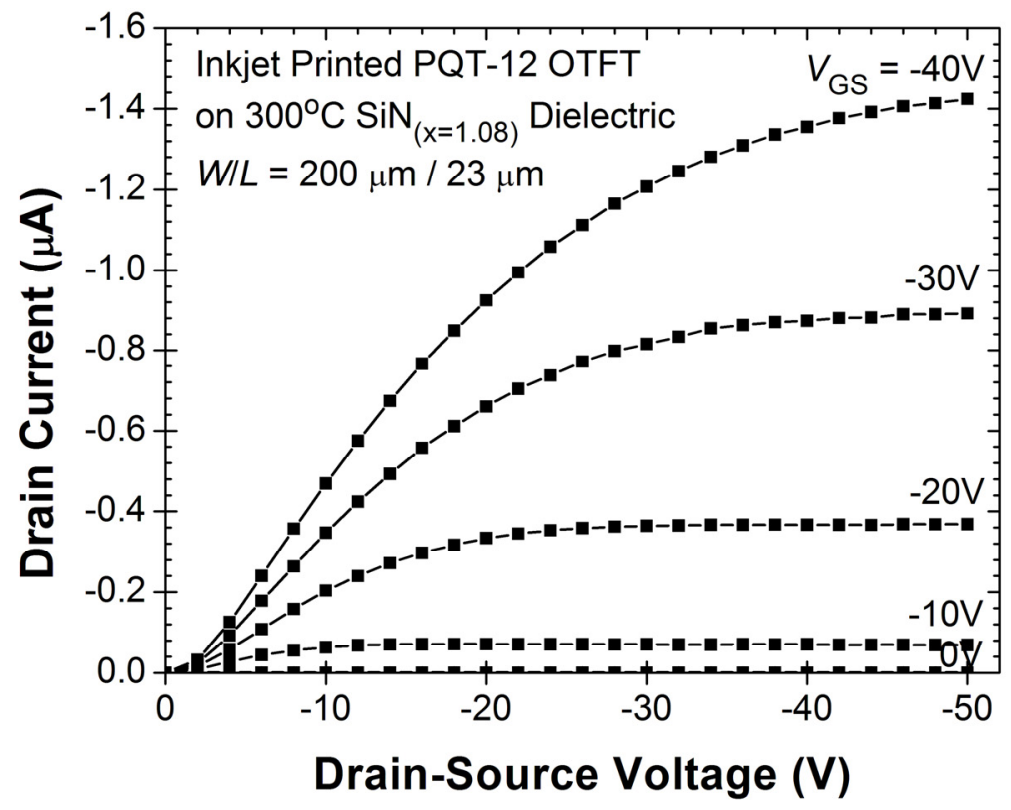

Figure 3.16. (a) Transfer and (b) output characteristics of a fully-patterned bottom-gate bottom-contact PQT-12 OTFT with $\mathrm{SiN}_{\mathrm{x}}$ gate dielectric on glass substrate. Organic semiconductor layer was deposited by inkjet printing. 


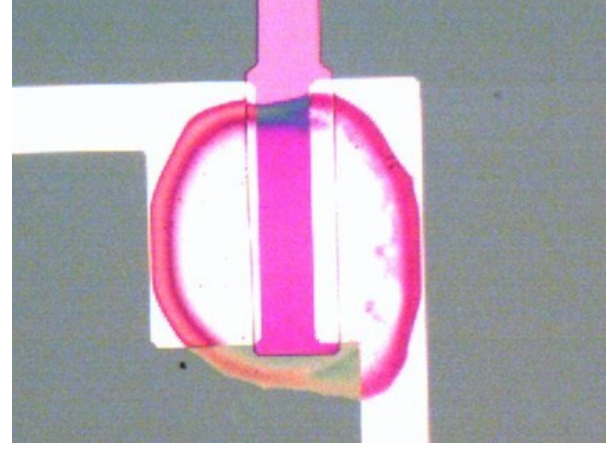

(a)

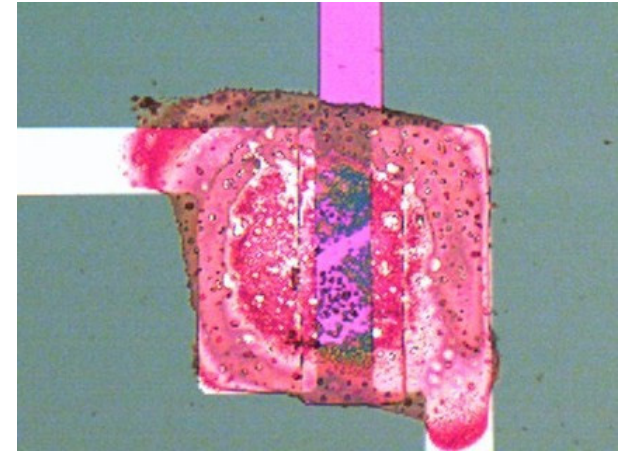

(b)

Figure 3.17. Photograph of OTFTs with inkjet printed organic semiconductor showing (a) a thin uniform layer and (b) a thicker non-uniform layer.

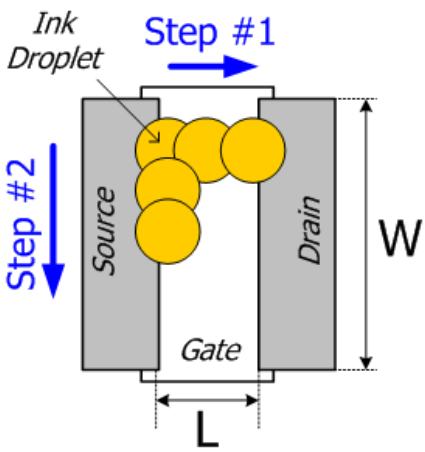

(a) Horizontal Printing

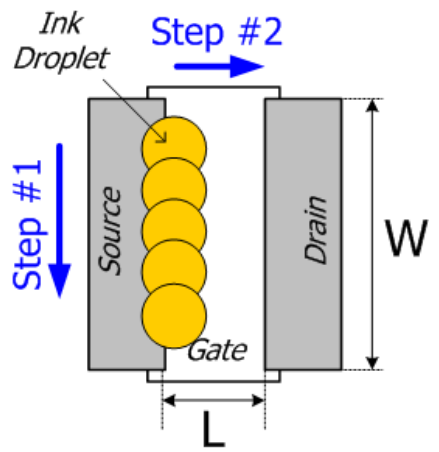

(b) Vertical Printing

Figure 3.18. Illustration of inkjet printing direction: (a) horizontal and (b) vertical printing.

Table 3.3. Experimental summary of the impact of selected printing parameters on the quality of inkjet printed PQT-12 organic semiconductor thin film.

\begin{tabular}{|lcl|}
\hline \multicolumn{1}{|c|}{$\begin{array}{c}\text { Inkjet Printing } \\
\text { Parameter }\end{array}$} & \multicolumn{1}{c|}{ Impact on Quality of Inkjet Printed Organic Semiconductor Layer } \\
\hline $\begin{array}{l}\text { Printing Direction } \\
\text { (see Figure 3.18) }\end{array}$ & $\square$ & $\begin{array}{l}\text { Vertical printing produces better device performance and less coffee ring } \\
\text { effect compared to horizontal printing }\end{array}$ \\
\hline $\begin{array}{l}\text { Nozzle } \\
\text { Temperature }\end{array}$ & $\square$ & $\begin{array}{l}\text { Increasing nozzle temperature }\left(\sim 60^{\circ} \mathrm{C}\right) \text { can prevent precipitation of } \\
\text { polymer solution and avoid blockage of cartridge nozzles }\end{array}$ \\
\hline $\begin{array}{l}\text { Substrate } \\
\text { Temperature }\end{array}$ & $\square$ & $\begin{array}{l}\text { Higher substrate temperature improves stability of ink after printing and } \\
\text { enhances quality of the printed line. }\left(60^{\circ} \mathrm{C} \text { was used) }\right.\end{array}$ \\
\hline $\begin{array}{l}\text { Printing Amplitude } \\
\text { (Voltage) }\end{array}$ & $\square$ & $\begin{array}{l}\text { Amplitude needs to be adjusted to deliver large and stable drops } \\
\text { Optimal jetting voltage varies with ink formulation } \\
\text { Higher amplitude needed for more viscous fluids and higher } \\
\text { concentration inks } \\
\text { Higher amplitude increases drop volume, jetting speed, line-width }\end{array}$ \\
\hline
\end{tabular}




\begin{tabular}{|lcl|}
\hline Printing Frequency & $\square$ & $\begin{array}{l}\text { Lowest jetting frequency gave stable drops with good registration } \\
\end{array}$ \\
& $\square$ & Higher frequency leads to misalignment of the printed layer \\
Drop Spacing & $\square$ & $\begin{array}{l}\text { Drop spacing (i.e., center to center distance between adjacent drops) } \\
\text { should be slightly smaller than drop size to ensure continuity of the } \\
\text { printed line or pattern }\end{array}$ \\
\hline
\end{tabular}

\subsubsection{Photolithography Scheme for Top-Gate and Dual-Gate OTFTs}

The fabrication scheme for photolithograhically-defined dual-gate OTFT based on a 5-mask process is outlined in Figure 3.19. This procedure can be adapted for fabrication of top-gate OTFT, with the omission of Step (1) for bottom-gate deposition and Steps (8)-(9) for via/interconnect. Observe that bottom-gate OTFT can also be realized with Steps (1)-(6) in Figure 3.19. Dual-gate and top-gate P3HT OTFTs have been demonstrated using this fabrication scheme [25][26][27]; device characteristics are presented later in this section. Advantages of top-gate and dual-gate structures are discussed in Section 2.3.2.

Our earliest attempts to fabricate fully-patterned and fully-encapsulated photolithograhically defined top-gate and dual-gate OTFTs utilized PECVD $\mathrm{SiN}_{\mathrm{x}}$ deposited at $75^{\circ} \mathrm{C}$ as the top gate dielectric and masking/passivation layer. Low temperature $\operatorname{SiN}_{x}$ was selected deliberately to ensure thermal compatibility with the organic semiconductor. The optimized processing conditions and material characterization of the $75^{\circ} \mathrm{C}$ PECVD $\mathrm{SiN}_{\mathrm{x}}$ were reported in [49]. For top-gate and dualgate OTFT designs, the deposition of the $\mathrm{SiN}_{\mathrm{x}}$ layer proceeds in two stages. First, a thin $\mathrm{SiN}_{\mathrm{x}}$ layer (50 nm thick) is formed and served as a masking layer for the organic semiconductor film. Then, the organic semiconductor film is patterned by dry etching to define active regions of the semiconductor. A second $\mathrm{SiN}_{\mathrm{x}}$ layer (150 nm thick) is deposited over the entire wafer and encapsulated the organic semiconductor layer. The top $\operatorname{SiN}_{x}$ layer is selectively etched to open source/drain contact windows. Finally, top metallization is deposited to define the top-gate electrode and contact pads. 
(1)

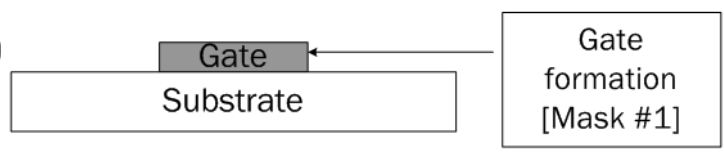

(2)

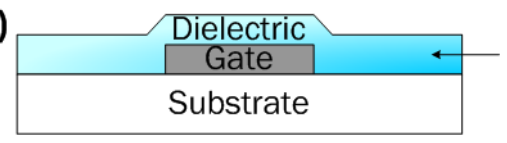

(3)

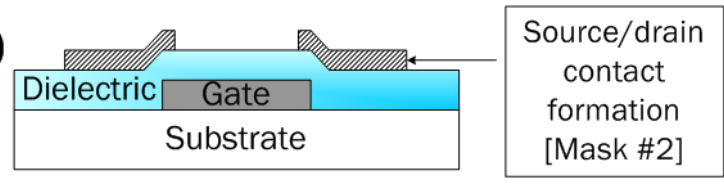

(4)

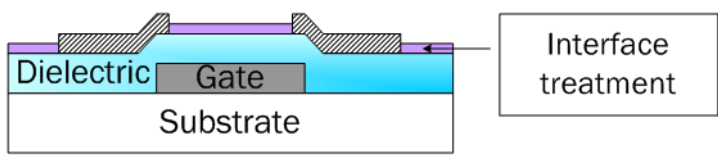

\section{(5)}

Polymer Semiconductor

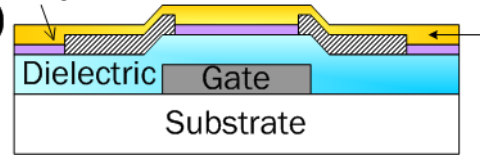

Gate dielectric deposition

Spin-coating of polymer semiconductor
(6)

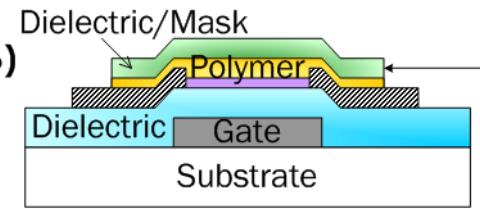

Masking layer deposition. Patterning of polymer layer [Mask \#3]

(7)

Dielectric/Passivation

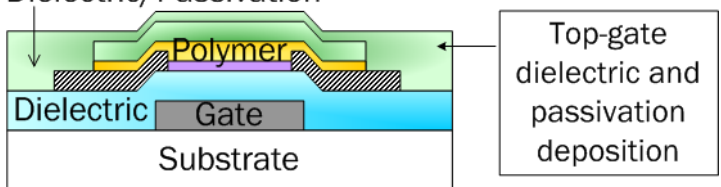

(8)

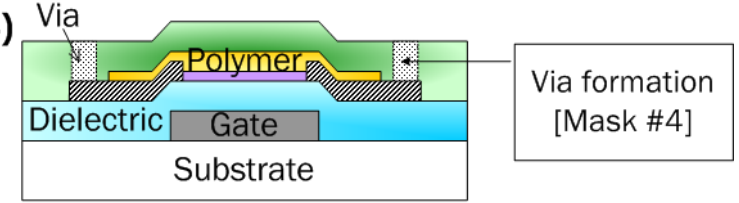

(9)

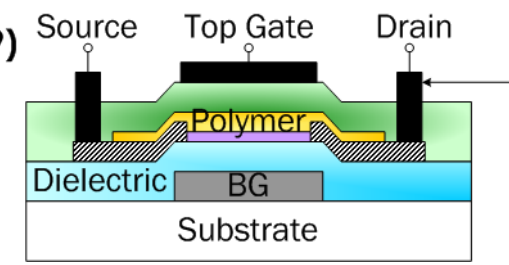

Top metal deposition [Mask \#5]

Figure 3.19. Fabrication sequence of dual-gate OTFT by a 5-mask photolithography process. Top-gate OTFT can be fabricated by excluding steps (1), (8) and (9).

\subsubsection{Top-Gate OTFT}

Electrical characteristics of a photolithographically-defined top-gate P3HT OTFT on glass are presented in Figure 3.20. The glass substrate surface was treated with OTS SAM prior to deposition of the P3HT semiconductor layer. Low-temperature PECVD SiN ${ }_{x}$ served as the top gate dielectric while providing a simultaneous solution for encapsulation of the organic semiconductor layer. The device demonstrated p-type field-effect transistor behavior and gate leakage current below 5 pA. However, the top-gate OTFT exhibited low drain current, limited mobility and low on/off current ratio. Possible causes of the limited device performance are analyzed in Section

\subsubsection{3.}


(a)

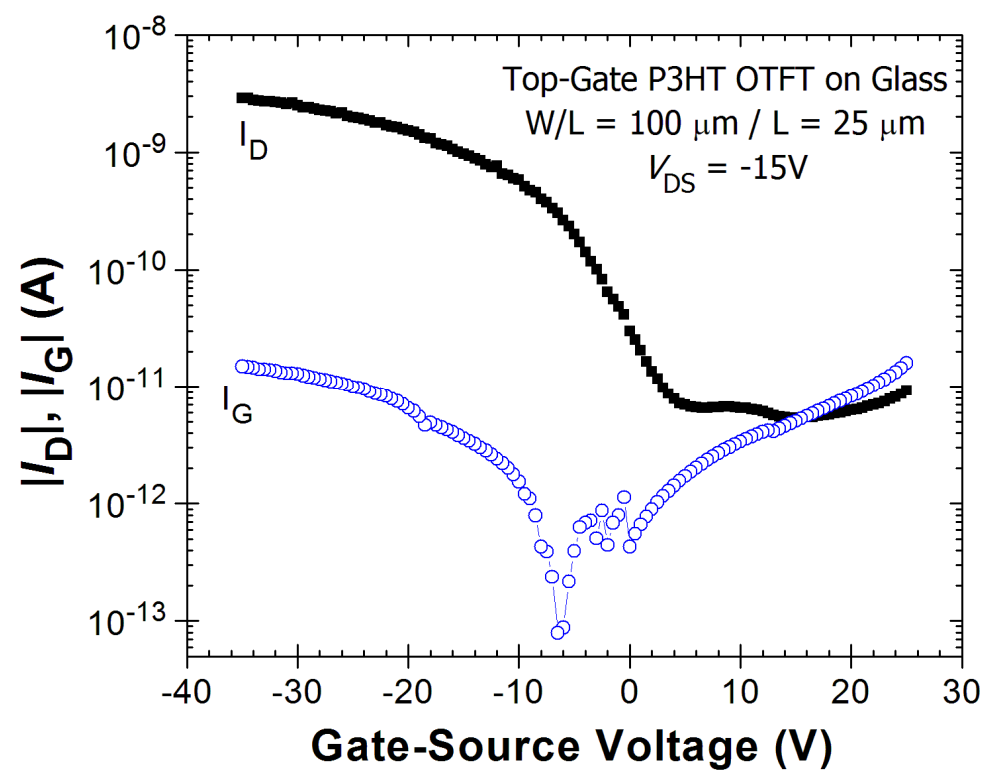

(b)

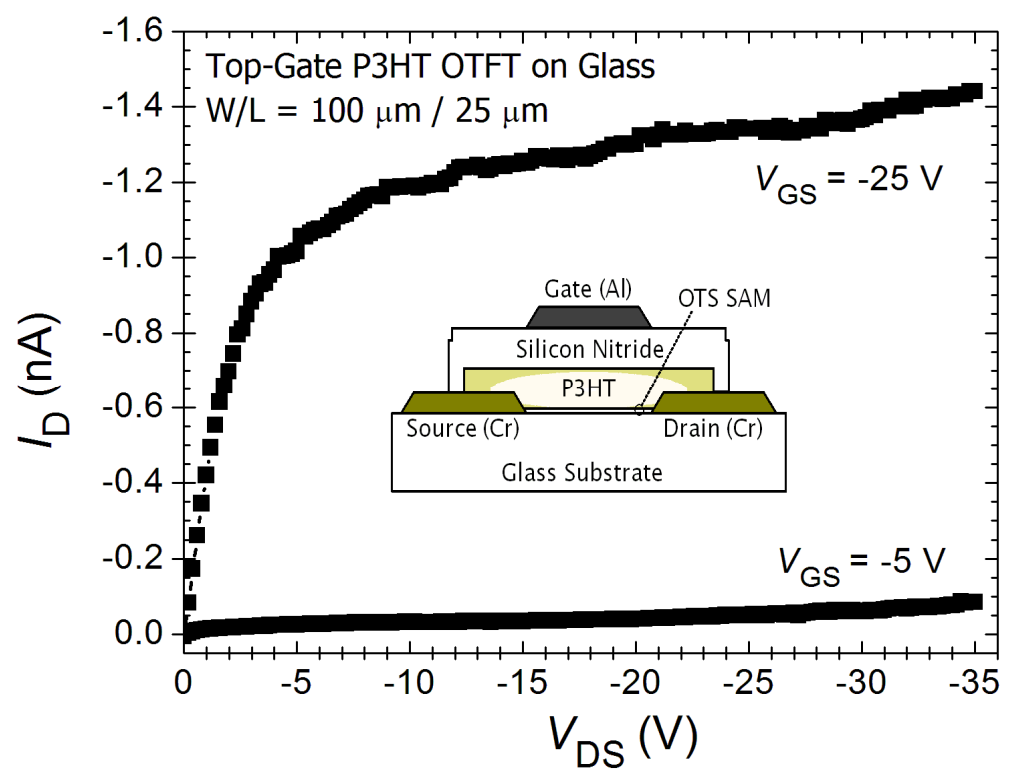

Figure 3.20. Top-gate P3HT OTFT on glass substrate, fabricated by photolithography. (a) Transfer characteristics (ID- $\left.V_{G S}\right)$. (b) Output characteristics ( $\left.I_{D}-V_{D S}\right)$.

An advantage offered by the top-gate structure is that the source/drain contact layer is the first layer to be defined on the substrate. This first layer is generally the easiest to pattern since it is not inhibited by and does not need to contend with the topology of other device layers; thus, there is more flexibility on selecting an appropriate patterning technique to achieve small $L$ for enhancing $\mu_{\mathrm{FE}}$. Another feature of top-gate OTFT is that the gate dielectric can provide encapsulation and 
protection of the organic semiconductor layer. Encapsulation is essential because most organic materials are chemically sensitive to environmental influences. Nevertheless, there is a number of process integration challenges associated with the top-gate OTFT design. First, the gate dielectric and gate electrode have to be deposited and structured on top of the organic semiconductor layer, and this process must preserve the organic material. Secondly, vertical interconnects and vias between the conductive layers necessitate developing a compatible etching process for the organic layer. These issues were addressed in our process, in which low temperature $\mathrm{SiN}_{\mathrm{x}}$ was selected as a passivating gate dielectric to ensure thermal conformity with the organic semiconductor, and a tailored etch recipe was developed for patterning the polymer layer with excellent compatibility.

\subsubsection{Dual-Gate OTFT}

This thesis research demonstrated one of the first dual-gate OTFTs reported in the literature. Our initial dual-gate OTFTs were prepared on oxidized $\mathrm{Si}$ wafer, with a thermal $\mathrm{SiO}_{2}$ layer as the bottom-gate dielectric and PECVD $\operatorname{SiN}_{\mathrm{x}}$ as the top-gate dielectric and passivation [27]. The fabrication process of this $\mathrm{SiO}_{2} / \mathrm{P} 3 \mathrm{HT} / \mathrm{SiN}_{\mathrm{x}}$ dual-gate OTFT is similar to that outlined in Figure 3.19, except these OTFTs consisted of a uniform bottom-gate provided by the highly doped Si substrate; thus, the bottom-gate deposition step is neglected. Figure 3.21(a) shows the transfer characteristic of a dual-gate OTFT as a function of the top-gate voltage $\left(V_{\mathrm{TG}}\right)$, with the bottom-gate $\left(V_{\mathrm{BG}}\right)$ biased at $10 \mathrm{~V}$. The output characteristics of the same device at various top-gate voltages are displayed in Figure 3.21 (b). The device demonstrated p-type transistor behavior, with field effect mobility $\left(\mu_{\mathrm{FE}}\right)$ of $10^{-5} \mathrm{~cm}^{2} / \mathrm{V}-\mathrm{s}$, threshold voltage $\left(V_{\mathrm{T}}\right)$ of $-1.9 \mathrm{~V}$, on/off current ratio $\left(I_{\mathrm{ON}} / I_{\mathrm{OFF}}\right)$ of $10^{3}$, and inverse subthreshold slope $(S)$ of $1.8 \mathrm{~V} / \mathrm{dec}$. The top gate leakage current was in the range of $10^{-14}-10^{-12} \mathrm{~A}$, indicating good electrical characteristics of the $75^{\circ} \mathrm{C} \mathrm{SiN}_{\mathrm{x}}$ passivation dielectric. On the other hand, the bottom-gate component displayed poor field-effect transistor behavior, and large leakage current was observed through the bottom-gate $\mathrm{SiO}_{2}$ dielectric. Further assessments indicated that comparable dielectric leakage was present in OTFTs with $\mathrm{SiN}_{\mathrm{x}}$ as the bottom-gate dielectric, and that the leakage current became significant only after polymer deposition on the bottom-gate dielectric. Similar dielectric leakage phenomenon was reported by Raja et al. [50][51]. The large increase in dielectric leakage currents as a result of polymer deposition was attributed to the displacement of dopant ions or impurities present in the polymer film, as well as impurity contamination of the oxide from the polymer-chloroform solution. (A brief discussion of the limitations of commercially available polymer semiconductor is presented in Section 2.4.1.) More 
in-depth investigation of this matter is needed. It is believed that purification of the commerciallypurchased polymer material prior to deposition and selection of an appropriate solvent are necessary to alleviate this leakage problem.

(a)

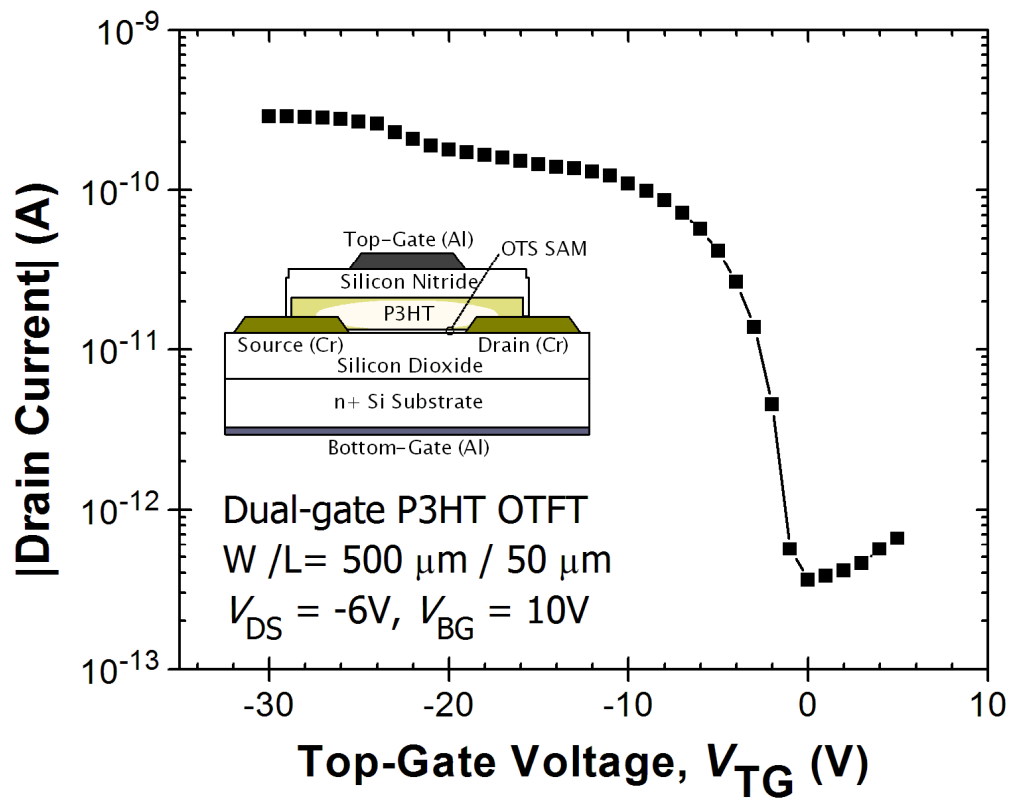

(b)

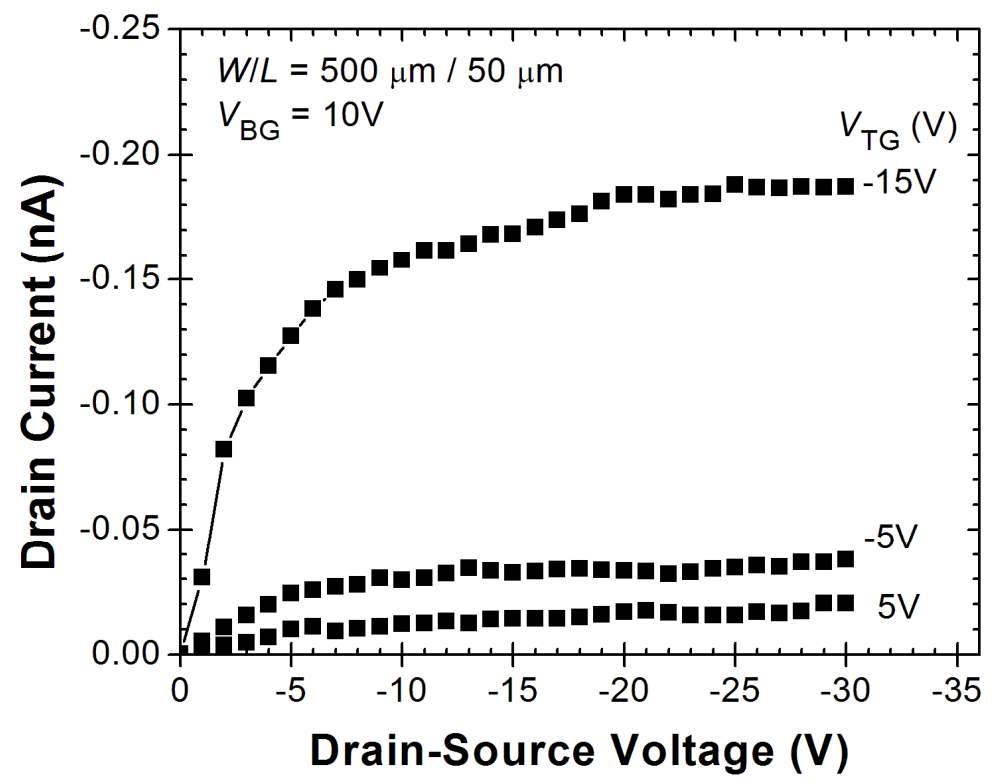

Figure 3.21. Dual-gate P3HT OTFT on $\mathrm{Si}$ wafer. Bottom-gate dielectric is $\mathrm{SiO}_{2}$, and top-gate dielectric is PECVD SiN $N_{x}$. (a) Transfer characteristics $\left(I_{D}-V_{T G}\right)$ of the dual-gate OTFT as a function of top-gate voltage. (b) Output characteristics ( $\left.I_{D}-V_{D S}\right)$ of the dual-gate OTFT at various top-gate voltages. 
A valuable/interesting property of dual-gate OTFTs is that the voltage bias on the bottom-gate has a distinct influence on the threshold voltage, subthreshold slope, on-current, and leakage current of the top-gate TFT. A larger negative $V_{\mathrm{BG}}$ resulted in a larger $\left|I_{\mathrm{D}}\right|$ and positive shift in $V_{\mathrm{T}}$. Likewise, there is a dependence of the bottom-gate TFT characteristics on the top-gate bias. Similar dual-gate effects were reported in [52]. With the ability to control selected TFT parameters, the dual-gate OTFT structure is attractive for circuit applications that demand high threshold voltage control and good reliability. The dual-gate OTFT also lends itself as a highly functional test structure for characterization of density of states at the top and bottom interfaces of the active organic and dielectric layers, to evaluate the interface integrity and provide insight into the underlying transport mechanisms. Another promising application of dual-gate OTFT is seen in AMOLED display circuits where vertical integration is desirable to achieve a high aperture ratio and a high on-pixel integration density. Another promising application of dual-gate OTFT is to implement a vertically stacked active-matrix backplane for displays or imagers; vertical integration is desirable to achieve a high aperture ratio and a high on-pixel integration density. This concept is discussed in Section 7.4.1.

\subsubsection{Analysis}

The limited performance (i.e., low current drive, mobility, current on/off ratio) of these preliminary photolithographically-defined top-gate and dual-gate OTFTs can be attributed to various factors, including environmental sensitivity of the polymer semiconductor, process-induced degradation, non-optimized dielectric-semiconductor interfaces, and large contact resistance. First, the poor field-effect mobility and on/off current ratio, relative to other P3HT OTFTs reported in the literature [53][54], are likely linked to the degradation of P3HT due to exposure to air. P3HT is sensitive to oxygen and water molecules, and becomes p-type doped upon absorption of atmospheric oxygen. Consequently, the material experiences an increase in free carrier density and a higher conductivity [55]. These changes translate to higher off-current, lower on/off current ratio, and negligible field-effect and gate-modulation effects in OTFT. Our observations are consistent with the data reported by Han et al., where they noted a decrease in mobility from $10^{-3} \mathrm{~cm}^{2} / \mathrm{V}-\mathrm{s}$ to $10^{-5} \mathrm{~cm}^{2} / \mathrm{V}$-s and a decay in on/off current ratio from $10^{4}$ to 10 following exposure of the P3HT OTFTs to air for only 15 minutes [55]. The rapid degradation of P3HT in air, during processing and measurement, also accounts for the reduced performance of our devices. Processing in inert 
environment, as well as purification of the polymer materials, should lead to enhanced OTFT performance.

Other potential factors contributing to the limited OTFT performance include process-induced degradation and inferior quality of the top $\mathrm{P} 3 \mathrm{HT} / \mathrm{SiN}_{\mathrm{x}}$ interface. Process-induced device

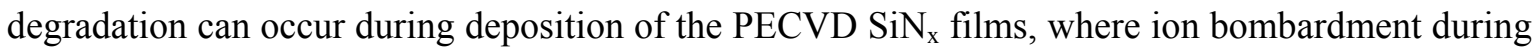
the initial deposition stage can adversely impact the $\mathrm{P} 3 \mathrm{HT} / \mathrm{SiN}_{\mathrm{x}}$ interface quality, and emission of UV radiation from plasma processing can alter the P3HT film properties. These observations are substantiated by the results reported in [56]. The effects of stress in the $\mathrm{SiN}_{\mathrm{x}}$ layer can also undermine the device performance. These concerns can be circumvented by selecting alternative top passivation dielectric materials with improved compatibility with the organic semiconductor layer. As demonstrated in Section 3.3.2, parylene is a viable candidate. An evaporated $\mathrm{SiO}_{2}$ layer and a solution-processed benzocyclobutene (BCB) layer are other possible options of passivation dielectric in photolithographically-defined OTFTs.

Contact effects constitute another source of the poor OTFT performance. Cr was used as the source/drain contacts in these preliminary dual-gate and top-gate OTFTs, due to unavailability of higher work function metals (e.g., Au) during early stages of research. The lower work function of $\mathrm{Cr}$ may cause injection barrier at the Cr-P3HT interface, and thus restricts device performance. Application of a higher work function contact material and optimization of the contact-polymer interface (by appropriate interface treatment) are expected to generate enhanced device characteristics in future experiments.

\subsubsection{Fabrication Scheme Comparisons}

The strengths and limitations of the above-described fabrication schemes and patterning techniques are summarized in Table 3.4. Table 3.5 compares the performance of OTFTs fabricated by the various integration schemes. The photolithography approach using indirect patterning with parylene buffer layer delivered the highest mobility, and thus, is currently the most robust method overall. However, inkjet printing presents a larger technological impact owing to its ease of patterning for the various device layers. Nonetheless, extensive optimization on printing parameters and processing conditions is needed to excel the performance of inkjet printed OTFTs. 
Table 3.4. Summary of strengths and weaknesses of the various OTFT integration approaches developed in this research. The approaches are grouped by the technique used for depositing/patterning organic layer: photolithography and inkjet printing.

\begin{tabular}{|c|c|c|c|}
\hline $\begin{array}{l}\text { Approaches to pattern } \\
\text { polymer semiconductor }\end{array}$ & Advantages & Challenges/Drawbacks & Reliability \\
\hline \multicolumn{4}{|l|}{ A. Photolithography } \\
\hline $\begin{array}{l}\text { A1) Direct patterning of } \\
\text { polymer semiconductor } \\
\text { (PQT-12) using photoresist }\end{array}$ & $\begin{array}{l}\text { One less deposition } \\
\text { step (compared to } \\
\text { indirect patterning) }\end{array}$ & $\begin{array}{l}\text { Adhesion issues between } \\
\text { PQT-12 and photoresist; } \\
\text { solvent processing may } \\
\text { affect PQT-12 }\end{array}$ & $\begin{array}{l}\text { Inconsistency } \\
\text { in } \\
\text { reproducibility }\end{array}$ \\
\hline $\begin{array}{l}\text { A2) Indirect patterning of } \\
\text { polymer semiconductor } \\
\text { using Parylene as } \\
\text { buffer/passivation layer }\end{array}$ & $\begin{array}{l}\text { Better processing } \\
\text { control and photoresist } \\
\text { handling than direct } \\
\text { patterning; highest } \\
\text { mobility amongst the } \\
\text { schemes reported }\end{array}$ & $\begin{array}{l}\text { Water/solvent may } \\
\text { penetrate under parylene } \\
\text { during photolithography } \\
\text { processing }\end{array}$ & Good \\
\hline $\begin{array}{l}\text { A3) Indirect patterning of } \\
\text { polymer semiconductor } \\
\text { using } \mathrm{SiN}_{\mathrm{x}} \text { as } \\
\text { buffer/passivation layer }\end{array}$ & $\begin{array}{l}\mathrm{SiN}_{\mathrm{x}} \text { is a good/stable } \\
\text { passivation material }\end{array}$ & $\begin{array}{l}\text { PECVD deposition of } \mathrm{SiN}_{\mathrm{x}} \\
\text { may induce damages in } \\
\text { polymer layer }\end{array}$ & Limited \\
\hline $\begin{array}{l}\text { B. Inkjet printing } \\
\text { (Hybrid photolithography- } \\
\text { inkjet printing scheme) }\end{array}$ & $\begin{array}{l}\text { Easiest patterning } \\
\text { method for organic } \\
\text { material; reduced } \\
\text { process complexity; } \\
\text { robust method. }\end{array}$ & $\begin{array}{l}\text { Resolution limitation; } \\
\text { uneven spreading of ink } \\
\text { may cause non-uniform } \\
\text { semiconductor in the TFT } \\
\text { channel }\end{array}$ & Good \\
\hline
\end{tabular}

Table 3.5. Comparing device performance of fully-patterned OTFTs fabricated by various approaches. Best values of field-effect mobility and on/off current ratio measured are shown.

\begin{tabular}{|lccc|}
\hline Approach & Mobility $\left(\mathrm{cm}^{2} / \mathrm{V}\right.$-s) & $\begin{array}{c}\text { On/off Current } \\
\text { Ratio }\end{array}$ & Remark \\
\hline $\begin{array}{l}\text { Photolithography: } \\
\begin{array}{l}\text { Direct patterning with } \\
\text { photoresist }\end{array}\end{array}$ & $0.003-0.008$ & $2.89 \times 10^{4}$ & $\begin{array}{c}\text { Low mobility and } \\
\text { on/off }\end{array}$ \\
\hline $\begin{array}{l}\text { Photolithography: } \\
\begin{array}{l}\text { Indirect patterning } \\
\text { with parylene buffer } \\
\text { layer }\end{array}\end{array}$ & $0.04-0.12$ & $1.89 \times 10^{6}$ & Highest mobility \\
\hline $\begin{array}{l}\text { Hybrid } \\
\text { photolithography and } \\
\text { inkjet-printing }\end{array}$ & $0.005-0.033$ & $1.84 \times 10^{7}$ & Highest On/off \\
\hline
\end{tabular}




\subsection{Summary and Contributions}

Establishment of robust and reliable OTFT integration strategies is a prerequisite for full-scale deployment of organic electronics manufacturing. The key challenge in this area is finding a reliable method to pattern functional organic materials. This chapter presents a number of OTFT fabrication schemes originated from this thesis work, which involved tailoring photolithography process for organic materials and exploring inkjet printing technique to enable robust and effective direct patterning of polymers. The research objective of this thesis is to exploit photolithography to enable immediate fabrication of highly integrated and high resolution OTFT circuits, allowing timely evaluation of device behavior and demonstration of integrated organic electronics. Inkjet printing is gradually incorporated into the fabrication process as the inkjet printing technology gains maturity and strength. The development of a hybrid photolithography-inkjet printing scheme serves as a technological bridge towards an all-printed fabrication process for the near future.

Key contributions highlighted in this chapter include:

- Development of integration schemes that enable fabrication of fully-patterned fullyencapsulated OTFTs and circuits.

- Design and formulation of an organic-friendly photolithography fabrication process, providing solutions that address concerns of chemical attacks and other process-induced damages to the organic layer.

- Demonstration of a hybrid photolithography-inkjet printing approach to allow robust fabrication of high-resolution OTFT devices. This development helps bridge the technological transfer to an all-printed process in the longer term.

- Realization of a variety of OTFT architectures using these integration schemes, including bottom-gate, top-gate and dual-gate OTFTs.

- Demonstration of the applicability and expandability of these integration strategies to other material systems (e.g., nanocomposite TFTs), providing a convenient platform to prototype and evaluate a wide range of new/novel material systems.

Table 3.6 presents an overall summary of the strengths and weaknesses of the patterning techniques considered in this thesis. A comparison of the pros and cons of the various techniques suggests that inkjet printing is the most promising method for patterning organic materials. However, additional research and development is needed to advance inkjet printing technique to facilitate well-controlled mass-production of high performance organic devices. Before inkjet 
printing technology can reach its full potential and maturity for electronics production, the hybrid photolithography-inkjet printing approach provides an appealing solution in the interim, as it enjoys the high resolution and registration advantages of photolithography, while benefitting from the convenient and robust deposition of patterned organic layer by inkjet printing. This hybrid scheme delivers delivering an integration strategy with workable manufacturing yields while lowering costs compared to conventional processes.

Table 3.6. Comparison of the strengths and drawbacks of the fabrication/patterning techniques considered in this thesis.

\begin{tabular}{|c|c|c|}
\hline $\begin{array}{l}\text { Patterning } \\
\text { Techniques }\end{array}$ & Strengths & Drawbacks \\
\hline $\begin{array}{l}\text { Shadow Mask } \\
\text { (Figure 3.2) }\end{array}$ & $\begin{array}{ll}\square & \text { Simple, robust process } \\
\square & \text { Enabled patterning of } \\
\text { evaporated source/drain } \\
\text { contacts \& organic films } \\
\square & \text { Cost-effective, large-area } \\
\square & \text { Direct deposition on the surface }\end{array}$ & $\begin{array}{ll}\otimes & \text { Limited feature resolution } \\
\otimes & \text { Difficult to pattern multi- } \\
\text { layers \& circuits } \\
\bigotimes & \text { Lack of alignment } \\
& \text { mechanism } \\
\bigotimes & \text { Not applicable solution- } \\
\text { processed polymers }\end{array}$ \\
\hline $\begin{array}{l}\text { Photolithography } \\
\text { (Figure 3.9, } \\
\text { Figure 3.19) }\end{array}$ & $\begin{array}{ll}\square & \text { High feature resolution } \\
\square & \text { Precise device definition } \\
\square & \text { Ease of integration, standard IC } \\
\text { compatible process } \\
\square & \text { Enable higher complexity } \\
\text { devices/circuits } \\
\square \quad \text { Mature technology } \rightarrow \text { provides a } \\
\text { good starting point for building } \\
\text { and studying OTFT circuits }\end{array}$ & $\begin{array}{ll} & \text { Higher process complexity } \\
\bigotimes & \begin{array}{l}\text { Issues with solvent } \\
\text { compatibility }\end{array} \\
\bigotimes & \begin{array}{l}\text { Requires a compatible and } \\
\text { robust masking layer }\end{array} \\
\text { Etching solutions and } \\
\text { developers may cause } \\
\text { damage to polymer films }\end{array}$ \\
\hline Inkjet printing & $\begin{array}{ll}\nabla & \text { Non-contact, additive printing } \\
& \text { process } \\
\square & \text { Direct patterning } \\
\square & \text { Minimum material consumption } \\
\square & \text { Low cost, fewer steps } \\
\square & \text { Custom circuit design }\end{array}$ & $\begin{array}{l}\otimes \\
\text { Typical system resolution: } \\
\sim 25 \mu \mathrm{m} \text {, limited by spreading } \\
\text { of ink droplet on the } \\
\text { substrate } \\
\bigotimes \quad \begin{array}{l}\text { Film non-uniformity and } \\
\text { discontinuity }\end{array} \\
\bigotimes \quad \begin{array}{l}\text { Requires precise control of } \\
\text { viscosity and careful choice } \\
\text { of solvents }\end{array}\end{array}$ \\
\hline $\begin{array}{l}\text { Hybrid } \\
\text { photolithography- } \\
\text { inkjet printing } \\
\text { (Figure 3.14) }\end{array}$ & $\begin{array}{ll}\square & \begin{array}{l}\text { Non-disruptive patterning of } \\
\text { polymer semiconductor layer by } \\
\text { inkjet printing }\end{array} \\
\square & \text { High resolution by } \\
\text { photolithography } \\
\square & \text { Bridges the transfer to an all- } \\
\text { printed fabrication process }\end{array}$ & $\begin{array}{l}\text { Reliant on photolithography } \\
\text { as a temporary solution for } \\
\text { defining critical dimensions } \\
\text { of the transistor. }\end{array}$ \\
\hline
\end{tabular}




\section{Bibliography}

[1] G. Horowitz, “Organic Field-Effect Transistors,” Adv. Mater. 10, 365 (1998).

[2] C.D. Dimitrakopoulos and D.J. Mascaro, "Organic thin-film transistors: A review of recent advances," IBM J. Res. \& Dev. 45(1), 111 (2001).

[3] I. Kymissis, C.D. Dimitrakopoulos, and S. Purushothaman, "Patterning pentacene organic thin film transistors," J. Vac. Sci. Technol. B 20(3), 956 (2002).

[4] H. Sirringhaus, T. Kawase, R.H. Friend, T. Shimoda, M. Inbasekaran, W. Wu, and E.P. Woo, "High-Resolution Inkjet Printing of All-Polymer Transistor Circuits," Science 290, $2123(2000)$.

[5] H. Jia, E.K. Gross, R. M. Wallace, and B.E. Gnade, "Patterning effects on poly(3hexylthiophene) organic thin film transistors using photolithographic processes," Organic Electronics 8, 44 (2007).

[6] T. Kawase, T. Shimoda, C. Newsome, H. Sirringhaus, and R.H. Friend, "Inkjet printing of polymer thin film transistors," Thin Solid Films 438, 279 (2003).

[7] G.E. Jabbour, R. Radspinner, and N. Peyghambarian, "Screen Printing for the Fabrication of Organic Light-Emitting Devices," IEEE J. Sel. Top. Quantum Electronics 7(5), 769 (2001).

[8] J.A. Rogers, Z. Bao, M. Meier, A. Dodabalapur, O.J.A. Schueller, and G.M. Whitesides, "Printing, molding, and near-field photolithographic methods for patterning organic lasers, smart pixels and simple circuits," Synthetic Metals 115, 5 (2000).

[9] J.A. Rogers, Z.N. Bao, V.R. Raju, "Nonphotolithographic fabrication of organic transistors with micron feature sizes," Appl. Phys. Lett. 72, 2716 (1998).

[10] M.L. Chabinyc, W.S. Wong, A. Salleo, K.E. Paul, and R.A. Street, "Organic polymeric thin-film transistors fabricated by selective dewetting," Appl. Phys. Lett. 81, 2460 (2002).

[11] S.M. Sze, VLSI Technology, McGraw-Hill, New York (1988).

[12] S.M. Sze, Semiconductor Devices, Physics and Technology, Wiley: New York (1985).

[13] Z. Bao, A. Dadabalapur, H.E. Katz, R.V. Raju and J.A. Rogers, "Organic Semiconductors for Plastic Electronics," IEEE-TAB New Technology Directions Committee (NTDC), 2001 IEEE Workshop on New and Emerging Technologies (2001).

[14] T.D. Anthopoulos, B. Singh, N. Marjanovic, N.S. Sariciftci, A.M. Ramil, H. Sitter, M. Colle, and D.M. de Leeuw, "High performance n-channel organic field-effect transistors and ring oscillators based on $\mathrm{C}_{60}$ fullerene films," Appl. Phys. Lett. 89, 213504 (2006).

[15] S.P. Tiwari, E.B. Namdas, V. Ramgopal Rao, D. Fichou, and S.G. Mhaisalkar, S.G., "Solution-Processed n-Type Organic Field-Effect Transistors With High on /off Current Ratios Based on Fullerene Derivatives," IEEE Electron Dev. Lett. 28, 880 (2007).

[16] A. Afzali, C.D. Dimitrakopoulos and T.L. Breen, "High-Performance, Solution-Processed Organic Thin Film Transistors from a Novel Pentacene Precursor,” J. Am. Chem. Soc. 124, 8812 (2002). 
[17] H. Sirringhaus, P.J. Brown, R.H. Friend, M.M. Nielsen, K. Bechgaard, B.M.W. LangeveldVoss, A.J.H. Spiering, R.A.J. Janssen, E.W. Meijer, P.T. Herwig, and D.M. de Leeuw, "Two-Dimensional Charge Transport in Self-Organized, High-Mobility Conjugated Polymers," Nature 401, 685 (1999).

[18] E. Menard, M.A. Meitl, Y. Sun, J.U. Park, D. J. Shir, Y.S. Nam, S. Jeon, and J.A. Rogers, "Micro- and Nanopatterning Techniques for Organic Electronic and Optoelectronic Systems," Chem. Rev. 107, 1117 (2007).

[19] P.F. Tian, V. Bulovic, P.E. Burrows, G. Gu, S.R. Forrest, and T.X. Zhou, "Precise Scalable Patterning of Vacuum-Deposited Organic Light Emitting Devices," J. Vac. Sci. Technol., A 17, 2975 (1999).

[20] C. Bartic, H. Jansen, A. Campitelli, and S. Borghs, " $\mathrm{Ta}_{2} \mathrm{O}_{5}$ as gate dielectric material for low-voltage organic thin-film transistors," Organic Electronics 3, 65 (2002).

[21] S.R. Forrest, "The path to ubiquitous and low-cost organic electronic appliances on plastic," Nature 428, 911 (2004).

[22] M.M. Ling, and Z.N., Bao, "Thin film deposition, patterning, and. printing in organic thin film transistors," Chem. Mater. 16, 4824 (2004).

[23] S.M. Yi, S.H. Jin, J.D. Lee, and C.N. Chu, "Fabrication of a high-aspect-ratio stainless steel shadow mask and its application to pentacene thin-film transistors," J. Micromech. Microeng. 15, 263 (2005).

[24] P.F. Baude, D.A. Ender, T.W. Kelley, D.V. Muyres, and S.D. Theiss, "Pentacene-based RFID transponder circuitry," 62nd Device Research Conference (DRC) Digest, pp. 227228 (2004)

[25] F.M. Li, Y. Vygranenko, S. Koul, and A. Nathan. "Photolithographically Defined Polythiophene OTFTs.” J. Vac. Sci. Technol. A 24, 657 (2006).

[26] F.M. Li, S. Koul, Y. Vygranenko, A. Sazonov, P. Servati and A. Nathan. "Fabrication of RR-P3HT-based TFTs using low-temperature PECVD silicon nitride." Mater. Res. Soc. Symp. Proc. 871E, I9.3.1 (2005).

[27] F.M. Li, S. Koul, Y. Vygranenko, P. Servati and A. Nathan. (2005) "Dual-Gate $\mathrm{SiO}_{2} / \mathrm{P} 3 \mathrm{HT} / \mathrm{SiN}_{\mathrm{x}}$ OTFT.” Mater. Res. Soc. Symp. Proc. 871E, I9.4.1 (2005).

[28] (a) http://www.conformal-coating.com/parylene_coating.htm.

(b) http://www.scscookson.com/parylene_knowledge/specifications.cfm

[29] “Ink-Jet Printing”, Hilord Chemical Corporation, (2003). http://www.hilord.com/InkJet.htm

[30] “UK in plastic electronics drive," BBC News, 3 January 2007. http://news.bbc.co.uk/2/hi/business/6227575.stm

[31] P. Calvert, "Inkjet Printing for Materials and Devices," Chem. Mater. 13, 3299 (2001).

[32] "Plastic Electronics," MicroDevice Science (MDS) Ltd. (2003). http://www.mdslsolutions.com/plastronics.htm

[33] J. Zhang, P. Brazis, A.R. Chowdhuri, J. Szczech and D. Gamota, "Investigation of Using Contact and Non-contact Printing Technologies For Organic Transistor Fabrication," Mat. Res. Soc. Symp. Proc. 725, 6.3.1 (2002). 
[34] Z. Bao, “Conducting Polymers - Fine Printing," Nature Materials 3, 137 (2004).

[35] J.Z. Wang, Z.H. Zheng, H.W. Li, W.T.S. Huck and H. Sirringhaus, "Dewetting of conducting polymer inkjet droplets on patterned surfaces," Nature Mater. 3, 171 (2004).

[36] T. Shimoda, K. Morii, S. Seki and H. Kiguchi, "Inkjet Printing of Light-Emitting Polymer Displays," MRS Bull. 28, 821-827. (2003).

[37] T.R. Hebner, C.C. Wu, D. Marcy, M.H. Lu and J.C. Sturm, "Ink-jet printing of doped polymers for organic light emitting devices," Appl. Phys. Lett. 72, 519 (1998).

[38] D. Kim, S. Jeong, S. Lee, B.K. Park, and J. Moon, J. "Organic thin film transistor using silver electrodes by the ink-jet printing technology," Thin Solid Films 515, 7692 (2007).

[39] H. Sirringhaus, T. Kawase and R.H. Friend, "High-Resolution Ink-Jet Printing of AllPolymer Transistor Circuits," MRS Bull. 26, 539 (2001).

[40] E.S. Burns, P. Cain, J. Mills, J. Wang and H. Sirringhaus, "Inkjet Printing of Polymer Thin-Film Transistor Circuits," MRS Bull. 28, 829 (2003).

[41] S.S. Speakman, G.G. Rozenberg, K.J. Clay, W.I. Milne, A. Ille, I.A. Gardner, E. Bresler and J.H.G. Steinke, "High performance organic semiconducting thin films: ink jet printed polythiophenes [rr-P3HT]," Organ. Electron. 2, 65 (2001).

[42] M. Plötnera, T. Wegener, S. Richter, S. Howitz, and W.J. Fischer "Investigation of ink-jet printing of poly-3-octylthiophene for organic field-effect transistors from different solutions," Syn. Metals 147, 299 (2004).

[43] A.C. Arias, J.H. Daniel, F. Endicott, R.A. Lujan, B.S. Krusor, "Flexible (ink) jet printed backplanes for displays: materials development and integration," Spring 2006 Meeting of the Materials Research Society, San Francisco; CA; USA (2006).

[44] F.M. Li, P. Dhagat, G.E. Jabbour, H.M. Haverinen, I. McCulloch, M. Heeney, and A. Nathan, "Polymer TFT without Surface Pre-Treatment on Silicon Nitride Gate Dielectric," Appl. Phys. Lett. 92, 3 pages (2008). (Article in Press).

[45] P. Beecher, P. Servati, A. Rozhin, A. Colli, V. Scardaci, S. Pisana, T. Hasan, A. J. Flewitt, J. Robertson, G. W. Hsieh , F. M. Li, A. Nathan, A. C. Ferrari and W. I. Milne, Ink-jet printing of carbon nanotube thin film transistors", J. Appl. Phys. 102, 043710 (2007).

[46] G.W. Hsieh, P. Beecher, F.M. Li, P. Servati, A. Colli, A. Fasoli, D. Chu, A. Nathan, B. Ong, J. Robertson, A.C. Ferrari, and W.I. Milne, "Formation of composite organic thin film transistors with nanotubes and nanowires," Physica E: Low-dimensional Systems and Nanostructures (2008). (Article in Press).

[47] (a) Dimatix Fujifilm. Dec 20, 2007. http://www.dimatix.com/.

(b) Dimatix Materials Printer Manual, pg 92-93.

[48] M. Kaneda, H. Ishizuka, Y. Sakai, J. Fukai, S. Yasutake, and A. Takahara, "Film Formation from Polymer Solution Using Inkjet Printing Method," AIChE Journal 53, 1100 (2007).

[49] C. McArthur, M. Meitine, and A. Sazonov, "Optimization of $75^{\circ} \mathrm{C}$ amorphous silicon nitride for TFTs on plastics,” Mater. Res. Soc. Proc. 769, 303 (2003). 
[50] M. Raja, G. Lloyd, N. Sedghi, S.J. Higgins, and B. Eccleston, "Critical considerations in polymer thin-film transistor (TFT) dielectrics,” Mater. Res. Soc. Symp. Proc. 725, P6.5.1 (2002).

[51] G. Lloyd, M. Raja, I. Sellers, N. Sedghi, R.D. Lucrezia, S. Higgins, and B. Eccleston, "The properties of MOS structures using conjugated polymers as the semiconductor," Microelec. Eng. 59, 323 (2001).

[52] S. Iba, T. Sekitani, Y. Kato, T. Someya, H. Kawaguchi, M. Takamiya, T. Sakuri, and S. Takagi, "Control of threshold voltage of organic field-effect transistors with double-gate structures," Appl. Phys. Lett. 87, 023509 (2005).

[53] Z. Bao, A. Dodabalapur, and A. J. Lovinger, "Soluble and Processable Regioregular Poly(3-hexylthiophene) for Thin Film Field-Effect Transistor Applications with High Mobility," Appl. Phys. Lett. 69, 4108 (1996).

[54] H. Sirringhaus, N. Tessler, and R.H. Friend, "Integrated Optoelectronic Devices Based On Conjugated Polymers," Science 280, 1741 (1998).

[55] J.I. Han, Y.H. Kim, S.K. Park, D.G. Moon, and W.K. Kim, "Stability of Organic Thin Film Transistors," Mat. Res. Soc. Symp. Proc. 814, I4.3.1 (2004).

[56] N. Kawashima, K. Nomoto, M. Wada, and J. Kasahara, "Organic-Inorganic Hybrid Encapsulation for P3HT Field-Effect Transistors," Mater. Res. Soc. Symp. Proc. 817E, I1.10.1 (2005). 


\section{Chapter 4}

\section{Gate Dielectric by Plasma Enhanced Chemical Vapor Deposition (PECVD)}

The demands on the gate dielectric layer in OTFTs are multiple, as summarized in Section 2.4.2. For instance, the material should form pinhole free film with high breakdown voltage and long term stability. Leakage current through the dielectric layer should be as low as possible to ensure low power consumption of the device. High dielectric constant is desirable for low-voltage operation and high current output. In the case of bottom-gate OTFTs, the dielectric layer presents a platform for the growth/formation of the organic semiconductor layer; thus, the surface properties of the gate dielectric play an important role in determining the molecular orientation, ordering, and microstructure of the organic semiconductor layer. These physical attributes are closely linked to the electrical properties of the semiconductor layer, which in turn influence the OTFT performance (e.g., field effect mobility, threshold voltage, on/off current ratio).

A key focus of this research is to explore the use of plasma enhanced chemical vapor deposited (PECVD) silicon nitride $\left(\mathrm{SiN}_{\mathrm{x}}\right)$ and silicon oxide $\left(\mathrm{SiO}_{\mathrm{x}}\right)$ films as gate dielectrics for OTFTs. PECVD dielectric materials have a number of appealing processing attributes, including relatively low deposition temperatures, large-area deposition capability, and high throughput. These attributes align with the technological drivers of organic electronics for low-cost flexible electronics application. Since the processing temperatures of most low-cost plastic substrates (e.g., PET, PEN, Kapton) are constrained to $150-200^{\circ} \mathrm{C}$ or below [1], materials deposited at plastic-compatible temperatures are a requisite for realization of flexible circuits. 
PECVD $\operatorname{SiN}_{\mathrm{x}}$ is particularly attractive because it possesses excellent dielectric properties and good dielectric strength. More importantly, $\mathrm{SiN}_{\mathrm{x}}$ has been a prevalent choice of gate and passivation dielectric material for active matrix thin film transistor (TFT) backplanes for flat panel displays and imagers, demonstrating the reliability and technological maturity of this material [2][3][4]. PECVD $\mathrm{SiO}_{\mathrm{x}}$ presents another gate dielectric option, offering the potential for lowtemperature and large-area deposition. By comparison, low temperature $\operatorname{SiN}_{x}$ surpasses low temperature $\mathrm{SiO}_{\mathrm{x}}$ in terms of electrical integrity with lower leakage current, fewer pinholes, higher breakdown field, higher stability, and less hysteresis [5]. Thus, the principal focus of our investigation is on the development of PECVD $\mathrm{SiN}_{\mathrm{x}}$ for OTFTs. Preliminary study on the application of PECVD $\mathrm{SiO}_{\mathrm{x}}$ gate dielectric in OTFTs is also conducted. In the past, researchers have reported limited OTFT performance with $\mathrm{SiN}_{\mathrm{x}}$ gate dielectric, which was attributed to surface roughness and unfriendly (or non-organic-friendly) interfaces of $\operatorname{SiN}_{\mathrm{x}}$. To enable pairing of $\mathrm{SiN}_{\mathrm{x}}$ with organic semiconductors and to enhance performance of related devices, our research approach is geared towards finding an organic-compatible $\mathrm{SiN}_{\mathrm{x}}$ film composition (or recipe) and developing a complementary interface treatment strategy. These are, in fact, the objectives for the dielectric investigation in this chapter and the interface engineering study in the next chapter.

Experimental details for our gate dielectric investigation are presented in Section 4.1, which include an overview of the PECVD $\mathrm{SiN}_{\mathrm{x}}$ deposition conditions and the analytical tools used for material characterization. The material properties of PECVD $\mathrm{SiN}_{\mathrm{x}}$ thin films are examined in Section 4.2, which provides a basis for analyzing the impact of different $\mathrm{SiN}_{\mathrm{x}}$ dielectrics on OTFT device performance. Section 4.3 investigates the electrical characteristics of PQT-12 OTFTs with different PECVD gate dielectrics. The investigation consists of four experimental components. The first experiment considers $\mathrm{SiN}_{\mathrm{x}}$ films of varying stoichiometry deposited at a substrate temperature of $300^{\circ} \mathrm{C}$ and evaluates the impact of film composition on the electrical performance of OTFTs; these results are reported in Section 4.3.1. Gate dielectrics deposited at a lower temperature is desirable for integration with plastic substrates for flexible electronic applications. $150^{\circ} \mathrm{C} \mathrm{SiN}$ gate dielectrics are assessed in Section 4.3.2, and the resulting device characteristics are compared with devices based on $300^{\circ} \mathrm{C} \operatorname{SiN}_{x}$ gate dielectric. The concept of stacked $\mathrm{SiN}_{\mathrm{x}}$ gate dielectric is explored in Section 4.3.3. OTFTs with PECVD $\mathrm{SiO}_{\mathrm{x}}$ gate dielectric are examined in Section 4.3.4. Finally, preliminary OTFTs fabricated on flexible plastic substrates are demonstrated in Section 4.3.5. 


\subsection{Experimental Details and Characterization Methods}

Amorphous hydrogenated silicon nitride films (abbreviated as $a-\mathrm{SiN}_{\mathrm{x}}: \mathrm{H}$ or $\mathrm{SiN}_{\mathrm{x}}$ ) are extensively used as dielectric or passivation materials in semiconductor device applications, including thin-film transistors (TFT), optoelectronic devices, metal-insulator-semiconductor (MIS) devices, and solar cells [6]-[11]. PECVD is a well-established technique for the deposition of $\mathrm{SiN}_{\mathrm{x}}$ films $[1][3][4][12]$. PECVD technology can accommodate low deposition temperatures and large area depositions, thus it presents exceptional potential for flexible and organic electronics fabrication. In TFT applications, PECVD $\mathrm{SiN}_{\mathrm{x}}$ has demonstrated excellent matching as gate dielectric for noncrystalline silicon TFTs (e.g., amorphous silicon, polycrystalline silicon, nanocrystalline silicon TFTs) [1][3][4][13]. For these silicon-based TFTs, nitrogen-rich $\mathrm{SiN}_{\mathrm{x}}$ gate dielectric (i.e., [N]/[Si] $>1.33$ ) is more preferable as it provides better interface properties with silicon thin films than silicon-rich $\operatorname{SiN}_{\mathrm{x}}[14]$.

This chapter examines the application of PECVD $\mathrm{SiN}_{\mathrm{x}}$ as gate dielectric for OTFTs. In particular, $\mathrm{SiN}_{\mathrm{x}}$ films of varying compositions were investigated to determine an optimal choice for organic semiconductor devices. The deposition conditions for $\mathrm{SiN}_{\mathrm{x}}$ films used in our experiment are identified in Section 4.1.1. The analytical tools and characterization techniques used to study the material properties of various $\mathrm{SiN}_{\mathrm{x}}$ films are discussed in Section 4.1.2.

\subsubsection{Deposition Conditions of PECVD Silicon Nitride $\left(\mathrm{SiN}_{\mathrm{x}}\right)$}

The $\mathrm{SiN}_{\mathrm{x}}$ films were deposited by a multi-chamber 13.56 MHz PECVD cluster tool with load lock, manufactured by MVSystems, Inc., Golden, CO. Two deposition temperatures were considered in this experiment: $300^{\circ} \mathrm{C}$ and $150^{\circ} \mathrm{C}$; the deposition conditions are summarized in Table 4.1. At a given substrate temperature, $\mathrm{SiN}_{\mathrm{x}}$ films of varying compositions were obtained by adjusting the ammonia $\left(\mathrm{NH}_{3}\right)$ to silane $\left(\mathrm{SiH}_{4}\right)$ gas flow ratio from 5 to 20 . All other deposition parameters (i.e., RF power and pressure) were fixed at values optimized for the standard recipe with $\mathrm{NH}_{3} / \mathrm{SiH}_{4}$ gas flow ratio of 20; this standard recipe gives optimal $\mathrm{SiN}_{\mathrm{x}}$ for non-crystalline silicon-based device applications. 
Table 4.1. Deposition conditions for PECVD $\operatorname{SiN}_{x}$ films prepared at substrate temperature of $300^{\circ} \mathrm{C}$ and $150^{\circ} \mathrm{C}$. SiN films were deposited in a parallel-plate PECVD reactor operating at an excitation frequency of $13.56 \mathrm{MHz}$.

\begin{tabular}{|cccccc|}
\hline $\begin{array}{c}\text { Substrate } \\
\text { Temperature }\left({ }^{\circ} \mathrm{C}\right)\end{array}$ & $\begin{array}{c}\text { Pressure } \\
\text { (mTorr) }\end{array}$ & $\begin{array}{c}\text { RF Power } \\
(\mathrm{W})\end{array}$ & $\begin{array}{c}\text { Duration } \\
\text { (min.) }\end{array}$ & $\begin{array}{c}\text { Gas Mixtures } \\
(\mathrm{sccm})\end{array}$ & $\begin{array}{c}\mathrm{NH}_{3} / \mathrm{SiH}_{4} \\
\text { Gas Flow Ratio }\end{array}$ \\
\hline 300 & 400 & 2 & 30 & $\begin{array}{c}\mathrm{NH}_{3}: 25-100 \mathrm{sccm}, \\
\mathrm{SiH}_{4}: 5 \mathrm{sccm}\end{array}$ & $20,15,10,5$ \\
150 & 1000 & 15 & 30 & $\begin{array}{c}\mathrm{NH}_{3}: 10-40 \mathrm{sccm}, \\
\mathrm{SiH}_{4}: 2 \mathrm{sccm}, \\
\mathrm{H}_{2}: 80 \mathrm{sccm}\end{array}$ & $20,15,10,5$ \\
\hline
\end{tabular}

\subsubsection{Thin Film Characterization Methods}

A number of analytical tools were used to characterize the structural and surface properties of the PECVD SiN $_{\mathrm{x}}$ dielectric films. A brief description of these tools is presented in this section. Fouriertransform infrared (FTIR) spectroscopy, ellipsometer, and x-ray photoelectron spectroscopy (XPS) are used to measure bulk properties of $\mathrm{SiN}_{\mathrm{x}}$ films. Atomic force microscopy (AFM), contact angle, and XPS are used for interface characterization. For the purpose of OTFT integration, interfacial properties play a crucial role in establishing device performance. Since the organic semiconductor layer is deposited on the dielectric surface under the influence of the physical and chemical interactions between organic and dielectric layer, the OTFT performance depends strongly on the semiconductor/dielectric interface properties.

\subsubsection{Fourier Transform Infrared Spectroscopy (FTIR)}

Chemical bonding structure of the $\mathrm{SiN}_{\mathrm{x}}$ films were studied by Fourier-transform infrared (FTIR) spectroscopy, using a Shimadzu FTIR-8400S spectrometer in transmission mode at normal incidence. FTIR measurements were done to examine changes in relative content of Si-H and N-H bonds in $\mathrm{SiN}_{\mathrm{x}}$ films as the deposition conditions are varied.

\subsubsection{Ellipsometry}

Ellipsometry was used to determine thickness and optical constants (refractive index $n$, coefficient of absorption $k$ ) of the PECVD $\operatorname{SiN}_{\mathrm{x}}$ thin films. Measurements were done using a WVASE32 spectroscopic ellipsometer, by J.A.Woollam Co., Inc. The measured refractive index was used to 
estimate the nitrogen-to-silicon ratio, $x=[\mathrm{N}] /[\mathrm{Si}]$, of the $\mathrm{SiN}_{\mathrm{x}}$ films; the procedure is described in Section 4.2.1.3.

\subsubsection{X-ray Photoelectron Spectroscopy (XPS)}

XPS, also known as Electron Spectroscopy for Chemical Analysis (ESCA), is a surface sensitive chemical analysis technique that can be used to analyze the surface chemistry of a material. It is a quantitative spectroscopic technique that measures the elemental composition, empirical formula, chemical state and electronic state of the elements that exist within a material's surface. In this chapter, XPS is used to study the surface chemical composition (i.e., elemental concentration) of $\mathrm{SiN}_{\mathrm{x}}$ thin films with varying compositions. In Chapter 5 and Chapter 6, XPS measurements are used to characterize changes in surface chemical composition after various types of surface treatments. The depth profile of $\mathrm{SiN}_{\mathrm{x}}$ samples was analyzed by in situ XPS ion beam sputtering with argon (Ar). The results are reported in Section 5.4.2, where the changes in elemental concentrations of oxygen $\left(\mathrm{O}_{2}\right)$ plasma treated $\mathrm{SiN}_{\mathrm{x}}$ samples were analyzed as a function of depth (or etch time) by interleaving XPS analysis and Argon ion sputtering at $3 \mathrm{keV}$. XPS measurements were obtained using VG Scientific ESCALab 250, with a monochromatic Al KR X-ray source $(1486.6 \mathrm{eV})$.

\subsubsection{Atomic Force Microscopy (AFM)}

The film surface morphology was studied using AFM with a Veeco Digital Instruments Dimension 3100 scanning probe microscope (SPM). The film surface was scanned using tapping mode. In this thesis, AFM was primarily used to characterize the surface roughness of the gate dielectric layer under various deposition and treatment conditions.

\subsubsection{Contact Angle Analysis}

Contact angle analysis characterizes the wettability (or surface energy) of a surface by measuring the surface tension of a solvent droplet at its interface with a homogenous surface. More specifically, contact angle measures the attraction of molecules within the droplet to each other versus the attraction or repulsion those droplet molecules experience towards the surface molecules. The contact angle is defined as the angle between the tangent to the drop's profile and 
the tangent to the surface at the intersection of the vapor, the liquid, and the solid, as illustrated in Figure 4.1. A large contact angle means that the surface is hydrophobic and has a low surface energy (Figure 4.1(a)). Contrarily, a small contact angle between solid surface and droplet indicates that the surface is hydrophilic and has a high surface energy (Figure 4.1(b)). Depending on the geometry and location of surface to be analyzed, a number of different techniques are available for contact angle characterization. The most commonly used technique is the static or sessile drop method, which is the chosen technique for this research.

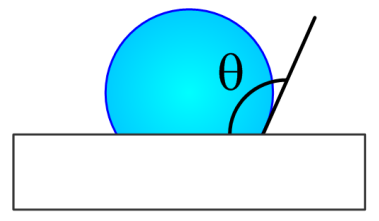

(a) Hydrophobic (large $\theta$ )

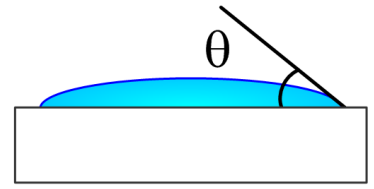

(b) Hydrophilic (small $\theta$ )

Figure 4.1. Contact angle ( $\theta)$ of surfaces with different wettability: (a) water droplet on a hydrophobic surface showing large contact angle, and (b) water droplet on a hydrophilic surface showing small contact angle.

Contact angle measurement provides a sensitive method for the detection and identification of functional groups on surface layer [15]. Hydrophobicity is a repulsive force between non-polar molecules and water, and is often linked to side chains of alkyl $(\mathrm{C}-\mathrm{H})$ groups. Thus, organic/polymer surfaces are typically hydrophobic. On the other hand, hydrophilicity of a surface is related to its affinity to water and its ability to form H-bonds, where polar components typically lead to hydrophilic properties. Glass, $\mathrm{SiO}_{2}$, and $\mathrm{SiN}_{\mathrm{x}}$ surfaces are typically hydrophilic. Also, oxygen plasma treatment results in hydrophilic surface with a decrease in water contact angle; this is due to the formation of polar component on the exposed surface.

Contact angle provides a measure of surface energy or tension. Low surface energy is preferable for organic thin film deposition because less energy is required for molecules to assemble into an energetically-stable and well-ordered film. There is a general consensus that a dielectric with low surface energy (i.e., large contact angle) is favorable for the subsequent growth/deposition of the organic semiconductor layer. Therefore, researchers experimented with a variety of interface treatment methods with the goal to increase the contact angle (or hydrophobicity) of the surface. In this thesis, contact angle is used to study the surface wettability of various types of gate dielectric 
and surface treatments. This information, along with other surface characterization data, is used to explain and analyze the OTFTs' electrical characteristics.

\subsection{Material Characterization of PECVD $\operatorname{SiN}_{\mathrm{x}}$ Films}

This section reports the material properties of various PECVD $\operatorname{SiN}_{\mathrm{x}}$ films. The discussion is grouped into three categories: bulk/structural properties (Section 4.2.1), surface properties (Section 4.2.2), and electrical properties (Section 4.2.3). In terms of bulk or structural properties, the chemical bonding structure of the $\mathrm{SiN}_{\mathrm{x}}$ films was studied FTIR spectroscopy. Refractive index was measured by ellipsometry. Elastic recoil detection analysis (ERDA) provided another method to evaluate chemical composition and relative atomic densities of the $\operatorname{SiN}_{x}$ films. The $\operatorname{SiN}_{x}$ film composition, denoted by nitrogen-to-silicon ratio $x=[\mathrm{N}] /[\mathrm{Si}]$, was estimated from refractive index and ERDA data. Film thickness and surface profiles were measured using Dektak 8 stylus profilometer (Veeco Instruments Inc.), after etching $\mathrm{SiN}_{\mathrm{x}}$ to form step profiles.

Surface wettability is characterized by sessile drop contact angle measurements. Surface topography (including surface roughness) was characterized AFM. Surface chemical composition was analyzed by XPS.

Electrical properties of $\mathrm{SiN}_{\mathrm{x}}$ films were measured on metal-insulator-semiconductor (MIS) structures, using a Keithley 4200 semiconductor characterization system. Parameters of interest include dielectric leakage current, breakdown field, and dielectric constant.

\subsubsection{Bulk/Structural Characterization}

\subsubsection{FTIR Spectroscopy}

The FTIR spectra of $300^{\circ} \mathrm{C}$ and $150^{\circ} \mathrm{C} \mathrm{SiN}_{\mathrm{x}}$ samples are presented in Figure 4.2 and Figure 4.3, respectively. The main absorption peaks visible in the spectra are due to Si-N bond stretching at $830-900 \mathrm{~cm}^{-1}$, Si-H bond stretching at $2150-2180 \mathrm{~cm}^{-1}$, and $\mathrm{N}-\mathrm{H}$ bond stretching at 3340 $3350 \mathrm{~cm}^{-1}$. As shown in Figure 4.2 and Figure 4.3, a decrease in $\mathrm{NH}_{3} / \mathrm{SiH}_{4}$ gas flow ratio appears to strengthen the $\mathrm{Si}-\mathrm{H}$ bond stretching mode, but weaken the N-H bond stretching mode, signifying a change in film composition as the gas ratio is varied. 


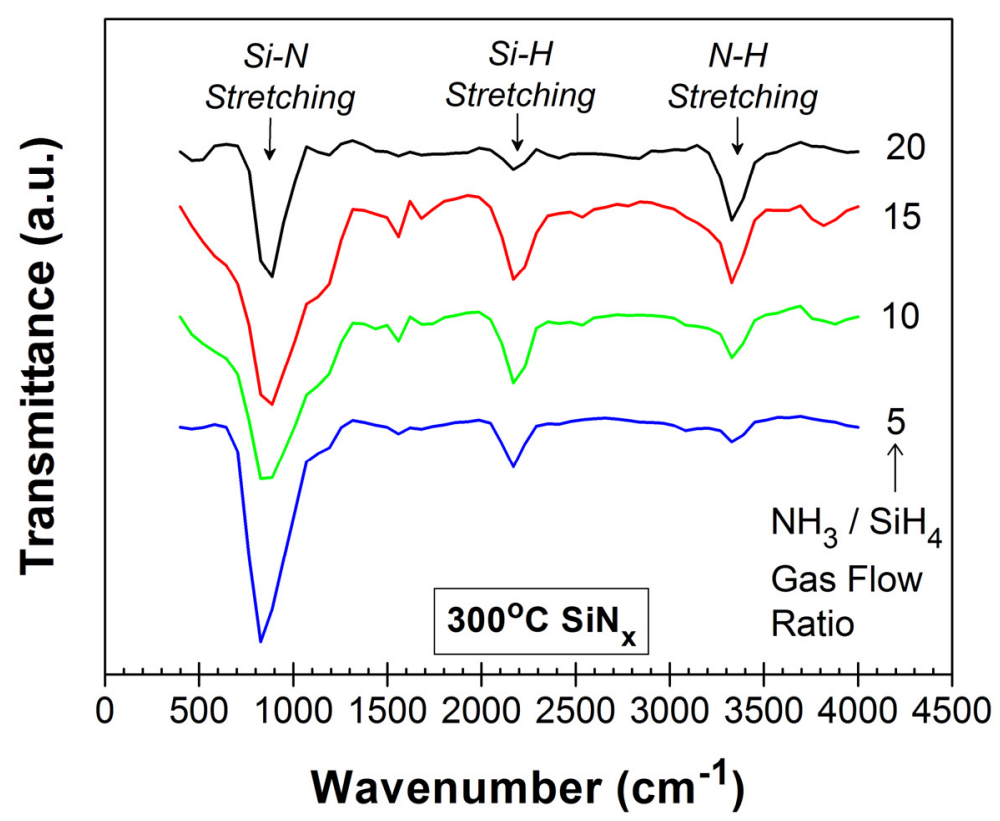

Figure 4.2. Fourier transform infrared (FTIR) spectroscopy of the $300^{\circ} \mathrm{C} \mathrm{PECVD} \mathrm{SiN}_{\mathrm{x}}$ films.

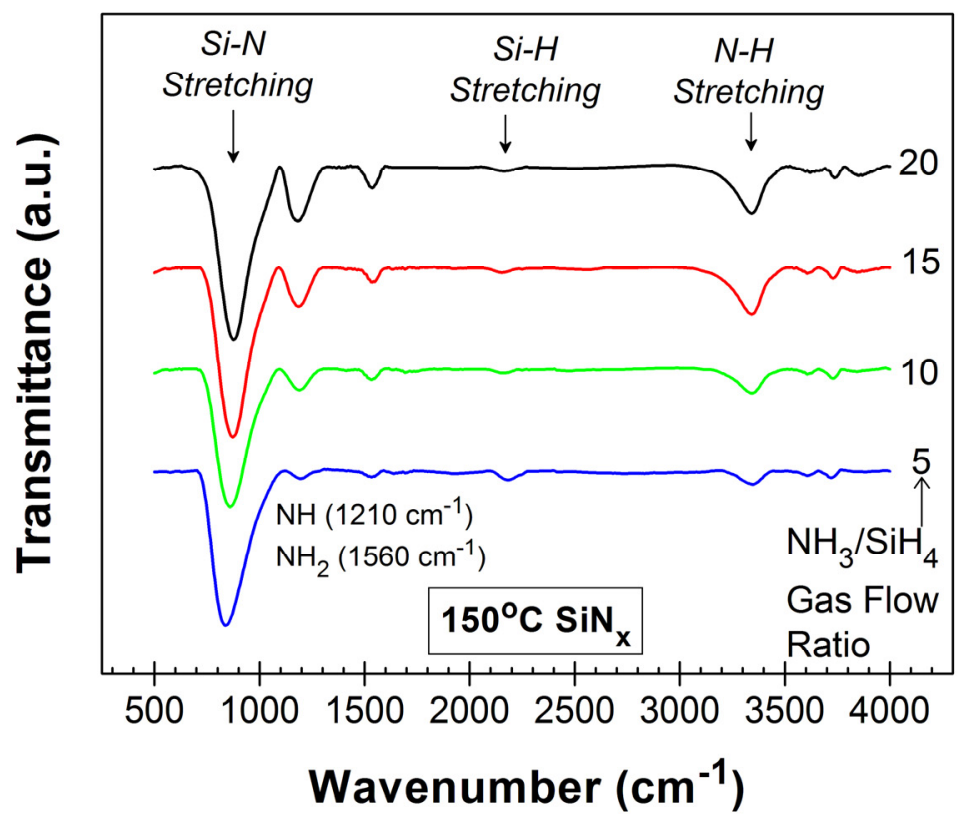

Figure 4.3. Fourier transform infrared (FTIR) spectroscopy of the $150^{\circ} \mathrm{C}$ PECVD SiN films. 
The $\mathrm{N}-\mathrm{H}$ and $\mathrm{Si}-\mathrm{H}$ bond densities were estimated following the method reported by Landford and Rand [16]. ${ }^{9}$ Figure 4.4 shows the normalized N-H and Si-H bond concentrations as a function of $\mathrm{NH}_{3} / \mathrm{SiH}_{4}$ gas flow ratio for films deposited at $150^{\circ} \mathrm{C}$. For higher values of $\mathrm{NH}_{3} / \mathrm{SiH}_{4}$ gas flow ratio, there is an increase in N-H bond concentration, but a decrease in Si-H bond concentration. This suggests that with increasing $\mathrm{NH}_{3} / \mathrm{SiH}_{4}$ gas flow ratio, the $\mathrm{SiN}_{\mathrm{x}}$ films are becoming more nitrogen-rich. Furthermore, Figure 4.4 shows that the N-H bond concentration is higher than Si-H bond concentration; this is in agreement with the data shown in Figure 4.8, where $150^{\circ} \mathrm{C} \mathrm{SiN}_{\mathrm{x}}$ samples are characterized by $[\mathrm{N}] /[\mathrm{Si}]>1$.

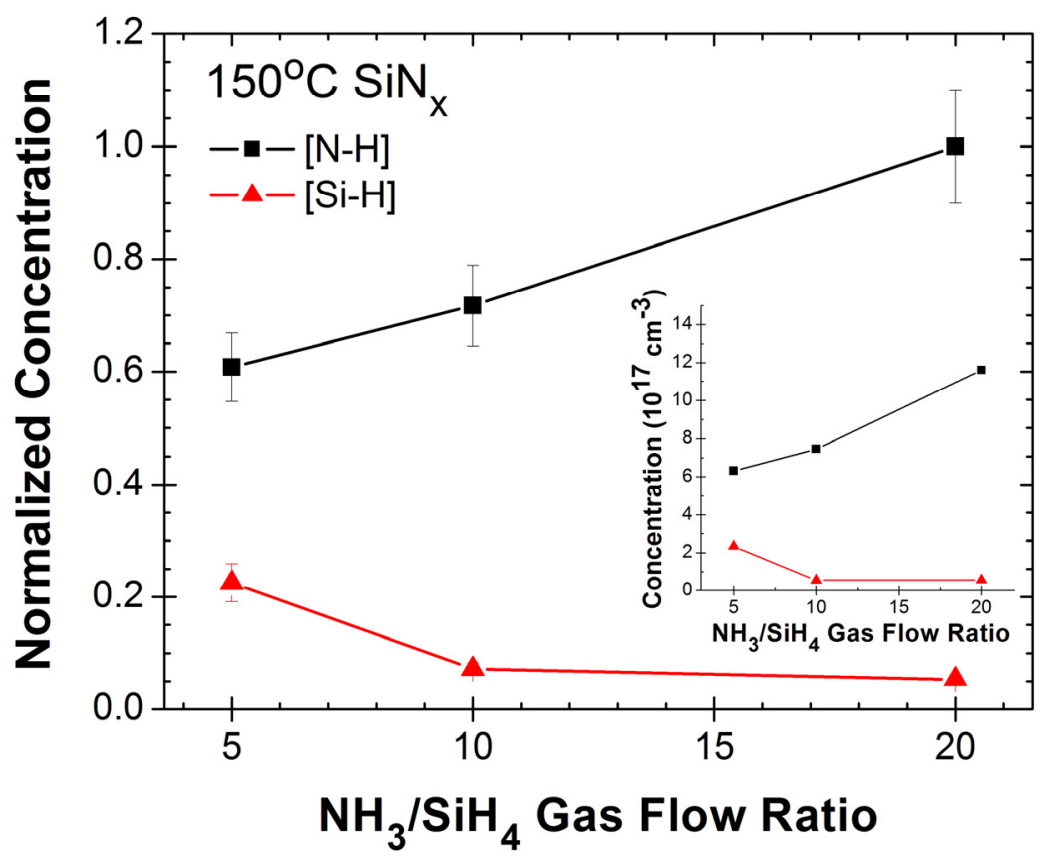

Figure 4.4. Normalized $\mathrm{N}-\mathrm{H}$ and $\mathrm{Si}-\mathrm{H}$ bond concentration as a function of $\mathrm{NH}_{3} / \mathrm{SiH}_{4}$ gas flow ratio for $\mathrm{SiN}_{\mathrm{x}}$ films deposited at $150^{\circ} \mathrm{C}$. Data is normalized to $[\mathrm{N}-\mathrm{H}]$ value for the sample deposited with $\mathrm{NH}_{3} / \mathrm{SiH}_{4}$ gas flow ratio of 20 .

The deposition recipes for $300^{\circ} \mathrm{C} \mathrm{SiN}$ films and $150^{\circ} \mathrm{C} \mathrm{SiN}$ films yield different concentration of $\mathrm{N}-\mathrm{H}$ and $\mathrm{Si}-\mathrm{H}$ bonds, as shown in Figure 4.5. At a given value of $\mathrm{NH}_{3} / \mathrm{SiH}_{4}$ gas flow ratio, $300^{\circ} \mathrm{C} \mathrm{SiN}{ }_{x}$ is characterized by a higher $\mathrm{Si}-\mathrm{H}$ bond concentration than $150^{\circ} \mathrm{C} \mathrm{SiN}_{\mathrm{x}}$, accompanied

\footnotetext{
${ }^{9}$ The extraction method was developed for a certain set of calibration factors. Thus, the calculated absolute bond density values may exhibit a certain degree of error. Nonetheless, the relative change in bond densities can still provide useful information on film properties.
} 
by a slight reduction in $\mathrm{N}-\mathrm{H}$ bond concentration. This observation coincides with the larger $\mathrm{Si}-\mathrm{H}$ peak observed in the FTIR spectra of $300^{\circ} \mathrm{C} \mathrm{SiN}_{\mathrm{x}}$ in Figure 4.2 when compared to the FTIR spectra for $150^{\circ} \mathrm{C} \mathrm{SiN}_{\mathrm{x}}$ in Figure 4.3. This observation also matches with the nitrogen-to-silicon $(x=$ $[\mathrm{N}] /[\mathrm{Si}]$ ) value extracted based on refractive index in Figure 4.8. The consistent trends observed from the various characterization methods substantiate the relative changes in nitrogen and silicon content as deposition conditions (i.e., gas flow ratio, substrate temperature) are varied.

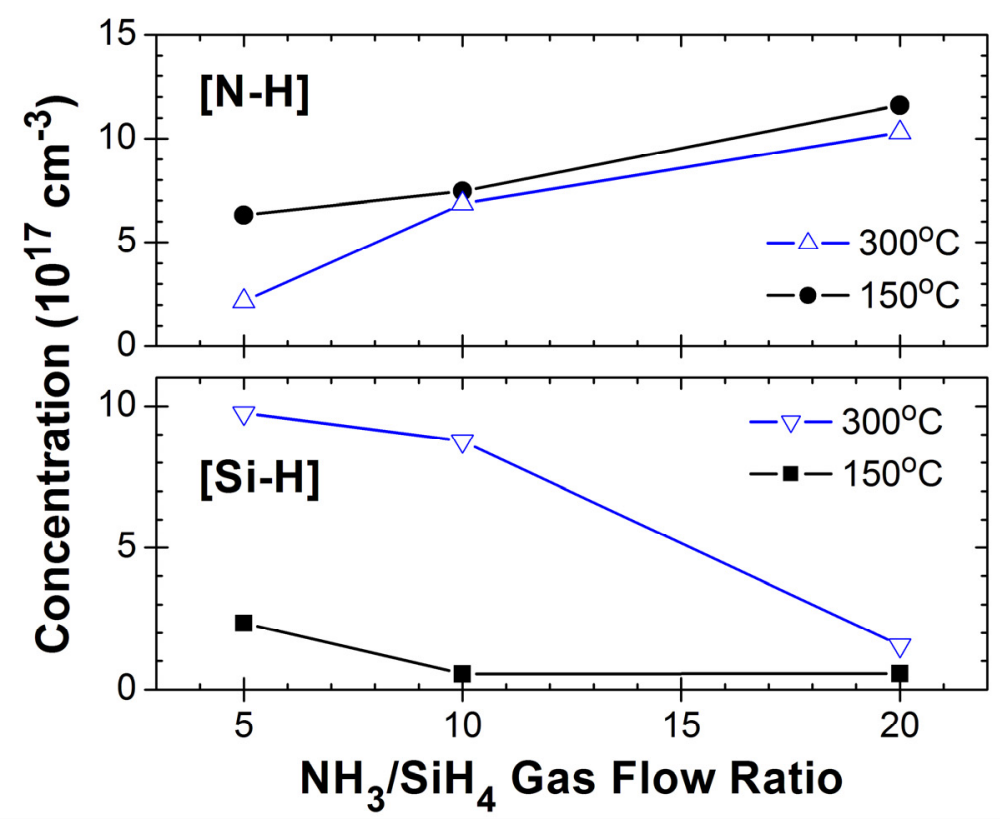

Figure 4.5. $\mathrm{N}-\mathrm{H}$ and $\mathrm{Si}-\mathrm{H}$ bond concentration as a function of $\mathrm{NH}_{3} / \mathrm{SiH}_{4}$ gas flow ratio for $\mathrm{SiN}_{x}$ films deposited at substrate temperatures of $150^{\circ} \mathrm{C}$ and $300^{\circ} \mathrm{C}$.

As the $\mathrm{NH}_{3} / \mathrm{SiH}_{4}$ gas flow ratio increases, the FTIR spectra in Figure 4.2 and Figure 4.3 indicate a shift of the Si-N stretching band peak to higher wavenumber. The changes in Si-N stretching band wavenumber as a function of $\mathrm{NH}_{3} / \mathrm{SiH}_{4}$ ratio for both $300^{\circ} \mathrm{C}$ and $150^{\circ} \mathrm{C}$ samples are plotted in Figure 4.6. The positive shift in Si-N band wavenumber typically coincides with an increase in N$\mathrm{H}$ bond density [12], which is consistent with our analysis here. The inset in Figure 4.6 plots the Si$\mathrm{N}$ wavenumber extracted from the FTIR spectra against the $[\mathrm{N}] /[\mathrm{Si}]$ calculated from refractive index (see discussion in Section 4.2.1.3). This trend again corroborates with the convention that higher Si-N wavenumber is associated with increase in nitrogen content in the $\mathrm{SiN}_{\mathrm{x}}$ film. 
Stochiometric silicon nitride is represented by $\mathrm{Si}_{3} \mathrm{~N}_{4}$, where $x=[\mathrm{N}] /[\mathrm{Si}]=4 / 3 \approx 1.33$. Nitrogenrich (N-rich) films are characterized by $x>1.33$, whereas silicon-rich (Si-rich) films have $x<1.33$. In our experiment, high $\mathrm{NH}_{3} / \mathrm{SiH}_{4}$ gas flow ratio produces nitrogen-rich films and low $\mathrm{NH}_{3} / \mathrm{SiH}_{4}$ gas flow ratio $(\sim 5)$ produces silicon-rich films. When low $\mathrm{NH}_{3} / \mathrm{SiH}_{4}$ gas flow ratio is used, the $\mathrm{SiH}_{4}$ species produce $\mathrm{Si}-\mathrm{Si}$ and $\mathrm{Si}-\mathrm{H}$ bonds in the growing film to form silicon-rich films [12].

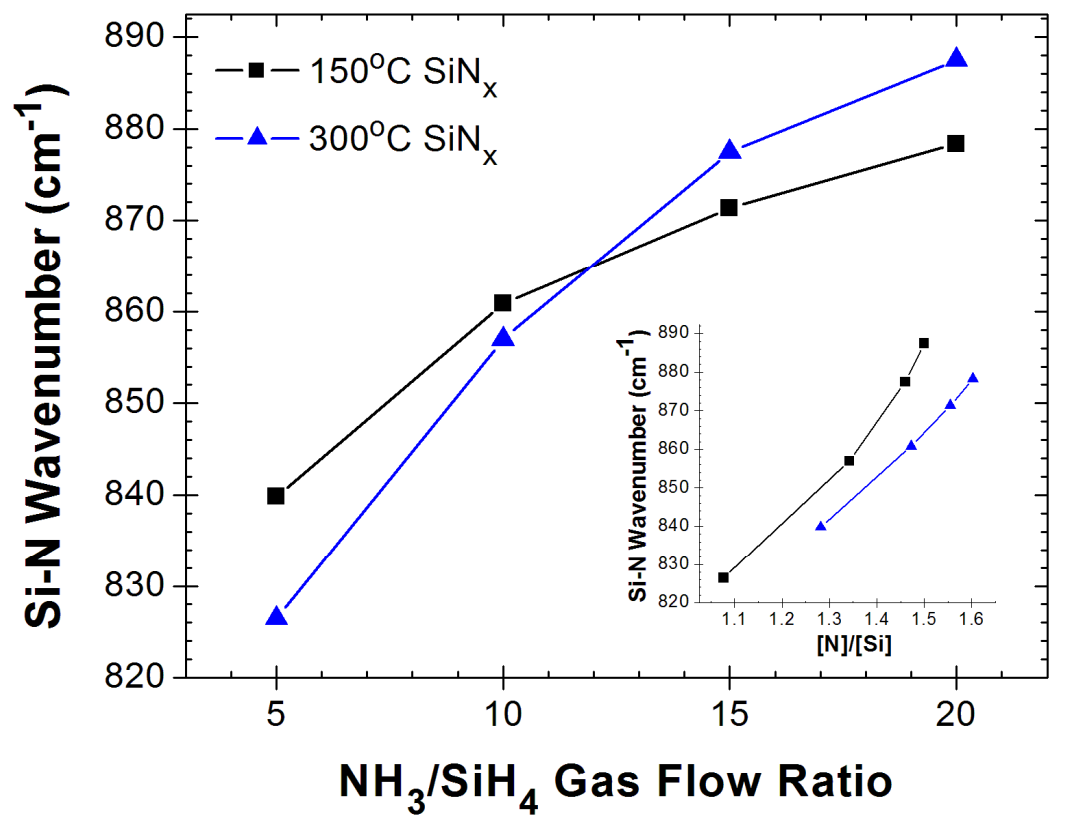

Figure 4.6. Wavenumber of the Si-N stretching band as a function of $\mathrm{NH}_{3} / \mathrm{SiH}_{4}$ gas flow ratio

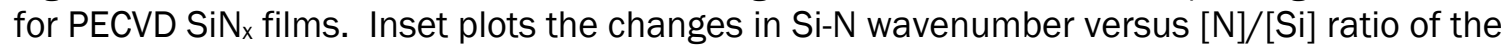
films.

\subsubsection{Refractive Index}

The influence of $\mathrm{NH}_{3} / \mathrm{SiH}_{4}$ gas flow ratio on refractive index $(n)$ of the $\mathrm{SiN}_{\mathrm{x}}$ films is shown in Figure 4.7. An increase in refractive index is observed with a decreasing $\mathrm{NH}_{3} / \mathrm{SiH}_{4}$ gas flow ratio for both $300^{\circ} \mathrm{C}$ and $150^{\circ} \mathrm{C}$ samples. Higher refractive index was measured for higher temperature nitrides at a given gas flow ratio. Increase of refractive index can be explained by the increase of the silicon (Si) content of the film. For example, it has been reported that a change in refractive index from 2.3 to 3.6 occurs for samples with composition varying from silicon nitride to amorphous silicon; clearly, the increase in refractive index is linked to an increase in $\mathrm{Si}$ concentration [12][17]. Thus, as the $\mathrm{NH}_{3} / \mathrm{SiH}_{4}$ gas flow ratio decreases, more $\mathrm{Si}$ is being 
incorporated in the film, corresponding to an increase in refractive index. Similarly, the higher refractive index in $300^{\circ} \mathrm{C}$ films signifies richer $\mathrm{Si}$ content than in the $150^{\circ} \mathrm{C}$ samples; this trend agrees well with the data in Figure 4.5 and is substantiated by XPS results in Figure 4.12. Wang et al. attributed the increase of refractive index to the densification of the film and change in composition, which also corresponds to a higher Si content [12].

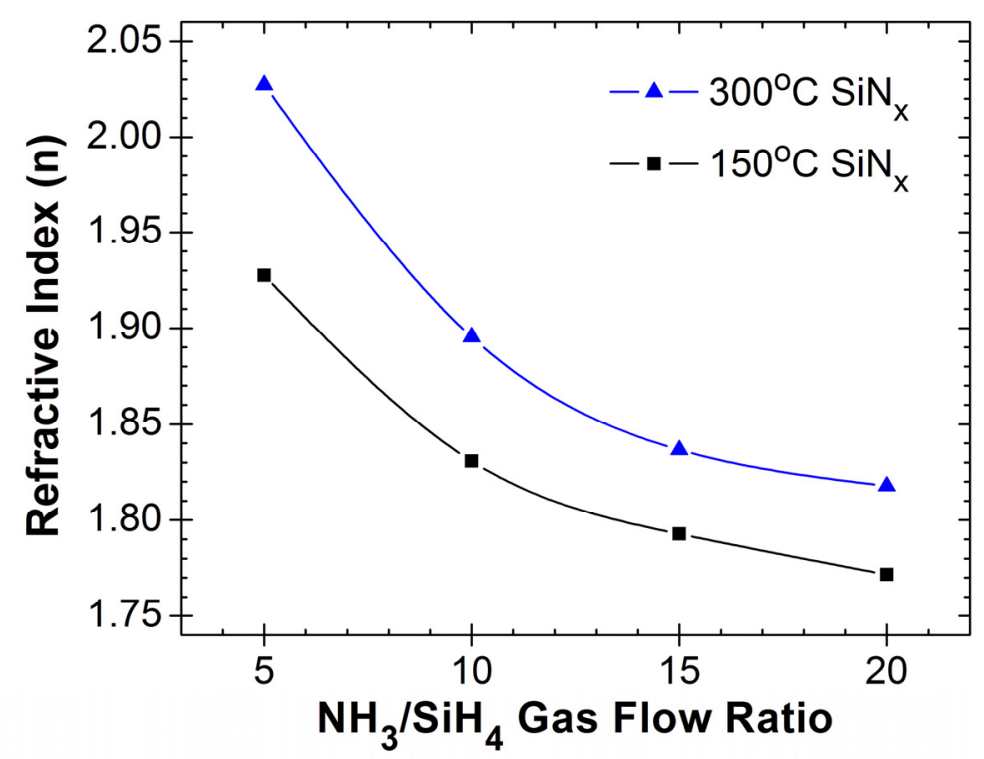

Figure 4.7. Refractive index at wavelength of $637 \mathrm{~nm}$ as a function of $\mathrm{NH}_{3} / \mathrm{SiH}_{4}$ gas flow ratio for $300^{\circ} \mathrm{C}$ and $150^{\circ} \mathrm{C} \mathrm{SiN}_{\mathrm{x}}$ samples.

\subsubsection{3 [N]/[Si] Ratio}

The stoichiometry $(x)$ of the $\operatorname{SiN}_{x}$ films is defined by the nitrogen to silicon ratio $([\mathrm{N}] /[\mathrm{Si}])$. It is possible to estimate the $[\mathrm{N}] /[\mathrm{Si}]$ ratio of $\mathrm{SiN}_{\mathrm{x}}$ films from the refractive index $(n)$ using the following formula [18][19]:

$$
\frac{[N]}{[S i]}=\frac{4}{3}\left[\frac{(3.3-n)}{(n-0.5)}\right] .
$$

Figure 4.8 plots the $[\mathrm{N}] /[\mathrm{Si}]$ ratio, calculated from refractive index (at the wavelength of $637 \mathrm{~nm}$ ), as a function of $\mathrm{NH}_{3} / \mathrm{SiH}_{4}$ gas flow ratio. As the $\mathrm{NH}_{3} / \mathrm{SiH}_{4}$ gas flow ratio changes from 5 to 20 , the $[\mathrm{N}] /[\mathrm{Si}]$ ratio of $\mathrm{SiN}_{\mathrm{x}}$ films increases, indicating an increase in nitrogen content relative to silicon. This trend is in agreement with the FTIR analysis in Section 4.1.2.1. 
$\mathrm{SiN}_{\mathrm{x}}$ films were also characterized using ERDA, which showed an increase of the $[\mathrm{N}] /[\mathrm{Si}]$ ratio from 1 to 1.3 for $300^{\circ} \mathrm{C} \mathrm{SiN}_{\mathrm{x}}$ when $\mathrm{NH}_{3} / \mathrm{SiH}_{4}$ gas flow changes from 5 to 20 . This trend concurs with the $[\mathrm{N}] /[\mathrm{Si}]$ data extracted from refractive index. Composition information deduced from XPS measurements showed identical dependence of $[\mathrm{N}] /[\mathrm{Si}]$ with gas flow ratio. Collectively, FTIR, EDRA, XPS, and refractive index measurements confirmed that as $\mathrm{NH}_{3} / \mathrm{SiH}$ gas flow ratio increases, the $\mathrm{SiN}_{\mathrm{x}}$ samples become more nitrogen rich, resulting in an increase in $[\mathrm{N}] /[\mathrm{Si}]$ ratio.

Comparing the effect of deposition temperature on $[\mathrm{N}] /[\mathrm{Si}]$ in Figure 4.8, samples deposited at higher deposition temperature displayed smaller $[\mathrm{N}] /[\mathrm{Si}]$ at a given gas flow ratio. This change in composition is also reflected by an increase of $\mathrm{Si}-\mathrm{H}$ bond concentration at higher deposition temperature in Figure 4.5. Higher deposition temperature enhances the incorporation of $\mathrm{Si}$ in the film with respect to $\mathrm{N}$, as evidenced by a decrease in $[\mathrm{N}] /[\mathrm{Si}]$ to indicate a more silicon-rich film. Additionally, higher temperature activates the release of non-bonded $\mathrm{H}$ and the breaking of weak $\mathrm{H}$ bonds so that $\mathrm{H}$ content decreases, and the relative amount of $\mathrm{N}$ and $\mathrm{Si}$ increases, resulting in a denser film [12].

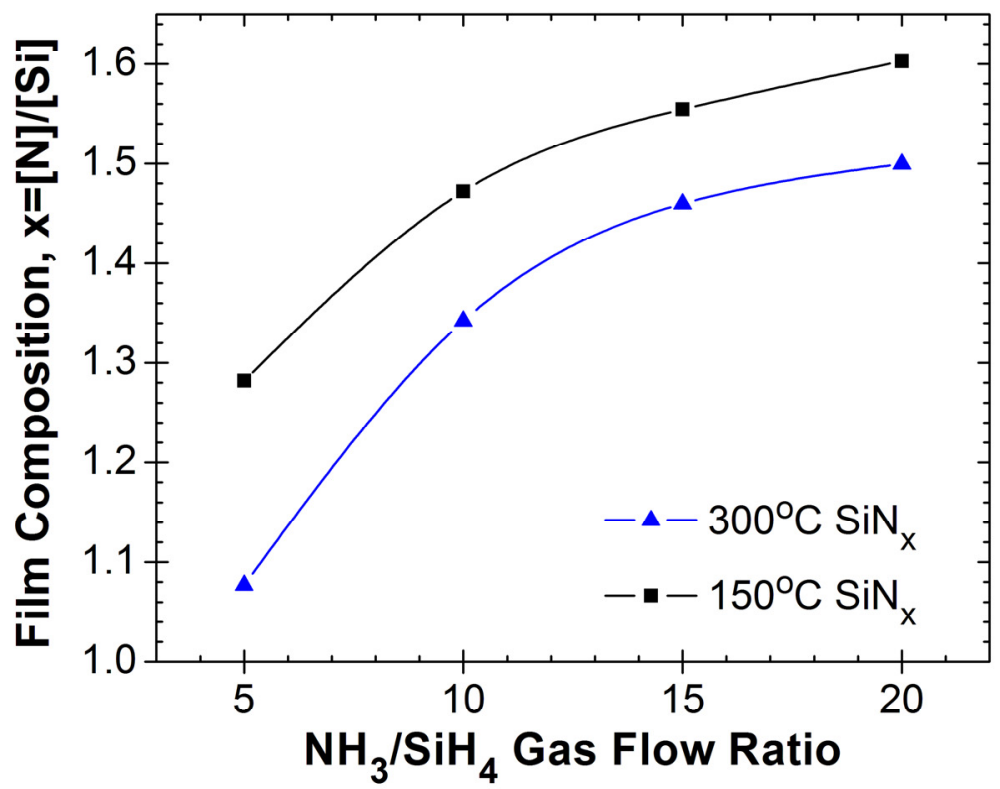

Figure 4.8. Nitrogen to silicon ratio ([N]/[Si]) of the $300^{\circ} \mathrm{C} \mathrm{SiN}_{\mathrm{x}}$ and $150^{\circ} \mathrm{C} \mathrm{SiN}_{\mathrm{x}}$ films as a function of gas flow ratio. 


\subsubsection{Surface Characterization}

\subsubsection{Contact Angle}

The surface wettability is a key parameter of interest in organic electronic devices because it affects the molecular ordering of the organic film. In the case of bottom-gate OTFTs, ideally, the dielectric surface should be tailored to allow the formation of a well-ordered organic layer, so that the density of grain boundaries in the organic semiconductor and scattering at the interface are minimized. An untreated $\mathrm{SiN}_{\mathrm{x}}$ surface is inherently hydrophilic. However, majority of published results suggest that hydrophobic surface is needed for the deposition of organic semiconductor to achieve higher

$\mu_{\mathrm{FE}}$. This mismatch can be resolved by proper surface treatment. Surface modification by alkyltrichlorosilane $\left(\mathrm{CH}_{3}\left(\mathrm{CH}_{2}\right)_{\mathrm{n}-1} \mathrm{SiCl}_{3}\right)$ self-assembled monolayer (SAM) is a popular choice for pre-treating hydroxylated dielectric surfaces, and has proven to significantly improve OTFT performance [20]. However, silane reagents often have poor adhesion on a bare $\mathrm{SiN}_{\mathrm{x}}$ surface due to the lack of hydroxyl groups. We address this limitation by pretreating the nitride samples with oxygen plasma prior to SAM deposition. It is believed that oxygen plasma exposure places hydroxyl groups on the nitride surface to facilitate attachment of alkyltrichlorosilane molecules. Our experiments suggested that the duration of oxygen plasma exposure must be carefully chosen to yield high quality OTS SAM; more details are reported in Chapter 5. Oxygen plasma exposure has been used in a similar context for modifying other dielectric surfaces in OTFT fabrication, as reported in the literature [21][22].

Contact angle measurements provide a means to evaluate the effectiveness of the surface modification recipe (i.e., oxygen plasma exposure followed by OTS SAM treatment). Figure 4.9 summarizes the contact angle data of $\mathrm{SiN}_{\mathrm{x}}$ films before and after surface treatment. There are two key observations:

1) The relatively small contact angle for the untreated/bare nitride indicates a hydrophilic surface. In contrast, the rise in contact angle across all samples after surface treatment signifies effective surface modification to promote hydrophobicity of the nitride surface.

2) A dependence of the contact angle on the $\mathrm{SiN}_{\mathrm{x}}$ film composition is evident for the untreated samples; the contact angle increases as the silicon content in the untreated nitride film increases. The dependence of contact angle on film composition 
diminishes after surface treatment, suggesting that the dielectric surface property becomes largely dictated by the properties of the OTS SAM.

In-depth discussions on the impact of interface treatment on $\mathrm{SiN}_{\mathrm{x}}$ surface properties and OTFT characteristics will be presented in Chapter 5 .

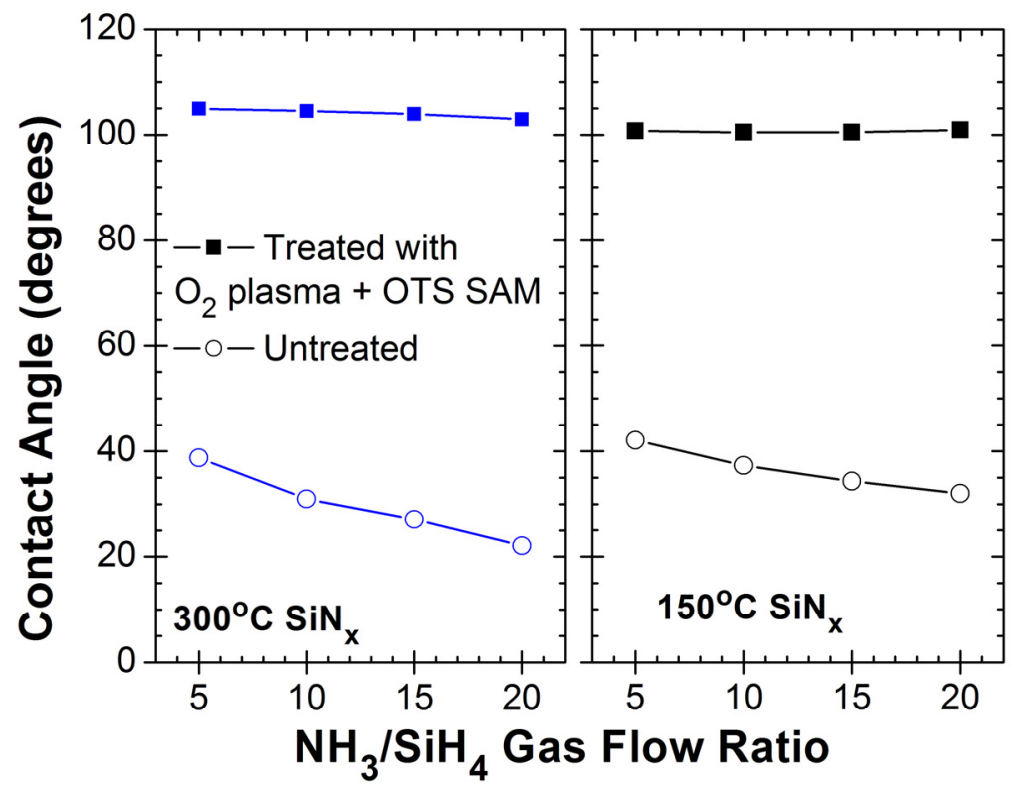

Figure 4.9. Water contact angle of $\operatorname{SiN}_{\mathrm{x}}$ surface before $(O)$ and after $(\boldsymbol{\square})$ surface treatment with oxygen plasma and OTS SAM, for four different $\mathrm{SiN}_{\mathrm{x}}$ films (denoted by $\mathrm{NH}_{3} / \mathrm{SiH}_{4}$ gas flow ratios).

\subsubsection{Surface Morphology \& Roughness}

Both the chemical and physical states of the gate dielectric surface affect OTFT performance. In terms of physical properties, surface roughness is particularly important because it hinders the movement of charge carriers. Rough dielectric surfaces can lead to formation of voids or discontinuities in the overlying organic semiconductor film and can cause surface scattering. There have been a number of reports on reduction in mobility with increasing dielectric surface roughness. Chabinyc et al. showed that the field-effect mobility of OTFTs decreases nearly exponentially with surface roughness of the gate dielectric [24]. Kim et al. evaluated changes in mobility of solution-processed OTFTs with interface roughness. They reported that below a critical roughness threshold, mobility remained constant for low values of the interface roughness; 
however, for roughness exceeding this threshold, a rapid drop in the mobility by orders of magnitude was observed [23].

AFM was used to characterize surface roughness of the $\mathrm{SiN}_{\mathrm{x}}$ samples. Figure 4.10 shows that as $\mathrm{NH}_{3} / \mathrm{SiH}_{4}$ gas flow ratio decreases (i.e., as film tends towards Si-rich), the surface roughness decreases. Another observation is that $150^{\circ} \mathrm{C} \mathrm{SiN}$ films have lower surface roughness than $300^{\circ} \mathrm{C}$ $\mathrm{SiN}_{\mathrm{x}}$ at a given gas flow ratio. These observations in surface roughness can offer valuable insights for analyzing/understanding OTFT characteristics; more discussions are presented in Section 4.3.1 and Section 4.3.2.

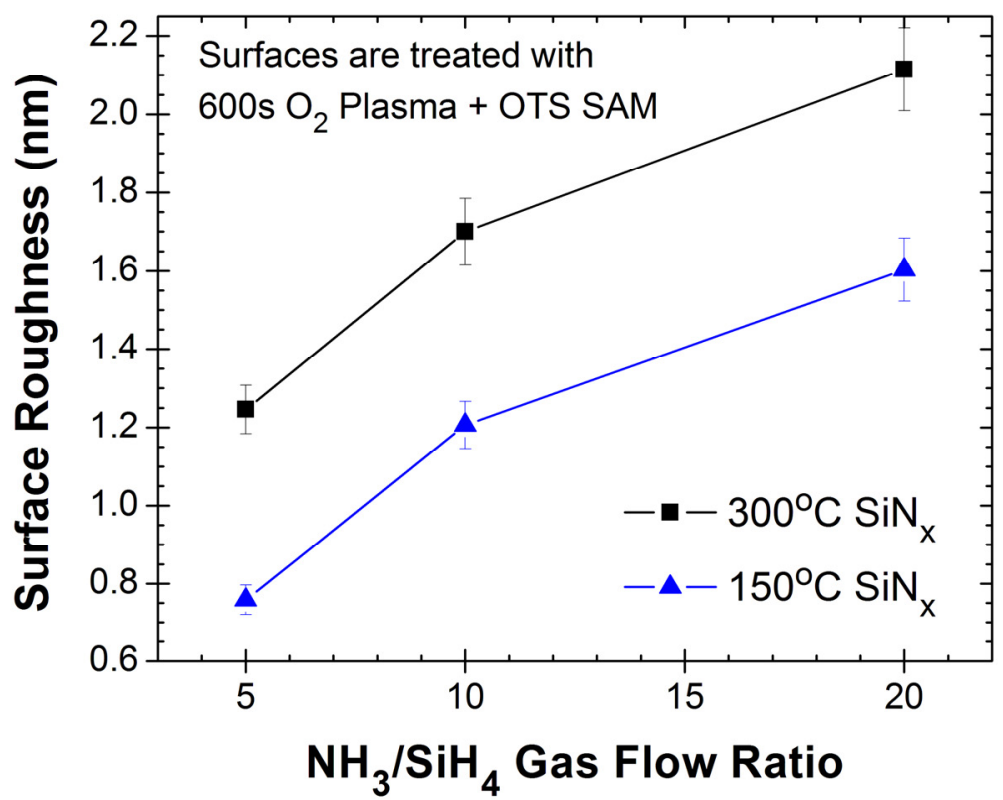

Figure 4.10. Surface roughness of $150^{\circ} \mathrm{C}$ and $300^{\circ} \mathrm{C} \mathrm{SiN}$ films as a function of $\mathrm{NH}_{3} / \mathrm{SiH}_{4}$ gas flow ratios, after surface treatment with $\mathrm{O}_{2}$ plasma and OTS SAM.

\subsubsection{Chemical Composition}

XPS was used to study the elemental and chemical composition of the $\operatorname{SiN}_{x}$ surfaces. Surface composition is particularly important when analyzing the dielectric-semiconductor interface because the type and density of atoms present at the surface determines the type of bonding that takes place. Consequently, the type of interface bonding influences the quality, microstructure and molecular ordering of the semiconductor layer, thus dictates the performance of OTFTs. XPS measurements were performed on various $300^{\circ} \mathrm{C} \mathrm{SiN}_{\mathrm{x}}$ and $150^{\circ} \mathrm{C} \mathrm{SiN}_{\mathrm{x}}$ samples; their XPS spectra 
were used to calculate the elemental distribution on the sample's surface. Figure 4.11(a) plots the atomic distribution at the surface of three $300^{\circ} \mathrm{C} \mathrm{SiN}$ films. The displayed trend is consistent with preceding discussions, where the sample labeled $x=1.08$ has the highest atomic percentage in $\mathrm{Si}$ $2 p$, indicating a higher surface concentration of Si than the other two samples $(x=1.34,1.50)$. This observation is in exact agreement with the definition of $x=[\mathrm{N}] /[\mathrm{Si}]$, where smaller $x$ means larger Si content.

When using $\mathrm{SiN}_{\mathrm{x}}$ as gate dielectric in OTFTs, surface treatment steps are performed to prepare the dielectric surface for deposition of the organic semiconductor layer. (Detailed investigation of dielectric surface treatments is discussed in Chapter 5.) Therefore, XPS is used to study the chemical composition of the $\mathrm{SiN}_{\mathrm{x}}$ surface after surface treatments to gain better insights on the desirable surface compositions for higher performance OTFTs. Figure 4.11(b) and Figure 4.11(c) plot the atomic distribution of $300^{\circ} \mathrm{C} \mathrm{SiN}$ films after $\mathrm{O}_{2}$ plasma treatment and $\mathrm{O}_{2}$ plasma/OTS SAM treatment, respectively. A significant increase in atomic $\%$ of $\mathrm{O} 1 \mathrm{~s}$ after $\mathrm{O}_{2}$ plasma treatment is observed by comparing Figure 4.11(a) and Figure 4.11(b)-(c). This is logical as $\mathrm{O}_{2}$ plasma treatment is expected to attach oxygen atoms on the surface, forming an oxidized nitride surface. Figure 4.11(c) also shows a large increase in C 1s content, attributable to the presence of OTS SAM. An interesting observation is that Si $2 p$ intensity remains highest for the $x=1.08$ sample even after $\mathrm{O}_{2}$ plasma or $\mathrm{O}_{2}$ plasma/OTS treatments. This Si-rich surface is believed to be a decisive factor for attaining high field-effect mobility for OTFTs on $\mathrm{SiN}_{\mathrm{x}}$ gate dielectric, which is explored or analyzed in-depth in Section 4.3. 
(a)

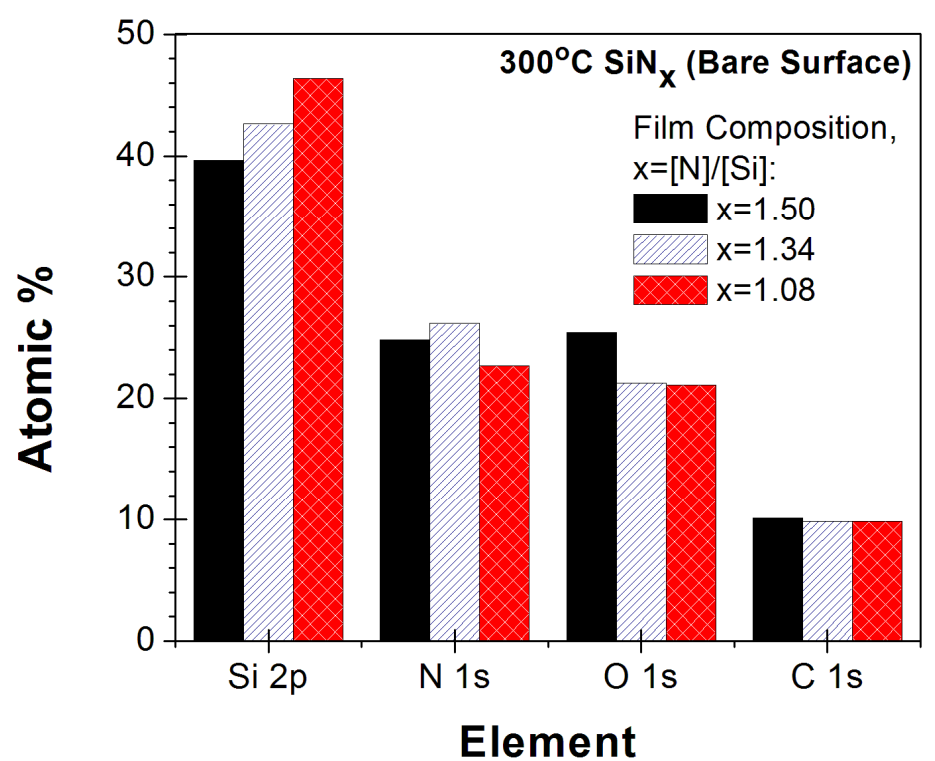

(b)

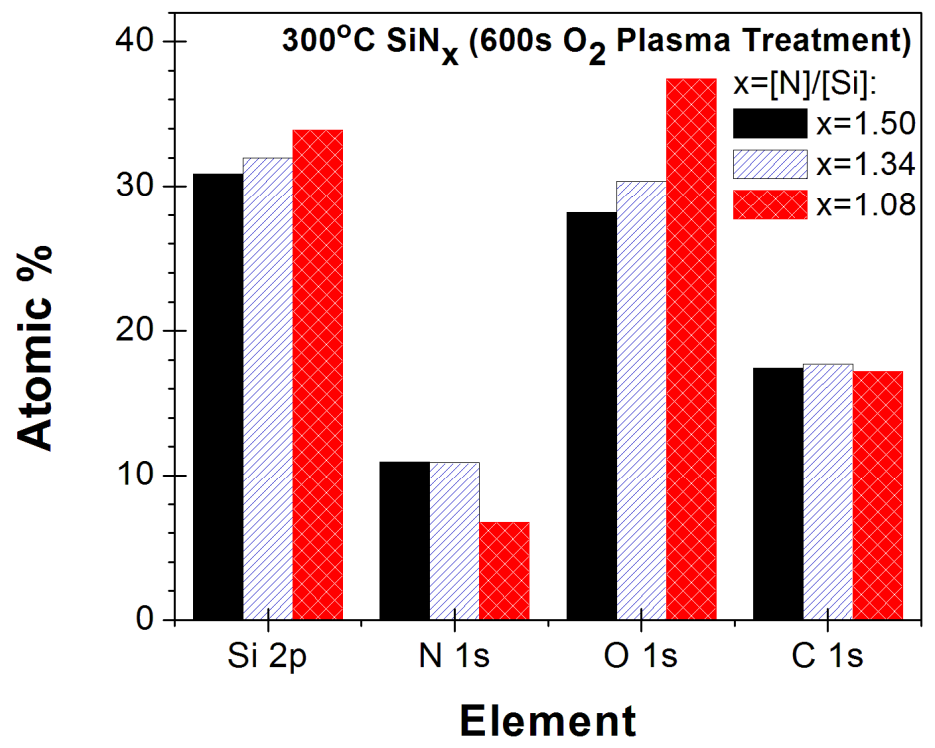


(c)

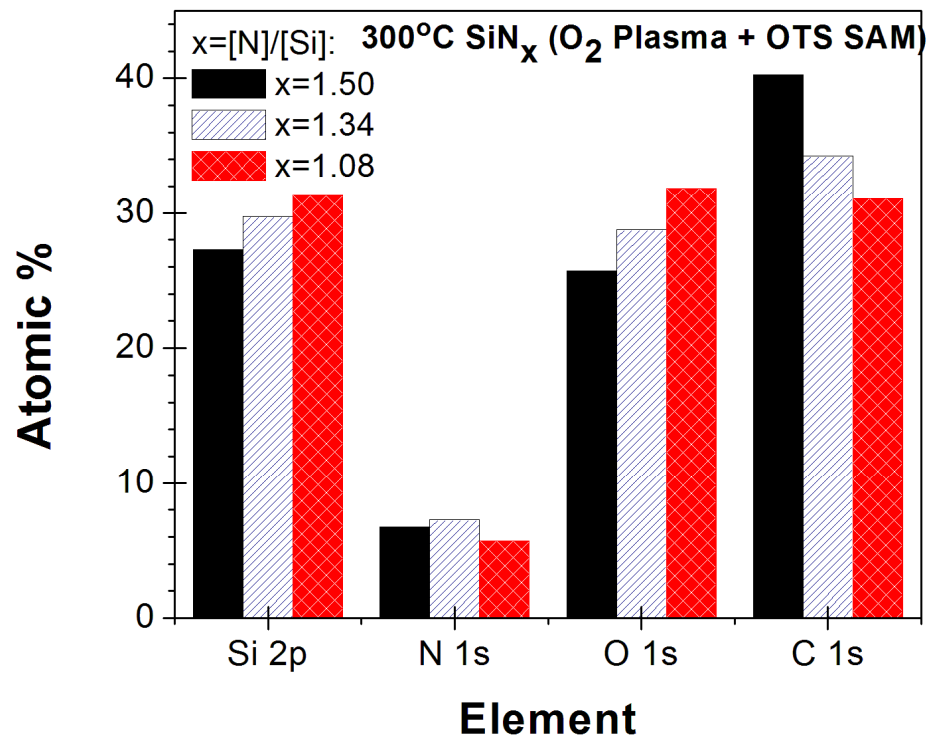

Figure 4.11. Chemical composition of three different $300^{\circ} \mathrm{C} \mathrm{SiN}$ films ( $\left.x=1.50,1.34,1.08\right)$, plotted as atomic distribution at the film surface as measured by XPS. Three surface conditions were considered for each of the $300^{\circ} \mathrm{C} \mathrm{SiN}$ films: (a) as-deposited (bare) surface, (b) $\mathrm{O}_{2}$ plasma treated surface, and (c) $\mathrm{O}_{2}$ plasma and OTS SAM treated surface

Figure 4.12 compares the chemical composition of $300^{\circ} \mathrm{C} \mathrm{SiN}_{\mathrm{x}}$ and $150^{\circ} \mathrm{C} \mathrm{SiN}_{\mathrm{x}}$ films, both deposited at the same $\mathrm{NH}_{3} / \mathrm{SiH}_{4}$ gas flow ratio. Higher $\mathrm{Si}$ concentration and lower $\mathrm{N}$ concentration (thus smaller $[\mathrm{N}] /[\mathrm{Si}]$ ) are observed in $300^{\circ} \mathrm{C} \mathrm{SiN}_{\mathrm{x}}$ when compared to $150^{\circ} \mathrm{C} \mathrm{SiN}_{\mathrm{x}}$. This is in agreement with the data in Figure 4.8, where a smaller $[\mathrm{N}] /[\mathrm{Si}]$ value is obtained for $300^{\circ} \mathrm{C} \mathrm{SiN}_{\mathrm{x}}$ than $150^{\circ} \mathrm{C} \mathrm{SiN}$, at a given $\mathrm{NH}_{3} / \mathrm{SiH}_{4}$ gas flow ratio. XPS of the $\mathrm{O}_{2}$ plasma treated surfaces are displayed in Figure 4.12(b); the increase in O 1s signal signifies successful attachment of oxygen species on the $\mathrm{SiN}_{\mathrm{x}}$ surface. 
(a)

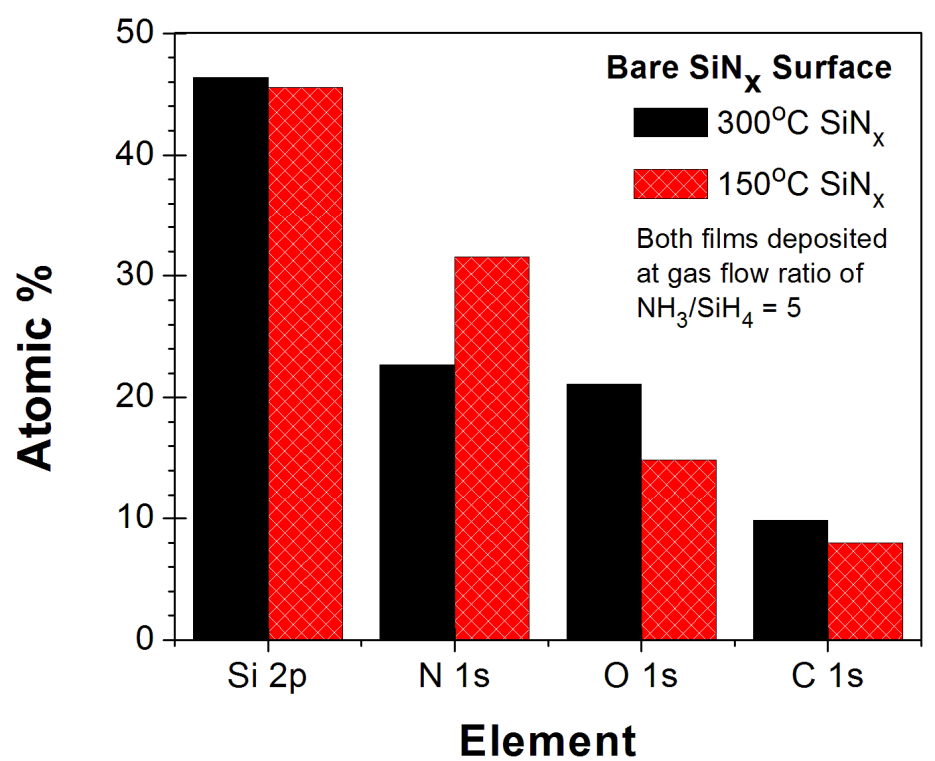

(b)

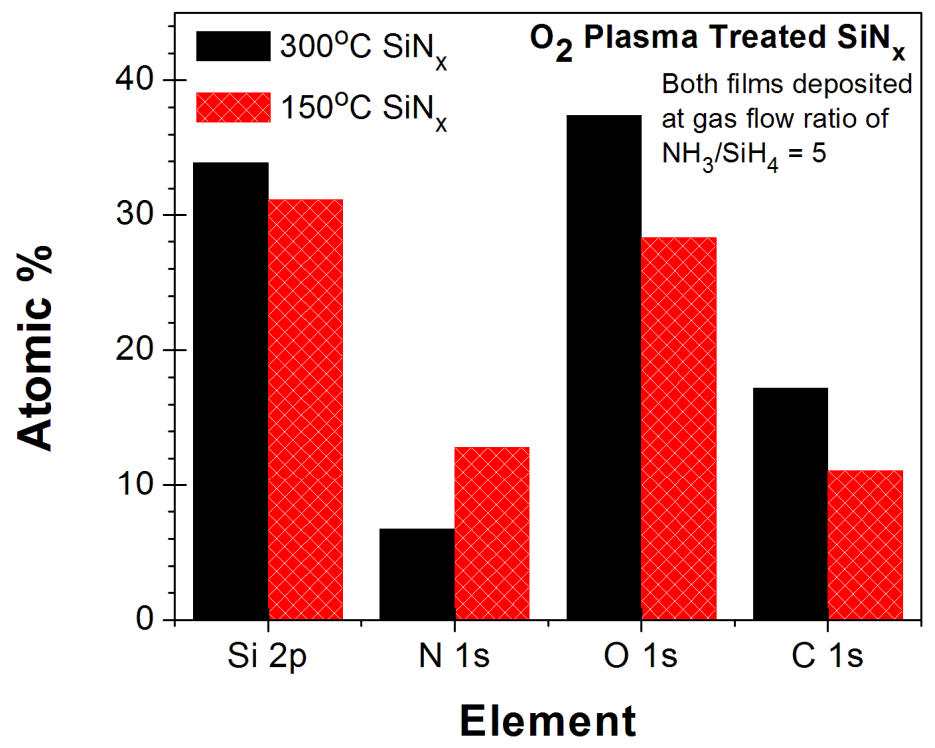

Figure 4.12. XPS measurement comparing chemical composition of silicon-rich $300^{\circ} \mathrm{C}$ and $150^{\circ} \mathrm{C} \mathrm{SiN}$ films deposited at gas flow ratio of $\mathrm{NH}_{3} / \mathrm{SiH}_{4}=5$. Two surface conditions are considered: (a) as-deposited (bare) and (b) $\mathrm{O}_{2}$ plasma treated surface. 


\subsubsection{Electrical Characterization}

Metal-insulator-semiconductor (MIS) capacitance structures were fabricated using $\mathrm{Al} / \mathrm{SiN}_{\mathrm{x}} / \mathrm{Si} / \mathrm{Al}$ configuration to evaluate the leakage current, electrical breakdown field, and dielectric constant of the nitride films by means of current-voltage $(I-V)$ and capacitance-voltage $(C-V)$ measurements.

\subsubsection{I-V Measurements}

Figure 4.13 and Figure 4.14 display the leakage current density as a function of electric field for $300^{\circ} \mathrm{C} \mathrm{SiN}_{\mathrm{x}}$ and $150^{\circ} \mathrm{C} \mathrm{SiN}_{\mathrm{x}}$ films, respectively. Leakage current density in the range $10-40 \mathrm{nA} / \mathrm{cm}^{2}$ for electric fields less than $2 \mathrm{MV} / \mathrm{cm}$ was observed across the different nitride films, and is comparable to results reported in [25][26]. Electric breakdown was not observed for $\mathrm{SiN}_{\mathrm{x}}$ films deposited with $\mathrm{NH}_{3} / \mathrm{SiH}_{4}$ gas flow ratio of 20 and 10, for fields up to $6 \mathrm{MV} / \mathrm{cm}$. However, lower breakdown field was measured for $\mathrm{SiN}_{\mathrm{x}}$ deposited at $\mathrm{NH}_{3} / \mathrm{SiH}_{4}=5$, suggesting a weakening of the dielectric strength as the $\mathrm{NH}_{3} / \mathrm{SiH}_{4}$ flow ratio decreases (i.e., for more silicon-rich films).

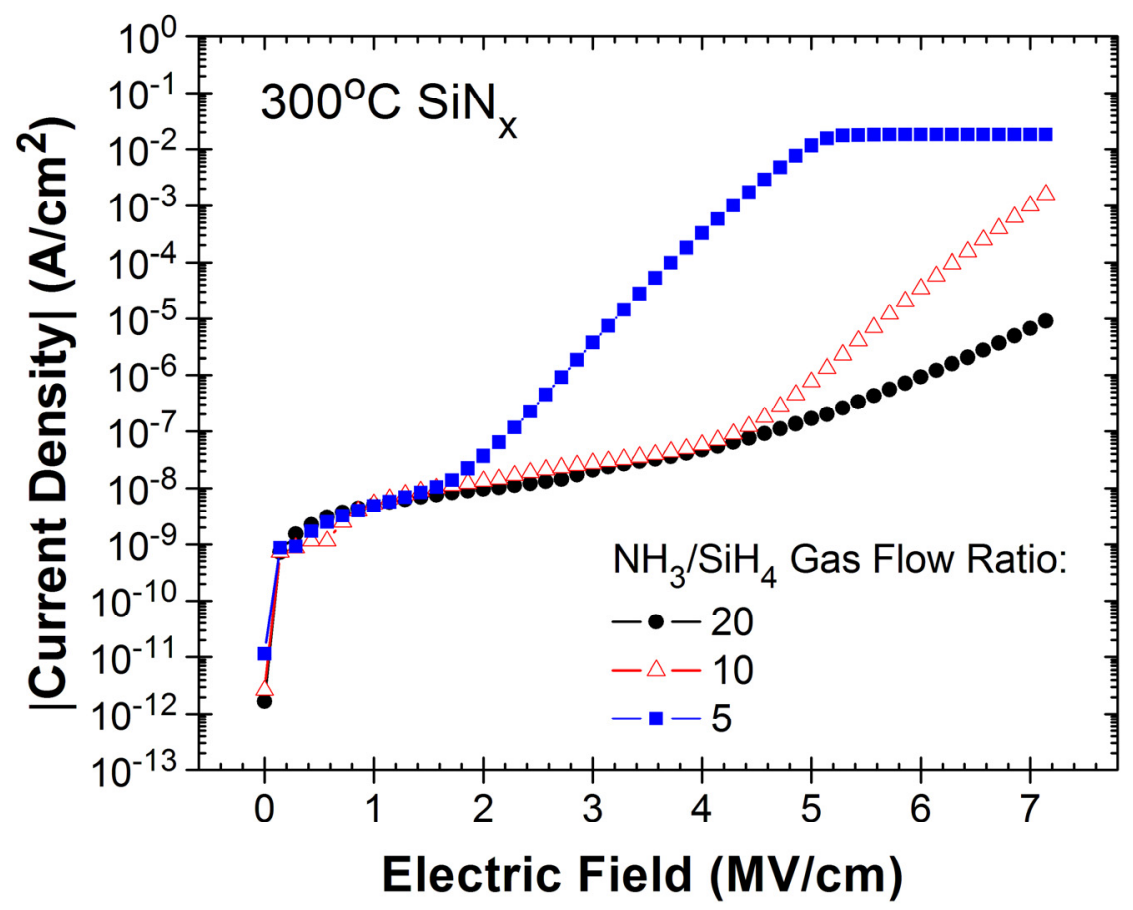

Figure 4.13. Leakage current density as a function of electric field for $300^{\circ} \mathrm{C} \mathrm{SiN}_{\mathrm{x}}$ dielectrics deposited at various $\mathrm{NH}_{3} / \mathrm{SiH}_{4}$ gas flow ratio. The film thickness is approximately $300 \mathrm{~nm}$. Measurements were performed using MIS capacitor structure. 


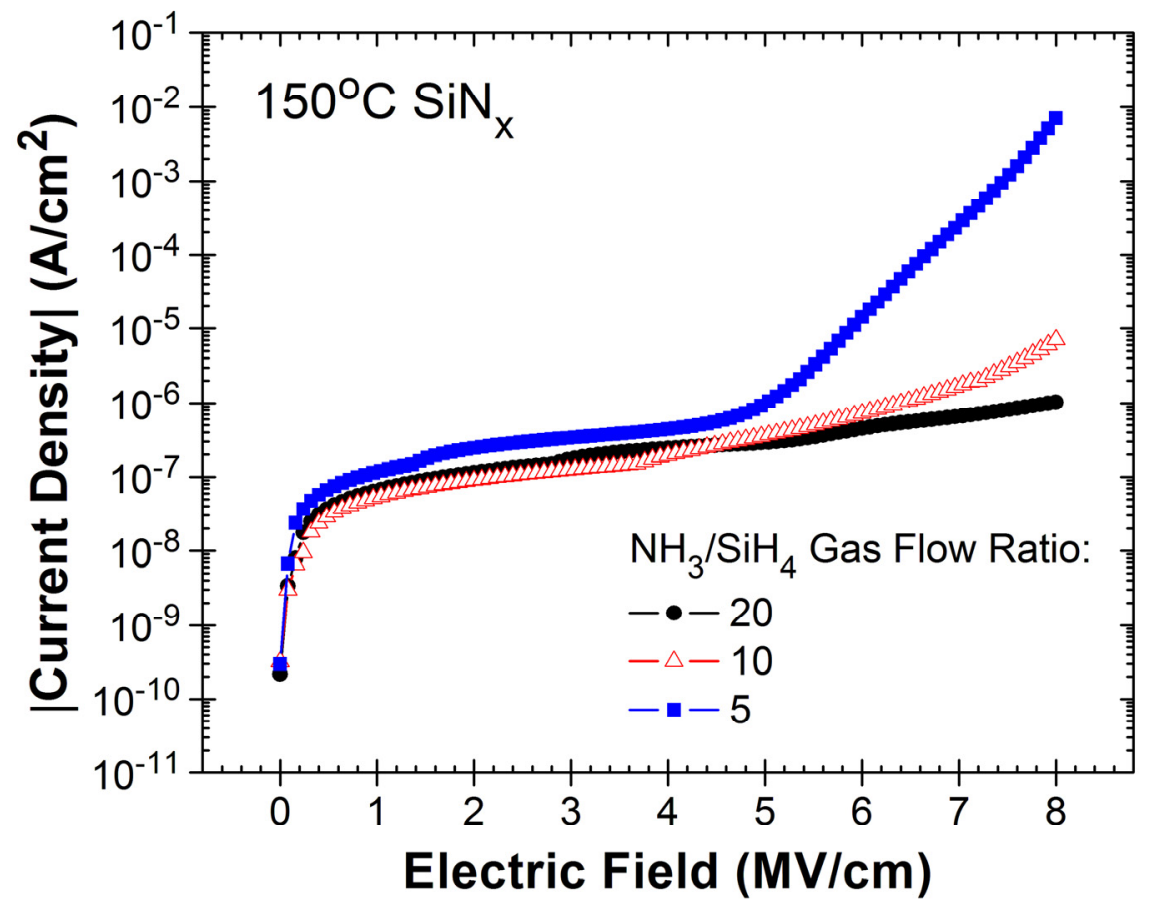

Figure 4.14. Leakage current density as a function of electric field for $150^{\circ} \mathrm{C} \mathrm{SiN}$ dielectrics deposited at various $\mathrm{NH}_{3} / \mathrm{SiH}_{4}$ gas flow ratio. The film thickness is approximately $300 \mathrm{~nm}$. Measurements were performed using MIS capacitor structure.

\subsubsection{C-V Measurements}

The relative dielectric constant $\left(\varepsilon_{\mathrm{r}}\right)$ of the various $\mathrm{SiN}_{\mathrm{x}}$ films was obtained from capacitance measurements, using the formula:

$$
C=\frac{\varepsilon_{0} \varepsilon_{r} A}{d}
$$

Figure 4.15 shows a decrease in dielectric constant with decreasing $\mathrm{NH}_{3} / \mathrm{SiH}_{4}$ gas flow ratio (i.e., increasing silicon content). $C-V$ measurements using MIS structures made with $300^{\circ} \mathrm{C}$ and $150^{\circ} \mathrm{C}$ $\mathrm{SiN}_{\mathrm{x}}$ are displayed in Figure 4.16 and Figure 4.17, respectively. Decrease in gas flow ratio (i.e., more silicon rich films) leads to a negative shift in flat band voltage $\left(V_{\mathrm{FB}}\right)$ and increase in hysteresis. Such hysteresis (i.e., voltage shift between forward and reverse $C$ - $V$ curves) is related to charge (electron) trapping within the $\mathrm{SiN}_{\mathrm{x}}$ bulk. Data showed more hysteresis for films deposited at lower $\mathrm{NH}_{3} / \mathrm{SiH}_{4}$ gas flow ratio (linked to film with smaller [N]/[Si] ratio), suggesting increase in charge trapping for more silicon-rich $\mathrm{SiN}_{\mathrm{x}}$ film. The observed hysteresis has been attributed to 
electron injection and trapping mechanisms in $\mathrm{SiN}_{\mathrm{x}}$, including: Fowler-Nordheim injection, trapassisted injection, constant-energy tunneling from silicon conduction band, direct tunneling from silicon valence band, and hopping at the Fermi level [27]. These charge trapping mechanisms also lead to higher leakage currents (especially at high electric fields); this can be observed in the $I-V$ characteristics in Figure 4.13 and Figure 4.14 for Si-rich $\mathrm{SiN}_{\mathrm{x}}$ films.

In conclusion, these electrical characteristics indicate that composition of the $\mathrm{SiN}_{\mathrm{x}}$ film, which depends on the deposition conditions, influences the electrical properties of the dielectric layer; this will in turn impact the OTFT device characteristics.

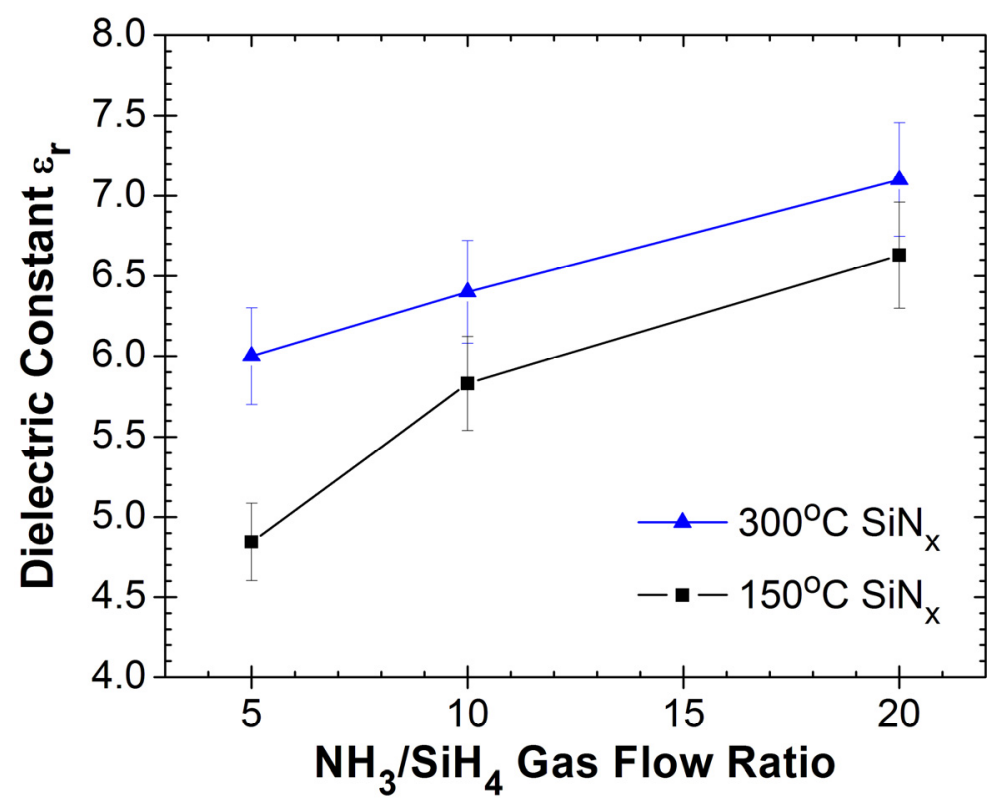

Figure 4.15. Dielectric constant ( $\left.\varepsilon_{r}\right)$ for $\mathrm{SiN}_{\mathrm{x}}$ films as a function of $\mathrm{NH}_{3} / \mathrm{SiH}_{4}$ gas flow ratio. 

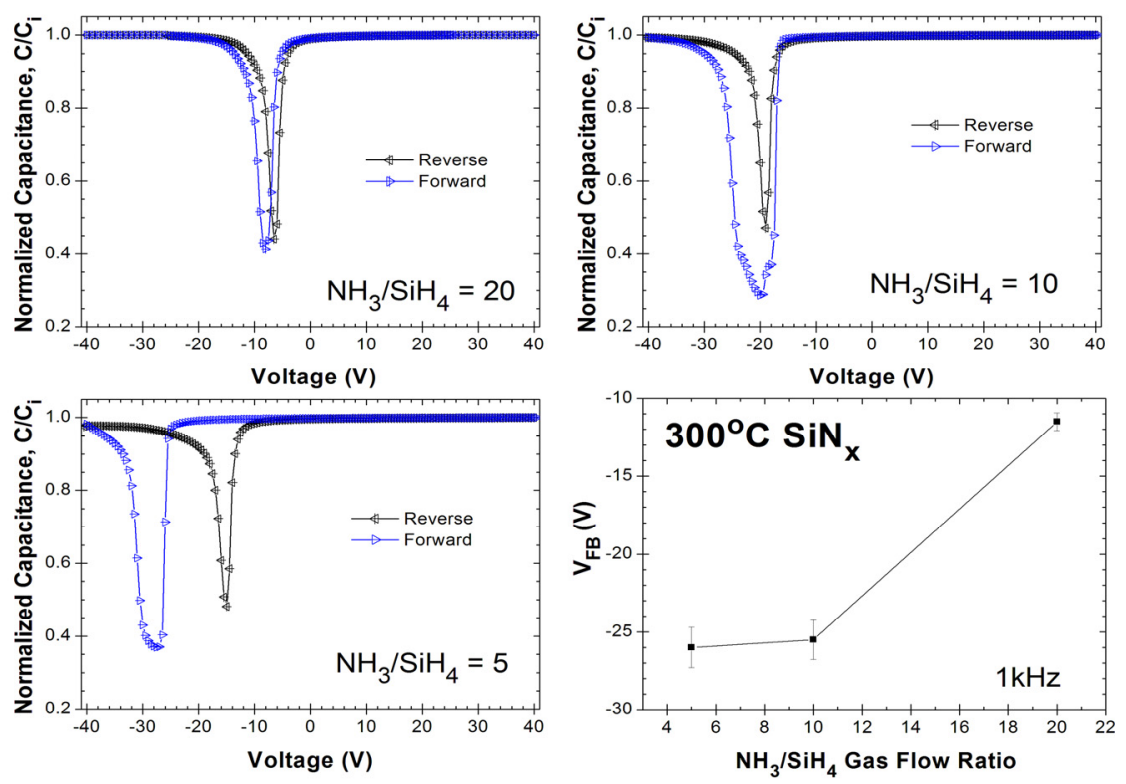

Figure 4.16. $\mathrm{C}-\mathrm{V}$ characteristics of various $300^{\circ} \mathrm{C} \mathrm{SiN}$ dielectrics at $1 \mathrm{kHz}$, in both forward (negative to positive) and reverse (positive to negative) sweep directions. Flatband voltage $\left(V_{\mathrm{FB}}\right)$ is extracted from forward curves and is plotted as a function of $\mathrm{NH}_{3} / \mathrm{SiH}_{4}$ gas flow ratio.
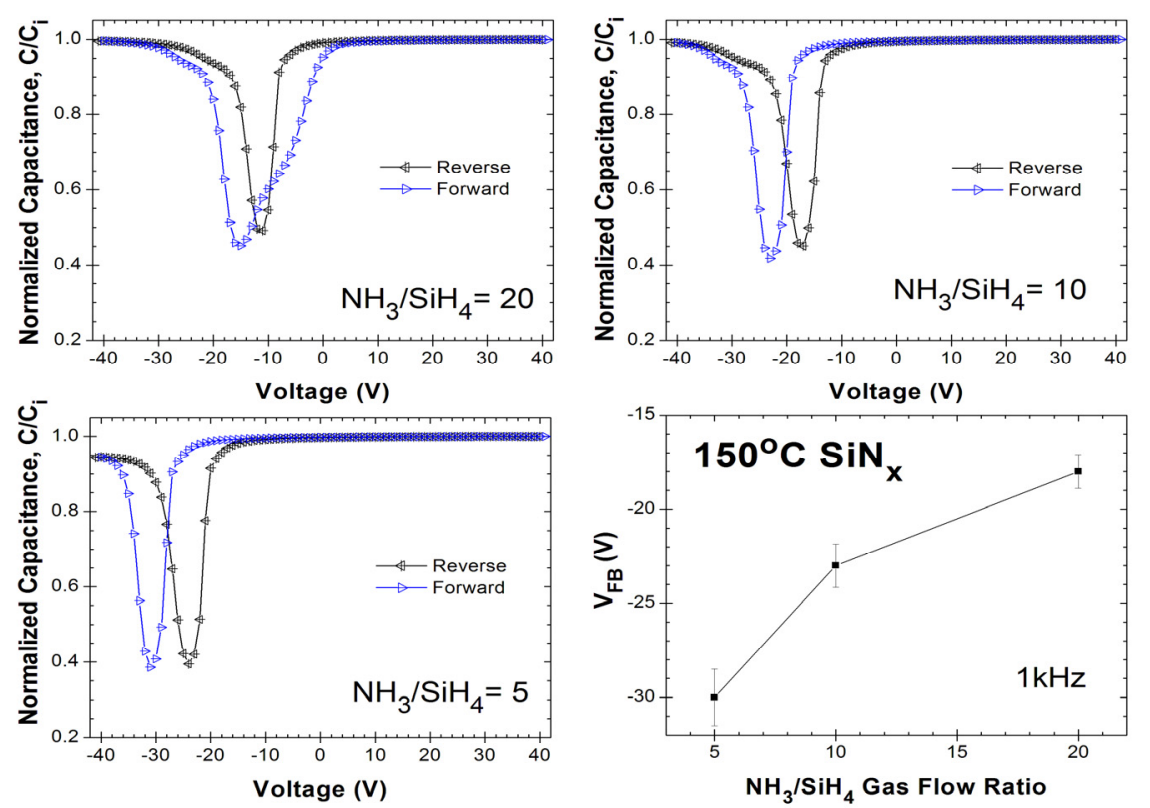

Figure 4.17. $\mathrm{C}-\mathrm{V}$ characteristics of various $150^{\circ} \mathrm{C} \mathrm{SiN}$ dielectrics at $1 \mathrm{kHz}$, in both forward (negative to positive) and reverse (positive to negative) sweep directions. Flatband voltage $\left(V_{\mathrm{FB}}\right)$ is extracted from forward curves and is plotted as a function of $\mathrm{NH}_{3} / \mathrm{SiH}_{4}$ gas flow ratio. 


\subsubsection{Summary}

Amorphous hydrogenated silicon nitride films have been deposited by the PECVD technique, using ammonia $\left(\mathrm{NH}_{3}\right)$ and silane $\left(\mathrm{SiH}_{4}\right)$ as precursor gases. The gas flow ratio has been varied in order to study their effect on the properties of the films, which was characterized by FTIR, ellipsometry, ERDA, XPS, AFM, and contact angle.

As the $\mathrm{NH}_{3} / \mathrm{SiH}_{4}$ gas flow ratio is increased (by increasing the $\mathrm{NH}_{3}$ partial pressure), measurements indicated an increase in nitrogen-to-silicon ratio $([\mathrm{N}] /[\mathrm{Si}])$. Changes in $\mathrm{SiN}_{\mathrm{x}}$ film composition result in different surface (e.g., contact angle, surface roughness, surface composition) and electrical properties (e.g., dielectric constant, breakdown field, leakage current). When these $\mathrm{SiN}_{\mathrm{x}}$ films are used as the gate dielectric in bottom-gate OTFTs, variation in surface states will influence the molecular ordering of the overlying organic semiconductor layer and the quality of the semiconductor-dielectric interface. These attributes have a strong bearing on the OTFT characteristics, including field-effect mobility, on/off current ratio, leakage current, threshold voltage, and subthreshold slope. Thus, proper control of the gate dielectric surface property is crucial for attaining higher performance OTFTs. In the next section, we examine the impact of changing the $\mathrm{SiN}_{\mathrm{x}}$ gate dielectric properties on OTFT performance.

\subsection{Electrical Characterization of OTFTs with PECVD Gate Dielectric}

PECVD SiN $x$ and $\mathrm{SiO}_{\mathrm{x}}$ thin films were evaluated as gate dielectrics for bottom-gate bottom-contact PQT-12 OTFTs. Devices were fabricated using the integration scheme outlined in Figure 3.8. Highly doped silicon wafers served as a common gate substrate. PECVD $\operatorname{SiN}_{x}$ or $\operatorname{SiO}_{x}(200-300$ $\mathrm{nm}$ thick) formed the gate dielectric layer. Source/ drain contacts, patterned by photolithography and a lift-off process, consisted of $60 \mathrm{~nm}$ of thermally evaporated $\mathrm{Au}$ on top of $5 \mathrm{~nm}$ of $\mathrm{Cr}$ adhesion layer. Au was used for the source/drain contacts because of its high work function $(\sim 5.1$ $\mathrm{eV}$ ), which theoretically should provide good matching to the HOMO of PQT-12 semiconductor, making hole injection from $\mathrm{Au}$ to PQT-12 more feasible. Solution processible poly(3,3"'didodecylquaterthiophene) (PQT-12) was used as the organic semiconductor layer. PQT-12 semiconductor was synthesized as previously reported in [28] with a number average relative molecular mass $\left(M_{\mathrm{n}}\right)$ and polydispersity of $17300 \mathrm{~g} / \mathrm{mol}$ and 1.32 . The PQT-12 films were spin- 
coated from a $0.3 \mathrm{wt} \%$ dispersion in 1,2-dichlorobenzene at a rate of $1000 \mathrm{rpm}$ onto substrates [29]. The as-cast films were then dried in a vacuum oven at $80^{\circ} \mathrm{C}$ and annealed at $140^{\circ} \mathrm{C}$, followed by a slow cooling to room temperature. Figure 4.18 illustrates the chemical structure of PQT-12. All processing and device characterization were carried out in atmosphere.

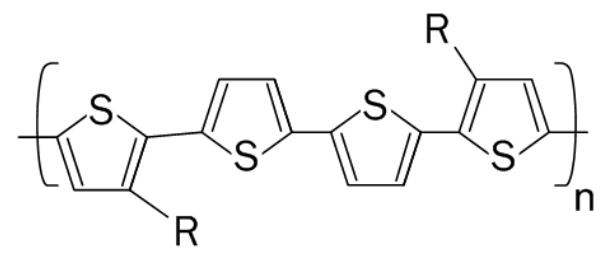

Figure 4.18. Chemical structure of poly(3,3'"-dialkylquarterthiophene) (PQT). In the case of PQT-12, alkyl side chain is $\mathrm{R}=\mathrm{n}-\mathrm{C}_{12} \mathrm{H}_{25}$. (Adapted from [28].)

Prior to deposition of the semiconductor layer, the dielectric surface was functionalized with a combination of oxygen $\left(\mathrm{O}_{2}\right)$ plasma treatment and an octyltrichlorosilane $\left(\mathrm{OTS}, \mathrm{CH}_{3}\left(\mathrm{CH}_{2}\right)_{7} \mathrm{SiCl}_{3}\right)$ self-assembled monolayer (SAM). $\mathrm{O}_{2}$ plasma treatment was done using a Phantom II Reactive Ion Etching (RIE) system by Trion Technology Inc., with chamber pressure at $150 \mathrm{mTorr}, \mathrm{O}_{2}$ gas flow rate at $30 \mathrm{sccm}$, and exposure duration of 600 seconds. OTS SAM was formed by immersing the substrates in a $0.1 \mathrm{M}$ of OTS in Toluene for $20 \mathrm{~min}$. at $60^{\circ} \mathrm{C}$. The Au electrodes were functionalized with 1-octanethiol SAM by immersing the substrates in a $0.01 \mathrm{M}$ solution in toluene solvent for $30 \mathrm{~min}$ at room temperature to improve the contact properties. (In-depth discussions on dielectric and contact surface treatments are presented in Chapter 5 and 6, respectively.) A simplified schematic cross-section diagram of the OTFT structure is shown in Figure 4.19.

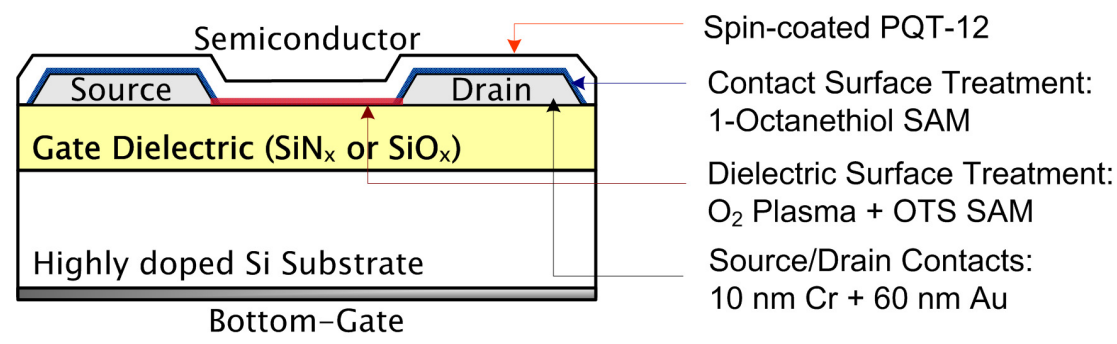

Figure 4.19. Schematic cross-section of the OTFT structure considered in this study.

Various PECVD films were investigated as the gate dielectric layer, including $300^{\circ} \mathrm{C} \mathrm{SiN}_{\mathrm{x}}$, $150^{\circ} \mathrm{C} \mathrm{SiN}$, and $200^{\circ} \mathrm{C} \mathrm{SiO}_{\mathrm{x}}$. The gate dielectric layers were deposited in a parallel-plate PECVD 
reactor operating at an excitation frequency of $13.56 \mathrm{MHz}$. The $300^{\circ} \mathrm{C} \operatorname{SiN}_{\mathrm{x}}(300 \mathrm{~nm}$ thick) of various compositions were prepared by adjusting the ammonia $\left(\mathrm{NH}_{3}\right)$ to silane $\left(\mathrm{SiH}_{4}\right)$ gas flow rate ratio from 5 to 20 . The $150^{\circ} \mathrm{C} \operatorname{SiN}_{\mathrm{x}}$ (300 nm thick) was deposited using a mixture of silane, ammonia, and hydrogen as process gases. The $200^{\circ} \mathrm{C} \mathrm{SiO}_{\mathrm{x}}(200 \mathrm{~nm}$ thick $)$ was deposited with a mixture of silane and nitrous oxide gases. Table 4.1 summarizes the deposition conditions used for PECVD SiN ${ }_{\mathrm{x}}$ films.

Electrical characterization of the OTFTs was carried out with a Keithley 4200-SCS parameter analyzer. Measurements were performed under ambient room conditions. From the measured transfer characteristic $\left(I_{\mathrm{D}}-V_{\mathrm{GS}}\right)$, transconductance $\left(g_{\mathrm{m}}\right)$ was calculated and was used for device parameter extraction as outlined in Section 2.2. Device parameters including effective field-effect mobility $\left(\mu_{\mathrm{FE}}\right)$, on/off current ratio $\left(I_{\mathrm{ON}} / I_{\mathrm{OFF}}\right)$, threshold voltage $\left(V_{\mathrm{T}}\right)$, and gate leakage current $\left(I_{\mathrm{G}}\right)$ are used to evaluate performance of OTFTs with different PECVD gate dielectric platforms. The various groups of PECVD gate dielectrics examined include $300^{\circ} \mathrm{C} \mathrm{SiN}_{\mathrm{x}}, 150^{\circ} \mathrm{C} \mathrm{SiN}_{\mathrm{x}}$, stacked $\mathrm{SiN}_{\mathrm{x}}, 200^{\circ} \mathrm{C} \mathrm{SiO}_{\mathrm{x}}$, and low-temperature dielectrics on plastic substrates.

\subsection{1 $\quad 300^{\circ} \mathrm{C} \mathrm{SiN}_{\mathrm{x}}$ Gate Dielectrics}

The first experiment evaluates OTFTs fabricated with $300^{\circ} \mathrm{C} \mathrm{SiN}_{\mathrm{x}}$ gate dielectric of varying film compositions. Figure 4.20(a) illustrates the transfer characteristics of a typical PQT-12 OTFT on a silicon-rich $\operatorname{SiN}_{\mathrm{x}}\left(x=1.08,300^{\circ} \mathrm{C}\right)$ gate dielectric. Parameter extraction from the saturation region $\left(V_{\mathrm{DS}}=-40 \mathrm{~V}\right)$ gives $\mu_{\mathrm{FE}}=0.092 \mathrm{~cm}^{2} / \mathrm{V}-\mathrm{s}, I_{\mathrm{ON}} / I_{\mathrm{OFF}}=10^{7}$, and $V_{\mathrm{T}}=-3.8 \mathrm{~V}$. The output characteristics show well-defined saturation behavior, as illustrated in Figure 4.20(b).

To examine the impact of $\mathrm{SiN}_{\mathrm{x}}$ film composition on OTFT performance, three sets of PQT-12 OTFTs were fabricated on $\mathrm{SiN}_{x}$ gate dielectrics with different $[\mathrm{N}] /[\mathrm{Si}]$ ratio $(x=[\mathrm{N}] /[\mathrm{Si}]=1.08$, $1.34,1.50)$ [30]. Each set of OTFTs comprised of devices with different channel geometries $(\mathrm{W} / \mathrm{L}$ ratio). Figure 4.21 compares the transfer characteristics, effective mobility, on/off current ratio, and threshold voltage as a function of the $[\mathrm{N}] /[\mathrm{Si}]$ ratio of the $\mathrm{SiN}_{\mathrm{x}}$ gate dielectrics. As the silicon content of the nitride dielectric increases (i.e., $[\mathrm{N}] /[\mathrm{Si}]$ ratio decreases), $I_{\mathrm{ON}}$ increases, $\mu_{\mathrm{FE}}$ increases, $I_{\mathrm{ON}} / I_{\mathrm{OFF}}$ increases, and $V_{\mathrm{T}}$ shifts in the negative direction. A $125 \%$ (or 2.25 times) increase in $\mu_{\mathrm{FE}}$ was observed using silicon-rich $\mathrm{SiN}_{\mathrm{x}}$ gate dielectric when compared to nitrogen-rich films. These results suggest that silicon-rich $\mathrm{SiN}_{\mathrm{x}}$ gate dielectric is a preferable choice for attaining OTFTs with improved $\mu_{\mathrm{FE}}, I_{\mathrm{ON}} / I_{\mathrm{OFF}}$, and current drive. 
(a)

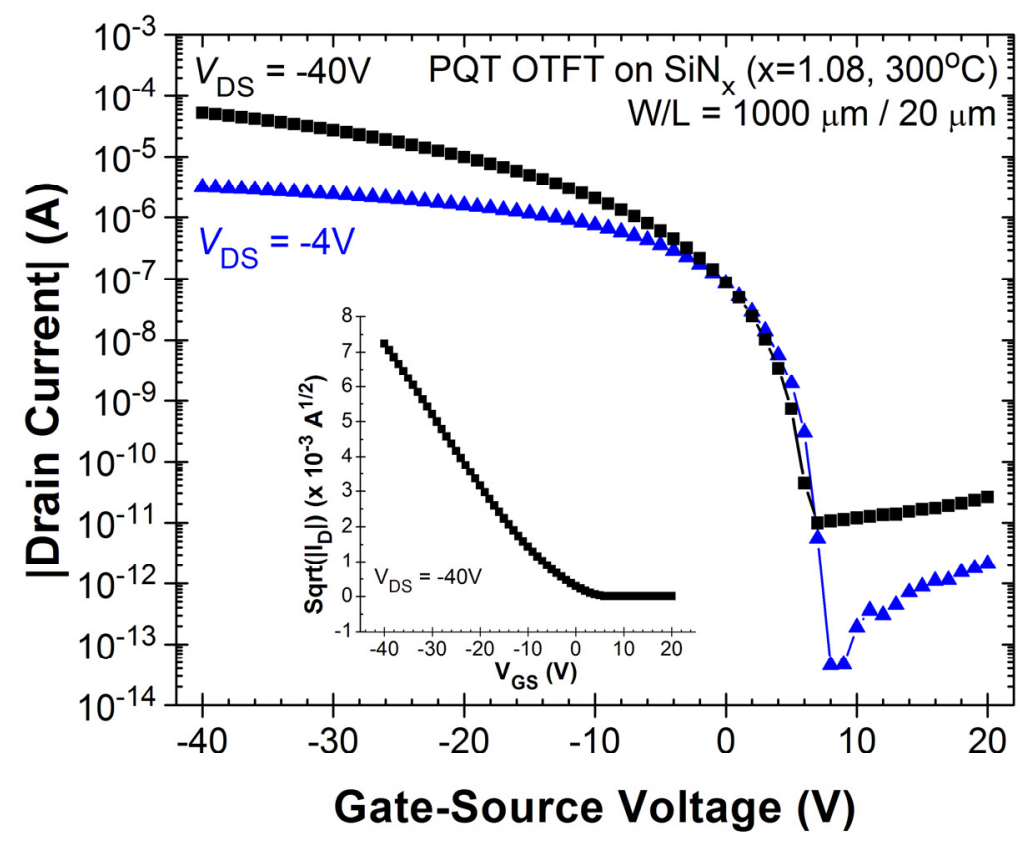

(b)

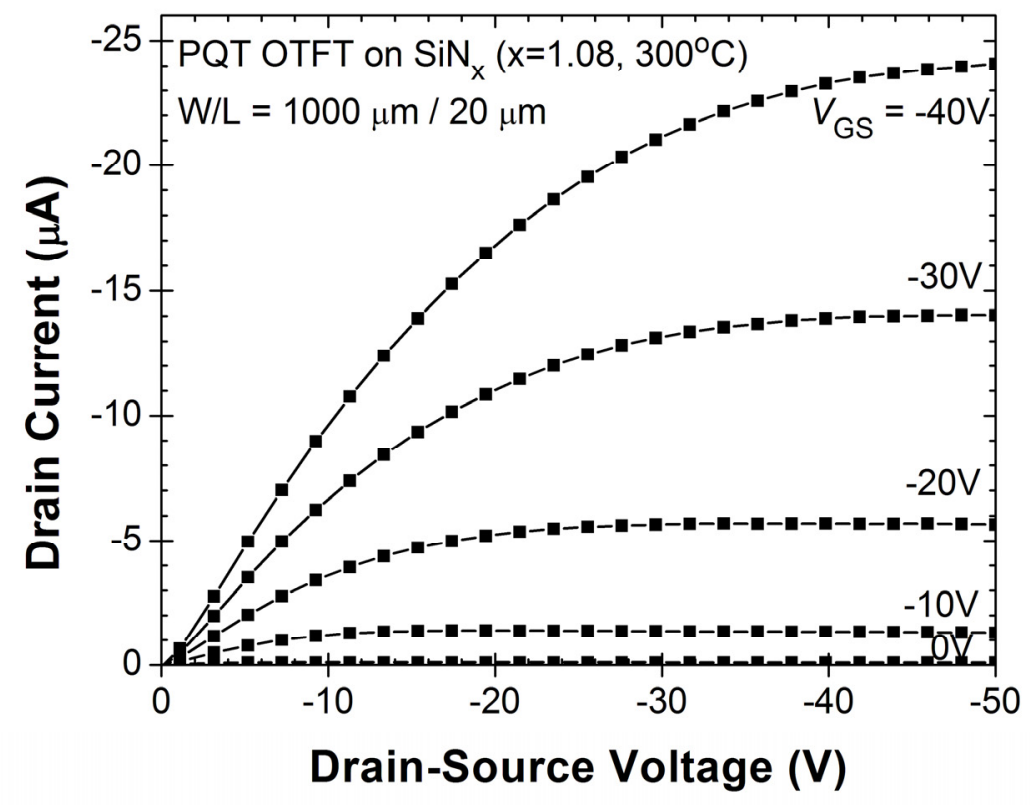

Figure 4.20. Electrical characteristics of PQT-12 OTFT on $300^{\circ} \mathrm{C} \mathrm{SiN}(x=1.08)$ gate dielectric with $W / L=1000 \mu \mathrm{m} / 20 \mu \mathrm{m}$ : (a) Transfer characteristics ( $\left.\log \left|I_{D}\right|-V_{G S}\right)$ and (b) output characteristics (ID- $\left.V_{D S}\right)$. Inset of (a) shows a well-behaved linear plot of $\sqrt{I_{D}}-V_{G S}$ in the saturation regime. 
(a)

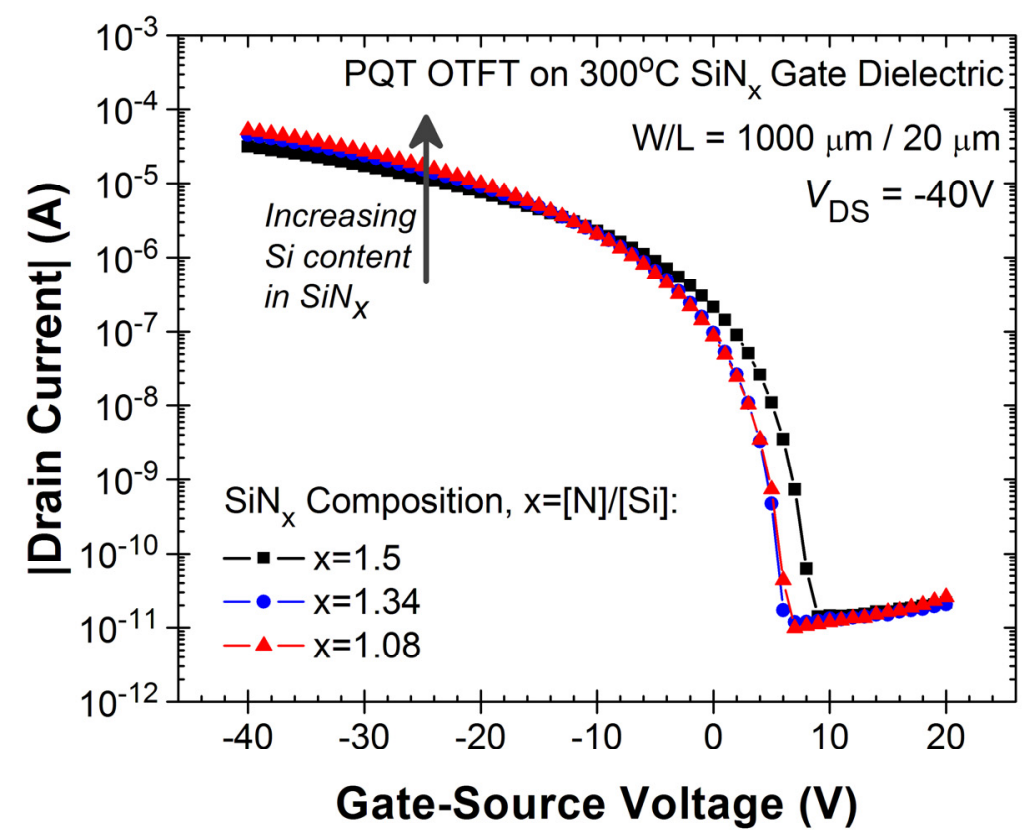

(b)

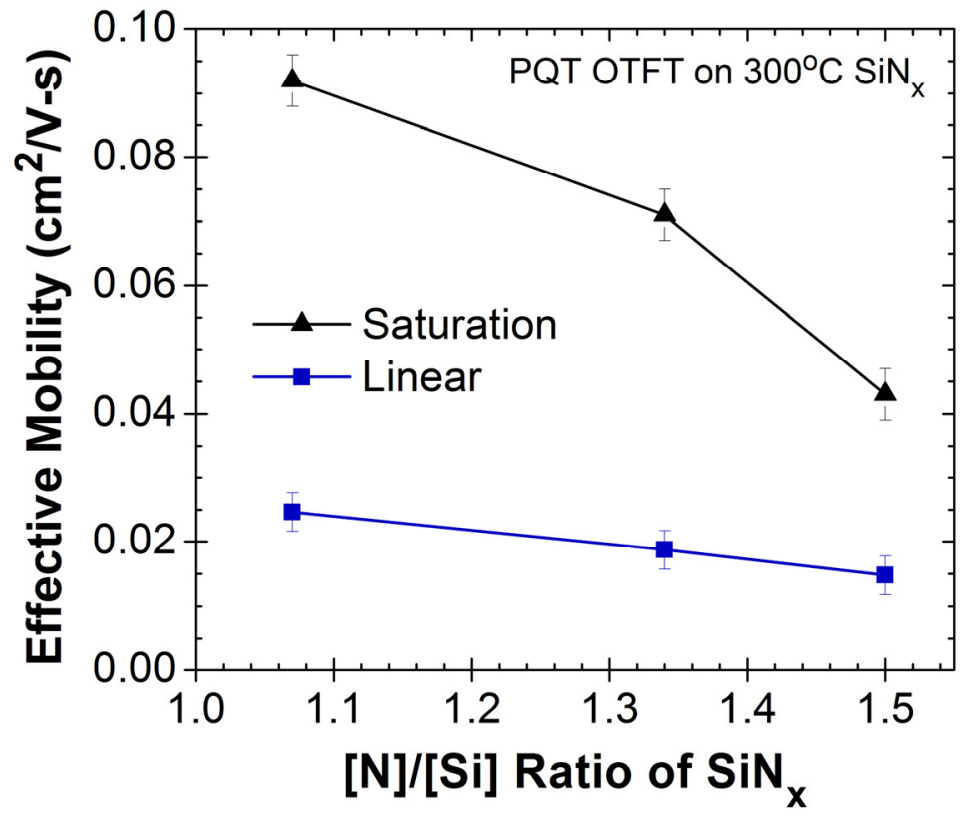


(c)

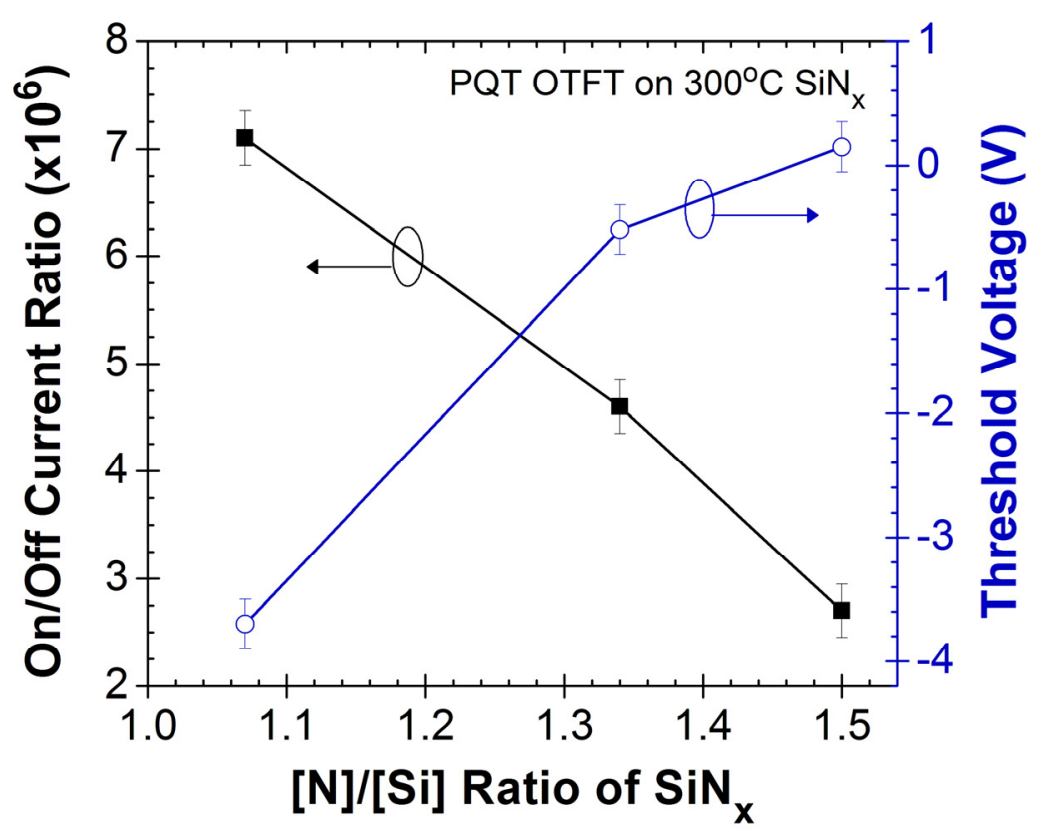

Figure 4.21. Comparison of PQT-12 OTFT characteristics on $300^{\circ} \mathrm{C} \mathrm{SiN}$ gate dielectric of varying film composition. (a) Transfer characteristics, (b) effective field-effect mobility, (c) on/off current ratio and threshold voltage as a function of the $[\mathrm{N}] /[\mathrm{Si}]$ ratio of $\mathrm{SiN}_{\mathrm{x}}$ film.

It is well recognized that a key criteria for attaining high mobility is to assemble an organic semiconductor film into a highly ordered molecular structure by a combination of clever molecular design and a properly prepared deposition surface [31][32][33][34]. The dependence of OTFT mobility on nitride composition observed here can be largely ascribed to differences in surface properties (e.g., composition, topography, surface energy) of the various nitride dielectrics. A higher density of silicon atoms appears to increase the density of OTS grafted on the $\mathrm{SiN}_{\mathrm{x}}$ surface, thus increasing the hydrophobicity of the dielectric surface (as seen in contact angle measurements in Figure 4.9). It has been reported that polycrystallites of regioregular polythiophenes typically self-organize into a highly oriented structure when deposited on a hydrophobic surface, which is the desired molecular arrangement for improved transistor performance [31].

AFM measurements in Section 4.2.2.2 revealed a decrease in surface roughness with decrease in $[\mathrm{N}] /[\mathrm{Si}]$ of $\mathrm{SiN}_{\mathrm{x}}$ film (i.e., towards silicon rich). This characteristic favorably supports the observed improvement in mobility for Si-rich $\mathrm{SiN}_{\mathrm{x}}$. This observation is consistent with the typical trend where increase in dielectric surface roughness results in reduced mobility [24].

Considering the surface chemical composition of the various $300^{\circ} \mathrm{C} \mathrm{SiN}_{\mathrm{x}}$ films plotted in Figure 4.11, there is a noticeable increase in $\mathrm{Si} 2 \mathrm{p}$ peak as the $[\mathrm{N}] /[\mathrm{Si}]$ ratio decreases. This result 
indicates increase in silicon content at the surface, which agrees perfectly with our extracted values of $[\mathrm{N}] /[\mathrm{Si}]$ ratio. The $\mathrm{Si} 2 \mathrm{p}$ peak remained the largest for the $\mathrm{Si}$-rich $\operatorname{SiN}_{\mathrm{x}}(x=1.08)$ sample relative to the other $\operatorname{SiN}_{\mathrm{x}}$ films $(x=1.50,1.34)$ even after $\mathrm{O}_{2}$ plasma and/or OTS SAM treatments, as indicated in Figure 4.11(b) and (c). It is hypothesized that the increased availability of Si atoms at the $\mathrm{SiN}_{\mathrm{x}}$ dielectric surface is a criteria for enhanced OTFT performance. It has been suggested that chemical interaction between the gate dielectric, SAM and organic semiconductor occurs via - $\mathrm{Si}-$ $\mathrm{O}-\mathrm{C}-$ bonds at the interface [35]. Therefore, a higher concentration of $\mathrm{Si}$ atoms at the interface facilitates and promotes bonding/interaction with the SAM and the organic semiconductor layer, leading to improved device performance for OTFTs with Si-rich $\mathrm{SiN}_{\mathrm{x}}$ gate dielectric. The role of $\mathrm{Si}$ and $\mathrm{O}$ atoms at the dielectric surface to facilitate bonding to SAM/organic is further highlighted in Figure 4.11(b) and (c), where $\mathrm{SiN}_{\mathrm{x}}$ sample with $x=1.08$ displays the highest $\mathrm{Si} 2 \mathrm{p}$ and $\mathrm{O} 1 \mathrm{~s}$ content compared to the other $\mathrm{SiN}_{\mathrm{x}}$ samples after $\mathrm{O}_{2}$ plasma and/or OTS treatments.

Based on the results reported in this experiment, it is concluded that improvement in PQT-12 OTFT characteristics observed with silicon-rich $\mathrm{SiN}_{\mathrm{x}}$ gate dielectric (as opposed to N-rich $\mathrm{SiN}_{\mathrm{x}}$ ) can be attributed to various surface properties of silicon-rich $\mathrm{SiN}_{\mathrm{x}}$ : (a) lowest surface energy (i.e., largest contact angle), (b) lowest surface roughness, and (c) highest [Si] and [O] content at the surface (after $\mathrm{O}_{2}$ plasma and $\mathrm{O}_{2}$ plasma/OTS treatment) when compared to the N-rich $\mathrm{SiN}_{\mathrm{x}}$ samples. A summary of these key surface qualities of silicon-rich $\mathrm{SiN}_{\mathrm{x}}$, along with their implications, is presented in Table 4.2.

Interestingly, the improvement in OTFT observed with silicon-rich $\mathrm{SiN}_{\mathrm{x}}$ is a new and unique discovery. This is because the opposite trend is typically seen in amorphous silicon or nanocrystalline silicon (nc-Si) TFTs, where nitrogen-rich $\mathrm{SiN}_{\mathrm{x}}$ is more preferable in silicon-based TFTs [13][14]. Possible rationalization to account for the different device behavior observed in OTFT and nc-Si TFT with the use of silicon-rich $\mathrm{SiN}_{\mathrm{x}}$ gate dielectric is presented in Table 4.3.

Our experimental results demonstrate enhancements in static operation of OTFTs with siliconrich $\operatorname{SiN}_{\mathrm{x}}$ gate dielectric. More detailed evaluation on the electrical stability and dynamic characteristics of OTFTs is needed to gain a more complete understanding on the overall impact of $\mathrm{SiN}_{\mathrm{x}}$ composition on OTFT performance. An attempt to assess stability of these OTFTs was made. However, it was difficult to isolate the effect of electrical stress from the effect of environmental stress on OTFT characteristics using our measurement setup (under ambient conditions). Specially designed environmental chambers (with separate control on levels of humidity, air, oxygen and 
vacuum) can facilitate thorough assessment of the impact of Si-rich $\mathrm{SiN}_{\mathrm{x}}$ gate dielectric on OTFT's electrical stability.

Table 4.2. Unique surface properties of silicon-rich $\mathrm{SiN}_{\mathrm{x}}$ (when compared to nitrogen-rich $\mathrm{SiN}_{\mathrm{x}}$ ) to account for the improved mobility in PQT-12 OTFTs. The qualities quoted are measured on $\mathrm{O}_{2}$ plasma and OTS SAM treated Si-rich $\mathrm{SiN}_{\mathrm{x}}$ surfaces.

\begin{tabular}{|c|c|c|}
\hline $\begin{array}{l}\text { Surface } \\
\text { Properties of Si- } \\
\text { rich } \mathrm{SiN}_{\mathrm{x}}\end{array}$ & Mechanisms & Implications \\
\hline $\begin{array}{l}\text { Larger contact } \\
\text { angle }\end{array}$ & $\begin{array}{l}\text { Si-rich } \mathrm{SiN}_{\mathrm{x}} \text { has more Si-Si and } \mathrm{Si}-\mathrm{H} \\
\text { bonds, which are less polar (or } \\
\text { electronegative) than the N-H and Si-N } \\
\text { bonds in N-rich } \mathrm{SiN}_{\mathrm{x}}\end{array}$ & $\begin{array}{l}\text { Large contact angle } \rightarrow \text { lower } \\
\text { surface energy } \rightarrow \text { more } \\
\text { energetically favorable and stable }\end{array}$ \\
\hline $\begin{array}{l}\text { Lower surface } \\
\text { roughness }\end{array}$ & $\begin{array}{l}\text { More Si species are available for bonding } \\
\text { with OTS via Si-O-C bonds, thus Si-rich } \\
\text { SiNx facilitates formation of a smoother } \\
\text { OTS layer }\end{array}$ & $\begin{array}{l}\text { Less interface scattering; fewer } \\
\text { (physical) disruption for the } \\
\text { overlying organic layer }\end{array}$ \\
\hline More Si-O bonds & $\begin{array}{l}\text { More Si-O bonds are available for bonding } \\
\text { with polymer semiconductor via Si-O-C to } \\
\text { generate an oxide-like surface } \\
\text { composition }\end{array}$ & $\begin{array}{l}\text { Favorable for deposition of OTS } \\
\text { and polymer semiconductor }\end{array}$ \\
\hline
\end{tabular}

Table 4.3. Impact of silicon-rich $\mathrm{SiN}_{\mathrm{x}}$ gate dielectric on OTFT and nc-Si TFT.

\begin{tabular}{|c|c|c|}
\hline $\begin{array}{l}\text { Impact of Si-rich } \\
\text { SiN }_{x} \text { Gate } \\
\text { Dielectric on: }\end{array}$ & $\begin{array}{c}\text { Electric } \\
\text { Characteristics }\end{array}$ & Possible Mechanisms \& Explanations \\
\hline Organic TFT & $\begin{array}{l}\text { Higher } \mu \mathrm{FE} \text { and } \\
{\text { ION } / I_{\text {OFF }}}\end{array}$ & $\begin{array}{l}\text { Device improvements with Si-rich } \mathrm{SiN}_{\mathrm{x}} \text { can be } \\
\text { attributed to interfacial \& microstructure effect! } \\
\text { Enhanced dielectric-semiconductor interface } \\
\text { between } \mathrm{SiN}_{\mathrm{x}} \text { and PQT-12 } \\
\text { Si-rich } \mathrm{SiN}_{\mathrm{x}} \text { provides a more organic-friendly dielectric } \\
\text { surface (larger contact angle, lower surface } \\
\text { roughness) to facilitate well-ordered organic layer } \\
\text { formation } \\
\text { Even if } \mathrm{Si} \text {-rich } \mathrm{SiN}_{\mathrm{x}} \text { may possess more } \mathrm{Si-Si} \text { dangling } \\
\text { bonds, their effect might be masked by } \mathrm{O}_{2} \text { plasma } \\
\text { and OTS SAM treatment }\end{array}$ \\
\hline $\begin{array}{l}\text { Nanocrystalline } \\
\text { silicon (nc-Si) } \\
\text { TFT }\end{array}$ & $\begin{array}{l}\text { Lower } \mu_{\mathrm{FE}} \text { and } \\
\text { loN/loFf }\end{array}$ & $\begin{array}{l}\text { Reduced device performance with } \mathrm{Si}-\text {-rich } \mathrm{SiN}_{\mathrm{x}} \text { can be } \\
\text { linked to electrical and defect-related effect! } \\
\text { Poorer dielectric-semiconductor interface between } \\
\text { Si-rich } \mathrm{SiN}_{\mathrm{x}} \text { and } \mathrm{nc}-\mathrm{Si} \\
\text { Higher interface trap density } \\
\text { More pronounced charge trapping effects with Si-rich } \\
\mathrm{SiN}_{\mathrm{x}} \text { (Si-Si dangling bonds) }\end{array}$ \\
\hline
\end{tabular}




\subsection{2 $\quad 150^{\circ} \mathrm{C} \mathrm{SiN}_{\mathrm{x}}$ Gate Dielectrics}

Due to constraints in processing temperatures of most low-cost plastic substrates (e.g., PET, PEN, Kapton), the maximum fabrication temperature for transistors on these substrates is typically in the range, $150-200^{\circ} \mathrm{C}[1]$; this temperature restriction also applies to gate dielectric deposition. PECVD $\mathrm{SiN}_{\mathrm{x}}$ is of particular interest for flexible electronics in view of their excellent dielectric properties even at low temperature. In this section, the electrical performance of PQT-12 OTFTs on $150^{\circ} \mathrm{C} \mathrm{SiN}$ gate dielectric is evaluated.

Figure 4.22 depicts the transfer characteristics of a typical device on $150^{\circ} \mathrm{C} \mathrm{SiN}_{\mathrm{x}}(x=1.60)$. The device parameters in saturation region $\left(V_{\mathrm{DS}}=-40 \mathrm{~V}\right)$ are $\mu_{\mathrm{FE}}=0.06 \mathrm{~cm}^{2} / \mathrm{V}-\mathrm{s}, I_{\mathrm{ON}} / I_{\mathrm{OFF}}=1.3 \times 10^{8}, V_{\mathrm{T}}$ $=5.74 \mathrm{~V}$, and $I_{\mathrm{G}}$ in the range of $10^{-10} \mathrm{~A}$. Well-behaved saturation characteristics can be observed in the output characteristics in Figure 4.22(b); however, contact resistance is evident from the nonlinear behavior at low drain voltages near the origin.

Figure 4.23 compares the effective mobility and on/off current ratio of PQT-12 OTFT with various $150^{\circ} \mathrm{C} \mathrm{SiN}_{\mathrm{x}}$ gate dielectrics, plotted as a function of gas flow ratio. The results showed an improvement in mobility towards more Si-rich films (i.e., lower $\mathrm{NH}_{3} / \mathrm{SiH}_{4}$ gas flow ratio). This trend is consistent with outcomes for OTFTs with $300^{\circ} \mathrm{C} \mathrm{SiN}_{\mathrm{x}}$ gate dielectric (see Figure 4.21). More importantly, Figure 4.23 demonstrated that PQT-12 OTFTs implemented on $150^{\circ} \mathrm{C} \mathrm{SiN}_{\mathrm{x}}$ possessed higher mobility and on/off current ratio when compared to its $300^{\circ} \mathrm{C} \mathrm{SiN}_{\mathrm{x}}$ counterpart! The improvement can be attributed to larger contact angle and smaller surface roughness of $150^{\circ} \mathrm{C}$ $\mathrm{SiN}_{\mathrm{x}}$ films when compared to $300^{\circ} \mathrm{C} \mathrm{SiN}_{\mathrm{x}}$, as illustrated in Figure 4.9 and Figure 4.10, respectively. Larger contact angle implies lower surface energy, which is favorable for deposition of subsequent organic layers. A smoother dielectric surface provides a more agreeable platform for formation of higher quality organic semiconductor layer and dielectric-semiconductor interface, and hence enhanced device characteristics. An attempt to compare the chemical composition of $300^{\circ} \mathrm{C}$ and $150^{\circ} \mathrm{C} \mathrm{SiN}_{\mathrm{x}}$ film surfaces via XPS was made. Unfortunately, the resulting data in Figure 4.12 was unable to provide sufficient justification to account for the observed OTFT trends.

Overall, these results showcase promising potential for implementing OTFT circuits with our low temperature $150^{\circ} \mathrm{C} \mathrm{SiN}_{\mathrm{x}}$ for flexible electronic applications. Nonetheless, more extensive electrical characterization to examine the OTFTs' electrical stability, lifetime and dynamic behavior are needed to gain more insights on the overall device performance. 
(a)

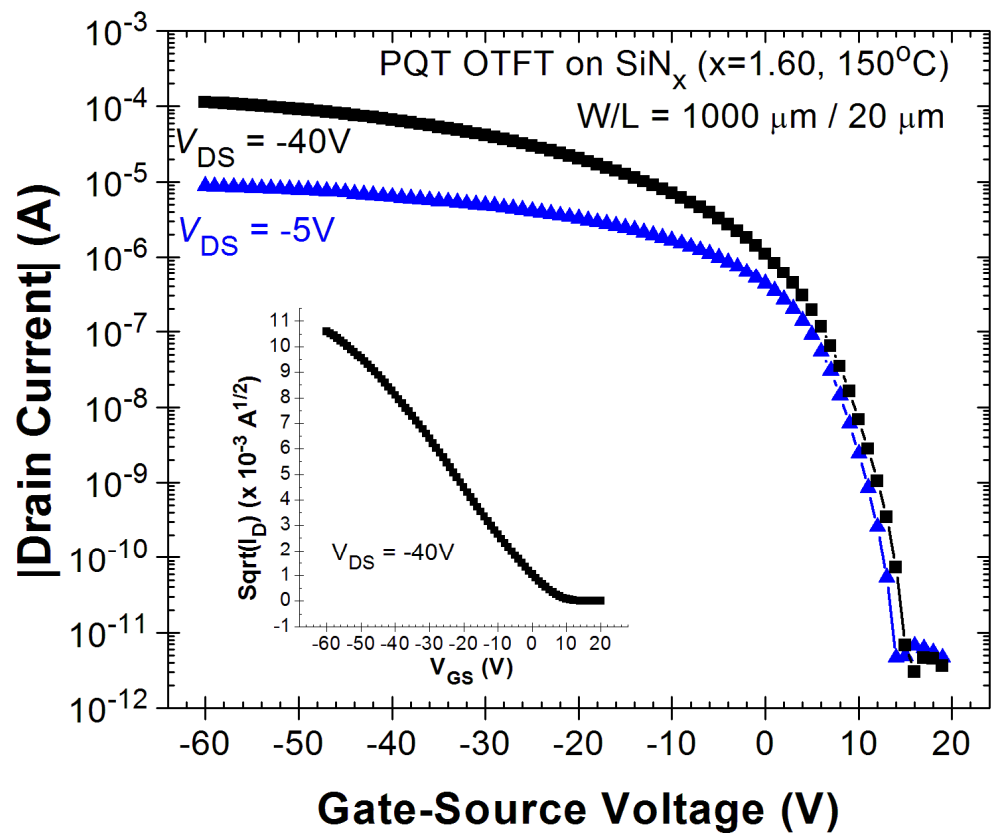

(b)

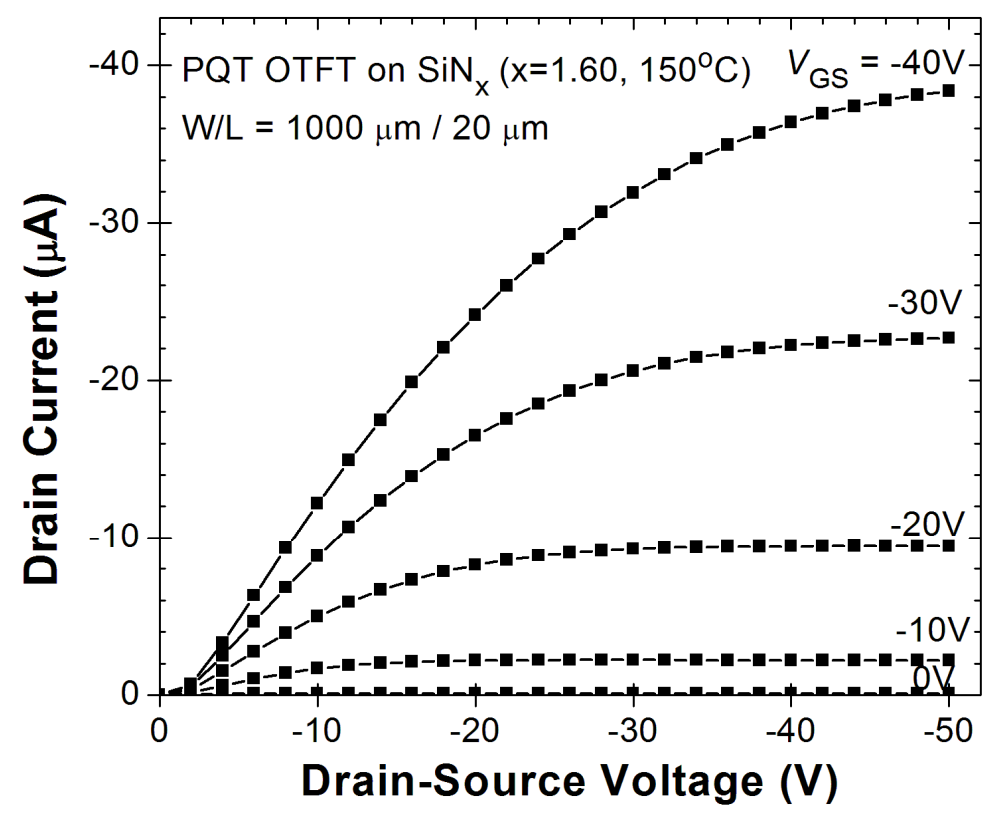

Figure 4.22. Electrical characteristics of PQT-12 OTFT on $150^{\circ} \mathrm{C}$ PECVD $\mathrm{SiN}_{x}(x=1.60)$ gate dielectric with $W / L=1000 \mu \mathrm{m} / 20 \mu \mathrm{m}$ : (a) transfer characteristics $\left(\log \left|I_{\mathrm{D}}\right|-V_{\mathrm{GS}}\right)$ and (b) output characteristics (ID-VD). Inset of (a) shows a well-behaved linear plot of $\sqrt{I_{D}}-V_{G S}$ in the saturation regime. 
(a)

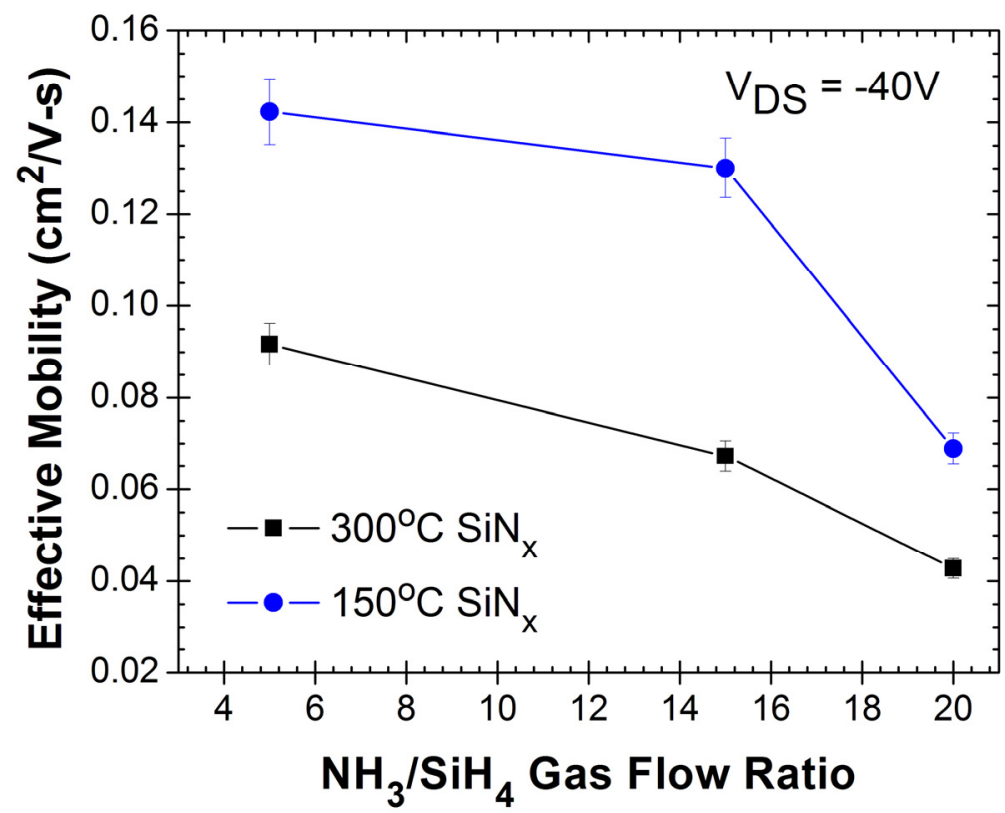

(b)

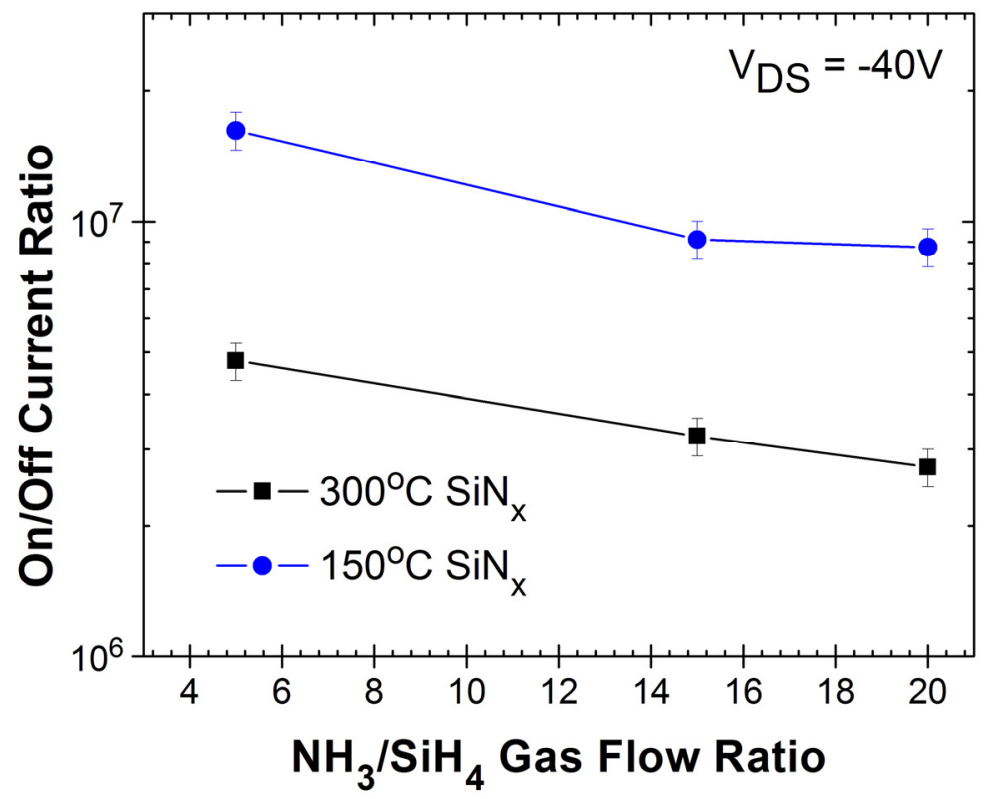

Figure 4.23. (a) Effective field-effect mobility and (b) on/off current ratio of PQT-12 OTFT (W/L $=1000 \mu \mathrm{m} / 20 \mu \mathrm{m}$ ) plotted as a function of $\mathrm{NH}_{3} / \mathrm{SiH}_{4}$ gas flow ratio for $150^{\circ} \mathrm{C}$ and $300^{\circ} \mathrm{C}$ $\mathrm{SiN}_{\mathrm{x}}$ gate dielectric. Data corresponds to measurements in saturation $\left(V_{D S}=-40 \mathrm{~V}\right)$ region. 


\subsubsection{Stacked $\mathrm{SiN}_{\mathrm{x}}$ Gate Dielectrics}

Electrical characterization of the $\mathrm{SiN}_{\mathrm{x}}$ films in Section 4.2 demonstrates that $\mathrm{N}$-rich $\mathrm{SiN}_{\mathrm{x}}$ has lower leakage current, higher breakdown field and less hysteresis than Si-rich $\mathrm{SiN}_{\mathrm{x}}$ films. However, OTFT characterization in Section 4.3.1 and Section 4.3.2 reveals that a Si-rich $\mathrm{SiN}_{\mathrm{x}}$ gate dielectric is preferred for OTFTs because it leads to higher mobility, higher on/off current ratio, and larger current driving capability. Our analysis showed that these device improvements are largely ascribed to a more organic-friendly interface with Si-rich $\mathrm{SiN}_{\mathrm{x}}$. In an attempt to combine the best qualities from both types of $\mathrm{SiN}_{\mathrm{x}}$ films, stacked $\mathrm{SiNx}$ gate dielectrics are considered. This design takes advantage of the excellent bulk properties of $\mathrm{N}$-rich $\mathrm{SiN}_{\mathrm{x}}$ and the desirable surface properties of Si-rich $\mathrm{SiN}_{\mathrm{x}}$. OTFTs featuring stacked $\mathrm{SiN}_{\mathrm{x}}$ gate dielectric are compared against OTFTs with unilayer $\mathrm{SiN}_{\mathrm{x}}$ gate dielectric (i.e., results from Section 4.3.1), to investigate possible improvements that can be achieved with the stacked dielectric structure.

Figure 4.24 depicts a cross-sectional diagram of OTFT incorporating stacked $\mathrm{SiN}_{\mathrm{x}}$ gate dielectric. The stacked dielectric film consisted of a Si-rich top layer and an N-rich bottom layer. It was deposited in a single/continuous deposition run by adjusting the gas flow ratio at a selected time during the deposition. Two combinations of stacked $\mathrm{SiN}_{\mathrm{x}}$ dielectric were tested for OTFTs, obtained by varying the "ratio of deposition time using N-rich recipe to Si-rich recipe." A description of these two stacked $\mathrm{SiN}_{\mathrm{x}}$ samples is presented in Table 4.4. For this experiment, $300^{\circ} \mathrm{C}$ $\mathrm{SiN}_{\mathrm{x}}$ deposition recipes were used, where $\mathrm{N}$-rich and $\mathrm{Si}$-rich refers to recipes with gas flow ratio $\mathrm{NH}_{3} / \mathrm{SiH}_{4}$ of 20 and 5, respectively. Please refer to Table 4.1 for specific deposition parameters.

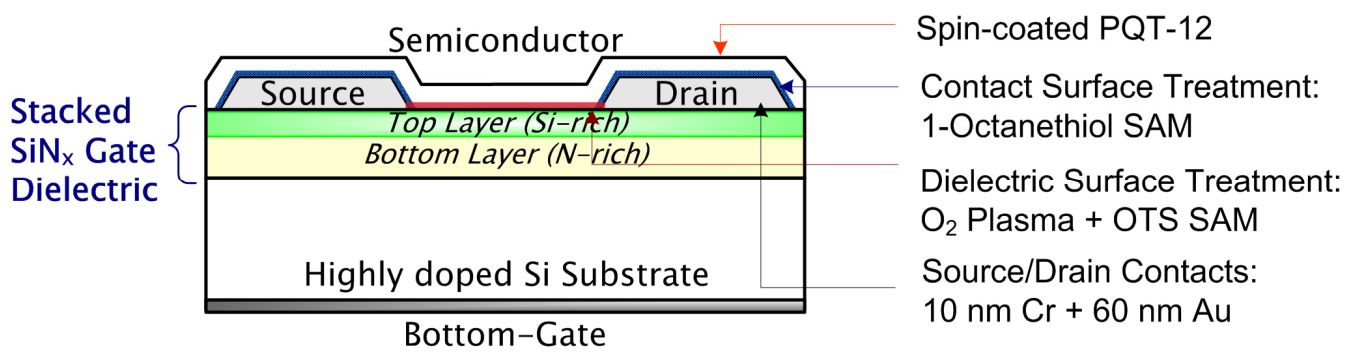

Figure 4.24. Schematic cross-section of the OTFT structure with stacked $\mathrm{SiN}_{\mathrm{x}}$ gate dielectric.

Figure 4.25 shows the FTIR spectrum of the two stacked $\mathrm{SiN}_{\mathrm{x}}$ films. Both spectra display a larger N-H stretching peak than Si-H peak, implying that the bulk of the film is N-rich. Comparing the two stacked samples, Stacked[2:1] displayed a larger Si-H peak than Stacked[5:1]. This is 
logical as the Stacked[2:1] sample is designed to have a larger volume of Si-rich layer than the Stacked[5:1] sample. Material characterization confirmed the properties of these stacked $\operatorname{SiN}_{\mathrm{x}}$ films are immediate between $\mathrm{N}$-rich $\mathrm{SiN}_{\mathrm{x}}$ and $\mathrm{Si}$-rich $\mathrm{SiN}_{\mathrm{x}}$. As shown in Table 4.5, the dielectric constant, refractive index, and extracted $[\mathrm{N}] /[\mathrm{Si}]$ value of stacked $\mathrm{SiN}_{\mathrm{x}}$ films fall within the data interval bounded by N-rich $\operatorname{SiN}_{\mathrm{x}}$ and Si-rich $\mathrm{SiN}_{\mathrm{x}}$.

Table 4.4. Description of stacked $\mathrm{SiN}_{\mathrm{x}}$ gate dielectric samples.

\begin{tabular}{|c|c|c|c|c|c|}
\hline \multirow[t]{2}{*}{ Sample ID } & \multirow[t]{2}{*}{$\begin{array}{l}\text { Ratio of deposition time } \\
\text { using N-rich recipe to } \\
\text { Si-rich recipe }\end{array}$} & \multicolumn{2}{|c|}{$\begin{array}{c}\text { Estimated } \\
\text { Thickness (nm) }\end{array}$} & \multicolumn{2}{|c|}{$\begin{array}{c}\text { Estimated } \\
\% \text { Thickness }\end{array}$} \\
\hline & & N-rich & Si-rich & N-rich & Si-rich \\
\hline $\operatorname{SiN}_{x}-$ Stacked[2:1] & $2: 1$ & 200 & 100 & $67 \%$ & $33 \%$ \\
\hline SiNx-Stacked [5:1] & $5: 1$ & 250 & 50 & $83 \%$ & $17 \%$ \\
\hline
\end{tabular}

Note: $\mathrm{N}$-rich recipe refers to films deposited using $\mathrm{NH}_{3} / \mathrm{SiH}_{4}$ gas flow ratio of 20; Si-rich recipe refers to films deposited using $\mathrm{NH}_{3} / \mathrm{SiH}_{4}$ gas flow ratio of 5 . SiNx deposition was performed at substrate temperature of $300^{\circ} \mathrm{C}$, RF power of $2 \mathrm{~W}$, pressure of $400 \mathrm{mTorr}$, and total duration of $30 \mathrm{~min}$. The estimated thickness is based on the crude assumption that deposition rate is constant.

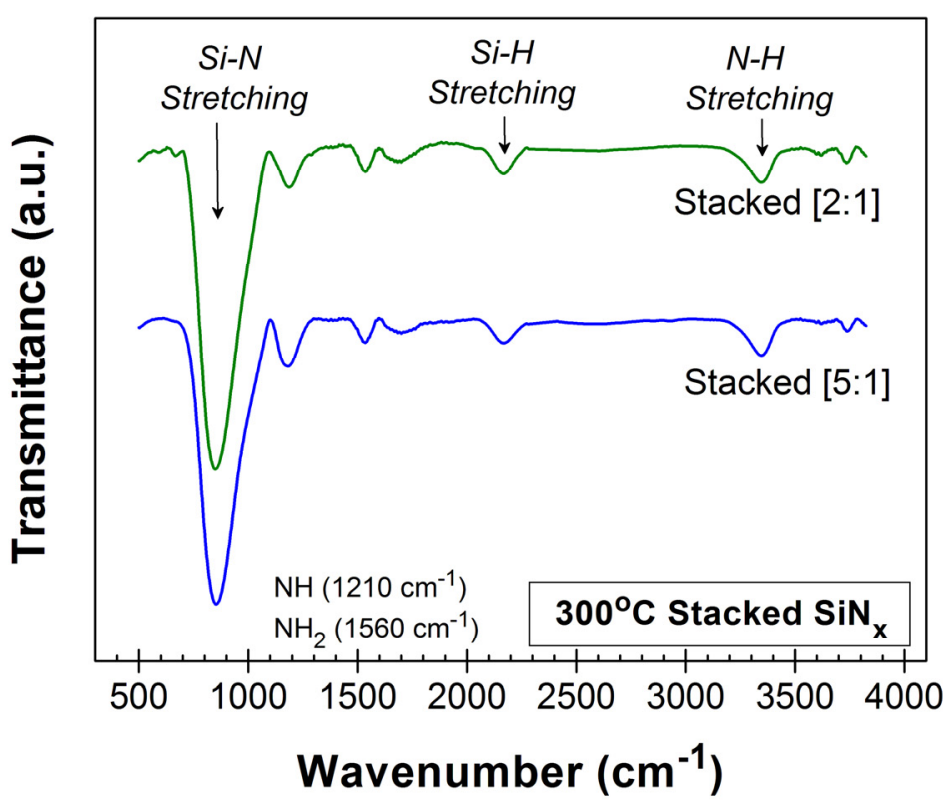

Figure 4.25. Fourier transform infrared (FTIR) spectroscopy of the stacked $\mathrm{SiN}_{\mathrm{x}}$ films, deposited by PECVD at substrate temperature of $300^{\circ} \mathrm{C}$. 
Table 4.5. Material characterization data for stacked $\mathrm{SiN}_{\mathrm{x}}$ films, including refractive index, $[\mathrm{N}] /[\mathrm{Si}]$ ratio, and dielectric constant. Data for Si-rich $\mathrm{SiN}_{\mathrm{x}}$ and $\mathrm{N}$-rich $\mathrm{SiN}_{\mathrm{x}}$ are included for comparison.

\begin{tabular}{|lccc|}
\hline SiN $_{\mathrm{x}}$ Sample ID & Refractive Index $(\mathrm{n})$ & {$[\mathrm{N}] /[\mathrm{Si}]$} & Dielectric Constant \\
\hline Si-rich SiN $_{\mathrm{x}}$ & 2.049 & 1.077 & 5.816 \\
\hline $\mathrm{SiN}_{\mathrm{x}}-\mathrm{Stacked}[2: 1]$ & 2.039 & 1.093 & 5.862 \\
\hline $\mathrm{SiN}_{\mathrm{x}}$-Stacked [5:1] & 2.045 & 1.083 & 5.865 \\
\hline N-rich SiN $_{\mathrm{x}}$ & 1.818 & 1.130 & 6.341 \\
\hline
\end{tabular}

The effective field-effect mobility of PQT-12 OTFTs with stacked $\mathrm{SiN}_{\mathrm{x}}$ gate dielectric is summarized in Figure 4.26. Higher mobility is attained with the $\operatorname{SiN}_{\mathrm{x}}$-Stacked[2:1] sample than the $\mathrm{SiN}_{\mathrm{x}}$-Stacked[5:1] sample. This suggests that thicker Si-rich top layer is preferred for enhanced mobility. When compared to unilayer $\operatorname{SiN}_{\mathrm{x}}$, OTFTs with $\mathrm{SiN}_{\mathrm{x}}$-Stacked[2:1] demonstrated improved mobility over N-rich gate dielectric. However, Si-rich $\mathrm{SiN}_{\mathrm{x}}$ gate dielectric provides superior mobility compared to stacked $\mathrm{SiN}_{\mathrm{x}}$ gate dielectrics. These results clearly showed that $\mathrm{Si}$-rich $\mathrm{SiN}_{\mathrm{x}}$ remains as the leading choice of gate dielectric for achieving high mobility OTFTs.

For ongoing research, more detailed device characterization and analysis should be performed to identify potential strengths of this stacked structure. It is predicted that OTFTs fabricated on N-rich $\mathrm{SiN}_{\mathrm{x}}$ or stacked $\mathrm{SiN}_{\mathrm{x}}$ gate dielectric can offer advantages in terms of reduced hysteresis, improved dielectric breakdown strength, enhanced AC/transient properties and improved electrical stability. Additional studies to understand the growth mechanisms of the stacked structure and the material properties of the transition region from N-rich to Si-rich would be beneficial. The deposition of stacked $\mathrm{SiN}_{\mathrm{x}}$ was executed continuously by simply changing the gas flow ratio after specific time duration. It is interesting to examine if a momentary pause/break after deposition of the N-rich layer before restarting deposition for Si-rich layer would alter OTFT characteristics. An intermittent break (e.g., few minutes) may help to purge the chamber and promote growth of a higher quality Si-rich $\mathrm{SiN}_{\mathrm{x}}$ at the interface. A better understanding of the growth process will allow more strategic design or optimization of the deposition scheme, to fulfill the intention of maximizing device performance by combining benefits of $\mathrm{N}$-rich $\operatorname{SiN}_{x}$ and Si-rich $\operatorname{SiN}_{x}$ in a stacked dielectric system. 


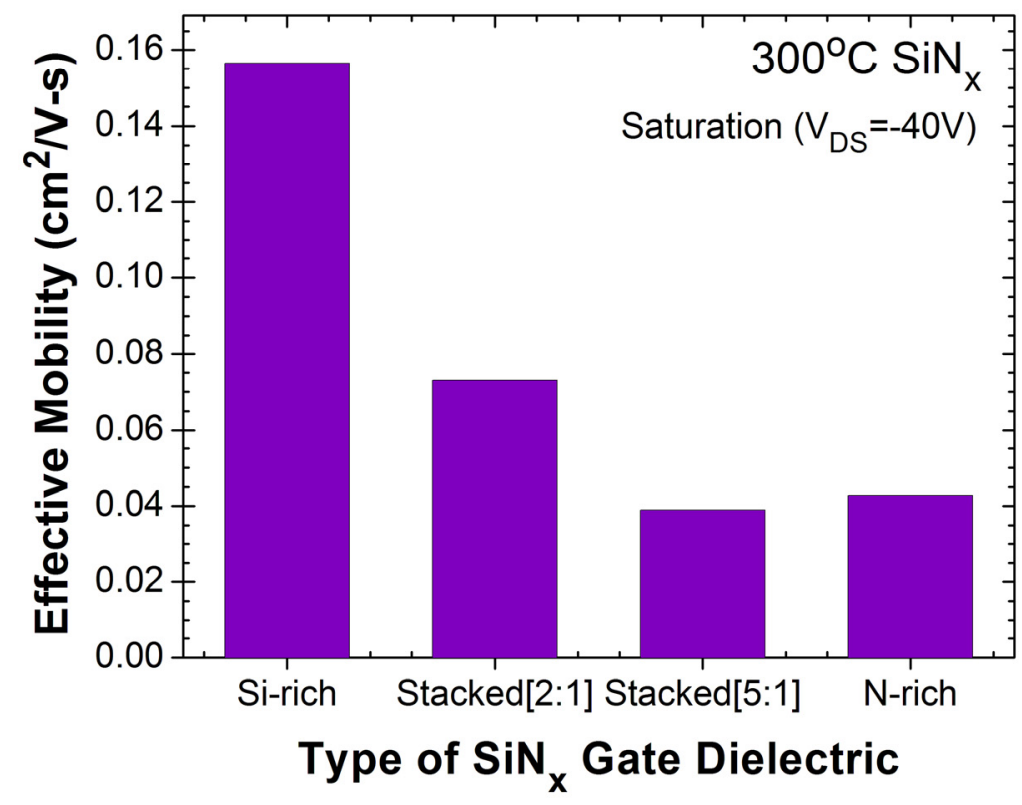

Figure 4.26. Effective field-effect mobility of PQT-12 OTFT with stacked $\mathrm{SiN}_{\mathrm{x}}$ gate dielectrics. Mobility of OTFT with Si-rich $\operatorname{SiN}_{x}(x=1.08)$ and N-rich $\operatorname{SiN}_{x}(x=1.50)$ is also shown for comparison.

\subsection{4 $200^{\circ} \mathrm{C} \mathrm{SiO}_{\mathrm{x}}$ Gate Dielectrics}

PECVD $\mathrm{SiO}_{x}$ presents an alternative low temperature gate dielectric option for large area flexible electronics integration. Most of the early research on OTFTs employed thermal silicon dioxide $\left(\mathrm{SiO}_{2}\right)$ on highly-doped silicon wafer as a platform for material and device characterization; these experiments showed that excellent dielectric-semiconductor interface properties can be achieved between $\mathrm{SiO}_{2}$ and organic layer [20][28][32]. However, the use of thermal $\mathrm{SiO}_{2}$ limits OTFT circuit integration because of the continuous gate structure (with the silicon wafer); more importantly, growth of thermal $\mathrm{SiO}_{2}$ involves a high temperature process that is not amenable to the low-temperature processing objectives of OTFTs. Therefore, it is of interest to explore PECVD $\mathrm{SiO}_{\mathrm{x}}$ as the gate dielectric for OTFTs. While being compatible with processing temperatures of plastic substrates, low temperature $\mathrm{PECVD}_{\mathrm{SiO}}$ can present a similar interface platform as thermal oxide to deliver high performance OTFTs. However, PECVD oxides are typically prone to poorer bulk properties than PECVD $\operatorname{SiN}_{x}$ [5], which may lead to high leakage current, low breakdown fields, hysteresis effects, and $V_{\mathrm{T}}$ instability, in OTFTs. Thus, there is an ongoing research endeavor to enhance quality of PECVD $\mathrm{SiO}_{\mathrm{x}}$ thin film. 
Transfer and output characteristics of a PQT-12 OTFT on $\mathrm{SiO}_{\mathrm{x}}$ gate dielectric are shown in Figure 4.27. Parameter extraction from the saturation characteristics $\left(V_{\mathrm{DS}}=-30 \mathrm{~V}\right)$ gives $\mu_{\mathrm{FE}}=0.34$ $\mathrm{cm}^{2} / \mathrm{V}-\mathrm{s}, I_{\mathrm{ON}} / I_{\mathrm{OFF}}=2.3 \times 10^{8}$, and $V_{\mathrm{T}}=-5.04 \mathrm{~V}$. The output characteristics in Figure 4.27(b) demonstrate well-defined saturation behavior and reasonable contact properties [37]. It is worthy to highlight that PECVD $\mathrm{SiO}_{x}$ produced relatively high mobility PQT-12 OTFT devices, in comparison to devices with PECVD $\operatorname{SiN}_{\mathrm{x}}$ (as reported in previous sections). The enhanced mobility indicates that the PECVD $\mathrm{SiO}_{\mathrm{x}}$ film, following appropriate interface treatment, delivers the needed surface platform for formation of a highly-ordered PQT-12 semiconductor layer, which is vital for attaining high field-effect mobility [36][38]. Despite the improvement in mobility, OTFTs on $\mathrm{PECVD} \mathrm{SiO}_{\mathrm{x}}$ gate dielectric exhibited high gate leakage current (from an initial value of $10^{-9} \mathrm{~A}$ to $10^{-6} \mathrm{~A}$ ) at high negative $V_{\mathrm{GS}}$ following successive electrical bias. PECVD $\mathrm{SiO}_{\mathrm{x}}$ thin films deposited at low processing temperatures are typically susceptible to electrical integrity issues; the low mass density of $\mathrm{SiO}_{\mathrm{x}}$ films often leads to considerable leakages [5]. Also, bias-induced shift in $V_{\mathrm{T}}$ is apparent in the saturation and linear transfer characteristics in Figure 4.27(a). This instability can be related to the higher density of fixed charge in the bulk $\mathrm{SiO}_{\mathrm{x}}$, charge trapping at the organicoxide interface, and leakage paths in $\mathrm{SiO}_{\mathrm{x}}$.

In summary, PQT-12 OTFTs with $\mathrm{SiO}_{\mathrm{x}}$ gate dielectric offer superior mobility compared to devices on $\mathrm{SiN}_{\mathrm{x}}$. However, $\mathrm{SiO}_{\mathrm{x}}$ is more susceptible to higher gate leakage, lower dielectric breakdown, and reduced electrical stability. Thus, for applications requiring low deposition temperatures, PECVD $\mathrm{SiN}_{\mathrm{x}}$ is currently the preferred choice, as it offers superior electrical integrity in terms of lower leakage current, fewer pinholes, higher breakdown field, higher stability, and reduced hysteresis.

For future research, additional effort to improve the quality and integrity of $\mathrm{PECVD}_{\mathrm{SiO}}$ is needed to make $\mathrm{SiO}_{\mathrm{x}}$ a feasible candidate for OTFT applications. In addition, it is of interest to explore a stacked $\mathrm{SiN}_{\mathrm{x}} / \mathrm{SiO}_{\mathrm{x}}$ dielectric structure, with $\mathrm{SiN}_{\mathrm{x}}$ serving as a bottom bulk layer and $\mathrm{SiO}_{\mathrm{x}}$ as a top interface layer. This configuration takes advantage of the excellent bulk properties of $\mathrm{SiN}_{\mathrm{x}}$ to ensure low gate leakage current, high dielectric breakdown strength and good dielectric stability; this is combined with the preferential surface properties of $\mathrm{SiO}_{\mathrm{x}}$ to form quality dielectricsemiconductor interface for delivering high mobility OTFTs. 
(a)

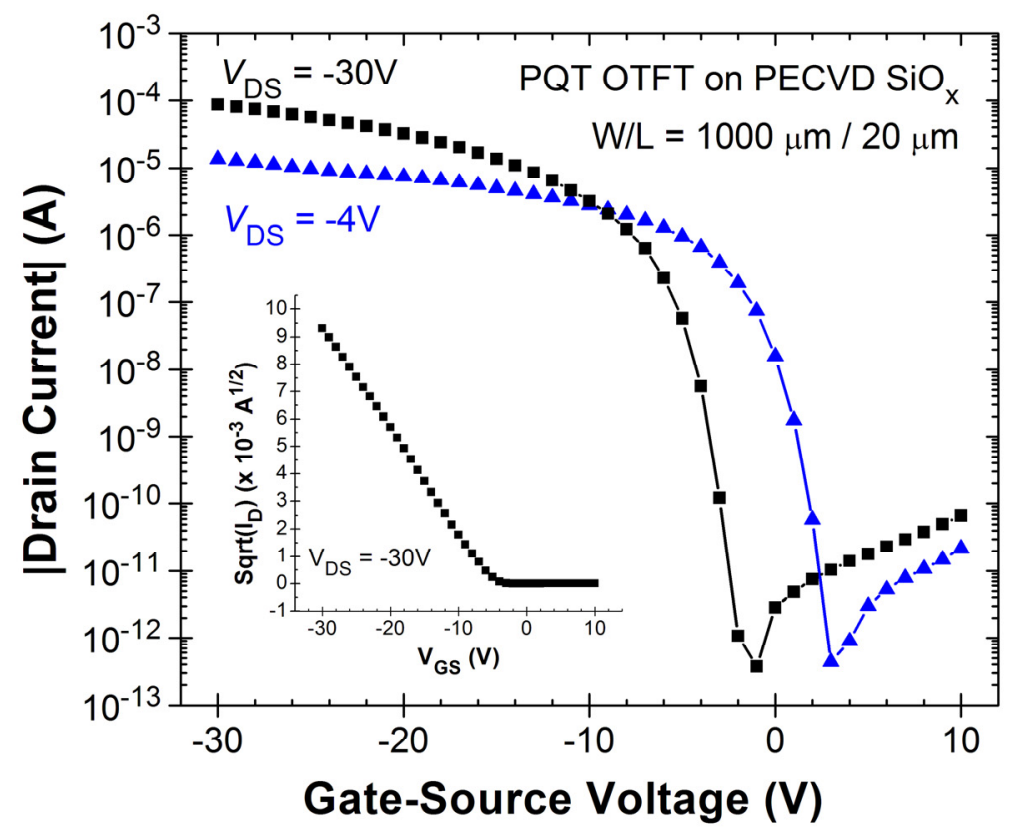

(b)

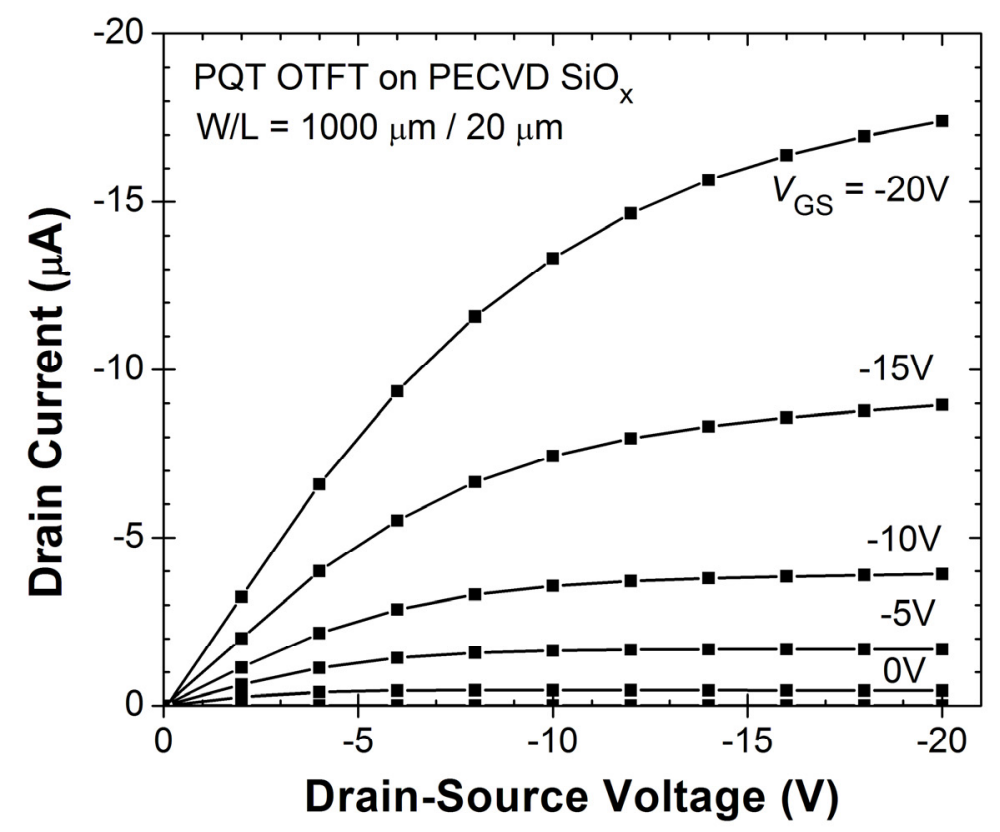

Figure 4.27. Electrical characteristics of PQT-12 OTFT on PECVD SiO $x$ gate dielectric with $W / L$ $=1000 \mu \mathrm{m} / 20 \mu \mathrm{m}:(a)$ transfer characteristics $\left(\log \left|I_{\mathrm{D}}\right|-V_{\mathrm{GS}}\right)$ and (b) output characteristics $\left(I_{\mathrm{D}}-V_{\mathrm{DS}}\right)$. Inset of (a) shows a linear plot of $\sqrt{I_{D}}-V_{G S}$ in saturation regime. 


\subsubsection{OTFTs on Plastic Substrates}

PQT-12 OTFTs fabricated with low temperature PECVD gate dielectrics have demonstrated very promising performance on rigid substrates, as presented in Section 4.3.2 and Section 4.3.4 for $150^{\circ} \mathrm{C} \mathrm{SiN}_{\mathrm{x}}$ and $200^{\circ} \mathrm{C} \mathrm{SiO}_{\mathrm{x}}$, respectively. This section examines the application of low temperature PECVD dielectrics for demonstration of OTFTs on plastic substrates. Table 4.6 lists the various pairs of gate dielectric and plastic substrate considered in this study, including $150^{\circ} \mathrm{C}$ $\mathrm{SiN}_{\mathrm{x}}$ gate dielectric on poly(ethylene terephthalate) (PET) substrate, $150^{\circ} \mathrm{C} \mathrm{SiN}_{\mathrm{x}}$ on Kapton (polyimide) substrate, and $180^{\circ} \mathrm{C} \mathrm{SiO}_{\mathrm{x}}$ on Kapton substrate. ${ }^{10}$ Bottom-gate top-contact OTFT configuration, as illustrated in Figure 4.28, was used for this investigation. The source/drain Au contacts were defined using the shadow mask technique, to simplify the fabrication process and to avoid any process-induced degradation of devices on plastic substrates. The dielectric surface was pretreated with OTS SAM only; $\mathrm{O}_{2}$ plasma treatment was omitted for preliminary tests to eliminate possible plasma-induced damage of the plastic substrate.

Table 4.6. Various plastic substrates and PECVD gate dielectric employed for PQT-12 OTFT fabrication in this study. The corresponding effective field effect mobility $\left(\mu_{\mathrm{FE}}\right)$, on/off current ratio ( ION/IOFF) and threshold voltage $\left(V_{\mathrm{T}}\right)$, extracted in the saturation region, are shown.

\begin{tabular}{|c|c|c|c|c|c|c|}
\hline ID & Substrate & $\begin{array}{l}\text { PECVD Gate } \\
\text { Dielectric }\end{array}$ & $\begin{array}{c}\mu_{\mathrm{FE}} \\
\left(\mathrm{cm}^{2} / \mathrm{V}-\mathrm{s}\right)\end{array}$ & lon/loff & $V_{T}(\mathrm{~V})$ & Remarks \\
\hline \#1 & PET & $\begin{array}{l}150^{\circ} \mathrm{C} \mathrm{SiN} x \\
(x=1.60)\end{array}$ & 0.0092 & $6.8 \times 10^{3}$ & 6.7 & $\begin{array}{l}\text { Higher } \mu_{F E} \text { but lower loN/loFF } \\
\text { compared to Kapton substrate }\end{array}$ \\
\hline \#2 & Kapton & $\begin{array}{l}150^{\circ} \mathrm{C} \mathrm{SiN} x \\
(x=1.60)\end{array}$ & 0.0051 & $1.6 \times 10^{6}$ & 5.0 & $\begin{array}{l}\text { Higher lon/loFf but lower } \mu_{F E} \\
\text { compared to PET substrate }\end{array}$ \\
\hline \#3 & Kapton & $180^{\circ} \mathrm{C} \mathrm{SiOx}$ & 0.0076 & $3.1 \times 10^{5}$ & -3.3 & $\begin{array}{l}\text { Very low yield. High gate } \\
\text { leakage current. Susceptible to } \\
\text { breakdown. }\end{array}$ \\
\hline
\end{tabular}

\begin{tabular}{|l|l|}
\hline \multicolumn{1}{|c|}{ Source } & Contacts: $60 \mathrm{~nm} \mathrm{Au}$ \\
\hline Polymer Semiconductor $(\mathrm{PQT}-12)$ & Dielectric Surface Treatment: \\
\hline Gate Dielectric $\left(\mathrm{SiN}_{\mathrm{x}}, \mathrm{SiO}_{\mathrm{x}}\right)$ & OTS SAM \\
\hline Bottom-Gate (Al) & \\
\hline Plastic Substrate (Kapton, PET) & \\
\hline
\end{tabular}

Figure 4.28. Schematic cross-section of the bottom-gate top-contact OTFT on plastic substrate.

\footnotetext{
${ }^{10}$ Kapton ${ }^{\circledR}$ is a polyimide film developed by DuPont
} 
Figure 4.29, Figure 4.30, and Figure 4.31 display the transfer and output characteristics of PQT12 OTFTs on various plastic substrates and PECVD gate dielectrics. The effective field-effect mobility, on/off current ratio and threshold voltage extracted from these measurements are summarized in Table 4.6. Each dielectric-substrate combination has its strengths and weaknesses.

PQT-12 OTFTs with PECVD $\mathrm{SiO}_{\mathrm{x}}$ gate dielectric on Kapton exhibited higher mobility than devices with $\mathrm{SiN}_{\mathrm{x}}$ dielectric on Kapton. The higher mobility can be attributed to the favorable $\mathrm{SiO}_{\mathrm{x}}$-polymer interface. However, the device yield for the $\mathrm{SiO}_{\mathrm{x}}$ on Kapton substrate is very low Moreover, the $\mathrm{SiO}_{\mathrm{x}} /$ Kapton sample is highly prone to gate leakage current $\left(10^{-9} \mathrm{~A}\right.$ range $)$ and dielectric breakdown (occurs around $40 \mathrm{~V}$ for $200 \mathrm{~nm}$ thick $\mathrm{SiO}_{\mathrm{x}}$ ). The offset of the $I_{\mathrm{D}}-V_{\mathrm{DS}}$ curve from the origin $\left(V_{\mathrm{DS}}=0 \mathrm{~V}\right)$ in Figure 4.31(b) is a result of large gate leakage current in the device.

On the contrary, OTFTs fabricated with PECVD $\mathrm{SiN}_{\mathrm{x}}$ dielectric on plastic substrates displayed much better dielectric strength and lower dielectric leakage current compared to $\mathrm{SiO}_{\mathrm{x}}$, but at the expense of reduced mobility. Comparing $\mathrm{SiN}_{\mathrm{x}} /$ Kapton with $\mathrm{SiN}_{\mathrm{x}} / \mathrm{PET}$, devices on Kapton showed higher on/off current ratio, better saturation characteristics and linear contact behavior (Figure 4.30); meanwhile, devices on PET showed higher mobility (Figure 4.29). Similar levels of gate leakage current were observed for the $\mathrm{SiN}_{\mathrm{x}} /$ Kapton and $\mathrm{SiN}_{\mathrm{x}} / \mathrm{PET}$ samples.

Overall, there is a substantial reduction in device performance for OTFTs on plastic substrates when compared to OTFTs on rigid substrates. Considering PQT-12 OTFTs with $150^{\circ} \mathrm{C} \mathrm{SiN}_{\mathrm{x}}$ gate dielectric, $\mu_{\mathrm{FE}}$ decreased by a factor of 10 when the substrate was changed from a rigid Si wafer to a flexible Kapton substrate. This reduction can be attributed to the higher surface roughness of $\mathrm{SiN}_{\mathrm{x}}$ films on plastic substrates than $\mathrm{SiN}_{\mathrm{x}}$ on $\mathrm{Si}$ substrates, as indicated in Table 4.7. In general, plastic substrates display higher surface roughness; passivation layer (e.g., BCB) can be used to smoothing the substrate roughness. Chabinyc et al. showed that the field-effect mobility of OTFTs decreases nearly exponentially with surface roughness of the gate dielectric [24]. Another possible explanation for the reduction in mobility is the omission of $\mathrm{O}_{2}$ plasma treatment for the devices on plastic substrate. As we will see in the next chapter, $\mathrm{O}_{2}$ plasma treatment plays a critical role for achieving high mobility OTFTs with $\mathrm{SiN}_{\mathrm{x}}$ gate dielectric.

Table 4.7. Surface roughness of $150^{\circ} \mathrm{C}$ PECVD SiN $\mathrm{N}_{\mathrm{x}}$ on Si wafer and plastic substrate.

\begin{tabular}{|lc|}
\hline Substrate & Surface Roughness \\
\hline Silicon wafer & $0.210 \mathrm{~nm}$ \\
\hline Kapton & $3.37 \mathrm{~nm}$ \\
\hline
\end{tabular}


(a)

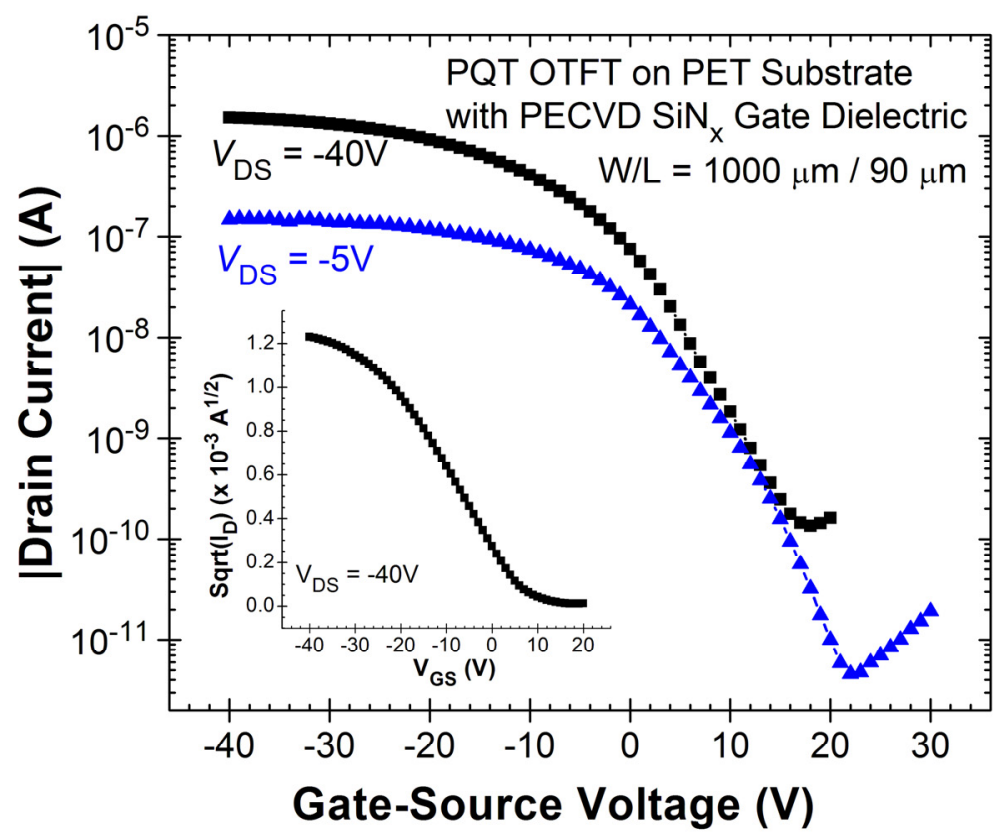

(b)

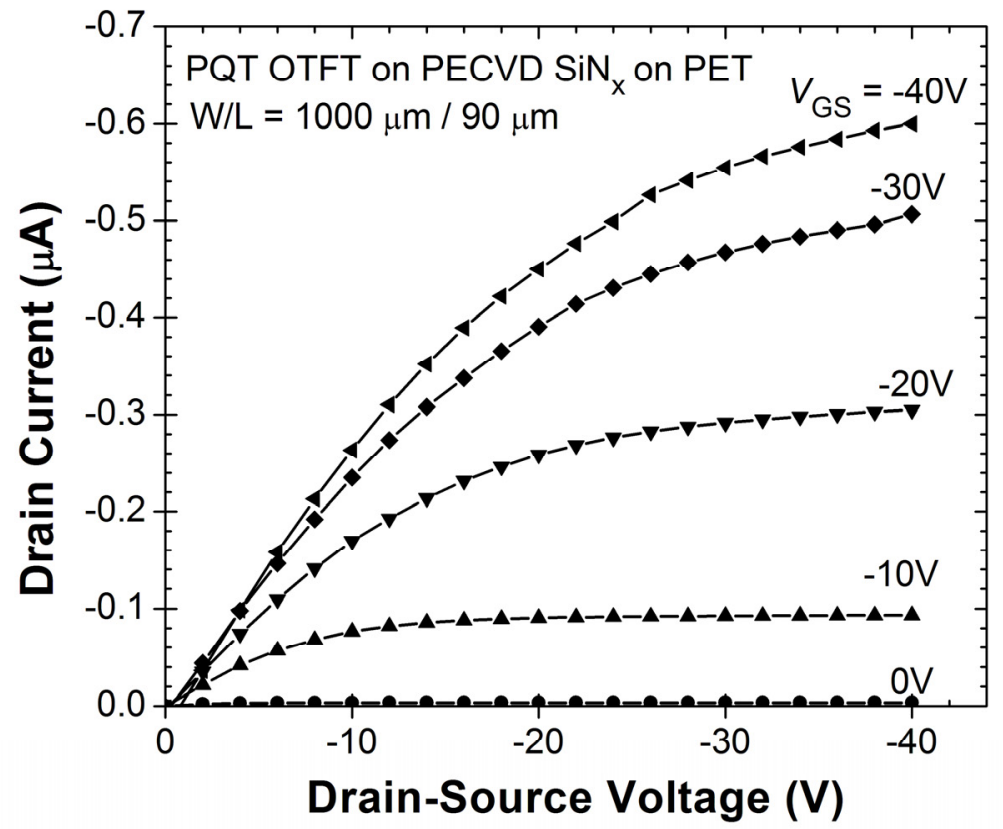

Figure 4.29. (a) Transfer and (b) output characteristics of PQT-12 OTFT on PET substrate with PECVD SiN gate dielectric $(W / L=1000 \mu \mathrm{m} / 90 \mu \mathrm{m})$. 
(a)

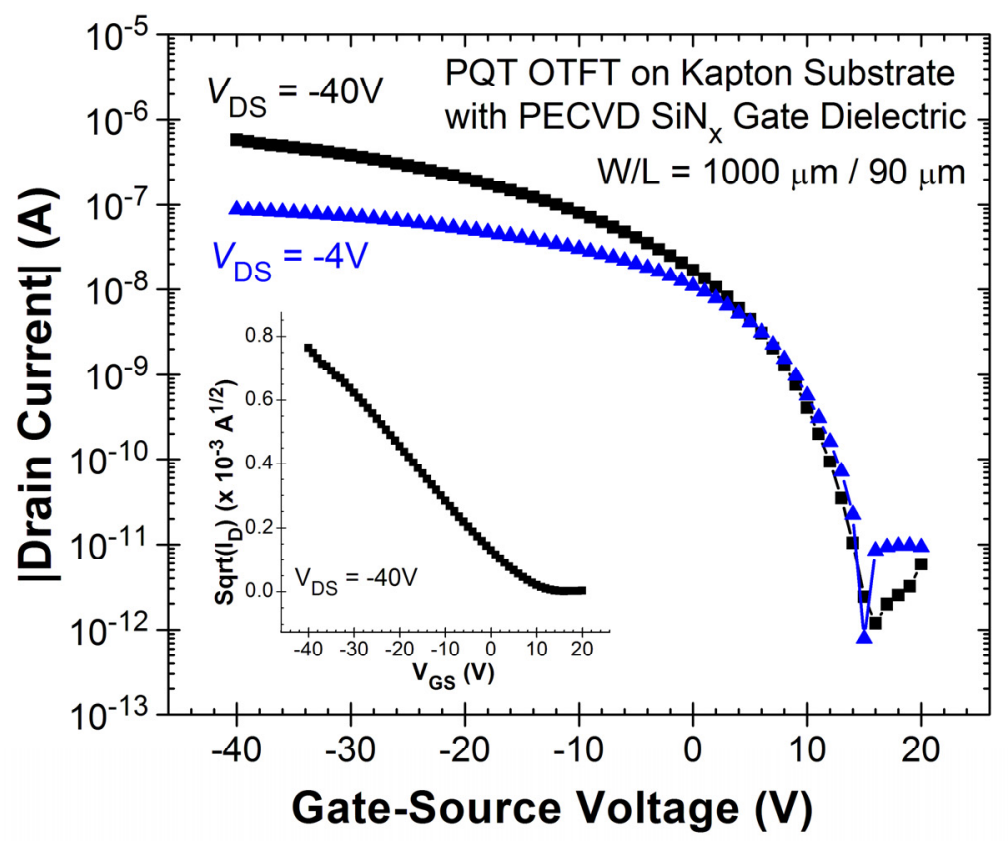

(b)

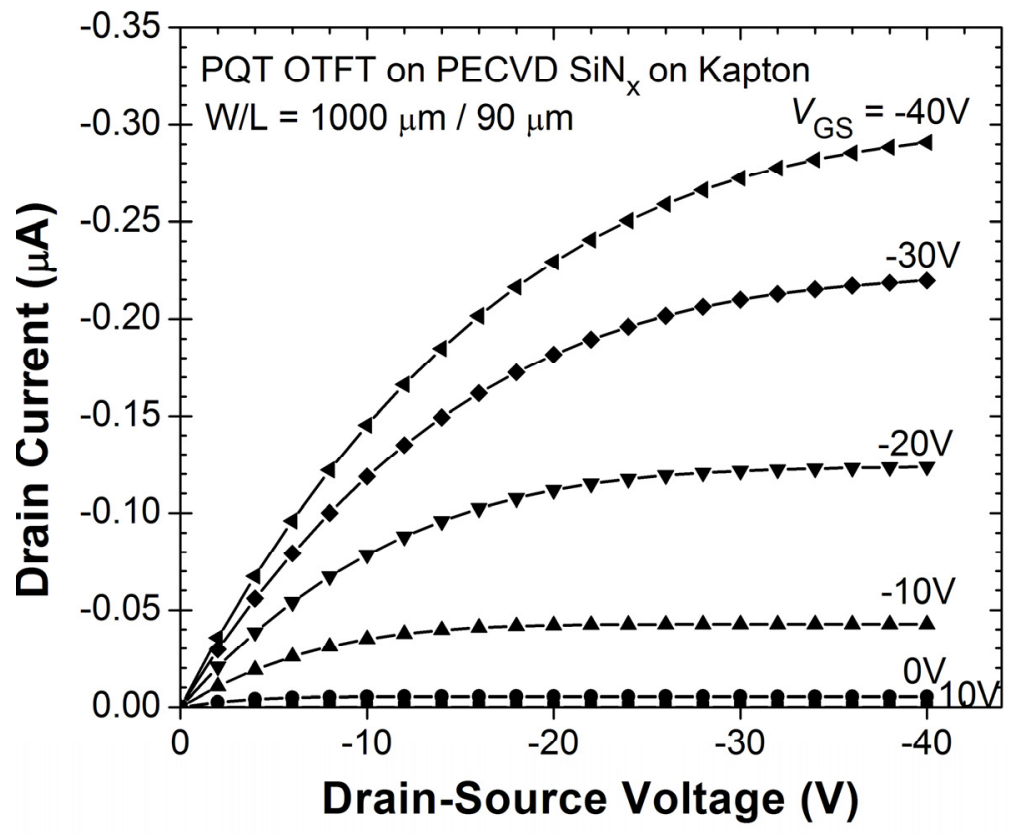

Figure 4.30. (a) Transfer and (b) output characteristics of PQT-12 OTFT on Kapton substrate with PECVD SiN ${ }_{x}$ gate dielectric $(W / L=1000 \mu \mathrm{m} / 90 \mu \mathrm{m})$. 
(a)

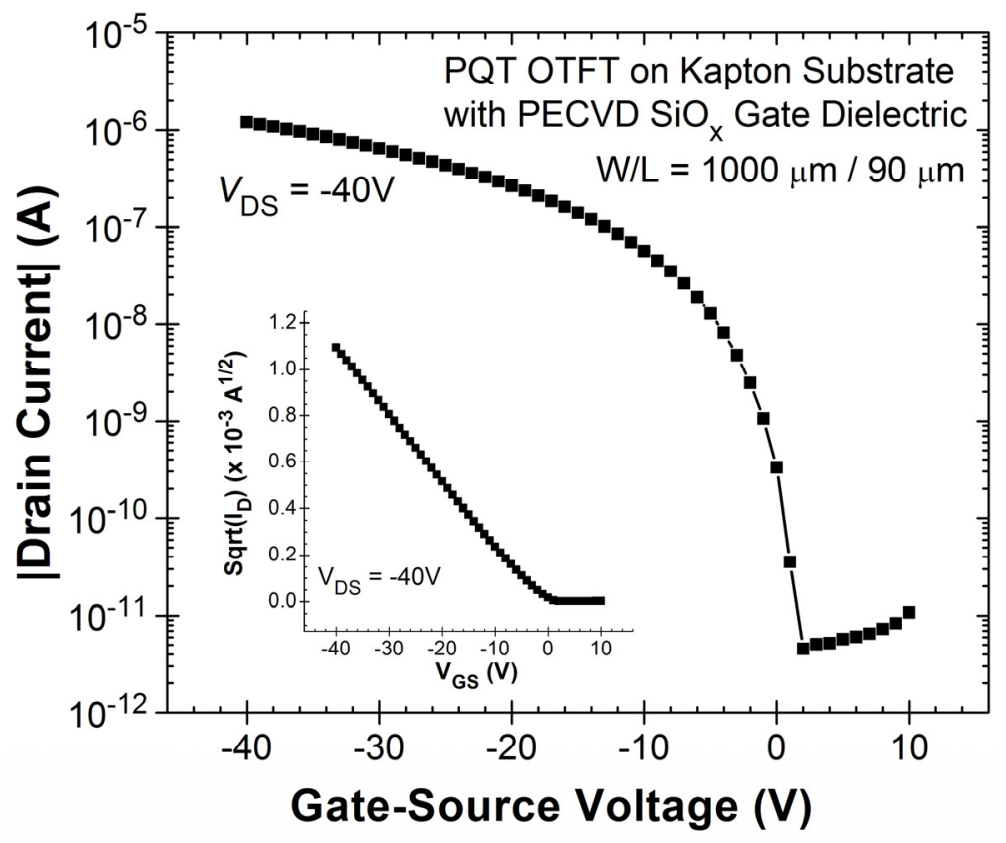

(b)

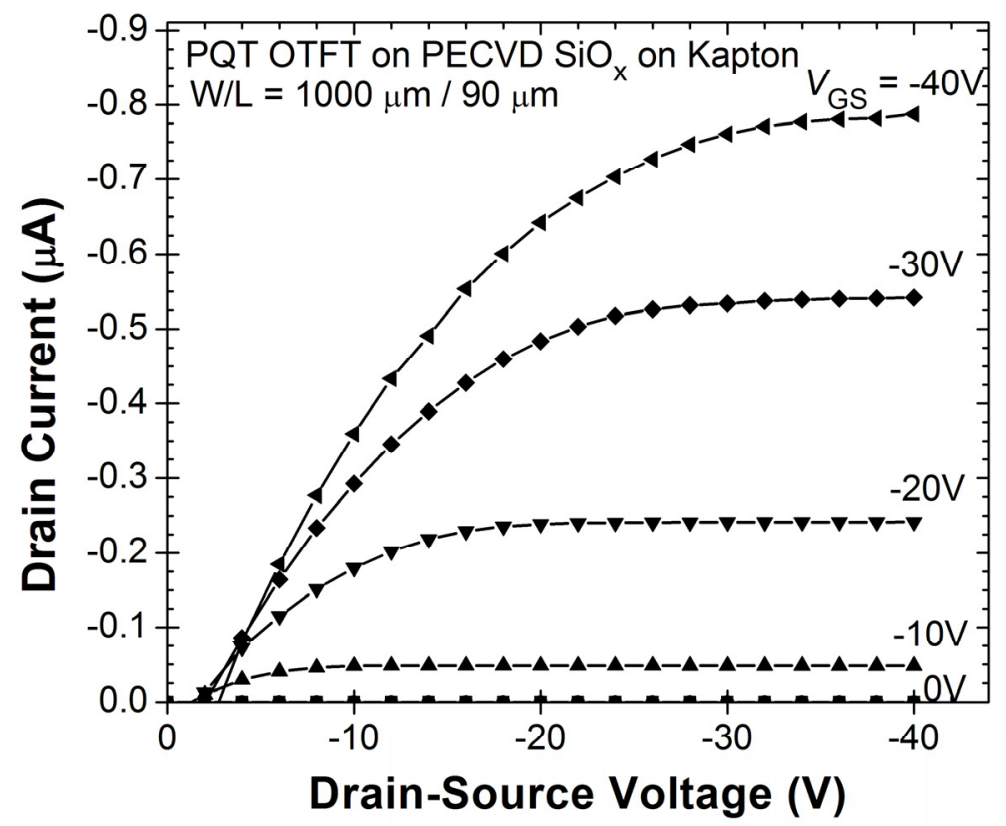

Figure 4.31. (a) Transfer and (b) output characteristics of PQT-12 OTFT on Kapton substrate with PECVD $\mathrm{SiO}_{x}$ gate dielectric $(W / L=1000 \mu \mathrm{m} / 90 \mu \mathrm{m})$.

In summary, this experiment demonstrated the feasibility of fabricating OTFTs with PECVD gate dielectric on plastic substrates. Preliminary devices showed functional devices, but the performance was inferior to devices on a rigid substrate. Surface roughness of plastic substrate is one of the key reasons for limited mobility. It is recommended that ongoing research to focus on 
optimizing the properties of low temperature gate dielectric on plastic substrates to improve dielectric properties (e.g., leakage, breakdown, stability), and on reducing the surface roughness of the resulting substrate via passivation techniques.

\subsection{Summary and Contributions}

PECVD $\mathrm{SiN}_{x}$ and $\mathrm{SiO}_{\mathrm{x}}$ thin films were studied as gate dielectrics for PQT-12 OTFTs, with the objective to develop materials for organic circuit integration on rigid and flexible substrates. The major observations from the investigations reported in this chapter are summarized in Table 4.8. Improvements in field-effect mobility and on/off current ratio were observed as the silicon content in the $\mathrm{SiN}_{\mathrm{x}}$ gate dielectric increases, suggesting that silicon-rich $\mathrm{SiN}_{\mathrm{x}}$ dielectrics are preferable over nitrogen-rich $\mathrm{SiN}_{\mathrm{x}}$. Two sets of OTFTs were evaluated, one with $300^{\circ} \mathrm{C} \mathrm{SiN}_{\mathrm{x}}$ gate dielectrics, and one with $150^{\circ} \mathrm{C} \mathrm{SiN}_{\mathrm{x}}$ gate dielectrics. While the same film composition dependency was observed in both sets of OTFTs, OTFTs with $150^{\circ} \mathrm{C} \mathrm{SiN}_{\mathrm{x}}$ gate dielectrics demonstrated higher mobility than devices with $300^{\circ} \mathrm{C} \mathrm{SiN}_{\mathrm{x}}$ films. A strong correlation between the electrical characteristics of the OTFTs and the surface mechanics of the $\mathrm{SiN}_{\mathrm{x}}$ films was observed. It was determined that contact angle, surface roughness and surface chemical composition of the $\operatorname{SiN}_{\mathrm{x}}$ gate dielectric have an overriding impact on OTFT characteristics. Thus, it is concluded that the composition of the $\mathrm{SiN}_{\mathrm{x}}$ film influences the dielectric surface properties, which in turn affects the quality of the dielectricsemiconductor interface and the molecular ordering of the overlying organic semiconductor layer; altogether, these factors present a strong bearing on the OTFT performance. These relationships are summarized in Figure 4.32.

PQT-12 OTFTs fabricated on PECVD $\mathrm{SiO}_{\mathrm{x}}$ displayed superior mobilities compared to devices on $\mathrm{SiN}_{\mathrm{x}}$. This improvement is largely ascribed to organic-friendly surface properties of the $\mathrm{SiO}_{\mathrm{x}}$ film. However, electrical integrity and stability of low-temperature PECVD $\mathrm{SiO}_{x}$ are a concern. Extensive process and material optimization are necessary to make PECVD $\mathrm{SiO}_{\mathrm{x}}$ useful for practical applications. Preliminary OTFTs on plastic substrates were demonstrated; further process development is expected to generate enhanced device performance, taking us one step closer to the realization of flexible electronics. Overall, these results demonstrated the viability of using PECVD $\mathrm{SiN}_{\mathrm{x}}$ and $\mathrm{SiO}_{\mathrm{x}}$ as gate dielectrics for OTFT circuit integration, especially considering the vast and well-established infrastructure already in place for displays. The low temperature and large area 
deposition capabilities of PECVD $\mathrm{SiN}_{\mathrm{x}}$ and $\mathrm{SiO}_{\mathrm{x}}$ films present good compatibility and technological potential to facilitate integration of OTFT circuits on plastic substrates.

Further optimization of the PECVD gate dielectric composition and the dielectric-organic layer interface property using combinatorial techniques [39], may further improve OTFT performance and boost PECVD $\mathrm{SiN}_{\mathrm{x}}$ and $\mathrm{SiO}_{\mathrm{x}}$ as a serious gate dielectric alternative over the high temperature thermal $\mathrm{SiO}_{2}$, especially for applications on flexible plastic substrates. As we will see in the next chapter, by optimizing the interface treatment conditions, additional improvements (with mobility as high as $0.2 \mathrm{~cm}^{2} / \mathrm{V}$-s with $150^{\circ} \mathrm{C} \mathrm{SiN}_{\mathrm{x}}$ gate dielectric) can be achieved.

Table 4.8. Summary of key observations on comparative study of OTFT with different PECVD gate dielectrics.

\begin{tabular}{|c|c|c|}
\hline & Description & Key Observations \\
\hline Part A & $300^{\circ} \mathrm{C} \mathrm{SiN} x$ & $\begin{array}{ll} & \mathrm{SiN}_{\mathrm{x}} \text { with smaller [N]/[Si] (i.e., Si-rich film) delivers gives higher } \\
\mu_{\mathrm{FE}}, \text { higher loN/loFf, smaller } \mathrm{V}_{\mathrm{T}} \text {. } \\
\mathrm{As}[\mathrm{N}] /[\mathrm{Si}] \text { decreases, contact angle increases (slightly), surface } \\
\text { roughness decreases, atomic \% of } \mathrm{Si} \text { at the surface increases; } \\
\text { thus rendering an interface more favorable for OTFTs. }\end{array}$ \\
\hline Part B & $150^{\circ} \mathrm{C} \mathrm{SiN} x$ & $\begin{array}{l}\text { Similar to } 300^{\circ} \mathrm{C} \mathrm{SiN}_{x} \text {, mobility increases as Si-content in } \mathrm{SiN}_{x} \\
\text { increases. } \\
\\
150^{\circ} \mathrm{C} \mathrm{SiN}{ }_{x} \text { dielectric delivers higher mobility than } 300^{\circ} \mathrm{C} \mathrm{SiN}_{x} .\end{array}$ \\
\hline Part C & $\begin{array}{l}\text { Stacked } \mathrm{SiN}_{\mathrm{x}} \\
\text { Gate Dielectric }\end{array}$ & $\begin{array}{l}\text { Performance with stacked dielectrics are inferior/poorer than } \\
\text { unilayer Si-rich } \mathrm{SiN}_{x} \text { dielectric. }\end{array}$ \\
\hline Part D & $200^{\circ} \mathrm{C} \mathrm{SiO} x$ & $\begin{array}{l}\text { Achieved mobility as high as } 0.5 \mathrm{~cm}^{2} / \mathrm{V}-\mathrm{s} \\
\text { However, PECVD } \mathrm{SiO}_{x} \text { has poorer dielectric integrity than PECVD } \\
\mathrm{SiN}_{\mathrm{x}} \mathrm{SiO} \text { x has lower breakdown fields, higher leakage currents, } \\
\text { and lower yield/reproducibility. }\end{array}$ \\
\hline Part E & $\begin{array}{l}\text { PECVD Dielectric } \\
\text { on Plastic } \\
\text { Substrates }\end{array}$ & $\begin{array}{l}\text { Mobility is 1-2 orders lower on plastic substrates than on rigid } \\
\text { substrate, accompanies by a reduction in device yield. } \\
\text { More work is needed to optimizing the processing procedure for } \\
\text { device fabrication on plastic substrates. }\end{array}$ \\
\hline
\end{tabular}

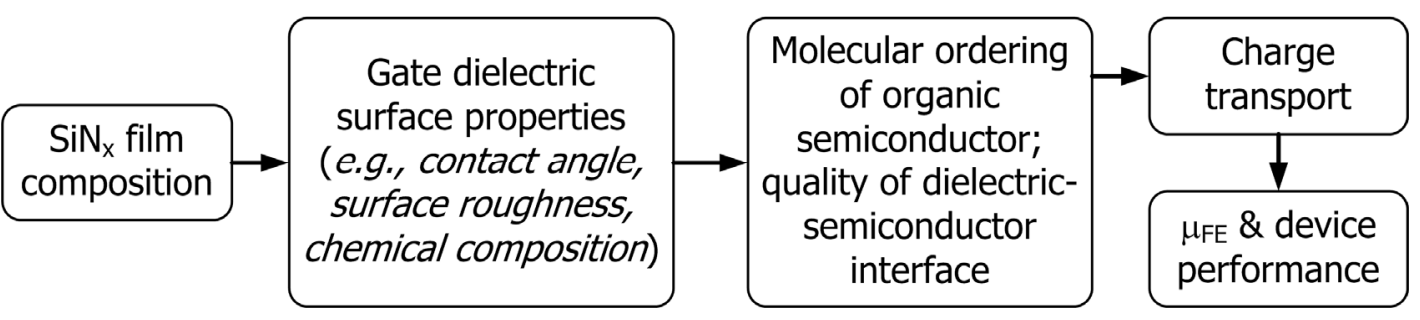

Figure 4.32. Flowchart illustrating the relationship between $\mathrm{SiN}_{\mathrm{x}}$ film composition, interface properties, and OTFT performance. 
As a continuation of this research to generate further device improvements and to develop additional understanding of device behavior, the following investigations are recommended:

- Expanding the range of deposition recipes of PECVD $\mathrm{SiN}_{\mathrm{x}}$, to evaluate if more siliconrich films (e.g., films with $[\mathrm{N}] /[\mathrm{Si}]<1$ ) can generate additional device improvements;

- $\mathrm{AC} /$ transient measurements and long term stress measurements to examine impact of $\mathrm{SiN}_{\mathrm{x}}$ gate dielectric composition on OTFT's dynamic characteristics and stability;

- In-depth evaluation of all device parameters to draw decisive conclusion on the impact of Si-rich $\mathrm{SiN}_{\mathrm{x}}$ gate dielectric on the overall OTFT performance.

- Further material development for $\mathrm{PECVD} \mathrm{SiO}_{\mathrm{x}}$ to improve dielectric integrity;

- Investigate stacked gate dielectric structure composed of $\mathrm{SiO}_{\mathrm{x}}$ as top-layer and Si-rich $\mathrm{SiN}_{\mathrm{x}}$ as bottom/bulk-layer. $\mathrm{SiO}_{\mathrm{x}}$ surface appears to render a favorable dielectricsemiconductor interface to achieve high device mobility. $\mathrm{SiN}_{\mathrm{x}}$ provides good dielectric integrity, high breakdown strength, and low leakage current. 


\section{Bibliography}

[1] W. Macdonald, "Engineered films for display technologies," J. Mater. Chem. 14, 4-10 (2004).

[2] D. Stryakhilev, A. Sazonov, and A. Nathan, "Amorphous Silicon Nitride Deposited at $120^{\circ} \mathrm{C}$ for OLED-TFT Arrays on Plastic Substrates," J. Vac. Sci. Technol. A 20, 1087 (2002).

[3] S. Wagner, H. Gleskova, I. C. Cheng, and M. Wu, "Silicon for thin-film transistors," Thin Solid Films 430, 15 (2003).

[4] C. McArthur, M. Meitine, and A. Sazonov, "Optimization of $75^{\circ} \mathrm{C}$ Amorphous Silicon Nitride for TFTs on Plastics,” Mat. Res. Soc. Symp. Proc. 769, 303 (2003).

[5] M. Meitine and A. Sazonov, "Low Temperature PECVD Silicon Oxide for Devices and Circuits on Flexible Substrates," Mat. Res. Soc. Symp. Proc. 769, 165 (2003).

[6] L. Cai, A. Rohatgi, S. Han, G. May, and M. Zou, "Investigation of the properties of plasma-enhanced chemical vapor deposited silicon nitride and its effect on silicon surface passivation," J. Appl. Phys. 83, 5885 (1998).

[7] D. Knipp, R.A. Street, A. Völkel, and J. Ho, "Pentacene thin film transistors on inorganic dielectrics: Morphology, structural properties, and electronic transport,” J. Appl. Phys. 93, 347 (2003).

[8] R. Peláez, E. Castán, S. Dueñas, J. Barboll, E. Redondo, I. Mártil, and G. González-Díaz, "Electrical characterization of electron cyclotron resonance deposited silicon nitride dual layer for enhanced $\mathrm{Al} / \mathrm{SiN}_{\mathrm{x}}: \mathrm{H} / \mathrm{InP}$ metal-insulator-semiconductor structures fabrication," J. Appl. Phys. 86, 6924 (1999).

[9] M.C. Hugon, F. Delmotte, B. Agius, and J.L. Courant, "Electrical properties of metalinsulator-semiconductor structures with silicon nitride dielectrics deposited by low temperature plasma enhanced chemical vapor deposition distributed electron cyclotron resonance," J. Vac. Sci. Technol. A 15, 3143 (1997).

[10] W.M.M. Kessels, J. Hong, F.J.H. van Assche, J.D. Moschner, T. Lauinger, W.J. Soppe, A.W. Weeber, D.C. Schram, and M.C.M. van de Sanden, "High-rate deposition of a$\mathrm{SiN}_{\mathrm{x}}: \mathrm{H}$ for photovoltaic applications by the expanding thermal plasma," J. Vac. Sci. Technol. A 20, 1704 (2002).

[11] A. Rohatgi and J.W. Jeong, "High-efficiency screen-printed silicon ribbon solar cells by effective defect passivation and rapid thermal processing," Appl. Phys. Lett. 82, 224 (2003).

[12] L. Wang, H. S. Reehal, F. L. Martinez, E. San Andres, and A. del Prado, "Characterization of nitrogen-rich silicon nitride films grown by the electron cyclotron resonance plasma technique," Semcond. Sci. Technol. 18 (7), 663 (2003).

[13] M. R. Esmaeili-Rad, F. Li, A. Sazonov, and A. Nathan, "Stability of nanocrystalline silicon bottom-gate thin film transistors with silicon nitride gate dielectric," J. Appl. Phys. 102, 064512 (2007). 
[14] F.L. Martínez, E. San Andrés, A. del Prado, I. Mártil, D. Bravo, and F. López, "Temperature effects on the electrical properties and structure of interfacial and bulk defects in Al/SiN $: H /$ Si devices," J. Appl. Phys. 90, 1573 (2001).

[15] B. Youn, D. Kim and S. Jeon, "Improvement of Interfacial Performance on Silicone Insulating and Semiconducting Interface by Plasma Treatment," 2005 IEEE Conference on Electrical Insulation \& Dielectric Phenomena (CEIDP), pp.75-78, (2005).

[16] W.A. Landford and M. J. Rand, "The hydrogen content of plasma-deposited silicon nitride," J. App. Phys. 49, 2473 (1978).

[17] P.V. Bulkin, P.L. Swart, and B.M. Lacquet, "Electron cyclotron resonance plasma deposition of $\mathrm{SiN}_{\mathrm{x}}$ for optical applications," Thin Solid Films 241, 247 (1994).

[18] H. Mäckel, and R. Lüdemann, "Detailed study of the composition of hydrogenated SiNx layers for high-quality silicon surface passivation,” J. App. Phys. 92, 2602 (2002).

[19] E. Bustarret, M. Bensouda, M. C. Habrard, J. C. Bruyère, S. Poulin, and S. C. Gujrathi, "Configurational statistics in a- $\mathrm{Si}_{\mathrm{x}} \mathrm{N}_{\mathrm{y}} \mathrm{H}_{\mathrm{z}}$ alloys: A quantitative bonding analysis," Phys. Rev. B 38, 8171 (1988-II).

[20] L. L. Kosbar, C. D. Dimitrakopoulos, and D. J. Mascaro, "The Effect of Surface Preparation on the Structure and Electrical Transport in an Organic Semiconductor," Mater. Res. Soc. Symp. Proc. 665, C10.6.1 (2001).

[21] C. Song, B. Koo, S. Lee and D. Kim, "Characteristics of Pentacene Organic Thin Film Transistors with Gate Insulator Processed by Organic Molecules," Jpn. J. Appl. Phys. 41, 2730 (2002).

[22] M.M. Sung, G.J. Kluth, and R. Maboudian, "Formation of alkylsiloxane self-assembled monolayers on $\mathrm{Si}_{3} \mathrm{~N}_{4}$, , J. Vac. Sci. Technol. A 17(2), 540 (1999).

[23] D.H. Kim, Y.D. Park, Y. Jang, H. Yang, Y.H. Kim, J.I. Han, D.G. Moon, S. Park, T. Chang, C. Chang, M. Joo, C.Y. Ryu, and K. Cho, "Enhancement of field-effect mobility due to surface-mediated molecular ordering in regioregular polythiophene thin film transistors," Adv. Funct. Mater. 15(1), 77 (2005).

[24] M.L. Chabinyc, R. Lujan, F. Endicott, M.F. Toney, I. McCulloch, and M. Heeney, "Effects of the surface roughness of plastic-compatible inorganic dielectrics on polymeric thin film transistors," Appl. Phys. Lett. 90, 233508 (2007).

[25] K.J. Park and G.N. Parsons, "Bulk and interface charge in low temperature silicon nitride for thin film transistors on plastic substrates," J. Vac. Sci. Technol. A 22(6), 2256 (2004).

[26] W.S. Lau, S.J. Fonash, and J. Kanicki, "Stability of electrical properties of nitrogen-rich, silicon-rich, and stoichiometric silicon nitride films," J. App. Phys. 66, 2765 (1989).

[27] M.J. Powell, "Charge trapping instabilities in amorphous silicon-silicon nitride thin-film transistors," App. Phys. Lett. 43, 597 (1983).

[28] B.S. Ong, Y. Wu, P. Liu, and S. Gardner, "High-Performance Semiconducting Polythiophenes for Organic Thin-Film Transistors," J. Am. Chem. Soc. 126, 3378 (2004).

[29] B.S. Ong, Y. Wu, P. Liu, and S. Gardner, "Structurally Ordered Polythiophene Nanoparticles for High-Performance Organic Thin Film Transistors," Adv. Mater. 17, 1141 (2005). 
[30] F.M. Li, Y. Wu, B.S. Ong, A. Nathan, "Organic thin-film transistor integration using silicon nitride gate dielectric," App. Phys. Lett. 90,133514 (2007).

[31] R. A. Street, "Conducting Polymers: The Benefit of Order," Nature Materials 5, 171 (2006).

[32] Z. Bao, A. Dodabalapur, and A. J. Lovinger, "Soluble and processable regioregular poly(3hexylthiophene) for thin film field-effect transistor applications with high mobility," Appl. Phys. Lett. 69, 4108 (1996).

[33] R. J. Kline, M. D. McGehee, and M. F. Toney, "Highly oriented crystals at the buried interface in polythiophene thin-film transistors," Nature Materials 5, 222 (2006).

[34] H. Sirringhaus, N. Tessler, and R. H. Friend, "Integrated Optoelectronic Devices Based On Conjugated Polymers," Science 280, 1741 (1998).

[35] M.F. Calhoun, J. Sanchez, D. Olaya, M.E. Gershenson, and V. Podzorov, "Electronic functionalization of the surface of organic semiconductors with self-assembled monolayers," Nature Materials 7, 84-89 (2008).

[36] M. Shtein, J. Mapel, J. B. Benziger, and S. R. Forrest, "Effects of film morphology and gate dielectric surface preparation on the electrical characteristics of organic-vapor-phasedeposited pentacene thin-film transistors," Appl. Phys. Lett. 81, 268 (2002).

[37] F.M. Li, Y. Wu, B.S. Ong, Y. Vygranenko, and A. Nathan, "Study of PECVD Silicon Nitride and Silicon Oxide Gate Dielectrics for Organic Thin-Film Transistor Circuit Integration," Mater. Res. Soc. Symp. Proc. 1003E, 1003-O06-49 (2008).

[38] A. Salleo, M. L. Chabinyc, M. S. Yang, and R. A. Street, "Polymer thin-film transistors with chemically modified dielectric interfaces," Appl. Phys. Lett. 81, 4383 (2002).

[39] L.A. Lucas, D.M. DeLongchamp, B.M. Vogel, E.K. Lin, M.J. Fasolka, D.A. Fischer, I. McCulloch, M. Heeney, and G.E. Jabbour, "Combinatorial screening of the effect of temperature on the microstructure and mobility of a high performance polythiophene semiconductor," Appl. Phys. Lett. 90, 012112 (2007). 


\section{Chapter 5}

\section{Dielectric Interface Engineering}

The overall OTFT performance is largely dictated by the properties and quality of device material layers (e.g., semiconductor, dielectric, contact, etc.). Of nearly equal importance is the interfacial interaction and material compatibility between device layers. In particular, the operation of OTFTs is strongly dependent on the properties of two device interfaces:

(i) The interface between semiconductor and gate dielectric, where charge transport takes place in the semiconductor layer, and

(ii) The interface between semiconductor and source/drain contacts, where charge injection occurs from the source/drain contacts into the semiconductor.

Optimization and modification of these interfaces for device improvement have been an active area of OTFT research [1][2][3]. This thesis focuses on engineering these two critical device interfaces to improve device performance. Interface treatment techniques for the dielectric-semiconductor interface are examined in this chapter and the contact-semiconductor interface is addressed in Chapter 6. The investigations are tailored to bottom-gate bottom-contact PQT-12 OTFTs on $\mathrm{SiN}_{\mathrm{x}}$ gate dielectric with Au contacts.

The purpose of this work is to improve performance of PQT-12 OTFTs by systematically introducing and applying various surface treatments to the dielectric-semiconductor $\left(\mathrm{SiN}_{\mathrm{x}}-\mathrm{PQT}\right)$ interface. Four types of dielectric surface conditions are investigated: no treatment (i.e., bare $\mathrm{SiN}_{\mathrm{x}}$ ), oxygen $\left(\mathrm{O}_{2}\right)$ plasma treatment, octyltrichlorosilane (OTS, $\left.\mathrm{CH}_{3}\left(\mathrm{CH}_{2}\right)_{7} \mathrm{SiCl}_{3}\right)$ self-assembled monolayer (SAM) treatment, and dual $\mathrm{O}_{2}$ plasma/OTS treatment. Correlations between surface properties (e.g., wettability, surface roughness, chemical composition) and field-effect mobility of OTFTs are analyzed. This work demonstrates that by suitably optimized dielectric surface 
treatment, the field-effect mobility of PQT-12 on $\mathrm{SiN}_{\mathrm{x}}$ gate dielectric can be enhanced by nearly 30 times over untreated devices, resulting in mobility as high as $0.2 \mathrm{~cm}^{2} / \mathrm{V}$-s. The studies reveal that surface wettability and roughness of the gate dielectric are decisive parameters that control device performance.

\subsection{Background}

High field-effect mobility is generally associated with a high degree of structural order of the organic semiconductor films. For bottom-gate OTFTs, the organic semiconductor film is deposited onto the dielectric layer under the influence of physical and chemical interactions between the semiconductor and dielectric layer. Furthermore, since charge transport in the active channel typically occurs in the first few monolayers of the semiconductor closest to the interface [4][5], it is expected that the surface state of the underlying dielectric layer has a significant influence on the charge carrier mobility of the OTFTs. In turn, the quality of the dielectric-semiconductor interface and the molecular ordering of the organic semiconductor layer have a strong bearing on the OTFT characteristics, including field-effect mobility $\left(\mu_{\mathrm{FE}}\right)$, on/off current ratio $\left(I_{\mathrm{ON}} / I_{\mathrm{OFF}}\right)$, subthreshold slope $(S)$, threshold voltage $\left(V_{\mathrm{T}}\right)$, and leakage current $\left(I_{\text {leak }}\right)$. Thus, proper control of the gate dielectric surface properties (both physical and chemical properties) is crucial for attaining higher performance OTFTs.

Ideally, the dielectric surface should provide an environment to allow formation of a wellstacked and highly-ordered organic film, so that the disorder phases in the organic film and scattering at the interface are minimized. To tailor the interface to achieve these objectives, this thesis focuses on two surface treatment approaches: self-assembled monolayers (SAMs) [6][7] and oxygen plasma treatment [8]. A background overview of these two techniques is presented next.

\subsubsection{Self Assembled Monolayer (SAM)}

SAMs are highly ordered, two-dimensional structures that form spontaneously on a variety of surfaces, and serve as an excellent surface modification system. Depending on the application, different interface properties can be achieved by tuning the interfacial properties through varying the rigidity, length, and terminal functional group of the molecule. This in turn influences the stacking, alignment, packing, conformation, uniformity, polarity, and charge density of the surface 
[1]. In general, molecules that form SAMs contain a head group, a body and an end/terminal group. A simple illustration of the three main parts of a SAM system is given in Figure 5.1(a). The most common and expedient method to deposit SAMs is by immersing a substrate into a dilute solution of SAM precursor molecules dissolved in an organic solvent [9]. The process is self-limiting and the resulting film is a dense organization of molecules (see Figure 5.1(b)). The orientation of the individual molecules and structure are dependent on the substrate material's structure and cleanliness. SAMs are useful as passivating layers, for the controlled modification of surface properties, and for the surface functionalization in molecular growth processes.
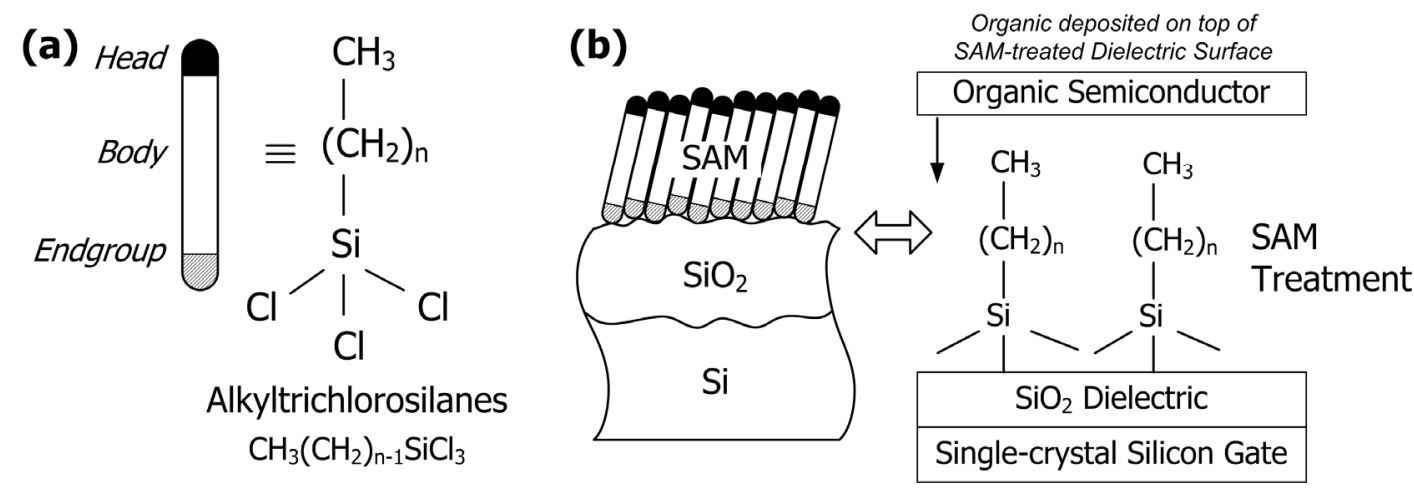

Figure 5.1. (a) Simplified illustration of three main parts of a SAM system, and (b) the formation of alkylsiloxane SAM by adsorption of alkyltrichlorosilanes from solution on a $\mathrm{Si}_{-} \mathrm{SiO}_{2}$ substrate (adapted from [9]).

In the context of OTFTs, SAMs are used to chemically modify the gate dielectric surface prior to deposition of the organic semiconductor layer. The formation of organic thin films using the selfassembly technique refers to the spontaneous formation of an ordered monolayer of organic molecules on a surface. Alkylsilane SAMs on oxide surfaces have been widely used as molecular platforms in the fabrication of OTFTs, especially to control the orientation, morphology and grain size of the organic semiconductor films, as well as to aid charge transport. Within the alkyltrichlorosilane $\left(\mathrm{CH}_{3}\left(\mathrm{CH}_{2}\right)_{\mathrm{n}-1} \mathrm{SiCl}_{3}\right)$ family, octyltrichlorosilane (OTS, $\left.\mathrm{n}=8\right)$ and octadecyltrichlorosilane (ODTS, $\mathrm{n}=18$ ) are frequently studied for pre-treating the $\mathrm{SiO}_{2}$ surfaces. Hexamethyldisilazane (HMDS) is another popular surface modifier for $\mathrm{SiO}_{2}$. Metal surfaces can also be treated with SAMs to enhance the characteristics of bottom-contact OTFTs. SAMs of alkanethiols $\left(\mathrm{CH}_{3}\left(\mathrm{CH}_{2}\right)_{\mathrm{n}-1} \mathrm{SH}\right)$, such as 2-Mercapto-5-Nitro-Benzimidazole ( $\mathrm{MNB}, \mathrm{C}_{7} \mathrm{H}_{5} \mathrm{~N}_{3} \mathrm{O}_{2} \mathrm{~S}$ ), have been applied on metal contct surfaces, which have shown to improve the quality of the 
overlying organic semiconductor film, reduce contact resistance and enhance field-effect mobility in OTFTs [7][10][11].

The formation of organic SAMs typically reduces the surface energy of gate dielectric or metal electrodes, rendering a more favorable environment for the deposition of organic semiconductor molecules. Numerous papers have reported enhancement in semiconductor film quality and in OTFT performance after ODTS SAM treatment of the $\mathrm{SiO}_{2}$ dielectric surface [6][7]. For instance, Song et al. studied the effect of various treatment techniques on the performance of bottom-gate pentacene OTFT with $\mathrm{SiO}_{2}$ gate dielectric on a $\mathrm{Si}$ substrate [11]. The treatments considered include: annealing, iodine doping, MNB SAM, HMDS SAM, and ODTS SAM. The most effective treatments were MNB, HMDS and ODTS SAMs, which generated two to three orders increase in mobility compared to the as-deposited samples. $\mathrm{SiO}_{2}$ gate dielectric treated with ODTS SAM delivered the highest mobility, highest on/off current ratio, the lowest leakage current, and close to zero threshold voltage. ODTS SAM forms a hydrophobic surface and reduces the surface tension of $\mathrm{SiO}_{2}$, thus promoting good molecular ordering of the subsequently-deposited organic semiconductor layer and prohibiting penetration of moisture into the organic layer (i.e., ODTS can act as a moisture inhibitor). The investigations reported in this thesis use OTS SAM; previous experiments have found that OTS SAM yields higher mobility than ODTS SAM for PQT-12 OTFTs on $\mathrm{SiO}_{2}$ gate dielectric.

Studies have shown that depending on the properties of the substrate surface that result from SAM treatment, the organic polymer semiconductor molecules may adopt two different orientations: perpendicular (edge-on) or parallel (face-on) to the dielectric surface (Figure 5.2) [12]. Increased field-effect mobility was often reported when the polymer nanocrystals are oriented perpendicularly (i.e., edge-on) with respect to the dielectric substrate compared to those with parallel (face-on) orientation.

Additional evidence on the influence of substrate properties on molecular orientation and grain morphology of the organic semiconductor is found in studies on vacuum deposited pentacene. It was shown that pentacene molecules stand almost perpendicular to the substrate when deposited onto flat, inert (or hydrophobic) substrates (e.g., polymeric dielectric). In contrast, if the substrate is more reactive (or hydrophilic), pentacene molecules tend to lie flat on the substrate due to increased pentacene-substrate interaction [1]. The enhancement in device mobility on SAMmodified surfaces was attributed to increased grain size of the semiconductor, high molecular surface mobility and reduced interactions with a hydrophobic substrate surface. 
(a)

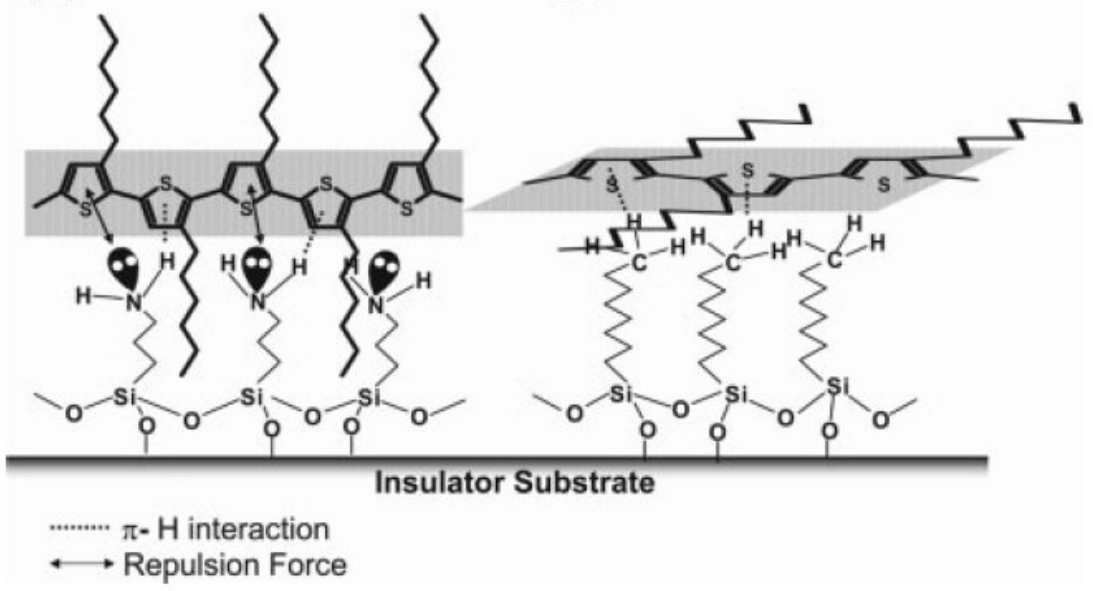

(b)

Figure 5.2. Schematics of different P3HT chain orientations, (a) edge-on and (b) face-on, according to interfacial characteristics (adapted from [12]).

The general consensus among the initial research in OTFT is that an inert hydrophobic substrate surface is most desirable for the formation of high quality, well-ordered organic semiconductor layers to deliver higher device performance. Interestingly, a more exhaustive review of scientific literature revealed that the preferable conditions of the dielectric surface to give high carrier mobility vary with the choice of material system; two examples are given in Table 5.1. In the case of pentacene, it was reported that inert surfaces (e.g., non-polar) are favorable for higher mobility [13][14]. In contrast, a polar surface was observed to attain higher mobility for P3HT devices, as reported by Kim et al. [12]. But in either case, higher mobility is observed when the organic semiconductor molecules adapted an edge-on or perpendicular orientation with respect to the substrate surface.

A potential concern of using SAM treatment is that it involves a wet deposition process, which requires immersing OTFT substrates in a SAM solution. In some instances, the immersion process may remove the contact metals, and the solvent may attack the plastic substrates (which are employed for the flexible electronics). To circumvent these wet processing issues, surface treatment based on dry process is explored. We investigate oxygen plasma treatment as an alternative approach for dielectric surface modification. This technique is discussed next. 
Table 5.1. The impact of surface conditions on the molecular orientation of organic semiconductor (e.g., pentacene and P3HT).

\begin{tabular}{|llll|}
\hline Semiconductor & Edge-on Orientation & Face-on Orientation & Reference \\
\hline Pentacene & $\begin{array}{l}\text { Inert (non-polar) surfaces } \\
\rightarrow \text { higher } \mu\end{array}$ & $\begin{array}{l}\text { Reactive }(\text { polar) surface } \\
\rightarrow \text { lower } \mu\end{array}$ & {$[13][14]$} \\
P3HT & $\begin{array}{l}\text { Surfaces with polar endgroups } \\
\rightarrow \text { higher } \mu\end{array}$ & $\begin{array}{l}\text { Surfaces without polar endgroups } \\
\rightarrow \text { Lower } \mu\end{array}$ & {$[12]$} \\
\hline
\end{tabular}

\subsubsection{Oxygen Plasma Treatment}

The effects of oxygen $\left(\mathrm{O}_{2}\right)$ plasma treatment on the performance of bottom-gate pentacene OTFT with thermal $\mathrm{SiO}_{2}$ gate dielectric were studied by Lee et al. [8]. Prior to pentacene deposition, the substrates were treated with $\mathrm{O}_{2}$ plasma using reactive ion etching (RIE) equipment. Compared to the as-deposited OTFTs, the electrical characteristics of OTFTs with $\mathrm{O}_{2}$ plasma treated gate dielectric are superior, with a 10 times increase in field-effect mobility, an order of magnitude increase in $I_{\mathrm{D}}$, higher on/off current ratio, sharper subthreshold slope, and reduction in device resistance (including channel resistance and contact resistance) [8]. It was concluded that $\mathrm{O}_{2}$ plasma treatment facilitated the growth of larger pentacene grains and enhanced ordering of the pentacene molecules, resulting in a decrease in the total device resistance and a lowering of the contact resistance [8]. These improvements can be ascribed to a plasma cleaning effect, where removal of surface defects and contaminants by ashing typically occurs at the initial phase of plasma exposure. The enhancement of grain size and molecular ordering can also be attributed to the modification of surface potential energy. A lower surface potential energy barrier produces a larger diffusion length of pentacene molecules on the dielectric surface, thus larger grains are formed and crystallinity of the pentacene film is improved. Plasma treatment also improved device uniformity across the wafer [8]. However, excessive exposure can cause a reduction in the fieldeffect mobility, threshold voltage and off-current [8]. In summary, $\mathrm{O}_{2}$ plasma treatment with a suitably controlled exposure time can produce OTFTs with enhanced, as well as uniform, performance across the wafer.

Wang et al. have shown that $\mathrm{O}_{2}$ plasma treatment of an organic polymer gate dielectric can be used to systematically modify the threshold voltage $\left(V_{\mathrm{T}}\right)$ of pentacene OTFTs at the process level [15]. They reported that $\mathrm{O}_{2}$ plasma treatment resulted in a positive $V_{\mathrm{T}}$ shift in pentacene OTFTs, but accompanied by an increase in field effect mobility. The shift in $V_{\mathrm{T}}$ was attributed to process- 
induced trap states, more specifically, an increase in fixed charges and mobile charges, at the semiconductor-dielectric interface.

\subsubsection{Basic of Plasma Processing (Etching)}

To understand the effect of $\mathrm{O}_{2}$ plasma treatment on surface properties, an overview of plasma processing technology is provided here. By definition, plasma is a partially ionized gas consisting of positively and negatively charged radicals and neutral species. Sometimes considered as the fourth state of matter, it is highly conductive due to the presence of charged particles and is magnetically controllable. Plasma technology is widely employed in the microelectronics industry in many dry etching or thin-film deposition systems. The ability of plasmas to transfer the lithographically defined patterns into underlying layers reliably and controllably, etch anisotropically without requiring crystallographic orientation at low or intermediate pressures makes them very attractive for most dry etching mechanisms.

The basic principle of dry etching by plasma involve ionizing a gas to create highly reactive positively and negatively charged species, which react chemically with the material to be etched; the reactions form volatile compounds that can be removed [16]. The Trion Phantom II RIE/ICP hybrid system was used in this project for oxygen plasma treatment of the gate dielectric [17]. During reactive ion etching (RIE) processing, process gases flow through the chamber, and RF power is applied between the two electrodes to generate a plasma (where highly reactive radicals, electrons, photons and ions are generated through ionization of the feed gas by electron impact dissociation). The chemically reactive species diffuse to the surface of the wafer and adsorb to the wafer surface. The chemical species undergo surface diffusion and react with the material to be etched forming volatile products. These volatile products desorb from the surface and are transported away from the chamber. Ion bombardment of the wafer also takes place through the generation of a DC bias on the lower electrode. The DC bias is a potential drop between the plasma and the wafer that accelerates ions towards the wafer to produce directional etching, as illustrated in Figure 5.3. A reactive ion etch mechanism is also called ion-beam assisted radical etching. Depending on the etch mechanism, plasma etch profiles can vary from isotropic to anisotropic (i.e., directional). The anisotropy level is controlled by the plasma conditions. The rate of etching or the rate of reaction in plasma processing is sensitive to various system parameters, including DC bias, RF power, pressure, and gas flow rate. 
RIE Configuration: with asymmetric capacitively coupled electrodes

(a)

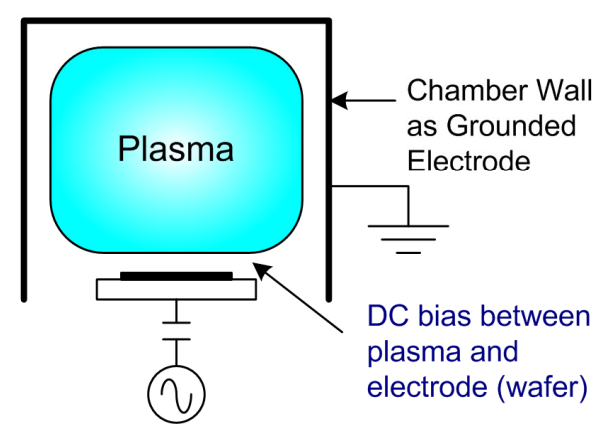

(b)

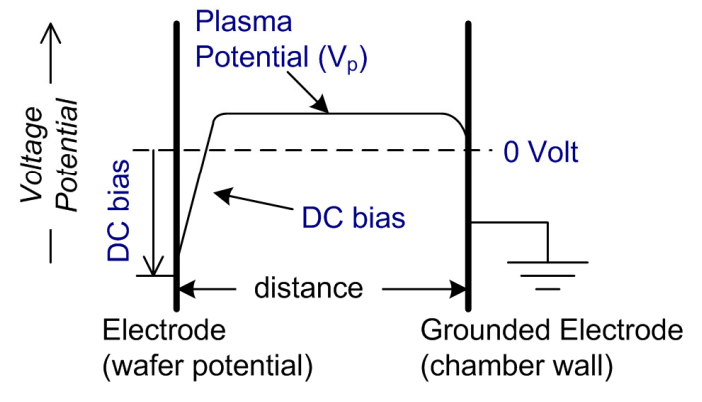

Figure 5.3. (a) Diagram of a RIE system. (b) Potential distribution as a function of distance between electrodes. Wafer is placed on a smaller electrode, which is biased negatively relative to the plasma. Ions accelerate from plasma to wafer due to a DC bias electric field.

RIE power is specified by an externally applied DC bias parameter. Under the influence of the DC bias, either positive or negative ions (depending on whether the DC bias is negative or positive) are accelerated toward the electrode where the substrate is mounted. The directed motion imparted to the ions by the DC bias, as well as the chemical reactivity of the ions, results in the selective, anisotropic chemical etching of the substrate. The magnitude of the DC bias at the electrode significantly affects, for example, the degree of the anisotropic etching (the smaller the magnitude of the DC bias, the lower the degree of anisotropic etching) and thus the shape of the etch profile. In addition, the magnitude of the DC bias significantly affects the etch selectivity between a substrate layer to be etched and material layers not to be etched, e.g., the masking material or other layers of substrate material. For example, a DC bias just sufficient to impose directionality on the reactive species will draw the reactive species to the electrode at a speed sufficient to cause sputter removal of a relatively small amount of masking material or substrate material which is not to be removed. However, an increase in the magnitude of the DC bias significantly above that required to impose directionality will result in the sputter removal of an undesirably large amount of material which is not to be removed. Consequently, proper control of DC bias can help to achieve a desired etch profile, e.g., an essentially vertical, inclined, or curved etch profile, to avoid undesirably large reductions in etch selectivity, and to avoid unintentional removal of material [18].

The plasma density becomes low in a RIE system especially when it is operated at low pressures, reducing the etch rates. Higher plasma densities can be generated with an inductively-coupled plasma (ICP) system, which heats the plasma inductively by the electric fields generated by a coil wrapped around the discharge chamber without coupling it to the substrate [16]. Apart from the 
high plasma density, ICP provides lower ion energy and the capability to operate at lower chamber pressures of less than $10 \mathrm{mTorr}$. Moreover, ion fluxes and ion energy can be controlled separately, thus increasing flexibility in optimizing etch responses. The higher plasma density afforded by the ICP system improves etch rate, anisotropicity, profile selectivity and uniformity, places less stringent requirements on operating pressures, and reduces radiation damage from RIE [19]. Thus, in a hybrid ICP-RIE system, ICP is employed to generate a high concentration of reactive species which increases the etch rate, whereas a separate RF bias (i.e., RIE mode) is applied to the substrate to create directional electric fields that accelerate reactive species towards the substrate to achieve more anisotropic etch profiles. As ICP-RIE mode provides high density without increasing the DC bias self voltage, it is an intrinsically low damage process.

$\mathrm{O}_{2}$ plasmas are commonly employed in wafer cleaning procedures. Oxygen radicals react with organic contaminants on the wafer surface, forming volatile organic compounds (e.g., $\mathrm{H}_{2} \mathrm{O}, \mathrm{CO}$ or $\mathrm{CO}_{2}$ ) that are desorbed from the surface and removed from the plasma. These reactions are believed to contribute to changes in OTFT performance upon $\mathrm{O}_{2}$ plasma treatment. For the present research, $\mathrm{O}_{2}$ plasma treatment of the $\mathrm{SiN}_{\mathrm{x}}$ surfaces was performed using an ICP/RIE system. It was found that the choice of ICP or RIE mode for $\mathrm{O}_{2}$ plasma generation affects device performance; more discussions will be presented in Section 5.4.

\subsection{Experimental Details}

Bottom-gate bottom-contact PQT-12 OTFTs with PECVD $\mathrm{SiN}_{\mathrm{x}}$ gate dielectric were used for this dielectric interface engineering study. The OTFTs were fabricated on highly-doped silicon substrates serving as the gate, with thermally-evaporated $\mathrm{Cr}-\mathrm{Au}$ source/drain contacts, and solution-processed PQT-12 as the active organic semiconductor layer. The fabrication sequence was outlined in Figure 3.8. Two dielectric surface treatment techniques were investigated: $\mathrm{O}_{2}$ plasma and OTS SAM. $\mathrm{O}_{2}$ plasma exposure was done using the Phantom II Reactive Ion Etching (RIE) system, a plasma etch system designed by Trion Technology Inc. OTS SAM was formed by immersing the substrates in a $0.1 \mathrm{M}$ of OTS in Toluene for $20 \mathrm{~min}$. at $60^{\circ} \mathrm{C}$, followed by rinsing with toluene and isopropanol. These treatments were done on the $\mathrm{SiN}_{\mathrm{x}}$ gate dielectric, prior to deposition of the PQT-12 layer. A simplified cross section structure of the OTFT is illustrated in Figure 5.4. 
The study on dielectric surface engineering is divided into two parts, as summarized in Table 5.2. The first experiment, reported in Section 5.3, examines the impact of individual dielectric surface treatment techniques, as well as their combination, on OTFT performance. The combination of $\mathrm{O}_{2}$ plasma and OTS treatment was considered, as it is believed that $\mathrm{O}_{2}$ plasma treatment can hydroxylate or oxidize the $\mathrm{SiN}_{\mathrm{x}}$ surface, to assist the growth of SAM through a silanisation reaction [4]. The second experiment, reported in Section 5.4, investigates the effect of $\mathrm{O}_{2}$ plasma exposure conditions (i.e., duration and power) on device performance, to determine an optimal $\mathrm{O}_{2}$ plasma exposure recipe for our devices. Electrical characterization of the OTFTs was carried out with a Keithley 4200-SCS parameter analyzer, under ambient conditions. For each dielectric surface treatment condition, at least five OTFTs with different $W / L$ were fabricated and tested, and the average value of the device parameter is reported below. The parameter extraction procedure is presented in Section 2.2. Surface properties were characterized using contact angle measurements for surface wettability, AFM measurements for surface roughness, and XPS measurements for chemical composition. The relationships between electrical properties and surface properties are analyzed to establish a better understanding on device behaviour. Please refer to Section 4.1.2 for an overview of the various material characterization techniques.

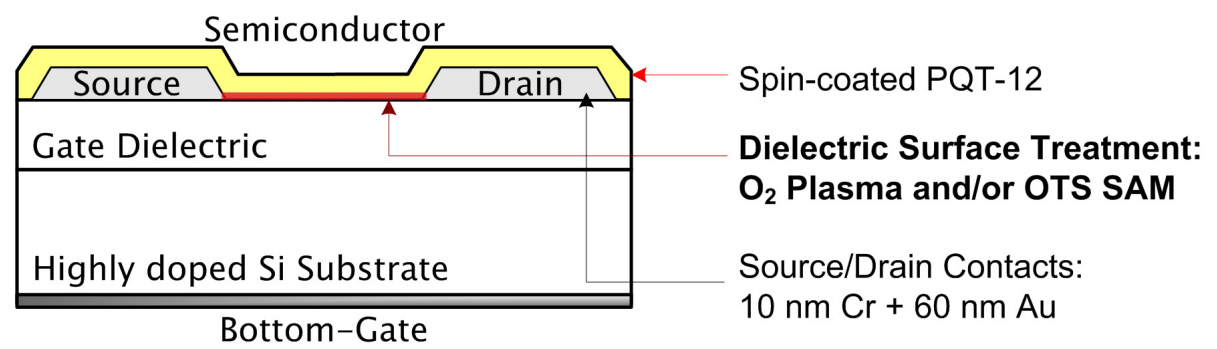

Figure 5.4. Schematic cross-section of the OTFT structure considered in this dielectric-surface treatment study.

Table 5.2. Dielectric-semiconductor interface engineering experiments.

\begin{tabular}{|lll|}
\hline & Description & Detail \\
\hline Part A & Comparison of transistors with & Examine device improvement resulting from $\mathrm{O}_{2}$ \\
(Section 5.3) & and without surface treatment & plasma, OTS SAM, and their combinations \\
\hline $\begin{array}{l}\text { Part B } \\
\text { (Section 5.4) }\end{array}$ & Effect of $\mathrm{O}_{2}$ Plasma Exposure & Evaluate impact of exposure duration and power \\
\hline
\end{tabular}




\subsection{Impact of Dielectric Surface Treatments}

The first experiment evaluates the impact of different dielectric surface treatments on OTFT performance. Four types of devices were prepared as outlined in Table 5.3. The electrical characteristics of the various OTFTs are compared to evaluate the effectiveness of each treatment recipe. The dielectric surface properties are characterized to investigate how these surface characteristics correlate with the observed device behavior.

Table 5.3. Dielectric surface treatment experiment: sample descriptions.

\begin{tabular}{|ll|}
\hline Sample ID & Description of the Dielectric Surface Treatment \\
\hline None or Untreated & As-deposited $\mathrm{SiN}_{\mathrm{x}}$ dielectric surface, without any treatment \\
\hline $\mathrm{O}_{2}$ Plasma & $\begin{array}{l}\text { Substrate exposed to } \mathrm{O}_{2} \text { plasma } \\
(60 \mathrm{sec}, 100 \mathrm{mTo} \text { Tr, } 34 \mathrm{~W} \text {. Refer to Recipe }(\mathrm{I}) \text { in Table } 5.6)\end{array}$ \\
\hline OTS & $\begin{array}{l}\text { Substrate treated with OTS SAM treatment } \\
\left(0.1 \mathrm{M} \text { of OTS in Toluene for } 20 \text { min. at } 60^{\circ} \mathrm{C}\right)\end{array}$ \\
\hline $\mathrm{O}_{2}$ Plasma /OTS & Substrate first exposed to $\mathrm{O}_{2}$ plasma $(60 \mathrm{~s})$, followed by OTS SAM \\
\hline
\end{tabular}

\subsubsection{Electrical Characterization}

The transfer and output characteristics of PQT-12 OTFTs on $300^{\circ} \mathrm{C} \mathrm{SiN} \mathrm{N}_{\mathrm{x}}(x=1.08)$ gate dielectric with different dielectric surface treatments are presented in Figure 5.5. As seen from the output characteristics, for a given gate voltage, drain current increases by more than one order of magnitude from the untreated sample to the $\mathrm{O}_{2}$ plasma/OTS treated sample. Using the extraction method outlined in Section 2.2, the changes in various device parameters, extracted from the OTFT characteristics in Figure 5.5, are summarized in Figure 5.6 and Table 5.4. The data shows an obvious improvement in field-effect mobility and on/off current ratio after surface treatment (as shown in Figure 5.6). This is accompanied by a reduction in inverse subthreshold slope (as shown in Table 5.4), suggesting improved dielectric-semiconductor interface properties as a result of the treatments. $V_{\mathrm{T}}$ shifts to increasingly positive voltages after surface treatment, and is possibility related to electron trapping at the interface.

When compared to untreated devices, remarkable improvement in mobility is observed after dielectric surface treatments. In particular, the combinatorial treatment using $\mathrm{O}_{2}$ plasma and OTS SAM generates the largest enhancement, where the mobility increased by 27 times $(2614 \%)$ 
(Figure 5.6(b)). Figure 5.7 plots the improvement factor in mobility of the treated device relative to the untreated samples (i.e., calculated using "mobility of treated device" / "mobility of untreated device"). To understand and account for these device variations, interface properties are examined to seek scientific insights.

(a)

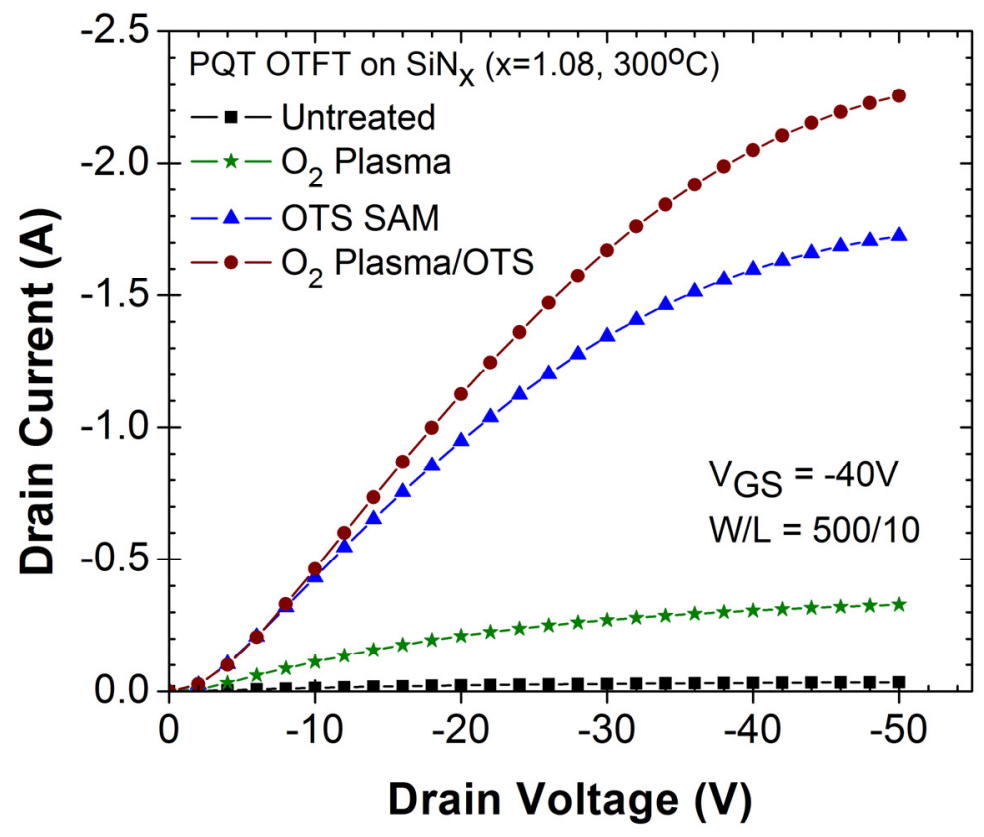

(b)

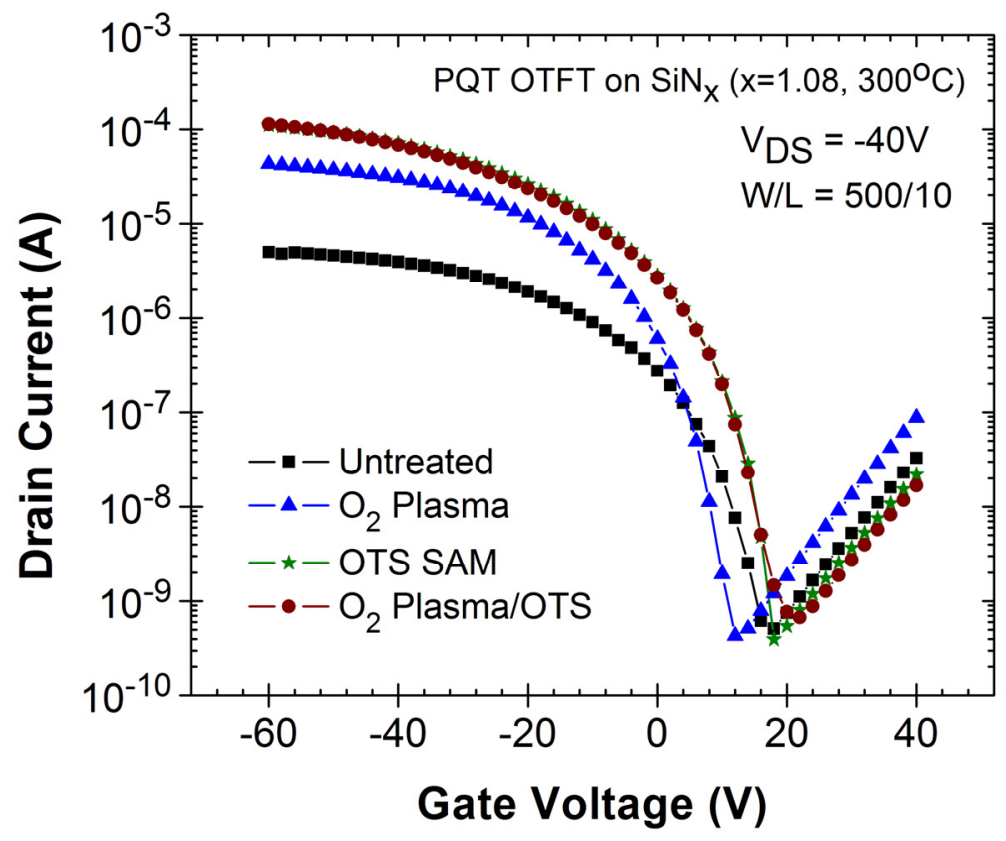


(c)

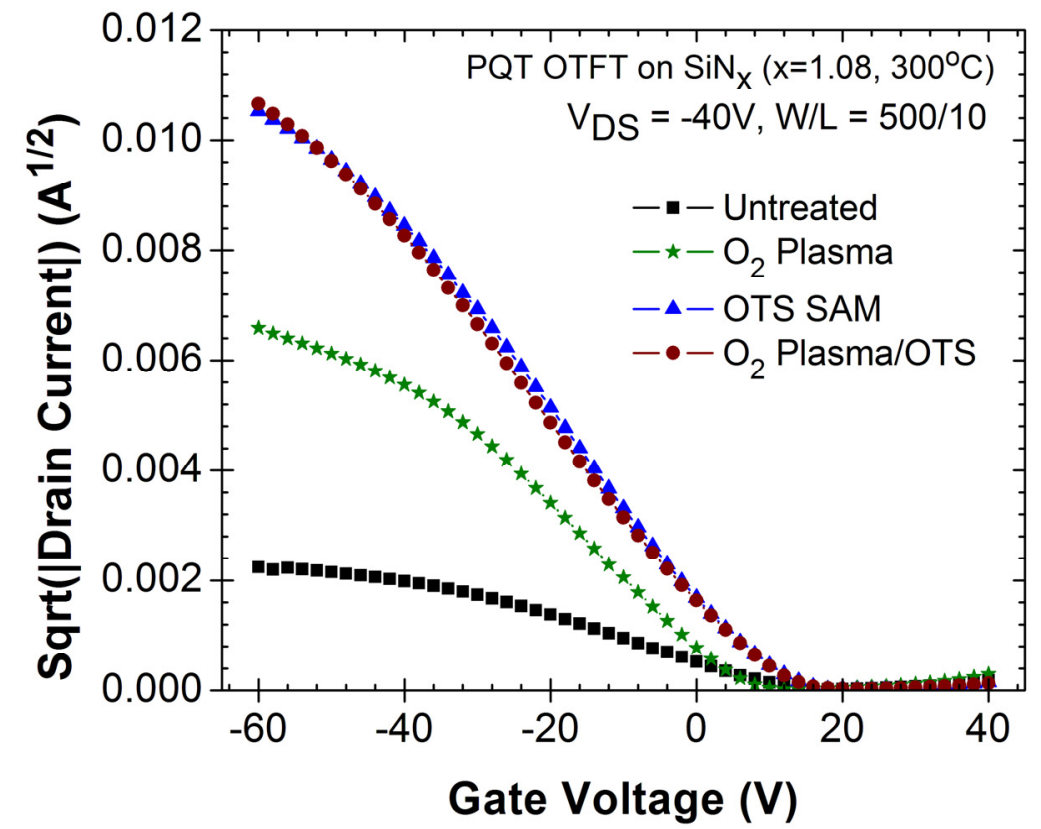

Figure 5.5. (a) Output and (b,c) transfer characteristics of PQT-12 OTFTs on $300^{\circ} \mathrm{C} \mathrm{PECVD}$ $\operatorname{SiN}_{x}(x=1.08)$ with different dielectric surface treatments. The transfer characteristics are shown on semi-logarithmic scale in (b) and as the square root of the drain current in (c).

Table 5.4. Changes in threshold voltage $V_{\mathrm{T}}$, switch-on voltage $V_{\mathrm{so}}$, and inverse subthreshold slope $S$ of OTFTs with different dielectric surface treatments. Data are extracted from PQT-12 OTFTs on $300^{\circ} \mathrm{C} \mathrm{SiN}_{x}(x=1.08)$ gate dielectric, with $W / L=500 \mu \mathrm{m} / 10 \mu \mathrm{m}$, in the saturation region $\left(V_{D S}=-40 V\right)$ (see Figure 5.5).

\begin{tabular}{|lccc|}
\hline Treatment & $V_{\mathrm{T}}(\mathrm{V})$ & $V_{\text {so }}(\mathrm{V})$ & $\mathrm{S}(\mathrm{V} / \mathrm{dec})$ \\
\hline Untreated & 3.54 & 18 & 4.28 \\
\hline $\mathrm{O}_{2}$ Plasma & 5.04 & 12 & 3.08 \\
\hline OTS & 9.46 & 18 & 2.84 \\
\hline O $_{2}$ Plasma / OTS & 9.19 & 20 & 3.81 \\
\hline
\end{tabular}


(a)

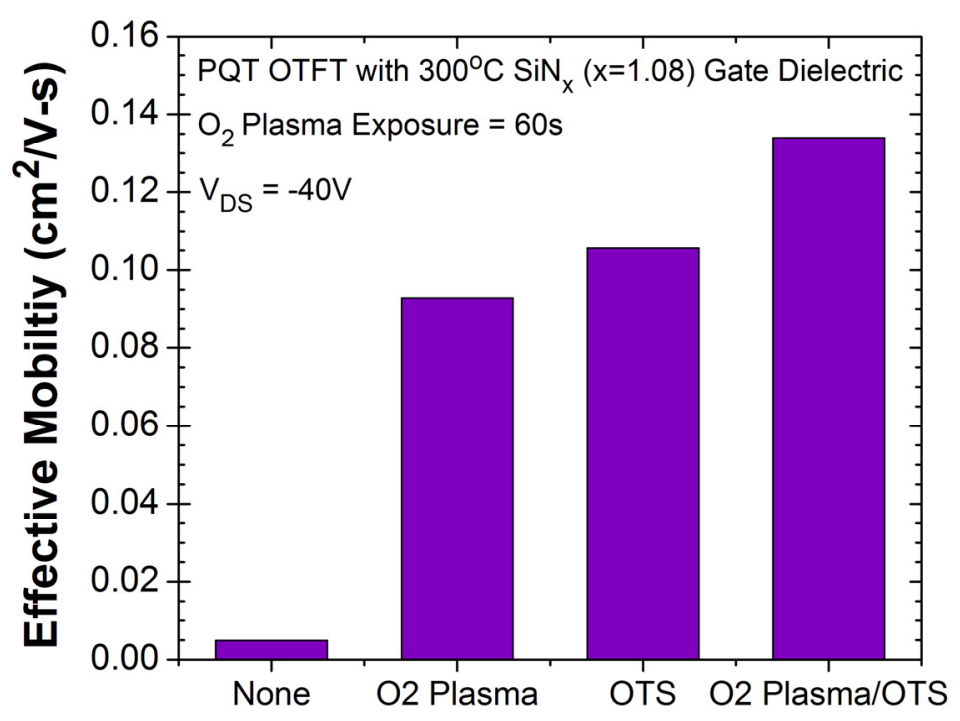

Dielectric Surface Treatment

(b)

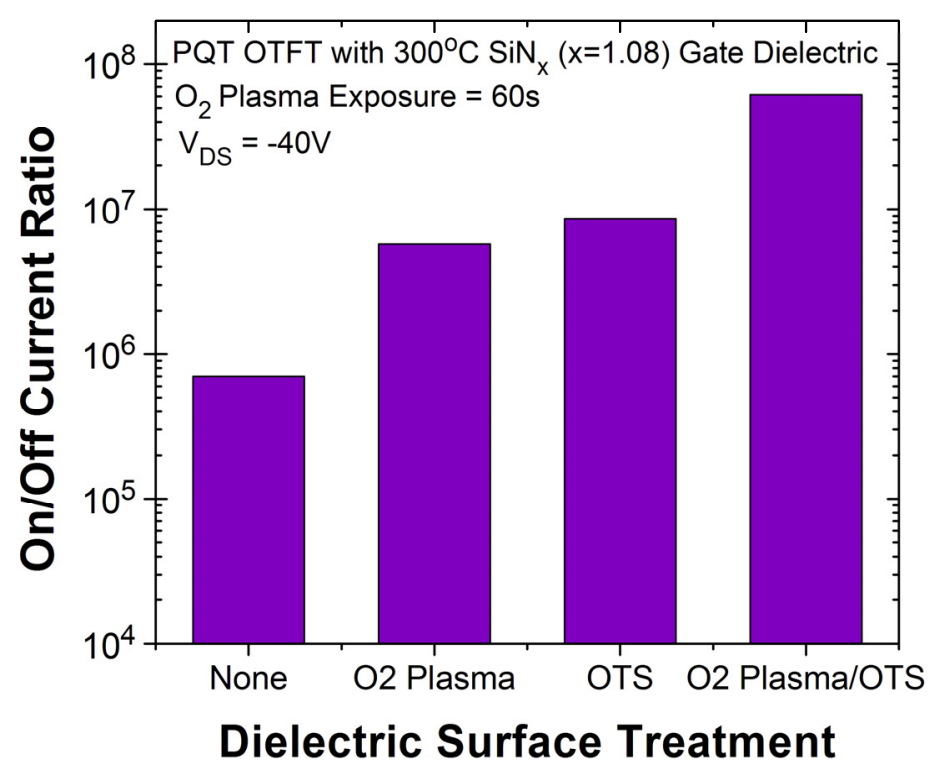

Figure 5.6. (a) Effective mobility and (b) on/off current ratio of PQT-12 OTFT with $\mathrm{SiN}_{\mathrm{x}}$ gate dielectrics under various dielectric surface treatment conditions. Measurements were collected in saturation region. Each data point corresponds to an average value from three to six devices. 


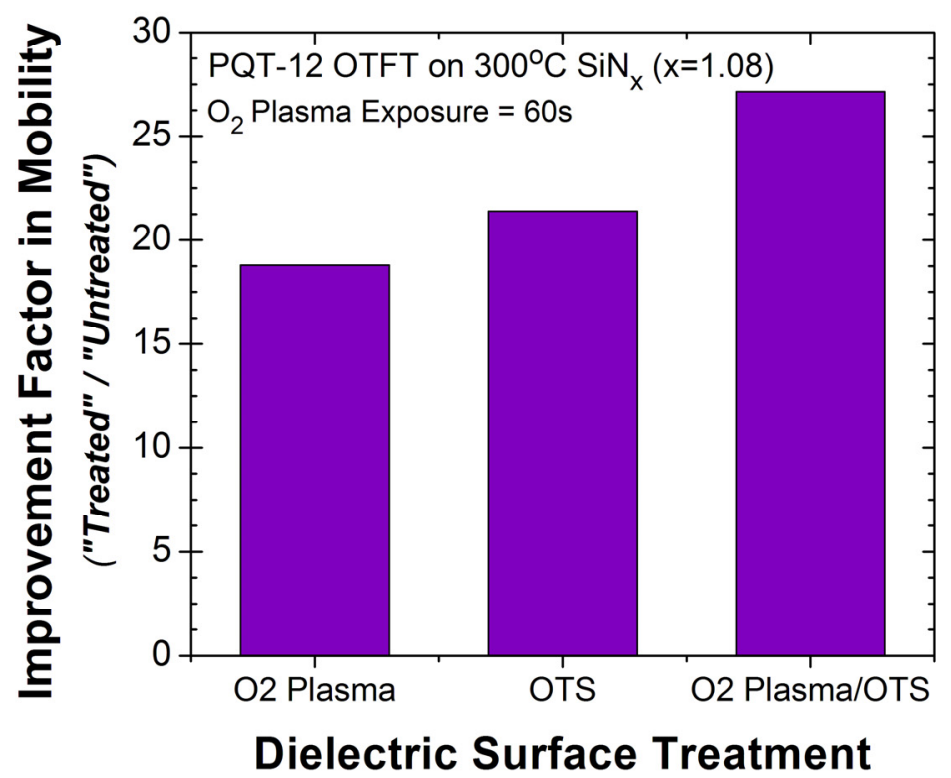

Figure 5.7. Factor of improvement in effective mobility, relative to untreated devices, for PQT12 OTFT after various types of dielectric surface treatments. Measurements were collected in saturation region.

\subsubsection{Interface Characterization}

The electrical characterization data indicated that the dielectric surface treatments have a very strong influence on the OTFT performance. In this section, the dielectric surface properties (e.g., contact angle, roughness, and composition) of the samples are analyzed, in an attempt to establish correlations with the observed electrical characteristics. Results unveiled that dielectric surfaces with large contact angle and low surface roughness are desirable for OTFTs. However, in some scenarios, they may behave as competing processes where the relative impact of one attribute may override the other.

\subsubsection{Contact Angle}

Figure 5.8 displays photographs from water contact angle measurement of a $\mathrm{SiN}_{\mathrm{x}}$ surface upon various surface treatment conditions; the measurement data is plotted in Figure 5.9. Compared to an untreated $\mathrm{SiN}_{\mathrm{x}}$ surface, an $\mathrm{O}_{2}$ plasma treated sample (Figure 5.8(b)) displayed a smaller contact angle, indicating hydrophilic properties. In contrast, samples with OTS treatment (Figure 5.8(c,d)) 
showed larger contact angle values, indicating hydrophobic surface states. The untreated $\mathrm{SiN}_{\mathrm{x}}$ is relatively hydrophilic, as indicated by its small contact angle value. Hydrophobicity is linked to the polarity (and thus the difference in electronegativity) of the bonds/molecules. Si-O, Si-N and N-O bonds are polar, leading to hydrophilic surface for (bare) $\mathrm{SiO}_{2}$ and $\mathrm{SiN}_{\mathrm{x}}$. Si-C and C-C bonds are weakly polar (or non-polar), thus OTS and polymer surfaces are hydrophobic.

(a) Untreated, $\theta=42.8^{\circ}$

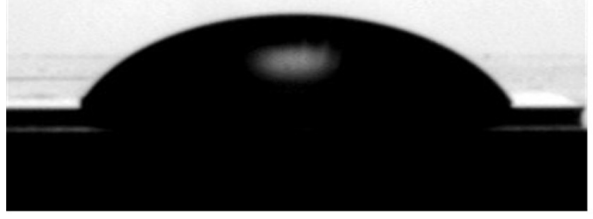

(c) OTS, $\theta=104.8^{\circ}$

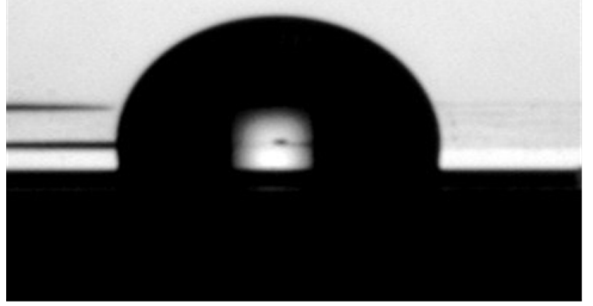

(b) $\mathrm{O}_{2}$ Plasma, $\theta=32.2^{\circ}$

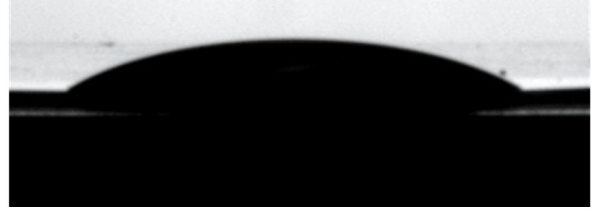

(d) $\mathrm{O}_{2}$ Plasma/OTS, $\theta=106.4^{\circ}$

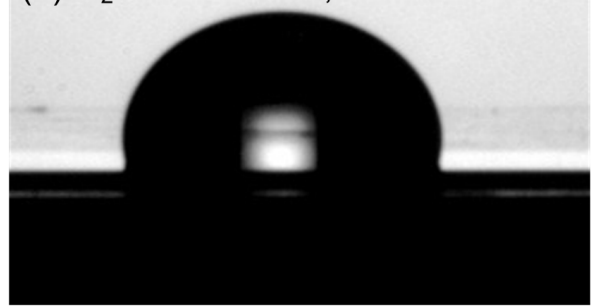

Figure 5.8. Photographs from contact angle measurement of $\mathrm{SiN}_{\mathrm{x}}$ surface after various dielectric surface treatments.

In general, most inorganic oxide and nitride surfaces (e.g., $\mathrm{SiO}_{2}$ and $\mathrm{SiN}_{\mathrm{x}}$ ) show hydrophilic properties, whereas most of organic materials (e.g., polythiophene) show hydrophobic properties. This mismatch in surface wettability/energy is believed to hinder the formation of well-ordered organic semiconductor molecules on inorganic dielectric surfaces. To overcome this mismatch, OTS SAMs are used to modify the dielectric surface from hydrophilic to hydrophobic; this modification can facilitate the formation of organic semiconductor layer with enhanced crystallinity and ordering, and thus resulting in increased carrier mobility [6].

On the other hand, $\mathrm{O}_{2}$ plasma treatment leads to a reduction in contact angle, indicating that the surface becomes more polar (or hydrophilic). $\mathrm{O}_{2}$ plasma treatment can have multiple effects on surface properties. Very short $\mathrm{O}_{2}$ plasma exposure duration (or initial stages of the exposure) typically causes removal of organic contaminations from the dielectric surface; contaminants may originate from wet cleaning procedures or from the environment [15]. Removal of organic compounds reduces contact angle. Furthermore, $\mathrm{O}_{2}$ plasma can generate polar groups (e.g., O-H, 
$\mathrm{Si}-\mathrm{O}, \mathrm{N}-\mathrm{O})$ on the surface, thus the sample acquires an hydrophilic character after $\mathrm{O}_{2}$ plasma treatment [16].

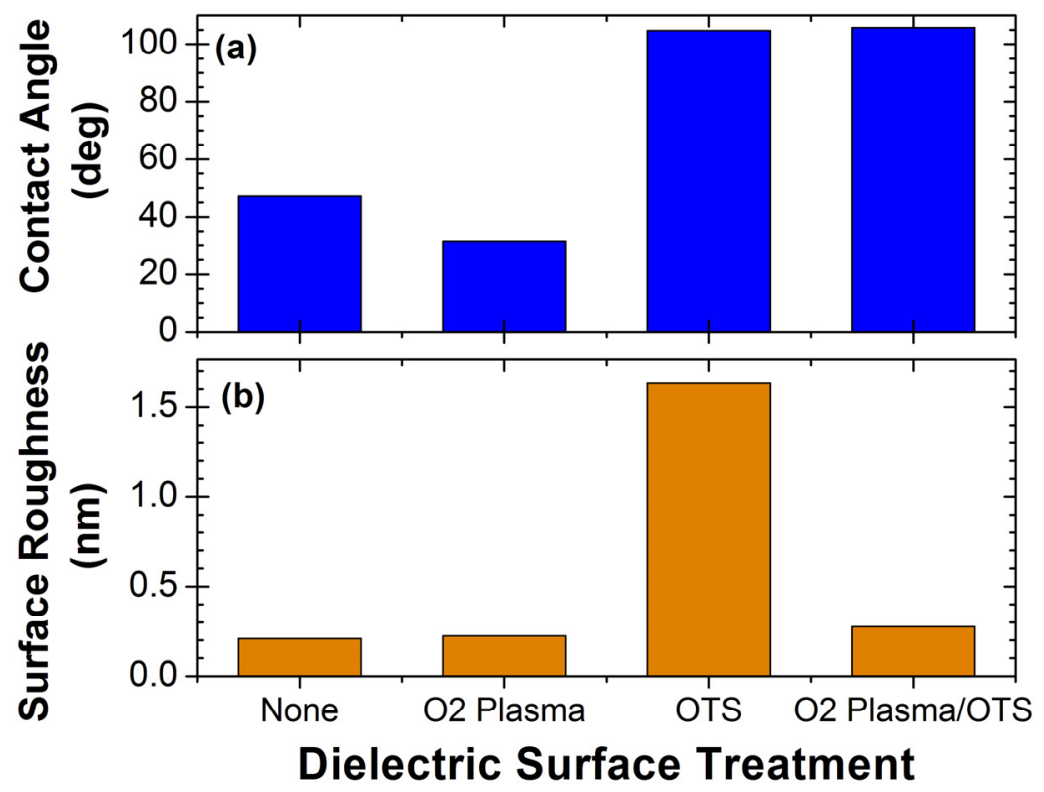

Figure 5.9. (a) Water contact angle measurement and (b) mean surface roughness of $\mathrm{SiN}_{\mathrm{x}}$ surfaces after various dielectric surface treatments.

Most literature reported that hydrophobic surfaces (i.e., large contact angle) are favorable for formation of well-ordered organic semiconductor film, and have a positive impact on mobility [7]. This tendency is reflected in our experiment where OTS treatment displays the large contact angle and generates high device mobility, relative to the untreated sample. An interesting and striking observation in our experiment is that there is an $18 \mathrm{x}$ improvement in mobility in $\mathrm{O}_{2}$ plasma treatment (despite its hydrophilic surface) when compared to an untreated OTFT, as shown in Figure 5.7. These results suggest that contact angle alone cannot give an accurate prediction of device behavior. In fact, interplay between contact angle, surface roughness, chemical composition, and other factors has a collective influence on the microstructure of the overlying organic semiconductor layer and on the interface quality. These factors are discussed next. In addition, deviation from the general hydrophobic-mobility trend is possible depending on the material system, as discussed in Section 5.1.1 (Table 5.1). 


\subsubsection{Surface Roughness}

Surface roughness affects the performance of TFTs. It can influence the quality of the semiconductor layer, affect charge transport in the channel, and cause interface scattering. Recent work by Chabinyc et al. showed that the field-effect mobility of OTFTs decreases nearly exponentially with surface roughness of the gate dielectric [20]. The surface roughness data is summarized in Figure 5.9, and the AFM surface topography photographs in Figure 5.10. Interestingly, compared to the untreated $\mathrm{SiN}_{\mathrm{x}}$ sample, $\mathrm{O}_{2}$ plasma treatment (Figure 5.10(b)) led to a reduction in surface roughness; the smoother surface is believed to be a key reason for the improved mobility observed in $\mathrm{O}_{2}$ plasma treated OTFTs. In contrast, OTS treatment (Figure $5.10(\mathrm{c})$ ) resulted in an increase in surface roughness. The higher surface roughness of the OTStreated $\mathrm{SiN}_{\mathrm{x}}$ is speculated to be due to a more sporadic formation of OTS SAM on the bare $\mathrm{SiN}_{\mathrm{x}}$ surface. On the other hand, combinatorial treatment with $\mathrm{O}_{2}$ plasma and OTS SAM (Figure $5.10(d))$ generated a much smoother surface, suggesting that pre-exposure of $\mathrm{SiN}_{\mathrm{x}}$ to $\mathrm{O}_{2}$ plasma is critical for formation of a smooth high quality OTS SAM on $\mathrm{SiN}_{\mathrm{x}}$. It is hypothesized that $\mathrm{O}_{2}$ plasma exposure places hydroxyl groups on the surface, which are needed to facilitate attachment of OTS molecules on $\mathrm{SiN}_{\mathrm{x}}$. Overall, it is concluded that the combination of $\mathrm{O}_{2}$ plasma and OTS treatment is the most preferable dielectric surface treatment approach; it delivers the highest TFT mobility, along with largest contact angle and relatively low surface roughness for the dielectric surface.

Altogether, these results showed that a variety of surface parameters must be taken into account to explain OTFT behavior. In most cases, higher mobility is achieved with increasing contact angle and decreasing surface roughness [20]. However, we conclude here that it is the combinatorial or collective effect of these parameters (along with many others) that dictate the resulting device performance. Table 5.5 compares the changes in field-effect mobility, contact angle and surface roughness across samples with different dielectric surface treatments. The champion device (with highest mobility) is attained with the combinatorial $\mathrm{O}_{2}$ plasma/OTS treatment, where the dielectric surface is simultaneously characterized by large contact angle and low surface roughness. The moderate mobility observed in the $\mathrm{O}_{2}$ plasma sample can be attributed to the low surface roughness, despite its low contact angle (or high surface energy). On the other hand, the high mobility observed in OTS sample is attributed to its low surface energy (i.e., high contact angle), despite its higher surface roughness. Therefore, depending on the particular sample or material system, the effect of surface roughness may prevail over that of surface energy, and vice versa. In 
addition, it is important to consider other surface properties that might influence OTFT characteristics; surface chemical composition is examined next.
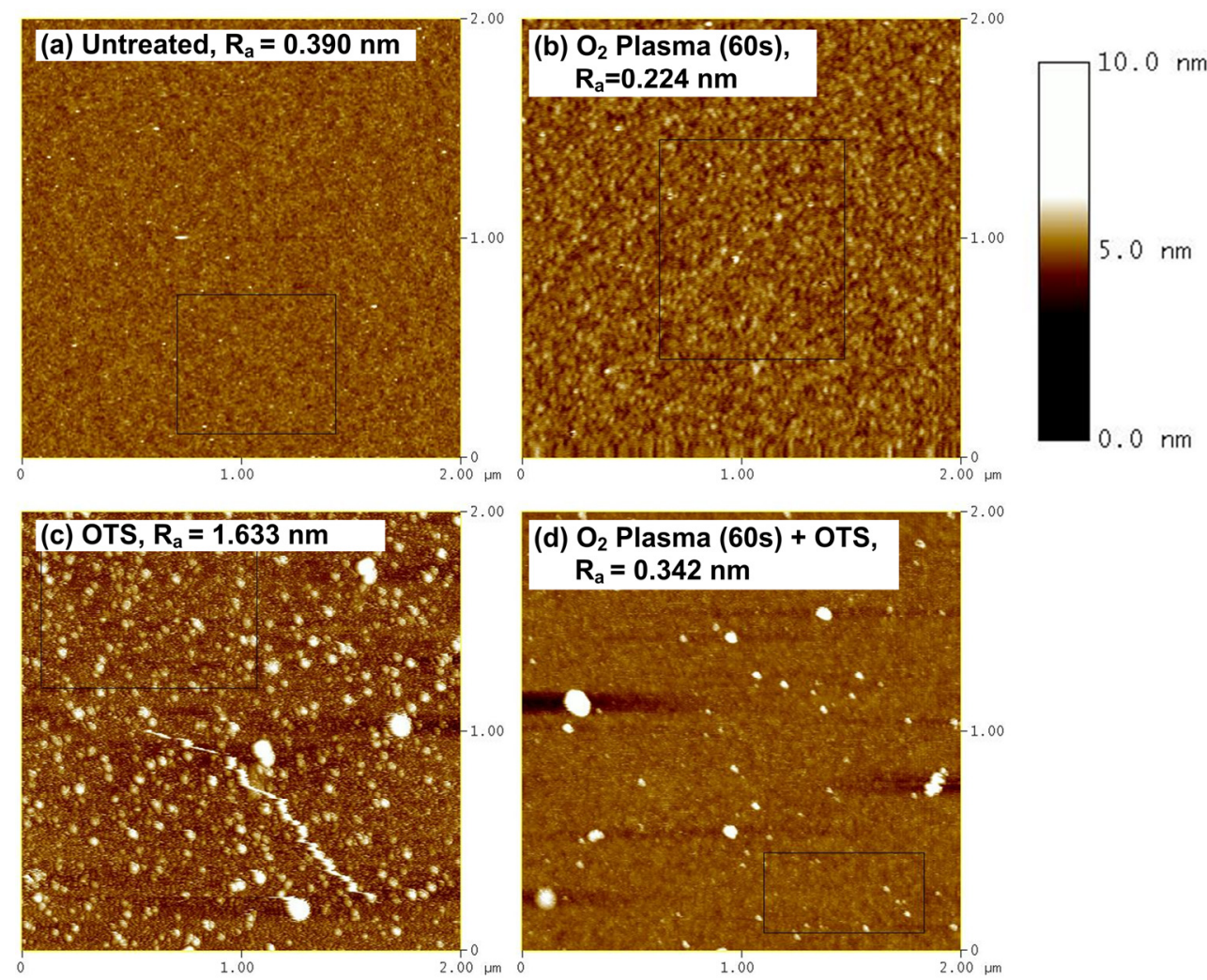

Figure 5.10. AFM images showing surface topography of $\mathrm{SiN}_{\mathrm{x}}$ surface after various dielectric surface treatments. The mean surface roughness $\left(R_{a}\right)$ is indicated.

Table 5.5. Comparison of effective field-effect mobility, contact angle and surface roughness for samples with different dielectric surface treatment. Data shown is obtained by averaging results from a number of samples.

\begin{tabular}{|lcccl|}
\hline Treatment & $\begin{array}{c}\mu_{\mathrm{FE}} \\
\left(\mathrm{cm}^{2} / \mathrm{V}-\mathrm{s}\right)\end{array}$ & $\begin{array}{c}\text { Contact } \\
\text { Angle, } \theta\end{array}$ & $\begin{array}{c}\text { Surface } \\
\text { Roughness }(\mathrm{nm})\end{array}$ & \multicolumn{1}{c|}{ Remark } \\
\hline Untreated & 0.0049 & $47.2^{\circ}$ & 0.21 & $\begin{array}{l}\text { Low } \theta+\text { Low roughness } \\
\rightarrow \text { Low } \mu\end{array}$ \\
\hline O2 Plasma & 0.093 & $31.35^{\circ}$ & 0.224 & $\begin{array}{l}\text { Low } \theta+\text { Low roughness } \\
\rightarrow \text { moderate } \mu\end{array}$ \\
\hline OTS & 0.106 & $104.77^{\circ}$ & 1.633 & $\begin{array}{l}\text { High } \theta+\text { High } \\
\text { roughness } \rightarrow \\
\text { moderate } \mu\end{array}$ \\
\hline O2 $_{2}$ Plasma / OTS & 0.134 & $105.73^{\circ}$ & 0.276 & $\begin{array}{l}\text { High } \theta+\text { Low } \\
\text { roughness } \rightarrow \text { High } \mu\end{array}$ \\
\hline
\end{tabular}




\subsubsection{Chemical Composition}

It is interesting to observe in Table 5.5 that both the "untreated" and " $\mathrm{O}_{2}$ plasma" samples are characterized by low contact angle (hydrophilic surface) and low surface roughness, but the $\mathrm{O}_{2}$ plasma treated device has much higher mobility than the untreated device. The difference in device behavior can be related to elemental chemical composition of the interface. The atomic composition at the $\mathrm{SiN}_{\mathrm{x}}$ surface was studied by XPS, and the results are shown in Figure 5.11. A key distinction between the untreated sample and the treated samples is that the former surface is dominated by $\mathrm{Si}$ and $\mathrm{N}$, whereas the latter surfaces are dominated by $\mathrm{Si}, \mathrm{O}$ and $\mathrm{C}$. As such, despite "untreated" and " $\mathrm{O}_{2}$ plasma" samples have similar surface roughness and contact angle, the $\mathrm{O}_{2}$ plasma surface has richer $\mathrm{Si}, \mathrm{O}, \mathrm{C}$ content than untreated surface. The presence of Si-O or Si-C bonds are believed to improve formation of the subsequent organic layer; thus, resulting in an improved dielectric-semiconductor interface.

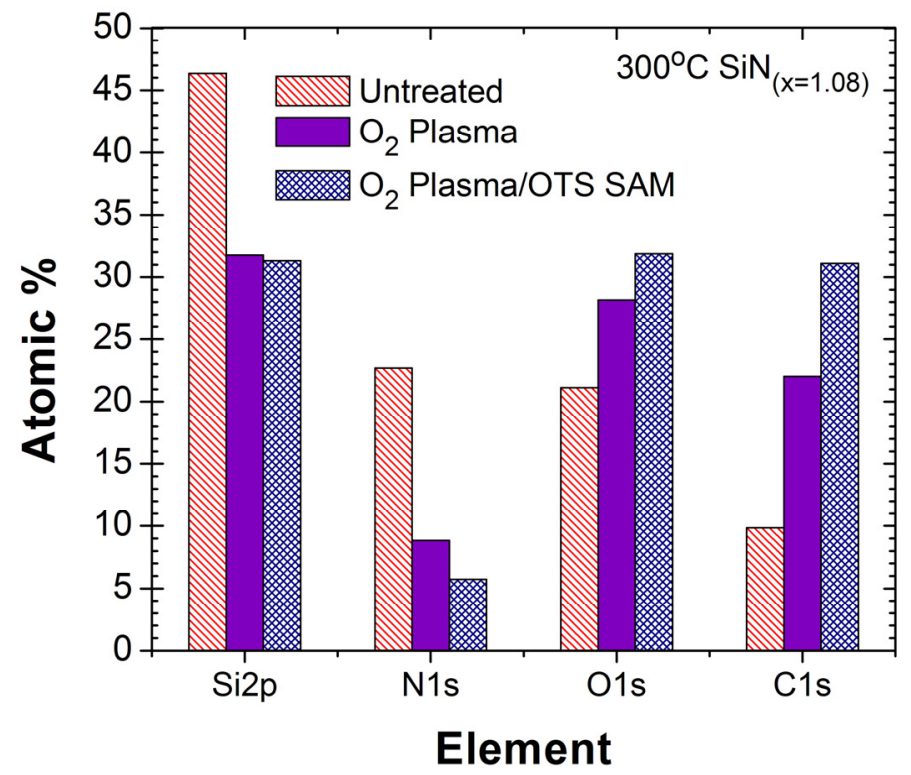

Figure 5.11. Atomic composition from XPS measurements of $\mathrm{SiN}_{\mathrm{x}}$ surfaces with different surface treatments. 


\subsubsection{Analysis}

Compared to results published in the literature [4], the relatively large improvement in mobility with $\mathrm{O}_{2}$ plasma treatment (as shown in Figure 5.6 and Figure 5.7) is quite remarkable because it has been reported that a hydrophobic dielectric surface is necessary for achieving high mobility OTFTs. A hydrophobic surface, characterized by low mean free surface energy, provides a favorable platform for organic semiconductor molecules to assemble into a well-ordered film [13]. As noted in Figure 5.9, OTS treatment leads to a hydrophobic surface (large contact angle), whereas $\mathrm{O}_{2}$ plasma treatment produces a hydrophilic surface (small contact angle). Therefore, the observation that $\mathrm{O}_{2}$ plasma gives nearly comparable mobility as OTS treatment is a unique discovery/breakthrough. Although the exact reason for this somewhat unconventional behavior is not completely clear, a few possible explanations are proposed here to account for the various observations in this experiment.

- $\underline{O}_{2}$ plasma vs. OTS: The $\mathrm{O}_{2}$ plasma treated device has comparable mobility to the OTS SAM treated device. The observation that $\mathrm{O}_{2}$ plasma has considerably smaller surface roughness than OTS SAM provides an agreeable justification for the high mobility in $\mathrm{O}_{2}$ plasma devices (see Figure 5.7). On the other hand, the small contact angle of $\mathrm{O}_{2}$ plasma treated surface (i.e., hydrophilic, high surface energy) does not corroborate with this mobility trend.

- $\quad$ OTS vs. $\mathrm{O}_{2}$ plasma/OTS: There might be imperfections in the OTS SAM when formed on bare $\mathrm{SiN}_{\mathrm{x}}$ surface. Since a hydroxylated surface is typically needed for the formation of OTS, bare $\mathrm{SiN}_{\mathrm{x}}$ might not provide the most favorable environment for high quality OTS SAM formation. By exposing the $\mathrm{SiN}_{\mathrm{x}}$ surface to $\mathrm{O}_{2}$ plasma prior to OTS treatment, the quality of OTS SAM is improved. As observed in Figure 5.7, surface roughness for OTS on $\mathrm{O}_{2}$ plasma treated $\mathrm{SiN}_{\mathrm{x}}$ is considerably lower than OTS on bare $\mathrm{SiN}_{\mathrm{x}}$, suggesting the positive contribution or effectiveness of $\mathrm{O}_{2}$ plasma treatment on formation of high quality OTS SAM. The higher surface roughness for OTS on bare $\mathrm{SiN}_{\mathrm{x}}$ likely compromises the mobility in OTS devices when compared to $\mathrm{O}_{2}$ plasma/OTS devices. In terms of surface energy, contact angle measurements showed very little difference between OTS surface and $\mathrm{O}_{2}$ plasma/OTS surfaces; this suggests the samples have comparable surface energy (or wettability).

- Untreated vs. $\mathrm{O}_{2}$ plasma: Both untreated $\mathrm{SiN}_{\mathrm{x}}$ surface and $\mathrm{O}_{2}$ plasma treated surface are characterized by low surface roughness and low contact angle (i.e., hydrophilic surface). However, the $\mathrm{O}_{2}$ plasma treated device showed significantly greater mobility than that of the untreated device. Therefore, surface roughness and surface energy alone cannot completely 
capture the interface properties to explain/understand OTFT behavior. Additional insights can be gained from studying the chemical composition of the surface. The XPS data in Figure 5.11 shows that the treated samples are characterized by a higher $\mathrm{O} 1 \mathrm{~s}$ concentration and a significantly lower Si 2 p concentration compared to untreated sample. It is believed that an oxide-like surface is beneficial to the assembly of the organic semiconductor layer. $\mathrm{O}_{2}$ plasma treatment produces an oxynitride surface, where more $\mathrm{Si}-\mathrm{O}$ bonds are present compared to the bare $\mathrm{SiN}_{\mathrm{x}}$ surface. The dominance of $\mathrm{Si}-\mathrm{O}$ bonds on the $\mathrm{O}_{2}$ plasma treated surface enhances the dielectric-semiconductor interface properties compared to bare $\operatorname{SiN}_{\mathrm{x}}$ (which is characterized by mainly Si-N bonds). This key distinction in surface composition accounts for the enhanced device mobility observed in the $\mathrm{O}_{2}$ plasma treated OTFT compared to untreated OTFT. In addition, $\mathrm{O}_{2}$ plasma treatment may improve the charge injection properties at the contacts to contribute to enhanced device performance. Recent studies reported a reduction in hole injection barrier at the Au-pentacene interface by $\mathrm{O}_{2}$ plasma, leading to an increase in linear mobility for pentacene OTFTs [21].

- Fluorinated $\mathrm{O}_{2}$ plasma treated surface: The XPS data for $\mathrm{O}_{2}$ plasma treated surface showed a very peculiar feature: a very weak presence of fluorine. It is speculated that fluorine species were introduced onto the surface during $\mathrm{O}_{2}$ plasma exposure by the RIE process. There is a possibility that the presence of fluorine or the combinatorial effect of fluorine and oxygen on the $\mathrm{SiN}_{\mathrm{x}}$ surface is particularly favorable for organic semiconductors. More controlled experiments must be conducted to justify this hypothesis. Nonetheless, this unique phenomenon of "fluorinated surface treatment" warrants more research attention to exploit its potential for practical use.

The above analyses confirmed that the OTFT device characteristics are influenced by surface roughness, surface energy, and surface composition. From this investigation, it is concluded that the ideal dielectric surface for bottom-gate OTFTs is characterized by low surface roughness, low surface energy (i.e., high contact angle), and a Si-O like surface composition; this combination leads to higher mobility for PQT-12 OTFTs on $\mathrm{SiN}_{\mathrm{x}}$ gate dielectric. GIXRD can be performed on the devices to compare the molecular microstructure of PQT-12 on the various treated surface, to understand how molecular ordering of PQT-12 vary on different surfaces. 


\subsection{Impact of Oxygen Plasma Exposure Conditions}

The objective of this experiment is to systematically evaluate the effect of $\mathrm{O}_{2}$ plasma exposure conditions on PQT-12 OTFT performance, and to determine the optimal exposure conditions for achieving high mobility devices. The first experiment examines the impact of exposure duration; the second experiment considers the impact of exposure power. Bottom-gate bottom-contact transistors with a Si-rich $150^{\circ} \mathrm{C} \operatorname{SiN}_{\mathrm{x}}(\mathrm{x}=1.28)$ gate dielectric and pre-patterned source/drain contacts serves as the device platform for this experiment. Prior to deposition of PQT-12, all dielectric surfaces were pre-treated with $\mathrm{O}_{2}$ plasma and OTS SAM and the Au contacts were treated with 1-Octanethiol SAM. Three $\mathrm{O}_{2}$ plasma exposure recipes were used and are listed in Table 5.6. The effects of $\mathrm{O}_{2}$ plasma treatment were analyzed by examining the variation of performance parameters such as field-effect mobility, on/off current ratio, subthreshold slope, threshold voltage, and on-set voltage with respect to the plasma exposure time. The physical (e.g., surface roughness) and chemical properties (e.g., contact angle, chemical composition) of the $\mathrm{O}_{2}$ plasma treated $\mathrm{SiN}_{\mathrm{x}}$ surface are analyzed for elucidation of the observed TFT behavior.

Table 5.6. Various $\mathrm{O}_{2}$ plasma recipes used in this experiment, with chamber pressure at 150 mTorr, and $\mathrm{O}_{2}$ gas flow rate at $30 \mathrm{sccm}$. RIE and ICP is abbreviation for reactive ion etching and inductively coupled plasma, respectively. DC bias directly controls the RIE power.

\begin{tabular}{|lcccc|}
\hline O $_{2}$ Plasma Recipe & DC Bias (V) & ICP Power (W) & RIE Power (W) & $\begin{array}{c}\text { Exposure Duration } \\
\text { (seconds) }\end{array}$ \\
\hline Recipe (I): RIE only & -300 & 0 & 34 & $0-900$ \\
\hline Recipe (II): ICP only & 0 & 300 & 0 & 200,600 \\
\hline Recipe (III): RIE + ICP & -150 & 300 & 9 & 600 \\
\hline
\end{tabular}

\subsubsection{Electrical Characterization}

\subsubsection{Impact of Exposure Duration}

The study on $\mathrm{O}_{2}$ plasma exposure duration was executed using Recipe (I) listed in Table 5.6. The transfer and output characteristics of PQT-12 OTFTs (with $W / L=500 \mu \mathrm{m} / 10 \mu \mathrm{m}$ ) for different $\mathrm{O}_{2}$ plasma exposure durations are shown in Figure 5.12. Table 5.7 summarizes the device parameters extracted from the OTFT characteristics in Figure 5.12. The data revealed an improvement in field-effect mobility and on/off current ratio as the $\mathrm{O}_{2}$ exposure duration increases from $0 \mathrm{sec}$ to 80 sec; this is accompanied by a reduction in inverse subthreshold slope, suggesting improved 
dielectric-semiconductor interface properties as a result of the treatments. However, for exposure duration from $80 \mathrm{sec}$ to $900 \mathrm{sec}$, a gradual reduction in mobility and on/off current ratio is observed. There is a clear shift in threshold voltage $V_{\mathrm{T}}$ and switch-on voltage $V_{\text {So }}$ towards higher positive voltages as $\mathrm{O}_{2}$ plasma exposure duration increases. This shift is likely related to $\mathrm{O}_{2}$ plasma induced electron trapping at the interface [22]. Additional analysis is presented in Section 5.4 .3

A set of 5 samples (with various $W / L$ ) were tested for each exposure duration conditions. The average effective mobility and on/off current ratio for each set of devices, as a function of $\mathrm{O}_{2}$ plasma duration, are plotted in Figure 5.13. The plots show a distinct peak near $80 \mathrm{sec}$, where maximum field effect mobility and on/off current ratio were observed. At exposure duration of 80 sec, effective mobility of $0.216 \mathrm{~cm}^{2} / \mathrm{V}$-s was measured, which represents a 6.3 times (or $530 \%$ ) increase in mobility compared to devices without $\mathrm{O}_{2}$ plasma exposure (i.e., exposure time $=0 \mathrm{sec}$ ). (Note: all devices were treated with OTS SAM.) For lengthened exposure ( $>80 \mathrm{sec}$ ), $\mu_{\mathrm{FE}}$ gradually decreases and plateaus at $0.05 \mathrm{~cm}^{2} / \mathrm{V}$-s. Figure $5.13(\mathrm{~b})$ shows that shorter exposure duration also favors higher on/off current ratio. In the following/subsequent discussion, $\mathrm{t}=80 \mathrm{sec}$ is referred to as the "turn-around" point. Potential interface mechanisms responsible for the observed OTFT behavior are analyzed in Section 5.4.2.

(a)

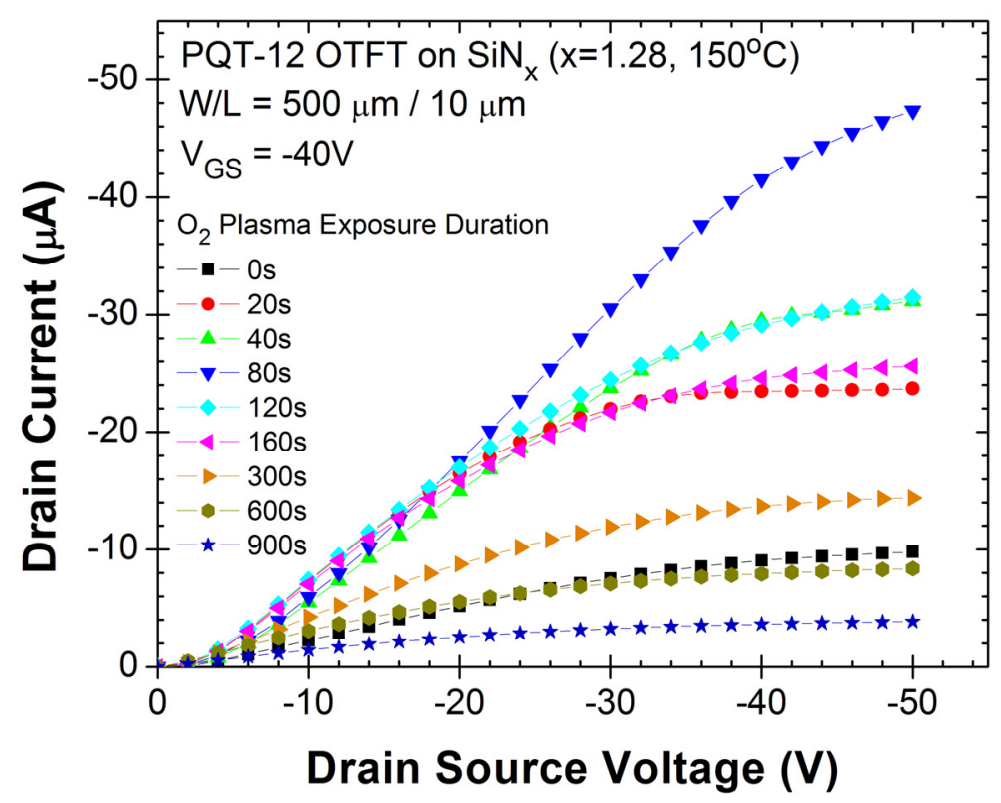


(b)

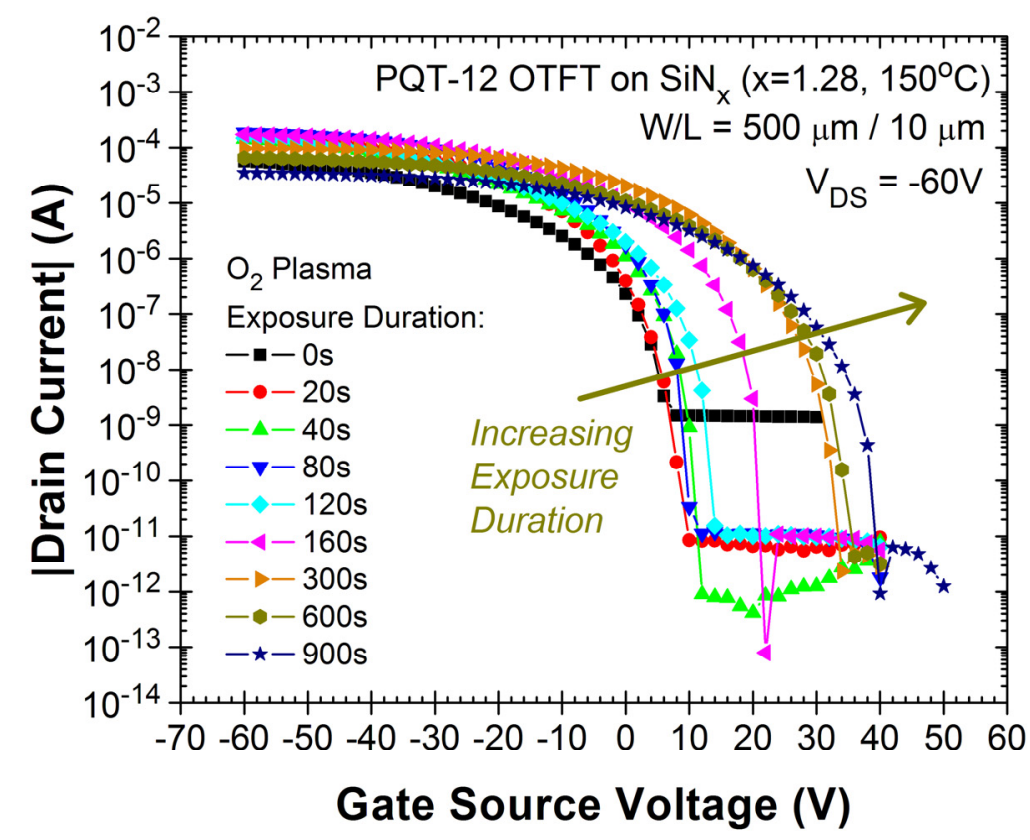

(c)

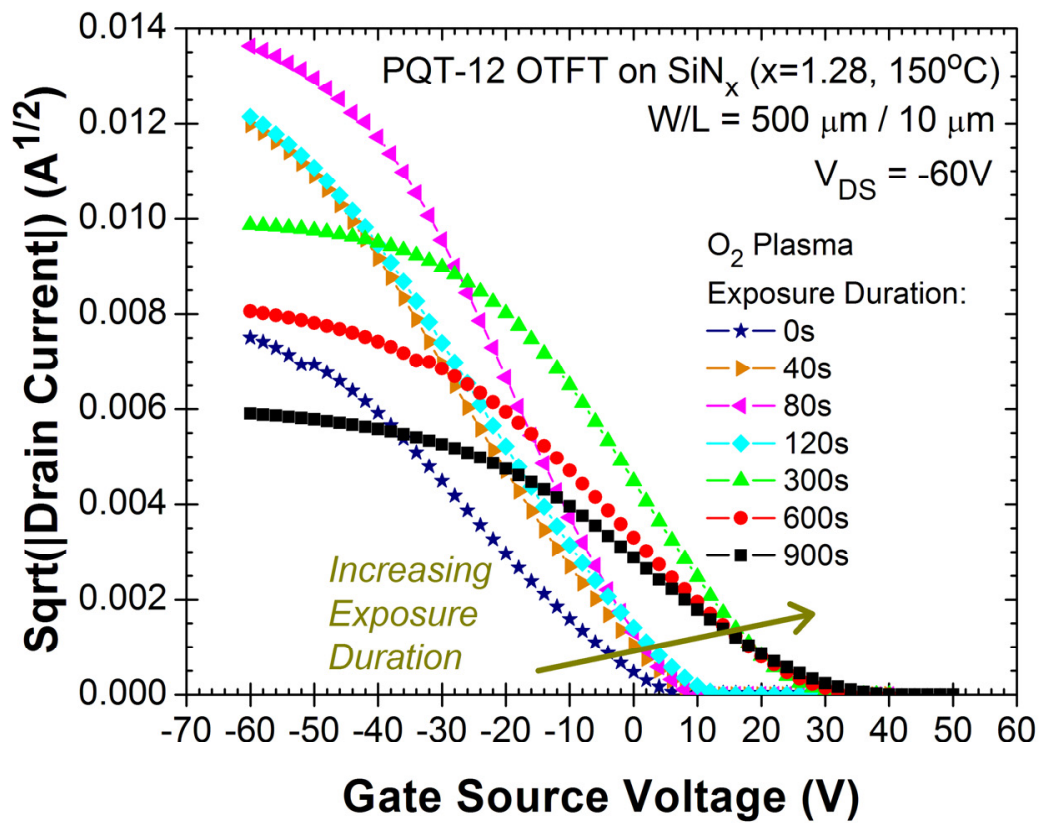

Figure 5.12. (a) Output characteristics and (b,c) transfer of PQT-12 OTFTs on $150^{\circ} \mathrm{C}$ PECVD $\operatorname{SiN}_{x}(x=1.28)$ gate dielectric treated with different $\mathrm{O}_{2}$ plasma exposure duration. The transfer characteristics are shown on semi-logarithmic scale in (b) and as the square root of the drain current in (c). All samples were treated with $\mathrm{O}_{2}$ plasma, OTS SAM and 1-Octanethiol SAM. 
Table 5.7. OTFT device parameters as a function of $\mathrm{O}_{2}$ plasma exposure duration. Data are extracted from PQT-12 OTFTs on $150^{\circ} \mathrm{C} \mathrm{SiN} \mathrm{N}_{\mathrm{x}}(\mathrm{x}=1.28)$ gate dielectric, with $\mathrm{W} / \mathrm{L}=500 \mu \mathrm{m} /$ $10 \mu \mathrm{m}$, in the saturation region $\left(V_{D S}=-60 \mathrm{~V}\right)$ (refer to Figure 5.12).

\begin{tabular}{|cccccc|}
\hline $\begin{array}{c}\text { Duration } \\
(\mathrm{sec})\end{array}$ & $\begin{array}{c}\mu_{\mathrm{FEE}} \\
\left(\mathrm{cm}^{2} / \mathrm{V}-\mathrm{s}\right)\end{array}$ & $V_{\mathrm{T}}(\mathrm{V})$ & $V_{\text {so }}(\mathrm{V})$ & loN $/$ loFF & $\mathrm{S}(\mathrm{V} / \mathrm{dec})$ \\
\hline 0 & 0.0476 & -2.33 & 8 & $4.06 \times 10^{4}$ & 3.067 \\
40 & 0.1481 & -2.13 & 10 & $2.70 \times 10^{7}$ & 1.587 \\
80 & 0.1746 & 1.33 & 12 & $2.15 \times 10^{9}$ & 0.830 \\
160 & 0.1307 & 10.90 & 22 & $1.04 \times 10^{8}$ & 1.290 \\
\hline 300 & 0.0912 & 19.59 & 34 & $4.17 \times 10^{7}$ & 1.691 \\
600 & 0.0416 & 21.25 & 36 & $2.04 \times 10^{7}$ & 1.586 \\
900 & 0.0247 & 24.18 & 40 & $3.76 \times 10^{7}$ & 3.081 \\
\hline
\end{tabular}

\subsubsection{Impact of Exposure Power}

The influence of $\mathrm{O}_{2}$ plasma exposure power on the effective mobility of PQT-12 OTFTs is illustrated in Figure 5.14. An increase in effective mobility is observed as RIE power increases (from $0 \mathrm{~W}$ to $34 \mathrm{~W}$ ). It is hypothesized that as RIE power increases, there is an increase in ion energy of reactive ions impinging on the wafer; as a result, more oxygen species become attached/adsorbed on the substrate surface. This explanation can be verified by XPS measurements (see Section 5.4.2.3). The enhanced attachment of oxygen species on the surface is believed to facilitate and enhance subsequent formation of the OTS SAM and PQT-12 layer on the $\mathrm{SiN}_{\mathrm{x}}$ gate dielectric.

The results also reveal that $\mathrm{O}_{2}$ plasma generated by the RIE mode of operation is more favorable than that generated by the ICP mode for improving device mobility. RIE mode creates a stronger drift field that directs the ions onto the substrate that ICP mode. This increases the reactivity of the $\mathrm{SiN}_{\mathrm{x}}$ surface, enhancing the bonding with OTS SAM on the $\mathrm{O}_{2}$ plasma treated $\mathrm{SiN}_{\mathrm{x}}$ surface. In addition, Figure 5.14 reveals that a $200 \mathrm{sec}$ exposure leads to higher mobility than $600 \mathrm{sec}$ exposure, for the two recipes plotted here. This substantiates the previous observation where shorter exposure duration leads to higher mobility. 
(a)

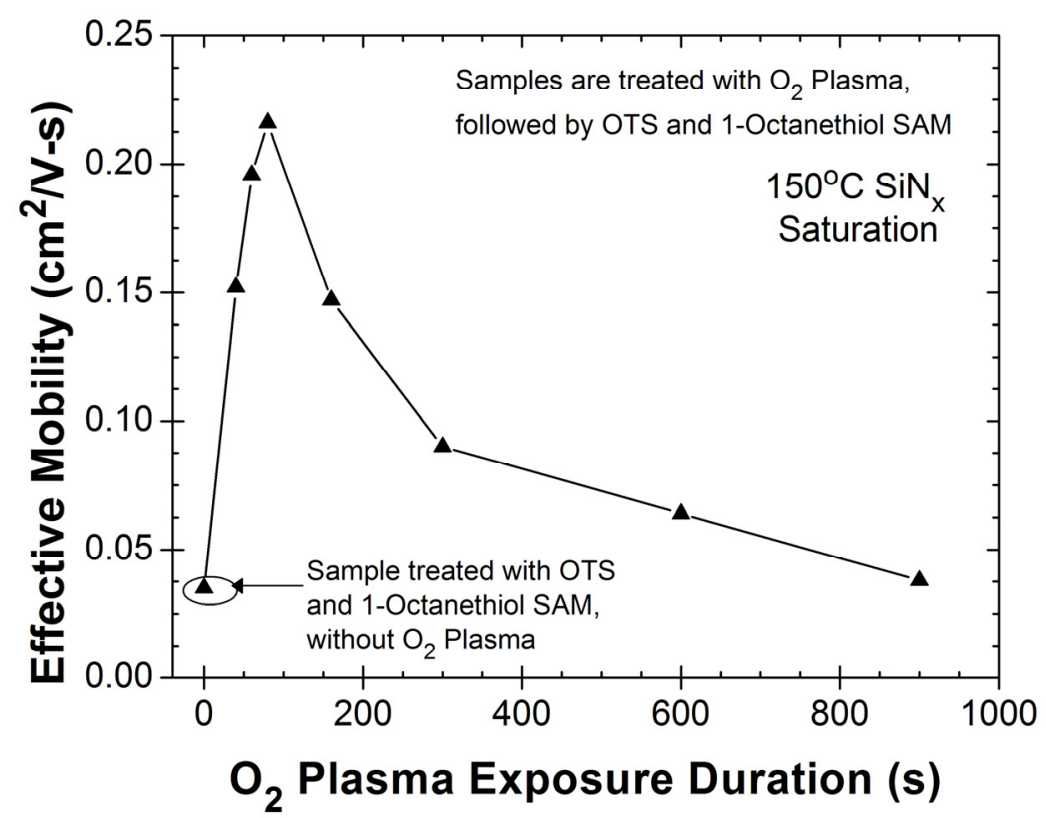

(b)

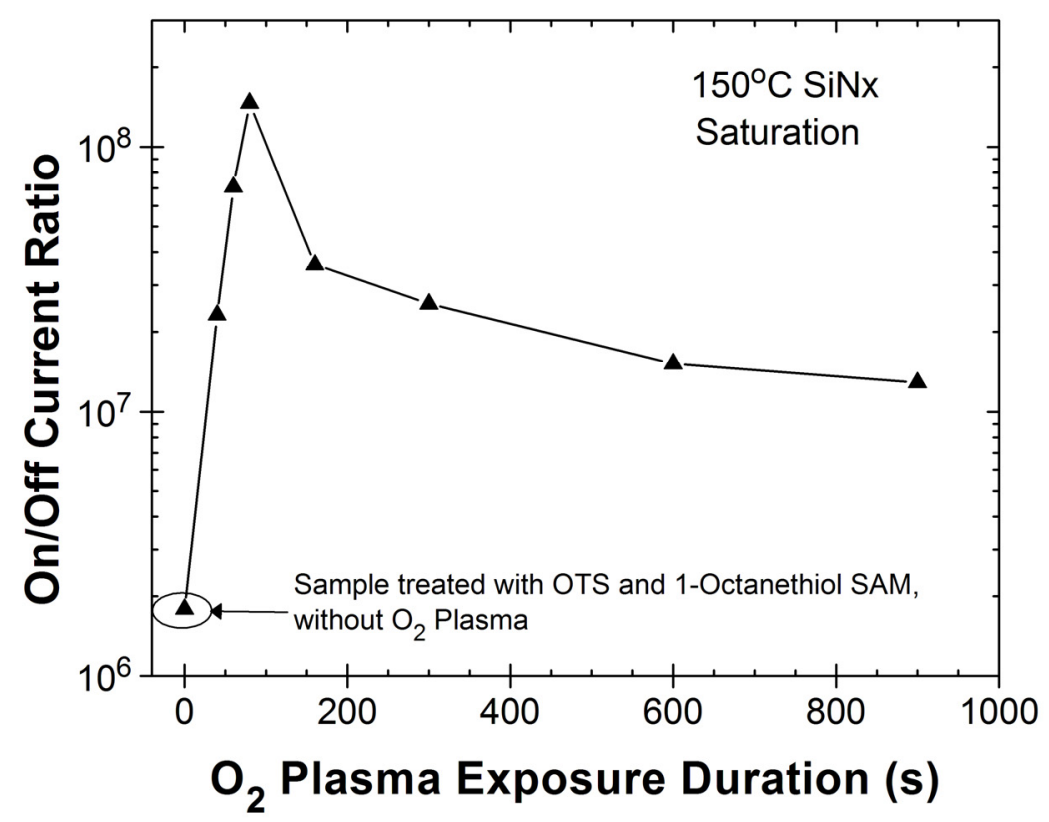

Figure 5.13. (a) Effective mobility and (b) on/off current ratio as a function of $\mathrm{O}_{2}$ plasma exposure duration of PQT-12 OTFT with $150^{\circ} \mathrm{C} \mathrm{SiN}{ }_{x}(x=1.28)$ gate dielectric. Data was collected in the saturation region. All samples were treated with $\mathrm{O}_{2}$ plasma, OTS, and 1octanethiol. Here, each data point corresponds to an average value from three to six devices. 


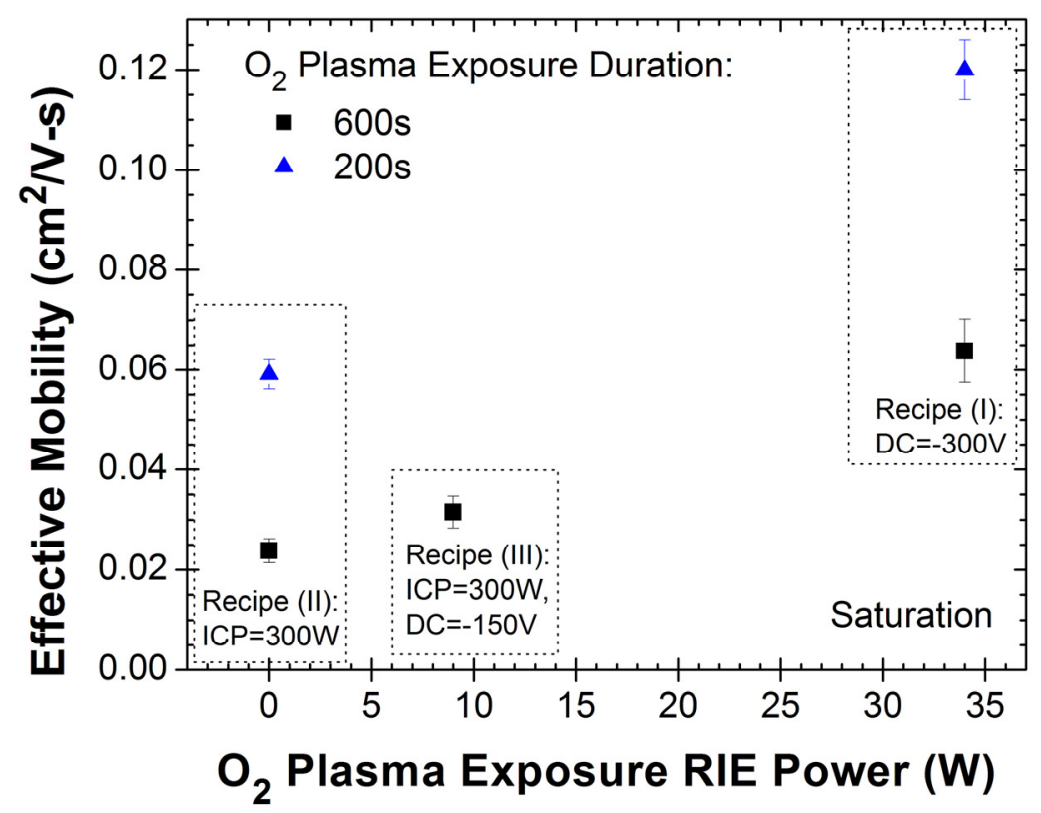

Figure 5.14. Effective mobility as a function of $\mathrm{O}_{2}$ plasma exposure power of PQT-12 OTFT with $\mathrm{SiN}_{\mathrm{x}}$ gate dielectric biased in saturation region. All samples were treated with $\mathrm{O}_{2}$ plasma and OTS SAM.

\subsubsection{Interface Characterization}

Contact angle, atomic force microscopy (AFM), and X-ray photoelectron spectroscopy (XPS) were used to study the interface properties of $\mathrm{O}_{2}$ plasma treated $\mathrm{SiN}_{\mathrm{x}}$. The interface characterization data are correlated with electrical data to account for the mechanisms underlying the observed OTFT characteristics.

\subsubsection{Contact Angle}

Figure 5.15 shows the changes in the water contact angle $(\theta)$ value of the $\operatorname{SiN}_{\mathrm{x}}$ surface as a function of $\mathrm{O}_{2}$ plasma exposure duration. Two sets of samples are considered: first set considers devices treated with $\mathrm{O}_{2}$ plasma only, and the second set considers devices treated with $\mathrm{O}_{2}$ plasma and OTS SAM. In the case of samples with a combinatorial treatment of $\mathrm{O}_{2}$ plasma/OTS SAM, the surfaces are hydrophobic $\left(\theta>107^{\circ}\right)$ and the contact angle is weakly dependent on $\mathrm{O}_{2}$ plasma exposure duration. As exposure duration increases from 0 to $900 \mathrm{sec}$, the change in contact angle is less than 
$5^{\circ}$. No conclusive/definite correlation can be drawn between contact angle data and field-effect mobility for these $\mathrm{O}_{2}$ plasma/OTS samples.

On the other hand, for samples treated with $\mathrm{O}_{2}$ plasma only, there is a distinctive dependence of contact angle on exposure duration. Figure 5.15 shows that the contact angle is smallest at exposure time around $80 \mathrm{sec}$, which corresponds to the turnaround point observed in the OTFT's mobility vs. $\mathrm{O}_{2}$ plasma exposure time curve in Figure 5.13(a). However, a logical correlation between the two sets of data is currently unclear. Intuitively, mobility should depend on the dynamics of dielectric surface after $\mathrm{O}_{2}$ plasma/OTS SAM treatment. Yet, the contact angle of the $\mathrm{O}_{2}$ plasma/OTS surface showed very little dependence on $\mathrm{O}_{2}$ plasma exposure duration, and hence cannot provide sufficient justification for the observed mobility behavior. Similarly, the contact angle data displayed no obvious dependence on $\mathrm{O}_{2}$ plasma exposure power for the $\mathrm{O}_{2}$ plasma/OTS surfaces. Therefore, these contact angle observations are unable to provide a clear explanation of the observed changes in mobility under different $\mathrm{O}_{2}$ plasma exposure conditions. Further analysis via surface roughness and surface chemistry measurements may offer insight on the underlying mechanism for the "turnaround" behavior observed in the OTFT electrical characteristics.

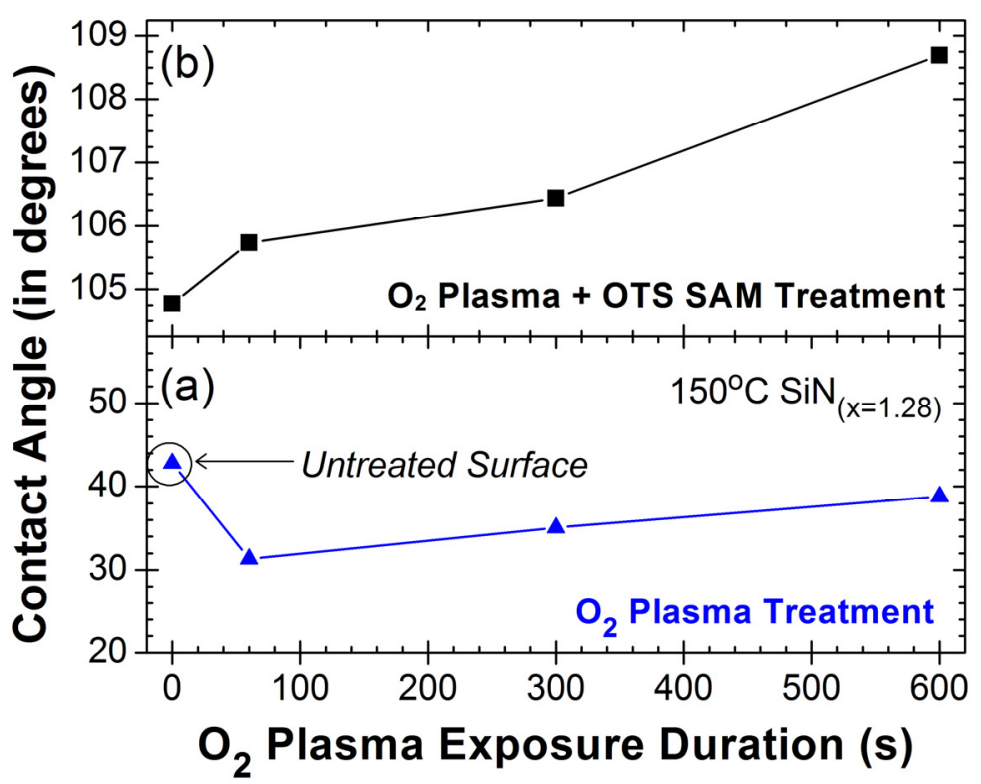

Figure 5.15. Water contact angle of $150^{\circ} \mathrm{C} \mathrm{SiN}_{\mathrm{x}}(\mathrm{x}=1.28)$ surface treated with (a) $\mathrm{O}_{2}$ plasma only and (b) $\mathrm{O}_{2}$ plasma/OTS SAM, as a function of $\mathrm{O}_{2}$ plasma exposure duration. 


\subsubsection{Surface Roughness}

Figure 5.16 plots the surface roughness of $\mathrm{SiN}_{\mathrm{x}}$ as a function of $\mathrm{O}_{2}$ plasma exposure duration, for two sets of samples: (a) $\mathrm{O}_{2}$ plasma treatment only, and (b) $\mathrm{O}_{2}$ plasma/OTS SAM combo treatment. Overall, bare $\mathrm{SiN}_{\mathrm{x}}$ and $\mathrm{O}_{2}$-plasma treated $\mathrm{SiN}_{\mathrm{x}}$ displayed very smooth surfaces, with measured surface roughness in the range of $0.192 \mathrm{~nm}$ to $0.224 \mathrm{~nm}$. There is a slight decrease in surface roughness with prolonged $\mathrm{O}_{2}$ plasma exposure. In contrast, we observed a larger variation in surface roughness for samples treated with $\mathrm{O}_{2}$ plasma/OTS; more importantly, a "turnaround" effect is apparent in the data. Here, a minimum surface roughness is observed near $\mathrm{O}_{2}$ plasma exposure duration of $80 \mathrm{sec}$, which corresponds precisely to the point of maximum mobility in Figure 5.14(a). Generally, as surface roughness decreases, OTFT mobility increases [20]. This behavior is evident by correlating the roughness data of $\mathrm{O}_{2}$ plasma/OTS sample in Figure 5.16 with the mobility data in Figure 5.14.

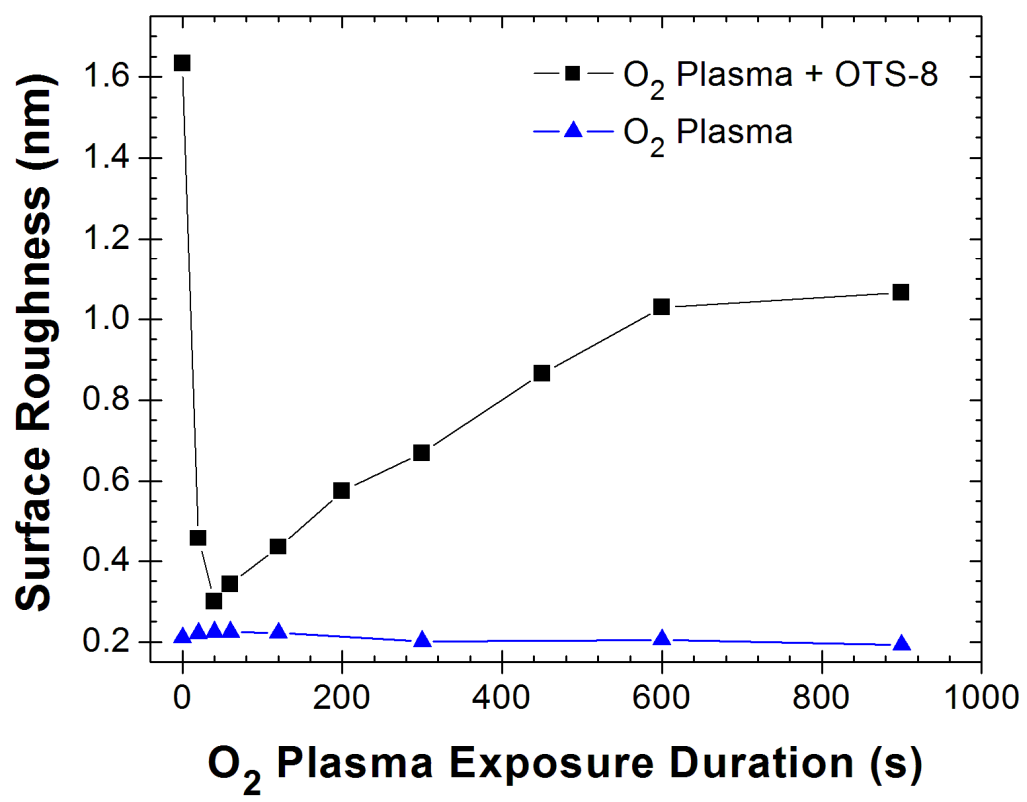

Figure 5.16. Surface roughness as a function of $\mathrm{O}_{2}$ plasma exposure duration for $\mathrm{SiN}_{\mathrm{x}}$ surface treated with $\mathrm{O}_{2}$ plasma and OTS SAM. Measurements performed using tapping-mode AFM.

These samples are treated with $\mathrm{O}_{2}$ plasma Recipe (I) in Table 5.6.

Figure 5.17 shows the surface roughness of $\mathrm{SiN}_{\mathrm{x}}$ samples treated with $\mathrm{O}_{2}$ plasma/OTS SAM as a function of $\mathrm{O}_{2}$ plasma exposure power. With increasing RIE power, surface roughness increases. Since higher energy ions are accelerated towards the substrate surface at higher RIE power, this 
potentially creates a rougher surface. The results also indicated that longer exposure duration (at $600 \mathrm{sec}$ ) generates a rougher surface (than at $200 \mathrm{sec}$ ), consistent with the observations in Figure 5.16. Interestingly, the roughness data follows an opposite trend to mobility. While higher RIE power enhances mobility (Figure 5.14), it is linked to higher surface roughness (Figure 5.17). This behavior diverges from the typical trend reported in the literature, stating that higher surface roughness degrades mobility. Such divergence can be attributed to other surface mechanisms that might impose a more dominant impact on device characteristics than surface roughness. The chemical composition of the surfaces is examined in the next subsection to seek insights in this matter.

In summary, AFM data reveal an increase in surface roughness with $\mathrm{O}_{2}$ plasma exposure time and power. During exposure, oxygen radicals may preferentially remove weak Si-Si bonds and form $\mathrm{Si}-\mathrm{O}$ bonds at the surface [25], thus influencing the roughness and chemical reactivity of the surface. Surface roughness plays an important role in the molecular ordering and quality of the overlying organic semiconductor layers; the increased surface roughness may limit the quality of OTFTs.

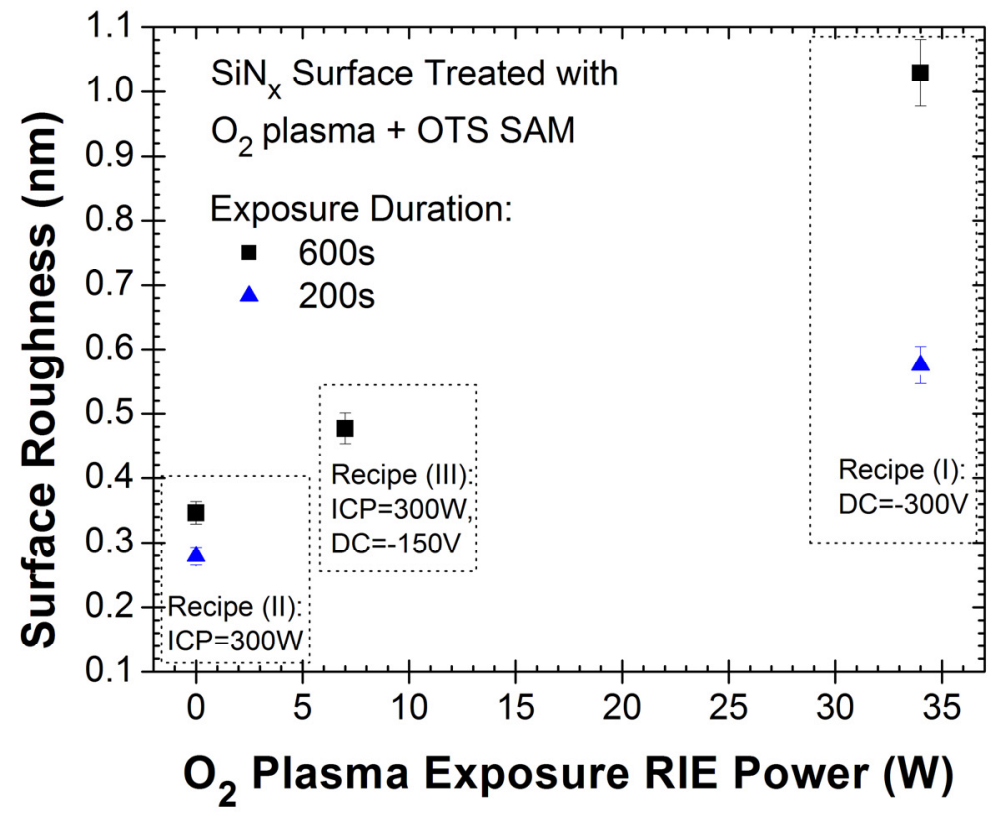

Figure 5.17. Surface roughness as a function of $\mathrm{O}_{2}$ plasma exposure power for $\mathrm{SiN}_{\mathrm{x}}$ surface treated with $\mathrm{O}_{2}$ plasma and OTS SAM. Measurements performed using tapping-mode AFM. 


\subsubsection{Chemical Composition}

Figure 5.18(a) compares the XPS survey spectrum of the untreated $\mathrm{SiN}_{\mathrm{x}}$ surface and the $\mathrm{O}_{2}$ plasma treated $(80 \mathrm{sec}, 600 \mathrm{sec}) \mathrm{SiN}_{\mathrm{x}}$ surfaces. Upon $\mathrm{O}_{2}$ plasma exposure, the $\mathrm{SiN}_{\mathrm{x}}$ dielectric surface experienced an increase in oxygen $(\mathrm{O} 1 \mathrm{~s})$ and carbon $(\mathrm{C} 1 \mathrm{~s})$ peaks, accompanied by a decrease in nitrogen (N 1s) and silicon (Si 2p) peaks. Figure 5.18(b) indicates that the $\mathrm{O} 1 \mathrm{~s}$ atomic percentage is highest for exposure duration of $80 \mathrm{sec}$; this is well-matched with to the point of maximum effective mobility in Figure 5.14(a).

The XPS data revealed that the untreated (as-deposited) $\mathrm{SiN}_{\mathrm{x}}$ surface is dominated by $\mathrm{Si}-\mathrm{N}$ bonds, while the $\mathrm{O}_{2}$ plasma treated $\mathrm{SiN}_{\mathrm{x}}$ surface becomes more dominated by $\mathrm{Si}-\mathrm{O}$ bonds. Thorough inspection of the $\mathrm{Si} 2 \mathrm{p}$ spectrum indicates the $\mathrm{Si}-\mathrm{O}$ peak becomes larger than the $\mathrm{Si}-\mathrm{N}$ peak after $\mathrm{O}_{2}$ plasma treatment. This means that the $\mathrm{SiN}_{\mathrm{x}}$ surface is oxidized. The $\mathrm{SiN}_{\mathrm{x}}$ surface acquires an oxide-like or oxynitride-like character upon $\mathrm{O}_{2}$ plasma exposure, which is highly favorable for deposition of OTS SAM and organic semiconductor layers. This oxide-like interface, along with the reduced surface roughness (Figure 5.16), of the $\mathrm{O}_{2}$ plasma $\left(80 \mathrm{sec}\right.$ ) treated $\mathrm{SiN}_{\mathrm{x}}$ favorably supports the observation that $\mathrm{O}_{2}$ plasma treatment creates a desirable platform for OTFTs, thus yielding improved effective mobility (Figure 5.14). Additional analysis of the XPS data is presented next. Collectively, the lower surface roughness and the higher Si-O content observed at $80 \mathrm{sec}$ of $\mathrm{O}_{2}$ plasma exposure of $\mathrm{SiN}_{\mathrm{x}}$ surface provide solid explanation for the maximum mobility at this turnaround point.

\section{$\underline{\text { Detection of Fluorine and Alumina on } \mathrm{O}_{2}} \underline{\text { Plasma Treated } \mathrm{SiN}_{\underline{x}}} \underline{\text { Surface }}$}

Peculiarly, XPS spectra of the $\mathrm{O}_{2}$ plasma treated $\mathrm{SiN}_{\mathrm{x}}$ surface in Figure 5.18 disclosed the presence of fluorine (F 1s) and aluminum ( $\mathrm{Al} 2 \mathrm{p}$ ). The intensities of the $\mathrm{F} 1 \mathrm{~s}$ and $\mathrm{Al} 2 \mathrm{p}$ peaks are relatively weak compared to other components, but increase with $\mathrm{O}_{2}$ plasma exposure duration. The unexpected presence of these elements is speculated to be a consequence of contamination from the plasma chamber/equipment. A Trion Phantom II RIE/ICP hybrid system was used to execute $\mathrm{O}_{2}$ plasma treatment. This equipment is routinely used to run a variety of semiconductor process recipes (e.g., etching, cleaning) which may involve $\mathrm{CF}_{4}$ and $\mathrm{SF}_{6}$ as process gases. Residual fluorine compounds in the chamber may react with the sample surface during $\mathrm{O}_{2}$ plasma exposure; this accounts for the detection of fluorine on the $\mathrm{O}_{2}$ plasma treated $\mathrm{SiN}_{\mathrm{x}}$ surface. 
(a)

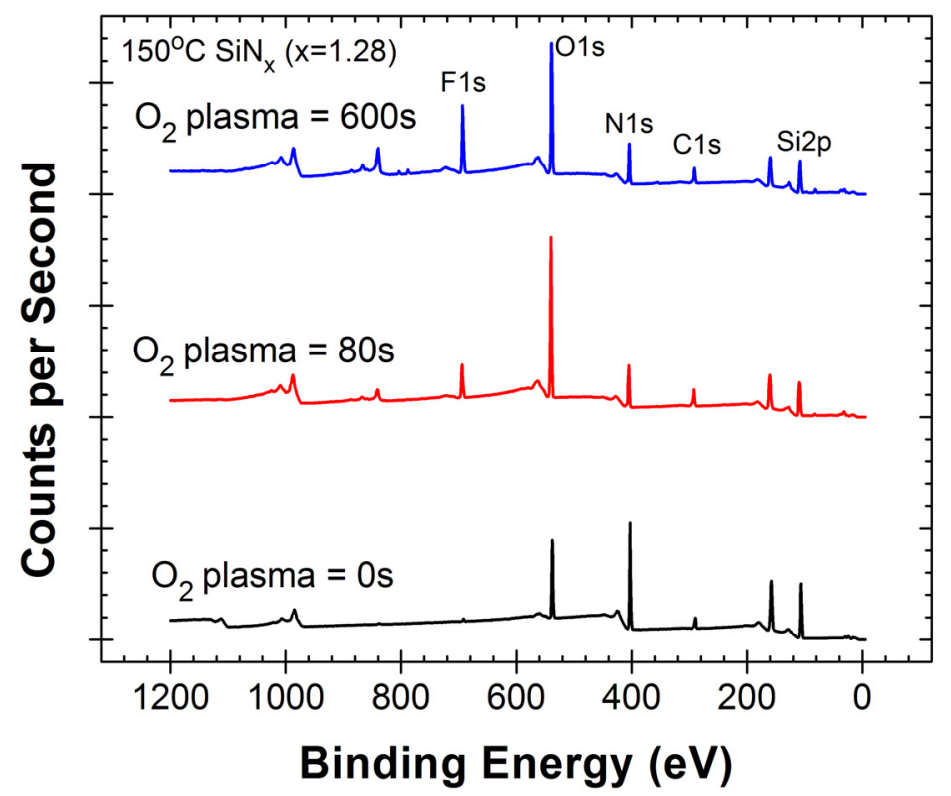

(b)

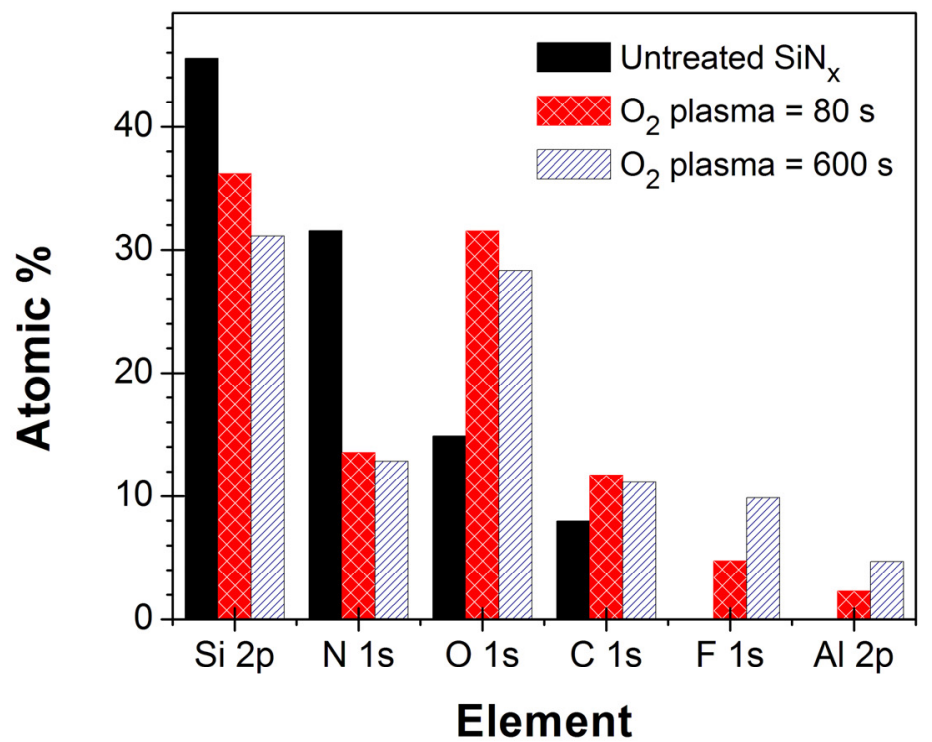

Figure 5.18. (a) XPS spectrum in survey scan mode of an untreated $\mathrm{SiN}_{\mathrm{x}}$ surface and $\mathrm{O}_{2}$ plasma treated $\mathrm{SiN}_{\mathrm{x}}$ surfaces after $80 \mathrm{sec}$ and $600 \mathrm{sec}$ exposure. (b) Chemical composition (represented by atomic percentage) of the $\mathrm{SiN}_{x}$ top surface extracted from (a). $\mathrm{O}_{2}$ plasma exposure was done using Recipe (I) in Table 5.6.

Regarding sources of aluminum contamination, the RIE chamber is equipped with anodized Al (i.e., alumina) as electrodes, and the sample/wafer is placed on the bottom electrode. Due to nature of the RIE/plasma processes, physical sputtering is inevitable; this causes the anodization to 
deteriorate overtime, exposing the underlying aluminum electrode to plasma. As a result, unintentional sputtering of aluminum may occur, which is manifested as aluminum contamination on the samples surface. XPS measurements were repeated on three batches of samples over the course of two years; traces of $\mathrm{F}$ and $\mathrm{Al}$ were still detected despite diligent attempts to decontaminate the chamber via physical and chemical cleaning methods.

An interesting speculation is that the presence of $\mathrm{F}$ and $\mathrm{Al}$ actually functionalizes the interface in a manner that positively contributes to OTFT performance. Analysis of the F 1s region suggests the possibility of Si-F and C-F bonding or $\mathrm{AlF}_{3}$ compounds, while the Al $2 p$ spectrum displayed characteristics of $\mathrm{Al}_{2} \mathrm{O}_{3}$. SAMs of fluorinated silanes have recently been investigated for surface modification of the dielectric surfaces in OTFTs [23][24]. Examples of fluorinated SAMs include (tridecafluoro-1,1,2,2-tetrahydrooctyl)trichlorosilane (FTS) and (tridecafluoro-1,1,2,2tetrahydrooctyl)triethoxysilane. Kobayashi et al. reported that perfluoroalkylsilane SAMs (F-SAM) with fluorine groups generated the highest mobility for p-type pentacene OTFTs compared to devices treated with alkylsilane SAMs $\left(\mathrm{CH}_{3}\right.$-SAM) or aminoalkylsilane SAM $\left(\mathrm{NH}_{2}\right.$-SAM) [23]. Calculation of the molecular dipole indicated that when molecules are uniformly aligned, F-SAMs generate a local electric field that enhances accumulation of holes in the channel, when compared to the $\mathrm{CH}_{3}$-SAMs. This agrees well with the enhanced p-type carrier conduction in pentacene and the positive $\mathrm{V}_{\mathrm{T}}$ shift observed in devices treated with F-SAMs [23].

Podzorov and co-workers reported larger improvement in rubrene transistor properties when using SAM with fluorine end-groups [24]. They observed that organosilane SAMs with larger fluorine content induced a higher surface conductivity owing to the larger electron-withdrawing ability of fluorinated compounds (when compared to non-fluorinated compounds). Such SAMinduced conductivity is due to a ground-state charge transfer at the interface, and the degree of charge transfer (or conductivity) of SAM-functionalized samples depends on the electronwithdrawing ability of organosilane molecules [24].

Moreover, plasma fluorinated surfaces have been shown to lower surface energy, due to formation of C-F bonds [26]. Fluorinated surface also displayed improved stability, where the presence of the C-F functionalities hinders the adsorption of $\mathrm{O}_{2}, \mathrm{~N}_{2}, \mathrm{CH}_{4}$ and $\mathrm{CO}$ gases. These characteristics are favorable in the case of OTFTs, where a reduction of surface energy and inhibition of $\mathrm{O}_{2}$ adsorption of the fluorinated surface can improve quality of the organic semiconductor layer and enhance device stability, respectively. 
It is hypothesized that our fluorinated interface provoked similar improvement in PQT-12 OTFTs. It is speculated that the substantial improvement seen in our $\mathrm{O}_{2}$ plasma treated samples is related to the unique presence of $\mathrm{F}$ and $\mathrm{Al}$ at the interface. This speculation is substantiated by the fact that the degree of improvement achieved by our $\mathrm{O}_{2}$ plasma treatment on $\mathrm{SiN}_{\mathrm{x}}$, which outperformed other $\mathrm{O}_{2}$ plasma treated OTFT devices reported in the literature (specifically, for devices without SAM treatment) [8]. Our $\mathrm{O}_{2}$ plasma treatment resulted in 18x improvement in mobility relative to untreated device (Figure 5.7). In comparison, Lee et al. reported a 9.3x improvement in mobility for their $\mathrm{O}_{2}$ plasma treated pentacene OTFTs on $\mathrm{SiO}_{2}$ gate dielectric [8]. Further experiments are needed to systematically study the impact of F and Al on interface properties and on OTFT characteristics, in order to develop a better understanding on the role of fluorine/alumina-functionalized dielectric interface.

\section{$\underline{\text { Effect of }}_{2} \underline{\text { Plasma Exposure Power }}$}

As reported in Section 5.4.1.2, a higher RIE power of $\mathrm{O}_{2}$ plasma exposure gives higher effective mobility of the OTFT device. Contact angle and surface roughness measurements were unable to account for this exposure power dependence. Changes in chemical composition of the $\mathrm{O}_{2}$ plasma treated $\mathrm{SiN}_{\mathrm{x}}$ surface as a function of RIE power are plotted in Figure 5.19. The results revealed that higher RIE power generated more oxygen species on the surface. It appears that higher exposure power rendered a more oxidized surface, which is believed to be favorable for subsequent binding of organosilane SAM or organic semiconductor layers. As the bias power increases, there is an increased in energy of reactive ions impinging on the wafer. This increase can partly be certified from the experiment, where the oxygen content increased with RIE bias power. However, more indepth studies are needed to develop a better understanding of the underlying science/mechanism. 


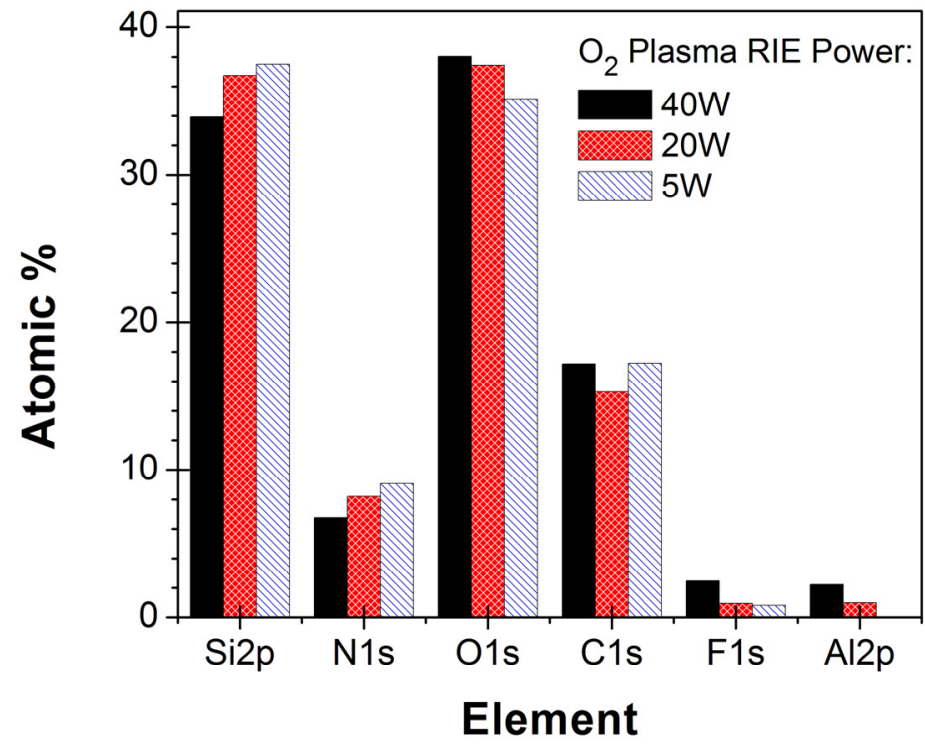

Figure 5.19. Changes in chemical composition (represented by atomic percentage) of $\mathrm{O}_{2}$ plasma treated $\mathrm{SiN}_{\mathrm{x}}$ surface at various exposure powers.

\subsubsection{XPS Depth Profile Analysis}

Figure 5.20 compares the corresponding Si 2p, N 1s and O 1s XPS spectra as a function of the total sputtering time for three $\mathrm{SiN}_{\mathrm{x}}$ samples treated with different $\mathrm{O}_{2}$ plasma durations. For this XPS depth analysis, the estimated sputtering rate using Ar ions is $1 \mathrm{~nm} / \mathrm{min}$. For untreated $\mathrm{SiN}_{\mathrm{x}}$, XPS depth profile measurements indicated that $\mathrm{Si} 2 \mathrm{p}$ and $\mathrm{N} 1 \mathrm{~s}$ peak intensities are quite constant with changes in sputtering time (or depth), suggesting homogeneous film properties from the surface region towards the bulk. $\mathrm{O} 1 \mathrm{~s}$ is detected at the top surface of untreated $\mathrm{SiN}_{\mathrm{x}}$, which might originate from the ambient/environment. This $\mathrm{O} 1 \mathrm{~s}$ peak quickly disappears after $60 \mathrm{sec}$ of sputtering, suggesting this is only a surface phenomenon.

The atomic distribution in Figure 5.20(d) (bottom panel) showed that the untreated $\mathrm{SiN}_{\mathrm{x}}$ sample is consistently characterized by a dominance of $\mathrm{Si}$ and $\mathrm{N}$ entities (i.e., Si-N bonds) from the surface to the bulk. This is in distinct contrast to the $\mathrm{O}_{2}$ plasma treated $\mathrm{SiN}_{\mathrm{x}}$ samples, displayed in the top and middle panels of Figure 5.20(d). For the $80 \mathrm{sec}$ and $600 \mathrm{sec} \mathrm{O}_{2}$ plasma treated $\mathrm{SiN}_{\mathrm{x}}$, their top surface (i.e., at sputtering time of $0 \mathrm{sec}$ ) is dominated by $\mathrm{O}$ and $\mathrm{Si}$. This reaffirms our previous analysis that the surface becomes oxidized after $\mathrm{O}_{2}$ plasma exposure. In addition, their Si $2 \mathrm{p}$ spectrum shows features of $\mathrm{Si}-\mathrm{O}$ bonds. Interestingly, this oxide-like characteristic penetrates 
deeper into the $\mathrm{SiN}_{\mathrm{x}}$ film as $\mathrm{O}_{2}$ plasma exposure duration increases. The $\mathrm{O}$ 1s spectra in Figure 5.20 (c) revealed deeper penetration of oxygen into the bulk (as indicated by the intensity of the $\mathrm{O}$ 1s peak with increasing sputtering time) for the $600 \mathrm{sec}$ sample when compared to the $80 \mathrm{sec}$ sample. Therefore, the Si-O or oxide like sub-region extends deeper into the film with increasing $\mathrm{O}_{2}$ plasma exposure. Considering the Si $2 \mathrm{p}$ and N 1s spectra in Figure 5.20(a)-(b), the 600 sec sample showed a more gradual transition of these peak intensities with sputtering time compared to the $80 \mathrm{sec}$ sample; meanwhile, the $80 \mathrm{sec}$ sample displayed a more brisk transition to the steadystate peak intensity with sputtering time. This behavior corroborates the conclusion that a thicker oxide-like sub-region is present in $\mathrm{SiN}_{\mathrm{x}}$ samples with longer $\mathrm{O}_{2}$ plasma exposure.

(a)

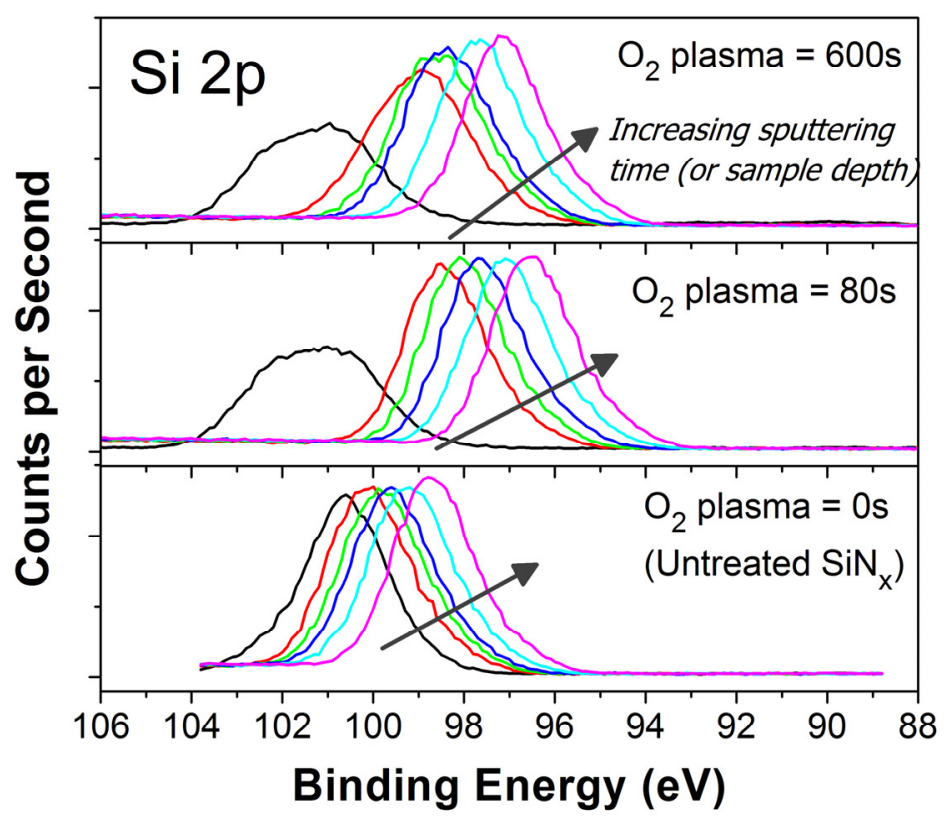


(b)

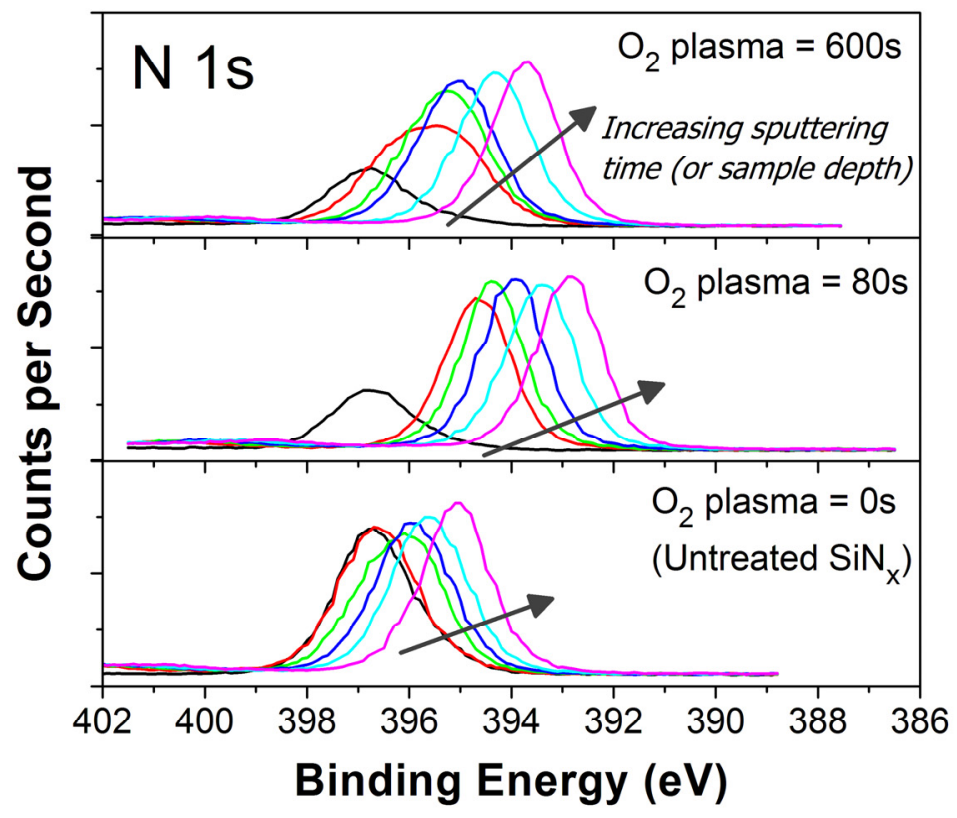

(c)

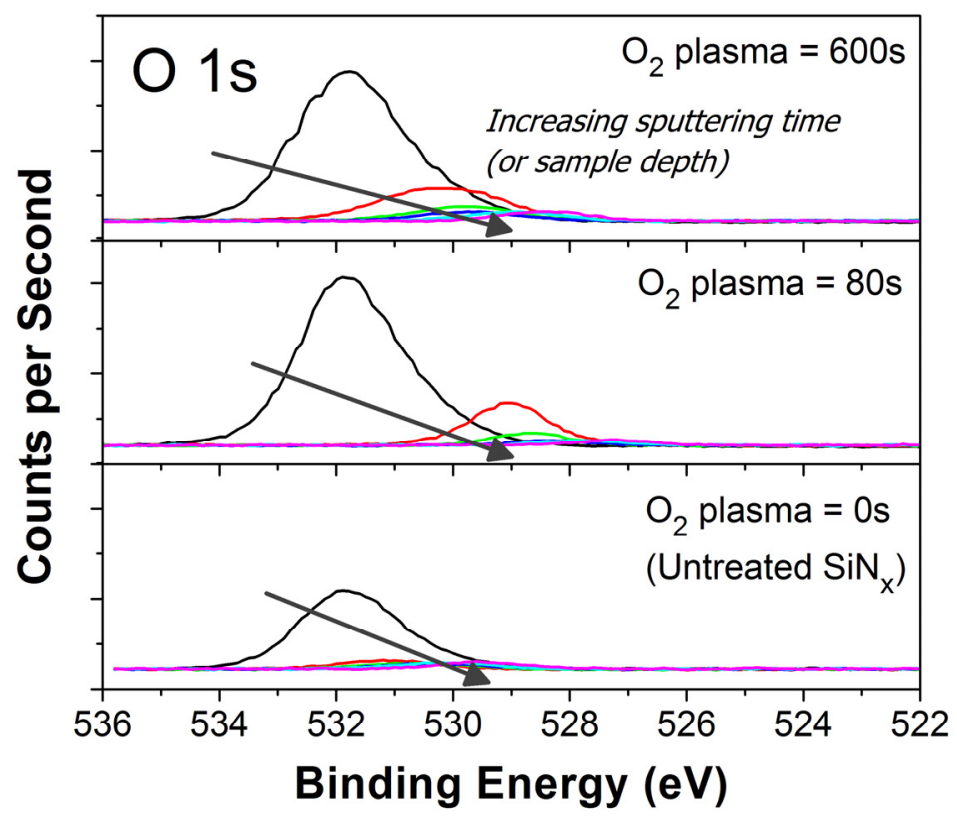


(d)

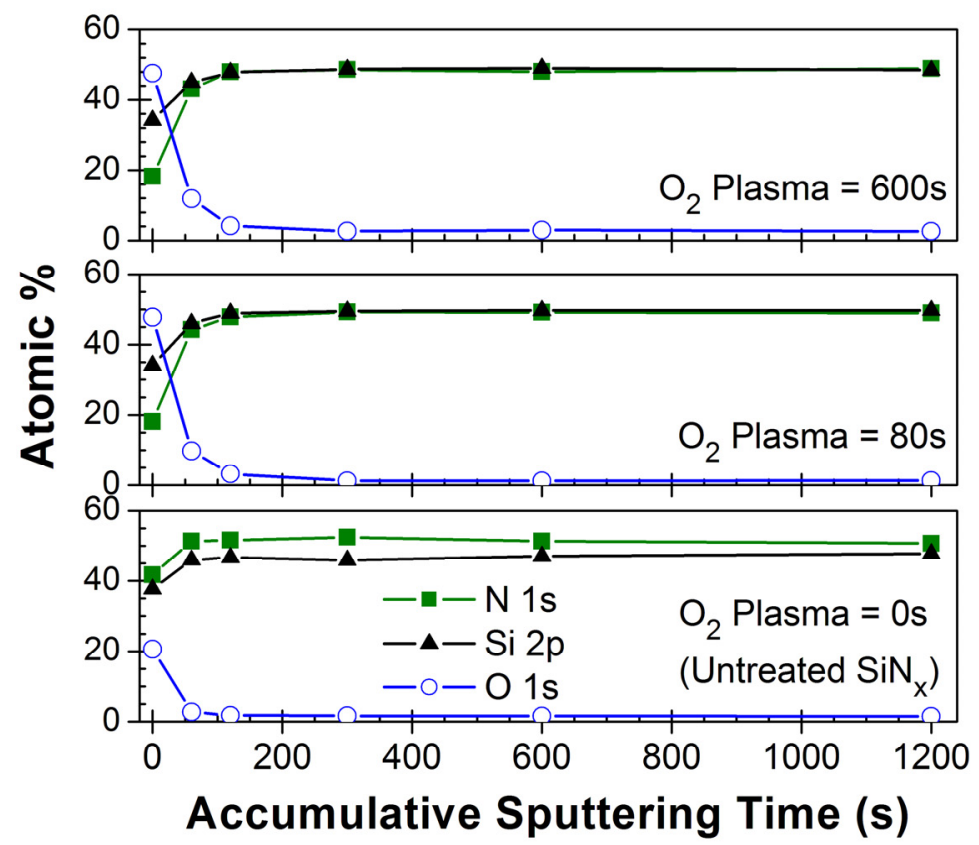

Figure 5.20. (a) Si $2 p$, (b) N 1s, and (c) 0 1s spectra of $150^{\circ} \mathrm{C} \mathrm{SiN} \operatorname{Si}_{x}(x=1.28)$ upon successive ion sputtering in XPS depth-profiling measurement. (d) Atomic percentage as a function of sputtering time, calculated based on XPS spectra in (a)-(c). Three $\mathrm{SiN}_{\mathrm{x}}$ samples were considered: as-deposited (untreated) $\mathrm{SiN}_{\mathrm{x}}$, and $80 \mathrm{sec}$ and $600 \mathrm{sec}_{2}$ plasma treated $\mathrm{SiN}_{\mathrm{x}}$.

\subsubsection{Analysis and Discussion}

Overall, a positive influence on OTFT performance was observed with $\mathrm{O}_{2}$ plasma treatment. However, to maximize the improvements in OTFTs, the plasma exposure time must be optimized and well-controlled. Excessive exposure duration can cause a "turn-around effect" in the fieldeffect mobility and on/off current ratio. The turnaround effect in the variation of field-effect mobility with plasma exposure time was also observed by Lee and coworkers for pentacene OTFT on silicon dioxide gate dielectric [8]. Additionally, they observed a decrease in the standard deviation of device parameters with oxygen plasma exposure time, resulting in enhanced uniformity of device parameters across the wafer.

Lee and coworkers proposed that oxide charge is responsible for the observed variation in device parameters. Upon $\mathrm{O}_{2}$ plasma treatment, the oxide charges in the $\mathrm{SiO}_{2}$ gate dielectric initially decrease due to UV-enhanced reduction and then increase due to the high energy particle bombardment [8]. The as-grown silicon oxide usually contains positive charges within the order of $10^{10}-10^{12} \mathrm{~cm}^{-2}$ depending on the growth process. The bridging oxygen vacancy defect 
$\mathrm{O}_{3} \equiv \mathrm{Si} \bullet \bullet \mathrm{Si} \equiv \mathrm{O}_{3}$ named $\mathrm{E}^{\prime}$ center is known to be responsible for the positive charges. It has been reported that the oxide charge is varied depending on the plasma condition and exposure time [27]. Under the soft plasma conditions used in [27], oxide charges decreased for 3-4 min after plasma treatment began, where the time depended on the plasma condition, and then increased again, resulting in the "turn-around" effect. A plausible origin for the decrease of oxide charge is the UV-enhanced reduction of positive charge (annihilation of $\mathrm{E}^{\prime}$ centers or generation of negative compensating charges by vacuum UV photons of the plasma). It appears that change in oxide charges induced by the UV plasma component was the dominating factor up to the turn-around point. As plasma treatment proceeds, the relative influence of the UV component decreased and the effect of high energy particles bombardment became dominant which resulted in an increase of oxide charge. The relative contribution of these two components depended on the specific plasma conditions [2]. Therefore, the plasma treatment up to the turn-around time was found to be effective for performance enhancement in pentacene OTFTs.

For the investigation reported in this thesis, XPS results indicated an oxide-like or oxynitridelike $\mathrm{SiN}_{\mathrm{x}}$ surface upon $\mathrm{O}_{2}$ plasma exposure. Moreover, depth analysis by XPS revealed the presence of Si-O bonds deeper in the $\mathrm{SiN}_{\mathrm{x}}$ as $\mathrm{O}_{2}$ plasma exposure duration increases. Therefore, oxygen species penetrate into the $\mathrm{SiN}_{\mathrm{x}}$ films with longer exposure duration, creating an oxide-like subregion in the $\mathrm{SiN}_{\mathrm{x}}$ bulk, near the interface. Owing to the presence of Si-O characteristics in our $\mathrm{SiN}_{\mathrm{x}}$ dielectric, plasma/UV-induced changes in oxide charge species, proposed by Lee et al. in [27], can be a relevant mechanism contributing to the observed changes in OTFT behavior as a function of $\mathrm{O}_{2}$ plasma exposure duration.

It has been reported that plasma treatment can activate and clean surfaces, thus enabling stronger bonds with subsequent layers and reducing impurity-induced degradation in the interface's electrical properties [25]. This is reflected in our contact angle measurements of the $\mathrm{O}_{2}$ plasma treated $\mathrm{SiN}_{\mathrm{x}}$ surfaces, which showed that even with short exposure time, the surfaces were activated to generate a hydrophilic surface. This implies the effectiveness of $\mathrm{O}_{2}$ plasma treatment for surface activation is related to substituting carbon surface contaminations with hydroxyl groups and chemically active dangling bonds.

The contact angle of $\mathrm{O}_{2}$ plasma treated $\mathrm{SiN}_{\mathrm{x}}$ displayed a weak dependence on the exposure duration, where the contact angle was smallest for $80 \mathrm{sec}$ of exposure (Figure 5.15). The observed contact angle trend cannot provide sufficient proof to explain the observed TFT data as a function of exposure duration. However, contact angle is sensitive to the physical state of the surface (e.g., 
roughness). Thus, surface roughness measurements might be able to shed light on the observed contact angle and TFT mobility trend. Figure 5.16 shows that $80 \mathrm{sec}$ of exposure gives minimum surface roughness for $\mathrm{O}_{2}$ plasma/OTS treated. This matches precisely with the point of maximum mobility in Figure 5.14. With longer plasma exposure duration, the surface roughness of $\mathrm{O}_{2}$ plasma/OTS treated samples was observed to increase. The rougher surface accounts for the reduced mobility at longer exposure duration. Because the surface roughness plays an important role in the quality of the overlying polymer semiconductor layers and efficiency of charge transport, increased surface roughness typically degrades the quality of OTFTs.

Oxygen radicals during plasma treatment may preferentially remove weak Si-Si bonds and break $\mathrm{Si}-\mathrm{O}$ bonds at the surface, leading to a rougher surface. Choi et al. studied the effects of $\mathrm{O}_{2}$ plasma treatment on Si and glass substrates, and reported that the activated oxygen reacts with surface impurities, and reaction products were desorbed in the form of volatile materials as follows [25]:

$$
\mathrm{C}_{x} \mathrm{H}_{y} \mathrm{O}_{z}+\left(\mathrm{O}_{2}^{*}, \mathrm{O}\right) \rightarrow \mathrm{CO}_{2}+\mathrm{H}_{2} \mathrm{O} \text {. }
$$

$\mathrm{C}_{\mathrm{x}} \mathrm{H}_{\mathrm{y}} \mathrm{O}_{\mathrm{z}}$ represents a general hydrocarbon molecule and $\left(\mathrm{O}_{2}{ }^{*}, \mathrm{O}\right)$ is chemically activated oxygen. These oxygen radicals preferentially modify $\mathrm{Si}-\mathrm{O}$ bonds at the top of the $\mathrm{SiO}_{2}$ surface, and cause the generation of chemically active dangling bonds and hydroxyl groups at the surface [25]. These chemical changes in bonding structure agree with the hydrophilic surface properties observed in oxygen-plasma-activated $\mathrm{SiN}_{\mathrm{x}}$ surface.

Table 5.8 summarizes the effect of $\mathrm{O}_{2}$ plasma exposure conditions on electrical properties of the OTFT and the surface properties of the $\mathrm{SiN}_{\mathrm{x}}$ dielectric. Altogether, our results suggested that the chemical states (e.g., surface energy, composition), physical states (e.g., surface roughness), and electrical states of the dielectric surface collectively influence the resulting device performance. In some instances, they operate coherently/consistently to enhance device performance. However, in other occasions, these mechanisms may compete to impose opposing impact on device properties.

In conclusion, $\mathrm{O}_{2}$ plasma treatment is an effective way to enhance the performance of OTFTs on $\mathrm{SiN}_{\mathrm{x}}$ gate dielectric if the plasma exposure time and exposure conditions are appropriately optimized and well-controlled. The effect of $\mathrm{O}_{2}$ plasma treatment on $\mathrm{SiN}_{\mathrm{x}}$ dielectric is to introduce an oxide-like interface, rendering a dielectric surface more favorable for subsequent binding to alkylsilane SAMs or organic semiconductor films. 
Table 5.8. Summary of the impact of $\mathrm{O}_{2}$ plasma exposure duration and RIE power on surface properties of $\mathrm{SiN}_{x}$ and on OTFT device mobility. $t^{*}$ denotes the "turnaround point", defined as the exposure duration that generated maximum effective mobility.

\begin{tabular}{|c|c|c|c|c|c|}
\hline \multirow{2}{*}{$\begin{array}{l}\mathrm{O}_{2} \text { Plasma Exposure } \\
\text { Parameter }\end{array}$} & \multicolumn{2}{|c|}{ Contact Angle } & \multicolumn{2}{|c|}{ Surface Roughness } & \multirow{2}{*}{$\begin{array}{l}\text { Effective } \\
\text { Mobility }\end{array}$} \\
\hline & $\mathrm{O}_{2}$ plasma & $\begin{array}{l}\mathrm{O}_{2} \text { plasma } \\
+ \text { OTS }\end{array}$ & $\mathrm{O}_{2}$ plasma & $\begin{array}{c}\mathrm{O}_{2} \text { plasma } \\
+ \text { OTS }\end{array}$ & \\
\hline \multicolumn{6}{|c|}{ Duration: with increasing exposure time.. } \\
\hline For $t<t^{*}$ & Small $\downarrow$ & Small $\uparrow$ & Small $\uparrow$ & $\downarrow \downarrow$ & 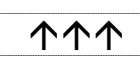 \\
\hline For $t>t^{*}$ & Small $\uparrow$ & Small $\uparrow$ & Small $\downarrow$ & 个个 & $\downarrow \downarrow$ \\
\hline \multicolumn{6}{|l|}{ RIE Power: } \\
\hline As power increases & Unchanged & Unchanged & & $\uparrow$ & $\uparrow$ \\
\hline
\end{tabular}

\subsection{Summary and Contributions}

The influence of dielectric surface treatment with OTS SAM and $\mathrm{O}_{2}$ plasma exposure was analyzed by electrical measurements of PQT-12 OTFTs with $\mathrm{SiN}_{\mathrm{x}}$ gate dielectric. Interface properties were characterized to establish correlation with TFT characteristics. A key contribution of this investigation is that surface treatment using a combination of $\mathrm{O}_{2}$ plasma and OTS SAM increased the effective field-effect mobility by $\sim 30 \mathrm{x}$, with saturation mobility up to $0.22 \mathrm{~cm}^{2} / \mathrm{V}$-s. Our results showed the $\mathrm{SiN}_{\mathrm{x}}$ surface must be pre-treated with $\mathrm{O}_{2}$ plasma prior to OTS SAM formation in order to generate a smooth and effective OTS layer. The adhesion of OTS on bare $\mathrm{SiN}_{\mathrm{x}}$ is more sporadic as indicated by a higher surface roughness. In contrast, there is large reduction in surface roughness when OTS is formed on $\mathrm{O}_{2}$ plasma treated $\mathrm{SiN}_{\mathrm{x}} \cdot \mathrm{O}_{2}$ plasma exposure provides a hydroxylated surface to facilitate attachment of OTS molecules on $\mathrm{SiN}_{\mathrm{x}}$.

Another impactful observation is the turnaround effect in $\mathrm{O}_{2}$ plasma treatment. It is recognized that the $\mathrm{O}_{2}$ plasma exposure time must be carefully controlled to ensure optimal quantity of hydroxyl groups are created on the surface for maximizing OTFT's mobility. The surface properties (studied by means of contact angle and surface roughness measurements) of $\mathrm{SiN}_{\mathrm{x}}$ varied with the $\mathrm{O}_{2}$ plasma exposure time. There is a turnaround effect with $\mathrm{O}_{2}$ plasma treatment; for this particular investigation, the turnaround point occurs at exposure duration of $80 \mathrm{sec}$. With $80 \mathrm{sec}$ of $\mathrm{O}_{2}$ plasma exposure and OTS SAM treatment, the OTFT demonstrated the highest mobility, and the $\mathrm{SiN}_{\mathrm{x}}$ gate dielectric exhibited the smallest surface roughness and largest contact angle.

This investigation also demonstrated that high mobility $\left(\sim 0.1 \mathrm{~cm}^{2} / \mathrm{V}-\mathrm{s}\right)$ PQT-12 OTFT on $\mathrm{SiN}_{\mathrm{x}}$ can be achieved even in the absence of OTS SAM. The $\mathrm{SiN}_{\mathrm{x}}$ surface was only subjected to $\mathrm{O}_{2}$ plasma treatment. This is a striking observation (or breakthrough) as SAM is generally considered 
a critical element for OTFTs. The ability to achieve high mobility in the absence of SAM can potentially simplify the OTFT fabrication process. Table 5.9 provides a summary of the key experimental observations from this chapter.

An extension of this work to generate further device improvements or advance understanding of device behavior may include:

- Expanding the investigation to additional $\mathrm{O}_{2}$ plasma exposure parameters, such as power, pressure, gas flow;

- Exploring fluorination as an interface treatment technique. Possible methods to functionalize the $\mathrm{SiN}_{\mathrm{x}}$ gate dielectric surface with fluorine include using fluorinated SAMs, fluorine plasma treatment, or deposition of a fluorinated-SiN $\mathrm{N}_{\mathrm{x}}$ gate dielectric.

- Long term stress measurements to study effect of different surface treatments on stability of OTFTs.

Table 5.9. Summary of key observations from dielectric interface engineering experiments.

\begin{tabular}{|c|c|c|}
\hline & Description & Key Observations \\
\hline Part A & $\begin{array}{l}\text { Comparison of TFTs } \\
\text { with and without } \\
\text { dielectric surface } \\
\text { treatment }\end{array}$ & $\begin{array}{ll} & \mu_{\mathrm{FE}} \text { is enhanced in presence of } \mathrm{O}_{2} \text { plasma and OTS } \\
\text { (compared to untreated devices) } \\
\text { Combination of } \mathrm{O}_{2} \text { plasma \& OTS yields the highest } \mu_{\mathrm{FE}} \\
(\sim 30 \mathrm{x} \text { improvement over untreated device) } \\
\text { Single } \mathrm{O}_{2} \text { plasma treatment led to } \sim 18 \mathrm{x} \text { increase in } \mu_{\mathrm{FE}} \\
\text { compared to untreated device }\end{array}$ \\
\hline Part B & $\begin{array}{l}\text { Effect of } \mathrm{O}_{2} \text { plasma } \\
\text { exposure conditions }\end{array}$ & $\begin{array}{l}\mathrm{O}_{2} \text { plasma exposure of } 80 \mathrm{sec} \text { gives the highest } \mu_{\mathrm{FE}} \\
\text { For longer exposure }(\mathrm{t}>80 \mathrm{~s}), \mu_{\mathrm{FE}} \text { dropped \& eventually } \\
\text { plateau. } \\
\text { Higher plasma exposure RIE power gives higher } \mu_{\mathrm{FE} .} \mathrm{O}_{2} \\
\text { plasma generated by RIE mode produced TFTs with higher } \\
\mu \mathrm{FE} \text { than ICP mode. }\end{array}$ \\
\hline
\end{tabular}




\section{Bibliography}

[1] Y.D. Park, J.A. Lim, H.S. Lee, and K. Cho, "Interface engineering in organic transistors," Materials Today 10(3), 46-54 (2007).

[2] S. C. Lim, S. H. Kim, J.E. Lee, M. K. Kim, D. J. Kim, and T. Zyung, "Surface-treatment effects on organic thin-film transistors," Synthetic Metals 148 (1), 75 (2005)

[3] N. Hiroshiba, R. Kumashiro, N. Komatsu, Y. Suto, H. Ishii, S. Takaishi, M. Yamashita, K. Tsukagoshi, and K. Tanigaki, "Surface Modifications using Thiol Self-Assembled Monolayers on Au Electrodes in Organic Field Effect Transistors," Mater. Res. Soc. Symp. Proc. 965, 0965-S08-03 (2007).

[4] S. Grecu, M. Roggenbuck, A. Opitz and W. Brütting, "Differences of interface and bulk transport properties in polymer field-effect devices," Organic Electronics 7(5), 276 (2006).

[5] C. Tanase, E.J. Meijer, P.W.M. Blom and D.M. de Leeuw, "Local charge carrier mobility in disordered organic field-effect transistors," Organic Electronics 4(1), 33 (2003).

[6] T.N. Jackson, Y.Y. Lin, D.J. Gundlach, and H. Klauk, "Organic Thin-Film Transistors for Organic Light-Emitting Flat-Panel Display Backplanes,” IEEE J. Sel. Top. Quantum Electronics 4(1), 100 (1998).

[7] L.L. Kosbar, C.D. Dimitrakopoulos, and D.J. Mascaro, "The Effect of Surface Preparation on the Structure and Electrical Transport in an Organic Semiconductor," Mater. Res. Soc. Symp. Proc. 665, C10.6.1 (2001).

[8] M. Lee and C. Song, "Oxygen Plasma Effects on Performance of Pentacene Thin Film Transistor,” Jpn. J. Appl. Phys. 42, 4218 (2003).

[9] S.R. Wasserman, Y-T Tao, and G.M. Whitesides, "Structure and Reactivity of Alkylsiloxane Monolayers Formed by Reaction of Alkyltrichlorosilanes on Silicon Substrates," Langmuir 5, 1074 (1989).

[10] M.M. Sung, G.J. Kluth, and R. Maboudian, "Formation of alkylsiloxane self-assembled monolayers on Si3N4," J. Vac. Sci. Technol. A 17(2), 540 (1999).

[11] C. Song, B. Koo, S. Lee and D. Kim, "Characteristics of Pentacene Organic Thin Film Transistors with Gate Insulator Processed by Organic Molecules," Jpn. J. Appl. Phys. 41, 2730 (2002).

[12] D.H. Kim, Y.D. Park, Y. Jang, H. Yang, Y.H. Kim, J.I. Han, D.G. Moon, S. Park, T. Chang, C. Chang, M. Joo, C.Y. Ryu, and K. Cho, "Enhancement of field-effect mobility due to surface-mediated molecular ordering in regioregular polythiophene thin film transistors," Adv. Funct. Mater. 15(1), 77 (2005).

[13] J. Veres, S. Ogier, G. Lloyd, and D. de Leeuw, "Gate Insulators in Organic Field-Effect Transistors," Chem. Mater. 16, 4543 (2004).

[14] K. Ihm, B. Kim, T.H. Kang, K.J. Kim, M.H. Joo, T.H. Kim, and S.S. Yoon, "Molecular orientation dependence of hole-injection barrier in pentacene thin film on the Au surface in organic thin film transistor," Appl. Phys. Lett. 89, 033504 (2006). 
[15] D. Lu, Y. Wu, J. Guo, G. Lu, Y. Wang and J. Shen, "Surface treatment of indium tin oxide by oxygen-plasma for organic light-emitting diodes," Mater. Sci. Eng. B 97, 141-144 (2003).

[16] Handbook of plasma processing technology: fundamentals, etching, deposition, and surface interactions, Noyes Publications, USA (1990).

[17] Trion Phantom II RIE/ICP system manual, Trion Technology, Inc. (2006).

[18] "Method for fabricating devices with DC bias-controlled reactive ion etching," United State Patent 4496448 (1985). www.freepatentsonline.com/4496448.html

[19] "ICP Source - A Brief Summary", Trion Technical Papers and Resources, 2004.

[20] M. L. Chabinyc, R. Lujan, F. Endicott, M. F. Toney, I. McCulloch, and M. Heeney, "Effects of the surface roughness of plastic-compatible inorganic dielectrics on polymeric thin film transistors," Appl. Phys. Lett. 90, 233508 (2007).

[21] W.K. Kim and J.L. Lee, "In Situ Analysis of Hole Injection Barrier of $\mathrm{O}_{2}$ Plasma-Treated Au with Pentacene Using Photoemission Spectroscopy," Electrochem. Solid-State Lett. 10(3), H104-H106 (2007).

[22] A. Wang, I. Kymissis, V. Bulovic, and A.I. Akinwande, "Process control of threshold voltage in organic FETs," IEDM 04-381, pp.15.5.1 (2004).

[23] S. Kobayashi, T. Nishikawa, T. Takenobu, S. Mori, T. Shimoda, T. Mitani, H. Shimotani, N. Yoshimoto, S. Ogawa, and Y. Iwasa, "Control of carrier density by self-assembled monolayers in organic field-effect transistors," Nature Materials 3, 317 (2004).

[24] M.F. Calhoun, J. Sanchez, D. Olaya, M.E. Gershenson, and V. Podzorov, "Electronic functionalization of the surface of organic semiconductors with self-assembled monolayers," Nature Materials 7, 84-89 (2008).

[25] S.W. Choi, W.B. Choi, Y.H. Lee, B.K. Ju, and B.H. Kim, "Effect of oxygen plasma treatment on anodic bonding," J. of Korean Phys. Soc. 38 (3), 207 (2001).

[26] M. Nakahara, K. Ozawa and Y. Sanada, "Change in the chemical structures of carbon black and active carbon caused by $\mathrm{CF}_{4}$ plasma irradiation," J. Mater. Sci. 291646 (1994).

[27] A. Paskaleva and E. Atanassova, "Bulk oxide charge and slow states in $\mathrm{Si}_{-} \mathrm{SiO}_{2}$ structures generated by RIE-mode plasma," Microelectron. Reliab. 40, 2033 (2000). 


\section{Chapter 6}

\section{Contact Interface Engineering}

Interface control is indispensible for realization of high performance OTFTs. The two most critical interfaces in OTFTs are the dielectric-semiconductor interface and the contact-semiconductor interface. The former interface was examined in Chapter 4 and 5. This chapter focuses on the latter. The quality of contact-semiconductor interface is important because charge injection into the OTFT channel occurs precisely at this interface. For efficient charge injection, ohmic contact is desirable. In a transistor, ohmic behavior is characterized by a linear relation between $I_{\mathrm{D}}$ and $V_{\mathrm{DS}}$ at small $V_{\mathrm{DS}}$ biases. To achieve ohmic contacts, one of the fundamental requirements is energetic matching/alignment of the Fermi level of the source/drain metal contacts to the HOMO or LUMO levels of the p-type or n-type organic semiconductor layer, respectively. In the case of p-type organic semiconductors, good matching is possible using contacts with high work function (e.g., gold). Fermi level of the high work function metal electrodes lies in the proximity of the HOMO level of p-type organic semiconductor, thus rendering a small energy barrier at the interface to facilitate efficient hole injection. This energy matching approach provides a simple guideline for contact material selection. However, in practice, physical interaction (e.g., interface dipole) between materials is more complex and may induce an energy barrier at the contact-semiconductor interface that limits charge injection [1]. A more in-depth discussion on charge injection is presented in Section 6.1.1. To improve contact properties, interface modification using SAMs of charge transfer complexes can potentially lead to larger drain currents and better current saturation comparing to non-modified contacts [2]. Use of alkanethiol SAMs for metal contact treatment is reviewed in Section 6.1.2. 
The SAM contact treatment method was evaluated on PQT-12 OTFTs. The experiment is divided into two parts. Part One investigates the impact of $\mathrm{Au}$ contact modification by 1octanethiol $\left(\mathrm{CH}_{3}\left(\mathrm{CH}_{2}\right)_{7} \mathrm{SH}\right)$ SAMs on device performance of PQT-12 OTFTs; the results are presented in Section 6.3. Part Two examines the influence of execution sequence of the surface treatment steps on device behavior; the outcomes are discussed in Section 6.4. Interface characteristics (e.g., wettability, surface roughness, chemical composition) are analyzed, where correlation with the electrical properties of the OTFTs are drawn to gain a better understanding on device behavior.

\subsection{Background}

\subsubsection{Charge Injection}

The electrical performance of OTFTs is not only limited by the intrinsic carrier mobility of the organic semiconductor, but by the efficiency of injecting and extracting charge carriers at the source and drain contacts. Contact resistance $\left(R_{\mathrm{C}}\right)$ provides a measure of charge injection efficiency in an OTFT. A basic parameter that determines charge injection in a device is the injection barrier at the contact-semiconductor interface, as illustrated in Figure 2.18. This injection barrier is given by the energy difference between the Fermi-level of the contact and the conduction or valence band of the semiconductor. Low energy barriers $\left(\varphi_{B}=I_{P}-\Phi_{M}\right)$ necessitate matching the work function $\left(\Phi_{\mathrm{M}}\right)$ of the contact metal with the ionization potential (IP $\left.\mathrm{S}_{\mathrm{S}}\right)$ of the semiconductor; this is one of the fundamental criteria for high charge injection efficiency. Organic devices are often prone to large contact resistance owing to the relatively large band-gap $(\sim 2 \mathrm{eV})$ and high ionization potential $(\sim 5$ $\mathrm{eV}$ ) of most organic materials; as result, they form Schottky barriers with various metals [3].

Figure 6.1 shows a simplified energy level diagram of the contact-semiconductor interface. When a metal and a p-type semiconductor are brought in contact, their Fermi levels $\left(E_{\mathrm{F}}\right)$ line up in equilibrium. For a low work function metal (Figure 6.1(a)), the valence band of a p-type semiconductor bends downward, which creates a barrier for hole conduction and results in a high contact resistance. For a high work function metal (Figure 6.1(a)), the valence band of a p-type semiconductor bends upward, which reduces the barrier for hole conduction and results in an improved contact. Therefore, to alleviate contact resistance in p-type OTFTs, high work function metals should be selected for source/drain contacts. Burgi et al. extracted the contact resistance of 
P3HT and F8T2 OTFTs made with different metal contacts [4], and observed that metals with higher work function generate lower contact resistance for bottom-contact P3HT OTFT and poly(9,9dioctyl-fluorene-co-bithiophene) (F8T2) OTFT. The data also revealed that organic semiconductors with higher ionization potential (e.g., F8T2) are more susceptible to higher contact resistance [4]. These results are summarized in Table 6.1 .
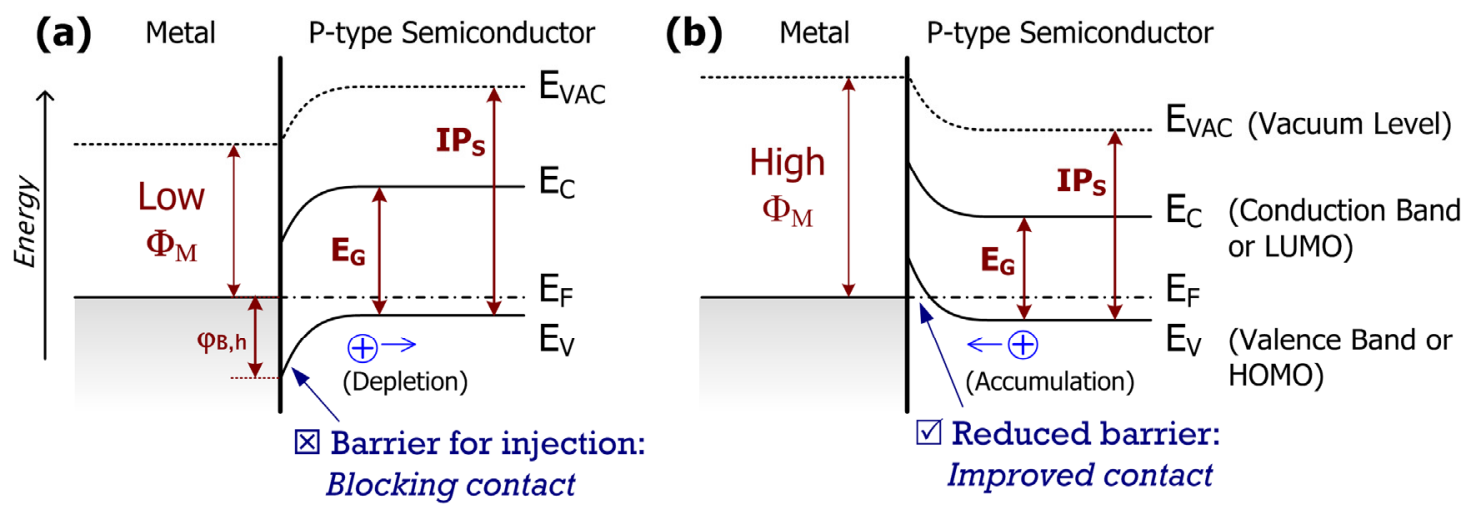

Figure 6.1. Energy level diagrams for metal and p-type semiconductor contact interface (assuming negligible interface states). (a) Small work function metal leads to interface barrier and poor contact. (b) Large work function metal leads to reduced interface barrier and improved contact.

Table 6.1. Source contact resistance $R$ s of bottom-contact P3HT and F8T2 OTFTs made with different metal contacts $\left(T=300 \mathrm{~K}, V_{\mathrm{GS}}=-20 \mathrm{~V}\right)$. IPS is the ionization potential of the semiconductor, $\Phi_{\mathrm{M}}$ is the measured work function of the metal, and $\varphi_{\mathrm{B}}$ is the estimated Schottky barrier height at the metal/organic interface (adapted from [4]).

\begin{tabular}{|ccccc|}
\hline Organic Semiconductor & S/D Contact & $\Phi_{\mathrm{M}}(\mathrm{eV})$ & $\varphi_{\mathrm{b}}(\mathrm{eV})$ & $R \mathrm{~s}(\mathrm{k} \Omega-\mathrm{cm})$ \\
\hline P3HT $(\mathrm{IPs}=5.0 \mathrm{eV})$ & $\mathrm{Au}$ & 5.2 & 0.0 & $<5$ \\
\hline $\mathrm{P} 3 \mathrm{HT}$ & $\mathrm{Ag}$ & $4.89 \pm 0.10$ & 0.1 & $\approx 15$ \\
\hline $\mathrm{P} 3 \mathrm{HT}$ & $\mathrm{Cr}-\mathrm{Au}$ & & & 22 \\
\hline $\mathrm{P} 3 \mathrm{HT}$ & $\mathrm{Cu}$ & $4.72 \pm 0.10$ & 0.3 & 320 \\
\hline P3HT & $\mathrm{Cr}$ & $4.68 \pm 0.10$ & 0.3 & 5400 \\
\hline F8T2 $(\mathrm{IPs}=5.5 \mathrm{eV})$ & $\mathrm{Au}$ & 5.2 & 0.3 & 13000 \\
\hline F8T2 & $\mathrm{Cr}-\mathrm{Au}$ & & & $>70000$ \\
\hline P3HT & $\mathrm{Al}$ & $4.05 \pm 0.10$ & 1.0 & $\infty$ \\
\hline
\end{tabular}

It is generally believed that the alignment of molecular energy levels with respect to the Fermilevel of contacts is of utmost importance for charge carrier injection and device efficiency. Charge carrier injection barriers are often estimated by assuming vacuum level alignment across interfaces, as explained above. However, recent research studies have cast doubts on the accuracy of this 
simple model due to the identification/detection of interfacial dipoles across many interfaces [5][6]. Consequently, charge injection barriers in the presence of interfacial dipoles can differ by more than $1 \mathrm{eV}$ from the values estimated from the vacuum level alignment approach. Possible origins of interfacial dipoles include charge transfer, formation of chemical bonds, or a "push-back" of electrons into the metal bulk after molecule adsorption [7]. Therefore, the contact-semiconductor interface mechanisms are more complex than expected. Detailed analytical techniques such as ultraviolet photoelectron spectroscopy (UPS) can be used to conscientiously study these interfacial mechanisms to allow proper analysis and modeling of OTFT behavior.

In addition to the effects of energy level alignment and interfacial dipole at the contactsemiconductor interface, a number of other factors can influence the charge injection efficiency and contact resistance in an OTFT, including device configuration [8], device geometry (e.g., channel length) [4], processing technique [9], and doping level of the semiconductor layer [10]. In terms of structural dependence of contact resistance, smaller contact resistance was reported in top-contact OTFTs owing to the relatively larger contact areas at the contact-semiconductor interface compared to bottom-contact structures [8]; more descriptions are presented in Section 2.3.1. Process-induced damage, associated with metal deposition on top of the organic semiconductor layer to form topcontact OTFTs, can also influence contact resistance. Metals are typically deposited by evaporation or by sputtering. The evaporation of metals onto organic materials can lead to in-diffusion of the metal, changes in the morphology of the organic material, and possible disruption of chemical bonds in the polymer chains [9]. These effects can lead to large interfacial resistances in OTFTs, which can subsequently increase contact resistance and undermine device performance. Processing conditions must be optimized to inhibit chemical, physical, and morphological changes induced by metal deposition on the organic semiconductor material. Contacts formed by solution-processible organic conductors can circumvent the aforementioned process-induced contact effects. However, more extensive development is necessary to improve the quality and patterning techniques for contacts based on soluble organic conductors.

Contact resistance $\left(R_{\mathrm{C}}\right)$ in OTFTs is typically in the range of $10 \mathrm{k} \Omega-\mathrm{cm}$ to $10 \mathrm{M} \Omega-\mathrm{cm}$, and is larger than those in inorganic transistors (e.g., for amorphous silicon TFTs, $R_{\mathrm{C}}$ is usually in the $\mathrm{k} \Omega$ $\mathrm{cm}$ range) [4]. Unlike inorganic FETs based on silicon, the source and drain contacts in OTFTs are not easily optimized by conventional processes, such as selective semiconductor doping or metal alloying. For example, the absence of a highly doped source/drain contact regions in OTFTs compared to silicon MOSFETs, as shown in Figure 3.1, is one of the primary causes for larger 
contact resistance in OTFTs. If highly doped contact regions or layers can be implemented in the OTFT structure, significant enhancement in charge injection efficiency and reduction in contact resistance can be expected.

More research efforts are needed to minimize contact resistance in OTFT; large contact resistance can severely limit device performance, perhaps to an extent where the speed of organic integrated circuits may not be limited by the intrinsic carrier mobility of the organic semiconductor, but by the contact resistance of the OTFTs. Large contact effects can limit the current-carrying ability of the device, lead to underestimations of important device parameters (e.g., mobility), and restrict the utility of OTFTs in active-matrix backplanes due to longer charging time $(\tau \sim R C)$ of an individual pixel. Therefore, the nature of interface between an organic semiconductor and source/drain contact is critical to the performance of organic electronics.

To promote charge injection and address contact resistance issues, this thesis investigates SAM treatment of the semiconductor/contact interface; this concept is reviewed in Section 6.1.2. Alternatively, developing techniques to enable selective doping of the source/drain contact regions of OTFTs (similar to inorganic transistors), or incorporating a charge injection layer at the contacts (similar to OLEDs) can offer additional routes to enhance contact properties of OTFTs.

\subsubsection{Alkanethiol SAM on Metals}

Thiol (also referred to as mercaptan) is a compound that contains the functional group composed of a sulfur atom and a hydrogen atom (-SH). Alkanethiol refers to group of compounds where thiol group is a substituent on an alkane. SAMs of thiols on gold and other metals have emerged as one of the most important classes of surface coatings, and are used in a variety of applications [11]. Alkanethiols are widely used to prepare highly ordered monolayers whose wetting properties can be controlled by changing the chemical nature of the terminal groups. Surfaces modified by alkanethiols can be made hydrophilic by introduction of polar groups (e.g., - $\mathrm{OH},-\mathrm{COOH},-$ $\mathrm{CONH}_{2}$ ), or hydrophobic by non-polar groups (e.g., $\left.-\mathrm{CH}_{3},-\mathrm{OCH}_{2} \mathrm{CF}_{2} \mathrm{CF}_{3},-\mathrm{O}\left(\mathrm{CH}_{2}\right)_{\mathrm{m}} \mathrm{CH}_{3}\right)$ [1]. By using more complex functionalities, SAMs can be made (bio)chemically reactive, adhesive or biologically inert. For example, long chain alkanethiols can produce a highly packed and ordered surface, rendering a membrane-like microenvironment that is suitable for immobilizing biological molecules in biosensing applications [11]. Development of nanoelectronic devices also exploits alkanethiol SAMs, where SAMs are used to position and pattern molecular components selectively 
on surfaces. One example is soft lithography with SAMs for creation of small (nano-sized) twoand three-dimensional chemical patterns on material surfaces [11]. Another application of thiol SAMs is for protection of metal against corrosion. Thick barriers of thiols can block electron transfer and hinder the transport of water, oxygen, and aggressive ions to the metal surface. Barrier properties of SAMs are largely determined by the length and organization of the alkyl chains. Metal surfaces covered by long-chain alkanethiols have demonstrated enhanced corrosion resistance [11]. These examples exemplify the versatility of alkanethiol SAMs. Alkanethiol SAMs can provide a basis for many scientific and technological applications, including nano-fabrication, biological recognition, molecular electronics, analytical and sensory applications; moreover, they can be used as molecular lubricants, protective coatings, or templates for crystal nucleation and growth [10].

SAMs of alkanethiols are often used to control interactions of metal surfaces because Sulfur compounds (from thiol) typically have a strong affinity to transition metal surfaces [11]. Other attractive attributes of alkanethiol SAMs include stability, ease of preparation/modification and high degree of order. In the liquid phase method of forming SAMs, the molecules are dissolved in an organic solvent and they adsorb spontaneously to the substrate material. When SAM is formed by immersing a gold surface in a solution of an alkyl thiol, the - SH group of thiol molecule experiences deprotonation (i.e., hydrogen atom is abstracted from thiol molecule) at the surface and the molecule binds to the surface through the $\mathrm{S}$ atom, forming a strong covalent $\mathrm{Au}-\mathrm{S}$ bond. Figure 6.2 shows a pictorial illustration of highly ordered alkanethiol SAM on Au surface. Stability, packing, and ordering of the SAMs are determined by two interacting factors: interfacial linkage between sulfur and gold (i.e., surface interactions), and the lateral van der Waals attractions between hydrocarbon chains (i.e., interchain interactions). Typically, the longer the hydrocarbon chains of the deposited thiol, the greater the stability of the resulting monolayer [10].

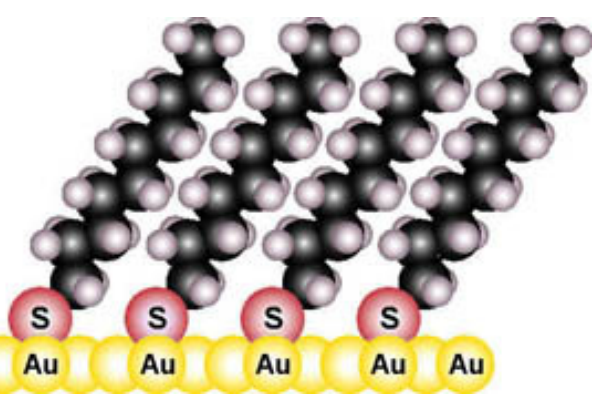

Figure 6.2. Pictorial illustration of alkanethiol SAM on Au surface. (Adapted from [10].) 
In OTFTs, alkanethiol SAMs have been applied as surface modifier for the Au source/drain contacts. Some researchers have reported improved molecular ordering, grain size, and adhesion of the organic semiconductor on modified Au surfaces. Thus, with proper material combination, thiol SAMs can improve charge injection at the metal contacts and enhance device performance [12]. However, there are cases where little or no influence/difference was observed with thiol SAM treatment [13][14]. Therefore, the effects of thiol SAMs are not clear at moment. More extensive studies are needed to clarify the real influences and establish an effective methodology for SAM treatment of source/drain contacts for practical OTFT applications.

\subsection{Experimental Details}

Bottom-gate bottom-contact PQT-12 OTFTs with $\mathrm{SiN}_{\mathrm{x}}$ gate dielectric and $\mathrm{Au}$ source/drain contacts (with thin $\mathrm{Cr}$ adhesion layer) were used for this contact interface engineering experiment. The device fabrication scheme is outlined in Figure 3.8. The basic criteria for material selection of source/drain contacts include chemical stability and energy compatibility with the organic semiconductors to form ohmic contacts for efficient charge injection. Au was selected for this experiment because of its high conductivity, excellent operational stability, and particularly its large work function. The work function of $\mathrm{Au}\left(\Phi_{\mathrm{M}} \approx 5.1 \mathrm{eV}\right)$ is energetically similar to the

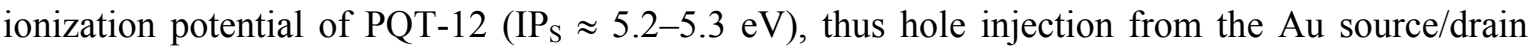
contacts to PQT-12 semiconductor is theoretically feasible. For selected devices with contact surface treatment by thiol SAM, the Au contacts were functionalized by immersing the substrates in a $0.01 \mathrm{M}$ 1-octanethiol $\left(\mathrm{CH}_{3}\left(\mathrm{CH}_{2}\right)_{7} \mathrm{SH}\right)$ solution in toluene for $30 \mathrm{~min}$. at room temperature. A cross sectional diagram of the device structure is shown in Figure 6.3.

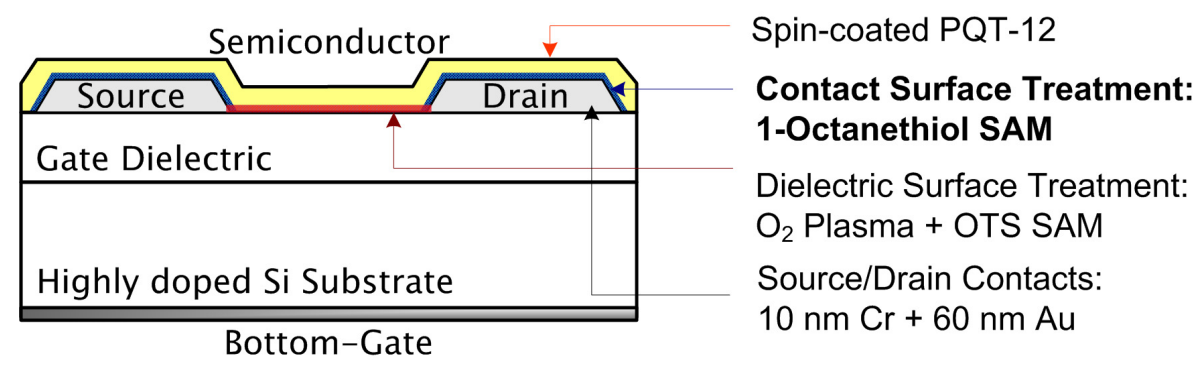

Figure 6.3. Schematic cross-section of the OTFT structure considered in this contact surface treatment study. 
The purpose of this study is to evaluate the impact of octanethiol SAM contact surface treatment on PQT-12 OTFTs characteristics, particularly the effect on device contact resistance. Two experiments were conducted, as summarized in Table 6.2. The first experiment (Section. 6.3) evaluates the effects of incorporating thiol SAM treatment on four samples with different dielectric surface treatment conditions; this is an extension of the investigation reported in Section 5.3. The second experiment (Section 6.4) assesses the effect of changing the execution sequence of surface treatment steps on device performance. The OTFT electrical characteristics and the interface characterization data are analyzed to establish correlations between electrical, chemical and physical characteristics of the devices; this can contribute to a better understanding of the mechanisms responsible for the changes in device characteristics due to octanethiol SAM contact surface treatment. The TFT parameters are extracted following the procedure outlined in Section 2.2. An overview of the various analytical techniques use for surface characterization is provided in Section 4.1.2.

Table 6.2. Contact-semiconductor interface engineering experiments.

\begin{tabular}{|lll|}
\hline & Description & Detail \\
\hline $\begin{array}{l}\text { Part A } \\
\text { (Section 6.3) }\end{array}$ & $\begin{array}{l}\text { Effect of addition of } \\
\text { octanethiol SAM treatment }\end{array}$ & $\begin{array}{l}\text { Compare device characteristics after adding thiol } \\
\text { treatment, for various samples with varying } \\
\text { dielectric surface conditions }\end{array}$ \\
\hline $\begin{array}{l}\text { Part B } \\
\text { (Section 6.4) }\end{array}$ & $\begin{array}{l}\text { Effect of sequence/order of } \\
\text { SAM surface treatment }\end{array}$ & $\begin{array}{l}\text { Evaluate how the execution order of SAM } \\
\text { treatments using 1-Octanethiol and OTS affect } \\
\text { transistor performance }\end{array}$ \\
\hline
\end{tabular}

\subsection{Impact of Contact Surface Treatment by Thiol SAM}

The first experiment evaluates the impact of adding 1-octanethiol SAM contact surface treatment step to PQT-12 OTFT fabrication under four dielectric surface modification conditions:

(i) No dielectric surface treatment: "none",

(ii) $\mathrm{O}_{2}$ plasma exposure for 60 seconds,

(iii) OTS SAM,

(iv) $\mathrm{O}_{2}$ plasma exposure for 60 seconds followed by OTS SAM.

The dielectric surface treatment procedures are discussed in Chapter 5. For the scenarios considered here, thiol SAM treatment was performed after completion of dielectric surface 
treatment(s), and before PQT-12 semiconductor deposition. Electrical characterization of the resulting OTFTs is reported in Section 6.3.1, and the interface characteristics are analyzed in Section 6.3.2.

\subsubsection{Electrical Characterization}

Figure 6.4 displays the effect of thiol SAM treatment on the mobility and on/off current ratio of PQT-12 OTFT on $150^{\circ} \mathrm{C} \operatorname{SiN}_{\mathrm{x}}(x=1.60)$ gate dielectric. For devices without dielectric surface treatment (labeled "none"), the incorporation of thiol produced an increase in mobility by $\sim 0.01$ $\mathrm{cm}^{2} / \mathrm{V}$-s (141\% improvement). In contrast, for devices with dielectric pretreatment, there was a small drop in mobility by $0.01-0.014 \mathrm{~cm}^{2} / \mathrm{V}-\mathrm{s}(\sim 8 \%-26 \%$ reduction) after thiol treatment. Presence of thiol appears to improve on/off current ratio for most samples, as shown in Figure 6.4(b). The consistency of these results was verified by repeating the study on another set of PQT-12 OTFTs with $300^{\circ} \mathrm{C} \mathrm{SiN}{ }_{x}(x=1.08)$ gate dielectric. Similar trends in mobility are observed in Figure 6.5, where the presence of thiol led to an increase in mobility for devices with untreated dielectric (i.e., "none"), but a reduction in mobility for devices with pre-treated dielectrics.

A number of interesting device behaviors can be identified from an analysis of the mobility data in Figure 6.4 and Figure 6.5:

- For devices without dielectric surface treatment (labeled "none"), an improvement in mobility is seen after thiol treatment in both Figure 6.4 and Figure 6.5. It is hypothesized that thiol treatment is favorable for previously-untreated devices (i.e., bare $\mathrm{SiN}_{\mathrm{x}}$ and bare $\mathrm{Au}$ surfaces). Possible explanations for this hypothesis are presented below.

- Channel related: Improvement in the extracted mobility value may stem from (a) an actual enhancement in charge transport, or (b) mobility's dependence on contact resistance due to artifact/limitation of the TFT extraction model. In the former, thiol treatment may be (unexpectedly) modifying the bare $\mathrm{SiN}_{\mathrm{x}}$ surface in a manner that enhances the dielectricsemiconductor interface and charge transport properties. However, there is a conventional belief by chemists that thiol only chemisorbs on $\mathrm{Au}$ surfaces, and there should be no interaction with $\mathrm{SiN}_{\mathrm{x}}$. This notion is challenged by the contact angle data in Figure 6.8, which revealed that thiol treatment indeed altered the $\operatorname{SiN}_{\mathrm{x}}$ surface wettability in our experiment. More discussion on this will follow in Section 6.3.2.1. In the latter scenario, 
when TFT becomes less contact limited, the extraction mobility may increase due to oversimplified modeling of OTFT characteristics.

- Contact related: Thiol SAM treatment on bare $\mathrm{Au}$ contacts is effective for improving contact injection properties. Hence, effect of contact-limited mobility is reduced, leading to noticeable device improvement compared to untreated devices.

- For devices with dielectric pretreatment, a decrease in mobility was observed after thiol treatment in both Figure 6.4 and Figure 6.5. Thus, thiol treatment is not as favorable for devices pre-treated with $\mathrm{O}_{2}$ plasma and OTS SAM. Possible causes of this behavior are speculated below.

- Channel related: The reduction in mobility suggests interaction of thiol with the pretreated $\mathrm{SiN}_{\mathrm{x}}$ dielectric surface in a way that disturbs the device performance. It is speculated that traces of thiol molecules aggregated on the pretreated $\mathrm{SiN}_{\mathrm{x}}$ surface, which hinders the quality of the semiconductor channel and the dielectric-semiconductor interface. Contact angle measurements revealed that thiol treatment indeed imposed a small change in the surface wettability of OTS-treated or $\mathrm{O}_{2}$-plsama-treated $\mathrm{SiN}_{\mathrm{x}}$ surface, as shown in Figure 6.8. An attempt was made to study the surface composition of thiol-treated surfaces via XPS. However, the sulfur concentration in the thiol monolayer was too minuscule to permit reliable detection and analysis using XPS.

- Contact related: The intention of thiol treatment is to improve charge injection at the contact-semiconductor interface, which should reduce contact resistance and lead to an overall improvement in device performance (e.g., higher current conduction). This thiolinduced improvement is not obvious for devices with pretreated dielectric. One possibility is that the $\mathrm{O}_{2}$ plasma and OTS dielectric treatments modified the Au contacts, thus hindering the formation of high-quality thiol SAM on Au. It is generally believed that OTS does not self-assemble on $\mathrm{Au}$. This is confirmed by contact angle measurements where negligible changes in contact angle were observed after subjecting Au to OTS treatment. On the other hand, recent studies showed evidence that $\mathrm{O}_{2}$ plasma can modify the work function and interface dipole of $\mathrm{Au}$ surfaces. Kim et al. observed a reduction in hole injection barrier at the interface by $\mathrm{O}_{2}$ plasma, leading to an increase in linear mobility for pentacene OTFTs [21]. These results confirmed the (unintentional) changes in Au contact properties during dielectric surface treatment by $\mathrm{O}_{2}$ plasma in our PQT-12 OTFT devices. However, there is currently few or no report on the effect of thiol on $\mathrm{O}_{2}$ plasma treated $\mathrm{Au}$. 
(a)

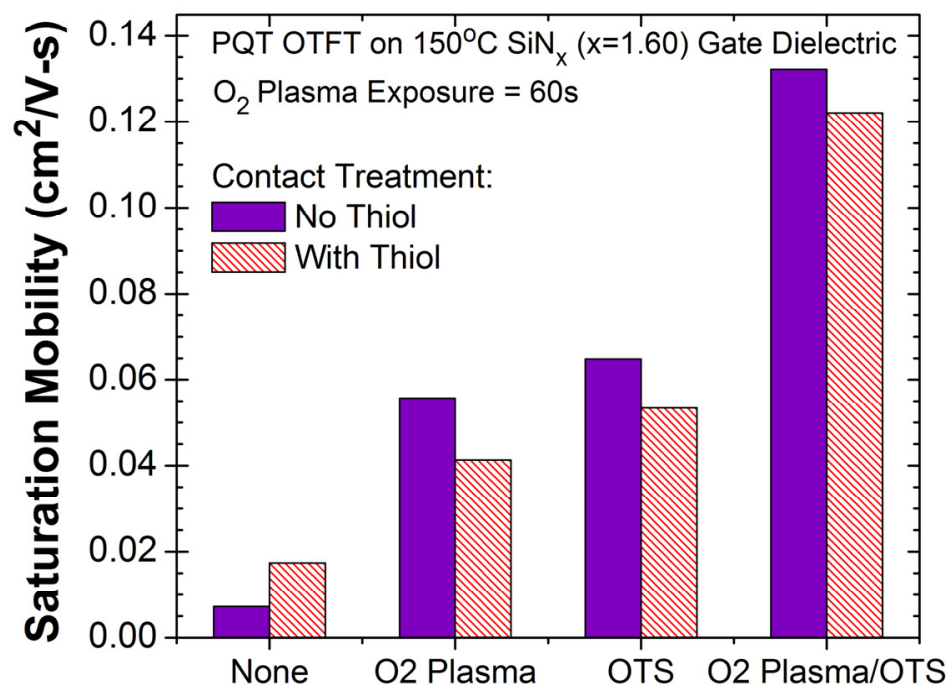

\section{Dielectric Surface Treatment}

(b)

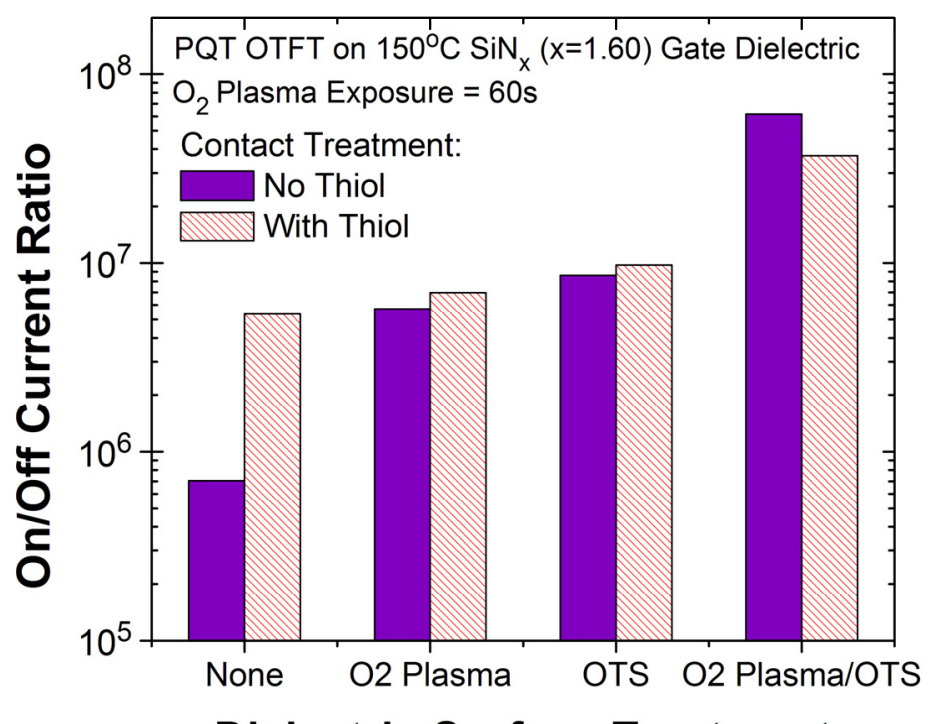

\section{Dielectric Surface Treatment}

Figure 6.4. (a) Effective mobility and (b) on/off current ratio of PQT-12 OTFT with $150^{\circ} \mathrm{C} \mathrm{SiN} \mathrm{N}_{\mathrm{x}} \mathrm{x}$ $=1.60$ ) gate dielectrics in the absence ("no thiol") or presence ("with thiol") of 1-octanethiol SAM modification of Au contact surfaces. Four scenarios of gate dielectric surface treatments were considered. Measurements collected in saturation region. 


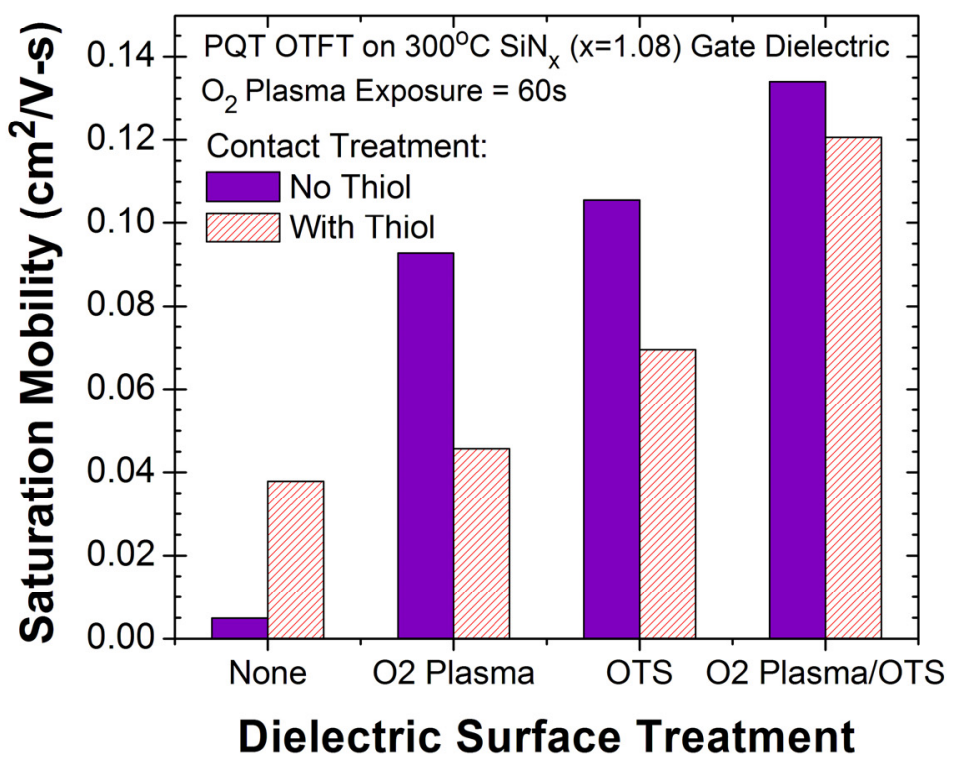

Figure 6.5. Effective mobility of PQT-12 OTFT with $300^{\circ} \mathrm{C} \mathrm{SiN}(x=1.08)$ gate dielectrics in the absence ("no thiol") or presence ("with thiol") of 1-octanethiol SAM modification of Au contact surfaces. Four scenarios of gate dielectric surface treatments were considered. Measurements were collected in saturation region of OTFT operation.

Contact resistance provides a good measure for analyzing the contact-semiconductor interface properties in OTFTs. Contact resistance was calculated following the extraction method published in [16] and outlined in Section 2.2.2. Figure 6.6 plots the constant (linear) component of the contact resistance in PQT-12 OTFTs with various surface treatment conditions. Devices treated with thiol exhibited higher contact resistance than devices without thiol. A consistent trend between mobility (Figure 6.4) and contact resistance (Figure 6.6) is observed and is summarized in Figure 6.7. Here, thiol-treated devices have lower mobility and higher contact resistance than their no-thiol counterparts. Interestingly, these observations are contrary to initial expectation that thiol SAM can improve charge injection and reduce contact resistance [12].

The accuracy of the contact resistance extraction requires further examination. In most of the existing TFT models, contact resistance is often modeled with a mobility-dependent component. As a result, higher mobility devices might have a smaller extracted contact resistance. Undeniably, the extracted mobility and contact resistance data in Figure 6.6 are susceptible to error introduced by mobility-dependent contact resistance. However, the percentage change in contact resistance 
differs considerably from the percentage change in mobility for each pair of devices, as tabulated in Table 6.3. Therefore, we can conclude that the observed trend in contact resistance is justifiable.

Our observation that thiol-treated devices have higher contact resistance is contradictory to general belief that SAM would reduce contact resistance. One possible reason for this deviation is that the thiol SAM might be imperfect due to pre-processing conditions. As such, instead of enhancing contact injection, it might introduce a barrier that hinders charge injection [17], and thus leads to higher contact resistance. More detailed experimentation is required to confirm these hypotheses. In addition, employment of alternate TFT contact resistance models should be employed to confirm results reported here [18][19][20][21][22]. In the next section, interface properties of the thiol-treated surfaces are examined to seek insights into the observed OTFT behavior.

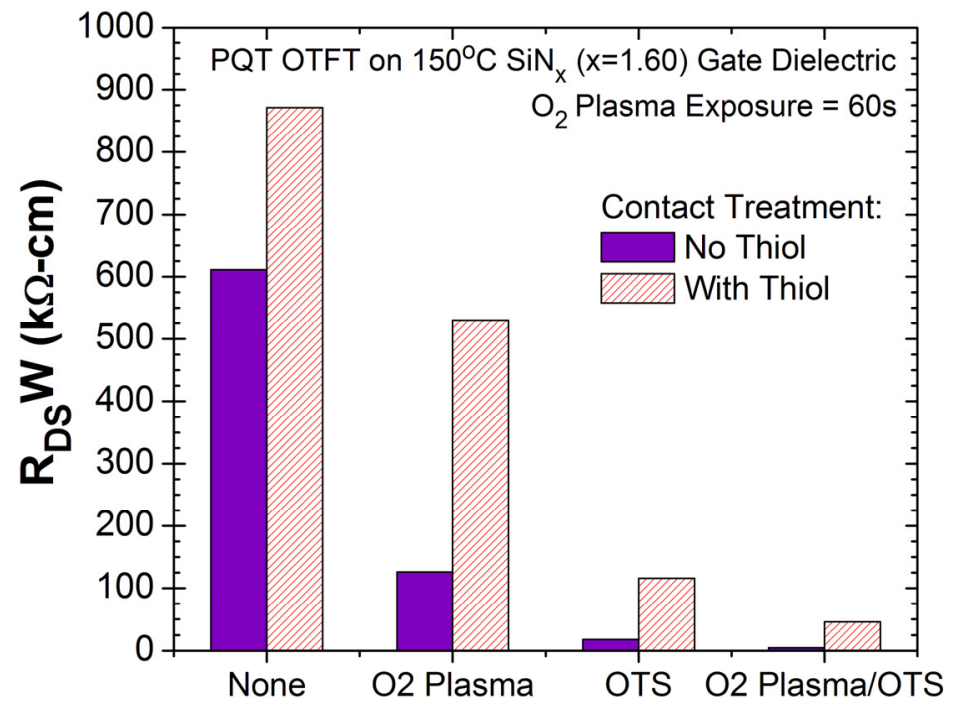

Dielectric Surface Treatment

Figure 6.6. Contact resistance of $\mathrm{PQT}-12$ OTFT on $150^{\circ} \mathrm{C} \mathrm{SiN} \mathrm{N}_{\mathrm{x}}(x=1.60)$ gate dielectrics in the absence ("no thiol") or presence ("with thiol") of 1-octanethiol SAM modification of Au contact surfaces. Four scenarios of gate dielectric surface treatments were considered. Measurements were collected in linear region of OTFT operation. 


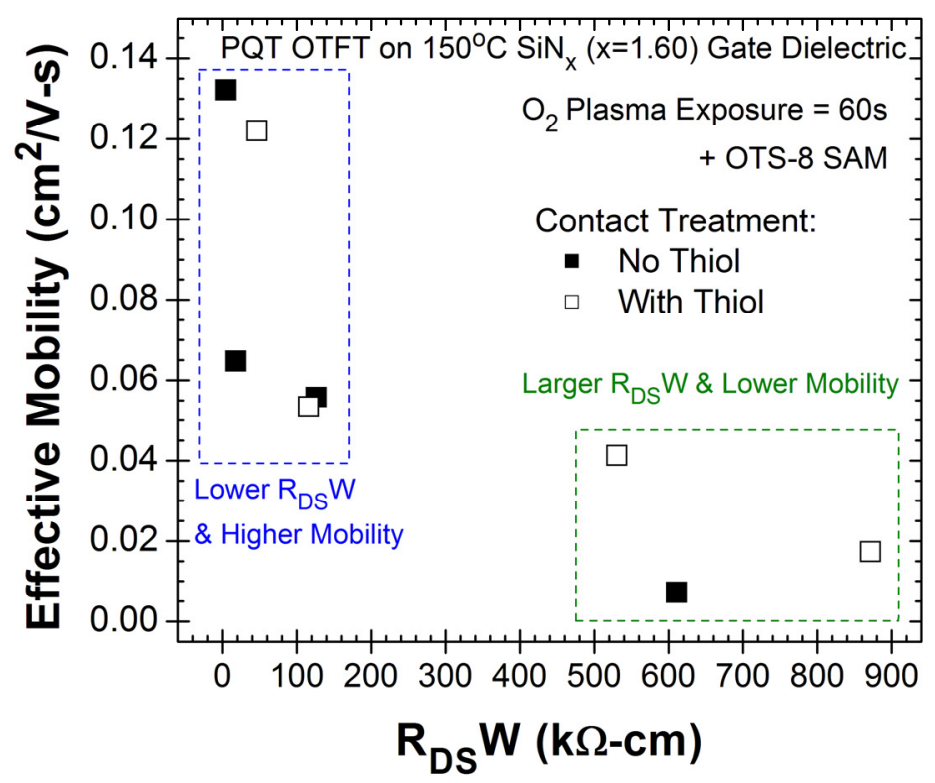

Figure 6.7. Saturation mobility versus contact resistance of PQT-12 OTFT on $150^{\circ} \mathrm{C} \operatorname{SiN}_{x}(x=$ 1.60) gate dielectrics.

Table 6.3. Percentage change in mobility and contact resistance for PQT-12 OTFT on $150^{\circ} \mathrm{C}$ $\operatorname{SiN}_{x}(x=1.60)$, based on data in Figure 6.5 and Figure 6.6, respectively. \% Change is calculated as "(Thiol - NoThiol)/NoThiol".

\begin{tabular}{|lcc|}
\hline Dielectric Surface Treatment & \% Change in Mobility & \% Change in RDsW \\
\hline None & $141.1 \%$ & $42.7 \%$ \\
\hline $\mathrm{O}_{2}$ Plasma & $-25.8 \%$ & $545.1 \%$ \\
\hline OTS & $-20.8 \%$ & $320.5 \%$ \\
\hline $\mathrm{O}_{2}$ Plasma/OTS & $-7.6 \%$ & $905.2 \%$ \\
\hline
\end{tabular}

\subsubsection{Interface Characterization}

\subsubsection{Contact Angle}

Contact angle measurements were done on various $\mathrm{SiN}_{\mathrm{x}}$ samples with or without 1-octanethiol SAM treatment in order to study the impact of thiol on the dielectric surface properties and correlate the results with the observed device characteristics. It is generally conceived that alkanethiol SAM modifies Au surfaces only, and there should be minimal or no interaction with $\mathrm{SiN}_{\mathrm{x}}$ [1]. However, contrary to conventional intuition, Figure 6.8 shows a consistent reduction in contact angle on various $\mathrm{SiN}_{\mathrm{x}}$ surfaces after 1-octanethiol SAM treatment. This reduction in contact 
angle for thiol-treated devices correlates well with the decrease in mobility (Figure 6.4 and Figure 6.5), since dielectric surface with higher contact angle (or hydrophobicity) is typically linked to higher device mobility [2][7][13].

However, the interaction between $\operatorname{SiN}_{\mathrm{x}}$ and thiol is unclear. Does thiol actually chemisorb to form SAM to provide "real" surface modification? Or does the change in contact angle merely reflect the presence of traces of thiol residues on the $\operatorname{SiN}_{\mathrm{x}}$ surface? At present, scientific reports/evidence on thiol- $\mathrm{SiN}_{\mathrm{x}}$ bonding cannot be found; most literature reported thiol-Au bonding only (since sulfur molecules in thiol has a strong affinity for $\mathrm{Au}$ ). More extensive experiments are needed to clarify the interaction between thiol and $\mathrm{SiN}_{\mathrm{x}}$.

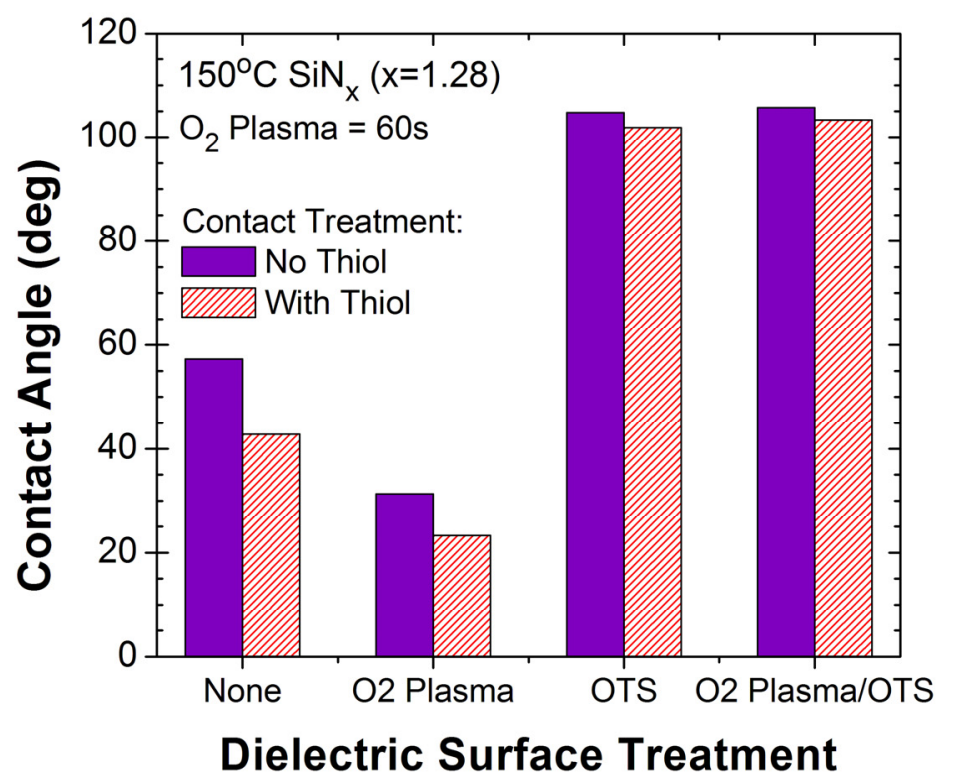

Figure 6.8. Water contact angle of $150^{\circ} \mathrm{C} \mathrm{SiN} x(x=1.28)$ surface after various types of dielectric surface treatment, and in the absence or presence of 1-octanethiol exposure.

\subsubsection{Surface Roughness}

The effect of 1-octanethiol SAM treatment on surface roughness of $\mathrm{SiN}_{\mathrm{x}}$ is illustrated in Figure 6.9. Comparing the data for " $\mathrm{O}_{2}$ plasma + OTS" and " $\mathrm{O}_{2}$ plasma + OTS + thiol", thiol treatment led to an increase in surface roughness for samples with $\mathrm{O}_{2}$ plasma and OTS treatments. This trend was observed consistently for samples subjected to different $\mathrm{O}_{2}$ plasma exposure durations. Although experimental error must be considered, the consistency observed in each set of samples presents a 
convincing argument that the thiol treatment step is indeed modifying the $\mathrm{SiN}_{\mathrm{x}}$ surface physically or chemically, as noted by changes in surface roughness and contact angle, respectively.

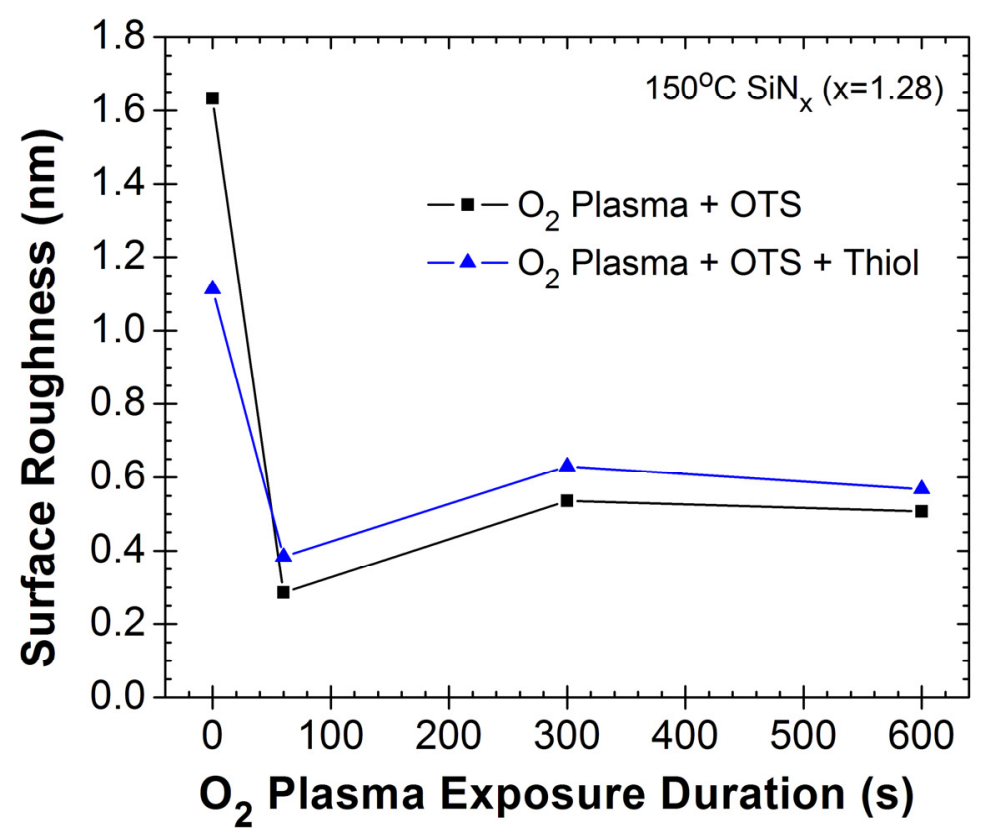

Figure 6.9. Mean surface roughness measured by AFM for $\mathrm{SiN}_{\mathrm{x}}$ surfaces after $\mathrm{O}_{2}$ plasma/OTS SAM surface treatment, in the absence or presence of 1-octanethiol SAM treatment.

\subsubsection{Chemical Composition}

Figure 6.10 compares the elemental distribution on the $\operatorname{SiN}_{\mathrm{x}}$ surface without and with the thiol treatment step. A small decrease in Si $2 \mathrm{p}$ and $\mathrm{C} 1 \mathrm{~s}$ intensities is observed after thiol treatment, which may be related to the low Si and C content in thiol. However, the \% change is small $(\sim 1-6 \%$ after addition of thiol), thus the data must be interpreted with caution to take experimental errors into consideration. The intensity of the sulfur (S 2p) peak from thiol compound was too low to be accurately detected by XPS. Thus, we cannot draw any conclusive statement from these XPS data. 


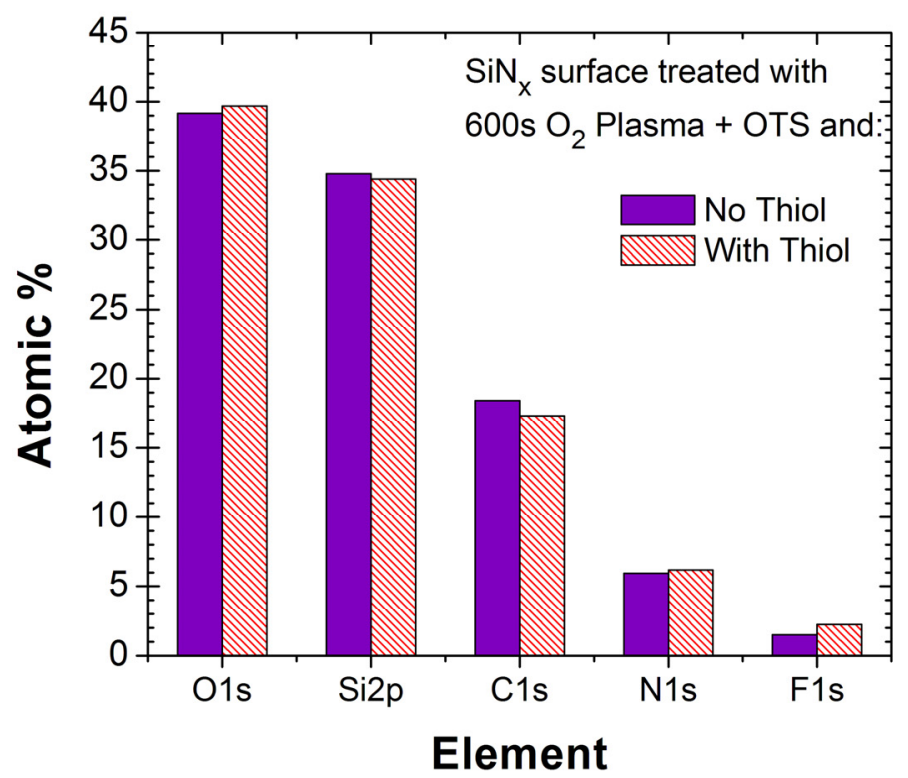

Figure 6.10. Atomic percentage detected by XPS on $\mathrm{SiN}_{\mathrm{x}}$ surface after $\mathrm{O}_{2}$ plasma/OTS SAM surface treatment, in the absence or presence of 1-octanethiol treatment. (Experimental error $= \pm 5 \%)$.

\subsubsection{Analysis}

The key observations upon incorporating 1-octanethiol SAM contact surface treatment in the fabrication of PQT-12 OTFTs are:

- Reduced field-effect mobility and increased contact resistance in the OTFT;

- Reduced contact angle and increased surface roughness of the $\mathrm{SiN}_{\mathrm{x}}$ gate dielectric surface.

It has been reported that OTFT mobility decreases when the dielectric surface becomes less hydrophobic (i.e., smaller contact angle) and when the surface roughness increases [26]. Therefore, the interface characterization data display good correlation to justify the OTFT electrical characteristics.

The most striking observation in this experiment is that the incorporation of thiol treatment actually degrades device performance, which is contrary to expectation. Therefore, the device performance was compromised by the addition of thiol SAM for the PQT-12 OTFT devices investigated in this thesis. It has been reported that the application of thiol SAMs on Au contacts can improve OTFT performance [12][11][28]. This improvement was attributed to an enhancement in semiconductor film morphology near the contact-semiconductor interface or the effect of the 
interfacial dipole induced by the SAM. However, such improvement was not observed in the present experiment, particularly for the case where devices were subjected to dielectric surface treatment prior to thiol SAM contact surface treatment. The increase in contact resistance after thiol treatment suggests the formation of a contact barrier layer at the Au-PQT interface. The reduction in mobility after thiol treatment for the pretreated devices indicates that the combination of " $\mathrm{O}_{2}$ plasma + thiol" and "OTS + thiol" unfavorably modify device interfaces in a manner that limits charge injection or charge transport. The precise mechanisms responsible for the reduced performance in thiol-treated devices are currently unclear. A number of possible explanations for the observed device behavior are presented below.

- Modification of $\mathrm{Au}$ from $\mathrm{O}_{2}$ plasma exposure: Previous reports on successful device improvement by thiol treatment are assumed to be on bare Au surface. However, for the fabrication of our bottom-gate bottom-contact devices, the $\mathrm{Au}$ surface is predisposed to $\mathrm{O}_{2}$ plasma and OTS modification during the dielectric treatment steps. These pretreatment steps may influence how thiol self-assembled onto $\mathrm{Au}$ surface. The effect of $\mathrm{O}_{2}$ plasma exposure on $\mathrm{Au}$ was reported by $\mathrm{Kim}$ et al, where they observed $\mathrm{O}_{2}$ plasma treated $\mathrm{Au}$ contacts led to enhanced hole injection and increased mobility for pentacene OTFTs when compared to bare Au contacts [21]. The band structure at the Au-pentacene interface was quantitatively studied, and they found the work function of $\mathrm{Au}$ increased from 4.65 to $5.28 \mathrm{eV}$ as the Au surface was treated with $\mathrm{O}_{2}$ plasma. The corresponding interface dipoles were $-0.30 \mathrm{eV}$ for bare $\mathrm{Au}$ and $0.71 \mathrm{eV}$ for $\mathrm{O}_{2}-\mathrm{Au}$, respectively. Accordingly, the hole injection barrier at the Au-pentacene interface reduced from 0.45 to $0.15 \mathrm{eV}$ by the $\mathrm{O}_{2}$ plasma before the deposition of pentacene, leading to an increase in linear field-effect mobility for pentacene OTFTs [21]. This report confirmed that the Au contacts in the PQT-12 OTFTs studied in this thesis were indeed modified by $\mathrm{O}_{2}$ plasma exposure; and more importantly, $\mathrm{O}_{2}$ plasma treatment should enhance contact properties of our TFTs. This agrees with the dramatic reduction in contact resistance shown in Figure 6.6 for the $\mathrm{O}_{2}$ plasma treated sample (no thiol) when compared to the untreated device. Therefore, $\mathrm{O}_{2}$-Au contacts display superiority over bare-Au contacts in PQT12 OTFTs.

- Ineffective formation of thiol SAM on $\mathrm{O}_{2}$ plasma treated Au: Thiol on $\mathrm{O}_{2}-\mathrm{Au}$ may behave differently from thiol on bare-Au. Reports on device enhancement by thiol SAM were based on bare $\mathrm{Au}$ surfaces [12][11][28]. On the other hand, the effect of thiol SAM on $\mathrm{O}_{2}$-Au surfaces for OTFTs has not been published. Lahio et al. studied the influence of initial oxygen 
(contamination from atmosphere) on formation of thiol layers [11]. They observed that the adsorption of thiol removes oxygen from the surface (i.e., oxygen concentration reduced after thiol treatment), and that the presence of initial oxygen on Au reduces the amount of thiol on the surface. These results provide evidence that the presence of oxygen indeed affects the adsorption of thiol on Au surfaces. More extensive studies are needed to examine behavior or formation of thiol SAM on $\mathrm{O}_{2}$-Au surface.

- Thiol-induced contact barrier: The extracted contact resistance values in Figure 6.6 displayed a consistent increase in contact resistance after thiol treatment, regardless of the type of dielectric surface treatment. It is hypothesized that the thiol SAM introduced a barrier layer that hinders charge injection at the contacts, thus resulting in increased in contact resistance. This notion of a thiol-induced contact injection barrier is substantiated by a recent study on pentacene OTFTs with $\mathrm{Au}$ electrodes modified by 1-hexadecanethiol $\left(\mathrm{C}_{16} \mathrm{H}_{33} \mathrm{SH}\right)$ [17], which revealed the presence of tunneling barriers by the insulating $\mathrm{C}_{16} \mathrm{H}_{33} \mathrm{SH}$ at the interfaces between the $\mathrm{Au}$ contacts and pentacene thin film [17].

- Unoptimized processing conditions: Device improvements may be possible if thiol SAM treatment recipes is reoptimized for device subjected to $\mathrm{O}_{2}$ plasma or OTS pretreatments. The thiol processing conditions used in our experiment were designed for bare $\mathrm{Au}$ surface. However, since our device fabrication scheme involves other pretreatment steps that might inadvertently alter the $\mathrm{Au}$ surface prior to thiol treatment, special considerations are required. Hiroshiba et al. found that OTFT characteristics are very sensitive to the preparation condition of thiol SAMs modified surfaces (e.g., chemical formulation and substituent groups of the thiol compound, concentration and preparation duration, etc.) [12]. Longer treatment duration may form a "perfect" but thicker thiol layer, which may create a barrier for carrier injection in OTFTs. In contrast, imperfect modifications with a short thiol preparation time delivered improved TFT action and improved charge injection compared to untreated devices! In our case, the thiol treatment conditions might not be optimal for the devices under study, and thus may create an additional barrier for carrier injection. More in-depth studies to understand the interfacial kinetics at the thiol $/ \mathrm{O}_{2} / \mathrm{Au}$ or thiol/OTS $/ \mathrm{O}_{2} / \mathrm{Au}$ junctions are needed to develop a suitable contact treatment recipe.

Based on the above observations, it is concluded that 1-octanethiol SAM treatment should be omitted for bottom-gate bottom-contact PQT-12 OTFTs fabricated using the processing conditions reported in this thesis. More thorough studies must be conducted to clarify the underlying 
mechanisms responsible for the reduced performance upon incorporation of the thiol treatment step. Nonetheless, it is believed that with proper treatment conditions, thiol SAM can potentially enhance contact properties and improve OTFT device performance.

\subsection{Impact of Execution Sequence of Surface Treatment}

This section examines the question: "does the order or sequence in which surface treatments are executed affect device performance?" In this experiment, two sets of devices were prepared by varying the execution order of 1-octanethiol and OTS SAM treatments, as summarized in Table 6.4. The sample labeled "Thiol/OTS" was prepared by performing thiol treatment on the prepatterned substrate first, followed by OTS treatment. The sample labeled as "OTS/Thiol" was first treated with OTS SAM, followed by a thiol treatment step. PECVD $\operatorname{SiN}_{\mathrm{x}}$ gate dielectrics were considered in this experiment. All surfaces were pretreated with $\mathrm{O}_{2}$ plasma (60 s) prior to OTS and 1-octanethiol SAM treatments.

Table 6.4. Experiment on execution sequence of OTS and thiol surface treatments.

\begin{tabular}{|c|c|c|}
\hline Sample Name & Treatment Sequence & Key Observations \\
\hline $\begin{array}{l}\text { OTS/Thiol } \\
\text { (i.e., OTS first, } \\
\text { Thiol on top) }\end{array}$ & $\begin{array}{l}\text { OTS 1st } \\
\text { Thiol 2nd } \\
\text { PQT-12 last }\end{array}$ & $\begin{array}{l}\text { Higher } \mu_{\mathrm{FE}} \& l_{\mathrm{ON}} / l_{\mathrm{OFF}} \\
\text { Larger } \theta_{\text {contact }} \\
\text { Smaller surface roughness }\end{array}$ \\
\hline $\begin{array}{l}\text { Thiol/OTS } \\
\text { (i.e., thiol first, } \\
\text { OTS on top) }\end{array}$ & $\begin{array}{l}\text { Thiol 1st } \\
\text { OTS 2nd } \\
\text { PQT-12 last }\end{array}$ & $\begin{array}{l}\text { Smaller } \mu_{\mathrm{FE}} \& \text { lon } / \text { loFF } \\
\text { Smaller } \theta_{\text {contact }} \\
\text { Larger surface roughness }\end{array}$ \\
\hline
\end{tabular}

\subsubsection{Electrical Characterization}

OTFTs with various geometries were measured, and the average effective field-effect mobility and on/off current ratio in the saturation region are displayed in Figure 6.11. The results showed that OTS/Thiol devices delivered higher mobility (by $27 \%$ or $1.3 \mathrm{x}$ ) and on/off current ratio (by $124 \%$ or 2.3x) than Thiol/OTS devices. One speculation for the superior characteristics with OTS/Thiol devices is that OTS SAM is deposited directly on the $\mathrm{SiN}_{\mathrm{x}}$ surface in this configuration. As such, a better quality OTS SAM can be formed to effectively improve the dielectric-semiconductor interface (assuming thiol does not adhere on OTS surface). In the Thiol/OTS configuration, residues of thiol might remain on the $\mathrm{SiN}_{\mathrm{x}}$ surface, which might hinder the formation of a highly 
ordered OTS SAM. Consequently, this can interfere with the formation of a well-ordered PQT-12 semiconductor layer in the channel, thus reducing device performance.

\subsubsection{Interface Characterization}

To account for the observed device behavior, interfacial studies are conducted to examine the surface chemistry of OTS/Thiol and Thiol/OTS samples.

\subsubsection{Contact Angle}

Results from contact angle measurements are shown in Figure 6.12. The OTS/Thiol surface displays a larger contact angle ( $\left.\theta_{\text {contact }}\right)$ than the Thiol/OTS surface. This agrees with the observed mobility trend, where higher contact angle is linked to higher mobility. However, the difference in contact angle ( $\left.\Delta \theta_{\text {contact }}\right)$ between the two samples is very small (less than $\left.1^{\circ}\right)$; thus, this data must be analyzed with caution to take experimental error into consideration.

\subsubsection{Surface Roughness}

OTS/Thiol sample displays lower surface roughness than Thiol/OTS. This is concurrent with the OTFT data, where higher mobility in OTS/Thiol sample is linked to lower surface roughness. The higher surface roughness of the Thiol/OTS sample may be linked to possible thiol residues on $\mathrm{SiN}_{\mathrm{x}}$ surface that obstruct the formation of high quality OTS SAM. As a result, the Thiol/OTS sample has higher surface roughness and lower contact angle; these characteristics correlate well with the lower mobility observed in the corresponding OTFT. 
(a)

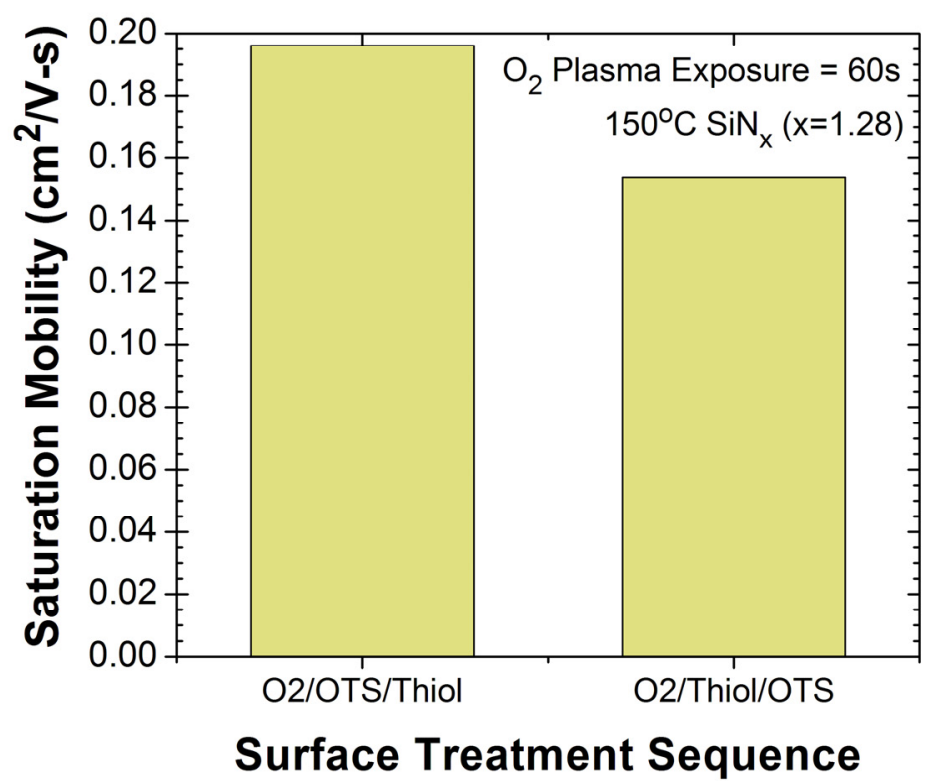

(b)

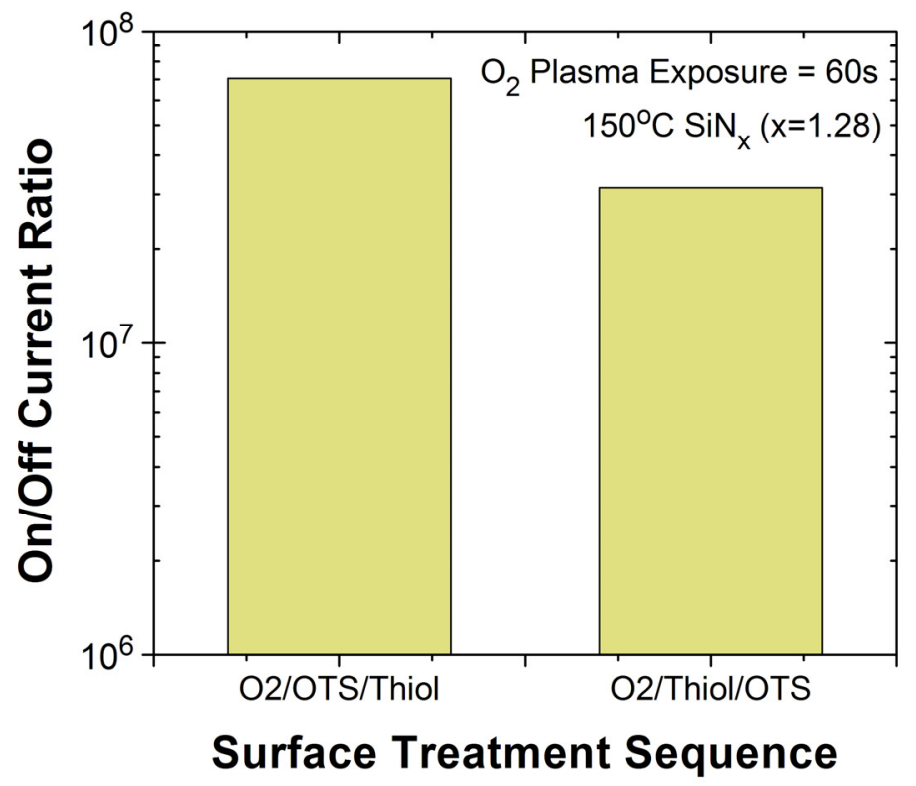

Figure 6.11. Comparison of (a) effective mobility and (b) on/off current ratio of PQT-12 OTFTs subjected to different execution sequence of OTS and Thiol SAM treatment. 


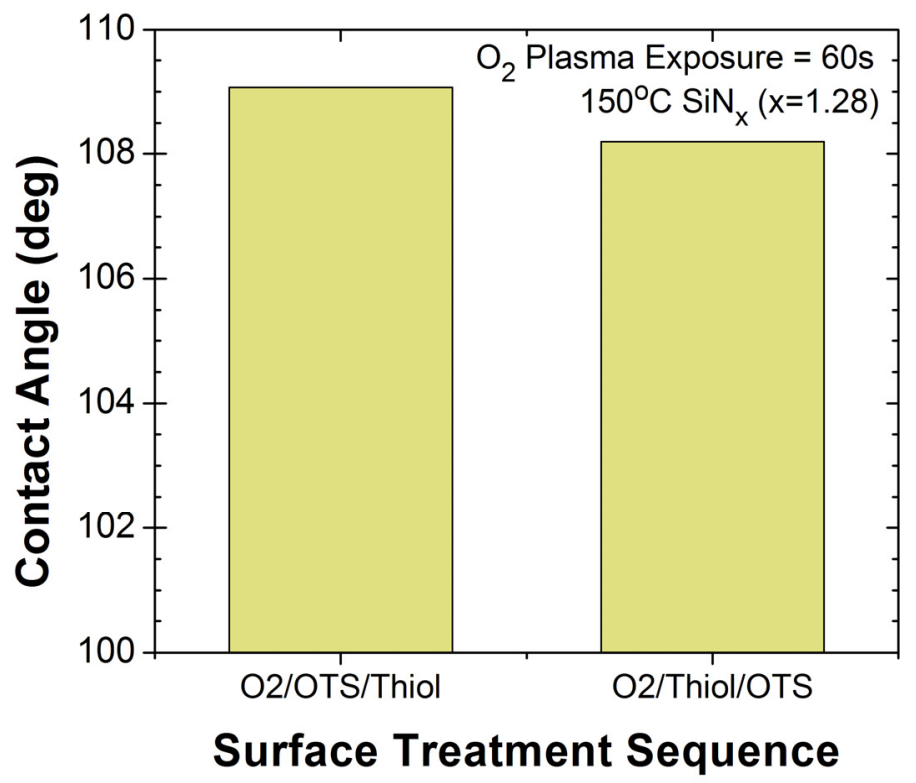

Figure 6.12. Water contact angle for Thiol/OTS and OTS/Thiol treated $\mathrm{SiN}_{\mathrm{x}}$ surfaces.

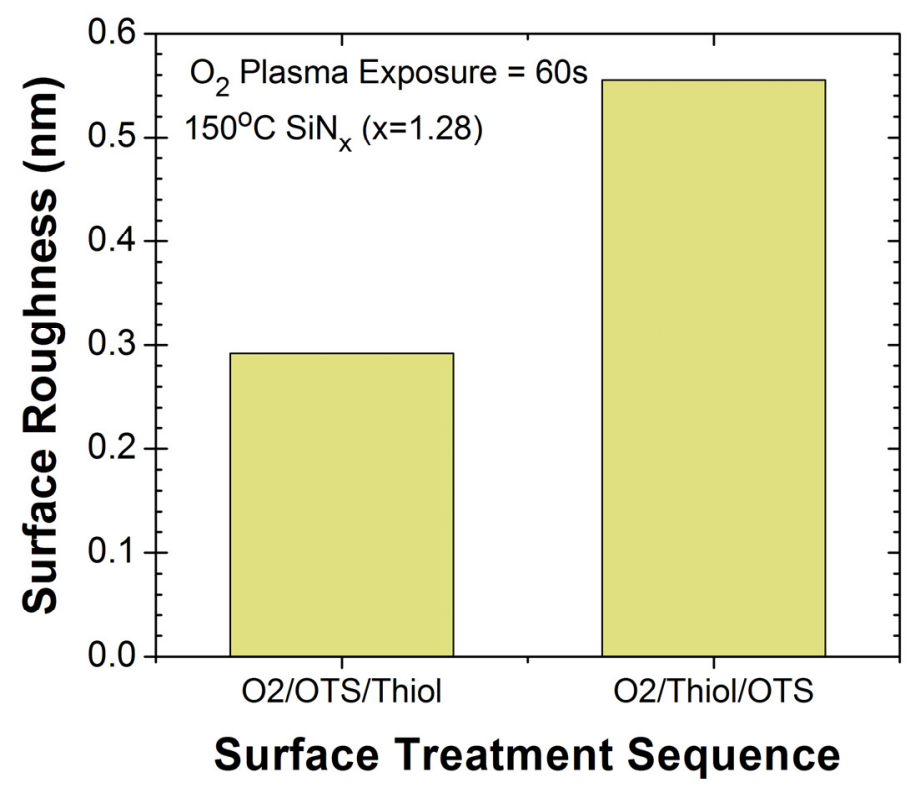

Figure 6.13. Surface roughness for Thiol/OTS and OTS/Thiol treated $\mathrm{SiN}_{\mathrm{x}}$ surfaces. 


\subsubsection{Chemical Composition}

XPS was used to analyze the chemical composition of the OTS/Thiol and Thiol/OTS surfaces. Figure 6.14 showed that the atomic compositions for the two samples are very similar. Thiol/OTS has a larger C $1 \mathrm{~s}$ concentration, and can be attributed to characteristic of the top OTS layer. However, it was be difficult to collect reliable data from XPS to quantitatively compare the characteristics of thiol SAM on the treated surfaces. The atomic concentration of sulfur from the Thiol SAM was too minuscule to generate a strong XPS signal. Thus, no conclusive statements can be drawn from the XPS measurements.

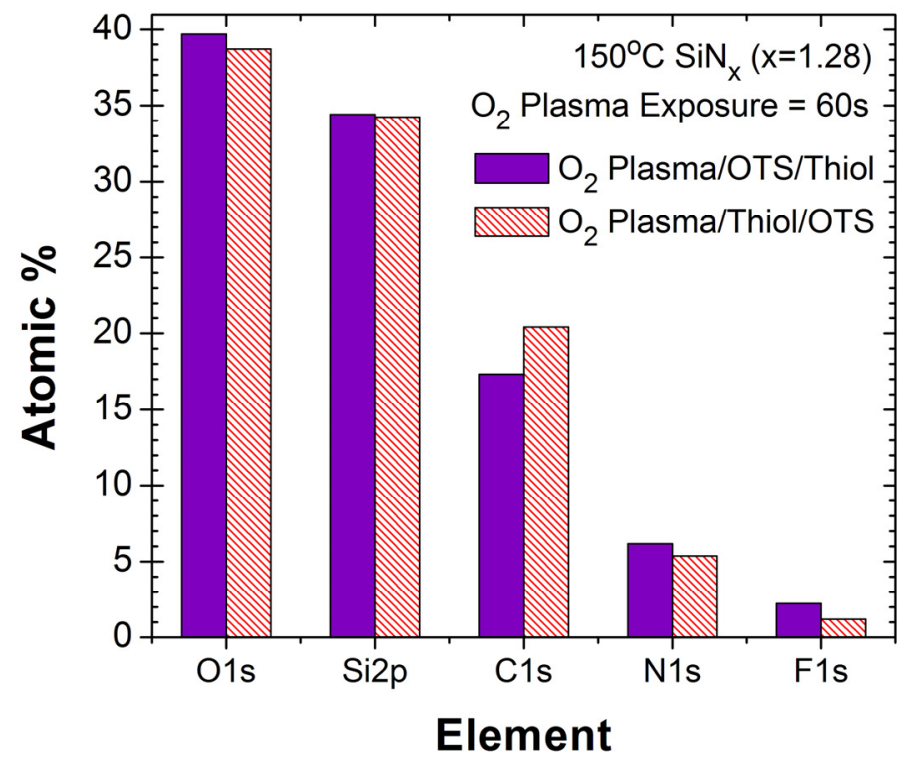

Figure 6.14. Atomic percentage detected by XPS on the surface of $\mathrm{SiN}_{\mathrm{x}}$ for different execution sequences of OTS and 1-Octanethiol SAM treatments.

Overall, this experiment demonstrates that the execution order or placement of a thiol layer affects the surface energy of the dielectric surface. The key observations are summarized in Table 6.4. For the $\mathrm{SiN}_{\mathrm{x}}$ gate dielectric and OTFT process sequence used in this experiment, it is more preferable to perform thiol treatment after OTS SAM treatment (i.e., OTS/Thiol is better than Thiol/OTS). However, combining the observations from Section 6.3 and Section 6.4, it is best to omit the thiol treatment step for our PQT-12 OTFTs on $\mathrm{SiN}_{\mathrm{x}}$ gate dielectric to deliver better device performance. 


\subsection{Summary and Contributions}

The influence of Au contact surface treatment with 1-octanethiol SAM was analyzed by electrical and interface measurements of PQT-12 OTFTs. For devices on "bare/untreated $\mathrm{SiN}_{\mathrm{x}}$ ", thiol SAM surface treatment led to increased field-effect mobility by up to $140 \%$. However, for devices with dielectric surface pre-treatment, mobility decreases by up to $25 \%$ after addition of thiol. It is interesting to observe that the percentage reduction in mobility, or sensitivity, is smaller for OTStreated devices compared to $\mathrm{O}_{2}$ plasma-treated devices. Perhaps devices with an OTS-treated surface are less sensitive to thiol treatment; since OTS is already hydrophobic, there is a higher chance that thiol does not stick or interact with OTS. On the other hand, devices with only $\mathrm{O}_{2}$ plasma are more sensitive to the addition of thiol; this can be attributed to modification of the bare $\mathrm{Au}$ surface by oxygen plasma prior to thiol treatment. While a correlation is observed between field-effect mobility and contact angle, XPS measurements were unable to provide useful insight because the atomic concentration of sulfur from thiol monolayer was below the detection limit.

The key observations from this contact interface engineering investigation are summarized in Table 6.5. Combining these results, the recommendation is to exclude the 1-octanethiol treatment step to bring forth better device performance for PQT-12 OTFTs on $\mathrm{SiN}_{\mathrm{x}}$ gate dielectric. The 1octanethiol SAM treatment conditions applied in this work might not be optimal for substrates pretreated with $\mathrm{O}_{2}$ plasma and OTS SAM. It is recommended that future work to consider optimizing the processing parameters for thiol treatment, and/or exploring other thiol compounds that can provide a better fit to the material system being investigated.

Although we do not have a precise understanding of the mechanisms responsible for the device trends observed here, this experiment allows us to gain new knowledge on how different surface treatment conditions affect device performance and to learn new insight on ways to generate device improvements. Hypotheses were proposed to account for the observed behavior; more in-depth device experiments are needed to justify these speculations, which is beyond the scope or timeframe of this thesis research. Nonetheless, the experimental results and analyses documented in this thesis can provide some useful guidelines/insights for further optimization of interface modification recipes for OTFTs, with the potential to generate more intriguing device improvements. 
Table 6.5. Summary of key observations from contact interface engineering experiments.

\begin{tabular}{|c|c|c|}
\hline & Description & Key Observations \\
\hline Part A & $\begin{array}{l}\text { Effect of thiol SAM } \\
\text { for contact surface } \\
\text { treatment }\end{array}$ & $\begin{array}{l}\text { Thiol improves } \mu_{\mathrm{FE}} \text { for devices without any other } \\
\text { pretreatments (compared to untreated devices) } \\
\text { Addition of thiol decreases mobility and increase contact } \\
\text { resistance for devices with prior dielectric surface } \\
\text { treatments. } \\
\text { Recommendation to omit thiol treatment step for devices } \\
\text { considered here. Further optimization of the contact } \\
\text { surface treatment process (e.g., processing parameters) or } \\
\text { investigation of a more fitting thiol compound should be } \\
\text { considered }\end{array}$ \\
\hline Part B & $\begin{array}{l}\text { Effect of execution } \\
\text { sequence of surface } \\
\text { treatments }\end{array}$ & $\begin{array}{l}\text { OTS/Thiol configuration (i.e., OTS first and thiol last) gives } \\
\text { higher mobility, higher contact angle and smaller surface } \\
\text { roughness than Thiol/OTS configuration (i.e., thiol first, OTS } \\
\text { last). }\end{array}$ \\
\hline
\end{tabular}




\section{Bibliography}

[1] N. J. Watkins, Q.T. Le, S. Zorba, L. Yan, Y. Gao, S.F. Nelson, C.S Cuo, and T.N. Jackson, "Photoemission characterization of interfaces between $\mathrm{Au}$ and pentacene," Proc. SPIE 4466, 1 (2001).

[2] S.H. Kim, J.H. Lee, Y.S. Yang, and T. Zyung, "Device Fabrications of Organic Thin-Film Transistors," Mol. Cryst. Liq. Cryst. 405, 137 (2003).

[3] Y. Chen, I. Shih and S. Xiao, "Effects of $\mathrm{FeCl}_{3}$ doping on polymer-based thin film transistors," J. Appl. Phys. 96(1), 454 (2004).

[4] L. Burgi, T.J. Richards, R.H. Friend, and H. Sirringhaus, "Close look at charge carrier injection in polymer field-effect transistors,” J. Appl. Phys., 94(9), 6129 (2003).

[5] H. Ishii, K. Sugiyama, E. Ito and K. Seki, "Energy Level Alignment and Interfacial Electronic Structures at Organic/Metal and Organic/Organic Interfaces," Adv. Mater. 11, 605 (1999).

[6] X. Crispin, V. Geskin, A. Crispin, J. Cornil, R. Lazzaroni, W.R. Salaneck, and J.L. Brédas, "Characterization of the Interface Dipole at Organic/ Metal Interfaces," J. Am. Chem. Soc. 124, 8131 (2002).

[7] N. Koch, A. Elschner, R.L. Johnson, and J.P. Rabe, "Energy level alignment at interfaces with pentacene: metals versus conducting polymers," Appl. Surf. Sci. 244, 593 (2005).

[8] M. Halik, H. Klauk, U. Zschieschang, G. Schmid, W. Radlik, S. Ponomarenko, S. Kirchmeyer, and W. Weber "High-mobility organic thin-film transistors based on $\alpha, \alpha$ 'didecyloligothiophenes," J. Appl. Phys. 93(5), 2977 (2003).

[9] T.W. Lee, J. Zaumseil, Z. Bao, J.W.P. Hsu, and J.A. Rogers, "Organic light-emitting diodes formed by soft contact lamination," PNAS 101(2), 429 (2004).

[10] D.B.A. Rep, A.F. Morpurgo, and T.M. Klapwijk, "Doping-dependent charge injection in regioregular poly(3-hexylthiphene," Organic Electronics 4, 201 (2003).

[11] T. Laiho and J.A. Leiro, "Influence of initial oxygen on the formation of thiol layers," Appl. Surf. Sci. 252, 6304 (2006).

[12] N. Hiroshiba, R. Kumashiro, N. Komatsu, Y. Suto, H. Ishii, S. Takaishi, M. Yamashita, K. Tsukagoshi, and K. Tanigaki, "Surface Modifications using Thiol Self-Assembled Monolayers on Au Electrodes in Organic Field Effect Transistors," Mater. Res. Soc. Symp. Proc. 965, 0965-S08-03 (2007).

[13] J. Wang, D. J. Gundlach, A. J. Benesi, and T. N. Jackson, "High Mobility Polymer Thin Film Transistors Based on Copolymers of Thiophene and 3-hexylthiophene," 41st Electronic Materials Conf. Dig. p.16 (1999).

[14] S.H. Kim, J.H. Lee, S.C. Lim, Y.S. Yang, and T. Zyung, "Improved Contact Properties for Organic Thin-Film Transistors Using Self-Assembled Monolayer,” Jpn. J. Appl. Phys. 43, L60 (2004). 
[15] W.K. Kim and J.L. Lee, "In Situ Analysis of Hole Injection Barrier of $\mathrm{O}_{2}$ Plasma-Treated $\mathrm{Au}$ with Pentacene Using Photoemission Spectroscopy," Electrochem. Solid-State Lett. 10(3), pp. H104 (2007).

[16] P. Servati, Amorphous Silicon TFTs for Mechanically Flexible Electronics, Ph.D. Thesis, University of Waterloo, Canada (2004).

[17] N. Kawasaki, Y. Ohta, Y. Kubozono, and A. Fujiwara, "Hole-injection barrier in pentacene field-effect transistor with $\mathrm{Au}$ electrodes modified by $\mathrm{C}_{16} \mathrm{H}_{33} \mathrm{SH}$," Appl. Phys. Lett. 91, 123518 (2007).

[18] A. Ahnood, P. Servati, M.R Esmaeili-Rad, F.M. Li, A. Sazonov, and A. Nathan, "Extraction of Non-Ohmic Contact Resistance in Nanocrystalline Silicon Thin Film Transistors," 2008 Spring MRS Meeting Abstract A16.6. San Francisco, USA. March 24$28,2008$.

[19] D.J. Gundlach, L. Zhou, J.A. Nichols, T.N. Jackson, P.V. Necliudov, and M.S. Shur, "An experimental study of contact effects in organic thin film transistors," J. Appl. Phys. 100, 024509 (2006)

[20] D. Natali, L. Fumagalli, and M. Sampietro. "Modeling of organic thin film transistors: Effect of contact resistances," J. Appl. Phys. 101, 014501 (2007).

[21] R. A. Street and A. Salleo, "Contact effects in polymer transistors," Appl. Phys. Lett. 81, 2887 (2002).

[22] M.L. Chabinyc, "Characterization of semiconducting polymers for thin film transistors," J. Vac. Sci. Technol. B 26, 445 (2008)

[23] J. Veres, S. Ogier, G. Lloyd, and D. de Leeuw, "Gate Insulators in Organic Field-Effect Transistors," Chem. Mater. 16, 4543 (2004).

[24] S. C. Lim, S. H. Kim, J.E. Lee, M. K. Kim, D. J. Kim, and T. Zyung, "Surface-treatment effects on organic thin-film transistors," Synthetic Metals 148 (1), 75 (2005)

[25] L.L. Kosbar, C.D. Dimitrakopoulos, and D.J. Mascaro, "The Effect of Surface Preparation on the Structure and Electrical Transport in an Organic Semiconductor," Mater. Res. Soc. Symp. Proc. 665, C10.6.1 (2001).

[26] R. J. Kline, D. M. DeLongchamp, D. A. Fischer, E. K. Lin, M. Heeney, I. McCulloch, and M. F. Toney, Appl. Phys. Lett. 90, 062117/1 (2007).

[27] C. Song, B. Koo, S. Lee and D. Kim, "Characteristics of Pentacene Organic Thin Film Transistors with Gate Insulator Processed by Organic Molecules," Jpn. J. Appl. Phys. 41, 2730 (2002).

[28] M. Kawasaki, S. Imazeki, M. Ando, and M. Ohe, "Bottom contact organic thin-film transistors with thiol-based SAM treatment," Proceedings - Electrochemical Society, v PV 2004-15, Thin Film Transistor Technologies VII - Proceedings of the International Symposium, pp. 249 (2005). 
Chapter 7. OTFT Circuits and Systems

\section{Chapter 7 OTFT Circuits and Systems}

As discussed in Chapter 1, OTFTs are aimed for applications requiring large-area coverage, structural flexibility, low-temperature processing and low cost. Applications envisioned for OTFTs include large-area flexible displays, electronic paper (e-paper), disposable electronics, low-cost and low-end electronic devices such as RFID tags and smart cards [1]. While inorganic semiconductors already serve in many of these systems, all of these applications can benefit from the potential cost reduction and simpler integration offered by organic semiconductor technology. To implement practical organic electronic devices, integration of OTFTs into circuits to perform switching, logic, or amplification functions is compulsory. Some of the desirable functional specifications imposed on OTFTs for circuit applications are reviewed in Section 7.1. This chapter discusses the development of OTFT circuits, with emphasis on two main application fields: active matrix display backplanes and RFID tags; these technologies are reviewed in Section 7.2.1 and Section 7.2.2, respectively. A number of OTFT circuits were fabricated, by incorporating the technical knowledge acquired from our investigations on gate dielectric, interface treatment, and integration strategies; the experimental outcomes are presented in Section 7.3.

\subsection{OTFT Requirements for Circuit Applications}

Desirable qualities/characteristics of an OTFT include: high field-effect mobility, high on/off drain current ratio, low leakage current, minimal threshold voltage shift, and sharp subthreshold slope [2]. These general requirements are discussed in Section 2.2.3. Depending on the specific circuit 
application, the demands imposed on certain TFT parameters are more stringent. Table 7.1 reviews application-specific demands for TFT. For AMLCDs, TFT behaves as a switch, and thus, minimal leakage is the most critical. On the other hand, current drive and on/off current ratio are of great importance in AMOLED displays, since the TFT must provide sufficient current output to drive OLED for light emission. For RFID tags, speed is the most critical parameter. In most cases, the threshold voltage $\left(V_{\mathrm{T}}\right)$ should be as low as possible for large dynamic range (in displays) and for low voltage operation. Also, good device stability is demanded in all applications. These application-specific requirements can be addressed via choice of material, device structure, and/or the associated fabrication method, as analyzed in this section. In particular, the discussion focuses on speed, current-driving capability, leakage current, and stability requirements for OTFTs.

Table 7.1. A concise comparison of OTFT characteristics demanded by various applications. The most critical parameter(s) in each application is identified by the symbol $\diamond$.

\begin{tabular}{|lccc|}
\hline OTFT Parameter & $\begin{array}{c}\text { AMLCD } \\
\text { (TFT as switch) }\end{array}$ & $\begin{array}{c}\text { AMOLED } \\
\text { (TFT as active } \\
\text { driver) }\end{array}$ & $\begin{array}{c}\text { RFID tags, } \\
\text { Smart Cards }\end{array}$ \\
\hline Operating Speed & Not as critical & Not as critical & High $^{\diamond}$ \\
Leakage (lleak) & Low $^{\diamond}$ & Low $^{\diamond}$ & Low \\
Current Drive (loN) & Not as critical & Large $^{\diamond}$ & Large \\
Threshold Voltage $\left(V_{T}\right)$ & Low & Low & Low \\
On/off Ratio (loN/loFF) & Large & Large & Large \\
Stability & High & High & High \\
\hline
\end{tabular}

\section{Speed:}

In RFID and memory devices, the operating speed of the integrated circuit is a critical parameter. OTFT circuits must operate sufficiently fast to deliver the data rates demanded by these applications. The maximum speed at which a transistor circuit can operate is limited by the time it takes for the charge carriers to transit from the source contact through the channel to the drain contact [3]. This is known as the transit time $(\tau)$, which is also described as the time it takes for the accumulation layer to be emptied of charges through the drain after $V_{\mathrm{G}}$ has been switched off. Transit time is often expressed using maximum switching frequency $\left(f_{\max }\right)$ :

$$
\frac{1}{\tau}=f_{\max } \approx \frac{\mu \cdot V_{D S}}{L^{2}}
$$

Note that the value of $f_{\max }$ calculated with the above formula provides an upper bound. In practice, $f_{\max }$ is lower due to parasitic capacitances and other non-ideal effects. 
For OTFT based on soluble polymer semiconductor, assuming $\mu_{F E}=10^{-1} \mathrm{~cm}^{2} / \mathrm{V}-\mathrm{s}, V_{\mathrm{DS}}=10 \mathrm{~V}$ and $L=10 \mu \mathrm{m}$, then $f_{\max } \approx 1 \mathrm{MHz}$. Other scenarios are summarized in Table 7.2. Therefore, the upper speed limit for these OTFTs is on the order of $1 \mathrm{MHz}$ due to limitations on transistor mobility, gate length and patterning methods. (For comparison, inorganic electronics work with $\mathrm{GHz}$ frequencies. Thus, it is clear that organic electronics should be aimed for low-cost and lowend products rather than high-performance electronics.) From an application perspective, the refresh rate of reflective displays is around $40-85 \mathrm{~Hz}$, thus OTFT technology should be able to fulfill these requirements. In the case of RFID tags, the logic circuit component operates around $100 \mathrm{kHz}$, which is deliverable by existing OTFTs. However, the rectification stage of RFID tags is expected to operate at $13.56 \mathrm{MHz}$, thus faster OTFTs are needed.

Table 7.2. Calculation of maximum intrinsic switching frequency for OTFTs.

\begin{tabular}{|ccccc|}
\hline Scenario & $\mu_{\mathrm{FE}}\left(\mathrm{cm}^{2} / \mathrm{V}-\mathrm{s}\right)$ & $V_{\mathrm{DS}}(\mathrm{V})$ & $L(\mu \mathrm{m})$ & Calculated $f_{\max }$ \\
\hline $\begin{array}{c}\# 1 \\
\text { (e.g., P3HT, } \\
\text { shadow mask) }\end{array}$ & 0.01 & 10 & 100 & $1 \mathrm{kHz}$ \\
\hline $\begin{array}{c}\# 2 \\
\text { (e.g., P3HT) }\end{array}$ & 0.01 & 10 & 10 & $100 \mathrm{kHz}$ \\
\hline $\begin{array}{c}\# 3 \\
\text { (e.g., PQT-12) }\end{array}$ & 0.1 & 10 & 10 & $1 \mathrm{MHz}$ \\
\hline $\begin{array}{c}\# 4 \\
\text { (e.g., pentacene) }\end{array}$ & 1 & 10 & 10 & $10 \mathrm{MHz}$ \\
\hline
\end{tabular}

As indicated by Eqn. (2.4), high $\mu_{\mathrm{FE}}$ and small $L$ are required to improve speed (i.e., increase $\left.f_{\max }\right) \cdot \mu_{\mathrm{FE}}$ is largely determined by the quality and microstructure of the organic semiconductor material. A higher degree of molecular ordering results in a higher $\mu$. Researchers have been actively working on enhancing mobility via a variety of approaches, including synthesis/development of new materials, improving interfaces and material systems, etc. [4]. Since $f_{\max } \propto 1 / L^{2}$, reducing $L$ should have a large impact on speed improvement. $L$ is usually dictated by the fabrication technique or processing technology. In a conventional lateral OTFT structure, it is difficult to define $L$ less than $5 \mu \mathrm{m}$ as it becomes challenging to delineate the source and drain contacts without extensive overlap with the gate (ideally, the overlap should be minimized to ensure low parasitic capacitance) [3]. Researchers are actively exploring innovative processing techniques to overcome these resolution limitations. Vertical OTFT structure, as shown in Figure 7.1, can provide an alternative means of achieving $L$ in the submicron scale. In vertical OTFT, $L$ is 
defined by the dielectric film thickness, which can be readily controlled by precise timing of the deposition process. Therefore, a vertical OTFT structure can facilitate further improvement in switching speed. Preliminary demonstration of vertical OTFT can be found in [5] and [6].

It should be noted that Eqn. (2.4) represents an upper limit for $f_{\max }$. Parasitic capacitances (e.g., overlap capacitance from the overlap of the source/drain electrodes with the gate electrode) may be present in the transistor, which charge/discharge when $V_{\mathrm{GS}}$ is switched on/off, thus limiting the switching frequency of an OTFT. In order to maximize speed, it is important to minimize these parasitic capacitances, via proper device design, optimized patterning procedure and suitable material choices. In addition to achieving maximal speed, other desired OTFT characteristics for RFID applications include: small overlap capacitance, good off-current behavior (important since RFID tags are power-constrained devices), and good stability (important for analog circuit operation).

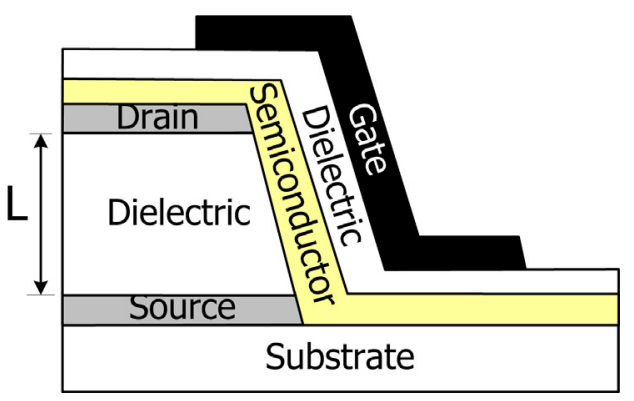

Figure 7.1. Cross sectional diagram of a vertical OTFT structure.

\section{Leakage:}

In active matrix display backplanes (e.g., AMLCD, AMOLED), the desirable OTFT characteristics include low off-current (i.e., leakage), low gate leakage current, sufficient drain current, small gate overlap capacitance, and good device uniformity [64]. Minimum leakage current is particularly important in order to retain charge in the pixel during the "off" state of the transistor. To minimize leakage current, it is important to control the quality of gate dielectric, and minimize the interface state density and interfacial stress. Also, isolation between transistors on the same substrate is needed by patterning the semiconductor layer, in order to reduce parasitic leakage and cross-talk. 


\section{Current Drive Capacity:}

In AMOLED displays, the output current drive of the OTFT is also critical. Since OLEDs are current-driven devices, the OTFT must have sufficient output current to provide the needed brightness for the OLED element. To get large output drain current, the transconductance $\left(g_{\mathrm{m}}\right)$ of the transistor must be considered. $g_{\mathrm{m}}$ represents the change in $I_{\mathrm{D}}$ for a given change in $V_{\mathrm{G}}$. For large $g_{\mathrm{m}}$ (and thus large output current), $\mu_{\mathrm{FE}}$ and $W / L$ ratio of the transistor should be large, as dictated by the following relationship:

$$
g_{m}=\left(\frac{\partial I_{D}}{\partial V_{G}}\right)_{V_{D}=\text { const }}=\frac{W C_{i}}{L} \mu_{F E} V_{D} .
$$

Alternatively, since $C_{\mathrm{i}}=\varepsilon_{0} \varepsilon_{\mathrm{r}} A / d$, choosing high-k dielectric or using thinner gate dielectric can increase $C_{\mathrm{i}}$, thus promoting larger $g_{\mathrm{m}}$.

\section{Stability:}

Most OTFT applications demand good device stability. This necessitates maintaining good stability of the organic material even under environmental stress or operational stress. Most organic materials are sensitive to environmental parameters, including air, humidity, moisture, oxygen, light, etc. Tailoring the chemical structure of the organic material during synthesis is a possible route to improve intrinsic environmental stability. Encapsulation methods provide an extrinsic technique to address stability issues. Operational instability in OTFTs, such as threshold voltage shift $\left(\Delta V_{\mathrm{T}}\right)$, should also be taken into account. $\Delta V_{\mathrm{T}}$ is often observed in OTFTs, and becomes significant after prolonged gate bias. $\Delta V_{\mathrm{T}}$ is typically caused by the creation of metastable defects in the band-gap, and the charge trapping in gate and passivation dielectric layers. The extent of charge trapping is determined by the quality of dielectric layer and the dielectric-semiconductor interface state density. The interface treatment techniques, examined in Chapter 5 and Chapter 6 , provide possible routes to enhance the interface quality, and perhaps improve device stability. 


\subsection{Applications}

\subsubsection{Displays}

One of the most promising applications of OTFTs is their use as an on-pixel switching element in active-matrix displays, similar to the functions that are currently fulfilled by a-Si:H TFTs in AMLCD and AMOLED display applications. Active-matrix backplane electronics based on OTFTs are attractive because of the large-area capability and low-cost advantage of organic technology, as well as the opportunity to realize a new generation of flexible, lightweight displays and electronic paper [8]. The incorporation of OTFTs in active-matrix displays have recently been demonstrated with a polymer-dispersed liquid crystal or with an electrophoretic material as a display element [19][64]. Development of OTFT-driven AMOLED displays is also in progress, and integrated OTFT-OLED smart pixels have been reported [8][11][29]. OTFT-OLED integration is appealing because it suggests the potential to manufacture inexpensive and flexible display modules with completely functional organic materials.

Table 7.3 summarizes some of the display prototypes demonstrated using OTFTs; most of the existing prototypes were built using pentacene OTFTs. The E-paper and LCD were made with OTFT matrix array and the AMOLED with dot patterns [12]. Since the OTFT serves as an active element, the AMOLED is very sensitive to non-uniformity in OTFT performance across the array, which can lead to non-uniformity in display's brightness. The non-uniformity often arises from the grain size distribution of polycrystalline organic semiconductors. On the other hand, OTFTs are more accommodating for LCD or E-paper because OTFTs act mainly as switches and the key performance requirement is high on/off current ratio.

Table 7.3. Reported display prototypes using OTFTs [12].

\begin{tabular}{|lllll|}
\hline Application & Semiconductor & Specification & Author Organization & Ref. \\
\hline E-paper & $\begin{array}{l}\text { Polyfluorene-based } \\
\text { polymer (inkjet printing) }\end{array}$ & $60 \times 80$ pixel on PET & $\begin{array}{l}\text { Plastic Logic (UK) \& E-ink } \\
\text { (USA) }\end{array}$ & {$[13]$} \\
E-paper & $\begin{array}{l}\text { Pentacene (solution- } \\
\text { process) }\end{array}$ & QVGA on PEN & Philips (Netherlands) & {$[14]$} \\
LCD & Pentacene & $\begin{array}{l}1.4 \text { in. } 80 \times 80 \text { RGB } \\
\text { on glass }\end{array}$ & Hitachi (Japan) & {$[15]$} \\
LCD & Pentacene & $64 \times 128$ on plastic & ERSO/ITRI (Taiwan) & {$[16]$} \\
LCD & Pentacene & $\begin{array}{l}12 \text { in. full color XGA } \\
\text { on glass }\end{array}$ & Samsung Elec. (Korea) & \\
OLED & Pentacene & $8 \times 8$ pixels on glass & Pioneer (Japan) & {$[17]$} \\
OLED & Pentacene & $4 \times 4$ pixels on PC & NHK (Japan) & {$[18]$} \\
\hline
\end{tabular}


In this thesis, the development of OTFT pixel circuits for active matrix display backplanes is of interest. OTFT-driven pixel circuits were developed and demonstrated; the circuit designs were inspired by existing pixel circuits built using a-Si:H TFTs [19]. Some of the pixel circuit architectures and results for OTFT-based pixel circuits are discussed in Section 7.3.5.

\subsubsection{RFID Tags}

In recent years, there has been significant interest in the development of radio frequency identification (RFID) tags for its versatile detecting and tracking capabilities. RFID technology provides an automatic way to collect product, place, time, or transaction data quickly and easily without human intervention or error. This technology is expected to dramatically improve automation, inventory control, distribution, shipment, tracking, and purchasing operations, provided they are cheap enough to be widely deployed [20]. Depending on the intended application, the RFID system will operate at different frequency bands, as listed in Table 7.4. In general, higher system frequency allows for longer read range, but with the trade-off of higher costs. For lower-end applications, RFID tags are viewed as a promising alternative to today's barcode technology. RFID tags can provide more comprehensive data collection/storage and can be read from a distance; in contrast, barcode provides limited identification information and requires in-line detection [20].

Table 7.4. Frequency bands and applications of RFID systems [20].

\begin{tabular}{|lll|}
\hline Frequency Band & $\begin{array}{l}\text { RFID System } \\
\text { Characteristics }\end{array}$ & Example Applications \\
\hline $\begin{array}{l}\text { Low: } \\
100-500 \mathrm{kHz}\end{array}$ & $\begin{array}{l}\text { Short read range, } \\
\text { Inexpensive }\end{array}$ & $\begin{array}{l}\text { Access control, Animal } \\
\text { identification, Inventory control }\end{array}$ \\
$\begin{array}{l}\text { Intermediate: } \\
10-15 \mathrm{MHz}(13.56 \mathrm{MHz})\end{array}$ & Medium read range & Access control, Smart cards \\
$\begin{array}{l}\text { High: } \\
850-950 \mathrm{MHz}, 2.4-5.0 \mathrm{GHz}\end{array}$ & $\begin{array}{l}\text { Long read range, High } \\
\text { reading speed, Line of sight } \\
\text { required, Expensive }\end{array}$ & $\begin{array}{l}\text { Railroad car monitoring, } \\
\text { Toll collection systems }\end{array}$ \\
\hline
\end{tabular}

A typical RFID system consists of a "reader" that uses an antenna to transmit radio energy to interrogate a "tag" or "transponder". In its simplest form, a RFID tag is composed of an antenna attached to an integrated circuit (IC). The IC carries information that identifies an item to which the 
tag is attached. Antenna coils are used to magnetically couple the RF energy from the reader into the tag. This energy, emitted by the reader, is used to power the tag and provide bi-directional communications between the reader and the tag. After extracting the data stored in the tag's IC, the retrieved information is directed back to the reader, from where it can be fed to a computer for processing [20]. The setup of a simple RFID system is shown in Figure 7.2(a). Figure 7.2(b) describes the major functional blocks inside a RFID tag.

Organic electronics technology is an appealing choice for RFID tags because it can enable the low-cost manufacturing of thin and flexible tags. At present, organic RFID tags are still in their early stage of development, with some initial progresses reported in [21][22][23][24]. OTFT-based RFID tags are targeted for inventory control and item-level tracking, which operate in the low to medium frequency ranges (refer to Table 7.4). For these lower-end applications, the logic circuitry of RFID tag (i.e., "Control Logic" component in Figure 7.2(b)) usually operates in the vicinity of $100 \mathrm{kHz}$ [21]. These data rates are expected to be deliverable by OTFTs. However, the RFID tag has a front end (i.e., "RF Interface" in Figure 7.2(b)) that must handle rectification and operate at the frequency (e.g., $13.56 \mathrm{MHz}$ ) of the incoming RF signal. The rectification stage at the interface needs to convert the absorbed RF energy into DC power to run to the entire RFID tag. Designing organic circuits to operate at this high frequency is a major challenge; this will require clever and innovative designs to overcome speed limitations of OTFTs.

(a)

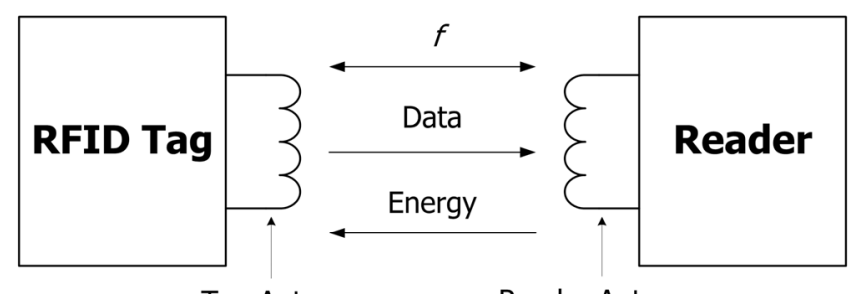

Tag Antenna

Reader Antenna

(b)

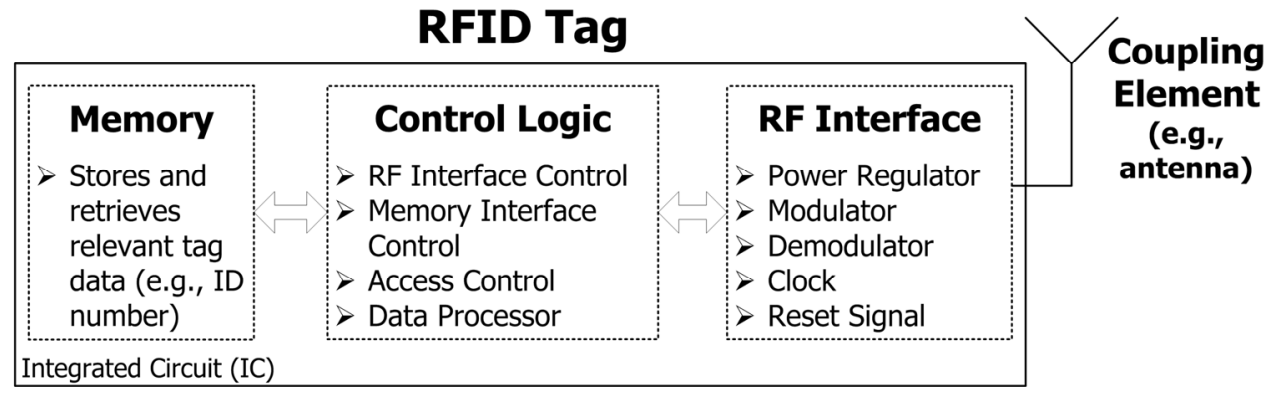

Figure 7.2. (a) Typical setup of a RFID system. (b) Key modules/components of a RFID tag [20][21]. 
Figure 7.3 illustrates a simple approach to implement the rectification stage of RFID tags, which uses diodes in a half-wave rectifying configuration. This configuration is commonly used by silicon-based RFID tags. However, organic semiconductor based diodes often fall short of the required performance necessary to establish sufficient rectification at high frequencies $(>100 \mathrm{kHz})$ and high voltages. In many cases, the peak input voltage in a tag can reach well over $50 \mathrm{~V}$ [22]. Thus, the rectification stage has to provide sufficient breakdown resistance at these levels, while maintaining a sufficient output voltage to operate the tag's logic circuitry. In order to provide sufficient forward bias current to run the circuit, the diode needs to be relatively large to compensate for the low vertical mobilities seen in most organic diodes. Rectification stages based on organic diodes have been reported by PolyIC [24].

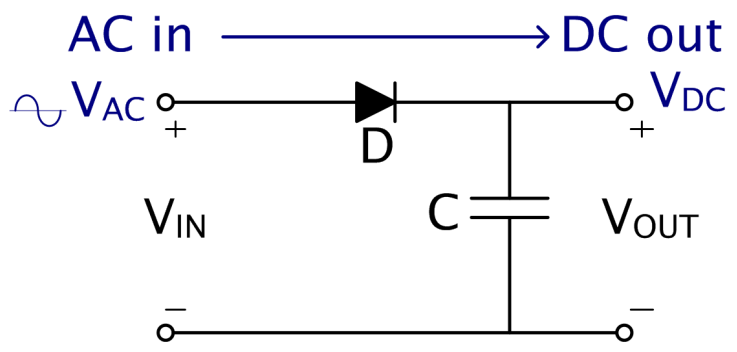

Figure 7.3. A simple diode-based half-wave rectification stage for RFID tag applications [22].

To address the limitations of organic-based diodes, the 3M Company introduced an $\mathrm{AC}$ powering scheme for the RFID tag that eliminated the rectification stage [22]. This concept is illustrated in Figure 7.4, where the 1-bit OTFT RFID tag uses AC power from a tuned antenna to directly power the logic circuitry, without an intermediate rectification stage. The RF interface of the tag consists of a LC resonant tank, which absorbs RF energy from the reader antenna to generate the AC power required for the tag circuitry. A basic property of the LC tank is its ability to store energy in the form of AC power that oscillates/resonates at a particular frequency, $f=1 /(2 \pi \sqrt{L C})$. A ring oscillator and NOR gate, powered directly from the LC tank, generates a pulse signal whenever the two input nodes of NOR gate are at logic level ' 0 '; this occurs once per cycle. This pulse signal is then buffered and sent to a large inverter at the output modulation stage. This output stage serves to amplitude modulate the RF signal absorbed by the tag's antenna according to the data stored in the tag. The modulated signal is subsequently detected by the reader, and relevant information can be retrieved. This 1-bit OTFT RFID tag design has been realized 
using pentacene OTFTs patterned with polymeric shadow masks and powered by near-field coupling at RF of $125 \mathrm{kHz}$ [22]. An 8-bit RFID transponder circuitry, based on an extension of this design, has also been reported [22]. Higher operating frequencies can be achieved with RFID tag using this AC powering scheme, when compared to RFID tag that uses organic diodes in a rectification stage configuration.

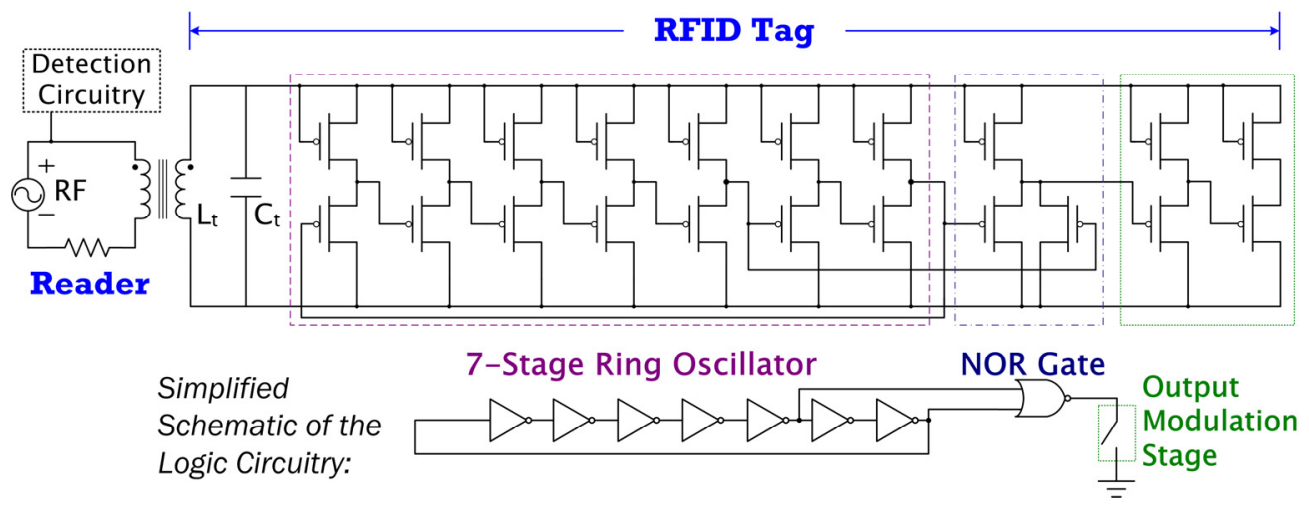

Figure 7.4. Circuit schematic of a 1-bit OTFT based RFID tag. Power is coupled into the tag circuit using inductive coupling between the reader and the tag. $L_{t}$ and $C_{t}$ form a resonant tank that powers the tag circuitry directly, without a separate rectification stage. The lower figure shows a scaled-down schematic of the logic circuitry [22].

The development of OTFT circuits for RFID tag applications is one of the interests for this doctoral research. To facilitate this development, immense research effort was dedicated to creating a reliable processing scheme for fabricating OTFT circuits. Fabrication of simple organic circuits including inverters, current mirrors, source followers and ring oscillators were recently completed. Preliminary experimental results are reported in the next section to demonstrate feasibility of our approach. By gaining a better understanding on the behavior of simple organic circuits, circuits of higher complexity (e.g., logic gates, memory) can be designed to account for non-idealities and metastabilities that are present in OTFT circuitries. Figure 7.5 outlines our strategic progression for the development of OTFT-based RFID tag circuitries. The key circuit components of a RFID tag (as identified in Figure 7.2(b)) are included in this diagram. Integration of these circuit components with an appropriate RF interface and antenna can provide the necessary technology to realize organic-based RFID tags. 


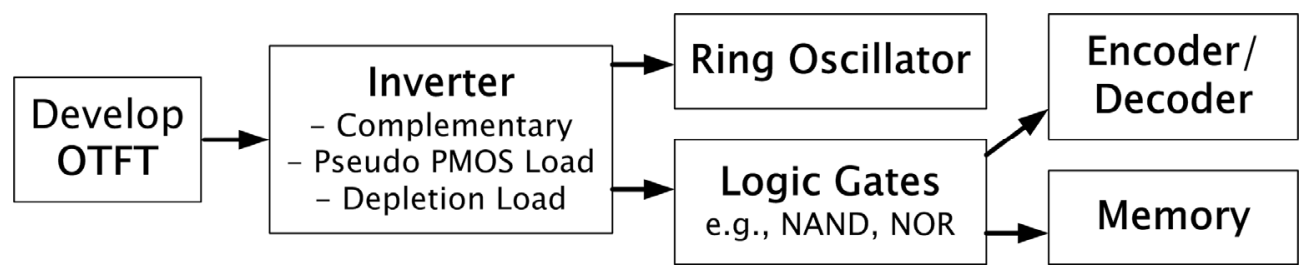

Figure 7.5. Development strategy for OTFT-based RFID tag circuitries for this thesis.

\subsection{Circuit Demonstration}

A key objective of this doctoral research is the integration of OTFTs into practical circuit applications. This necessitates a well-developed and reliable fabrication process that can consistently produce OTFTs with good performance, careful selection of material systems for optimal device performance, a systematic approach for device characterization, and a comprehensive model that can predict device behavior. Synergy between these research efforts (with results reported in the preceding chapters) enables the fabrication of simple OTFT circuits presented in this section. Preliminary experimental results are presented here for inverters, current mirrors, ring oscillators and various display pixel circuits to demonstrate the feasibility of our approach. Detailed circuit characterization, analysis, and optimization are needed to enable design and fabrication of more complex and application-specific circuits. These steps are crucial for promoting further advancements in organic electronics. The enormous potential to provide very low cost solutions makes organic integrated circuits a new, challenging frontier for electronics.

\subsubsection{Fabrication Schemes}

The OTFT circuits reported here were implemented with fully-patterned bottom-gate bottomcontact PQT-12 OTFTs. These circuits were fabricated using three processing schemes:

- Scheme \#1: 4-mask photolithography scheme with direct patterning of the organic semiconductor layer, where photoresist was deposited directly on the organic semiconductor for photolithographic patterning (see Figure 3.9).

- Scheme \#2: 4-mask photolithography scheme with indirect patterning of the organic semiconductor layer, where the organic semiconductor was passivated with a parylene buffer layer prior to photolithographic patterning (see Figure 3.9). 
- Scheme \#3: hybrid photolithography-inkjet printing scheme, where the organic semiconductor layer was deposited/patterned by inkjet printing (see Figure 3.14).

Detail discussion of these fabrication schemes are presented in Chapter 3. Each method has its own strengths and limitations. Device performance may vary depending on the fabrication scheme used, which can lead to slight variations in the circuit characteristics. Figure 7.6 illustrates a photograph of an array of OTFT circuits fabricated using the photolithography scheme with indirect patterning using parylene on a 3" glass wafer.

Please note the main objective of the following results is to demonstrate functional OTFT circuits, evaluate initial performance, and study feasibility of the fabrication approaches. The intent is to compile and integrate the knowledge/experience gained throughout this doctoral research on fabrication techniques, dielectric material optimization, interface modification and device characterization to produce organic circuits for practical applications. The results reported here are the first demonstrations, to-date, of PQT-12 OTFT circuits. As such, circuit performance has not been optimized, and there is definitely room for improvement.

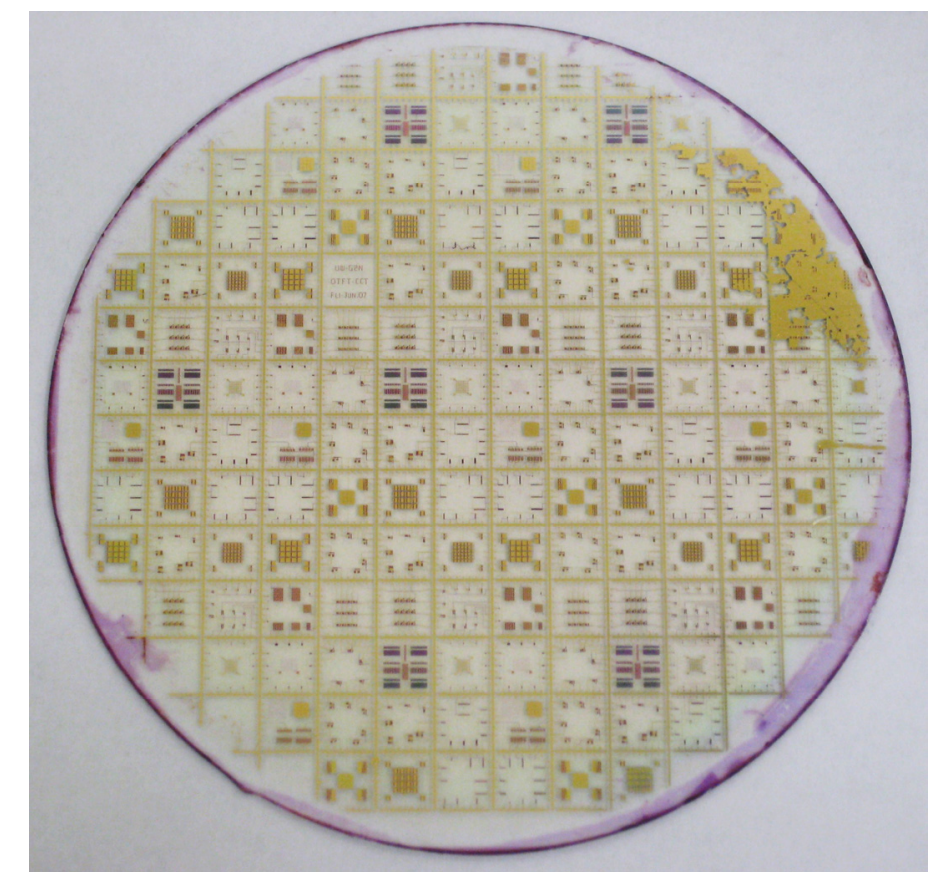

Figure 7.6. Photograph of a variety of OTFT circuits on a 3" glass wafer, fabricated using the integration schemes developed in this thesis. In this photo, circuits were fabricated using photolithography approach with parylene passivation (scheme \#2). 


\subsubsection{Inverters}

The simple inverter circuit is a key building block for many digital electronic circuits and for analog amplifying circuits. An inverter circuit outputs a voltage representing the opposite logiclevel or polarity as its input. Figure 7.7(a) displays a circuit schematic and top-view photograph of a PMOS inverter with saturated load, built using p-channel PQT-12 OTFT; its cross-sectional diagram is shown in Figure 7.7(c) to illustrate the interconnections. An alternative inverter design with depletion-mode transistor is displayed in Figure 7.7(b). In both designs, T1 serves as the driver (or input transistor) and T2 acts as load. In the ideal case, T1 is a transistor with infinite off resistance (for $\left|V_{\mathrm{GS}}\right|<\left|V_{\mathrm{T}}\right|$ ) and a finite on-resistance (for $\left|V_{\mathrm{GS}}\right|>\left|V_{\mathrm{T}}\right|$ ). When $V_{\mathrm{IN}}$ is at a voltage that turns $\mathrm{T} 1$ on (e.g., $\left.V_{\mathrm{IN}}=-40 \mathrm{~V}=V_{\mathrm{SS}}\right), V_{\mathrm{OUT}}$ is pulled up towards $V_{\mathrm{DD}}$ (i.e., $V_{\mathrm{IN}}=$ low, $V_{\mathrm{OUT}}=$ high). When $V_{\mathrm{IN}}$ is at a voltage that turns $\mathrm{T} 1$ off (e.g., $\left.V_{\mathrm{IN}}=40 \mathrm{~V}=V_{\mathrm{DD}}\right), V_{\mathrm{OUT}}$ is pulled down towards $V_{\mathrm{SS}}$ (i.e., $V_{\mathrm{IN}}=$ high, $V_{\mathrm{OUT}}=$ low). Thus, the inverter circuit operates in two stable states.

(a) Inverter with Saturated Load

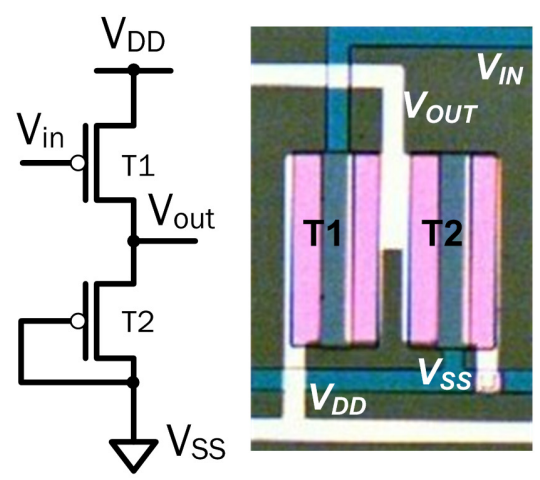

(b) Inverter with Depletion Load

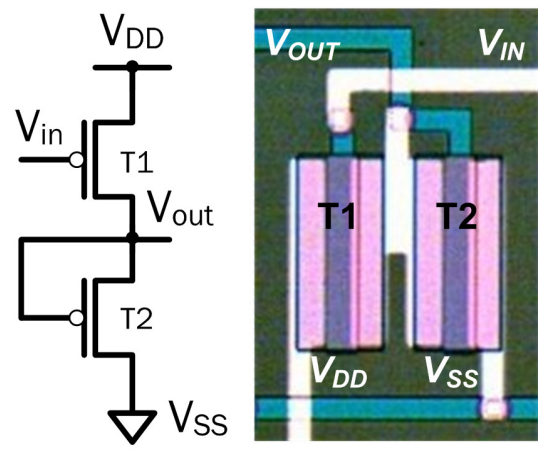

(c)

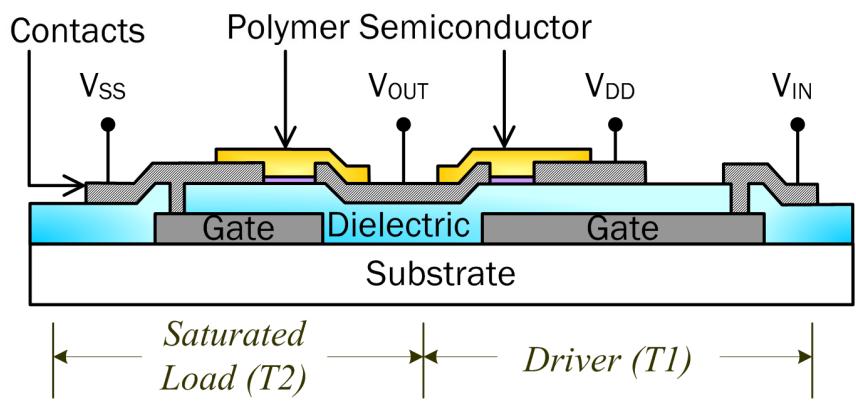

Figure 7.7. Schematic diagram and top-view photograph of OTFT inverter circuits with (a) saturation load and (b) depletion load. (c) Cross-section diagram of an inverter with saturated load. The photographs in (a) and (b) correspond to top-view layout of actual PQT-12 OTFT circuits $(W / L=200 \mu \mathrm{m} / 25 \mu \mathrm{m}$ for both $\mathrm{T} 1$ and $\mathrm{T} 2)$ fabricated by photolithography approach with parylene buffer/passivation layer (scheme \#2). 
The choice of load affects the inverter characteristics (e.g., gain, voltage level, noise margin) [25][26]. For the circuit in Figure 7.7(a) with a saturated load, $V_{\mathrm{GS} \_ \text {Load }}=V_{\text {DS_Load; }}$ thus, the load transistor is always in saturation $\left(\left|V_{\mathrm{GS}}-V_{\mathrm{T}}\right|<\left|V_{\mathrm{DS}}\right|\right)$ and gives a relatively constant/steady drain current. For the circuit in Figure 7.7(b) with depletion load, $V_{\mathrm{GS} \text { Load }}=0$; the load transistor is always "on" since the p-type OTFT typically has a positive turn-on voltage. Since $V_{\mathrm{GS} \_L a d}=0$ and $V_{\mathrm{DS} \_ \text {Load }}$ changes approximately between " $V_{\mathrm{DD}}-V_{\mathrm{SS}}$ " and $V_{\mathrm{SS}}$, the load transistor is subjected mainly to drain stress, which should experience much weaker bias-induced $V_{\mathrm{T}}$ shift (compared to gate induced bias stress) [27]. Reduction of bias-induced stress effects is crucial for stability and lifetime of OTFT circuits. Please refer to references [25] and [26] for detailed circuit analysis and comparison of the different inverter designs.

Figure 7.8 and Figure 7.9 shows the transfer voltage characteristics of a PQT-12 inverter with saturated load and with depletion load, respectively. These devices were fabricated using the photolithography scheme with photoresist passivation. Inversion operation is evident from these curves, and the inverter has sufficiently large gain. However, due to the positive switch-on voltage of PQT-12 OTFTs, the input and output levels do not match. Threshold voltage shift, leakage current, and the non-ideal subthreshold behavior of the OTFTs also contributed to deviation from the ideal inverter characteristics. Hysteresis was observed in the inverter characteristics, which is possibly due to mobile charges in the gate dielectric and material instability.

Figure 7.10 and Figure 7.11 present the voltage transfer characteristics of the PQT-12 OTFT inverter with saturated load, fabricated using photolithography with a parylene buffer/passivation layer and by inkjet printed PQT-12 layer, respectively. These results demonstrated the feasibility of fabricating functional OTFT inverter circuits using the various processing approaches developed in this research. The non-idealities observed in these electrical characteristics are due to lack of process optimization in these preliminary devices.

In summary, functional PQT-12 OTFT inverters are demonstrated here. To the author's knowledge, this is one of the first reports of PQT-12 inverter circuit in the scientific literature. These initial demonstrations provide a basic idea of the OTFT inverter characteristics. Device parameters can be extracted from these early experiments, which can be used to generate device models for circuit analysis and simulation purposes. With the ability to predict device and circuit behavior, proper circuit design techniques can be used (e.g., transistor sizing, layout) to create circuits with desirable inverter characteristics. Alternate circuit topography can be considered to 
address the shortcomings of OTFTs. For instance, to allow circuits to operate over a wide voltage range, regardless of switch-on voltage and hysteresis, inverters with active load and integrated level shifting can be considered [28]. A CMOS-type inverter, implemented with complementary p-type and n-type OTFTs, is expected to lead to improved device performance. Ambipolar OTFTs are a promising choice for implementing complementary logic circuits [17].

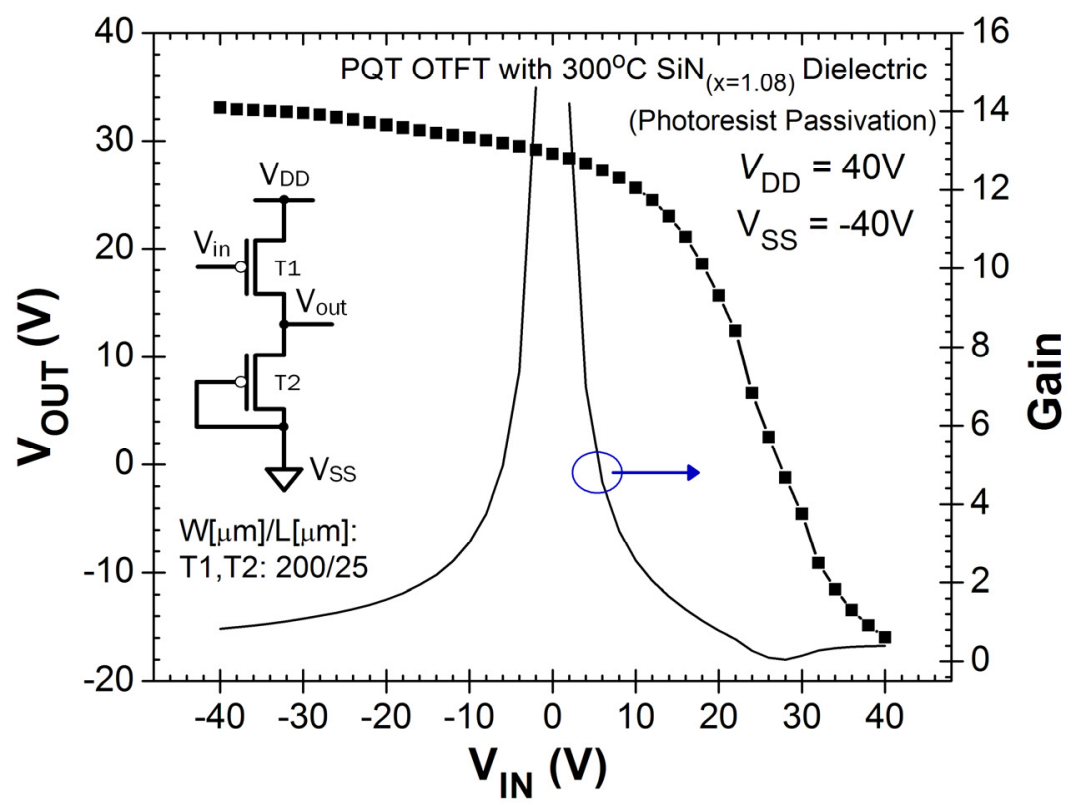

Figure 7.8. Voltage-transfer characteristics of a p-type inverter circuit with saturation load, fabricated using photolithographically-defined PQT-12 OTFT with photoresist as passivation (scheme \#1). 


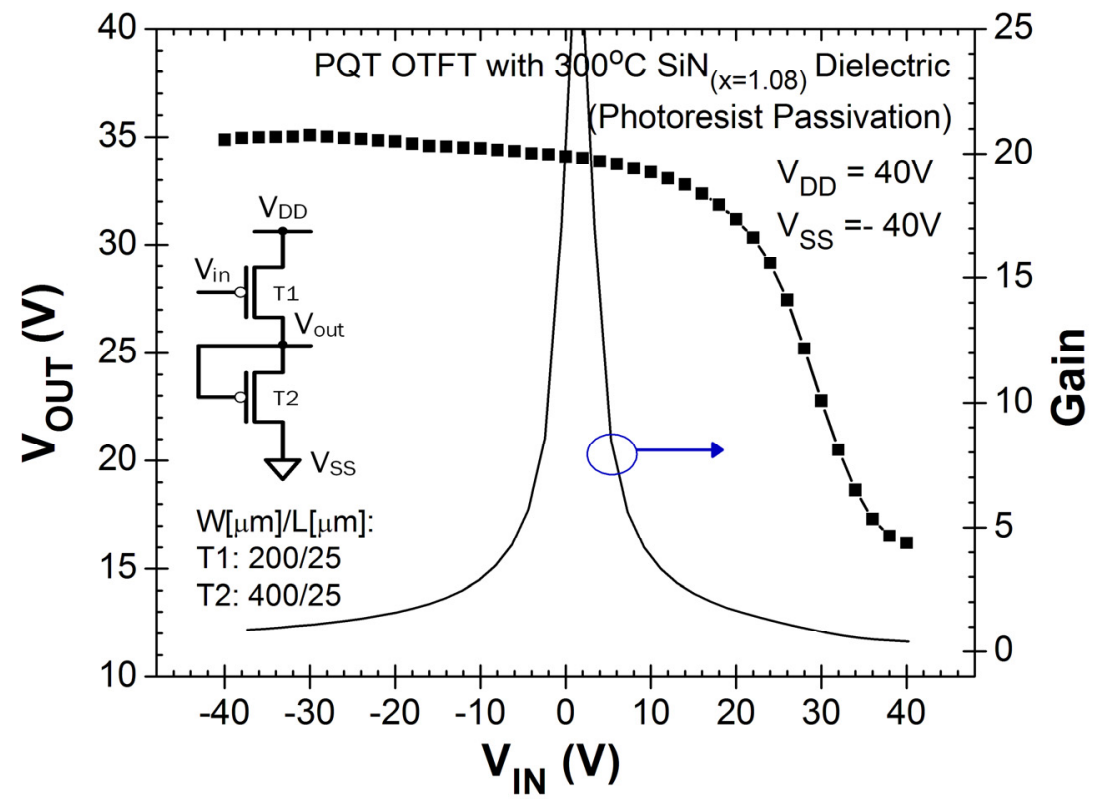

Figure 7.9. Voltage-transfer characteristics of a p-type inverter circuit with depletion load, fabricated using photolithographically-defined PQT-12 OTFT with photoresist as passivation (scheme \#1).

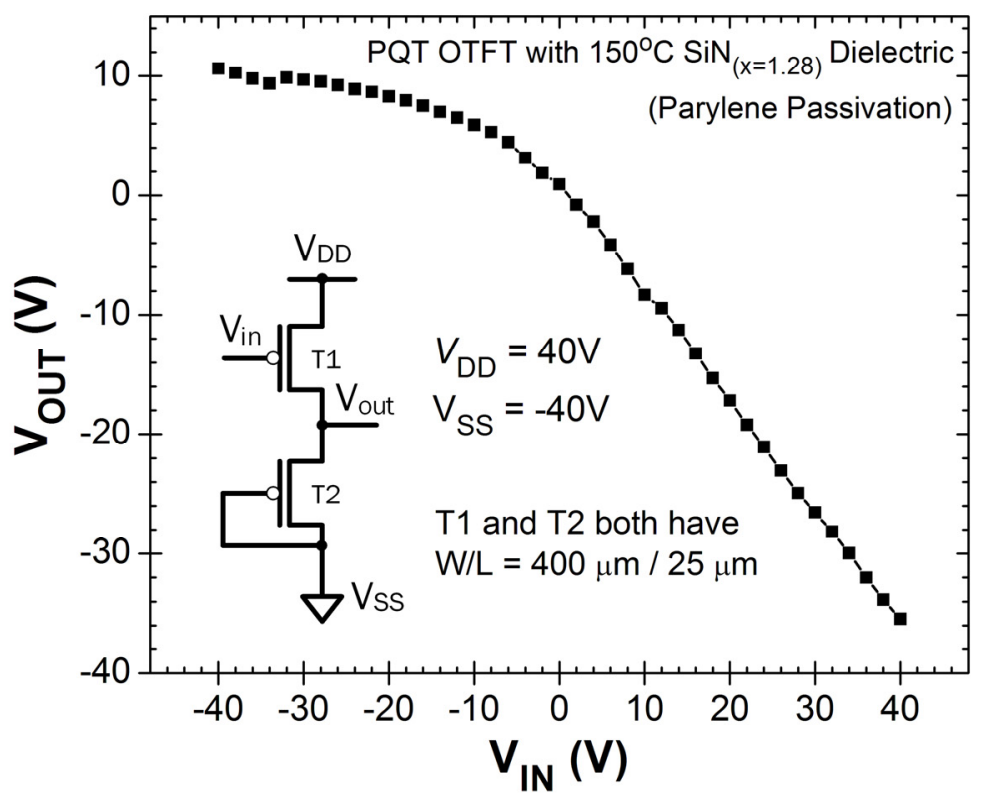

Figure 7.10. Voltage-transfer characteristics of a p-type inverter circuit with saturation load, fabricated using the photolithography approach with parylene passivation (scheme \#2). 


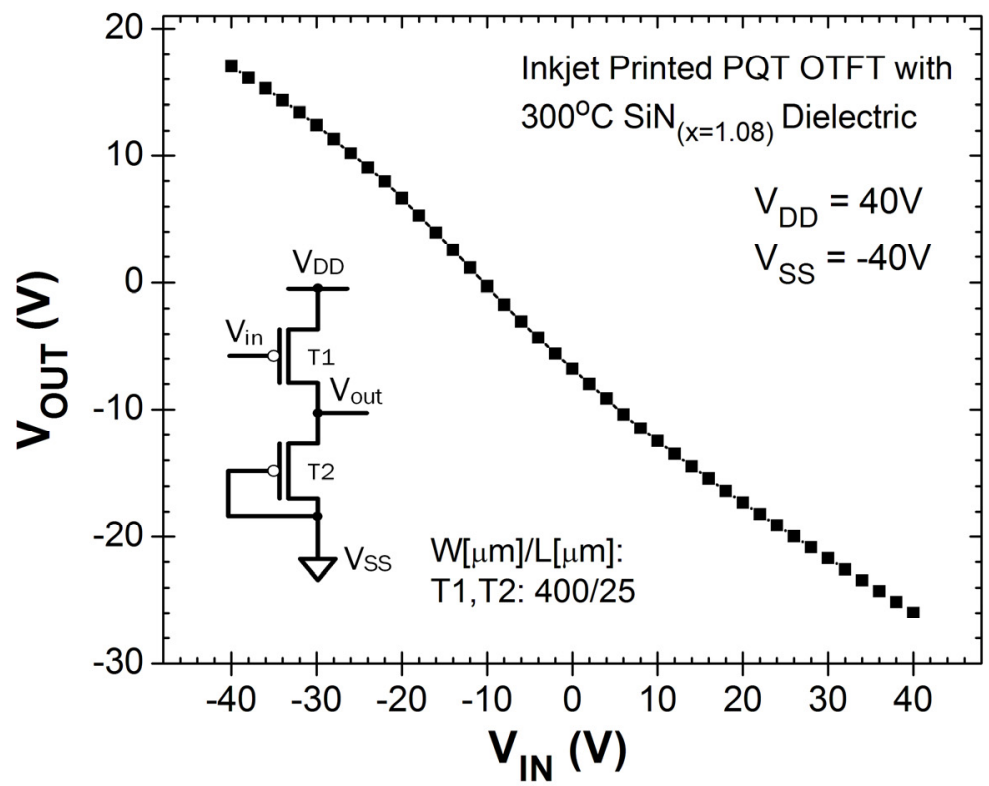

Figure 7.11. Voltage-transfer characteristics of a p-type inverter circuit with saturation load, fabricated with inkjet printed PQT-12 layer (scheme \#3).

\subsubsection{Current Mirrors}

Conceptually, an ideal current mirror is simply an ideal current amplifier. Figure 7.12 illustrates the simplest configuration of a MOS current mirror. In the ideal case,

$$
\frac{I_{\text {OUT }}}{I_{I N}}=\frac{(W / L)_{2}}{(W / L)_{1}},
$$

where $(W / L)_{1}$ and $(W / L)_{2}$ are the channel width to channel length ratio for $\mathrm{T} 1$ and $\mathrm{T} 2$, respectively. The ideal case assumes $\mathrm{T} 1$ and $\mathrm{T} 2$ are well matched (i.e., $k=\mu_{\mathrm{FE}} C_{\mathrm{i}}$ is identical) and $V_{\mathrm{DS}, 1}=V_{\mathrm{DS}, 2}$.

Figure 7.13 and Figure 7.14 display the transfer characteristics of PQT-12 OTFT current mirror circuits. These preliminary devices demonstrated "current mirror" or "current amplification" effects. The circuit in Figure 7.13 was constructed with two transistors of identical geometries, thus, theoretically, $I_{\mathrm{OUT}} / I_{\mathrm{IN}}=1$. The experimental value for $I_{\mathrm{OUT}} / I_{\mathrm{IN}}$ is 0.927 , which is in close proximity of theoretical value. The circuit in Figure 7.14 employed transistors with $(W / L)_{2} /(W / L)_{1}=$ 2 ; thus, theoretically, $I_{\mathrm{OUT}} / I_{\mathrm{IN}}=2$. This is well-matched with the experimental value of 1.856 . Therefore, proper current amplification function was achieved with these OTFT current mirror circuits. Deviation of the measured data from ideal transfer characteristics may derive from 
imperfections in the OTFT devices (e.g., large $V_{\mathrm{T}}$, leakage current, poor subthreshold slope, parasitic leakage, parasitic capacitances), and process-induced mismatches between devices (e.g., material non-uniformity, offset in mask alignment during photolithography exposure). Further process and design optimization should deliver improved device performance.

(a)

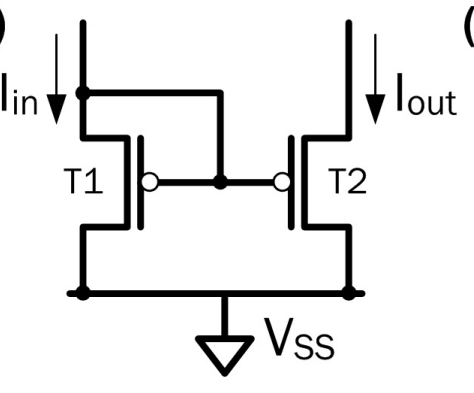

(b)

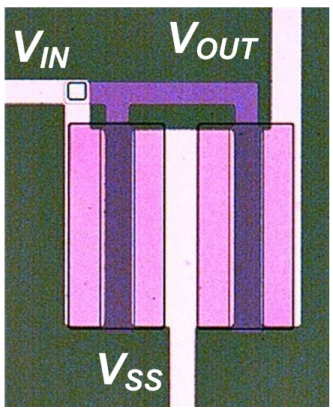

Figure 7.12. Current Mirror: (a) circuit schematic, and (b) micrograph showing top view of an actual circuit fabricated using hybrid photolithograph-inkjet method (scheme \#3). Photo shows $W / L=200 / 25$ for both $\mathrm{T} 1$ and $\mathrm{T} 2$.

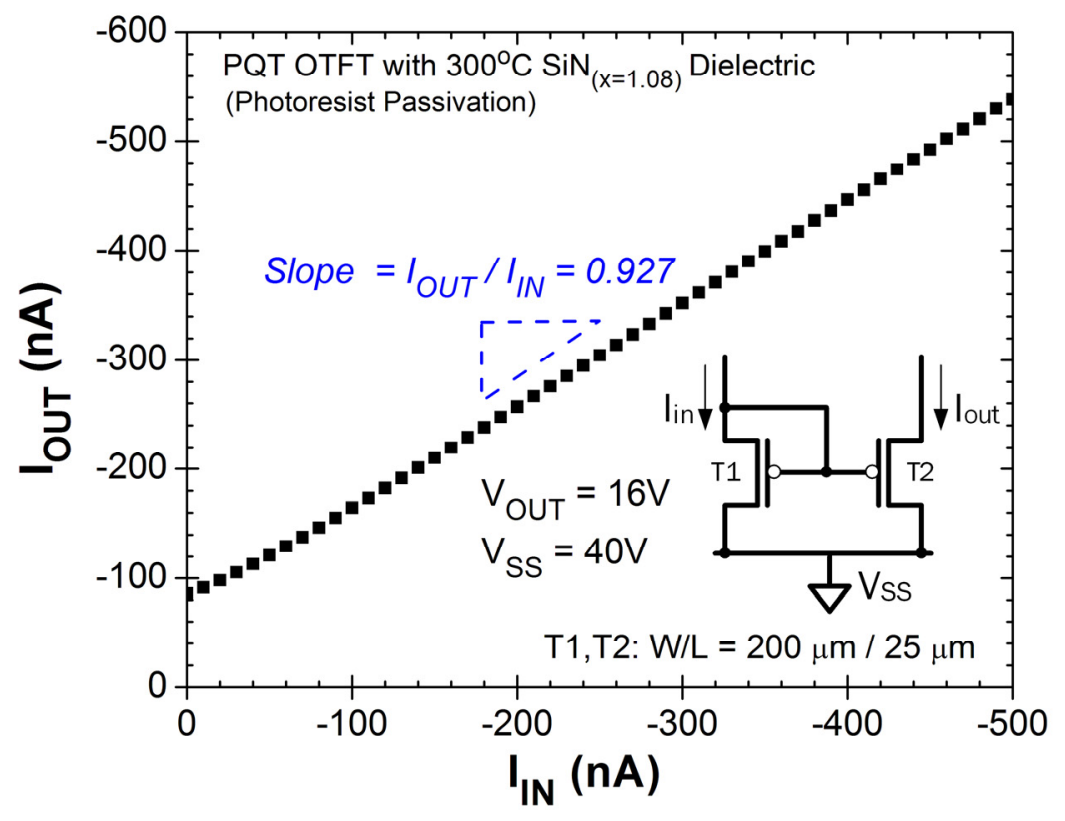

Figure 7.13. Transfer characteristic of current mirror circuits built using PQT-12 OTFTs on $\mathrm{SiN}_{\mathrm{x}}$ gate dielectric with $(W / L)_{2} /(W / L)_{1}=1$. Circuit was fabricated using the photolithography approach with photoresist passivation (scheme \#1). 


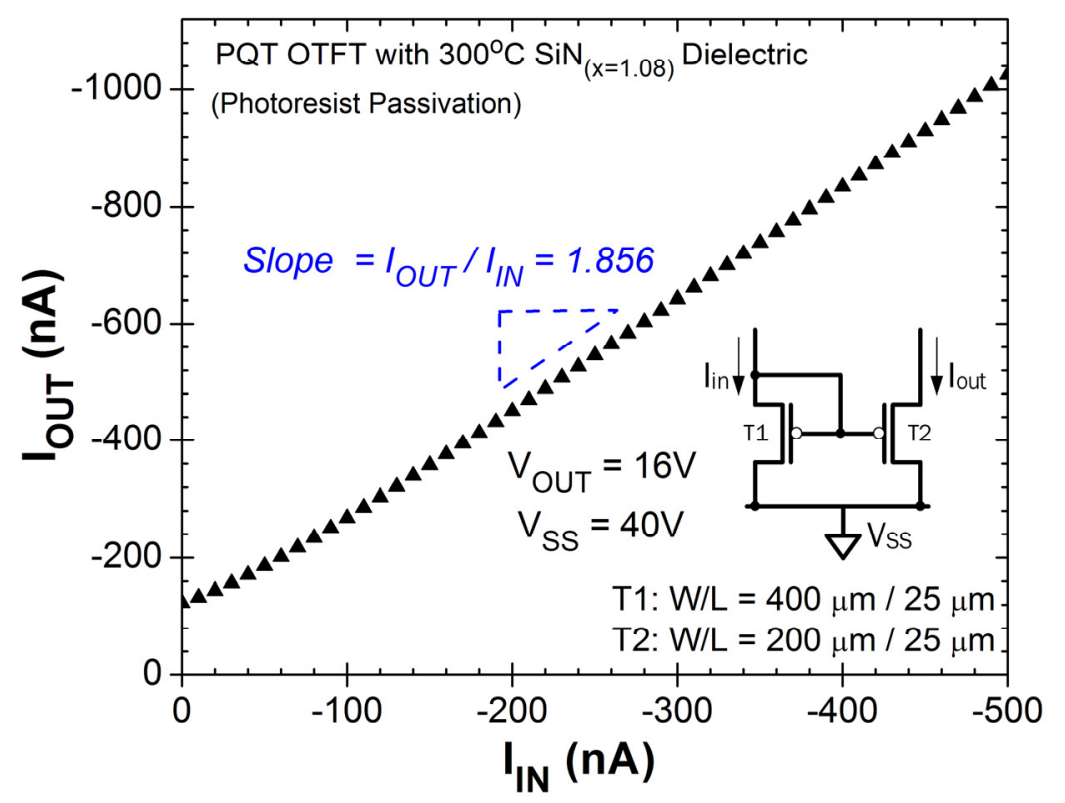

Figure 7.14. Transfer characteristic of current mirror circuits built using PQT-12 OTFTs on $\mathrm{SiN}_{\mathrm{x}}$ gate dielectric with $(W / L)_{2} /(W / L)_{1}=2$. Circuit was fabricated using the photolithography approach with photoresist passivation (scheme \#1).

\subsubsection{Ring Oscillators}

A ring oscillator is composed of an odd number of inverter stages, whose output oscillates between two voltage levels, representing high $\left(V_{\mathrm{DD}}\right.$, logic 1$)$ and low $\left(V_{\mathrm{SS}}\right.$, logic 0$)$. The inverters are connected in a chain, and the output of the last inverter is fed back into the first, as shown in Figure 7.15. Theoretically, a ring oscillator only requires power to operate; above a certain threshold voltage, oscillations begin spontaneously. The frequency of the oscillation depends on the gate delay of the inverter. To increase the frequency of oscillation, one may increase the applied voltage, use a smaller ring oscillator, adjust the $W / L$ of the transistors, or employ techniques to reduce gate delay of the inverter circuit. A ring oscillator circuit is commonly used to test the device delay for a given process. It can also be used to provide clocking functionality, which is a key element in RFID tags. 


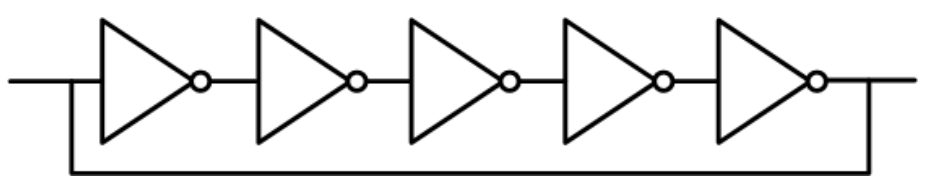

Figure 7.15. 5-stage ring oscillator symbol representation.

Figure 7.16 illustrates the ring oscillator circuits fabricated using PQT-12 OTFTs. Two types of inverter circuits were experimented for the implementation of a 5-stage ring oscillator: (a) inverter with saturation load, and (b) inverter with depletion load. Attempts were made to measure these circuits; however, preliminary testing was not possible due to limitations of the measurement setup and circuit performance. A higher sensitivity and lower capacitance probe is needed to avoid capacitive loading effects that might restrict accuracy measurements. An output buffer stage can be implemented to improve the sensitivity of the circuit/measurement setup.

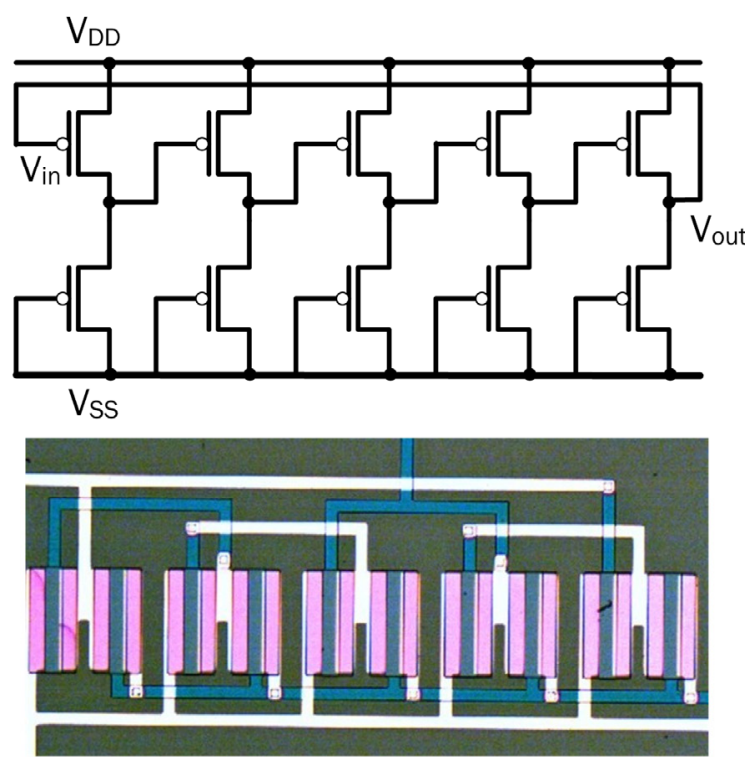

(a) Ring oscillator with saturation load

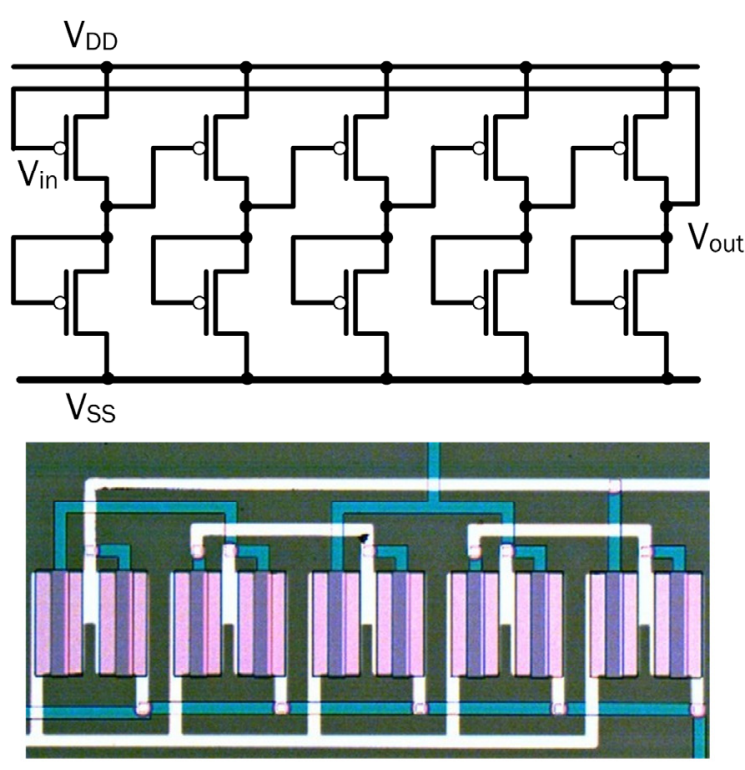

(b) Ring oscillator with depletion load

Figure 7.16. (a) 5-stage ring oscillator with saturation load. (b) 5-stage ring oscillator with depletion load. Circuit schematic and micrograph of the top-view of the fabricated structure (using scheme \#2) are also shown (W/L=200 $\mu \mathrm{m} / 25 \mu \mathrm{m}$ for the transistors shown). 


\subsubsection{Display Pixel Circuits}

Active matrix addressing, which is needed for high information content formats, involves a layer of backplane electronics based on TFTs to provide the bias voltage and drive current needed in each OLED pixel [19]. Figure 7.17 illustrates the concept of an active-matrix OLED (AMOLED) display backplane. OTFT backplane is a feasible candidate for small-area displays such as those needed in pagers, cell phones and other mobile devices, where performance and speed requirements are less stringent. The lower mobility associated with OTFTs can be compensated by scaling up the drive transistor in the pixel to provide the needed drive current, without necessarily compromising the aperture ratio. A number of AMOLED pixel circuits, originally designed for aSi:H TFT backplanes, are adapted to demonstrate the feasibility of OTFT pixel circuits in this research.

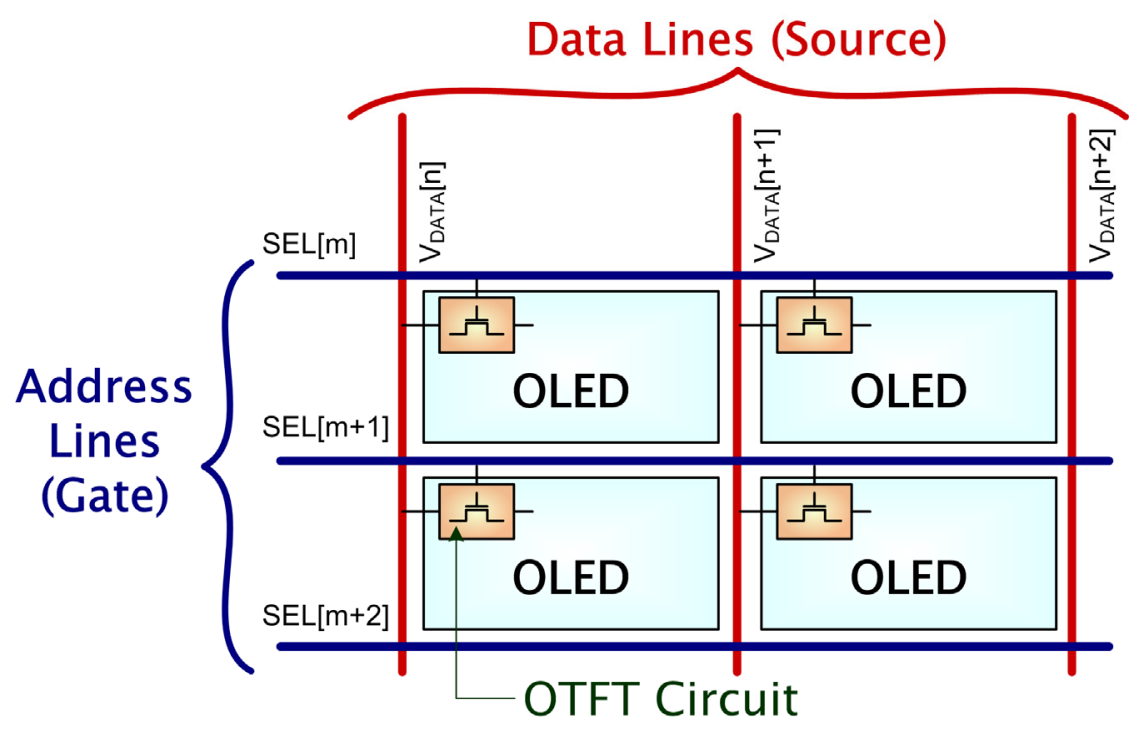

Figure 7.17. Active-matrix addressed backplane.

\subsubsection{Conventional 2-TFT Pixel Circuit}

The simplest pixel driver circuit is the 2-TFT voltage-programmed circuit shown in Figure 7.18. T2 serves as the switching transistor and T1 serves as the drive transistor. During the programming cycle, SEL is at a voltage that turns $\mathrm{T} 2$ on, and the voltage of $V_{\mathrm{DATA}}$ is transferred to $C_{\mathrm{S}}$ and charges up the gate node of T1 to a certain programming voltage $\left(V_{\mathrm{p}}\right)$. During the driving cycle, SEL is low and a current related to the programming voltage passes through the OLED. 
Figure 7.19 demonstrates the static operation of a conventional 2-T pixel circuit constructed with PQT-12 OTFTs, fabricated using a photolithographically defined polymer semiconductor layer with parylene buffer/passivation layer. When $V_{\mathrm{SEL}}=0 \mathrm{~V}, \mathrm{~T} 2$ turns on and $V_{\mathrm{DATA}}$ is passed to $C_{\mathrm{S}}$; this charges up $C_{\mathrm{S}}$ and turns on T1. As a result, T1 generates a current $I_{\mathrm{OUT}}$ to drive the OLED and the pixel is "on". On the other hand, when $V_{\mathrm{SEL}}=50 \mathrm{~V}$, T2 turns off; T1 should be off theoretically, and hence a smaller $I_{\text {OUT }}$ was measured. In this case, the pixel is considered "off". However, $I_{\text {OUT }}$ during off-state (i.e., $V_{\mathrm{SEL}}=50 \mathrm{~V}$ ) may originate from a number of sources, including leakage current through T2 which undesirably charges up $C_{\mathrm{S}}$, leakage current of $\mathrm{T} 1$, and residual charges in $\mathrm{C}_{\mathrm{S}}$ from previous programming cycle.

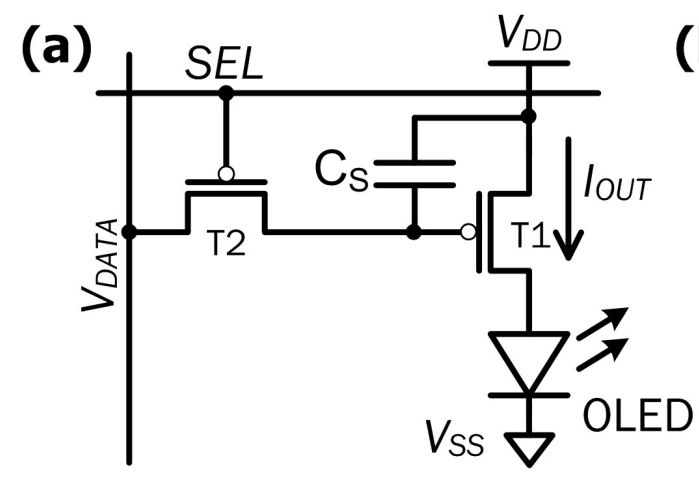

(b)

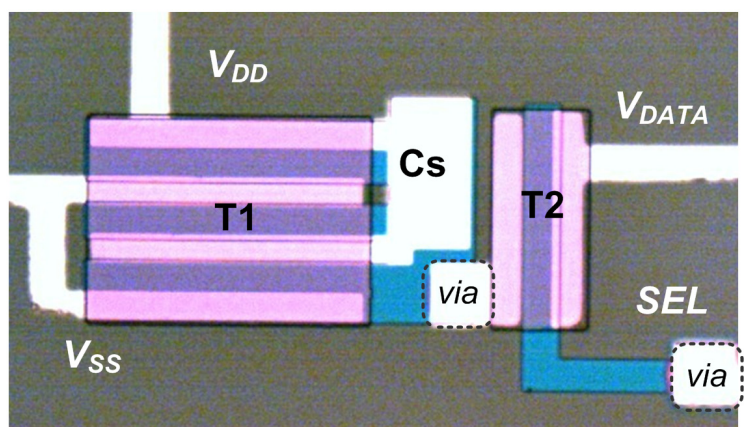

Figure 7.18. Conventional 2-TFT AMOLED pixel circuit: (a) schematic diagram, (b) micrograph of the top-view of the fabricated structure (using scheme \#2).

The same pixel circuit was fabricated using another photolithography scheme, whereby the PQT12 semiconductor layer was patterned directly by photoresist. The output characteristic of this circuit is displayed in Figure 7.20. Similar operation is observed here as explained above: when $V_{\mathrm{SEL}}=0 \mathrm{~V}$, pixel is "on" and a sizeable $I_{\mathrm{OUT}}$ conducts through OLED; when $V_{\mathrm{SEL}}=50 \mathrm{~V}$, pixel is "off" and a significantly smaller $I_{\mathrm{OUT}}$ is detected. Comparatively, the photoresist-passivated OTFT circuit (in Figure 7.20) has lower current drive than the parylene-passivated OTFT circuit (in Figure 7.19). As discussed in Chapter 3, devices fabricated using the photoresist-passivated approach are more susceptible to process-induced degradation, and hence, reduced device performance. $\mathrm{AC}$ or timing measurements are in progress to provide a more in-depth evaluation of the circuit performance.

Key advantages of this conventional 2-T voltage-programmed pixel circuit include its simplicity, ability to accommodate high aperture ratio displays (due to low transistor count per pixel), and low 
power. However, this simple design does not compensate for the $V_{\mathrm{T}}$ shift that is inherent in OTFTs. $\Delta V_{\mathrm{T}}$ is most pronounced in the drive TFT (i.e., T1) of an OLED pixel due to its continuous "on" state operation. The bias stress causes $\left|V_{\mathrm{T}}\right|$ to increase over time, and leads to a reduction in the drive current. These changes translate to a gradual decrease in brightness of the OLED with time, which undesirably degrades the quality of the display. Therefore, a circuit that can compensate for $\Delta V_{\mathrm{T}}$ is required to maintain the performance and improve the lifetime of the OTFT-driven OLED display. Pixel circuits with built-in compensation function are presented next.

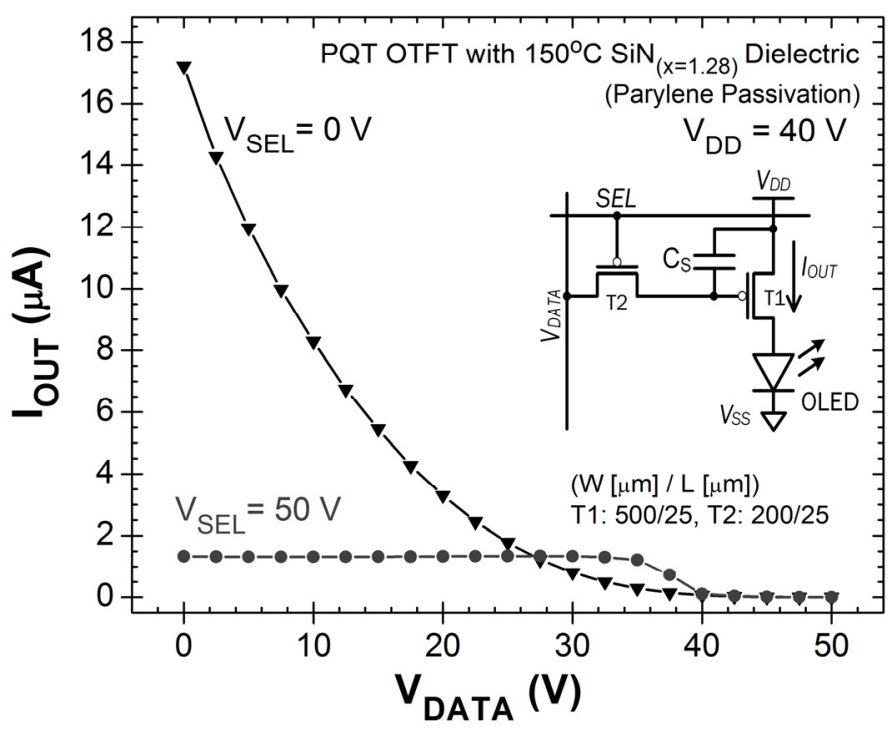

Figure 7.19. Current-voltage characteristic of a conventional 2-T pixel circuit for AMOLED, built using PQT-12 OTFTs on $\mathrm{SiN}_{\mathrm{x}}$ gate dielectric. Circuit was fabricated using the photolithography approach with parylene passivation (scheme \#2). 


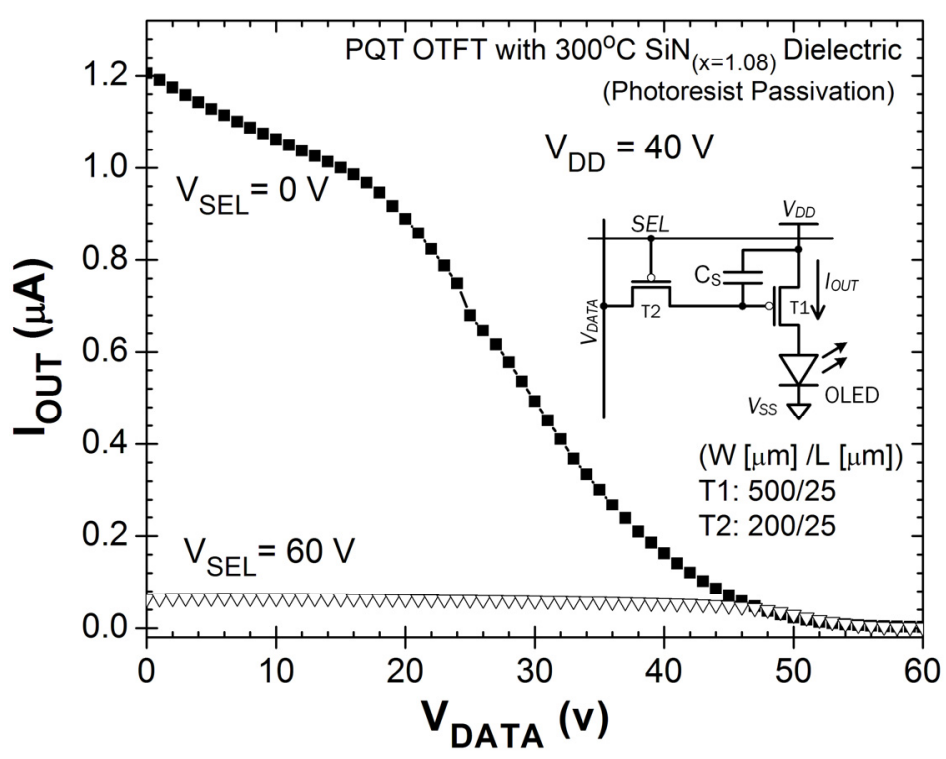

Figure 7.20. Current-voltage characteristic of a conventional 2-T pixel circuit for AMOLED, built using PQT-12 OTFTs on $\mathrm{SiN}_{\mathrm{x}}$ gate dielectric. Circuit was fabricated using the photolithography approach with photoresist passivation (scheme \#1).

\subsubsection{Compensating 2-TFT Pixel Circuit}

Although the conventional 2-TFT AMOLED voltage-programmed pixel circuit can provide high resolution and high yield, this 2-TFT pixel circuit is prone to image retention over time due to $V_{\mathrm{T}}$ shift in the OTFTs. An alternative stable driving scheme that can compensate for $V_{\mathrm{T}}$ shift in the 2TFT pixel circuit is considered here, derived from [30]. This compensating driving scheme not only preserves the simplicity of the 2-TFT pixel, it also demonstrates high uniformity and improved stability. Figure 7.21(a) presents a schematic of the compensating 2-TFT pixel circuit. A diode-connected TFT (TLD) and a capacitor $\left(C_{\mathrm{LD}}\right)$ are used to emulate the OLED. Since TLD is subjective to bias stress, its threshold voltage may shift, resembling OLED voltage shift in practical circuits [30]. PQT-12 OTFTs were used to implement this compensating 2-TFT pixel circuit design; a top-view microphotograph of a fabricated circuit is depicted in Figure 7.21(b). 
(a)

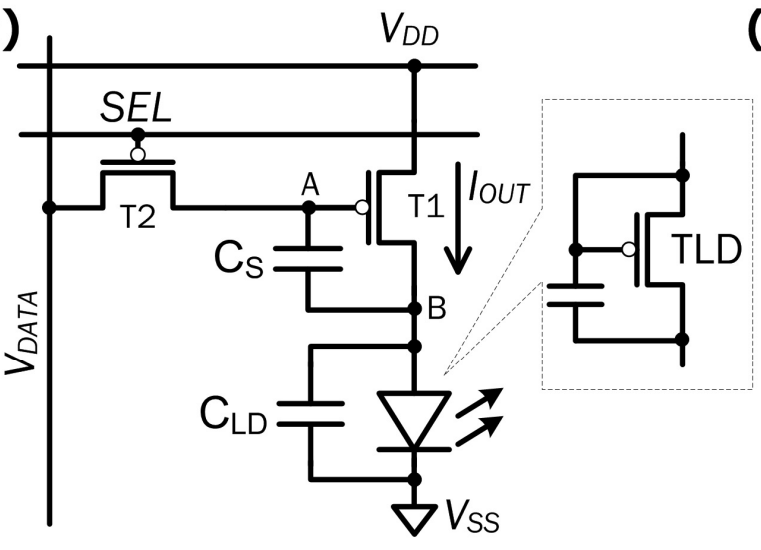

(b)

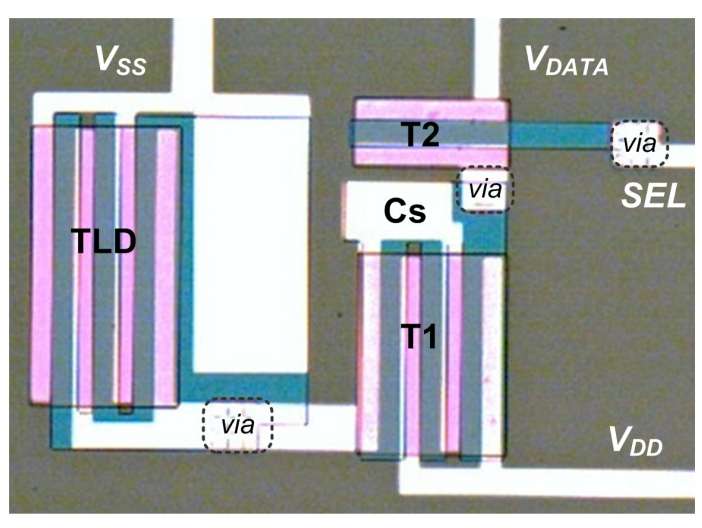

Figure 7.21. Compensating 2-TFT AMOLED pixel circuit: (a) schematic diagram, (b) micrograph of the top-view of the fabricated structure (using scheme \#2). A diode-connected TFT (TLD) and a capacitor $\left(\mathrm{C}_{\mathrm{LD}}\right)$ are used to emulate the OLED, Also, since the TLD is under stress, its threshold voltage increases, which resembles the OLED voltage shift.

Figure 7.22 presents the static output characteristics of the compensating 2-T pixel circuit constructed with PQT-12 OTFTs, fabricated using a photolithographically defined polymer layer with parylene buffer/passivation layer. When $V_{\mathrm{SEL}}=-10 \mathrm{~V}, \mathrm{~T} 2$ turns on and $V_{\mathrm{DATA}}$ is passed to $C_{\mathrm{S}}$; this charges up $C_{\mathrm{S}}$ and turns on T1. As a result, T1 generates a current $\mathrm{I}_{\mathrm{OUT}}$ to drive the OLED and the pixel is "on". On the other hand, when $V_{\mathrm{SEL}}=50 \mathrm{~V}, \mathrm{~T} 2$ turns off; hence, a small $I_{\mathrm{OUT}}$ was measured. In this case, the pixel is considered "off". $I_{\mathrm{OUT}}$ during off-state (i.e., $V_{\mathrm{SEL}}=50 \mathrm{~V}$ ) can be due to a number of factors: leakage current through $\mathrm{T} 2$ which undesirably charges up $C_{\mathrm{S}}$, leakage current of $\mathrm{T} 1$, residual charges in $C_{\mathrm{S}}$ from previous programming cycle. Overall, the plot shows proper operation of the circuit. 


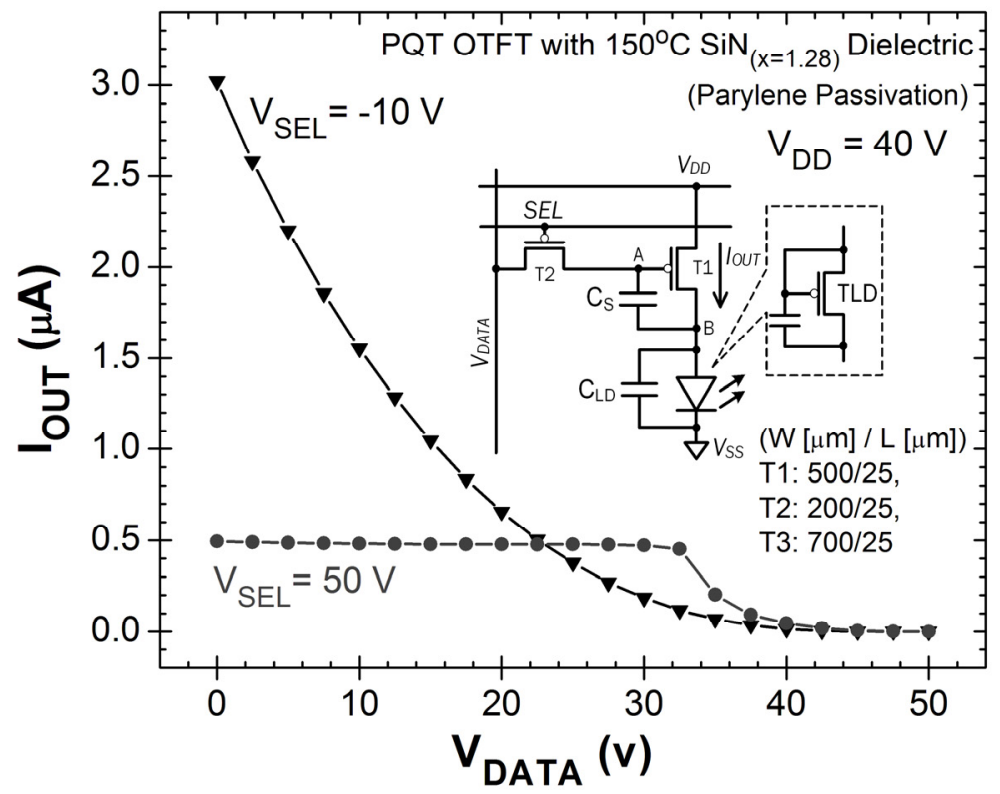

Figure 7.22. Input-output transfer characteristic of a compensating 2-T pixel circuit for AMOLED, built using PQT-12 OTFTs on $\mathrm{SiN}_{\mathrm{x}}$ gate dielectric. Circuit was fabricated using the photolithography approach with parylene passivation (scheme \#2).

To demonstrate the true benefits of this compensating pixel circuit, timing and stability measurements are required. The sophisticated driving scheme of this pixel is reported in [30]. Unfortunately, timing/stability data are unavailable at the time of thesis writing due to a number of experimental/ measurement limitations. One major challenge is related to wire bonding and packaging of OTFT circuit dies in dual in-line package (DIP) for circuit testing using a test board with properly-designed driving circuits. The standard wire bonding recipes caused degradation in OTFT performance, possibly due to stress and/or handing. Efforts to tailor a bonding recipe for OTFT circuits are currently underway. More discussion on back-end processing for organic electronics is available in Section 7.4.2.

A similar compensating circuit was implemented using a-Si TFT technology. Experimental results indicated that this compensating 2-TFT pixel circuit and its corresponding driving scheme delivered improved stability compared to the conventional 2-T pixel circuit [30]:

- After 15 days of operation, OLED current degradation in the new driving scheme was less than $11 \%$, compared to over $50 \%$ degradation for the conventional driving scheme.

- After a $70 \%$ change in the temperature, the current remained approximately constant in the new driving scheme, compared to up to $300 \%$ increase in current in the conventional driving 
scheme. The current the new driving scheme is less sensitive to temperature variations due to an internal feedback mechanism.

Therefore, the compensating 2-TFT pixel circuit offers improved stability against bias stress induced $V_{\mathrm{T}}$ shift in TFT and voltage shift of the OLED. Its simple 2-TFT configuration is favorable for displays demanding high aperture ratio. The main disadvantage is its complex driving scheme, and thus it is not as suitable for large area displays. Timing and stability measurements are in progress to provide a more in-depth evaluation of the circuit performance.

$4 \times 4$ arrays of PQT-12 OTFT pixel circuits have been fabricated using conventional 2-TFT pixel circuit design and the compensating 2-TFT pixel circuit design. Figure 7.23 displays a photograph from a section of such $4 \times 4$ array. Electrical measurements are in progress.

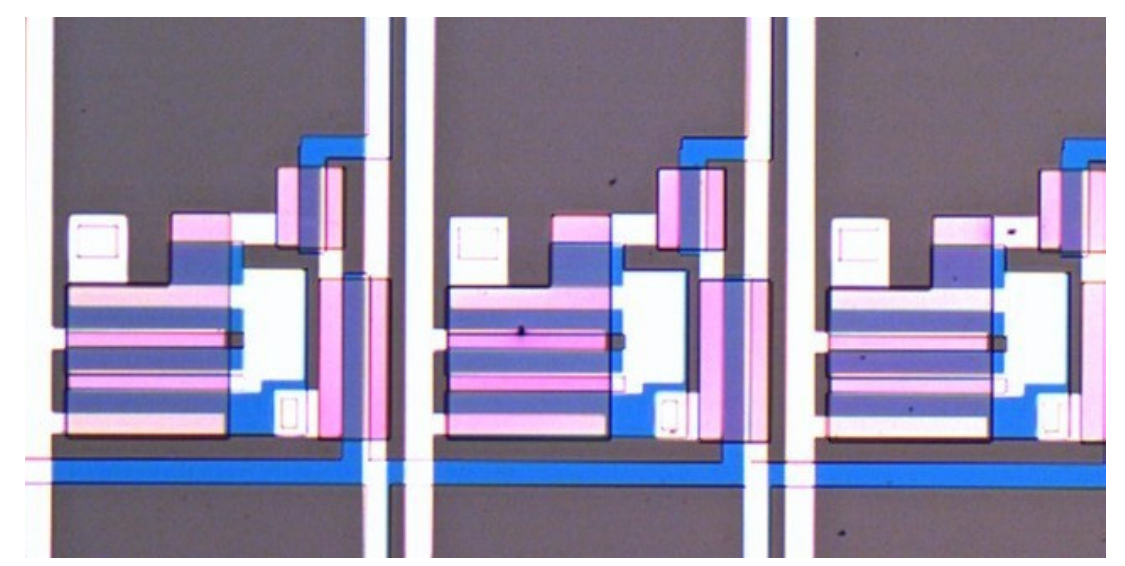

Figure 7.23. Photograph showing a section of a $4 \times 4$ array of AMOLED PQT-12 OTFT pixel circuits, based on compensating 2-TFT circuit design (using fabrication scheme \#2).

\subsubsection{4-TFT Current Mirror Pixel Circuit}

To overcome the problem of OLED current degradation due to bias induced $V_{\mathrm{T}}$-shift in the conventional 2-TFT pixel circuit, a current-programmed pixel circuit based on the current mirror circuit family can be used [31]. Figure 7.24(a) illustrates a current mirror-based $V_{\mathrm{T}}$-shift compensated 4-TFT OLED pixel circuit. T1 and T2 are drive transistors that constitute a current mirror pair, and T3 and T4 are switch transistors. When SEL becomes high (i.e., a pixel is selected by gate drivers), T3 and T4 conduct. Initially, $I_{\mathrm{DATA}}$ flows through T4 and charges up the storage capacitor $C_{\mathrm{S}}$ until T2 starts to conduct. The gate voltage of T2 keeps increasing until all of $I_{\mathrm{DATA}}$ passes through T3 and T2. This current is then mirrored to pass through $\mathrm{T} 1$ since the gates of $\mathrm{T} 1$ and T2 are connected. Thus, the OLED gets the desired current [19]. In this manner, the drive TFT 
does not have to remain "on" continuously, thus reducing the effects of bias-induced $V_{\mathrm{T}}$-shift. Furthermore, since $V_{\mathrm{GS}}$ of both drive TFTs is the same, the threshold voltages of T1 and T2 will shift equally and the output current will not be affected. Therefore, the OLED current in this circuit is independent of threshold voltage or mobility variation in the drive TFT provided it stays in the saturation region of operation.
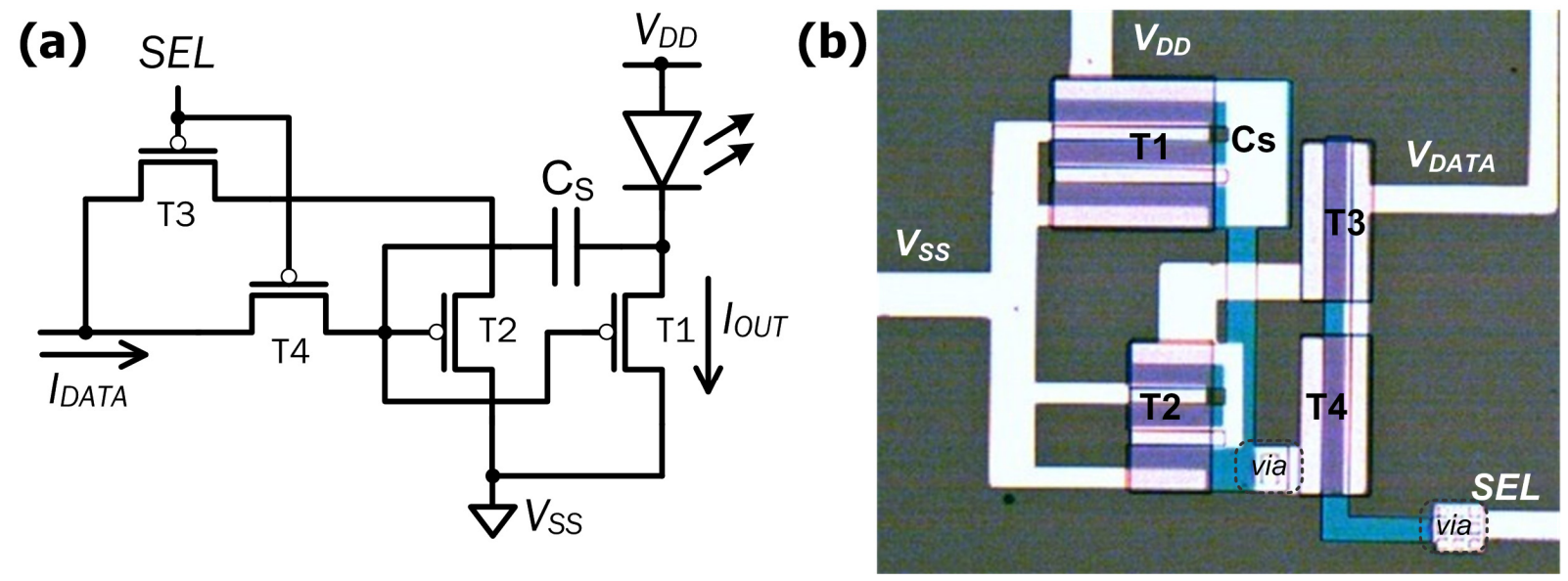

Figure 7.24. 4-TFT current mirror AMOLED pixel circuit: (a) schematic diagram, (b) photomicrograph of the top-view of the fabricated structure (using scheme \#2).

A 4-TFT current mirror pixel circuit has been fabricated using PQT-12 OTFTs. A photomicrograph displaying the top-view of the fabricate circuit is given in Figure 7.24(b). Electrical characterization was in progress at the time of thesis writing. Experimental results for a 4-OTFT pixel circuits will be reported in a scientific publication in the near future.

In summary, this current-programmed current mirror-based $V_{\mathrm{T}}$-shift compensated 4-TFT OLED pixel circuit presents advantages in terms of OLED current stability, insensitivity to $V_{\mathrm{T}}$ and $V_{\mathrm{OLED}}$, as well as insensitivity to temperature variation. The major shortcoming is that current programming is slow, thus this design is not suitable for medium and large area displays. A number of novel TFT pixel circuit designs and pixel addressing schemes have recently been proposed for a-Si TFT backplane technology to deliver enhanced performance [19][30][31][32][33][34]. Since a-Si and organic semiconductors share a number of similar traits (in which both are disordered semiconductors), one should adapt these innovative pixel circuit designs for OTFT technology. This will provide a means to gain better understanding on the strengths/weaknesses of OTFT pixel circuits, which will assist the development of reliable and high-performance OTFT pixel circuits for integration with AMOLED or other display backplanes. 


\subsection{Summary, Contributions and Outlook}

Organic circuits constructed using fully-patterned PQT-12 OTFTs with PECVD gate dielectric have been demonstrated. The results on organic circuits presented in this thesis are one of the first/early demonstrations of PQT-12 organic circuits in the scientific literature. Although further process optimization is necessary to improve circuit performance, the preliminary results here signify a promising outlook for the integration of OTFTs into practical electronic applications in the near future. These outcomes also confirmed the practicality of the photolithography and inkjet printing fabrication approaches for organic circuit implementation. As a next step, in addition to improving material, device and circuit performance, two novel recommendations to advance OTFT technology are proposed below.

\subsubsection{Active-Matrix Backplane Integration}

Following successful demonstration and evaluation of various OTFT pixel circuit designs, subsequent research efforts can be devoted to optimization of OTFT pixel circuits and development of OTFT pixel arrays for realization of display backplanes. The application of pixel circuits based on dual-gate OTFTs for vertically-integrated display backplanes is an interesting concept to explore, as depicted in Figure 7.25. In AMOLED displays, the data is stored in the pixel using a TFT circuit, which connects the OLED display device to the address, data, and power supply lines. In such applications, the aperture ratio, which is defined as the ratio of the active light-emitting area to the total pixel area, is a critical performance parameter. Higher aperture ratio is desirable as it improves the lifetime of the OLED, enhances the device efficiency, and ensures display quality [35]. One approach for maintaining high aperture ratio, even in the case of higher on-pixel integration density, is to vertically stack the active layers of the OLED on the backplane electronics, as illustrated in Figure 7.25. However, the presence of a continuous back electrode can induce a parasitic channel in TFTs, giving rise to high leakage current. A dual-gate TFT structure can effectively reduce this leakage component, because the voltage on the top-gate can be chosen to minimize the parasitic conduction induced in the TFTs [35][50]. Therefore, the use of dual-gate OTFTs for vertical integration of display applications can potentially improve the aperture ratio of the device, and permit shielding parasitic effects in the vertically integrated backplane electronics. 


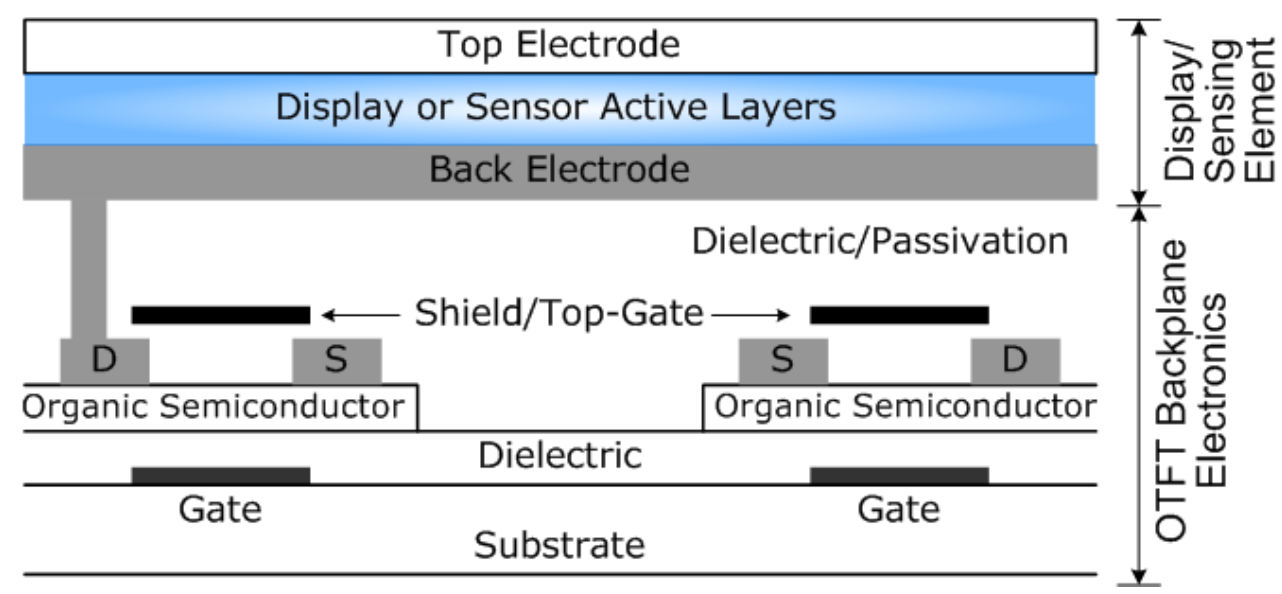

Figure 7.25. Architecture of a vertically integrated AMOLED display, featuring pixels with dualgate OTFTs.

Likewise, this vertical integration concept can lead to an intriguing implementation of sensor arrays for a variety of applications. Discrete OTFT-based gas sensors [37], light sensors, biosensors [38], temperature and respiration sensors for health monitoring [39], and humidity sensors [40], have been reported. Array implementation of these OTFT sensors using vertically stacked structures is expected to lead to enhanced sensitivity and/or aperture ratio. More interestingly, the concept of active-matrix arrays can be extended to nanocomposite TFT devices. Nanocomposite TFTs based on mixtures of polymer semiconductors, carbon nanotubes, Si nanowires, $\mathrm{ZnO}$ nanowires have been fabricated [41][42]. Unique properties of nanocomposite materials will open up new and exciting opportunities for diversified application areas.

\subsubsection{Back-End Process Integration: Bonding \& Packaging}

Most of the current/existing research in organic electronics has been directed towards front-end processing, which includes fabrication of transistors and circuits. To deliver a total manufacturing package for commercialization and deployment of organic electronics products, solutions for OTFT-compatible back-end process integration are in demand. Back-end processes include wafer dicing, die attaching/bonding, wire bonding, and encapsulation. In today's microelectronics industry, integrated circuit (IC) packaging is the final stage of semiconductor device fabrication, followed by IC testing. Packaged integrated circuits are mounted on a printed circuit board (PCB), 
where on-board or peripheral driving circuits are designed to deliver the necessary electronic device/system functionality.

In this research, while attempting to wire-bond and package OTFT circuits in dual in-line package (DIP) for extensive electrical testing (see Figure 7.26), degradation in OTFT performance was observed. Standard back-end processing recipes, from silicon ICs packaging, were applied. Experiments revealed that organic thin film materials are sensitive to these standard back-end processing conditions, including mechanical stress, pressure, sonication, heating, solvents, and handling. Efforts to tailor the wire bonding recipe for OTFT circuits are currently underway; initial process optimization showed improved device characteristics (i.e., less degradation). More research effort is needed to develop OTFT-specific back-end integration processes, where the sensitivities/requirements of organic electronics materials are properly addressed.

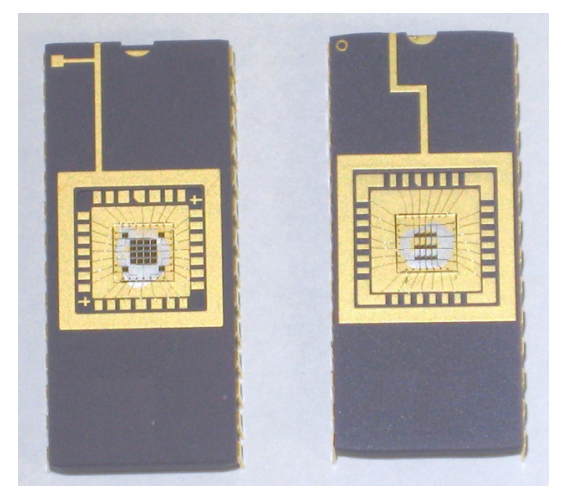

Figure 7.26. Photograph of packaged and wire-bonded OTFT circuits in a dual inline package (DIP). 


\section{Bibliography}

[1] E. Cantatore, "Organic Materials: A New Chance for Electronics?" Proceedings of the SAFE/IEEE Workshop, 27, November 2000.

[2] A. Nathan, "Large area thin film electronics", University of Waterloo, Waterloo, Ontario (2001).

[3] W. Clemens, W. Fix, J. Ficker, A. Knobloch, and A. Ullmann, "From polymer transistors toward printed electronics," J. Mater. Res. 19(7), 1963, (2004).

[4] A. Facchetti, "Organic semiconductors for organic transistors," Materials Today 10(3), 37 (2007).

[5] N. Stutzmann, R. H. Friend, and H. Sirringhaus, "Self-aligned, vertical-channel, polymer field-effect transistors," Science 299, 1881 (2003).

[6] Y. Chen and I. Shih, "Fabrication of vertical channel top contact organic thin film transistors," Organic Electronics 8, 655 (2007).

[7] T. Kawase, H. Sirringhaus, R.H. Friend et al., "Inkjet printing of polymer thin film transistors," Thin Solid Films 438, 279 (2003).

[8] G.H. Gelinck et al., "Flexible active-matrix displays and shift registers based on solutionprocessed organic transistors," Nat. Mat. 3, 106 (2004).

[9] H. Sirringhaus, N. Tessler, and R.H. Friend, "Integrated Optoelectronic Devices Based On Conjugated Polymers," Science 280, 1741 (1998).

[10] J.A. Rogers, Z. Bao, M. Meier, A. Dodabalapur, O.J.A. Schueller, and G.M. Whitesides, "Printing, molding, and near-field photolithographic methods for patterning organic lasers, smart pixels and simple circuits," Syn. Metals 115, 5 (2000).

[11] Z.L. Li, S.C. Yang, H.F. Meng, Y.S. Chen, Y.Z. Yang, C.H. Liu, S. F. Horng, C.S. Hsu, L.C. Chen, J.P. Hu, and R.H. Lee, "Patterning-free integration of polymer light-emitting diode and polymer transistor," Appl. Phys. Lett. 84(18), 3558 (2004).

[12] J. Jang, and S.H. Han, "High-performance OTFT and its application," Current Appl. Phys. 6, e12-e21 (2005).

[13] K. Reynolds, S. Burns, M. Banach, T. Brown, K. Chalmers, N. Cousins, L. Creswell, M. Etchells, C. Hayton, K. Jacobs, A. Menon, C. Ramsdale, W. Reeves, J. Watts, T. von Werne, J. Mills, C. Curling, H. Sirringhaus, K. Amundson, and M.D. McCreary, "Printing of Polymer Transistors for Flexible Active Matrix Displays," Proceedings of the 11th International Display Workshops (IDW '04), p. 367 (2004).

[14] E. Van Veenendaal, L. Schrijnemakers, M. Van Mil, P. Van Lieshout, F. Touwslager, G. Gelinck, and E. Huitema, "Rollable Displays and Integrated Drivers Based on Organic Electronics," Proceedings of the 11th International Display Workshops (IDW '04), p. 371 (2004).

[15] M. Ando, M. Kawasaki, S. Imazeki, Y. Sekiguchi, S. Hirota, H. Sasaki, S. Uemura, T. Kamata, in: The Proceedings of the 1st International TFT Conference (ITC '05), p. 178 (2005). 
[16] J.C. Ho, L.Y. Huang, T.S. Hu, C.C. Hsieh, W.K. Hwang, Y.W. Wang, W.L. Lin, H.Y. Cheng, T.H. Lin, M.C. Hsiao, Y.K. Wang, P.S. Wu, and C.C. Lee, Society for Information Display 2004 (SID’04), p. 1298 (2004).

[17] T. Chuman, S. Ohta, S. Miyaguchi, H. Satoh, T. Tanabe, Y. Okuda, M. Tsuchida, Society for Information Display 2004 (SID ’04), p. 45 (2004).

[18] Y. Inoue, Y. Fujisaki, T. Suzuki, S. Tokito, T. Kurita, M. Mizukami, N. Hirohata, T. Tada, S. Yagyu, "Active-Matrix OLED Panel Driven by Organic TFTs," Proceedings of the 11th International Display Workshops (IDW ’04), p. 335 (2004).

[19] A. Nathan, A. Kumar, K. Sakariya, P. Servati, K.S. Karim, D. Striakhilev, and A. Sazonov, "Amorphous Silicon Back-Plane Electronics for OLED Displays," IEEE J. Sel. Top. Quantum Electronics 10(1), 58 (2004).

[20] (a) www.research.ucla.edu/whatsbruin/2004/7/orfid/; (b) www.hi-point.com/how.html .

[21] R. Rotzoll, S. Mohapatra, V. Olariu, R. Wenz, M. Grigas, O. Shchekin, K. Dimmler, and A. Dodabalapur, "13.56 MHz Organic Transistor Based Rectifier Circuits for RFID Tags," Mater. Res. Soc. Symp. Proc. 871E, I11.6.1 (2005).

[22] (a) P.F. Baude, D.A. Ender, M.A. Haase, T.W. Kelley, D.V. Muyres, S.D. Theiss, "Pentacene-based radio-frequency identification circuitry," Appl. Phys. Letts., 82(22), 3964 (2003).

(b) P.F. Baude, D.A. Ender, T.W. Kelley, D.V. Muyres, and S.D. Theiss, "Pentacene-based RFID transponder circuitry," 62nd Device Research Conference (DRC) Digest, pp. 227 (2004)

[23] (a) V. Subramanian, P.C. Chang, J.B. Lee, S.E. Molesa, and S.K. Volkman, "Printed Organic Transistors for Ultra-low-cost RFID Applications," IEEE Polytronics 2004, pp. 67-71 (2004).

(b) D. Redinger, S. Molesa, R. Farshchi, and V. Subramanian, “An Ink-Jet Deposited Passive Components for RFID", IEEE Trans. on Electron Devices 51(12), 1978 (2004).

[24] D. Zipperer, W. Clemens, A. Ullmann, M. Boehm and W. Fix, "Polymer Based Rectifiers and Integrated Circuits for Printable RFID Tags," 2005 Mater. Res. Soc. Spring Meeting, Symposium I: Organic Thin-Film Electronics, I11.5 (2005).

[25] D.A. Neamen, Electronic Circuit Analysis and Design, $2^{\text {nd }}$ Edition, McGraw-Hill Science Engineering (2001).

[26] R.C. Jaeger and T. Blalock, Microelectronic Circuit Design, $2^{\text {nd }}$ Edition, McGraw Hill (2003).

[27] S.J. Zilker, C. Detcheverry, E. Cantatore, and D.M. de Leeuw, "Bias stress in organic thinfilm transistors and logic gates," Appl. Phys. Lett. 79, 1124 (2001).

[28] H. Klauk, M. Halik, U. Zschieschang, F. Eder, G. Schmid, and C. Dehm, "Pentacene organic transistors and ring oscillators on glass and on flexible polymeric substrates," Appl. Phys. Lett. 82, 4175 (2003).

[29] J. H. Schon, S. Berg, C. Kloc, and B. Batlogg, "Ambipolar Pentacene Field-Effect Transistors and Inverters," Science 287, 1022 (2000).

[30] G.R. Chaji and A. Nathan, "A Stable Voltage-Programmed Pixel Circuit for a-Si:H AMOLED Displays,” J. Display Tech. 2, 347 (2006). 
[31] A. Nathan, D. Striakhilev, P. Servati, K. Sakariya, A. Sazonov, S. Alexander, S. Tao, C.-H. Lee, A. Kumar, S. Sambandan, S. Jafarabadiashtiani, Y. Vygranenko, and I.W. Chan, "a-Si AMOLED Display Backplanes on Flexible Substrates," Mat. Res. Soc. Symp. Proc. 814, I3.1.1 (2004).

[32] G.R. Chaji and A. Nathan, "A Novel Driving Scheme for High-Resolution Large-Area aSi:H AMOLED Displays," IEEE $48^{\text {th }}$ Midwest Symposium on Circuit and Systems, 782 (2005).

[33] G.R. Chaji and A. Nathan, "Parallel Addressing Scheme for Voltage-Programmed ActiveMatrix OLED Displays," IEEE Trans. Elect. Device 54, 1095 (2007).

[34] A. Nathan, G. R. Chaji, and S. J. Ashtiani, "Driving schemes for a-Si and LTPS AMOLED displays," J. Display Technol. 1, , 267 (2005).

[35] P. Servati, S. Prakash, A. Nathan, and C. Py, "Amorphous silicon driver circuits for organic light-emitting diode displays," J. Vac. Sci. Technol. A 20, 1374 (2002).

[36] P. Servati, K.S. Karim, and A. Nathan, "Static Characteristics of a-Si:H Dual-Gate TFTs," IEEE Trans. Electron Devices 50, 926 (2003).

[37] J.B. Chang, V. Liu, V. Subramanian, K. Sivula, C. Luscombe, A. Murphy, J. Liu, and J.M.J. Fréchet, "Printable polythiophene gas sensor array for low-cost electronic noses," J. Appl. Phys. 100, 014506 (2006).

[38] G.M. Farinola, L. Torsi, F. Naso, P.G. Zambonin, L. Valli, M.C. Tanese, O. Hassan Omar, G. Giancane, F. Babudri, and F. Palmisano, "Chemical design, synthesis and thin film supramolecular architecture for advanced performance chemo- and bio-sensing organic field effect transistors," 2nd International Workshop on Advances in Sensors and Interface, pp.1-2 (2007).

[39] S. Jung, T. Ji, and V.K Varadan, "Point-of-care temperature and respiration monitoring sensors for smart fabric applications," Smart Mater. Struct. 15, 1872 (2006).

[40] Z.T. Zhu, J.T. Mason, R. Dieckmann, and G.G. Malliaras, "Humidity sensors based on pentacene thin-film transistors," Appl. Phys. Lett. 81, 4643 (2002).

[41] P. Beecher, P. Servati, A. Rozhin, A. Colli, V. Scardaci, S. Pisana, T. Hasan, A. J. Flewitt, J. Robertson, G. W. Hsieh , F. M. Li, A. Nathan, A. C. Ferrari and W. I. Milne, Ink-jet printing of carbon nanotube thin film transistors", J. Appl. Phys. 102, 043710 (2007).

[42] G.W. Hsieh, P. Beecher, F.M. Li, P. Servati, A. Colli, A. Fasoli, D. Chu, A. Nathan, B. Ong, J. Robertson, A.C. Ferrari, and W.I. Milne, "Formation of composite organic thin film transistors with nanotubes and nanowires," Physica E: Low-dimensional Systems and Nanostructures (2007) (Article in Press). 
Chapter 8. Conclusions

\section{Chapter 8}

\section{Conclusions}

Development of organic functional material has been an active area of research in the past two decades, and novel materials continue to evolve to bring new heights to device performance and functionality. Intrigued by their processability advantages, compatibility with large area flexible substrates and their unique material properties with potentially multi-dimensional/versatile functionalities, organic electronic materials can bring exciting and revolutionizing opportunities for flexible, light weight, low cost and disposable electronics. The objective of this thesis was to advance the area of OTFT research from an engineering and integration perspective, in which existing materials and techniques were exploited/engineered to deliver higher device performance and to demonstrate the feasibility of practical organic circuits. These objectives were addressed through experimental research in the following theme areas:

$\phi$ Device performance: optimization of PECVD gate dielectric properties and investigation of interface engineering methodologies to enhance PQT-12 OTFT performance;

$\phi$ Device manufacture: development of OTFT fabrication strategies to enable reliable device fabrication and circuit integration;

$\phi$ Device integration: demonstration of integration of PQT-12 OTFTs into functional circuits for active-matrix display and RFID applications.

The major outcomes from these investigations are highlighted next. 


\section{Device Performance}

With the aim to improve device performance and enhance field-effect mobility by tuning the gate dielectric composition and the interface properties, the following conclusions were drawn from the investigations of bottom-gate bottom-contact PQT-12 OTFTs on PECVD gate dielectrics:

$\phi$ The film composition of silicon nitride $\left(\mathrm{SiN}_{\mathrm{x}}\right)$ gate dielectric affects the electrical performance of PQT-12 OTFTs. Overall improvement in mobility, on/off current ratio, and gate leakage current was observed as silicon content in the $\mathrm{SiN}_{\mathrm{x}}$ gate dielectric increases. Interface characterization confirmed that silicon-rich $\mathrm{SiN}_{\mathrm{x}}$ gate dielectric, with appropriate surface modifications, presents a more desirable dielectric-semiconductor interface (with higher contact angle, lower surface roughness, and more Si-O bonds) for OTFT fabrication than nitrogen-rich $\mathrm{SiN}_{\mathrm{x}}$ gate dielectric.

$\phi \quad$ OTFTs fabricated with $150^{\circ} \mathrm{C}$ PECVD $\mathrm{SiN}_{\mathrm{x}}$ gate dielectric showed improved mobility and on/off current ratio compared to devices with $300^{\circ} \mathrm{C} \mathrm{SiN}$ gate dielectric. PQT-12 OTFTs on plastic substrates with $150^{\circ} \mathrm{C} \mathrm{SiN}_{\mathrm{x}}$ gate dielectric were demonstrated.

$\phi$ OTFTs with PECVD $\mathrm{SiO}_{x}$ gate dielectric showed higher mobility $\left(0.34 \mathrm{~cm}^{2} / \mathrm{V}-\mathrm{s}\right)$ than on PECVD $\mathrm{SiN}_{\mathrm{x}}$, indicating superior $\mathrm{SiO}_{\mathrm{x}}$-PQT interface properties. However, PECVD $\mathrm{SiO}_{\mathrm{x}}$ was more susceptible to dielectric breakdown at lower fields and higher gate leakage current. Further material optimization is necessary to strengthen the electrical integrity of $\mathrm{SiO}_{\mathrm{x}}$ films. PQT-12 OTFTs on plastic substrate with $180^{\circ} \mathrm{C}$ PECVD $\mathrm{SiO}_{\mathrm{x}}$ gate dielectric were demonstrated.

$\phi$ Interface treatment of PECVD $\mathrm{SiN}_{\mathrm{x}}$ gate dielectric by a combination of $\mathrm{O}_{2}$ plasma exposure and OTS SAM delivered the highest field-effect mobility for PQT-12 OTFT, in comparison to untreated $\mathrm{SiN}_{\mathrm{x}}, \mathrm{O}_{2}$-plasma-only-treated-SiN $\mathrm{x}$, and OTS-only-treated $\mathrm{SiN}_{\mathrm{x}}$. Interface characterization showed that the combination of $\mathrm{O}_{2}$ plasma and OTS treatments rendered a dielectric surface with large contact angle (i.e., low surface energy), low surface roughness, and abundance of Si-O bonds, which are desirable interface qualities for high performance OTFTs.

$\phi$ Investigation on the impact of exposure duration of $\mathrm{O}_{2}$ plasma for dielectric surface treatment on OTFT performance revealed a "turn-round" effect, where device characteristics (i.e., mobility and on/off current ratio) peaked at an exposure duration of $\sim 80$ seconds. $\mathrm{SiN}_{\mathrm{x}}$ dielectric surface treated with "80 seconds of $\mathrm{O}_{2}$ plasma exposure and OTS SAM" displayed the largest contact angle, lowest surface roughness, and largest 
concentration of Si-O bonds, when compared to samples subjected to either shorter or longer $\mathrm{O}_{2}$ plasma treatment. These surface attributes coincided with the peak device performance observed at 80 seconds of $\mathrm{O}_{2}$ plasma exposure, where $\mu_{\mathrm{FE}}$ as high as 0.22 $\mathrm{cm}^{2} / \mathrm{V}$-s was achieved. In addition, device mobility increased with higher RIE power of the $\mathrm{O}_{2}$ plasma exposure.

$\phi$ For devices with dielectric surface pretreatments, addition of 1-octanethiol contact treatment led to a reduction in device mobility and an increase in contact resistance. 1octanethiol SAM may be creating a charge injection barrier at the contact-semiconductor interface. Therefore, 1-octanethiol SAM treatment should be excluded for PQT-12 OTFTs fabricated using the process outlined in this thesis.

Altogether, with regards to the experimental conditions considered here, it was concluded that the combination of PECVD $\mathrm{SiN}_{\mathrm{x}}$ dielectric composition and surface treatment recipe that produces the highest performance PQT-12 OTFTs is: a silicon-rich $150{ }^{\circ} \mathrm{C} \mathrm{SiN}$. gate dielectric, with 80 seconds of $\mathrm{O}_{2}$ plasma exposure and OTS SAM as dielectric surface treatments, and with the omission of 1octanethiol contact surface treatment. A major outcome of this work is that it provides an economical means (and contrary to conventional perception) for organic transistor and circuit integration, by enabling use of the well-established PECVD infrastructure, yet not compromising the performance of electronics. The results demonstrated the viability of using PECVD $\operatorname{SiN}_{x}$ as the gate dielectric for OTFTs to deliver promising device performance. Furthermore, the low temperature and large area deposition capabilities of $\mathrm{SiN}_{\mathrm{x}}$ hold great promise for integration of OTFT circuits for large-area flexible electronics applications.

\section{Device Manufacture}

Establishment of robust, reliable, and scalable OTFT integration strategies is a prerequisite for full-scale deployment of organic electronics manufacturing. One of the key technological challenges lies in patterning of organic-based thin films. A number of OTFT fabrication schemes were developed in this thesis to produce organic circuits, including an all-photolithography processing scheme and a hybrid photolithography-inkjet printing processing scheme. The major conclusions drawn from the development/implementation of fabrication schemes for OTFT manufacture were:

\$ Photolithography enables precise definition/patterning of device layers with good resolution and registration. However, the adaption of photolithography for organic electronics 
fabrication requires judicious/meticulous planning and design of the processing sequence. It was concluded that photolithographic patterning of the organic semiconductor layer by incorporating a compatible buffer/passivation layer provides a viable approach to fabricate OTFTs. Fully-patterned bottom-gate parylene-passivated PQT-12 OTFTs displayed mobility comparable to unpassivated/unpatterned devices, validating the robustness of this photolithography approach. A variety of photolithographically-defined fully-patterned and fully-encapsulated bottom-contact OTFTs in bottom-gate, top-gate, and dual-gate configurations were also fabricated; this demonstrates the scalability/adaptability of our photolithography process for OTFT fabrication.

$\phi$ The hybrid photolithography-inkjet printing fabrication scheme combines the highresolution and multilayer-registration capabilities of photolithography with the direct and non-disruptive patterning of organic-based thin films by inkjet printing. OTFTs produced by this hybrid scheme with inkjet printed organic semiconductor exhibited reduced mobility compared to OTFTs with spin-coated organic semiconductor layer; the inferior performance is attributed to non-uniformity, inhomogeneity and discontinuity of the inkjet printed organic semiconductor layer. Device improvements can be achieved through improved control and optimization of printing parameters and ink formulation. It is concluded that this hybrid fabrication scheme is attractive because it presents a simple and effective interim method to fabricate OTFT circuits for preliminary evaluation and prototyping.

Although inkjet printing is envisioned to be one of the key manufacturing standards for future generations of organic electronics, our photolithography and inkjet printing integration schemes present an interim alternative to enable immediate fabrication of highly integrated and high resolution OTFT circuits, to facilitate timely evaluation of organic device/circuit behavior, and more importantly, to bridge the technological transfer and development towards an all-printed fabrication process for the near future.

\section{$\underline{\text { Device Integration }}$}

PQT-12 OTFT circuits, including inverters, current mirrors, and various display pixel circuits, were successfully fabricated using an all-photolithography integration schemes and a hybrid photolithography-inkjet printing integration approach. Optimized recipes for PECVD gate dielectric and interface treatments, as derived from our systematic investigations, were applied to implement these preliminary circuits. Proper circuit functionality was demonstrated, which proves 
the feasibility of practical organic circuits using the integration approaches developed in this research. Further process, device, and material optimizations are necessary to boost the performance of OTFT circuits and to deploy these integration strategies for larger scale implementation of organic circuits and systems. It was recommended that researchers draw attention to the development of compatible backend integration processes (e.g., die bonding and packaging), in order to provide a total manufacturing solution for deployment/commercialization of organic electronics in the near future.

The future looks very promising for organic electronics. Continued growth and development in this technological field can be anticipated, and is fueled by the promise of new products and applications that can be derived from electrically and optically active organic and hybrid materials. These include flexible displays for electronic newspapers, low-cost memory and logic devices, disposable products like smart luggage tags and RFID tags, bio-inspired detectors, and chemical or gas sensors. In order to fully realize the benefits of organic electronics, long-term research efforts and innovation are needed to provide functional organic materials with enhanced performance, processability, stability and lifetime. As material properties and integration strategies advance and mature over the next decades, organic electronics may displace traditional entrenched technologies in application areas requiring low cost, mechanical flexibility, and large area compatibility. There is little doubt that, with industry and academia pooling their resources, organic/plastic electronics will become part of our lives in the near future. 


\section{Chapter 9}

\section{Research Contributions}

This application-driven research is motivated by the need to advance organic electronics from fundamental research to reality, by contributing to OTFT integration for application development. This thesis focuses on advancing the area of OTFT research from an engineering/integration perspective, by applying existing materials, techniques, and scientific knowledge to deliver higher performance devices and demonstrate feasibility of practical organic circuits. This chapter summarizes the research contributions of this Ph.D. thesis that were described in detail in earlier chapters. These original contributions to the field of OTFT technology, as highlighted in Table 9.1, are grouped into four categories:

1) Fabrication/Integration Strategies

2) Gate Dielectric

3) Interface Engineering

4) Circuits and Systems 
Table 9.1. Summary of research contributions of this Ph.D. thesis.

\begin{tabular}{|c|c|c|c|}
\hline Contributions & Implications & Recommendations & $\begin{array}{l}\text { Industrial } \\
\text { Relevance }\end{array}$ \\
\hline \multicolumn{4}{|c|}{ 1) OTFT Fabrication/Integration Strategies } \\
\hline $\begin{array}{l}\text { Developed an OTFT- } \\
\text { compatible } \\
\text { photolithographic-based } \\
\text { fabrication process }\end{array}$ & $\begin{array}{l}\text { Enable integration of } \\
\text { higher complexity OTFT } \\
\text { devices \& circuits } \\
\text { Demonstrate that } \\
\text { photolithography of } \\
\text { organic materials is } \\
\text { possible, with sensible } \\
\text { design and planning }\end{array}$ & $\begin{array}{l}\text { Further development to } \\
\text { improve device } \\
\text { performance and yields } \\
\text { Expand scope of work } \\
\text { and evaluate other } \\
\text { masking materials and } \\
\text { patterning methods }\end{array}$ & $\begin{array}{l}\text { Provide an } \\
\text { immediate/interim } \\
\text { solution to } \\
\text { fabricate organic } \\
\text { circuits }\end{array}$ \\
\hline $\begin{array}{l}\text { Developed a hybrid } \\
\text { photolithography-inkjet } \\
\text { printing process }\end{array}$ & $\begin{array}{l}\text { Provide an immediate } \\
\text { solution to make high- } \\
\text { resolution OTFT } \\
\text { circuits, with efficient, } \\
\text { robust, direct } \\
\text { patterning of the } \\
\text { organic layer }\end{array}$ & $\begin{array}{l}\text { Processing technique } \\
\text { optimization } \\
\text { More in-depth research } \\
\text { into inkjet printing } \\
\text { technology to master } \\
\text { proper control of } \\
\text { processing parameters } \\
\text { for printing a wide } \\
\text { variety of materials }\end{array}$ & $\begin{array}{l}\text { Presents an interim } \\
\text { manufacturing } \\
\text { solution } \\
\text { Serve as a } \\
\text { technological- } \\
\text { bridge to an all- } \\
\text { printed } \\
\text { manufacturing } \\
\text { process }\end{array}$ \\
\hline \multicolumn{4}{|l|}{ 2) OTFT Gate Dielectric } \\
\hline $\begin{array}{l}\text { Integration of low } \\
\text { temperature PECVD as } \\
\mathrm{SiN}_{x} \text { gate dielectric for } \\
\text { OTFT; enabling } \\
\text { integration on plastic } \\
\text { substrates }\end{array}$ & $\begin{array}{l}\text { Demonstrate the } \\
\text { feasibility of using } \mathrm{SiN}_{x} \\
\text { for organic electronics } \\
\text { Show that } \mathrm{SiN}_{x} \text {-organic } \\
\text { interface can be } \\
\text { strategically modified } \\
\text { to deliver good } \\
\text { performance devices. }\end{array}$ & $\begin{array}{l}\text { Further improvement \& } \\
\text { optimization on the } \\
\text { quality of PECVD gate } \\
\text { dielectrics on plastic, to } \\
\text { enhance yield \& } \\
\text { integrity }\end{array}$ & $\begin{array}{l}\text { Enable functional } \\
\text { devices } \\
\text { Low temperature } \\
\text { and large area } \\
\text { deposition } \\
\text { capabilities of } \mathrm{SiN}_{\mathrm{x}} \\
\text { are promising for } \\
\text { large-area flexible } \\
\text { electronics } \\
\text { applications }\end{array}$ \\
\hline $\begin{array}{l}\text { Optimized } \mathrm{SiN}_{\mathrm{x}} \text { film } \\
\text { composition; concluded } \\
\text { that Si-rich } \mathrm{SiN}_{\mathrm{x}} \\
\text { delivered the highest } \\
\text { OTFT mobility, yielding a } \\
>2.25 \mathrm{x} \text { (or } 125 \% \text { ) } \\
\text { improvement in mobility } \\
\text { compared to N-rich } \mathrm{SiN}_{\mathrm{x}}\end{array}$ & $\begin{array}{l}\text { Present a technique } \\
\text { for improving device } \\
\text { performance, through } \\
\text { the discovery of a } \\
\text { dependence of OTFT } \\
\text { performance on SiNx } \\
\text { composition } \\
\text { Contribute to scientific } \\
\text { understanding }\end{array}$ & $\begin{array}{l}\text { - Investigate a larger } \\
\text { range of composition to } \\
\text { validate observed trend } \\
\text { - Study if there's a } \\
\text { "turnaround" or } \\
\text { "optimal" point, beyond } \\
\text { which device degrades. } \\
\text { - Examine effect of } \mathrm{SiN}_{\mathrm{x}} \\
\text { composition on OTFTs' } \\
\text { dynamic characteristics } \\
\text { and stability }\end{array}$ & $\begin{array}{l}\text { Enable functional } \\
\text { devices } \\
\text { Present a strategy } \\
\text { for improvements } \\
\text { in device } \\
\text { performance }\end{array}$ \\
\hline
\end{tabular}




\begin{tabular}{|c|c|c|c|c|c|c|}
\hline $\begin{array}{l}\text { Developed PECVD } \mathrm{SiO}_{x} \\
\text { gate dielectric for high } \\
\text { mobility PQT-12 OTFTs, } \\
\text { and demonstrated } \\
\text { integration on plastic } \\
\text { substrates }\end{array}$ & व & $\begin{array}{l}\text { Offer another PECVD } \\
\text { gate dielectric option } \\
\text { for OTFTs } \\
\text { More material options } \\
\text { means more possibility } \\
\text { to expand the } \\
\text { research potential for } \\
\text { specific needs of } \\
\text { different material } \\
\text { systems }\end{array}$ & 口 & $\begin{array}{l}\text { Work on improving } \mathrm{SiO}_{x} \\
\text { properties/integrity } \\
\text { (esp. leakage) } \\
\text { Study stacked } \mathrm{SiO}_{x} / \mathrm{SiN}_{x} \\
\text { gate dielectric structure } \\
\text { for device performance } \\
\text { enhancements }\end{array}$ & & $\begin{array}{l}\text { Present another } \\
\text { material options of } \\
\text { organic electronics } \\
\text { integration }\end{array}$ \\
\hline \multicolumn{7}{|c|}{ 3) OTFT Interface Engineering } \\
\hline $\begin{array}{l}\text { Developed a systematic } \\
\text { surface treatment } \\
\text { recipe for the } \mathrm{SiN}_{\mathrm{x}} \mathrm{PQT} \\
\text { interface }\end{array}$ & & $\begin{array}{l}\text { Enable integration of } \\
\text { SiNx and PQT-12 to } \\
\text { deliver high } \\
\text { performance OTFTs }\end{array}$ & a & $\begin{array}{l}\text { Expand the scope of } \\
\text { this investigation to } \\
\text { other treatment } \\
\text { techniques/materials }\end{array}$ & 口 & $\begin{array}{l}\text { Enable functional } \\
\text { devices }\end{array}$ \\
\hline $\begin{array}{l}\text { Determined a } \\
\text { sequence/combination } \\
\text { of surface treatment } \\
\text { steps to lead to }>27 x \\
\text { ( } 2614 \%) \text { increase in } \\
\text { mobility compared to } \\
\text { untreated device }\end{array}$ & a & $\begin{array}{l}\text { Present a technique } \\
\text { for improving device } \\
\text { performance } \\
\text { Advance scientific } \\
\text { understanding; } \\
\text { knowledge gained can } \\
\text { be useful for further } \\
\text { development of an } \\
\text { efficient interface } \\
\text { engineering technique }\end{array}$ & $\begin{array}{l}\square \\
\square\end{array}$ & $\begin{array}{l}\text { More in-depth analysis } \\
\text { Evaluate other } \\
\text { treatment options or } \\
\text { techniques to expand } \\
\text { scope of this research }\end{array}$ & 口 & $\begin{array}{l}\text { Present a strategy } \\
\text { for improvements } \\
\text { in device } \\
\text { performance }\end{array}$ \\
\hline $\begin{array}{l}\text { Determined an optimal } \\
\text { exposure duration for } \\
\mathrm{O}_{2} \text { plasma interface } \\
\text { treatment, resulting in } \\
>6 x(530 \%) \text { increase in } \\
\text { mobility. Higher RIE } \\
\text { power of } \mathrm{O}_{2} \text { plasma } \\
\text { exposure is also } \\
\text { desirable. }\end{array}$ & 口 & $\begin{array}{l}\text { Contribute to } \\
\text { development of an } \\
\text { optimized interface } \\
\text { engineering solution. } \\
\text { Advance scientific } \\
\text { understanding. }\end{array}$ & 口 & $\begin{array}{l}\text { Investigate impact of } \\
\text { other process } \\
\text { parameters (e.g., } \\
\text { pressure, power, flow } \\
\text { rate, ICP power, RIE } \\
\text { power) on device } \\
\text { performance. } \\
\text { Explore fluorination as } \\
\text { interface treatment } \\
\text { technique }\end{array}$ & 口 & $\begin{array}{l}\text { Present a strategy } \\
\text { for improvements } \\
\text { in device } \\
\text { performance }\end{array}$ \\
\hline
\end{tabular}




\begin{tabular}{|c|c|c|c|c|c|}
\hline $\begin{array}{l}\text { Observed 1-octanethiol } \\
\text { SAM contact treatment } \\
\text { causes undesirable } \\
\text { decrease in mobility and } \\
\text { increase in contact } \\
\text { resistance. Thus, thiol } \\
\text { treatment should be } \\
\text { omitted. }\end{array}$ & 口 & $\begin{array}{l}\text { Results suggest that } \\
\text { thiol treatment does } \\
\text { not always improve } \\
\text { performance as others } \\
\text { have claimed. } \\
\text { Selection of treatment } \\
\text { steps depends on the } \\
\text { overall process } \\
\text { sequence/conditions. } \\
\text { The impact of each } \\
\text { step should be } \\
\text { carefully evaluated. } \\
\text { Advance scientific } \\
\text { understanding. }\end{array}$ & व & $\begin{array}{l}\text { More analysis \& } \\
\text { characterization to } \\
\text { strengthen } \\
\text { understanding } \\
\text { Explore other contact } \\
\text { treatment methods } \\
\text { Optimize existing } \\
\text { treatment procedure to } \\
\text { lead to possible } \\
\text { improvements }\end{array}$ & $\begin{array}{l}\text { Present a strategy } \\
\text { for improvements } \\
\text { in device } \\
\text { performance }\end{array}$ \\
\hline
\end{tabular}

\section{4) OTFT Circuits}

$\begin{array}{ll}\text { Demonstrate OTFT } & \text { Present a process that } \\ \text { circuits with three } & \text { enables realization of } \\ \text { different fabrication } & \text { working organic circuit, } \\ \text { schemes based on } & \text { which is still one of the } \\ \text { photolithography and } & \text { pending challenges in } \\ \text { inkjet printing } & \text { the area of organic } \\ \text { technologies } & \text { electronics }\end{array}$

- Continual process development \& optimization.

- Experiment other innovative approaches

- Investigate n-type OTFT, to make complementary circuits
- Bring us one step closer to manufacturing of organic circuits for practical or commercial applications

\section{Demonstrate PQT-12}

OTFT circuits: including inverters, current mirrors, ring oscillators, display pixel circuits, imaging pixel circuits

\section{- Demonstrate} applicability of PQT-12 and $\mathrm{SiN}_{\mathrm{x}}$ for organic circuit applications

- Circuit characterization can strengthen scientific understanding of organic circuits and materials
Extensive circuit testing.

- Continual process development \& optimization.

- Fabricate a wider variety of circuits for different applications (e.g., RFID tags, chemical/gas sensors, bio-detectors, etc.)
Establish a template or platform for TFT/circuit fabrication that is compatible with a wide range of materials including organic semiconductors, nanomaterials, nanocomposites, etc.
- Enable convenient characterization of new materials and evaluation of the applicability for various circuits and/or device structures
- Roll-out more wafers, design a testing template, offer services to characterize new materials for other research groups
- Bring us one step closer to manufacturing of organic circuits for practical or commercial applications
- Provide research community a method to easily evaluate their materials (organic, nano-materials) in device or circuit configuration 


\subsection{List of Publications}

A list of relevant publications resulting from this $\mathrm{Ph}$.D. research is presented below.

- F.M. Li, Y. Wu, B.S. Ong, Y. Vygranenko, and A. Nathan, "Study of PECVD Silicon Nitride and Silicon Oxide Gate Dielectrics for Organic Thin-Film Transistor Circuit Integration," Material Research Society Symposium Proceeding 1003E, 1003-O06-49 (2008).

- F.M. Li, P. Dhagat, G.E. Jabbour, H.M. Haverinen, I. McCulloch, M. Heeney, and A. Nathan, "Polymer TFT without Surface Pre-Treatment on Silicon Nitride Gate Dielectric," Applied Physics Letter 92 (2008). (Accepted for publication. Article in press)

- G.W. Hsieh, P. Beecher, F.M. Li, P. Servati, A. Colli, A. Fasoli, D. Chu, A. Nathan, B. Ong, J. Robertson, A.C. Ferrari, and W.I. Milne, "Formation of composite organic thin film transistors with nanotubes and nanowires," Physica E: Low-dimensional Systems and Nanostructures, (2007). (Accepted for publication. Article in Press).

- M.R. Esmaeili-Rad, F.M. Li, A. Sazonov, and A. Nathan, "Stability of nanocrystalline silicon bottom-gate thin film transistors with silicon nitride gate dielectric," Journal of Applied Physics 102, 064512 (2007).

- P. Beecher, P. Servati, A. Rozhin, A. Colli, V. Scardaci, S. Pisana, T. Hasan, A. J. Flewitt, J. Robertson, G. W. Hsieh, F. M. Li, A. Nathan, A. C. Ferrari and W. I. Milne, Ink-jet printing of carbon nanotube thin film transistors", Journal of Applied Physics 102, 043710 (2007).

- F.M. Li, A. Nathan, Y. Wu, and B. Ong. "Organic Thin-Film Transistor Integration using Silicon Nitride Gate Dielectric," Applied Physics Letter 90, 133514 (2007).

- F.M. Li, Y. Vygranenko, S. Koul, and A. Nathan, "Photolithographically Defined Polythiophene OTFTs." Journal of Vacuum Science A: Vacuum, Surfaces, and Films 24, 657(2006).

- F.M. Li, S. Koul, Y. Vygranenko, P. Servati and A. Nathan, "Dual-Gate $\mathrm{SiO}_{2} / \mathrm{P} 3 \mathrm{HT} / \mathrm{SiN}_{\mathrm{x}}$ OTFT," Material Research Society Symposium Proceeding 871E, I9.4.1 (2005).

- F.M. Li, S. Koul, Y. Vygranenko, A. Sazonov, P. Servati and A. Nathan, "Fabrication of RRP3HT-based TFTs using low-temperature PECVD silicon nitride," Material Research Society Symposium Proceeding 871E, I9.3.1 (2005).

\section{Manuscripts in progress:}

- F.M. Li, Y. Wu, B. Ong, Y. Vygranenko, and A. Nathan, "Comparative Study of PECVD Gate Dielectric for Polymer Thin Film Transistor Integration." Work-in-progress, 2008.

- F.M. Li, Y. Wu, B. Ong, and A. Nathan, "Evaluation of Surface Treatment Type, Sequence, Duration, and Combination on Polymer Thin Film Transistors.” Work-in-progress, 2008.

- F.M. Li, Y. Wu, B. Ong, and A. Nathan, "Hybrid Inkjet-Photolithography Approach for Logic and Active-Matrix Pixel Polymer Circuits." Work-in-progress, 2008. 


\section{Glossary}

\section{Abbreviations:}

$\mathrm{AC}$

AFM

$\mathrm{Ag}$

$\mathrm{Al}$

$\mathrm{Al}_{2} \mathrm{O}_{3}$ or $\mathrm{AlO}_{\mathrm{x}}$

ALD

AMLCD

AMOLED

a-Si:H or a-Si

$\mathrm{Au}$

$\mathrm{BCB}$

C60

CMOS

CNT

CT

CTC

$\mathrm{Cu}$

C-V

CVD

D6HT

DC

DFH-4T
Alternating current

Atomic force microscopy

Silver

Aluminum

Aluminum oxide

Atomic layer deposition

Active-matrix liquid crystal display

Active-matrix organic light emitting diode

Amorphous silicon

Gold

Benzocyclobutene

Fullerene

Complementary metal oxide semiconductor

Carbon nanotube

Charge transfer

Charge transfer complex

Copper

Capacitance-voltage characteristics

Chemical vapor deposition

Dihexyl-sexithiophene

Direct current

Diperflurorohexylquarter-thiophene 


\begin{tabular}{|c|c|}
\hline DIP & Dual in-line package \\
\hline DOS & Density of states \\
\hline Dpi & Dots per inch \\
\hline EDM & Electro-discharge machining \\
\hline E-Paper & Electronic paper \\
\hline ERDA & Elastic recoil detection analyses \\
\hline $\mathrm{F}_{16} \mathrm{CuPc}$ & Hexadecafluoro-phthalocyanine \\
\hline F8T2 & Poly $(9,9$ '-dioctyl-fluorene-co-bithiophene) \\
\hline FTIR & Fourier transform infrared spectroscopy \\
\hline GIXRD & Grazing-(or glacing) incidence $\mathrm{x}$-ray diffraction \\
\hline $\mathrm{HF}$ & Hydrofluoric acid \\
\hline HMDS & Hexamethyldisilazane \\
\hline HOMO & Highest occupied molecular orbital \\
\hline IC & Integrated circuit \\
\hline $\mathrm{ICP}$ & Inductively coupled plasma \\
\hline IEEE & Institute of Electrical and Electronics Engineers \\
\hline IJP & Inkjet printing \\
\hline IP & Ionization potential \\
\hline $\mathrm{I}-\mathrm{V}$ & Current-voltage characteristics \\
\hline $\mathrm{LCD}$ & Liquid crystal display \\
\hline LUMO & Lowest unoccupied molecular orbital \\
\hline MIS & Metal-insulator-semiconductor \\
\hline MOS & Metal-oxide-semiconductor \\
\hline MNB & 2-Mercapto-5-Nitro-Benzimidazole \\
\hline Mo & Molybdenum \\
\hline MOSFET & Metal oxide semiconductor field effect transistor \\
\hline MTR & Multiple trapping and release model \\
\hline $\mathrm{N}_{2}$ & Nitrogen \\
\hline $\mathrm{NH}_{3}$ & Ammonia \\
\hline NMOS & N-channel or n-type metal oxide semiconductor \\
\hline NW & Nanowire \\
\hline $\mathrm{O}_{2}$ plasma & Oxygen plasma \\
\hline
\end{tabular}




\begin{tabular}{|c|c|}
\hline ODTS & Octadecyltrichlorosilane \\
\hline OFET & Organic field effect transistor \\
\hline OLED & Organic light emitting Diode \\
\hline OTFT & Organic thin film transistor \\
\hline OTS or OTS-8 & Octyltrichlorosilane \\
\hline P3HT & Poly(3-hexylthiophene) \\
\hline PA & Polyacetylene \\
\hline PANI & Polyaniline \\
\hline PBTTT & $\operatorname{Poly}(2,5$-bis(3-alkylthiophen-2-yl)thieno[3,2-b]thiophene) \\
\hline PCBM & Phenyl-C61-butyric acid methyl ester \\
\hline PECVD & Plasma enhanced chemical vapor deposition \\
\hline PEDOT:PSS & Poly(3,4-ethylene dioxythiophene) doped with polystyrene sulfonic acid \\
\hline PEN & Poly(ethylene naphthalate) \\
\hline PET & Poly(ethylene terephthalate) \\
\hline Ph.D. & Doctor of philosophy \\
\hline PI & Polyimide \\
\hline PMMA & Poly(methyl methacrylate) (PMMA) \\
\hline PPV & Poly(p-phenylene vinylene) or polyphenylene vinylene \\
\hline PQT & Poly(3,3"'-dialkylquarterthiophene) \\
\hline $\mathrm{Pt}$ & Platinum \\
\hline PT & Polythiophene \\
\hline PTV & Poly(thienylene vinylene) \\
\hline PVA & Polyvinyl acetate or polyvinyl alcohol \\
\hline R\&D & Research and Development \\
\hline RCA clean & $\begin{array}{l}\text { A standard set of wafer cleaning steps; RCA }=\text { Radio Corporation of } \\
\text { America }\end{array}$ \\
\hline RF & Radio frequency \\
\hline RFID & Radio frequency identification \\
\hline RIE & Reactive ion etching \\
\hline SAM & Self-assembled monolayer \\
\hline $\mathrm{SiH}_{4}$ & Silane \\
\hline $\mathrm{SiN}_{\mathrm{x}}$ & Silicon nitride \\
\hline
\end{tabular}




$\begin{array}{ll}\mathrm{SiO}_{2} & \text { Silicon dioxide } \\ \mathrm{SiO}_{x} & \text { Silicon oxide } \\ \mathrm{SnO}_{2} & \text { Tin oxide } \\ \mathrm{TFT} & \text { Thin film transistor } \\ \mathrm{TiO}_{2} & \text { Titanium oxide } \\ \mathrm{UV} & \text { Ultraviolet } \\ \mathrm{UW} & \text { University of Waterloo } \\ \mathrm{XPS} & \text { X-ray photoelectron spectroscopy } \\ \text { XRCC } & \text { Xerox Research Centre of Canada } \\ \text { ZnO } & \text { Zinc oxide }\end{array}$

\section{Mathematic Symbols:}

$\begin{array}{ll}\varphi_{\mathrm{B}} & \text { Injection barrier } \\ \Phi_{\mathrm{M}} & \text { Work Function of the electrode (metal) } \\ {[\mathrm{N}] /[\mathrm{Si}]} & \text { Nitrogen to silicon ratio, to describe stoichiometry or composition of } \mathrm{SiN}_{\mathrm{x}} \\ \mu_{\mathrm{FET}} & \text { Field effect mobility } \\ C_{\mathrm{i}} & \text { Gate capacitance per unit area } \\ C_{\mathrm{S}} & \text { Storage capacitor } \\ E_{\mathrm{G}} & \text { Band-gap energy } \\ f_{\mathrm{max}} & \text { Maximum switching frequency } \\ \mathrm{g}_{\mathrm{m}} & \text { Transconductance } \\ I_{\mathrm{D}} & \text { Drain current } \\ I_{\mathrm{G}} & \text { Gate current } \\ I_{\text {leak }} & \text { leakage current } \\ I_{\mathrm{OFF}} & \text { Off Current } \\ I_{\mathrm{ON}} & \text { On Current } \\ I_{\mathrm{ON}} / I_{\mathrm{OFF}} & \text { On/Off Current Ratio } \\ I_{\mathrm{S}} & \text { Source current } \\ \mathrm{IP}_{\mathrm{S}} & \text { ionization potential of the semiconductor } \\ L & \text { Channel length } \\ R_{\mathrm{CONTACT}} & \text { Contact resistance }\end{array}$




$\begin{array}{ll}S & \text { Inverse subthreshold slope (V/dec) } \\ \tau & \text { Transit time } \\ V_{\mathrm{BG}} & \text { Bottom-gate voltage } \\ V_{\mathrm{DD}} & \text { Positive supply voltage } \\ V_{\mathrm{DS}} & \text { Drain-source voltage } \\ V_{\mathrm{GS}} & \text { Gate-source voltage } \\ V_{\mathrm{ON}}, V_{\mathrm{SO}} & \text { Onset voltage or switch-on voltage } \\ V_{\mathrm{SS}} & \text { Negative supply voltage } \\ V_{\mathrm{T}} & \text { Threshold voltage } \\ V_{\mathrm{TG}} & \text { Top-gate voltage } \\ W & \text { Channel width }\end{array}$

\section{Definitions:}

(Definitions of selected terms were cited from Wikipedia webpage. http://en.wikipedia.org/wiki/Main_Page.)

Alkanes
(also Alkyl)

(CT complex):

Conductive Polymer:

(also Conducting

Polymer)
Chemical compounds that consist only of the elements carbon (C) and hydrogen (H) (i.e., hydrocarbons), wherein these atoms are linked together exclusively by single bonds (i.e., they are saturated compounds) without any cyclic structure (i.e. loops). An alkyl group is a functional group or side-chain that, like an alkane, consists solely of singly-bonded carbon and hydrogen atoms

An electron donor-electron acceptor complex, characterized by electronic transition(s) to an excited state. In this excited state, there is a partial transfer of elementary charge from the donor to the acceptor. A CT complex composed of the tetrathiafulvalene (TTF, a donor) and tetracyanoquinodimethane (TCNQ, an acceptor) was discovered in 1973. This was the first organic conductor to show almost metallic conductance.

Polymer that is made conducting, or "doped," by reacting the conjugated semiconducting polymer with an oxidizing agent, a reducing agent, or a protonic acid, resulting in highly delocalized polycations or polyanions. The conductivity of these materials can be tuned by chemical manipulation of the polymer backbone, by the nature of the dopant, by the degree of doping, and by blending with other polymers. Conductive polymer is an organic polymer semiconductor, or an organic semiconductor. 
Conjugated Polymer:

Dielectric:

(also Insulator)

Electrode:

(also Contact)

Insulator:

(also Dielectric)

Mobility:

(also Carrier mobility, Field-effect mobility, Effective mobility)

Organic Compounds:

Organic Electronics: (also Plastic

Electronics)
A system of atoms covalently bonded with alternating single and double carbon-carbon (sometimes carbon-nitrogen) bonds in a molecule of an organic compound. This system results in a general delocalization of the electrons across all of the adjacent parallel aligned p-orbitals of the atoms, which increases stability and thereby lowers the overall energy of the molecule.

A non-conducting substance, i.e. an insulator. Although "dielectric" and "insulator" are generally considered synonymous, the term "dielectric" is more often used when considering the effect of alternating electric fields on the substance while "insulator" is more often used when the material is being used to withstand a high electric field. Dielectric encompasses the broad expanse of nonmetals (including gases, liquids and solids) considered from the standpoint of their interaction with electric, magnetic, of electromagnetic fields. In this thesis, the terms "dielectric" and "insulator" are used interchangeably.

An electrical conductor (e.g., metallization) used to make contact with a nonmetallic part of a circuit (e.g., a semiconductor). The gate/source/drain metal layer of the TFT is referred to as electrode. The connection between the source/drain metal layer with the semiconductor layer (i.e., when we speak of the interface) is referred to as "contact". In this thesis, the terms "electrode" and "contact" are used almost interchangeably.

A material that resists the flow of electric current. It is an object intended to support or separate electrical conductors without passing current through itself. An insulation material has atoms with tightly bonded valence electrons. The term electrical insulation often has the same meaning as the term dielectric.

The state of being in motion. Carrier mobility is a quantity relating the drift velocity of electrons or holes to the applied electric field across a material; this is a material property. Field-effect mobility or effective mobility describes the mobility of carriers under the influence of the device structure in field-effect transistors. Field-effect mobility is device-specific, not material-specific, and includes effects such as contact resistances, surface effects, etc

Chemical compounds containing carbon-hydrogen $(\mathrm{C}-\mathrm{H})$ bonds of covalent character.

A branch of electronics that deals with conductive polymers, plastics, or small molecules. It is called 'organic' electronics because the polymers and small molecules are carbon-based, like the molecules of living things. This is as opposed to traditional electronics which relies on inorganic conductors such as copper or silicon. 
Organic Semiconductor: Any organic material that has semiconductor properties. Both short chain (oligomers) and long chain (polymers) organic semiconductors

(also Polymer

Semiconductor)

OTFT:

(also OFET)

Plastic:

Polymer: are known. There are two major classes of organic semiconductors, which overlap significantly: organic charge-transfer complexes, and various "linear backbone" polymers derived from polyacetylene. This thesis focuses on investigation of polymer organic semiconductors; thus, in most cases, the term "organic semiconductor" and "polymer semiconductor" are used interchangeably.

An organic-thin film transistor (OTFT) or organic field-effect transistor (OFET) is a field effect transistor using an organic semiconductor in its channel.

A general term for a wide range of synthetic or semi-synthetic polymerization products. Plastics are polymers, i.e., long chains of atoms bonded to one another.

A substance composed of molecules with large molecular mass composed of repeating structural units, or monomers, connected by covalent chemical bonds. 